\title{
Television Standards Conversion
}

Tim Borer

October 1992 
ProQuest Number: 11009909

All rights reserved

INFORMATION TO ALL USERS

The quality of this reproduction is dependent upon the quality of the copy submitted.

In the unlikely event that the author did not send a complete manuscript and there are missing pages, these will be noted. Also, if material had to be removed, a note will indicate the deletion.

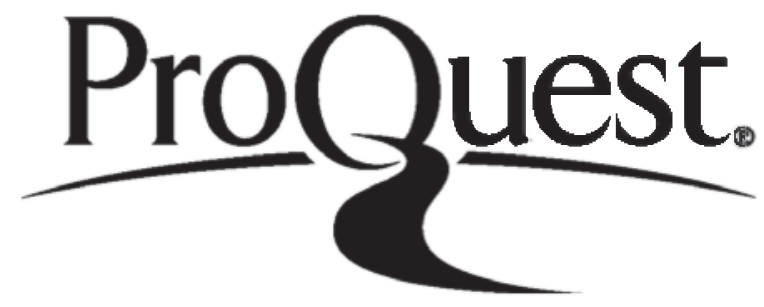

ProQuest 11009909

Published by ProQuest LLC (2018). Copyright of the Dissertation is held by the Author.

All rights reserved.

This work is protected against unauthorized copying under Title 17, United States Code Microform Edition (C) ProQuest LLC.

ProQuest LLC.

789 East Eisenhower Parkway

P.O. Box 1346

Ann Arbor, Ml $48106-1346$ 


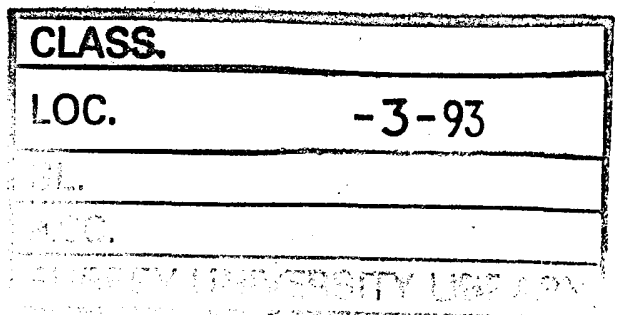

\section{Television Standards Conversion}

by

Tim Borer

A thesis submitted for the degree of

Doctor of Philosophy

Supervised by

Josef Kittler

Department of Electronic and Electrical Engineering

University of Surrey

Guildford, Surrey, GU2 5XH, U.K.

and

Ian Childs

Research Department

British Broadcasting Corporation

Kingswood Warren, Tadworth, Surrey, KT20 6NP, U.K. 


\section{Abstract}

This thesis investigates the process of television standards conversion. That is converting a television signal originated in one standard for display in another standard. A typical example of this process is converting between European television, with 625 lines and 25 frames/second, and American television, with 525 lines and 30 frames/second. Although European/American interconversion is the best known type of standards conversion many other types are becoming necessary or desirable. These other standards conversion processes are between the burgeoning number of standards for high definition television, computer graphics as well as conventional television.

The standards conversion process is, essentially, one of resampling a three dimensional sampled signal on a new sampling lattice. In one dimension the analogous process of sample rate changing is well understood. For standards conversion the theory of sample rate changing must be extended to three dimensions. Television standards conversion is much more difficult than sample rate changing an audio signal. This is partly because of the signal is 3 dimensional and partly because the sampling rates are orders of magnitude greater. The most significant problem of standards conversion, however, is the fact that television signals are undersampled spatially and, most significantly, temporally. Undersampling in television signals results in aliasing which confounds the assumptions underlying the theory of sample rate changing.

At the start of this work the state of the art in television standards conversion involved interpolation of the signal using a 16 tap, 2 dimensional, finite impulse response filter. The filter coefficients were determined empirically to minimise the picture artifacts caused by the aliasing inherent in the signal. The first purpose of the work described here was to analyse the standards conversion process and develop objective methods of optimising the performance of the existing type of standards converters. It was likely that even optimised standards converters of this type would generate undesirable artifacts in their output pictures. Therefore, the second purpose of the work was to develop improved techniques for standards conversion.

To optimise the performance of conventional standards converters it is necessary to analyse the component parts of the television signal chain. Of particular significance for standards conversion are the characteristics of real scenes and the way in which the eye perceives them. The thesis starts with an analysis of the television signal chain. Experimental work confirms previously published results regarding the spectral content of typical scenes and extends them from 2 to 3 dimensions. A model is derived to describe these results and a theoretical justification is given for this model. The frequency response of the human visual system is a important part of the television signal chain and a model is presented for this response, distilled from an extensive review of the literature. 
A new method is presented for optimising the performance of conventional standards converters using the preceding analysis of the television signal chain. The optimisation technique defines an 'ideal' interpolation filter response which simultaneously minimises picture impairments due to loss of detail and aliasing. For practical standards converters it is necessary to produce an optimal realisable filter which approximates the 'ideal' response. Several new techniques are presented for generating optimum, practical, finite impulse response filters. Although the filter design process is, essentially, performed in the frequency domain some of the filter design methods also allow the inclusion of time domain constraints.

There are many ways in which practical television standards conversion hardware can be built. In practice only a very few of the possibilities have been explored. The best way to build a standards converter depends on the type of conversion (eg whether the number of lines is increased or decreased) and the commercially available integrated circuit building blocks. The various ways in which standards converters can be built is examined in a unified and systematic way. This allows the best standards conversion architecture to be selected for a particular application.

Having considered the design and implementation of interpolation filters for standards converters, 2 examples are tested experimentally. The process of increasing the picture rate to reduce display flicker is investigated using software simulation and European/American interconversion is investigated using real time hardware. These experiments confirm that it is possible to objectively design filter coefficients for standards converters. The time consuming empirical design techniques used previously can thus be avoided. State of the art, empirically designed, filters for European/American interconversion provide near optimal performance for this type of standards conversion. However significant, undesirable, picture artifacts remain, prompting investigation of improved standards conversion techniques.

The worst aliasing in television signals is usually temporal aliasing engendered by the movement of objects within the scene. By analysing this motion and compensating for it, the worst effects of temporal aliasing may be ameliorated. This work investigates whether this can be done and the performance of such a motion compensated standards converter.

The most difficult part of the motion compensated approach to standards conversion is analysis of movement in a picture. The myriad different algorithms for motion estimation were reviewed and their suitability for motion compensation considered. One technique, that of 'phase correlation' was selected for experimental software simulation. The results of these experimental simulations both confirmed and extended previously published results, confirming the suitability of this algorithm for motion compensated standards conversion. In addition a new motion estimation algorithm was developed and simulated in software. This new algorithm appeared to have significantly better performance than the 'phase correlation' algorithm. 
Real time motion estimation hardware was designed and built based on the 'phase correlation' algorithm. This was interfaced to two different experimental, real time, motion compensated standards converters. One standards converter was designed for increasing the picture rate to reduce display flicker while the other was designed for European/American television conversion. These experimental real time systems were amongst the first to implement such techniques. Using this equipment is was possible to assess the performance of motion estimation and motion compensated standards conversion on a wide range of television pictures unfettered by the storage and processing constraints of computer systems.

Results from these experimental standards conversion systems were very encouraging. Initial results emphasised the importance of the motion estimation system and its interface to the interpolation hardware. Once modifications had been made to this interface very encouraging results were achieved for both types of standards conversion. The results suggest that much improved standards conversion can be achieved using motion compensation, albeit at the cost of considerable extra complexity. Improved standards conversion will become both increasingly possible and increasingly necessary as the fields of computing and communication converge. 


\section{Acknowledgements}

I would like to express my thanks to my two supervisors, Josef Kittler and Ian Childs, for their help and support in this research. Josef provided the opportunity for the research and Ian allowed me the freedom to pursue it, even though this was not always easy. Many thanks.

I must also thank my colleagues and friends Dick Storey, Graham Thomas and Steve Dabner, with whom I spent much time discussing, designing and building the motion estimation system described in this thesis. Thanks also to Martin Weston for some of his thoughts and ideas on motion estimation and filter design.

Many thanks to my wife Sally, who allowed me to write this thesis free from the all too friendly attention of our two young sons.

The research described here was undertaken as a collaboration between Surrey University and the BBC Research Department. Many thanks to the British Broadcasting Corporation, for their support and for providing the facilities which enabled me to undertake this work.

Thanks to Philips Research Laboratories and, in particular, Dave Parker and Mike Hulyer with whom I worked on motion compensated display field rate upconversion.

Thanks to the Vistek company for providing the experimental motion compensated standards converter. Thanks also to Snell \& Wilcox for the opportunity to test filter coefficients in their standards converter. 


\section{Contents}

1. Introduction $\ldots \ldots \ldots \ldots \ldots \ldots \ldots \ldots \ldots \ldots \ldots \ldots \ldots \ldots \ldots \ldots \ldots \ldots \ldots$

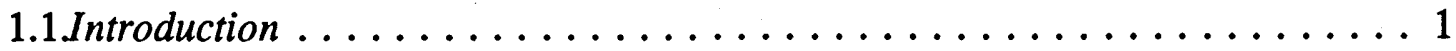

1.2.The problem of standards conversion $\ldots \ldots \ldots \ldots \ldots \ldots \ldots \ldots \ldots$

1.3.The historical development of standards conversion $\ldots \ldots \ldots \ldots \ldots \ldots 4$

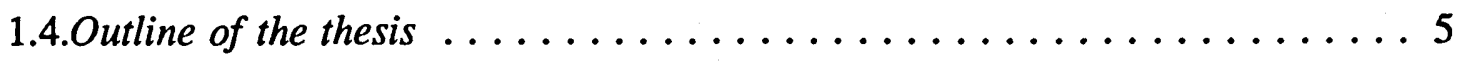

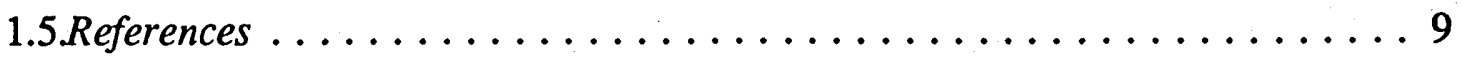

2. The Television Signal Chain $\ldots \ldots \ldots \ldots \ldots \ldots \ldots \ldots \ldots \ldots \ldots \ldots$

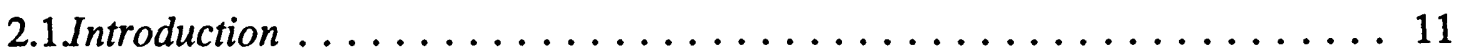

2.2.The Spectral Content of Scenes .................. 12

2.2.1. Experimental Investigation $\ldots \ldots \ldots \ldots \ldots \ldots \ldots \ldots \ldots$

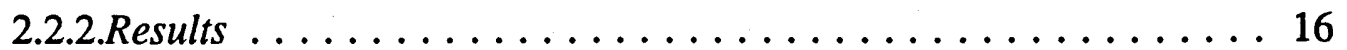

2.2.3.Discussion \& Conclusions .................... 19

2.2.4.Appendix : Fractal models for $3 D$ scene spectra . . . . . . 24

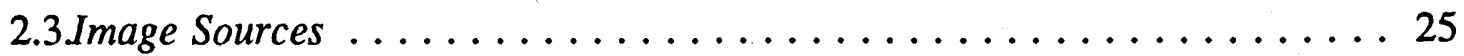

2.3.1.Cameras ......................... 27

2.3.1.1.Vacuum Tube Sensors ............... 28

2.3.1.2.Charge Coupled Devices ................. 29

2.3.2.Telecines ...................... 30

2.4.Display Devices ........................ 31

2.5.The Human Visual System . . . . . . . . . . . . . . 32

2.5.1.Frequency response of the stationary 'eye' $\ldots \ldots \ldots \ldots \ldots 34$

2.5.2. Chrominance response of the eye $\ldots \ldots \ldots \ldots \ldots \ldots \ldots . \ldots 38$

2.5.3.Effect of Motion ................... 40

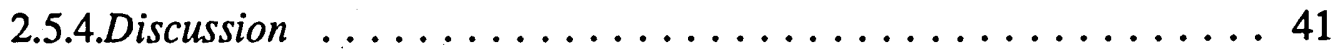

2.6.Summary ......................... 42

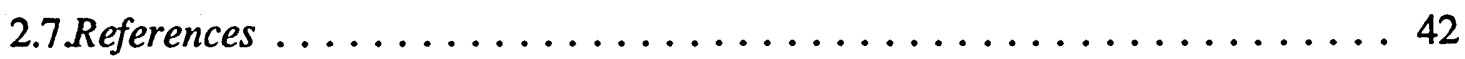

3. Filter Design for Standards Converters $\ldots \ldots \ldots \ldots \ldots \ldots \ldots \ldots \ldots \ldots$

3.1.Introduction ......................... 46

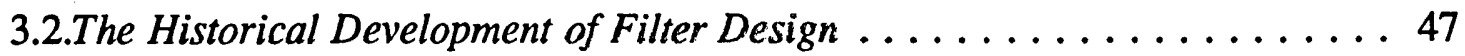

3.2.1.The Brillouin zone as an 'ideal' filter characteristic ....... 48

3.2.2. Frequency sampling design : fixed (frequency) sampling lattice . 50

3.2.3. Frequency sampling design : variable sampling sites ...... 52

3.2.4. Frequency sampling design : overdetermined specification .... . 54

3.3. 'Pseudo' Wiener Filters as 'Ideal' Filters . . . . . . . . . . . . 57 
3.4. 'Optimum' approximations to 'Ideal' Filters . . . . . . . . . . 61

3.4.1. Discrete LMS approximations . . . . . . . . . . . 61

3.4.2. Continuous LMS approximations . . . . . . . . . . . 62

3.4.3. Time domain constraints . . . . . . . . . . . . 64

3.4.4. Eigenfilters ...................... 66

3.5. The Design of Adaptive and Motion Compensated Filters . . . . . . . 69

3.6. Summary .......................... 72

3.7. References ......................... 73

4. The Implementation of Standards Converters $\ldots \ldots \ldots \ldots \ldots \ldots \ldots \ldots$

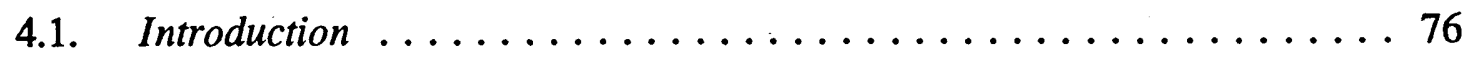

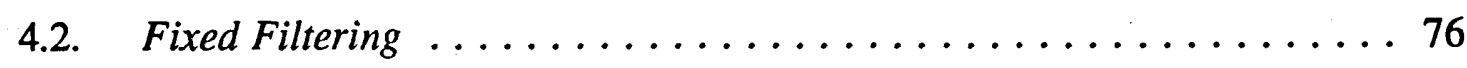

4.3. Sample Rate Changing .................... 78

4.4. Aperture Quantisation .................... 83

4.5. Multidimensional Filtering ................ 86

4.6. Scanning Standards ..................... 88

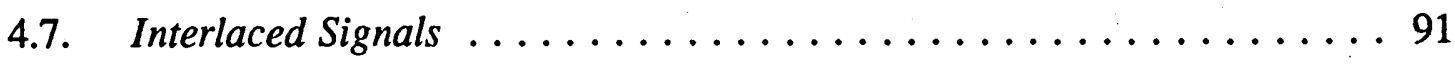

4.8. Motion Compensation ...................... 92

4.9. Summary \& Conclusions . . . . . . . . . . . . . . . . . . 94

4.10. References .......................... 95

4.11. Appendix : Calculating the aperture position ............ 96

5. The Performance of Conventional Standards Converters . . . . . . . . . . . . . 99

5.1. Introduction ........................ 99

5.2. Field rate upconversion . . . . . . . . . . . . . . . . . 99

5.2.1. Introduction . . . . . . . . . . . . . . 99

5.2.2. Filter Design ...................... 100

5.2.3. Experimental Procedure . . . . . . . . . . . . . . . 103

5.2.4. Results, Discussion \& Conclusions . . . . . . . . . . . . . 104

5.3. Intercontinental standards conversion . . . . . . . . . . . . . 104

5.3.1. Introduction . . . . . . . . . . . . . . . . 104

5.3.2. Filter Design ..................... 105

5.3.3. Experimental Procedure . . . . . . . . . . . . . . 107

5.3.4. Results, Discussion \& Conclusions . . . . . . . . . . . 107

5.4. Summary .......................... 110

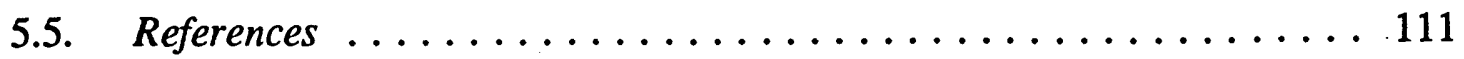

6. The Principles of Motion Compensation . . . . . . . . . . . . . . 112 
6.1. Introduction ......................... 112

6.2. The perception of motion in television pictures ............ 112

6.3. Motion compensated processing ................ 114

6.4. The Nyquist paradox ..................... 116

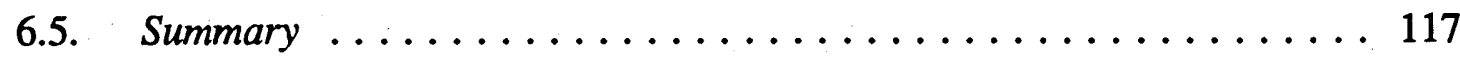

6.6. References ........................... 118

6.7. Appendix: The spectrum of a moving object $\ldots \ldots \ldots \ldots \ldots \ldots 118$

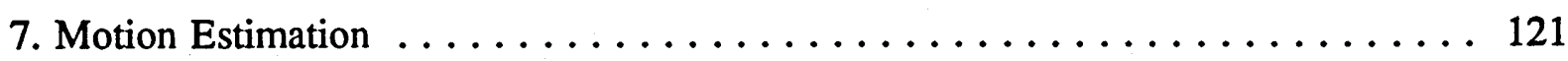

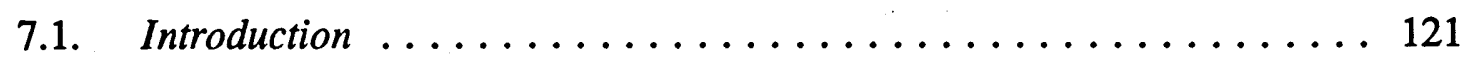

7.2. A Review of motion estimation algorithms ............. 125

7.2.1. Spatio-temporal constraint methods . . . . . . . . . . . 127

7.2.2. Region matching methods $\ldots \ldots \ldots \ldots \ldots \ldots \ldots \ldots \ldots$

7.2.3. Frequency domain methods . ............... 139

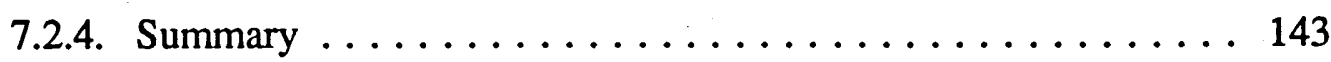

7.3 An experimental investigation of two motion estimation algorithms ... 143

7.3.1. Image Preprocessing . . . . . . . . . . . . . . 144

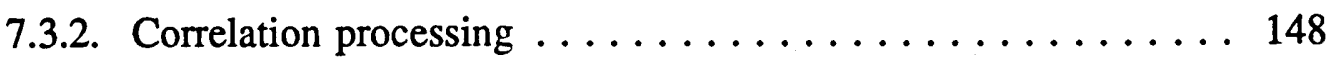

7.3.2.1. Phase Correlation ............... 148

7.3.2.2. Motion Correlation . .............. 149

7.3.3. Searching for correlation maxima $\ldots \ldots \ldots \ldots \ldots \ldots \ldots \ldots$

7.3.4. Results and Discussion .................. 154

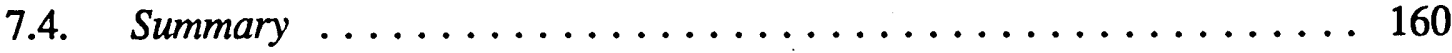

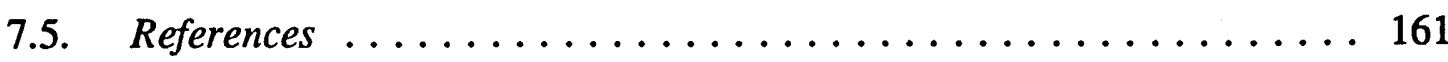

8. The Implementation of a Motion Estimator $\ldots \ldots \ldots \ldots \ldots \ldots \ldots \ldots \ldots$

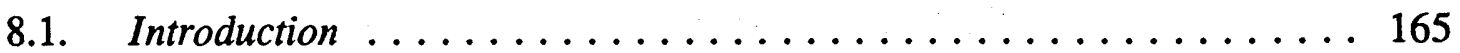

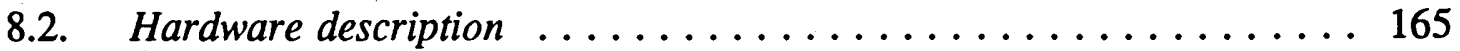

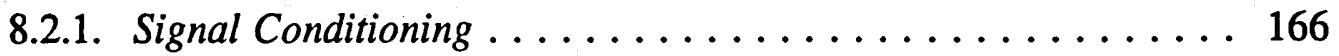

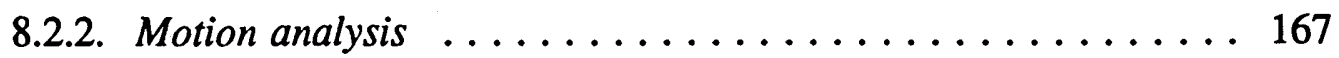

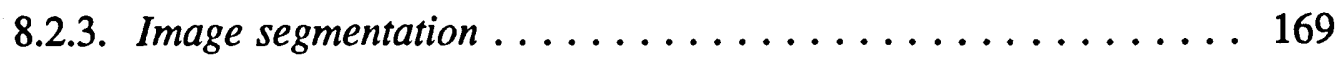

8.3. Results \& Discussion . . . . . . . . . . . . . . . 172

8.4. Summary \& Conclusions . . . . . . . . . . . . . . . 175

8.5. References .......................... 175

9. The Implementation of Motion Compensated Standards Conversion . . . . . . . 177

9.1. Introduction ....................... 177

9.2. Implementing Motion Compensation $\ldots \ldots \ldots \ldots \ldots \ldots \ldots \ldots \ldots$ 
9.3. The Interface between Motion Estimator and Standards Converter . . 180

9.4. Motion Compensated Display Field Rate Upconversion . . . . . . . . . 181

9.4.1. Implementation . . . . . . . . . . . . . . 182

9.4.2. Results, discussion and conclusions . . . . . . . . . 183

9.5. Motion Compensated, Intercontinental, Standards Conversion . . . . . 184

9.5.1. Implementation . . . . . . . . . . . . . . 185

9.5.2. Initial results $\ldots \ldots \ldots \ldots \ldots \ldots \ldots \ldots \ldots \ldots \ldots \ldots$

9.5.3. Improved motion estimation . . . . . . . . . . 187

9.5.4. Results, conclusions and discussion . . . . . . . . . . 188

9.6. Summary \& Conclusions . . . . . . . . . . . . . . . . . . . 191

9.7. References ........................... 192

10. Summary \& Conclusions . . . . . . . . . . . . . . . . . . 194

11. Appendix : Optimum Window Functions . . . . . . . . . . . . 203

11.1. Introduction ......................... 203

11.2. A Brief History of Window Functions . . . . . . . . . . . . . . 203

11.3. Applications of Window Functions . . . . . . . . . . . . . . . . . . 204

11.3.1. Filter design ................. 204

11.3.2. Signal segmentation ............... 206

11.4. Optimum Window Functions . . . . . . . . . . . . . . . . . 207

11.5. Multidimensional Window functions . . . . . . . . . . . . . . . 208

11.6. Filter Design Examples . . . . . . . . . . . . . . . . . . 210

11.7. Conclusions .......................... 212

11.8. References ........................... 213

11.9. Appendix 1: Some sub-optimal window functions . . . . . . . . 214

11.9.1. Rectangular Window .............. 215

11.9.2. Hamming Window ............... 216

11.9.3. Blackman Window .............. 216

11.9.4. Kaiser Window ................ 217

11.10. Appendix 2: Calculating optimum window functions ......... 218 


\section{Tables}

2. The Television Signal Chain

2.1. Model $1, \mathrm{x} / \mathrm{y}$ plane, gamma corrected pictures $\ldots \ldots \ldots \ldots \ldots$

2.2. Model 2, $\mathrm{x} / \mathrm{y}$ plane, gamma corrected pictures $\ldots \ldots \ldots \ldots \ldots$

2.3. Model 1, $\mathrm{x} / \mathrm{y}$ plane, linear pictures $\ldots \ldots \ldots \ldots \ldots \ldots \ldots \ldots$

2.4. Model $2, \mathrm{x} / \mathrm{y}$ plane, linear pictures $\ldots \ldots \ldots \ldots \ldots \ldots \ldots \ldots$

2.5. Model $1, \mathrm{x} / \mathrm{z}$ plane, gamma corrected pictures $\ldots \ldots \ldots \ldots$

2.6. Model 2, $x / z$ plane, linear pictures . . . . . . . . . . . . . . . 19

2.7. Model $1, \mathrm{x} / \mathrm{y}$ plane, gamma corrected pictures, from Chiminenti \& Picco . 22

2.8. Model 2, $\mathrm{x} / \mathrm{y}$ plane, gamma corrected pictures, from Chiminenti \& Picco . 22

2.9. Model parameters for the response of the human visual system . . . . . 37

3. Filter Design for Standards Converters

3.1. Frequency domain specification of a standards conversion filter . . . . . 52

4. The Implementation of Standards Converters

4.1. Filtering errors due aperture quantisation $\ldots \ldots \ldots \ldots \ldots \ldots$

4.2. Combinations of input and output scanning methods . . . . . . . . . 90

\section{Motion Estimation}

7.1. Comparison of peak location techniques . . . . . . . . . . . 153

7.2. Phase Correlation, horizontal pans . . . . . . . . . . . . 156

7.3. Phase Correlation, vertical tilts $\ldots \ldots \ldots \ldots \ldots \ldots \ldots \ldots \ldots$

7.4. Motion Correlation, horizontal pans . . . . . . . . . . . . . 157

7.5. Motion Correlation, vertical tilts . . . . . . . . . . . . . 157

7.6. Phase Correlation, effect of input noise . . . . . . . . . . . . 159

7.7. Motion Correlation, effect of input noise .............. 159

11. Appendix: Optimum Window Functions

11.1. Performance of 1D Windowed Filter Designs . . . . . . . . . . 211

11.2. Performance of $2 \mathrm{D}$ Windowed Filter . . . . . . . . . . . 212 


\section{Figures}

2. The Television Signal Chain $\ldots \ldots \ldots \ldots \ldots \ldots \ldots \ldots \ldots \ldots \ldots$

2.1. The signal chain

2.2. Mean measured spatial autocorrelation function

2.3. Mean measured spatio/temporal autocorrelation function

2.4. Signal chain within a typical colour television camera

2.5. Signal chain within a telecine

2.6. Formation of luminance and chrominance signals in the human visual system

2.7. Model of response of eye

2.8. Spatio/temporal response of the eye: model of Kelly's data

2.9. Spatio/temporal response of the eye: model of Robson's data

3. Filter Design for Standards Converters $\ldots \ldots \ldots \ldots \ldots \ldots \ldots \ldots \ldots \ldots$

3.1. 1 dimensional resampling theory

3.2. Sampling lattice conversion

3.3. Sampling and reciprocal lattice of interlaced television signal

3.4. Brillouin zones for $50 \mathrm{~Hz}$ and $60 \mathrm{~Hz}$ television signals

3.5. Possible Brillouin zones for interlaced television signals

3.6. Frequency sampling filter design

3.7. The signal chain

3.8. Standards conversion process

3.9. Alias contributions in $1 \mathrm{D}$ sample rate changing

3.10. The principle of adaptive processing

3.11. Typical motion detector

4. The Implementation of Standards Converters $\ldots \ldots \ldots \ldots \ldots \ldots \ldots \ldots$

4.1. Direct form realisation of an FIR filter

4.2. Transposed direct form realisation of an FIR filter

4.3. Non-pipelined FIR filter

4.3. Non-pipelined transposed FIR filter

4.5. 1 dimensional resampling theory

4.6. Sampling lattice conversion

4.7. Time varying input lattice filter

4.8. Timing relationship between input and output samples for input lattice filter

4.9. Time varying output lattice filter

4.10. Timing relationship between input and output samples for output lattice filter 
4.11. Aperture position generation for time varying filters

4.12. Non-pipelined input lattice filter

4.13. Non-pipelined output lattice filter

4.14. Examples of aperture quantisation

4.15. Example 2 dimensional (spatial) filter

4.16. Generalised input lattice filter

4.17. Generalised output lattice filter

4.18. Example segmentation of part of a $2 \mathrm{D}$ impulse response into coefficient blocks

4.19. Some possible coefficient block layouts

4.20. Spatial relationship between input and output scanning formats

4.21. Vertical/Temporal relationship between input and output scanning formats

4.22. Multidimensional aperture position generation

4.23. The four subsets of filter coefficients required for filtering interlaced pictures

4.24. The principle of motion compensated interpolation

4.25. Motion compensated input lattice filter

4.26. Motion compensated output lattice filter

4.27. Vector processing for motion compensation

5. The Performance of Conventional Standards Converters

5.1. Alias diagram for $50 \mathrm{~Hz}$ to $100 \mathrm{~Hz}$ upconversion

5.2. Typical scene (power) spectrum $(50 \mathrm{~Hz}$ to $100 \mathrm{~Hz}$ )

5.3. Aliased scene spectrum $(50 \mathrm{~Hz}$ to $100 \mathrm{~Hz})$

5.4. Response of ideal post filter $(50 \mathrm{~Hz}$ to $100 \mathrm{~Hz})$

5.5. Frequency response of the eye $(50 \mathrm{~Hz}$ to $100 \mathrm{~Hz})$

5.6. Aliased frequency response of the eye $(50 \mathrm{~Hz}$ to $100 \mathrm{~Hz})$

5.7. Response of ideal pre filter $(50 \mathrm{~Hz}$ to $100 \mathrm{~Hz})$

5.8. Response of ideal interpolation filter $(50 \mathrm{~Hz}$ to $100 \mathrm{~Hz})$

5.9. Weighting function for deriving practical filters $(50 \mathrm{~Hz}$ to $100 \mathrm{~Hz})$

5.12. Frequency response for frame repeat upconversion $(50 \mathrm{~Hz}$ to $100 \mathrm{~Hz})$

5.13. Frequency response for field repeat upconversion $(50 \mathrm{~Hz}$ to $100 \mathrm{~Hz})$

5.14. Frequency response bilinear interpolator for upconversion $(50 \mathrm{~Hz}$ to $100 \mathrm{~Hz})$

5.15. Alias diagram for $50 \mathrm{~Hz}$ to $60 \mathrm{~Hz}$ standards conversion

5.16. Typical scene (power) spectrum $(50 \mathrm{~Hz}$ to $60 \mathrm{~Hz})$

5.17. Aliased scene spectrum $(50 \mathrm{~Hz}$ to $60 \mathrm{~Hz})$

5.18. Response of ideal post filter $(50 \mathrm{~Hz}$ to $60 \mathrm{~Hz})$

5.19 . Frequency response of the eye $(50 \mathrm{~Hz}$ to $60 \mathrm{~Hz})$

5.20. Aliased frequency response of the eye $(50 \mathrm{~Hz}$ to $60 \mathrm{~Hz})$

5.21. Response of ideal pre filter $(50 \mathrm{~Hz}$ to $60 \mathrm{~Hz})$ 
5.22. Response of ideal interpolation filter $(50 \mathrm{~Hz}$ to $60 \mathrm{~Hz})$

5.23 . Weighting function for deriving practical filters $(50 \mathrm{~Hz}$ to $60 \mathrm{~Hz})$

5.24. Practical interpolation filter $(50 \mathrm{~Hz}$ to $60 \mathrm{~Hz})$

5.25. 'ACE' aperture, studio pictures $(50 \mathrm{~Hz}$ to $60 \mathrm{~Hz})$

5.26. 'ACE' aperture, sports pictures $(50 \mathrm{~Hz}$ to $60 \mathrm{~Hz})$

5.27. Alias diagram for $60 \mathrm{~Hz}$ to $50 \mathrm{~Hz}$ standards conversion

5.28. Typical scene (power) spectrum $(60 \mathrm{~Hz}$ to $50 \mathrm{~Hz})$

5.29. Aliased scene spectrum $(60 \mathrm{~Hz}$ to $50 \mathrm{~Hz})$

5.30. Response of ideal post filter $(60 \mathrm{~Hz}$ to $50 \mathrm{~Hz})$

5.31. Frequency response of the eye $(60 \mathrm{~Hz}$ to $50 \mathrm{~Hz})$

5.32. Aliased frequency response of the eye $(60 \mathrm{~Hz}$ to $50 \mathrm{~Hz})$

5.33. Response of ideal pre filter $(60 \mathrm{~Hz}$ to $50 \mathrm{~Hz})$

5.34. Response of ideal interpolation filter $(60 \mathrm{~Hz}$ to $50 \mathrm{~Hz})$

5.35. Weighting function for deriving practical filters $(60 \mathrm{~Hz}$ to $50 \mathrm{~Hz})$

5.36. Practical interpolation filter $(60 \mathrm{~Hz}$ to $50 \mathrm{~Hz})$

5.37. 'ACE' aperture, studio pictures $(60 \mathrm{~Hz}$ to $50 \mathrm{~Hz})$

5.38. 'ACE' aperture, sports pictures $(60 \mathrm{~Hz}$ to $50 \mathrm{~Hz})$

5.39. Spectral causes of alias impairments

6. The Principles of Motion Compensation . . . . . . . . . . . . . . . . . . 120

6.1. Response of a stationary eye to a stationary object

6.2. Response of a stationary eye to a moving object

6.3. Response of a tracking eye to a moving object

6.4. Response of the eye tracking a moving object in the eye's frame of reference

6.5. Conventional standards conversion

6.6. Motion compensated standards conversion

6.7. Idealised passband of non-motion compensated standards converter

6.8. Passband of a motion compensated standards converter for a slowly moving object

7. Motion Estimation 164

7.1. Hierarchical motion estimation

7.2. Recursive motion estimation

7.3. Estimating displacement from brightness differences

7.4. Motion estimation using block matching

7.5. Contour lines of a displaced frame difference function

7.6. Frequency response of horizontal prefilter

7.7. Frequency response of vertical prefilter 
7.8. Test image used for motion analysis

8. The Implementation of a Motion Estimator $\ldots \ldots \ldots \ldots \ldots \ldots$

8.1. Overall structure of the motion estimator

8.2. Signal conditioning for motion estimation

8.3. Motion analysis processing

8.4. Phase correlation processing

8.5. Calculation of the error function

8.6. Image segmentation for motion estimation

8.7. Image segmentation modes

8.8. Example output from phase correlator

8.9 to 8.16 Examples of error functions

8.17 to 8.24 Motion vectors presented as colours

9. The Implementation of Motion Compensated Standards Conversion . . . . . . . . . 193

9.1. Geometry of motion compensated standards conversion

9.2. Implementing picture shifts

9.3. Motion compensated standards converter

9.4. Conventional standards conversion

9.5. Motion compensated standards conversion

9.6. A circular buffer for implementing motion compensation

9.7. Basic interpolation cell

9.8. Interpolator structure

9.9. Forward \& backward assignment

9.10. Forward \& backward assignment

9.11 to 9.18 Original, uninterpolated test images

9.19 to 9.26 Conventional standards conversion (linear filtering)

9.27 to 9.34 Initial results of motion compensated standards conversion

9.35 to 9.42 Final results of motion compensated standards conversion

11. Appendix : Optimum Window Functions

11.1. Frequency responses with a transition band of \pm 1 sample

11.2. Frequency responses with a transition band of \pm 2 sample

11.3. Frequency responses with a transition band of \pm 3 sample

11.4. Frequency responses of two dimensional filters

11.5. Aperture of 81 point window 


\subsection{Introduction.}

When television systems were introduced in Europe and America different picture rates were chosen for the transmission of pictures. The picture rates were chosen to be related to the frequency of the electricity supplies in the two continents. This avoided problems with displays in the early receivers. Hence Europe adopted a $50 \mathrm{~Hz}$ field rate while America adopted a $60 \mathrm{~Hz}$ field rate. Inevitably it would, at some stage, become necessary to interconvert television programs between the European and American television standards. Television standards conversion is the process of converting television programs between different standards. The necessity for standards conversion was inevitable following the adoption of more than one television standard.

At the start of television services the difference between European and American standards was unimportant. Initially it was not possible to record television pictures and hence there was no question of American programs being shown in Europe. In recent times the advent of video recording and satellite communication has made the intercontinental transfer of television pictures an everyday necessity. In the last decade the number of standards use for the storage, transmission and display of moving pictures has greatly increased. In addition to the common $50 \mathrm{~Hz}$ and $60 \mathrm{~Hz}$ systems in use in Europe and America, we have 3 high definition television standards (European, American and Japanese), to provide much greater resolution, and also a plethora of computer graphics standards. With the increasing integration of global television systems and of television, computer graphics and communications there is a growing need to interconvert between all these standards. Hence the subject of standards conversion is assuming increasing importance in the fields of television, communications and computer systems.

The objectives of the research presented in this thesis were to study standards conversion and to investigate ways of improving the performance of these systems. The most common type of standards conversion is intercontinental conversion between European and American television standards. In spite of considerable research, spanning four decades, the processes commonly used for standards conversion still generate significant picture impairments. These impairments result from some profound problems due to the nature of television signals themselves. There is, thus, a very real need for improved methods of performing standards conversion. One technique, known as 'motion compensation', has received increasing attention since the early 1980's. This technique, if it can be made to work, provides the prospect of greatly improved standards conversion. The basis of motion compensation is to measure movement in television pictures and use this information to 
improve the standards conversion process. Much of the work of this research has focused on the technique of motion compensation as a way of improving standards conversion.

There are many different types of standards conversion. In addition to intercontinental standards conversion there is the interconversion between all the different standards mentioned above. Some standards conversion processes are between standards with the same field rate. These would include the interconversion between high definition and ordinary television, or between British 405 and 625 line standards. Such processes are concerned, primarily, with the preservation of spatial detail and movement is of secondary importance. Other standards conversion processes are between standards with different field rates. This group includes intercontinental standards conversion and also the conversion of film images to television. For these sort of conversions the preservation of good motion portrayal is at least as important as preservation of spatial detail. Standards conversions which involve a change of field rate are, arguably, more difficult to perform than those which do not. Nevertheless the same basic principles govern the operation of all these processes. It is standards conversion between different field rates which is of most interest in this thesis.

\subsection{The problem of standards conversion.}

One view of television signals is as a multidimensional sampled signal. Vertical and temporal sampling are inherent in the scanning process used to generate television pictures. Horizontal sampling is also introduced by digital signal processing systems. Standards conversion (and other processing in the television studio) may therefore be considered from the point of view of digital signal processing theory.

Standards conversion may be regarded as resampling a 3 dimension signal, sampled on an input lattice, onto a different output sampling lattice. The process is, therefore, the 3 dimensional analog of changing the sampling rate in one dimension. The theory of one dimensional sample rate changing is well understood and elucidated in many texts, eg. Oppenheim \& Schafer 1989 section 3.6 and Crochiere \& Rabiner 1988. Unfortunately the generalisation of one dimensional sample rate changing to 3 dimensional television signals is not straight forward.

Standards conversion may be analyzed in the space-time or the frequency domains. Early standards converters were based on space-time domain analysis and, for example, considered linear interpolation between adjacent field lines. In digital signal processing, however, it is usual to analyze systems in the frequency domain. It is in the frequency domain that some of the problems of television standards conversion become apparent. Analysis of standards conversion in the frequency domain led to significant improvements in the early 1980's (Clarke \& Tanton 1984). More recently, the use of motion compensation encourages a dual space-time/frequency domain analysis. 
The difficulties of standards conversion stem from the multidimensional signal, the nature of the television signal and the way it is perceived by the human visual system. The multidimensional nature of the signal, in itself, causes complication but no fundamental problems. However, one of the dimensions of the television signal is time. This has different units from the other two spatial dimensions and should be treated differently. This contrasts to still image processing, in which both (spatial) dimensions are equivalent. Viewed from a conventional signal processing viewpoint, television signals are somewhat undersampled vertically and can be grossly undersampled temporally. This is only partly due to limitations in technology. More fundamentally, the presence of even slow movement in pictures can generate large amplitudes of high spatio-temporal frequency components. In order to avoid this aliasing, very much faster field rates would be required. This would be extremely difficult technically and very expensive to achieve. Furthermore such a rapid picture rate seems unnecessary because aliasing is not usually apparent when viewing (unprocessed) television pictures.

The alias artifacts, inherent in television signals, only become apparent when the signal is processed. The only artifact that is usually visible in an unprocessed television signal is the sampling lattice itself (ie line structure and flicker). When television signals are processed the position of aliases are moved (in frequency) and become both visible and objectionable. Television signal aliases are typically apparent, after standards conversion, as artifacts such as judder and additional (small area) flicker.

The theory of one dimensional sample rate changing usually assumes that the input signal is free from aliasing. Since this is not the case for television signals, one dimensional sample rate changing theory cannot simply be extended to these signals. The difficulty of television standards conversion is essentially trying to perform sampling lattice conversion on an aliased signal.

The standards conversion process usually used is, essentially, a linear filtering operation. Unwanted repeat spectra, in the sampled input signal, are removed by an input post filter. Then a second, output prefilter, is used to remove frequency components which would otherwise alias in the sampled output signal. In practice these two filters are usually combined as a single composite filter. If the television signal were not aliased the design of the filters would be relatively straight forward. Without aliasing the filters required would be multidimensional, sharp cut, low pass filters. Aliasing in the television signal requires the use of more complicated filtering whose design is far from obvious. It is for this reason that further research was required. 


\subsection{The historical development of standards conversion.}

The problem of standards conversion has been considered by researchers since early in the development of television. One early researcher, Howe 1948, considers the need for conversion between 405 and 625 line television and also the possible requirement for conversion between different field rates. One of his proposed solutions, image transfer converters, later saw considerable use.

Image transfer converters take a simple and straight forward approach to standards conversion. Essentially a television camera, operating on the output standard, is focused on a CRT display operating on the input standard. Such converters were used both for conversion between signals with the same field rate (Lord 1953) and conversion between different field rates (Lord \& Rout 1964 and Jones et al 1964). The problem with this type of conversion was that the filtering action, implicit in the conversion, was very difficult to control. Temporal filtering could be adjusted by changing the decay time of the phosphor in the CRT. This decay time was a compromise between excessive multiple imaging and moving horizontal bands on the output picture. Spatial filtering could be adjusted by broadening the display spot (eg by using sinusoidal spot wobble). Such systems needed constant skilled maintenance. Overall the output picture quality was only acceptable in the absence of better techniques.

Later, the conversion between standards with the same field frequency was achieved by using electronic line stores (Rainger 1962, Rainger \& Rout 1966 and Jones 1965). These line store converters used capacitors to store a line of horizontal samples from a television signal. Line store converters were used for the conversion of 625 line to 405 line television. Although they required less maintenance than the image transfer converters they still provided little control over the interpolation filter.

To convert between different field rates considerable amounts of image storage are required. Electronic storage using capacitors, as in line store converters, was impractical for storing multiple fields for field rate converters. The first electronic field rate standards converters used (analogue) quartz delay lines (Edwardson 1968, Davies \& Edwardson 1971). Although better than image transfer converters they still had limited control of the interpolation filter, which was limited to a small number of filter taps with simple coefficients. Further more, since this was an analogue implementation, it required careful adjustment and maintenance to achieve the required stability.

By the early 1970's semiconductor technology had developed to the stage where standards converters using digital field stores were possible (Baldwin et al 1974, Baldwin et al 1976). These early digital standards converters were very constrained by storage requirements. While they were an improvement on quartz delay line standards converters they could still only use relatively simple interpolation filters. At the end of the 1970's digital technology had improved further, to the point where a more sophisticated interpolation filter 
could be used (Clarke \& Roe 1978, Astel \& Shelton 1980, Clarke \& Tanton 1984). This digital field rate standards converter used 4 taps on each of 4 fields to implement the interpolation filter. In order to design a good interpolation filter it was necessary to perform a frequency domain analysis of the problem rather than the time domain analysis used previously. This standards converter produced good results. Nevertheless the effects of aliasing (eg judder) could still easily be seen on standards converted pictures containing fast motion. Most commercial standards converters in use today use a very similar system.

The artifacts due to aliasing, which are still apparent in pictures from converters using 4 line, 4 field filters, have maintained the need for research into improved standards conversion algorithms. A promising technique for improved standards conversion is the use of motion compensation. The principle of the technique is to measure movement in the input picture and use this information to improve the standards conversion process. Although, potentially, there are great benefits from this technique, it is difficult to implement and is at the limit of the practical complexity for today's electronic technology. One of the first experimental implementations of motion compensation was to convert from high definition to ordinary television at different field rates (Tanaka et al 1986, Nishizawa \& Tanaka 1987). This used a fairly simple movement estimation technique. While it dramatically demonstrated the potential benefits of motion compensation it could not cope with some types of moving pictures. Lately, two motion compensated standards converters have become commercially available (Rutter 1990, Novak \& Weiss 1992). Again these machines can produce good results but they can also fail on some types of moving pictures. Generally the use of motion compensation has demonstrated great promise but, not yet, robust and reliable operation.

\subsection{Outline of the thesis.}

The purpose of this research was to investigate the process of standards conversion and improvements to the conventional algorithms. I have examined the use of both conventional (linear filtering), and motion compensated techniques. Two examples of standards conversion processes were chosen for particular consideration. These were field rate upconversion and intercontinental standards conversion. Field rate upconversion is the process of increasing the field rate of a television signal, to reduce flicker in the displayed pictures. Intercontinental standards conversion refers, in this context, to the interconversion of the $50 \mathrm{~Hz}$ and $60 \mathrm{~Hz}$ television standards used in Europe and America. Intercontinental standards conversion is complicated by the different standards used to code colour information. This aspect of the process is not considered in this thesis. My investigations were performed using component (luminance and colour difference) signals.

The thesis starts, in chapter 2 , by considering the signal chain in which the standards conversion process is embedded. That is the scene from which the pictures originate, the 
cameras used to detect them, the displays for showing then and the way images are perceived by the human visual system. This sets the process of standards conversion in context. Chapter 2 considers, in turn, the various parts of the signal chain. The important characteristics are those which affect the processing used for standards conversion. Although non-linear processing may be used for standards conversion, the algorithms which are practical using today's technology are, nevertheless, largely based on frequency domain analysis of signals. Therefore this chapter concentrates on the spectral characteristics of the components of the television chain.

The frequency domain characteristics of the television chain are useful for designing filters and for indicating how adaptive and motion compensated processing may be applied to standards conversion. For this purpose mathematical models of these characteristics are required. In Chapter 2 I present a number of such models, for different parts of the chain. These models both simplify and generalise the true characteristics to give an approximate mathematical description of reality. The experimental results I present confirm previously published results on the average (spatial) spectral content of scenes and extends those results to include the temporal frequency domain. A mathematical model of the results is presented, which fits the experimental data and is used in subsequent chapters. I also present a mathematical model of the frequency response of the human visual system. This model was derived from reviewing the published literature, much of which is aimed at understanding the human visual system rather than generating a useful mathematical model.

Armed with an analysis of the signal chain, in chapter 3 I consider the thorny question of filter design for standards conversion. The chapter examines the design of linear, symmetric, transversal interpolation filters for standards conversion. It starts by considering the historical development of filter design techniques for television. This is followed by a more precise description of the desired filter characteristics and ways in which they can be approximated. My aim was to take a fresh look and present an overview of filter design techniques, from the perspective of standards conversion. Previously filter design for standards conversion has tended to be heuristic in nature and has failed to take full account of aliasing in the television signal. In Chapter 3 I develop a general and objective way in which the desired specification for a standards conversion filter can be determined. I then consider a number of new ways in which this ideal specification can be approximated, by finite hardware, to produce an 'optimum' practical filter.

Where chapter 3 examines the determination of filter coefficients, chapter 4 examines efficient ways in which interpolation filters can be implemented in practice. I show that many different implementations of standards converters are possible and try to present a systematic and unified analysis of the possible processing architectures. Only a few of these possible architectures have been made as commercial products. This may be partly because no systematic description of the options was previously available. I hope that this chapter will 
allow the most efficient implementation to be selected for any particular standards conversion process.

In chapter 4 I start by considering 1 dimensional, single sample rate filtering. This is then progressively extended to eventually cover the multidimensional, multirate filtering which is required for standards conversion. In a single sample rate system, FIR filters can be implemented using either the direct or transposed filter structure. I show that, for multirate interpolation filters, the direct and transposed forms become different, but complementary, filter architectures. I have termed these two structures 'input' or 'output' lattice filters because the former stores input samples whereas the latter stores interpolated output samples. Fast, efficient signal processing is often performed with pipelined (systolic) architectures. These can be used for interpolation filters. In practice, however, standards converters often have to convert between sampling lattices in both directions using the same hardware. Pipelined filter architectures, where the data 'flows' through the hardware and the coefficients remain 'stationary', are not efficient for such bidirectional standards conversion. An alternative architecture, convenient for performing bidirectional standards conversion, is presented. In this alternative architecture the coefficients 'flow' through the hardware while the data remains 'stationary'. Finally chapter 4 considers the changes required for implementing motion compensated standards conversion. While input and output lattice filters are equally appropriate for non-motion compensated standards conversion, I show that input lattice filters have an advantage when used with motion compensation.

Chapter 5 describes experiments in which the performance of the new filter design techniques are compared to those used previously. The filter design techniques of chapter 3 are, in principle, applicable to any standards conversion process. It was important to determine that they performed well compared to filters used previously. The two examples of field rate upconversion and intercontinental standards conversion were investigated. In both cases the new filter designs had at least comparable performance to previous filter designs.

Impairments in pictures, standards converted using 'conventional' interpolation, prompted the investigation of possible improvements using motion compensation. In Chapter 6 I describe the principles and theory of motion compensation interpolation. I consider the way in which the human visual system responds to moving objects. By performing interpolation in a moving object's frame of reference, rather than a stationary frame of reference, I show that the problems of temporal aliasing are avoided. This is the principle of motion compensation.

One of the most critical parts of a motion compensated standards converter is the movement measurement system (the 'motion estimator'). The difficulty of motion estimation is probably demonstrated by the plethora of techniques described in the literature. In the first part of chapter 7 I present a review of the literature on motion estimation, from the perspective of standards conversion. I suggest that some motion estimation algorithms, 
successfully used in other applications, are less suitable for standards conversion. The second half of the chapter presents the results of experiments on two promising motion estimation algorithms. The first algorithm is 'phase correlation' which has been reported previously (Thomas 1987). The second is a new algorithm, which I have termed 'motion correlation', based on analyzing the image's 3 dimensional spectrum. The results I present here indicate the performance which might be achieved with typical 'real' pictures. This contrasts with previous results for phase correlation which have only applied to specific test images. The results using 'motion correlation' suggest it has superior performance to 'phase correlation'.

Having considered the theory of motion estimation, in chapter $8 \mathrm{I}$ describe the implementation, and results from, an experimental real time motion estimation system, built by myself and my colleagues. The motion estimator was based on phase correlation and designed to produce a distinct motion vector for each input pixel. Results are presented in the form of photographs of the output. These include the motion vectors, displayed as colours, superimposed on the input luminance signal.

The motion estimator of chapter 8 was used in combination with two, experimental, real time, motion compensated, standards conversion systems. As before the examples taken were of field rate upconversion and intercontinental standards conversion. In chapter 9 I first consider the practical application of motion vectors to standards conversion and the implications of revealed and obscured background in pictures. Particular consideration is given to the way in which a motion estimator can be interfaced to interpolation hardware. The motion-estimator/interpolator interface can have a significant effect on the quality of the standards converted pictures, as is demonstrated by some results. The results of motion compensated intercontinental standards conversion are presented in a series of photographs.

Chapter 10 presents a summary of the thesis and some conclusions from the investigation. Some suggestions are made for future research.

Finally in chapter 11 I present an analysis of theory of window functions. These functions are used in many applications of digital signal processing generally and for standards conversion in particular. The essential characteristic of a window function is to be of finite extent in both the space/time and frequency domains. While it is impossible to meet this requirement exactly, very good approximations are possible in practice. Historically 1 dimensional window functions were developed in a largely heuristic manner. In this chapter I present a new analysis of window functions. In particular I show how optimum multidimensional window functions can be designed which are non-zero in arbitrarily shaped regions of support in both the space/time and frequency domains. By using optimised window functions the amount of processing required for motion estimation and standards conversion can be significantly reduced. 


\subsection{References.}

1. Astel, J.M. \& Shelton W.T., 1980. Digital television standards converter with random access memory storing four fields. IBC 1980, IEE Conf. Pub. No. 191, September 1980.

2. Baldwin, J.L.E., Stalley, A.D., Coffey, J.A., Greenfield, R.L., Lever, I.R., \& Taylor J.H., 1974. DICE: The first intercontinental digital standards converter. Royal Television Society Journal September/October 1974.

3. Baldwin, J.L.E., Lever, I.R., Connolly, W.P., Corbyn, T.E., Greenfield, R.L., Thirlwall, A.C., Barrett, K.H., Taylor, J.H., Bellis, A., Carmen, P.R., Dunne, J.F., Ive, J.G., Wilkinsin, J.H., 1976. Digital video processing - DICE. IBA Technical Review No. 8., September 1976.

4. Clarke, C.K.P. \& Roe, G.D., 1978. Developments in standards conversion. IBC 1978, IEE Conf. Pub. No. 166, September 1978.

5. Clarke, C.K.P. \& Tanton, N.E., 1984. Digital standards conversion: interpolation theory and aperture synthesis. BBC Research Department Report, BBC RD 1984/20, December 1984.

6. Crochiere, R.E. \& Rabiner, L.R., 1988. Multirate processing of digital signals. Advanced Topics in Signal Processing, Lim, J.S. \& Oppenheim, A.V. (Editors), Prentice Hall, 1988, ISBN 0-13-013129-6 025, pp 123-198.

7. Davies, R.E. \& Edwardson, S.M., 1971. Electronic field store standards converter. Proc. IEE, Vol. 118, No. 3/4 March/April 1971.

8. Edwardson, S.M., 1968. An advanced form of field store standards converter. International Broadcasting Convention 1968, IEE Conf. Pub. No. 46, Part 1, September 1968.

9. Howe, A.B., 1948. On the conversion of television standards. BBC Research Department Report, Report No. T.019. Serial No. 1948/43.

10. Jones, A.H., Lent, S.J. \& Reed, C.R.G., 1964. Image transfer standards conversion: modulation due to difference between field frequencies. BBC Research Department Report, Report No. T.130. Serial No. 1964/9.

11. Jones, A.H., 1965. Line store standards conversion: The design of video transmission lines. BBC Research Department Report, Report No. T.150. Serial No. 1965/35.

12. Lord, A.V., 1953. Conversion of television standards. BBC Quarterly, Vol VIII, No. 2, Summer 1953.

13. Lord, A.V. \& Rout, E.R., 1964. A review of television standards conversion. BBC Engineering Monograph, No. 55, December 1964. 
14. Nishizawa, T. \& Tanaka, Y. 1987. Standards Conversion with HDTV. Proceedings of the Third International Colloquium on Advanced Television Systems, Ottawa, Canada, October 1987.

15. Nowak, T. \& Weiss, P., 1990. Vector. International Broadcast Engineer, Edition 249, January 1992, pp 20-24.

16. Oppenheim, A.V. \& Schafer, R.W., 1989. Discrete-time signal processing. Prentice Hall Inc, 1989, ISBN 0-13-216771-9.

17. Rainger, P., 1962. A new system of standards conversion. Report of the International Television Conference, London, 31st May - 7th June, 1962, IEE Conf. Rep. No. 5, IEE London, 1963, pp 170-172.

18. Rainger, P. \& Rout, E.R., 1966. Television standards converters using a line store. Proc. IEE, 1966, Vol. 113, No. 9, pp 1437-1456.

19. Rutter, P., 1990. The Oki Lt 2000 standards converter. International Broadcast Engineer, Vol. 21, Edition 235, January 1990, pp 26-27.

20. Tanaka, Y., Ohnura, T., Okada, K., Ohtsuka, Y., Kurita, T., Goshi, S., Ninomiya, Y. \& Nishizawa, T., 1986. HDTV-PAL standards converter. NHK Laboratories Note No. 326, January 1986.

21. Thomas, G.A., 1987. Television Motion Measurement for DATV and other Applications. BBC Research Department Report No BBC RD 1987/11. September 1987. 


\subsection{Introduction.}

This chapter provides an analysis of the parts of the television signal chain excluding processing. The subject of this thesis as a whole is to investigate the process of standards conversion. However, in order to discuss the image processing which can be used for standards conversion it is necessary to set that process in its correct context. The context for standards conversion is the other parts of the signal chain. That is the scene from which the pictures originate, the camera used to detect them, the displays for showing them and the way they are perceived by the human visual system.

Figure 2.1 shows the signal chain from origination to perception. The various parts of this signal chain (excluding processing) are discussed, in turn, in this chapter. The important characteristics of the parts of the chain are those which affect the processing used for standards conversion. Although non-linear processing may be used for standards conversion, the algorithms which are practical using today's technology are, nevertheless, largely based on frequency domain analysis of signals. Therefore this chapter concentrates on the spectral characteristics of components of the television chain.

The frequency domain characteristics of the television chain are useful for the design of filters and for indicating how adaptive and motion compensated processing may be applied to standards conversion. To be used for these purposes mathematical models of these characteristics are required. This chapter presents a number of such models, based on both new experimental results and reviewing the literature. These models both simplify and generalise the true characteristics to give an approximate mathematical description of reality.

The mathematical models of spectral characteristics, required for designing standards converters, have some particular characteristics. One characteristic is that they should provide a useful approximation even at higher frequencies. Unfortunately the higher frequencies are those which are both most difficult to measure and least reported in the literature. Great accuracy is not, however, required of these models. This is, in part, because the filter design algorithms (discussed in the next chapter) which use them are not particularly sensitive to the details of the models used. More importantly, however, each scene and observer is different and there are many different cameras and displays. Therefore models which are particularly accurate in one circumstance will not be accurate in another. What the models try to achieve is an approximation to the general characteristics of the parts of the television chain. 


\subsection{The Spectral Content of Scenes.}

This section is concerned with modelling the 'average' power spectrum of 'typical' scenes. This information is required in designing 'optimum' filters for standards conversion as described in the next chapter. These models also suggest ways in which adaptive processing can be applied to both conventional and motion compensated processing (see also chapter 3).

The power spectra of different scenes show considerable differences, as do the spectra which are 'local' to different parts of the same scene. All that can be done is to estimate the 'average' value of the power spectra of typical pictures. The spectra modelling techniques, described below, can also be extended to the case when the motion in the image has already been measured. This will be discussed in section 2.3 , and is useful in the design of filters for motion compensated processing.

The obvious method of estimating the 'average' spectrum is to fit a model to the measured mean power spectrum. This has two disadvantages arising from the nature of the available image data. The picture data will have been imaged with a camera and sampled, for storage and processing in a computer. The imaging process will, inevitably, have filtered the image with the transfer characteristic of the camera. Worse still, aliased spectra will have been introduced by the sampling process. These aliases will contaminate high frequency components of the measured power spectrum. The aliased spectra are of particular significance in this application, because filters designed using the spectral model are intended to minimise the subjective effect of aliasing. If the estimate of the model is seriously contaminated by the presence of aliases it would defeat the purpose of modelling the spectrum.

An alternative way of estimating the average power spectrum is via the image's autocorrelation function. The Wiener-Khintchine theorem (eg Kay 1988 p59) shows that an image's autocorrelation function is the Fourier transform of its power spectrum (and vice versa). The effects of filtering and sampling are somewhat different for the autocorrelation function than for direct calculation of the spectrum. The autocorrelation surface is convolved with the impulse response of the camera. This latter effect should have little effect if the autocorrelation function is smooth, because the camera's impulse response will be quite narrow. Hence modelling the power spectrum via the autocorrelation function has the advantage that power spectrum measurements are relatively independent of the frequency response of the camera. Aliasing components introduced by sampling contaminate measured values of the autocorrelation function by introducing spurious detail. If a smooth model is assumed for the autocorrelation function the fitting process will tend to ignore this spurious detail. Therefore fitting a model to the autocorrelation function is relatively insensitive to aliasing in the original image. 
Several different models can be fitted to the autocorrelation function of typical scenes. These, in turn, provide (via the Fourier transform) models of the power spectrum of typical scenes. A Gaussian model of the autocorrelation function might be the first to come to mind. However this clearly would not fit the data as can be seen from figures $2.2 \& 2.3$. These figures are measured autocorrelation functions averaged over several scenes. Three more suitable models which might be considered are;

Model 1:

$$
a(x, y, t)=\exp \left\{-\left(\frac{|x|}{\alpha}+\frac{|y|}{\beta}+\frac{|t|}{\gamma}\right)\right\}
$$

Model 2:

$$
a(x, y, t)=\exp \left\{-\sqrt{\left(\frac{x}{\alpha}\right)^{2}+\left(\frac{y}{\beta}\right)^{2}+\left(\frac{t}{\gamma}\right)^{2}}\right\}
$$

Model 3:

$$
a(x, y, t)=\exp \left\{-\sqrt[h]{\left(\frac{x}{\alpha}\right)^{q h}+\left(\frac{y}{\beta}\right)^{p h}+\left(\frac{t}{\gamma}\right)^{r h}}\right\}
$$

where $\mathrm{a}(\mathrm{x}, \mathrm{y}, \mathrm{t})$ is the autocorrelation function and where $\alpha, \beta, \gamma$ and $\mathrm{q}, \mathrm{p}, \mathrm{r} \& \mathrm{~h}$ are modelling parameters. Each of these models has previously been used as a model for the autocorrelation function (see Clarke 1985, p29-31). The first model is the autocorrelation of a first order, variables separable, Markov process. As such it is implicitly used in DCT (discrete cosine transform) coding. The DCT becomes the Karhunen-Loeve transform in the limit as $\alpha, \beta$ \& $\gamma$ tend to infinity (Clarke 1985 and Jayant \& Noll 1984). The Karhunen-Loeve transform is optimal for transform coding and the success of DCT coding indicates the validity of model 1. Despite this success model 1 nevertheless tends to underestimate the autocorrelation function on the diagonals. Model 2 gives a better approximation to the mean autocorrelation function particularly for large off axis lags (which are important for this application). Model 3 can provide the most accurate model (models 1 and 2 are special cases). The modelling parameters for model 3 are, however, difficult to measure and is also difficult to Fourier transform analytically. For these reasons only models 1 and 2 are considered further in this section. 


\subsubsection{Experimental Investigation.}

To determine the most appropriate model for scene spectra, and to measure the model parameters, an experimental investigation was undertaken. A variety of 'realistic' pictures (that is not test pictures or graphics) were selected. These first had their mean value subtracted from each pixel and were then divided by the standard deviation of the image. This procedure allowed the autocorrelation functions to be averaged in a meaningful way.

The autocorrelation functions were then calculated for each picture using the following equations.

$$
a(x, y, t)=k \sum_{l=0}^{L-|x|-1} \sum_{m=0}^{M-|y|-1} \sum_{n=0}^{N-|t|-1} g(l, m, n) g(l+x, m+y, n+t)
$$

where

$$
k=\frac{1}{(L-|x|)(M-|y|)(N-|t|)}
$$

and

$$
x=0,1,2 \ldots L ; y=0,1,2 \ldots . M ; t=0,1,2 \ldots . N
$$

This is known as the unbiased autocorrelation function. For a discussion of the statistical properties of estimates of autocorrelation values see Kay 1988. The mean value of the autocorrelation function for all the images were then calculated.

Autocorrelation functions were calculated for both gamma corrected and non-gamma corrected (ie linear) images. Gamma correlation is a non-linearity deliberately introduced by television cameras. Gamma correction is discussed further in section 2.3. Calculating autocorrelation functions for both types of image was intended to indicate whether the 'average' spectrum of linear pictures was significantly different from that of gamma corrected pictures. The pictures used were stored having been gamma corrected. Linear pictures were derived from the equivalent gamma corrected one by raising them to the power 2.2.

Models 1 and 2 (described in the introduction) were then fitted to the experimental data. The model fitting technique was very similar for both models. Considering model 1 , taking logs gives, for the nth point;

$$
\frac{\left|x_{n}\right|}{\alpha}+\frac{\left|y_{n}\right|}{\beta}+\frac{\left|t_{n}\right|}{\gamma}=-\ln (a(x, y, t))
$$

The calculation of the autocorrelation function for each different lag (delay) gives a similar equation. The set of all calculated autocorrelation values gives an overdetermined set of 
equations for the model parameters, ie;

$$
\boldsymbol{A} \boldsymbol{b}=\boldsymbol{c}
$$

where;

$$
\boldsymbol{A}=\left[\begin{array}{ccc}
\left|x_{1}\right| & \left|y_{1}\right| & \left|t_{1}\right| \\
\left|x_{2}\right| & \left|y_{2}\right| & \left|t_{2}\right| \\
\cdot & \cdot & \cdot \\
\cdot & \cdot & \cdot \\
\left|x_{N}\right| & \left|y_{N}\right| & \left|t_{N}\right|
\end{array}\right], \quad \boldsymbol{b}=\left[\begin{array}{c}
\frac{1}{\alpha} \\
\frac{1}{\beta} \\
\frac{1}{\gamma}
\end{array}\right], \quad \boldsymbol{c}=\left[\begin{array}{c}
-\ln \left(a\left(x_{1}, y_{1}, t_{1}\right)\right) \\
-\ln \left(a\left(x_{2}, y_{2}, t_{2}\right)\right) \\
\cdot \\
\cdot \\
-\ln \left(a\left(x_{N}, y_{N}, t_{n}\right)\right)
\end{array}\right]
$$

and $\mathbf{N}$ is the total number of points modeled. The unknown in this equation is the vector $\mathbf{b}$ which contains the modelling parameters $\alpha, \beta \& \gamma$. Hence if a 65 by 65 pixel section of the autocorrelation function were modelled then $\mathrm{N}=65^{2}$. That is we would have a set of 4225 equations in 3 unknowns.

The overdetermined set of equations (equations $8 \& 9$ ) for the model parameters can be solved, by minimising the mean square error, using the following equation.

$$
b=\left(A^{t} A\right)^{-1} A^{t} c
$$

The components of vector $\mathrm{c}$ are logarithms of the measured autocorrelation function. Thus the mean square logarithmic (rather than absolute) error is minimised. Therefore this modelling technique effectively minimises the percentage error when fitting the model. This is a desirable feature because many of the measured autocorrelation values are quite small. Minimising the absolute error would model the central (high value) part of the autocorrelation function at the expense of errors at higher lags. This technique gives more importance to the autocorrelation function away from its centre.

The parameters for model 2 were determined in a similar way to those for model 1 . With model 2 it is necessary to take the square of the logarithm of the measured autocorrelation function. This gives an equation which is equivalent to equation 7 for model 1, ie:

$$
\frac{x_{n}^{2}}{\alpha^{2}}+\frac{y_{n}^{2}}{\beta^{2}}+\frac{t_{n}^{2}}{\gamma^{2}}=-\ln ^{2}\left(a\left(x_{n}, y_{n}, t_{n}\right)\right)
$$

The overdetermined set of equations for the model parameters were again solved, in a least mean square sense, using equation 10 . 


\subsubsection{Results.}

Sets of mean two dimensional autocorrelation functions were calculated. The results given are for 625 line pictures (sampled according to CCIR Rec. 601, 1986). Two dimensions were used rather than three because the storage and processing requirements for calculating 3 dimensional autocorrelation functions were impractical. The two planes in the 3 dimensional autocorrelation function chosen were at $\mathrm{t}=0$ and at $\mathrm{y}=0$, that is the $\mathrm{x} / \mathrm{y}$ and the $\mathrm{x} / \mathrm{z}$ planes. Images 65 pixels square, of the central part of the autocorrelation functions, were used to generate the models (see discussion).

The results are shown graphically (figures $2.2 \& 2.3$ ) and as tabulated values. Table 2.1 shows model parameters and modelling error calculated for model 1 on the $x / y$ plane using gamma corrected pictures. These are given for the five images used and for the mean autocorrelation function. The model parameters for the individual images are given to indicate the spread between model parameters for real pictures. The modelling error is indicated by the root mean square error between the model and the measured values. The root mean square difference between the logarithms of the model and of the measured values is also given. This is an indication of the 'typical' fractional error. Tables 2.2, 2.3 and 2.4 give similar information for the second model and also for linear (not gamma corrected) images. Tables 2.5 and 2.6 give the results for the $x / z$ plane. Five different sequences, containing a variety of representative picture material were used for calculating the results in these two tables. Results are not given for the individual sequences in tables $2.5 \& 2.6$ as these are not representative (see discussion). 
Table 2.1 : Model 1, x/y plane, gamma corrected pictures.

\begin{tabular}{|c|c|c|c|c||}
\hline $\begin{array}{l}\text { Image } \\
\text { Name }\end{array}$ & $\alpha$ & $\beta$ & $\begin{array}{c}\text { RMS } \\
\text { absolute } \\
\text { error } \\
\left(/ \sigma^{2}\right)\end{array}$ & $\begin{array}{c}\text { RMS } \\
\text { logarithmic } \\
\text { error }\end{array}$ \\
\hline \hline Boat & 67.4 & 30.1 & 0.07 & 0.14 \\
\hline Dick & 53.4 & 54.1 & 0.05 & 0.09 \\
\hline Pond & 54.3 & 27.5 & 0.06 & 0.14 \\
\hline Toys & 21.4 & 22.5 & 0.04 & 0.14 \\
\hline Tree & 22.8 & 37.1 & 0.09 & 0.22 \\
\hline \hline MEAN & 39.0 & 32.2 & 0.07 & 0.14 \\
\hline
\end{tabular}

Table 2.2 : Model 2, x/y plane, gamma corrected pictures.

\begin{tabular}{|c|c|c|c|c||}
\hline $\begin{array}{c}\text { Image } \\
\text { Name }\end{array}$ & $\alpha$ & $\beta$ & $\begin{array}{c}\text { RMS } \\
\text { absolute } \\
\text { error } \\
\left(/ \sigma^{2}\right)\end{array}$ & $\begin{array}{c}\text { RMS } \\
\text { logarithmic } \\
\text { error }\end{array}$ \\
\hline \hline Boat & 44.0 & 27.0 & 0.08 & 0.14 \\
\hline Dick & 41.4 & 42.8 & 0.05 & 0.09 \\
\hline Pond & 36.4 & 24.1 & 0.06 & 0.14 \\
\hline Toys & 15.9 & 17.2 & 0.06 & 0.20 \\
\hline Tree & 19.2 & 27.3 & 0.08 & 0.20 \\
\hline \hline MEAN & 29.0 & 26.4 & 0.07 & 0.14 \\
\hline
\end{tabular}


Table 2.3 : Model 1, x/y plane, linear pictures.

\begin{tabular}{||c|c|c|c|c||}
\hline $\begin{array}{c}\text { Image } \\
\text { Name }\end{array}$ & $\alpha$ & $\beta$ & $\begin{array}{c}\text { RMS } \\
\text { absolute } \\
\text { error } \\
\left(/ \sigma^{2}\right)\end{array}$ & $\begin{array}{c}\text { RMS } \\
\text { logarithmic } \\
\text { error }\end{array}$ \\
\hline \hline Boat & 60.8 & 31.4 & 0.08 & 0.14 \\
\hline Dick & 51.0 & 52.1 & 0.07 & 0.10 \\
\hline Pond & 48.2 & 20.6 & 0.06 & 0.17 \\
\hline Toys & 23.3 & 26.8 & 0.04 & 0.10 \\
\hline Tree & 23.8 & 40.2 & 0.09 & 0.22 \\
\hline \hline MEAN & 37.7 & 31.8 & 0.07 & 0.14 \\
\hline
\end{tabular}

Table 2.4 : Model 2, x/y plane, linear pictures.

\begin{tabular}{|c|c|c|c|c||}
\hline $\begin{array}{c}\text { Image } \\
\text { Name }\end{array}$ & $\alpha$ & $\beta$ & $\begin{array}{c}\text { RMS } \\
\text { absolute } \\
\text { error } \\
\left(/ \sigma^{2}\right.\end{array}$ & $\begin{array}{c}\text { RMS } \\
\text { logarithmic } \\
\text { error }\end{array}$ \\
\hline \hline Boat & 41.4 & 27.8 & 0.08 & 0.14 \\
\hline Dick & 40.1 & 40.9 & 0.06 & 0.10 \\
\hline Pond & 30.4 & 18.5 & 0.06 & 0.05 \\
\hline Toys & 17.9 & 19.9 & 0.04 & 0.14 \\
\hline Tree & 20.0 & 29.5 & 0.08 & 0.17 \\
\hline \hline MEAN & 28.2 & 26.0 & 0.07 & 0.14 \\
\hline
\end{tabular}


Table 2.5 : Model 1, x/t plane, gamma corrected pictures.

\begin{tabular}{|c|c|c|c|c||}
\hline \hline $\begin{array}{l}\text { Image } \\
\text { Name }\end{array}$ & $\alpha$ & $\gamma$ & $\begin{array}{c}\text { RMS } \\
\text { absolute } \\
\text { error } \\
\left(/ \sigma^{2}\right)\end{array}$ & $\begin{array}{c}\text { RMS } \\
\text { logarithmic } \\
\text { error }\end{array}$ \\
\hline \hline MEAN & 41.1 & 14.8 & 0.05 & 0.14 \\
\hline
\end{tabular}

Table 2.6 : Model 2, x/t plane, linear pictures.

\begin{tabular}{|c|c|c|c|c||}
\hline $\begin{array}{c}\text { Image } \\
\text { Name }\end{array}$ & $\alpha$ & $\gamma$ & $\begin{array}{c}\text { RMS } \\
\text { absolute } \\
\text { error } \\
\left(/ \sigma^{2}\right)\end{array}$ & $\begin{array}{c}\text { RMS } \\
\text { logarithmic } \\
\text { error }\end{array}$ \\
\hline \hline MEAN & 24.2 & 13.2 & 0.05 & 0.14 \\
\hline
\end{tabular}

\subsubsection{Discussion \& Conclusions.}

Two models were fitted to the measured 'average' autocorrelation function of typical image data. Both models give a reasonable fit to the experimental data. Model 2 is probably preferable for use in designing filters as it gives a better representation of high diagonal frequencies. The model parameters are in close agreement with those give in Chimienti \& Picco 1988, Clarke 1985 (pages 24, 44 \& 59) and Jayant \& Noll (pages 37 \& 39).

These models of the autocorrelation function give the following power spectra ${ }^{1}$.

${ }^{1}$ The Fourier transform, $G(f)$, of a spherically symmetrical function $g(r)$ is given by (Champeney 1973),

$$
\mathscr{F}(g(r))=\iiint g(r) \exp (-j 2 \pi k . r) d r=4 \pi \int g(r) \operatorname{sinc}(2 \pi k r) r^{2} d r
$$

where $r=(x, y, z), k=(m, n, f), r=\sqrt{ }\left(x^{2}+y^{2}+z^{2}\right), k=\sqrt{ }\left(m^{2}+n^{2}+f^{2}\right)$ and $\operatorname{sinc}(\mu)=\sin (\mu) / \mu$. 
Model 1:

$$
|S(m, n, f)|=\frac{8 \alpha \beta \gamma}{\left(1+(2 \pi \alpha m)^{2}\right)\left(1+(2 \pi \beta n)^{2}\right)\left(1+(2 \pi \gamma f)^{2}\right)}
$$

Model 2:

$$
|S(m, n, f)|=\frac{8 \pi \alpha \beta \gamma}{\left(1+(2 \pi k)^{2}\right)} ; \quad k=\sqrt{\alpha^{2} m^{2}+\beta^{2} n^{2}+\gamma^{2} f^{2}}
$$

where $|S(m, n, f)|^{2}$ is the power spectral density at frequencies $m$ (horizontal), $\mathrm{n}$ (vertical) and $\mathrm{f}$ (temporal).

Both linear and gamma corrected pictures gave the same results to within experimental error. This, somewhat surprising, result is convenient since it allows the same spectral model to be used for both linear and gamma corrected pictures. This implies that the same filters can be used equally well for linear or gamma corrected video. Autocorrelation functions were not calculated for linear pictures in the $x / t$ plane.

The autocorrelation functions show considerable variation between scenes in the $x / t$ plane. Movement in scenes causes 'ridges' in the $x / t$ plane of the autocorrelation function. These 'ridges' can clearly be seen in the individual autocorrelation functions and so render modelling individual scenes rather meaningless. Nevertheless the mean autocorrelation function can be modelled fairly accurately. For these reasons only the parameters for the mean autocorrelation function in the $x / t$ plane have been given.

Only the central portions of the autocorrelation functions were used to calculate the model parameters. This information, at relatively low lags, contains the overall shape of the power spectrum, which is of interest for this application. The value of the autocorrelation function at higher lags contains information on the detail in the power spectrum. The mean power spectrum is expected to be smooth and contain little detail. High lag values of the autocorrelation function, which are noisy and include spurious detail in the power spectrum, are best ignored.

The modelling technique used explicitly excludes the zero frequency component of the power spectrum by removing it before the pictures are processed. Curiously the modelling technique appears to restore a non-zero D.C. component. This arises because the autocorrelation model decays to zero at high lags, whereas the measured autocorrelation function decays to a small negative value at high lags. The value of the measured 
autocorrelation at high lags would be about -0.002 in these experiments for typical results ${ }^{2}$. This is below the noise level of the measured values and so has little effect on the model that is fitted. This feature of the measured autocorrelation function could be accommodated by subtracting an offset from the model fitted. This would make little difference to the model parameters (and so has not been done), but would ensure that the power spectrum was zero at D.C.

It is interesting to note that model 2 corresponds to the (measured) power spectrum of a 3 dimensional function with fractal dimension of 3.5. For more details see appendix section 2.4.

These experiments have investigated the autocorrelation function of the luminance part of CCIR Rec 601 images. The power spectrum of the luminance part of the image is very significant in determining the optimum filtering to use for interpolation for standards conversion (see next chapter). This thesis concentrates on standards conversion of Rec 601 luminance images. Standards conversion of the chrominance part of the image is complicated by the encoding and decoding of the colour information required when using the usual "composite' ${ }^{3}$ television signals. Nevertheless the standards conversion of the chrominance part of the image or of high definition images (HDTV) should not be completely ignored. Fortunately Chimienti \& Picco (1988) have presented spatial model parameters for models $1 \& 2$ for a variety of image types. Their results are presented in tables 2.7 and 2.8 below.

${ }^{2}$ This value can be determined by adding an offset to the autocorrelation model such that the sum integrates to zero, ie:

$$
\int\left(a_{\text {model }}(x)+\text { offset }\right) d x=0 ; x=(x, y, t)
$$

So that;

$$
N_{a} \text { offset }=\int_{-\infty}^{+\infty} a_{\text {moded }}(x) d x
$$

where $\mathrm{N}_{\mathrm{a}}$ is the number of pixels in the autocorrelation surface, ie 4 times the number in the original image. For these experiments, for the autocorrelation function in the $x / y$ plane, number of pixels $=1140 \times 1152$.

${ }^{3}$ For broadcast television the luminance and chrominance parts of an image are frequency/phase division multiplexed into a single 'composite' signal. 
Table 2.7 : Model 1, x/y plane, gamma corrected pictures, from Chimienti \& Picco 1988.

\begin{tabular}{||c|c|c|c||}
\hline $\begin{array}{c}\text { Image } \\
\text { Type }\end{array}$ & $\alpha$ & $\beta$ & $\begin{array}{c}\text { RMS } \\
\text { absolute } \\
\text { error } \\
\left(/ \sigma^{2}\right)\end{array}$ \\
\hline \hline Rec. 601, Luminance & 38.6 & 40.7 & 0.04 \\
\hline Rec. 601, Chrominance & 41.7 & 21.6 & 0.09 \\
\hline HDTV, Luminance & 27.0 & 43.1 & 0.04 \\
\hline HDTV, Chrominance & 27.1 & 31.4 & 0.10 \\
\hline
\end{tabular}

Table 2.8 : Model 2, x/y plane, gamma corrected pictures, from Chimienti \& Picco 1988.

\begin{tabular}{||c|c|c|c||}
\hline $\begin{array}{c}\text { Image } \\
\text { Type }\end{array}$ & $\alpha$ & $\beta$ & $\begin{array}{c}\text { RMS } \\
\text { absolute } \\
\text { error } \\
\left(/ \sigma^{2}\right)\end{array}$ \\
\hline \hline Rec. 601, Luminance & 30.4 & 31.1 & 0.03 \\
\hline Rec. 601, Chrominance & 30.9 & 17.8 & 0.09 \\
\hline HDTV, Luminance & 22.1 & 31.5 & 0.03 \\
\hline HDTV, Chrominance & 21.5 & 24.3 & 0.09 \\
\hline
\end{tabular}

It is encouraging that these results are very similar to those obtained by this investigation where they can be compared.

The models presented in this section can be extended to cases where the movement of objects in the scene has been estimated (see chapter 7). Considering model 2 (as this seems most appropriate for filter design), the model would be modified to;

$$
a(x, y, t)=\exp \left(-\sqrt{\frac{(x-u t)^{2}}{\alpha^{2}}+\frac{(y-v t)^{2}}{\beta^{2}}+\frac{t^{2}}{\gamma^{2}}}\right)
$$




$$
|S(m, n, f)|=\frac{\sqrt{8 \pi \alpha \beta \gamma}}{\left(1+(2 \pi k)^{2}\right)} ; \quad k=\sqrt{\alpha^{2} m^{2}+\beta^{2} n^{2}+\gamma^{2}(f-m u-n v)^{2}}
$$

where $(u, v)$ is the measured velocity of the object in the picture.

The spatial model parameters, $\alpha \& \beta$, would be similar to those for the static model. The temporal parameter, $\gamma$, would vary depending on the motion measurement method and the character and velocity of the moving object.

Using the model of the autocorrelation function the parameter can be calculated from the displaced frame difference. The displaced frame difference is the RMS difference between adjacent frames (allowing for the measured motion) averaged over a small region of the image. Let the displaced frame difference be denoted by $\delta$. Then,

$$
\delta^{2}=\int(g(x, y, t)-g(x+u t, y+v t, t+T))^{2} d x ; \quad x=(x, y)
$$

where $\mathrm{g}$ is the signal (assumed zero mean) and $\mathrm{T}$ is the frame period. Expanding the square and using the definition of the normalised autocorrelation function we get;

$$
\delta^{2}=2 \sigma^{2}(1-a(u t, v t, T))
$$

where $\sigma^{2}$ is the signal variance. Substituting either model 1 or model 2 , and assuming that the temporal correlation parameter $T<\gamma$, we find;

$$
\gamma=2 T \frac{\sigma^{2}}{8^{2}}
$$

This equation relates the temporal model parameter $\gamma$ to the displaced frame difference and the signal energy (excluding D.C.) both of which are easily measured. Equation 18 is equally applicable to either of the models of the autocorrelation function.

The spectrum of an image sequence, after motion compensation, can be estimated (via the autocorrelation function) using equation 18. The estimated spectrum can then be used to design filters for motion compensated filtering. The value of parameter measured will be heavily dependant on the motion estimation technique used and the picture areas to which it is applied. 


\subsubsection{Appendix : Fractal models for $3 D$ scene spectra.}

The spectral density of a 3 dimensional fractal function is given by (Dennis \& Dessipris 1989 and Peitgen \& Saupe 1988).

$$
|S(m, n, f)|=\frac{1}{k^{(11-2 D) / 2}}=\frac{1}{k^{\mu}} ; \quad \mu=\frac{11-2 D}{2}
$$

where $k=2 \pi \sqrt{ }\left(m^{2}+n^{2}+f^{2}\right)$ with frequencies $m, n \& f$, and $D$ is the fractal dimension which can range between 3 and $4 . \mu$ can vary between $3 / 2$ and $5 / 2$.

When a fractal function is imaged with a real camera it will be limited in both extent and bandwidth. The measured signal must be contained within the aperture (window) of the imaging system. Any measurement of the spectrum will thus be convolved with the Fourier transform of the windowing aperture. This convolution limits the measured spectral density at D.C. by averaging the spectral density in a finite region around D.C. The convolution has little effect on the measured spectral density at high frequencies because the underlying spectrum varies smoothly at these frequencies. Similarly the finite bandwidth of the camera prevents the measured value of the autocorrelation function being infinite at zero lag.

As an example consider the case of a spectral density function of the form $S(k)=1 / \mathrm{k}^{2}$ (corresponding to a fractal dimension 3.5). This function can be related to its Fourier transform by the transform integral for spherically symmetric functions vis (Champeney 1973 $\mathrm{p} 46)$;

$$
\begin{aligned}
& S(k)=\iiint \exp (-j 2 \pi k \cdot r) s(r) d r=4 \pi \int_{0}^{\infty} s(r) r^{2} \operatorname{sinc}(2 \pi k r) d r \\
& s(r)=\iiint \exp (+j 2 \pi k . r) S(k) d k=4 \pi \int_{0}^{\infty} S(k) k^{2} \operatorname{sinc}(2 \pi k r) d k
\end{aligned}
$$

where the bold symbols represent vector quantities. Using these equations we find that $S(k)=1 / k^{2}$ and $s(r)=1 / r$ are a transform pair. Both these functions are infinite at D.C. and are of infinite extent. The effect of limiting then in space and frequency can be seen by multiplying both functions by, for example, a Gaussian window function. Considering first the effect of limiting the camera's aperture we have the modified spectral density $S^{\prime}(k)$ given by; 


$$
\begin{aligned}
S^{\prime}(k) & =4 \pi \int_{0}^{-} s(r) \exp \left(-r^{2} / \sigma_{r}^{2}\right) \operatorname{sinc}(2 \pi k r) r^{2} d r \\
& =\left(2 \pi^{2} \sigma_{r}^{2}\right) \exp \left(-\pi^{2} \sigma_{r}^{2} k^{2}\right) \Phi\left(1 / 2,3 / 2, \pi^{2} \sigma_{r}^{2} k^{2}\right)
\end{aligned}
$$

where $\Phi(\alpha, \beta, z)=1+\frac{\alpha z}{\beta 1 !}+\frac{\alpha(\alpha+1) z^{2}}{\beta(\beta+1) 2 !}+\frac{\alpha(\alpha+1)(\alpha+2) z^{3}}{\beta(\beta+1)(\beta+2) 3 !}+\ldots$ is the degenerate hypergeometric series (the integration is given in Gradshteyn \& Ryzhik 1965, p480 3.896 3). This equation shows that the modified spectral density function equals $2 \pi^{2} \sigma^{2}$ at D.C. The asymptotic expansion for $\Phi$ for large values of $z$ (Abramowitz \& Stegun 1965 p508) shows that the modified spectral density equals $1 / \mathrm{k}^{2}$ at large values of $\mathrm{k}$. Similarly the modified spatio-temporal domain function is given by (Gradshteyn \& Ryzhik 1965, p495 3.952 7);

$$
\begin{aligned}
s^{\prime}(r) & =4 \pi \int_{0}^{\infty} S(k) \exp \left(-k^{2} / \sigma_{k}^{2}\right) \operatorname{sinc}(2 \pi k r) k^{2} d k \\
& =\left(2 \pi^{3 / 2} \sigma_{k}\right) \exp \left(-\pi^{2} \sigma_{k}^{2} r^{2}\right) \Phi\left(1,3 / 2, \pi^{2} \sigma_{k}^{2} r^{2}\right)
\end{aligned}
$$

This shows that the value of $s^{\prime}(r)$ at D.C.is $2 \pi^{3 / 2} \sigma_{k}$ and $s^{\prime}(r)$ asymptotically approaches $\pi / r$ at high values of $r$. The case where both $S(r)$ and $S(k)$ are both of finite extent is more difficult to deal with and has not been attempted.

Measurements of the spectral density of a (3 dimensional) fractal function can be approximated by the form $1 /\left(1+k^{\mu}\right)$, where $\mu$ varies between $3 / 2$ and $5 / 2$. The spectral model described earlier (Model 2) has a value of $\mu=2$ corresponding to a fractal dimension of 3.5. That is, a fractal dimension in the middle of the permissible range. Modelling spectra with other fractal dimension would be interesting. This might be done by assuming a measured mean spectral density of the form $1 /\left(1+k^{\mu}\right)$ with a value of $\mu$ other than 2 . The approximation of equation 19 is a reasonable approximation to the measured spectral density at both D.C. and high frequencies.

\subsection{Image Sources.}

The second stage in the television chain is capturing the light emitted by a scene with an image sensor. Virtually all television signals are produced using one of three types of sensor. Firstly there are two types of sensors used in television cameras. The oldest of these two sensors is the vacuum tube, photoconductive sensor. This has a well developed technology and has been used for about half a century. Nevertheless vacuum tube sensors are still widely used and, indeed, are used by state of the art, high definition television cameras. 
The second type of camera sensor, which is increasingly widely used, is the solid state, charge coupled device (CCD) sensor, based on semiconductor technology. This comprises an array of independent photosensors. Another major source of television programs is film. In addition to the display of cinema style motion pictures, film is also frequently used for other types of program material. The optical film is converted to an electronic television signal by a 'telecine' machine. A good discussion of the general characteristics of cameras and telecines can be found in Childs 1990.

This section gives a overview of the characteristics of image sensors as they affect the process of standards conversion. Of major importance, as in the previous section, are the spectral characteristics of the imaging devices. The spectral characteristics of cameras are defined in terms of their frequency response. Different mechanisms limit the (spatio/temporal) resolution in the different types (vacuum tube, $\mathrm{CCD}$ and film) of camera. Ideally the different spectral characteristics of signals from different sources require different interpolation to produce optimum results for standards conversion.

In addition to frequency response there are other differences in the signals from different sources. A major difference between television and film cameras is that the former provide a true 'interlaced' source whereas the latter fundamentally give a 'sequential' signal. In a sequential (or 'progressive') source (such as film) all pixels in a frame are either sensed simultaneously or in strict sequence (or progression) from the top left of the picture to the bottom right. Interlaced sources, by contrast, scan all the odd lines first, followed by all the even lines. This produces one odd and one even 'field' for each 'frame'. There is still considerable debate about the relative merits of sequential and interlaced scanning. Good discussions of this debate can be found in Clarke 1987 and Thorpe \& Hanabusa 1990.

The difference between sequentially scanned film and interlaced signals from television cameras has significant practical consequences. Television pictures are usually displayed in an interlaced format. That is, all the odd lines in a frame are displayed first followed by the display of the even lines. When interlaced signals from television cameras are displayed in this way the major impairments are resolution loss, from both the camera and display, and flicker of the whole picture. Alias artifacts, due to scanning and sampling, are unobtrusive because they are coherent with the scanning structure. In particular movement appears smooth and natural. When sequential images from film are displayed interlaced, the natural time progression of sampling is disturbed. Even lines, which were sampled at the same time as odd lines, are now displayed at a latter time. This gives rise to juddery motion when the camera pans. Ideally a 'standards converter' would be used to convert between the sequential source format and the interlaced output format. This is not done because conventional standards converters cannot eliminate judder which is the main artifact of sequential to interlaced conversion. In the future motion compensated standards conversion 
may eliminate the judder introduced by converting sequential film to interlaced television. This type of problem is discussed in much more detail in later chapters.

The signal from both television cameras and telecines (used for film) undergo significant non-linear processing. One reason is that the ubiquitous 'cathode ray tube' (CRT) displays are not linear. The light intensity output from a CRT, I, is related to the input voltage, V, by;

$$
I \propto V^{\gamma}
$$

where $\gamma$, the display gamma, is a characteristic of the display and typically has a value of about 2.2. Correction must be made for this non linear characteristic of the display and it is preferable to do this in the camera (telecine) rather than the receiver. This minimises the cost of the receiver and also minimises the perceived noise in the displayed picture. The output of television cameras and telecines are thus compressed by raising to the inverse power of the display gamma.

It might be expected that such non-linearity would significantly affect the processing required for standards conversion. In practice, however, it makes little difference, wether linear or non-linear 'gamma corrected' signals are used. There are, perhaps, two reasons for this. Firstly, as seen in the previous section, most of the energy in typical scene spectral is concentrated near zero frequency. Non-linear gamma correction does not, therefore, significantly change the typical scene spectra. Hence 'optimum' interpolation filters (see next chapter), based on typical scene spectra, are equally applicable to linear or gamma corrected signals. Furthermore artifacts caused by gamma correction are likely to be masked by other standards conversion artifacts.

The remainder of this section describes the general spectral characteristics of television cameras and telecines.

\subsubsection{Cameras.}

The signal chain within a typical, colour, television camera is shown in figure 2.4 . The same basic signal chain is used for both vacuum tube and CCD sensors. Three sensors are used to detect the red, green and blue components of the image. The colour analysis of the camera is corrected by 'matrixing' 4 the linear signals from the 3 sensors. Hunt 1987 presents an excellent discussion of colour analysis for television. The linear signals are then compressed by the non-linear 'gamma corrector', to allow for the non-linearity of CRT

${ }^{4}$ The result of matrixing 3 colour component signals is to produce 3 outputs, each of which is a linear combination of the 3 inputs. This operation can thus be described by a matrix of weighting coefficients. 
displays, as discussed above. Usually some form of 'aperture correction' is provided, to partially correct for attenuation of high frequencies by the sensor. Finally the output of the camera is 'blanked' to remove disturbances occurring in the scanning flyback interval.

There are three components to the frequency response of television and film cameras. The spatial and temporal responses of the camera sensor are approximately independent. Hence the response of the camera sensor can be modelled as the product of spatial and temporal frequency responses. The third component to the overall response is that of the aperture corrector. The response of the aperture corrector, is designed to correct for losses in the sensor and is usually wholly spatial (ie with uniform temporal response). The details of the aperture corrector can vary considerably from purely horizontal filtering to more complex spatial filtering. Drewery 1990 provides a discussion of the types of filtering typically used for aperture correction. The response of the whole camera is, thus, of the form;

$$
C(m, n, f)=C_{s}(m, n) C_{t}(f) A(m, n)
$$

where $\mathrm{C}$ is the overall response of the camera, $\mathrm{C}_{\mathrm{s}}$ is the spatial response of the sensor, $\mathrm{C}_{\mathrm{t}}$ is the temporal response of the sensor and $A$ is the (spatial) response of the aperture corrector. The performance of all cameras is, of course, also affected by the optics preceding the sensor. The remainder of this sub-section deals with the frequency response of the two type of sensor.

\subsubsection{Vacuum Tube Sensors.}

The spatial resolution of a vacuum tube sensor is governed by the profile of the electron beam used to scan the photoconductive layer in the tube. The profile of the electron beam is the result of the complex interaction between the charge in the beam and on the target layer. The charge on the target depends on the illumination and also on how the target is discharged by the beam. Nevertheless, as an approximation, we may assume a perfect circular profile for the beam. This gives a Gaussian spatial frequency response (Thorpe \& Hanabusa 1990) ie;

$$
C_{s}(m, n)=\exp \left(-(\ln 2)\left(\frac{m^{2}}{m_{0}^{2}}+\frac{n^{2}}{n_{0}^{2}}\right)\right)
$$

where $m_{0}$ and $n_{0}$ are the horizontal and vertical $-6 \mathrm{~dB}$ frequencies respectively. For a typical 625 line, interlaced camera $m_{0}=216 \mathrm{c} / \mathrm{pw}$ and $n_{0}=156 \mathrm{c} / \mathrm{ph}$.

There are two factors governing the temporal (dynamic) response of vacuum tube sensors. The first, and most important factor, is the integration time of the sensor. This is the period over which light input is integrated to give the sensor output for each frame. The integration time of a tube camera depends on its maximum resolution. As the maximum 
resolution is increased less of the photosensitive area is discharged on each field and the integration time increases towards $40 \mathrm{~ms}$ (assuming a $50 \mathrm{~Hz}$ interlaced system). Tube cameras typically have an integration time of $20 \mathrm{~ms}$ because their resolution is sufficiently low to discharge the whole of the photosensitive layer in each field. The second factor governing a tube's dynamic response is its lag, which depends on the rate at which charge is left behind following a single electron beam scan. The temporal (or dynamic) resolution of the sensor is thus given by (Thorpe \& Hanabusa 1990),

$$
C_{t}(f)=\operatorname{sinc}\left(\pi T_{i} f\right) \frac{(1-b)}{1-\exp \left(-j 2 \pi T_{f} f\right)}
$$

where the first term is due to integration time $T_{i}$ (usually about 1 field period) and the second term is due to lag (b depends on tube construction), $T_{\mathrm{f}}$ is the field period.

\subsubsection{Charge Coupled Devices.}

The spatial resolution of a charge coupled device (CCD) sensor is determined by the geometry of sensing elements in the array. The rectangular shape of these elements makes the frequency response a product of sinc functions, ie;

$$
C_{s}(m, n)=\operatorname{sinc}\left(\frac{2 \pi m p_{x}}{M}\right) \operatorname{sinc}\left(\frac{2 \pi n p_{y}}{N}\right)
$$

where $\mathrm{M}, \mathrm{N}$ are the number of pixels per line and number of lines per frame respectively (eg $\mathrm{M}=864, \mathrm{~N}=625$ for 625 line CCIR Rec 601 television). The parameters $\mathrm{p}_{\mathrm{x}} \& \mathrm{p}_{\mathrm{y}}$ are the fraction (horizontal and vertical respectively) of space on the CCD actually occupied by the sensing elements. Typically $\mathrm{p}_{\mathrm{x}} \& \mathrm{p}_{\mathrm{y}}$ are about $70 \%$ although they can be much smaller. The frequency response of $C C D$ sensors extends well beyond the Nyquist limit that can be supported by scanning standard and sampling rate. Hence spatial aliasing tends to be more of a problem with CCD rather than vacuum tube sensors. Since this aliasing is produced in the sensor it permeates the whole of the television chain causing problems with various types of signal processing. It is therefore desirable to use some form of optical prefilter prior to the sensor and many of the more recent broadcast quality CCD cameras incorporate such a filter.

The dynamic (temporal frequency) response of CCD sensors is governed purely by the integration time. Therefore;

$$
C_{i}(f)=\operatorname{sinc}\left(\pi T_{i} f\right)
$$

where, as above, $T_{i}$ is the integration time of the sensor. Unlike tube sensors the integration time of $\mathrm{CCD}$ sensors can be controlled electronically. Typical integration times range from 
$1 \mathrm{~ms}$ to a maximum $20 \mathrm{~ms}$ (for $50 \mathrm{~Hz}$ interlaced output). Short integration times increase the response at high temporal frequencies giving rise to greater temporal aliasing. Short integration times are often used for sport and other fast moving scenes. With tube cameras temporal aliasing is seldom visible on pictures displayed directly (ie without standards conversion). Temporal aliasing is sometimes visible from CCD sensors, when a short integration time is used, and looks as if the scene had been illuminated with a stroboscopic light. Such pictures provide a severe test for standards converters.

\subsubsection{Telecines.}

Telecines are used to generate an electronic television signal from an optical film. The film is illuminated by a light source and the density of the film detected by a light sensor. The signal chain for a telecine is shown in figure 2.5. This is similar to the camera processing chain with two exceptions. Firstly colour correction is applied (using a colour correction matrix) to the logarithm of the signal from the sensors. This is more suitable for correcting the colour analysis of films (Hunt 1987). Gamma correction is achieved by the combination of logarithmic and exponential non-linearities either side of the colour correction matrix. The second difference is the addition of an sequential to interlace converter. This is required, as explained above, because film is essentially a sequential source but is shown on interlaced displays. The sequential to interlace converter does not, usually, perform interpolation but merely changes the order of the output pixels from the telecine to simulate an interlaced signal.

The resolution (both spatial and temporal) of film originated television signals is limited both by the initial filming process and by the telecine. Film cameras are limited by the MTF (modulation transfer function) of the film stock and the film processing and printing processes. This second factor may well be dominant in limiting the final film resolution. Since the overall frequency response of the image on the final film is due to the combined action of several lowpass processes it is fair to assume (from the central limits theorem) that the spatial response is approximately Gaussian. Although the spatial response is of the same form as for tube television cameras, the frequency response can be considerably broader. The temporal response of a film camera is governed by the shutter time. Film cameras typically have their shutter open for $50 \%$ of the frame period. Therefore for 25 frames/second film the integration time is about $20 \mathrm{~ms}$, the same as for tube cameras.

Two types of telecine machine, known as 'flying spot' and line array telecines, are in common use. The flying spot telecine illuminates the film using a CRT and senses the light using (three) photomultipliers. At any instant only a single point on the CRT emits light and hence a single point on the film is illuminated by the imaging optics. As the electron beam in the CRT scans the phosphor plane in the CRT a spot of light scans the film. Hence the 
output of the photomultipliers is a scanned television signal. By careful design flying spot telecines can achieve very high resolution and the resolution of the television signal may be limited by that of the film. Such high resolution may not be desirable as it increases the amount of aliasing in the signal. Therefore the output resolution from the telecine is usually lower than that on the film. In both cases the shape of the frequency response will be approximately Gaussian. Note that the telecine does not affect the temporal (dynamic) resolution of the television signal, which is completely governed by the film camera.

Solid state CCD sensors are used in 'line array telecines'. In these machines the film is illuminated and the image focused on a one dimensional (line array) CCD sensor. The line array sensor (aligned horizontally with respect to the image) detects a complete line of the image. Vertical scanning of the image is achieved by the motion of the film itself. The vertical resolution of the television signal is determined by the motion of the film and the width and integration time of the sensor. Since the distance travelled by the film in one integration period is approximately the same as the sensor width the vertical frequency response has an approximately $\operatorname{sinc}^{2}$ shape. Horizontally the resolution is limited by the film and the imaging optics in the telecine and will hence be approximately Gaussian. Horizontal sampling in a line array telecine is performed in the sensor itself. It is unlikely that the number of elements in the sensor will be the same as on a digitally sampled television line 5 . A one dimensional sample rate conversion must be used to input horizontal sampling rate to the correct output sampling rate. This might be regarded as a 'one dimensional' standards conversion problem. The interpolation filter used for this process must compromise between loss of resolution and horizontal aliasing as will be discussed, in great detail, in later chapters.

\subsection{Display Devices.}

Having produced a television signal and possibly processed it (eg standards conversion) the moving pictures must be displayed. The display device forms the penultimate stage of the television chain. Two display devices, cathode ray tubes (CRTs) and liquid crystal displays (LCDs) are used for television pictures. The CRT is by far the most common type of display but, as manufacturing technology improves, LCDs will probably become increasingly common. Both type of display can be used in direct view and projection display systems.

The display mechanism of CRTs is complicated and highly non-linear which makes assessment of its 'frequency response' very difficult. There are several sources of nonlinearity in CRT displays. The overall transfer characteristic of these displays is a power law

${ }^{5}$ This is because the telecine must be able to cope with film with a variety of different aspect ratios. 
with an index of about 2.2 (as discussed above). Therefore the concept of a frequency response only really applies to small signals and is dependant on the mean signal level. Resolution and brightness of the display are complementary, an increase in one resulting in a decrease in the other. This arises firstly because at high beam currents the electron beam tends to defocus itself reducing resolution. Secondly, to achieve higher brightness a broader beam must be used to avoid phosphor saturation. Furthermore the resolution decreases away from the centre of the display tube because of the beam dynamics. Despite these limitations the frequency response can be approximated by a Gaussian shape. That is, the spatial part of the display response, $D_{s}$, is approximately;

$$
D_{s}(m, n)=\exp \left(-(\ln 2)\left(\frac{m^{2}}{m_{0}^{2}}+\frac{n^{2}}{n_{0}^{2}}\right)\right)
$$

where $m_{0}$ and $n_{0}$ are horizontal and vertical $-6 \mathrm{~dB}$ frequencies respectively. Typically for a 625/50/2:1 CRT display $m_{0}=432 \mathrm{c} / \mathrm{pw}$ and $\mathrm{n}_{0}=312 \mathrm{c} / \mathrm{ph}$, ie twice the resolution of the camera. No great accuracy should be expected of this approximation.

The fast exponential decay of light from the phosphor gives a very wide temporal response to a CRT display. The CRT temporal response provides almost no post filtering of the displayed image. Post filtering relies on the temporal response of the human eye.

Liquid crystal displays are becoming increasingly available. LCDs do not emit their own light but control transmitted, or reflected, light using a variable polariser (the liquid crystal). They are much more linear than CRT displays. Hence gamma corrected signals must be linearised before display. LCDs are made of an array of independent display elements. Therefore they have a similar spatial frequency response to CCD cameras. The temporal response of LCDs varies with the technology used but is generally much narrower (slower response) than that of a CRT. This might be a problem on fast moving images but can be overcome using a pulsed light source.

\subsection{The Human Visual System.}

The final stage in the television chain is the perception of the image by the human visual system. This system is the undoubtedly the most complex part of the chain. (For convenience I shall sometimes refer to the human visual system as simply 'the eye'.) It is a non-linear sensor and there is considerable evidence to indicate that subjective brightness is a logarithmic function of light intensity. The (small signal) frequency response of the eye is inhomogeneous over the field of vision. Vision is most acute at the centre of the visual field and becomes much less acute towards the edge. Finally, and most problematically, the eye is an 'active' sensor. That is the visual system changes its response depending on the image. 
In particular the eye can follow or 'track' moving objects, a characteristic which completely alters its spatio-temporal frequency response.

The section investigates possible simple models for the small signal frequency response of the human visual system. This response is generally referred to as 'the frequency response of the eye'. Initially the models presented apply only to the luminance response of the stationary eye. They are then extended to include the eyes response to coloured stimuli. Having considered the response of the stationary eye the effects of its motion are considered. The models presented are intended to allow the processing used for standards conversion to be optimised (particularly filter design). For this purpose an all embracing model of the response of the eye is not required and a simpler model will suffice. The models presented here are distilled from a considerable number of papers on the subject (Budrikis 1973, Budrikis \& Lucas 1982, Burbeck \& Kelly 1980, Hauske \& Zetzsche 1989, Kelly 1972, 79, $83,85,89$, and Sporea \& Tonnquist 1989).

A brief discussion of the physiology of the eye is helpful in trying to model the characteristics of its response. Light is focused, by the lens at the front of the eye, to form an image on the retina at the back of the eye. The retina is a layer of light sensitive cells which convert light intensity to nerve impulses. There are two type of light sensitive cells in the eye, rods and cones. There are about 100 million rods in the retina which are responsible for low resolution, monochrome vision. They are sensitive to low levels of illumination and are useful for night vision. By contrast there are only about 7 million cone cells, which are concentrated in the central part of the retina called the fovea. The cones are responsible for high resolution colour vision, such as used for viewing television. There are 3 type of cone cells sensitive to red, green and blue light. These have an unequal distribution, $64 \%$ are red sensitive, $32 \%$ green sensitive and only $2 \%$ are blue sensitive (Sporea \& Tonnquist 1989). The sparsity of 'blue' cones results in relatively poor resolution for blue light. The output from cone cells is combined in various ways, by nerve cells in the retina, to give luminance (monochrome) and colour difference (red/green and yellow/blue) signals which are then processed further by the brain. The combination of signals from the cone cells is shown in figure 2.6 (Sporea \& Tonnquist 1989).

A principle assumption in modelling the eye is that the human visual response can be modelled as a linear system. This is very desirable because the image processing techniques using the model are based on linear filtering. Although, as mentioned above, the overall response of the eye is approximately logarithmic, this is probably due to adaption rather than an inherent non-linear characteristic. For a given ambient light level the eye's response can reasonably be approximated as linear over a limited range of brightness.

Several other assumptions are used to simplify the model. A typical illumination level is assumed for television pictures. This is not too severe an assumption as the response of the eye does not vary very greatly with light level. Two other assumptions are also made which, 
while not strictly necessary, simplify the model and provide sufficient accuracy for this application. The first is that the spatial frequency response is isotropic, that is it does not vary as a function of orientation. The second is the assumption of homogeneity, that is frequency response is not a function of displacement from the centre of the field of view. These latter two assumption are probably justified since the television image is likely to be focused on the fovea which is a small, relatively isotropic, region of the retina. If the image is focused on the fovea then the response to the television image will be almost entirely due to colour sensing cone cells rather than rod cells.

\subsubsection{Frequency response of the stationary 'eye'.}

This subsection discusses some possible models for the monochrome frequency response of a stationary eye. There is considerable published information on the response of the eye (eg Robson 1966, Kelly 1972 \& 79 and Terkla-McGrane et al 1988) and of ways to model it (eg Hauske \& Zetzsche 1989, Budrikis 1973, Budrikis \& Lucas 1982 and Kelly 1979). There seems to be general agreement that the human visual response is due to the interaction of excitatory and inhibitory processes in the first stages of neural processing in the visual system. The apparent logarithmic non-linearity of the eye is probably due to this interaction (Hauske \& Zetzsche 1989).

A model for the response of the eye is presented by Lucas \& Budrikis 1982. It seems suitable for the purpose of optimising processing for standards conversion, and similar models are given in Budrikis 1973. The structure of the model is shown in figure 2.7, where $1(x, y, t)$ is the light input and $s(x, y, t)$ is the subjective output. Both the input and output are functions of space and time where the spatial variables are angles subtended from the centre of the field of view. This model incorporates adaption, as the interaction of excitatory and inhibitory responses, and thereby 'explains' the apparent logarithmic amplitude response characteristic of the eye. The model can be described mathematically as,

$$
s(x, y, t)=g\left(\frac{l(x, y, t) \star e(x, y, t)}{\mu+l(x, y, t) \star i(x, y, t)}\right)
$$

where $e(x, y, t)$ is an excitatory impulse response, $i(x, y, t)$ is an inhibitory impulse response and * represents the convolution operation, $\mu$ is an inhibition threshold level and $g$ is a gain constant.

The model above can be linearised by considering small signals superimposed on a background level. Let the input consist of a single small sinusoid ie, 


$$
l(x, y, t)=l_{0}(1+\delta \exp (-j 2 \pi(m x+n y+f t)))
$$

where $\mathrm{l}_{0}$ is the background light level and $\delta$ is a small constant tending to zero in the limit. Substituting equation 32 into 31 , expanding as a polynomial in $\delta$ and truncating squares and higher powers gives;

$$
s(x, y, t)=\left(\frac{g l_{0}}{\mu+l_{0}}\right)(1+8(E(m, n, f)-k I(m, n, f)) \exp (-j 2 \pi(m x+n y+f t)))
$$

where $k=1_{0} /\left(\mu+1_{0}\right)$ and $E(m, n, f)$ and $I(m, n, f)$ are the Fourier transforms of the excitatory and inhibitory impulse responses.

Hence we can conclude that the large signal (D.C.) response is given by $g /\left(\mu+l_{0}\right)$ and the small signal (A.C.) response is given by;

$$
R(m, n, f)=\left(\frac{g}{\mu+L_{0}}\right)(E(m, n, f)-k I(m, n, f))
$$

That is the small signal frequency response of the eye is due to the difference between excitatory and inhibitory responses.

Certain characteristics would be expected, from the known physiology of the eye, of the excitatory and inhibitory parts of its luminance frequency response. It is thought that the excitatory and inhibitory parts of the luminance response are due to the combined response of a small cluster of cone cells. The cone cells contributing to the excitatory response are more localised than those contributing to the inhibitory response. Hence the excitatory response is of wider bandwidth than the inhibitory response. The spatial frequency responses of both parts of the overall response are due to the spatial distribution and connection of cones to the nerve cells which combine their outputs. It is thought that the spatial and temporal parts of the two responses may be considered approximately independent. That is we would expect both the excitatory and inhibitory parts of the frequency response to be variables separable with respect to spatial and temporal frequency. Also it is reasonable to expect the spatial response to be approximately isotropic.

To determine model parameters we must compare mathematical models to experimental data. Many suitable results have been published for 'threshold vision' in humans. The threshold response of the eye is measured by determining the smallest amplitude (spatio-temporal) sine wave which is 'just detectable'. Applying an input as given in equation 32 we measure the smallest 'detectable' value of $\delta$. In practice, of course, the probability of detecting a low level sinusoid increases with amplitude. The threshold level, $\delta$, is determined at some particular probability of detection (say $50 \%$ ). If the probability of detecting a given sinusoid (of frequencies $m, n \& f$ ) is $p$ then, 


$$
p=T\left(\delta l_{0}|R(m, n, f)|\right)
$$

where $\mathrm{T}$ is an (unspecified) monotonically increasing function. Inverting this equation and rearranging gives,

$$
\frac{1}{\delta}=\frac{l_{0}|R(m, n, f)|}{T^{-1}(p)}
$$

where $\mathrm{T}^{-1}$ is the inverse of function $\mathrm{T}$. This shows that the small signal frequency response can be measured as the inverse of the smallest detectable signal at a given frequency. Conveniently threshold vision frequency responses are usually plotted as $1 / \delta$ versus frequency.

The overall luminance frequency response of the eye is bandpass with respect to both spatial and temporal frequency. This because the excitatory response is essentially low pass, but of wider bandwidth than the low pass inhibitory response for both spatial and temporal frequencies. A number of attempts have been made to model the eye's response, assuming the excitatory/inhibitory model with variable separable excitatory and inhibitory responses (eg Budrikis 1973 and Burbeck \& Kelly 1980). Two examples of these are shown in figures 2.8 $\&$ 2.9. These are models of Kelly's data (1972) and Robson's data (1966). They correspond to the following equations for the excitatory and inhibitory impulse responses;

$$
\begin{aligned}
& e(x, y, t)=\frac{1}{2 \pi \sigma_{e}^{2} \tau_{e}} \exp \left(-\frac{x^{2}+y^{2}}{2 \sigma_{e}^{2}}\right) \exp \left(-\frac{t}{\tau_{e}}\right) \\
& i(x, y, t)=\frac{1}{2 \pi \sigma_{i}^{2} \tau_{i}} \exp \left(-\frac{x^{2}+y^{2}}{2 \sigma_{i}^{2}}\right) t \exp \left(-\frac{t}{\tau_{i}}\right)
\end{aligned}
$$

These impulse responses correspond to the following frequency responses;

$$
\begin{aligned}
& E(m, n, f)=\frac{\exp \left(-2 \sigma_{e}^{2} \pi^{2}\left(m^{2}+n^{2}\right)\right)}{1+j 2 \pi \tau_{e}} \\
& I(m, n, f)=\frac{\exp \left(-2 \sigma_{i}^{2} \pi^{2}\left(m^{2}+n^{2}\right)\right)}{\left(1+j 2 \pi f \tau_{i}\right)^{2}}
\end{aligned}
$$


The overall frequency response is given by;

$$
R(m, n, f)=\frac{g}{\mu+l_{0}}(E(m, n, f)-k I(m, n, f)) \quad ; k=\frac{l_{0}}{\mu+l_{0}}
$$

The parameters for these models are given in the table 2.9 .

Table 2.9: Model parameters for the response of the human visual system.

\begin{tabular}{|c|c|c|c|}
\hline Parameter & Unit & $\begin{array}{c}\text { Kelly's Data } \\
15.2 \mathrm{~mL}\end{array}$ & $\begin{array}{c}\text { Robson's Data } \\
6.3 \mathrm{~mL}\end{array}$ \\
\hline \hline $\mathrm{g}$ & ratio & 168 & 198 \\
\hline$\mu$ & $\mathrm{mL}$ & 0.45 & 0.03 \\
\hline$\sigma_{\mathrm{e}}$ & minutes of arc & 1.4 & 1.01 \\
\hline$\tau_{\mathrm{e}}$ & $\mathrm{ms}$ & 37 & 55 \\
\hline$\sigma_{\mathrm{i}}$ & minutes of arc & 8.3 & 5.58 \\
\hline$\tau_{\mathrm{i}}$ & $\mathrm{ms}$ & 46 & 55 \\
\hline
\end{tabular}

Note that studio monitors are set to about $50 \mathrm{~cd} / \mathrm{m}^{2}$ and domestic receivers are typically 4 times brighter. These correspond to brightness values in the range $16 \mathrm{~mL}$ to $64 \mathrm{~mL}\left(1 \mathrm{~cd} / \mathrm{m}^{2}\right.$ $=\pi / 10 \mathrm{~mL}$ ). Hence these models correspond to approximately the correct brightness for television.

The models above can only be considered to provide an approximation to the overall shape of the eyes frequency response. They do, however, correctly predict features of the eye's response such as the position of the maximum in the spatial response at $0 \mathrm{~Hz}$. There are probably two reasons for the poor absolute accuracy of these models. Firstly these models were derived to minimise computation. More complex excitatory/inhibitory responses such as;

$$
e(x, y, t) \text { or } i(x, y, t)=\frac{1}{2 \pi \sigma^{2}} \exp \left(-\frac{\left(x^{2}+y^{2}\right)}{2 \sigma^{2}}\right) \frac{\tau^{\frac{1}{2}}}{(2 \pi)^{\frac{1}{2}} t^{\frac{3}{2}}}
$$

ie, 


$$
E(m, n, f) \text { or } I(m, n, f)=\exp \left(-2 \sigma^{2} \pi^{2}\left(m^{2}+n^{2}\right)\right) \exp (-\sqrt{|2 \pi f \tau|})
$$

would probably result in a more precise model. The temporal function here occurs in lossless diffusion processes and accurately models (Kelly 1971 and Ives 1922) the high frequency asymptote for large area flicker responses. A more important reason why the models above did not fit the experimental data very precisely, is because small natural movements of the fixated eye significantly change its frequency response. The effect of movement of the eye is discussed in subsection 2.5.3

Stabilising the position of images, used as stimuli for threshold vision experiments, on the retina allows much more accurate responses to be measured. This was achieved by Kelly 1979. Test images were presented on a television screen. The position of the observers eye was continually monitored. Involuntary movements of the fixated eye were compensated for by moving the position of the image on the television screen. With the improved threshold vision measurements he developed an accurate model for the response of the stationary eye (Kelly 1979). The model he gives is;

$$
R(m, n, f)=\left(241+23.6\left|\ln \left(\frac{f}{\alpha m}\right)\right|^{3}\right) f m \exp \left(-\frac{f+\beta m}{\gamma}\right)
$$

where $\alpha=3$ degrees $/ \mathrm{s}, \beta=2$ degrees $/ \mathrm{s}$ and $\gamma=3.65 \mathrm{~Hz}$. Note that $1 / \mathrm{R}$ approximates the experimentally measured modulation ( $1 / \delta$ in equation 36$)$.

This subsection has discussed a number of models for the luminance frequency response of a stationary eye. The excitatory/inhibitory model provides the greatest insight into the workings of the eye. Nevertheless Kelly's model provides the most accurate description of the frequency response. The excitatory/inhibitory model is discussed extensively by Kelly 1983, 1989 and provides a model for the chrominance response of the stationary eye discussed in the next sub section.

\subsubsection{Chrominance response of the eye.}

This subsection discusses the relationship between the luminance and chrominance frequency response of the eye. This may be considered in terms of the excitatory/inhibitory model discussed in the previous subsection. An extension to this model was developed by Ingling \& Martinez-Uriegas (1985) to include both the achromatic (luminance) and chromatic (colour) response of the eye, based on the physiology of the retina. The achromatic frequency response of the eye is essentially bandpass whilst the chromatic response is essentially lowpass in nature. Although the achromatic and chromatic responses of the eye are very different, nevertheless they appear to be intimately related. 
The excitatory and inhibitory responses of the eye, which combine to give its overall response, appear to be due to different colour receptors. This gives a model, known as the colour opponent receptive field model, for the impulse response of a part of the retina which can be written as;

$$
\phi(x, y, t, \lambda)=e(x, y, t) R(\lambda)+i(x, y, t) G(\lambda)
$$

where $\phi$ is the overall impulse response, $e$ is the excitatory response and $i$ the inhibitory response. $R$ and $G$ are the spectral sensitivities of the red and green receptor cells (cones) respectively, as a function of the wavelength $(\lambda)$ of the light. This equation thus corresponds to a retinal receptive field with a red sensitive, excitatory, centre and a green sensitive, inhibitory, surround. Equation 46 can be rewritten as;

$$
\phi(x, y, t, \lambda)=\frac{1}{2}[(R+G)(e-i)+(R-G)(e+i)]
$$

The first term in this equation corresponds to an achromatic, bandpass response, while the second term corresponds to a chromatic, lowpass response. This can be seen since an achromatic stimulus has equal red and green components making the second term vanish. Similarly an isoluminance chromatic stimulus will make the first term vanish.

The colour opponent receptive field model, described above, is in good agrement with published psychophysical data. Burbeck and Kelly (1980) assumed that the model was indeed valid and tried to derive the chromatic response from the achromatic response measured previously by Kelly 1979. They derived excitatory and inhibitory responses from the achromatic response by considering the response at high and low frequencies. The excitatory and inhibitory responses were then added to, hopefully, approximate the chromatic response of the eye. There was good agreement between their model and chromatic responses directly measure by Kelly (1983). This provides good quantitative verification of the colour opponent receptive field model.

Given models of the excitatory and inhibitory responses for a retinal receptive field the achromatic response can be determined by subtraction and the chromatic response by addition. Equations 39 and 40 could provide suitable mathematical models for these responses.

Equation 46 'explains' the form of the achromatic and chromatic response of the eye, but not the phenomenon of colour vision. A single retinal receptive field is unable to distinguish between colours since different combinations of red and green light will evoke the same response. It is known, however (Ingling \& Martinez-Uriegas 1985), that there are green centred receptive fields as well as red centred ones. The response of a green centred receptive field is given by; 


$$
\Psi(x, y, t, \lambda)=\frac{1}{2}[(R+G)(e-i)-(R-G)(e+i)]
$$

Adding and subtracting equations 46 and 47 could provide the necessary separation of the retinal response into achromatic and chromatic channels. Therefore the combination of both red and green centred colour receptive fields provides a mechanism for the phenomenon of colour vision (Kelly 1989).

\subsubsection{Effect of Motion.}

The above model of the eye is over simplified because it does not take account of the effect of the eye's motion. The response of the eye is complicated because it is an 'active' sensor; it responds to scene content by moving. The response of the eye therefore depends on the movement of whatever is being observed. Furthermore, even if the eye is 'fixated' on a stationary object it still undergoes small involuntary eye movements known as drifts and saccades (Ditchburn 1973). The nature and magnitude of involuntary motion and the overall effects of motion are discussed in Kelly 1985.

When a object moves the eye tries to track the motion. This skews the response of the eye in the frequency domain. The response, $\mathrm{M}$, of a moving eye is given by;

$$
M(m, n, f, u, v)=R(m, n, f-m u-n v)
$$

where $\mathrm{R}$ is the response of the stationary eye and $\mathrm{u} \& \mathrm{v}$ are the horizontal and vertical velocities respectively. The model presented in the previous sub-sections is purely that for a stationary eye. From the point of view of standards conversion we need to know the average response of the eye taking into account its motion. A reasonable assumption, in the first instance, is that the eye tracks objects in a scene perfectly. Thus the average response is the expected value for response of the eye, given a probability distribution for the velocities of objects in a scene. The expected value is defined by;

$$
\begin{aligned}
\langle M(m, n, f, u, v)\rangle & =\int M(m, n, f, u, v) P(u, v) d u d v \\
& =\int R(m, n, f-m u-n v) P(u, v) d u d v
\end{aligned}
$$

where $\mathrm{P}(\mathrm{u}, \mathrm{v})$ is the probability density function of a velocity $(\mathrm{u}, \mathrm{v})$.

The average response of a moving eye can be derived for the particular case of an opponent receptive field model (excitatory/inhibitory model) and a Gaussian probability distribution. Assuming the response of the stationary eye is of the form;

where $S$ and $T$ represent the spatial and temporal parts of the excitatory and inhibitory responses denoted by subscripts e and i respectively, and assuming also that the probability 


$$
R(m, n, f)=S_{e}(m, n) T_{e}(f)+k S_{i}(m, n) T_{i}(f)
$$

distribution of velocities is Gaussian ie;

$$
P(u, v)=\frac{1}{2 \pi \sigma_{u} \sigma_{v}} \exp \left(-\left(\frac{u^{2}}{2 \sigma_{u}^{2}}+\frac{v^{2}}{2 \sigma_{v}^{2}}\right)\right)
$$

then, after some tedious integration, we find that;

$$
\begin{gathered}
\langle R(m, n, f, u, v)\rangle=S_{c}(m, n)\left(T_{c}(f) \star \frac{1}{\chi \sqrt{2 \pi}} \exp \left(-\frac{f^{2}}{2 \chi^{2}}\right)\right) \\
-k S_{i}(m, n)\left(T_{i}(f) \star \frac{1}{\chi \sqrt{2 \pi}} \exp \left(-\frac{f^{2}}{2 \chi^{2}}\right)\right)
\end{gathered}
$$

where the star represents convolution and $\chi=\sqrt{m^{2} \sigma_{u}^{2}+n^{2} \sigma_{v}^{2}}$.

The average response of a moving eye (equation 52) has some interesting features. The overall effect of eye movement (including involuntary movements) is to widen the frequency response and, particularly, to increase the response at low frequencies. The very low response of the stationary eye to low frequencies is masked by the eye's natural involuntary movements. The average excitatory and inhibitory responses are no longer spatially/temporally separable as they were in the model for the stationary eye. These effects may account for the poor precision of the models from Budrikis 1973 (equations $39 \& 40$ ), which were based on threshold responses to images which had not been stabilised on the retina. A further factor which should be borne in mind is that the dynamic acuity of a tracking eye decreases slightly with tracking velocity. This is not a very large effect corresponding to a reduction in acuity by a factor of 2 for velocities of about 20 to 25 degrees/s.

\subsubsection{Discussion.}

This section has described a number of models, taken from the literature, for the three dimensional frequency response of the human visual system. These provide an estimate of both the monochrome and colour response of the eye over the range of frequencies of interest in television systems. These models should be considered indicative of the frequency response rather than being particularly precise. This is true, not least, because there is considerable variation between the responses of particular individuals. Nevertheless these models are 
sufficiently accurate to allow some useful optimisation of standards conversion processing as described in subsequent chapters.

Some aspects of the general physiology and operation of the eye were also discussed in this section. Understanding of the operation of the eye allows standards converters to be designed to perform well for critical features of human vision. In particular the ability of the eye to 'track' moving objects profoundly effects its response. Such movement of the eye is, fundamentally, the reason why the technique of motion compensation (discussed in detail later) is of great significance to improved standards conversion.

\subsection{Summary.}

This chapter has presented a number of models for the response of parts of the television signal chain. The four parts of the chain considered here are the spectrum of the original scene and the responses of the camera, display and the observer's human visual system. These models are only approximate but, nevertheless, allow improved processing to be developed for standards conversion.

A pervasive problem in the analysis of the signal chain has been the non-linearities in the system. However, under the restricted viewing conditions for television these have less effect than might, at first, be supposed. The most significant non linearity is that of 'gamma correction'. This is deliberately introduced in the camera and a complementary non-linearity is present in the display. Processing is thus performed on images with non-linearly scaled brightness. This has little effect on the average spectrum of signals anywhere in the signal chain because most of the energy is clustered at low frequencies. Thus although gamma correction has an effect it does not, necessarily, degrade the processing. Another possible source of non-linearity is the eye itself. However the apparent non-linearity of the eye over its wide dynamic range is, perhaps, illusory. This is because the non-linearity is due to adaption and over a restricted range of brightness, as occurs for television, the eye's response is more nearly linear. Despite the non-linearities it is probably fair, as a first approximation, to treat the complete television chain as linear!

\subsection{References.}

1. Abramowitz, M. \& Stegun, I.A. Handbook of Mathematical Functions. National Bureau of Standards Applied Mathematics Series 55. Fourth printing 1965.

2. Bateman, H. \& the Staff of the Bateman Manuscript Project. Tables of Integral Transforms, Volume 1. McGraw-Hill Book Company, Inc. 1954. 
3. Budrikis, Z.L. 1973. Model Approximations to Visual Spatio-Temporal Sine-Wave Threshold Data. The Bell System Technical Journal, Vol. 52, No. 9, p1643-1667, November, 1973.

4. Budrikis, Z.L. \& Lukas, F.X.J 1982. Picture Quality Prediction based on a Visual Model. IEEE Transactions on Communications, Vol. Com-30, No. 7, p1679-1692 July 1982.

5. Burbeck, C.A. \& Kelly, D.H. 1980. Spatiotemporal characteristics of visual mechanisms: excitatory-inhibitory model. Journal of the Optical Society of America, Vol. 70, No. 9, September 1980, pp 1121-1126.

6. CCIR. 1986. Encoding parameters of digital television for studios. CCIR Recommendation 601-1, XVIth Plenary Assembly, Geneva 1986, XI-1, pp. 319-328.

7. Champeney, D.C. 1973. Fourier Transforms and their Physical Applications. Academic Press. ISBN 0-12-167450-9.

8. Childs, I., 1990. Telecine and Cameras. Digital television, Sandbank, C.P. (Editor), John Wiley \& Sons Ltd, 1990, ISBN 0-471-92360-5, pp 499-538.

9. Chimienti, A. \& Picco, R. 1988. HDTV Image Statistics: Analysis and Comparison with CCIR Rec.601 Standard Images. Signal Processing of HDTV, Proceedings of the Second International Workshop on Signal processing of HDTV, L'Aquila, Italy, 1988. Edited by L. Chiariglione. Elsevier Science Publishers B.V. ISBN 0-444-70518-X.

10. Clarke, R.J. 1985. Transform Coding of Images. Academic Press. ISBN 0-12-175730-7.

11. Clarke, C.K.P. 1987. Future television systems: Comparison of sequential and interlaced scanning. BBC Research Department Report BBC RD 1987/18.

12. Dennis, T.J. \& Dessipris, N.G. 1989. Fractal Modelling in Image texture Analysis. I.E.E. Proceedings, Vol. 136, Pt. F, No. 5, October 1989.

13. Ditchburn, R.W. 1973. Eye-movements and visual perception. Clarendon, Oxford 1973 p142.

14. Drewery, J.O., 1990. Digital filtering of television signals. Digital television, Sandbank, C.P. (Editor), John Wiley \& Sons Ltd, 1990, ISBN 0-471-92360-5, pp 215286.

15. Gradshteyn, I.S. \& Ryzhik, I.M. (forth edition prepared by Geronimus, Yu.V. \& Tseytlin, M.Yu.). Table of Integrals, Series, and Products. Academic Press 1965.

16. Hauske, G. \& Zetzsche, C. 1989. Principal Features of Human Vision in the Context of Image Quality Models. Third International Conference on Image Processing and its Applications (organised by the I.E.E.). Conference Publication No. 307, p102-106. Warwick 18-20 July 1989. ISBN 0852963823.

17. Hunt, R.W.G. 1987. The reproduction of colour in photography, printing and television. Fourth edition, Fountain Press. 
18. Ingling, Jr., C.R. \& Martinez-Uriegas, E. 1985. The spatiotemporal properties of the r-g X-Cell channel, Vision Research, No. 25, 1985, pp 33-38.

19. Ives, H.E. 1922. A theory of intermittent vision. Jou. Opt. Soc. Am. and Rev. Sci. Instr., 6, 1922, pp343-361.

20. Jayant, N.S. \& Noll, P. 1984. Digital Coding of Waveforms. Prentice Hall. ISBN 0-13-211913-7 01.

21. Kay, S 1988. Spectral Estimation. Advanced topics in signal processing, J.S. Lim \& A.V. Oppenheim (Editors), Prentice Hall, ISBN 0-13-013129-6 025, 1988, chapter 2.

22. Kelly, D.H. 1971. Theory of flicker and transient responses, I : Uniform fields. Journal of the Optical Society of America, Vol. 61, 1971, pp 537-546.

23. Kelly, D. H., 1972. Adaptation Effects on Spatio-Temporal Sine Wave Thresholds. Vision Res., 12, 1972, p. 89-101.

24. Kelly, D.H. 1979. Motion and vision I : Stabilized images of stationary gratings. Journal of the Optical Society of America, Vol. 69, No. 9, September 1979, pp 12661274.

25. Kelly, D.H. 1979. Motion and vision II : Stabilized spatio-temporal threshold surface. Journal of the Optical Society of America, Vol. 69, No. 10, October 1979, pp 13401349.

26. Kelly, D.H. 1983. Spatiotemporal variation of chromatic and achromatic contrast thresholds. Journal of the Optical Society of America, Vol. 73, No. 6, June 1983, pp 742-750.

27. Kelly, D.H. 1985. Visual processing of moving stimuli. Journal of the Optical Society of America, Vol. 2, No. 2, February 1985, pp 216-225.

28. Kelly, D.H. 1989. Spatial and temporal interactions in colour vision. Journal of Imaging Technology, Vol. 15, No. 2, April 1989, pp 82-89.

29. Kelly, D.H. 1989. Opponent-color receptive-field profiles determined from large-area psychophysical measurements. Journal of the Optical Society of America, Vol. 6, No. 11 , November 1989, pp 1784-1793.

30. Lim, J.S. \& Oppenheim, A.V. (Editors) 1988. Advanced Topics in Signal Processing. Prentice Hall. ISBN 0-13-013129-6 025.

31. Peitgen, H.O. \& Saupe, D. (Ed.) 1988. The Science of Fractal Images. Springer-Verlag, New York, 1988. Chapters 1 \& 2.

32. Robson, J. J., 1966. Spatial and Temporal Contrast Sensitivity Functions of the Visual System. J. Opt. Soc. Am., 56, 1966, p. 1141-1142.

33. Sporea, D.G. \& Tonnquist, G. 1989. From a physical color stimulus to a psychological color percept. SPIE, Vol. 1077, Human Vision, Visual Processing, and Digital Display, 1989, pp 56-63. 
34. Terkla-McGrane, C., Tulunay-Keesey, U. \& Ver Hoeve J.N., 1988. Threshold and Suprathreshold Spatio-temporal Response throughout Adulthood. Journal Optical Society of America A, Vol. 5, No. 12, p2191-2200, December 1988.

35. Thorpe, L.J. \& Hanabusa, T. 1990. If progressive scanning is so good, how bad is interlace? SMPTE Journal 1990, p972-986. 


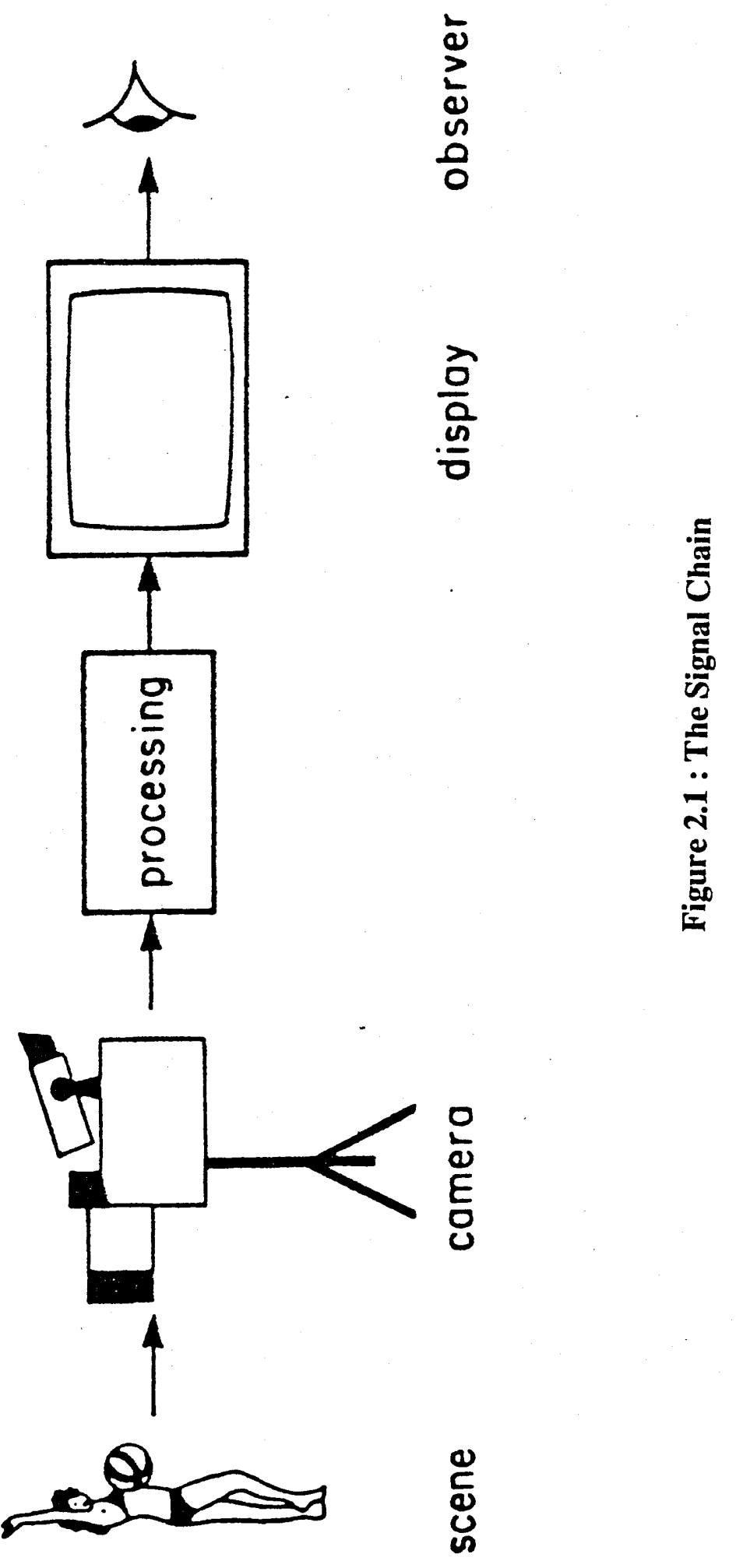




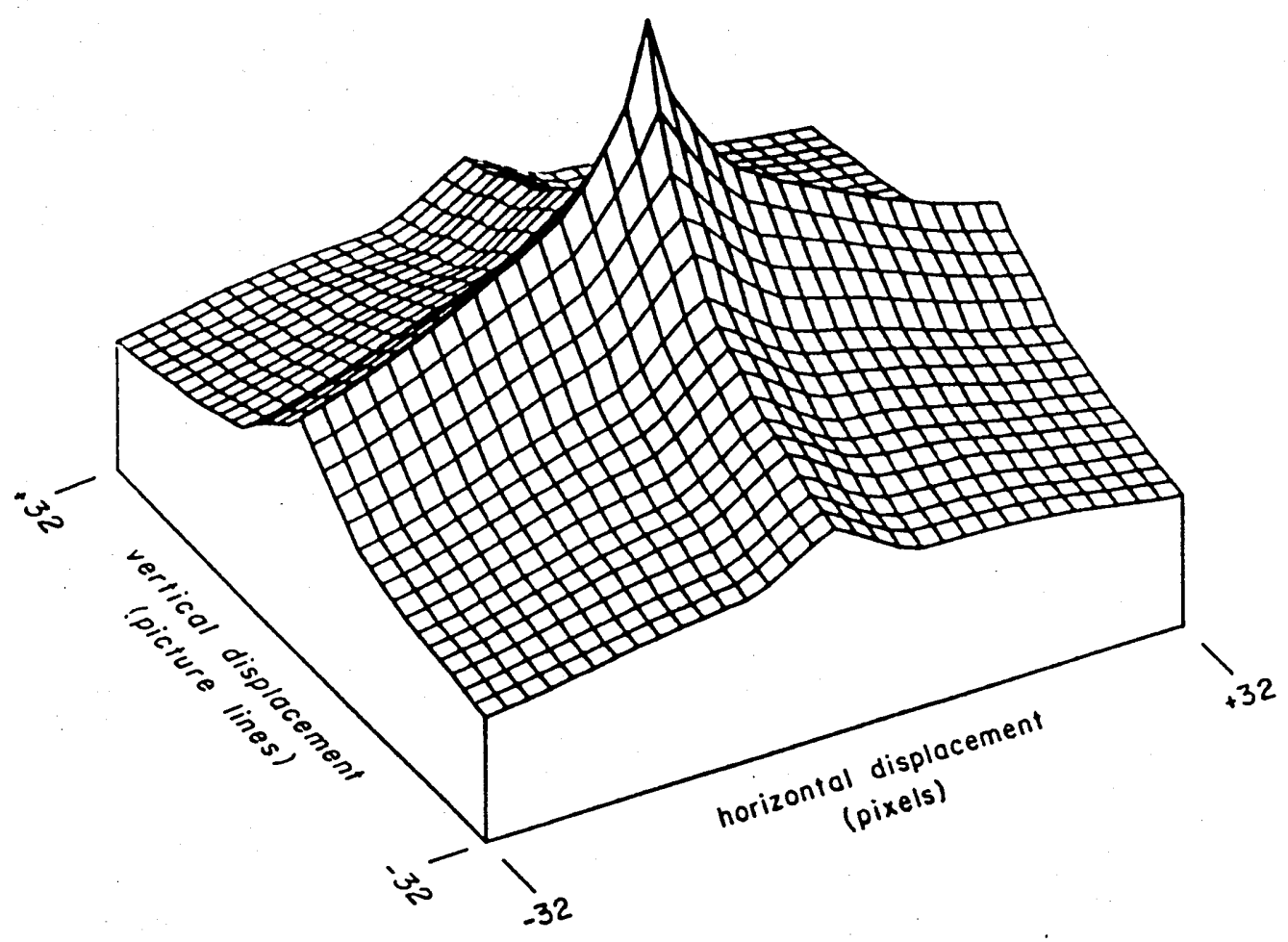

Figure 2.2 : Mean measured spatial autocorrelation function

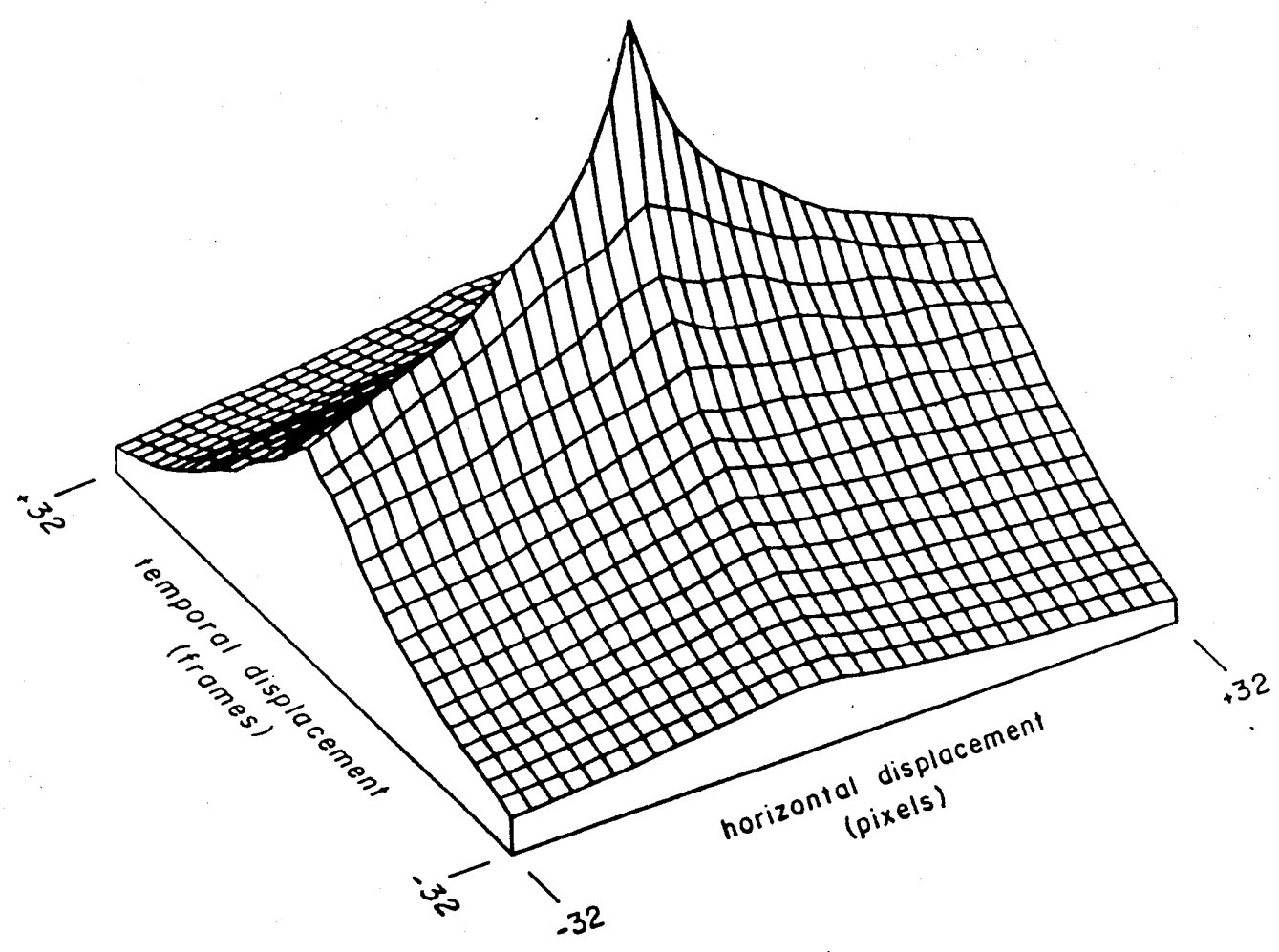

Figure 2.3 : Mean measured spatio/temporal autocorrelation function 

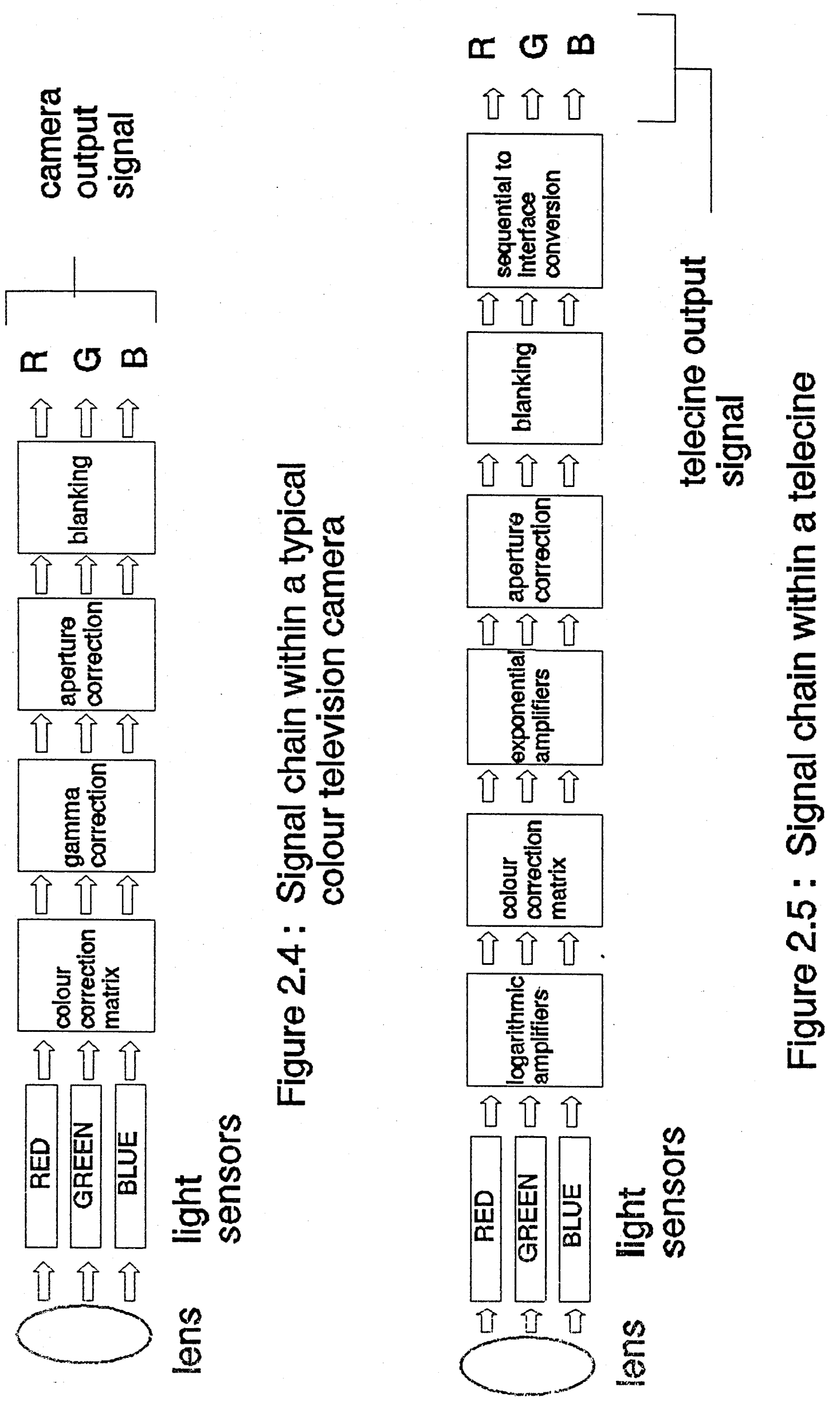


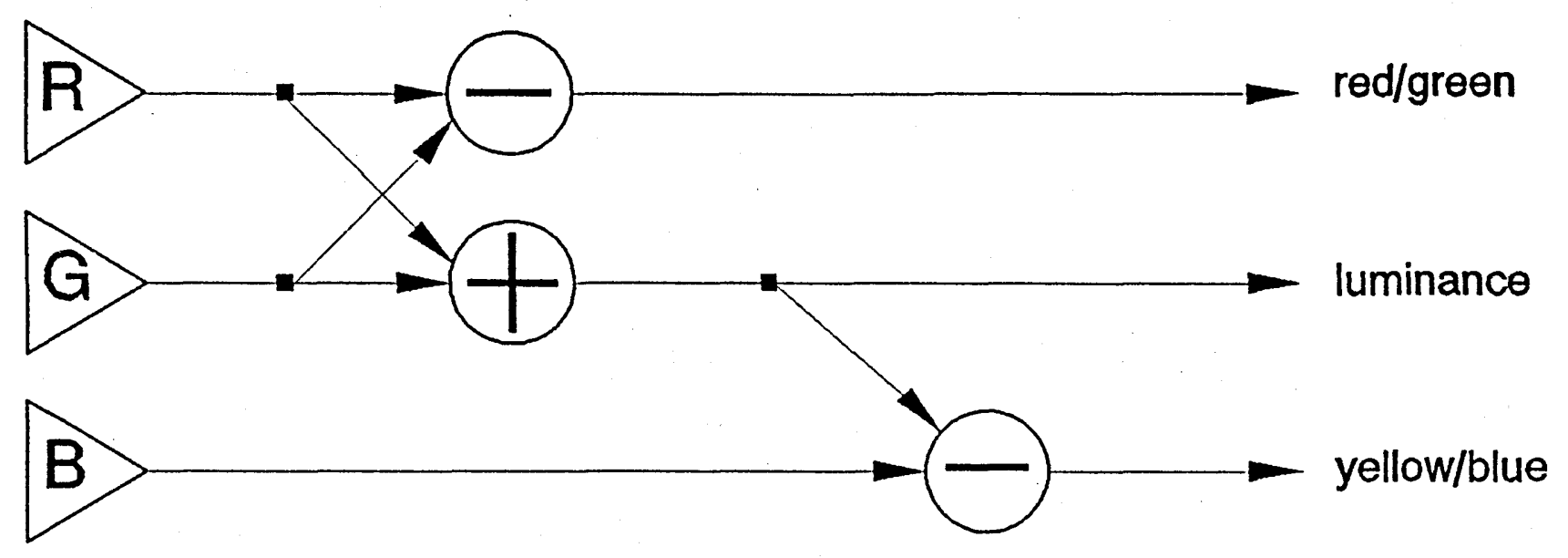

Light sensors in the retina

Figure 2.6: Formation of luminance and chrominance signals in the Human visual system

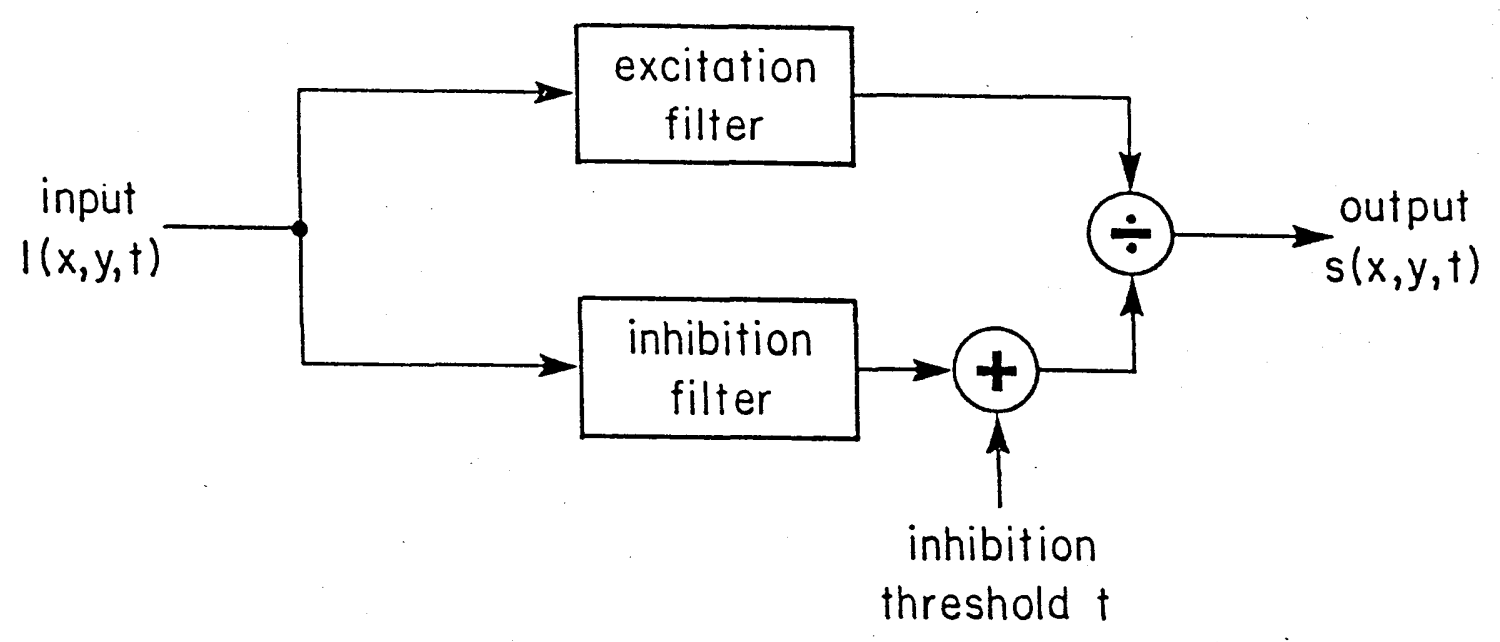

Figure 2.7 : Model Response of eye 


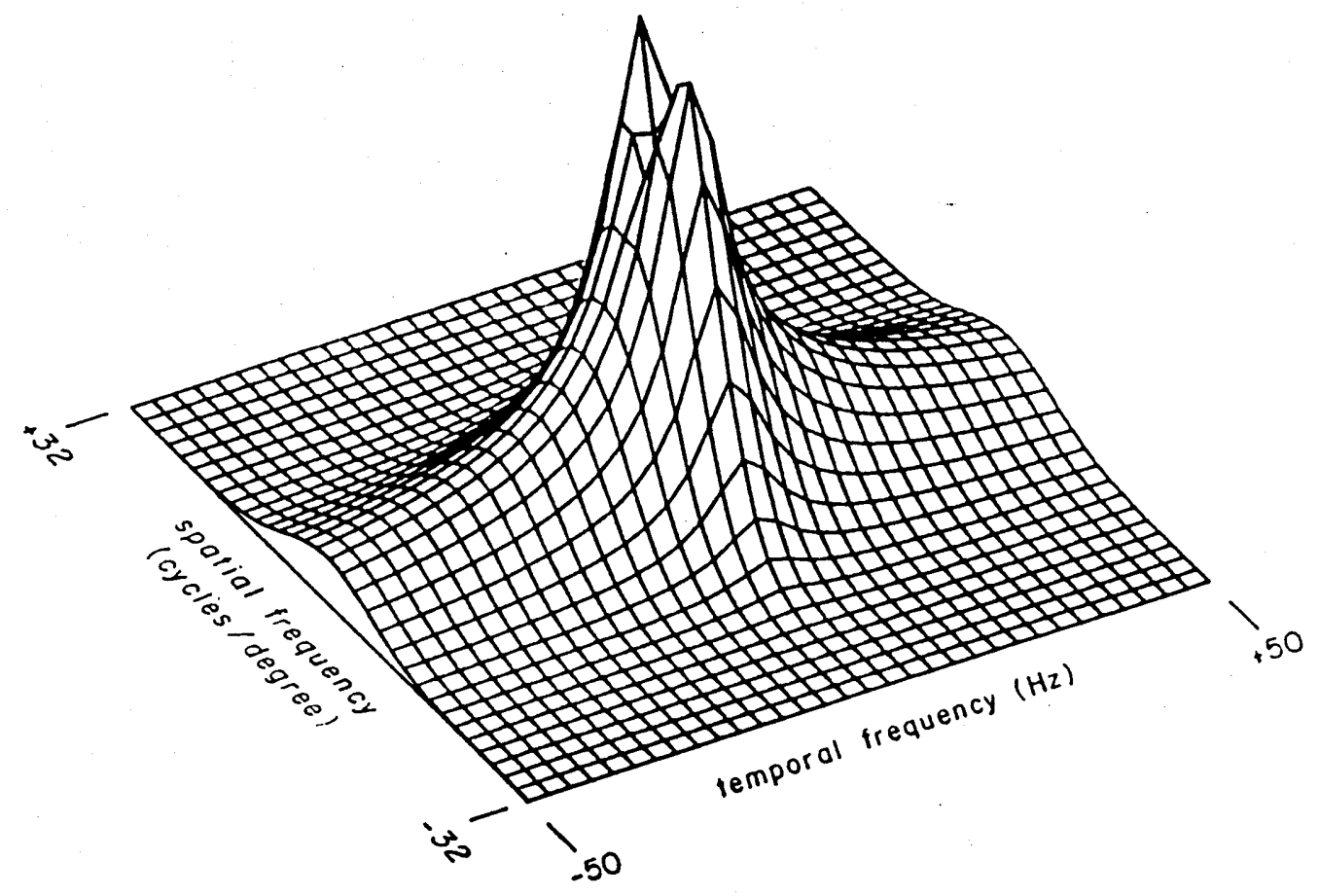

Figure 2.8 : Spatio/temporal response of the eye : model of Kelly's data at $15.2 \mathrm{~mL}$

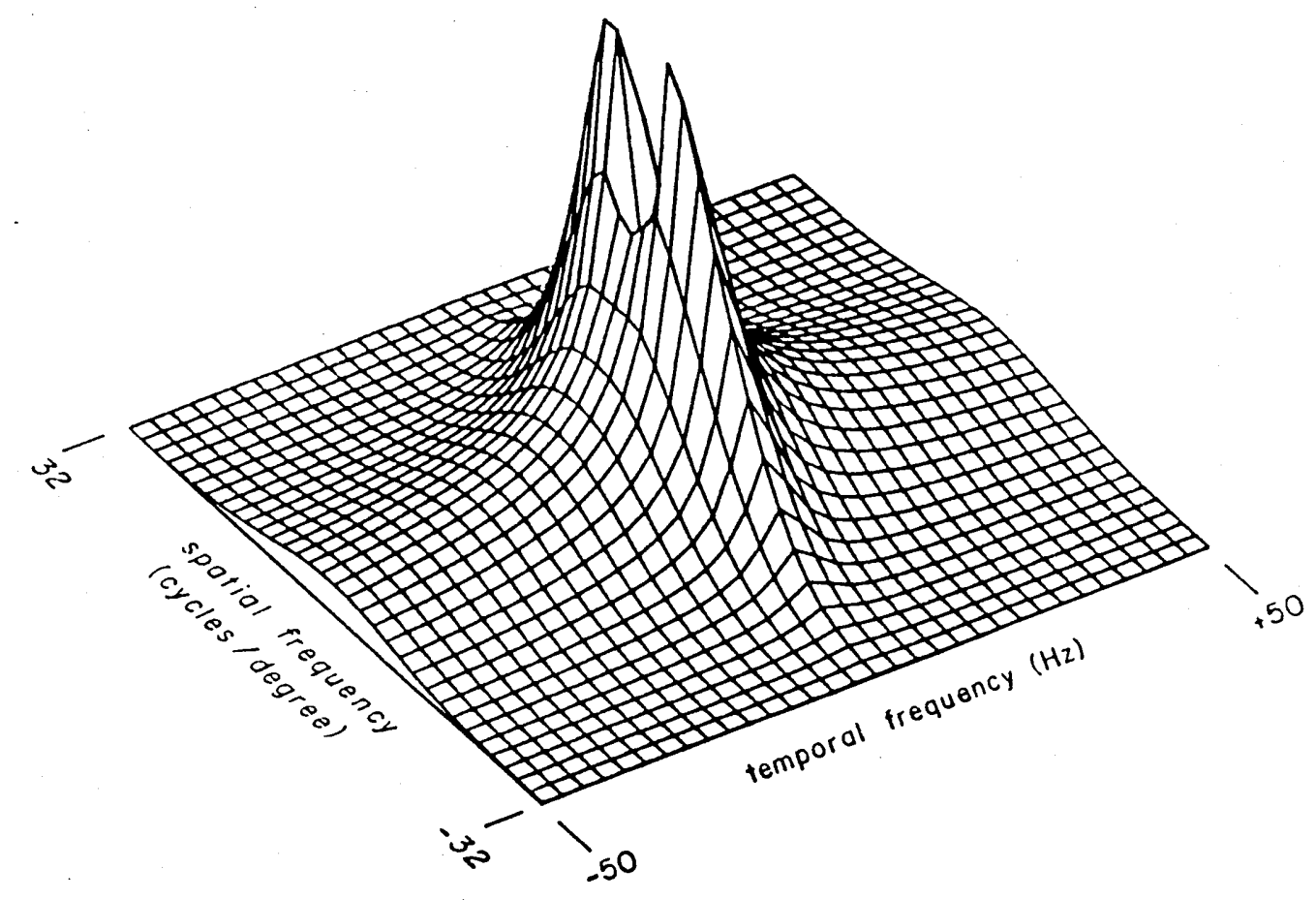

Figure 2.9 : Spatio/temporal response of the eye : model of Robson's data at $6.3 \mathrm{~mL}$ 


\subsection{Introduction.}

As the technology available to implement television standards converters has improved, so the need for good interpolation techniques has increased. This chapter examines the design of linear, symmetric, transversal interpolation filters for standards conversion. It starts by considering the historical development of filter design techniques for television. This is followed by a new and more precise formulation of the desired filter characteristics. It then presents a number of new ways in which these desired characteristics can be approximated in real hardware. Examples of the use of some of these filter design techniques are given in chapter 5 .

It is intended that, in addition to introducing new filter design techniques, this chapter will also provide an overview of filter design for standards conversion. It is assumed that the reader is familiar with the basic concepts of digital filtering, (multidimensional) Fourier transforms, lattices and reciprocal lattices etcetera. Good discussions of these topics, for the purposes of this chapter, can be found in Clarke 1990, Tonge 1981 and Pearson 1991.

Standards conversion is the process of interpolating a signal, sampled on an input lattice, at points on an output lattice. In one dimension this process is known as sample rate changing and is discussed, for example, in Oppenheim \& Schafer 1989 section 3.6 and Crochiere \& Rabiner 1988. Sample rate changing is, conceptually, a two stage process, as illustrated in figure 3.1. First the input signal is interpolated to give a continuous function. This continuous signal is then resampled at the appropriate output instants. Interpolation of the input to give a continuous function requires an input post-filter to remove repeat spectra (aliases) due to input sampling. Prior to output sampling, an output pre-filter should be applied to remove signal components which would otherwise alias in the sampled output.

In discrete time ('digital') systems the continuous signal is approximated by interpolation to a much higher sampling rate. This avoids the noise penalty of interconverting between the analogue and digital domains. The discrete time process of sampling lattice conversion is illustrated in figure 3.2. Conversion to the higher sampling rate is accomplished by 'padding with zeros', that is inserting zeros value samples between the input samples. Aliases due to padding with zeros are removed by (discrete time) filtering the signal. This 'interpolation filter' also removes frequency components which would otherwise alias when the 'supersampled' signal was resampled at the output rate.

Linear interpolation (described above) is essentially a filtering operation. Standards conversion is simply(!) sample rate changing in 2 or 3 dimensions. Filtering is applied to an input signal which has been padded with zeros. The filtered signal is then subsampled. The purpose of filtering is to minimise picture impairments due to both input and output sampling. 
The object of filter design, therefore, is to determine a filter characteristic which best achieves these objectives.

The use of transversal (finite impulse response) filters is almost universal for standards conversion $^{1}$ for two reasons. Firstly it is important that spatial video filtering exhibits a constant group delay (ie linear phase response). Non-linear group delay results in the spreading of edges in an image, producing a disturbing subjective picture impairment (Drewery 1990). Symmetric transversal filters inherently have constant group delay. Hence the design of symmetric transversal filters does not require the consideration of phase response. Recursive (infinite impulse response) filters can, of course, be designed with nearly constant group delay. The potential advantage of recursive filters is that they may require less computation to achieve a given frequency response. For (spatial) video processing, however, the computational advantage of recursive filters is likely to be small since a linear phase constraint is required. Furthermore recursive filters are considerably more difficult to design than transversal filters. The linear phase constraint does not seem to be so important in the temporal dimension. However, transversal filters have the further advantage that they give rise to convenient and efficient hardware implementations for interpolation (as described in the next chapter). For these reasons this chapter only considers the design of symmetric transversal filters.

Throughout this chapter explanations are given in terms of the lowest number of dimensions which is appropriate. Obviously standards conversion is usually a 2 dimensional (vertical-temporal) and sometimes a 3 dimensional problem. However, explanations in the minimum number of dimensions are usually briefer and clearer. Where extensions to higher dimensions are appropriate it is hoped that they will be obvious and straightforward.

\subsection{The Historical Development of Filter Design.}

This section is intended to describe a logical, historical development of techniques for designing video filters. The techniques presented in this section are mostly known in the literature (at least 1 dimensionally) but are described in the context of standards conversion. They are presented here in order to put later sections in context and to give a reasonably complete overview of filter design for this application.

${ }^{1}$ Recursive (infinite impulse response) filtering has, however, been used for temporal filtering for standards conversion. 


\subsubsection{The Brillouin zone as an 'ideal' filter characteristic.}

The most obvious way to approach filter design for standards conversion is to extend the ideas of one dimensional sample rate changing to multiple dimensions. The principle of sample rate changing is shown, for 1 dimension, in figures 3.1 \& 3.2. An input post-filter is used to remove repeat spectra in the sampled input. An output pre-filter then removes frequency components that would otherwise alias when the signal was sampled at the output rate. For a properly sampled signal, both input post- and output pre- filters are ideal low pass filters with unity gain passbands and zero gain stopbands. These two cascaded filters can be combined into a single filter, whose frequency response is the product of the two separate filters. This gives an ideal low pass filter whose cutoff frequency is the lowest of the, post and pre filter, cutoff frequencies. When increasing the sampling rate, the input pre-filter has the lowest cutoff frequency and the output post-filter is, essentially, redundant. When reducing the sampling rate the situation is reversed and the input post filter is redundant.

The corresponding situation in 2 dimensions is shown in figure 3.3. This shows the vertical temporal sampling lattice of an interlaced television signal and its reciprocal lattice. The reciprocal lattice is the pattern of repeat spectra in frequency space (see, for example, Pearson 1991 or Dubois 1985). Superimposed on the reciprocal lattice is an indication of the repeat spectra. Note that the shape of these spectra is purely diagrammatic and might more correctly (but inconveniently) be shown as elliptical.

The passbands of input post-filter and output pre-filter, in two dimensions correspond to the Brillouin zones of the input and output reciprocal lattices. These are shown in figure 3.4 for European and American television standards. They correspond to the low pass filters for 1 dimensional sample rate changing. The Brillouin zone (also known as the Voronoi cell, Dirichlet region, Wigner-Seitz cell or primitive bandwidth) is the set of all points, in the frequency domain, which are closer to the origin than to any other reciprocal lattice point.

The shape of the 'ideal' interpolation filter frequency passband may be considered to be the intersection of the Brillouin zones for the input and output lattices. This seems to be the view expressed by Tonge 1981 and Clarke \& Tanton 1984. The 'ideal' interpolation aperture (filter impulse response) is given by the Fourier transform of the ideal interpolation frequency response (which is unity in the passband and zero elsewhere).

The 'ideal' interpolation aperture, described above, is of infinite duration. To be of use in practical systems the interpolation aperture must be constrained to be of finite extent. This can be achieved by multiplying the ideal aperture by a finite extent window function. This use of window functions for filter design is discussed in detail in the appendix (chapter 11). The windowed interpolation aperture can then be sampled to give filter coefficients, as described in chapter 4. 
Practical (finite extent) interpolation apertures can be obtained by a variety of techniques, eg. the well known windowed filter design technique (Rabiner \& Gold 1975, Dudgeon \& Mersereau 1984) as described above. This is applied to a 2 dimensional low pass filter whose shape is defined by the intersection of the Brillouin zones for input and output lattices. Other methods can also be used to obtain approximations to the ideal interpolation aperture, for example equiripple (Dudgeon \& Mersereau 1984) or maximally flat (Tonge 1981) filter design techniques.

Unfortunately, for spatiotemporal interpolation, the definition of the Brillouin zone is somewhat arbitrary. This is because the spatial and temporal dimensions have fundamentally different units. By changing the equivalence between spatial and temporal sampling periods different shaped Brillouin zones are obtained, as shown in figure 3.5.

The output pre-filter removes frequency components which would otherwise alias in the output signal. It is desirable that spatial and temporal aliases have similar subjective effect. This can be achieved, for the output pre-filter, by deriving the equivalence between spatial and temporal sampling from the frequency response of the human visual system. This was suggested by Tonge 1982, who estimated an approximate equivalence of $1 \mathrm{~Hz}$ and 0.62 cycles per degree. Note that this equivalence is in terms of cycles per degree. Therefore the equivalence, in terms of cycles per picture height, as needed for filter design, varies with the distance of the viewer from the display.

The input post filter removes repeat spectra in the input signal. It is desirable that spatial and temporal aliases be rejected equally. Therefore, for the input post-filter, the equivalence between spatial and temporal sampling should be determined by the signal characteristics. The 'typical' auto-correlation function for the input signal (discussed in chapter 2) suggests an equivalence of $1 \mathrm{~Hz}$ and 12 or 13 cycles per picture height. In this case the ratio between spatial and temporal sampling changes with picture content. For moving pictures the temporal dimension should have more emphasis, while for still pictures the spatial dimension should be emphasised. This suggests the use of adaptive interpolation in which the aperture changes with picture content.

The use of the Brillouin zone has another drawback, in addition to the difficulty of its definition. its use, as described above, assumes that the signal does not, inherently, contain aliasing. That is that the signal was suitably filtered prior to sampling. This is very far from the case with video signals. In the absence of adequate pre filtering the basic assumptions in the use of the Brillouin zone do not, strictly speaking, apply.

Significant aliasing, both spatial and temporal, is inherent in most television signals. Adequate prefiltering, prior to sampling, is only performed horizontally. Vertically, the only prefilter is the spatial frequency response of the camera. This will certainly not have fallen to near zero by the vertical Nyquist frequency (312.5 cycles per picture height for European television, see chapter 2). Therefore significant aliasing can exist at higher vertical frequencies 
and even, in extreme cases, at D.C.. Temporally the situation is even worse, since high temporal frequencies can be generated by movement. Temporal prefiltering is only provided by the integration period of the camera (perhaps $20 \mathrm{~ms}$ for European television). With this type of temporal filtering only speeds of 1 pixel per field period can be accommodated (for the highest spatial frequencies) before aliasing occurs. In practice velocities of 32 pixels per field period are common in outside broadcasts!

With intrinsic aliasing in a signal the idea underlying the use of the Brillouin zone as an ideal filter characteristic does not apply. This idea is that signal and repeat spectra can be distinguished and repeat spectra (aliases) can be removed leaving only the true baseband spectrum. When the signal is aliased signal and repeat spectra can no longer be distinguished. Hence the use of the Brillouin zone technique is unlikely to give the best results for intrinsically aliased signals such as video. For this reason Clarke \& Tanton 1984 used a more heuristic approach based on the frequency sampling method described in the next section.

\subsubsection{Frequency sampling design : fixed (frequency) sampling lattice.}

Digital filters can be designed by specifying a number of constraints in the frequency domain. If the constraints are the specification of the frequency response, at a number of discrete frequencies, the technique is known as frequency sampling design. This, and following sub-sections, describe various aspects of frequency sampling design. Some aspects of frequency sampling filter design can be found in Parks \& Burrus 1987 and Lim 1990. Note that digital filters can also be designed to include time domain constraints and this is briefly touched on in sub-sections 3.4.3 \& 3.4.4.

The simplest type of frequency sampling design is when the desired frequency response is specified at regular intervals. For example, consider an $\mathrm{N}$ (odd) tap, 1 dimensional filter. The desired frequency response could then be specified at $N$ points, $2 / N^{\text {ths }}$ of the Nyquist frequency apart, ranging from minus to plus $(1-(1 / \mathrm{N}))$ times Nyquist frequency. This is illustrated, for $\mathrm{N}=7$, in figure 3.6.

Simple frequency sampling filter design can be understood by considering an $\mathrm{N}$ (odd) tap 1 dimensional filter. Let the frequency response specifications (ordered low to high frequency) be $R_{k}$, and the filter coefficients be $c_{n}$. If we knew the coefficients we could work out the frequency response from the sum;

$$
R_{k}=\sum_{k=-(N-1) / 2}^{+(N-1) / 2} c_{n} \exp (-j 2 \pi n k / N)
$$

where each term is due to a suitably located impulse response with a magnitude specified by the appropriate coefficients. That is, the frequency response of the filter is given by the 
discrete Fourier transform of the coefficients. Therefore, the coefficients can be determined from the frequency response specification by using the inverse discrete Fourier transform ie;

$$
c_{n}=\frac{1}{N} \sum_{k=-(N-1) / 2}^{+(N-1) / 2} R_{k} \exp (j 2 \pi n k / N)
$$

This is very convenient since discrete Fourier transforms can be implemented using fast Fourier transform algorithms (Brigham $1974 \& 1988$ ) requiring only of order $N \log N$ computations. Equations, similar to 1 and 2, can be derived for filters with an even number of taps (Parks \& Burrus 1987).

This frequency sampling design technique was used by Clarke \& Tanton 1984 for standards conversion. They used a vertical temporal filter, with an even number of taps both vertically and temporally, which was symmetric about the vertical and temporal axes. To perform interpolation, filter coefficients are required on a supersampled input lattice ${ }^{2}$ (see chapter 4). Clarke \& Tanton used a filter aperture extending over 8 picture lines (ie 4 field lines) and 4 field periods. The filter coefficients were specified on a lattice supersampled 16 times vertically and 8 times temporally with respect to the input lattice. Hence the frequency response of the filter can be specified at up to 16 times the vertical, and 8 times the temporal input Nyquist frequencies. For example, they specify a filter, suitable for conversion from European to American television, as in table 3.1 below $^{3}$. The left column specifies the vertical frequency in terms of cycles per picture height. The bottom row specifies the temporal frequency in Hertz. Other cells in the table contain (linear) frequency response values. Unspecified responses (above $312.5 \mathrm{c} / \mathrm{ph}$ or $50 \mathrm{~Hz}$ ) are zero.

In order to design a standards conversion filter, using this approach, an iterative, interactive technique can be used. First an initial frequency response specification is chosen, as in table 3.1 above. The coefficients corresponding to the specification are calculated. The filter is assessed on a range of representative picture material and the nature and severity of picture impairments is noted. These impairments are then related to the overall frequency response of the filter (ie including frequencies which were not specified), as in Clarke \& Tanton 1984. The frequency response specification is then modified to reduce the impairments and the procedure is repeated. After a number of iterations a good compromise can be reached between the severity of each type of impairment.

4.

${ }^{2}$ The filter coefficients can also be specified on a supersampled output lattice, see chapter

${ }^{3}$ This is Table 8, p41 from Clarke \& Tanton 1984. 
Table 3.1: Frequency domain specification of a standards conversion filter ${ }^{3}$.

\begin{tabular}{||c|c|c|c|c|c||}
\hline $312.5 \mathrm{c} / \mathrm{ph}$ & 0 & 0 & 0 & 0 & 0 \\
\hline $234.4 \mathrm{c} / \mathrm{ph}$ & 0.020 & 0 & 0 & 0 & 0 \\
\hline $156.3 \mathrm{c} / \mathrm{ph}$ & 0.500 & 0.125 & 0 & 0 & 0 \\
\hline $78.1 \mathrm{c} / \mathrm{ph}$ & 0.950 & 0.490 & 0.010 & 0 & 0 \\
\hline $0.0 \mathrm{c} / \mathrm{ph}$ & 1.000 & 0.700 & 0.100 & 0 & 0 \\
\hline & $0 \mathrm{~Hz}$ & $12.5 \mathrm{~Hz}$ & $25 \mathrm{~Hz}$ & $37.5 \mathrm{~Hz}$ & $50 \mathrm{~Hz}$ \\
\hline
\end{tabular}

This simple frequency sampling approach to filter design has both advantages and disadvantages. It is simple to implement the filter design software. The use of IDFT (inverse discrete Fourier transform) makes the calculation of the coefficients extremely quick. This speed facilitates its use in interactive filter design as described above. A disadvantage is that it is difficult to control the frequency response at frequencies not in the specification. This causes particular problems when, for example, the transition band of a filter lies between frequency specification points. Furthermore the coefficients are limited to be a rectangular array (cuboid array in 3 dimensions) if the IDFT is used. This may be inappropriate for some type of hardware implementation, particularly for filters for motion compensated interpolation. For these reasons more flexible methods of frequency sampling filter design can be used, as described below.

\subsubsection{Frequency sampling design : variable sampling sites.}

The frequency sampling technique of filter design can be applied in a more flexible manner than described above. Greater flexibility is possible if we relax the requirement that the frequency specification points are regularly spaced. This is useful in a number of situations. Particular points of interest in the filter's frequency response may not lie on regularly spaced frequencies. This would apply, for example, to the transition band of a circularly symmetric lowpass filter. In standards conversion the position of aliases, which should be suppressed, are unlikely to lie on regularly spaced frequencies. It is extremely difficult to create a null in the correct position using the frequency sampling technique of subsection 3.2.2. If the frequency response may be specified at any point then a null in the correct position may be specified directly.

It is also possible to relax the constraint that filter coefficients lie on a rectangular (or cuboid) array. This is useful when the cost of implementing the filter depends on the number 
of filter taps but not their position. This would typically be the case for motion compensated filtering.

In section 3.2.2 it was shown that the frequency response of a filter is easily calculated from its coefficients. This is true irrespective of the positions of the coefficients or the positions at which the frequency is sampled. In general, for an $\mathrm{N}$ tap, 1 dimensional filter, with coefficients $c_{n}$, we have a frequency response, $R_{k}$ given by;

$$
\boldsymbol{R}_{k}=\sum_{n=1}^{N} c_{n} \exp \left(-j 2 \pi f_{k} t_{n}\right) \quad ; \quad R_{k}=R\left(f_{k}\right) \text { \& } c_{n}=c\left(t_{n}\right)
$$

where $f_{k}$ is one at twice the Nyquist frequency, ie $f_{k}=1$ corresponds to 1 over the sampling interval. The $n^{\text {th }}$ coefficient is located at time $t_{n}$ (with units of sampling periods). Each term in the equation is due to the contribution from a single coefficient. Note that, in general, both the coefficients and the frequency response are complex. If the frequency response is specified at $\mathrm{N}$ arbitrary frequencies we have the following matrix equation;

$$
\boldsymbol{R}=\boldsymbol{P} \cdot \boldsymbol{c}
$$

where;

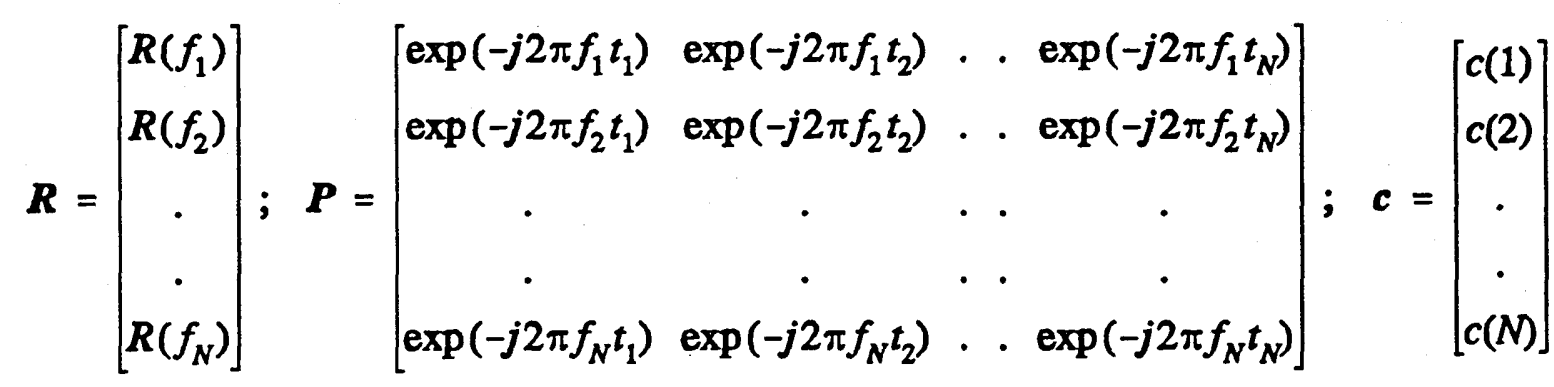

Hence, given a specification of the desired response $R(f)$ at $\mathrm{N}$ arbitrary frequencies, we can determine the coefficients by solving equation 4 , that is;

$$
\boldsymbol{c}=\boldsymbol{P}^{-1} \cdot \boldsymbol{R}
$$

When the frequency specifications are at regular intervals (as in sub-section 3.2.2) the phase matrix $\mathbf{P}$, in equation 5 , represents the discrete Fourier transform. Hence the inverse of $\mathbf{P}$ is the IDFT, and the matrix multiplication can be performed using an FFT algorithm. In the general case, however, equation 6 must be solved using more general methods for linear equations. These more general methods (eg Gaussian elimination) take of order $N^{3}$ computations rather than the $N \log N$ computations required for regularly spaced frequency 
sampling. Therefore this more flexible approach to frequency sampling filter design can take much longer to calculate than the simpler approach in the previous sub-section.

Again this approach to frequency sampling filter design has both advantages and disadvantages. It allows arbitrarily positioned coefficients. It also allows much more flexible specification of the filter's frequency response. This can make interactive filter design (as in sub-section 3.2.2) easier to perform. This type of filter design was used, for example, by $R$. Storey 1986, for a bandwidth reduction system. For standards conversion, however, there tend to be more coefficients than for other video processing applications. This is because the coefficients are required on a supersampled input (or output) lattice for interpolation. Hence this approach can be rather slow to calculate for standards conversion. Another drawback is that exactly $\mathrm{N}$ constraints must be specified. It would be more convenient if, having achieved a good approximation to the desired response, the response could be 'fine tuned' by adding further constraints. This idea leads to the further generalisation described in the next subsection.

\subsubsection{Frequency sampling design : overdetermined specification.}

Further generalisation of the frequency sampling technique of filter design is possible which increases its flexibility still further. As might be expected, this requires still more computation. Even a few years ago computationally intense filter design techniques may have been impractical. Rapid advances in computer technology, however, allows us to consider using such techniques.

It is not necessary to specify exactly $\mathrm{N}$ values of the desired frequency response, of an $\mathrm{N}$ tap transversal filter, in order to design a filter. If less than $\mathrm{N}$ frequency response values are specified the design is ambiguous (under specified). An infinite number of solutions are possible in this case, most of which will not be useful. If there are precisely $\mathrm{N}$ frequency response specifications the design problem is exactly specified and we have the situation of sub-section 3.2.3. If there are more than $\mathrm{N}$ frequency response specifications, it may not be possible to design a filter which exactly meets all the specifications. This is an overdetermined filter design problem. Nevertheless it is still possible to design a filter whose response approximates all the specifications.

The use of overdetermined filter specifications simplifies interactive filter design. Initially the design process might start with $\mathrm{N}$ frequency response specifications. The filter coefficients can be calculated and the overall frequency response (including the response at unspecified frequencies) can be examined. If the overall frequency response is not satisfactory the frequency response can be specified at a further frequency. The filter design process then calculates a 'best fit' response through all the specification points. By adding progressively 
more specifications the filter's frequency response can be 'tuned' to minimise (frequency domain) ripples, overshoots and to generate nulls and other features.

It is possible to specify how closely a desired frequency response specification is approximated at any point. It may, for example, be desirable to precisely specify the filter's frequency response at D.C.. By contrast the desired frequency response at, or near, a transition band can be much more loosely approximated. The closeness of the 'best fit' frequency response, at a specific frequency, can be improved by simply repeating the desired frequency response specification for that point. The more times a specification is repeated the more closely the 'best fit' frequency response will approximate the desired frequency at that point. It is more convenient to give a weighting associated with each desired frequency response specification. The weighting indicates how closely each specification should be approximated. The weighting is, essentially, the number of times each specification is repeated, though it need not be an integer.

The easiest way to generate a 'best fit' frequency response is to use a least mean square error approach. Assume there are $\mathrm{M}$ desired frequency specification points for an $\mathrm{N}$ tap filter $(M>N)$. The frequency response, at the $M$ frequencies, is given, as before (equation 3), by;

$$
\boldsymbol{R}=\boldsymbol{P} . \boldsymbol{c}
$$

where $\mathbf{R}$ is a vector of the frequency response values, $\mathbf{P}$ is a matrix of phase factors and $\mathbf{c}$ is a vector of the filter coefficients. Note that in this case, in contrast to sub-section 3.2.3, $\mathbf{R}$ and $\mathbf{P}$ now have $\mathrm{M}$ rather than $\mathrm{N}$ rows. Equation 7 cannot be solved directly because $\mathbf{P}$ is not square. However a least mean square approximation can be found by solving the 'normal equations' (see, for example, Jennings 1977) ie;

$$
P^{h} \boldsymbol{D}=\boldsymbol{P}^{k} \boldsymbol{P C}
$$

where $\mathbf{D}$ is the desired frequency response specifications, which are approximated by $\mathbf{R}$, the actual frequency response given by the coefficients c. Superscript h represents the Hermitian conjugate of the matrix, that is the complex conjugate of the transpose. This is equivalent to the transpose operation for a real matrix. From equation 8 we get;

$$
c=\left(P^{h} P\right)^{-1} P^{h} D
$$

The 'normal equations' (equation 8) provide a least mean square solution, that is they minimise $(\boldsymbol{D}-\boldsymbol{R})(\boldsymbol{D}-\boldsymbol{R})^{h}$. The weighting factors, described above, which control the accuracy of the approximation at each specification point, are included by premultiplying equation 7 by a weighting matrix ie; 


$$
\text { WD }=\text { WP.c }
$$

where;

$$
W=\left[\begin{array}{cccc}
W_{1} & 0 & \ldots & 0 \\
0 & W_{2} & \ldots & 0 \\
\cdot & \cdot & \ldots & \cdot \\
\cdot & \cdot & \cdots & \cdot \\
0 & 0 & . & W_{M}
\end{array}\right]
$$

where $W_{n}$ is the weighting of the $n^{\text {th }}$ desired frequency response specification.

This gives the following 'normal equations' for weighted, overdetermined, frequency sampling filter design;

$$
(W P)^{h} W D=(W P)^{h} W P c
$$

This may be rewritten, a little more clearly, as;

$$
P^{h} W^{2} D=P^{k} W^{2} P C
$$

If the filter's frequency response specifications are spaced in a rectangular (2D) or cuboid (3D) array, then the design process may be efficiently performed using FFT algorithms. This can be seen by considering the components of the matrices in equation 13 . $\mathbf{P}^{\mathrm{h}} \mathbf{W}^{2} \mathbf{D}$ is an $\mathbf{N}$ component vector $\left(\mathrm{N}=\right.$ number of filter taps) and $\mathbf{P}^{\mathrm{h}} \mathbf{W}^{2} \mathbf{P}$ is an $\mathbf{N x N}$ matrix whose components are given by;

$$
\begin{aligned}
& \left(\boldsymbol{P}^{h} W^{2} \boldsymbol{D}\right)_{k}=\sum_{m=1}^{M} W^{2}\left(f_{m}\right) D\left(f_{m}\right) \exp \left(j 2 \pi f_{m} t_{k}\right) \\
& \left(\boldsymbol{P}^{h} W^{2} \boldsymbol{P}\right)_{k, l}=\sum_{m=1}^{M} W^{2}\left(f_{m}\right) \exp \left(j 2 \pi f_{m}\left(t_{k}-t_{l}\right)\right)
\end{aligned}
$$

Provided that the specifications are equally spaced these matrix components can be calculated by FFT algorithms. Note also that $\mathbf{P}^{h} \mathbf{W}^{2} \mathbf{P}$ is a Toeplitz matrix (or block Toeplitz in multiple dimensions). There are fast algorithms for inverting this type of matrix (Jain '79, Kumar '85, Preis '72, Trench ' 64 , Zohar ' $69 \&$ 74). The use of these techniques can greatly reduce the amount of computation required for the design process.

Filter coefficients can be designed by generating a least mean squared approximation to an overdetermined frequency response specification. The least mean square approximation is the solution of a set of simultaneous equations. These simultaneous equations are the 'normal equations' (equation 13 above) derived from the response specification and the 
location of the coefficients. It is suggested, in texts on linear algebra and by Parks \& Burrus 1987 , that normal equations derived in this way may be ill-conditioned. Experimentally this does not appear to cause problems with realistic filter specifications. This method of filter design can be computationally expensive, especially for the large number of coefficients needed for standards conversion apertures. Nevertheless advances in computer technology make this approach increasingly attractive.

\section{3. 'Pseudo' Wiener Filters as 'Ideal' Filters.}

This section presents new ideas on how realistic filter specifications can be determined for practical television standards converters. The filter design techniques described in the previous section are either heuristic, or based on somewhat unrealistic assumptions about the nature of television signals. The use of the Brillouin zone concept for filter design largely ignores the aliasing inherent in television signals. The frequency sampling method of filter design does not include a objective specification but relies on the design engineer's idea of a 'good' filter. With the proliferation of different television standards the number of possible interconversions has greatly increased. It is therefore desirable to develop more objective techniques for standards conversion filter design. That is the aim of this, and subsequent, sections.

To determine the most suitable filter specification for standards conversion it is necessary to examine the entire signal chain. That is the path from the generation of the image by a scene to its perception by an observer. The signal path, for standards conversion, is illustrated in figure 3.7. An image is generated by a scene and converted into an electronic signal by a camera. The signal is processed, converted back to an image by the display and finally perceived by an observer.

There are a number of non-linearities in the signal chain, in addition to sampling. There are deliberate, complementary, non-linearities in television cameras and displays. This is generally referred to as gamma correction and is discussed in chapter 2 . The signal, $\mathbf{s}$, from

the camera is related to the light intensity, $l$, by $s=l^{1 / \gamma}$, where gamma (which typically equals 2.2) defines the non-linearity. The correct brightness, $b$, is restored in the display whose response is $\boldsymbol{b}=s^{\gamma}$. It is the non-linear, gamma corrected, signals which are usually processed. However the subjective effect of filtering gamma corrected, rather than linear, signals is quite small. Presumably this is because the 'typical' scene spectra are little changed by gamma correction (see chapter 2). A second source of non-linearity is the frequency response of the human visual system (or 'eye'), discussed in chapter 2. Although the response 
of the eye is highly non-linear overall, it may be approximated by a linear response over the brightness range of television signals.

Figure 3.8 illustrates the signal processing in a linearised, standards conversion, signal chain. The image is generated by the scene and filtered with the frequency response of the camera. The camera's signal is sampled on the input sampling lattice. The sampled signal is interpolated by the standards converter and resampled on the output sampling lattice. The resampled signal is filtered with the frequency response of the display. Finally the displayed image is perceived by the observer.

One impairment introduced in the standards conversion is loss of detail. This loss can be determined by examining figure 3.8. For simplicity a one dimensional signal is assumed. The ideal frequency response of the chain including the observers perception is simply;

$$
\text { ideal output }=S(f) \cdot E(f)
$$

where $S(f)$ is the spectrum of the scene and $E(f)$ is the frequency response of the human visual system (the 'eye').

The actual response of the system is given by;

$$
\text { actual output }=S(f) \cdot C(f) \cdot X(f) \cdot D(f) \cdot E(f)+\text { aliasing }
$$

Where $C(f)$ is the frequency response of the camera, $X(f)$ is the response of the interpolation filter and $D(f)$ is the response of the display.

Therefore the loss of resolution introduced by the standards conversion chain is;

$$
L(f)=(1-C(F) \cdot X(f) \cdot D(f)) \cdot S(f) \cdot E(f)
$$

where $L(f)$ is the loss at frequency $f$.

Another impairment of the signal passing through the standards conversion chain is the introduction of aliasing by the two sampling processes. There are three contributions to aliasing illustrated (for one dimension) in figure 3.9. Firstly there are aliases introduced by the input sampling process. An example of an input alias is $A_{\mathrm{in}}(f)$ in the figure. $A_{i n}(f)=C\left(f-f_{i n}\right) \cdot S\left(f-f_{i n}\right) \cdot X(f) \cdot D(f) \cdot E(f)$, where $\mathrm{f}_{\mathrm{in}}$ (equals 1 divided by the input sampling period) is twice the input Nyquist frequency. There are also aliases introduced by the output sampling process. For example, from the figure, $A_{\text {out }}(f)=C\left(f-f_{\text {out }}\right) \cdot S\left(f-f_{\text {out }}\right) \cdot X\left(f-f_{\text {out }}\right) \cdot D(f) \cdot E(f)$, where $\mathrm{f}_{\text {out }}$ (equals 1 divided by the output sampling period) is twice the output Nyquist frequency. Finally there are cross aliases 
due to output sampling acting on input repeat spectra (aliases). For example,

$$
A_{x}(f)=C\left(f-f_{\text {out }}+f_{\text {in }}\right) \cdot S\left(f-f_{\text {out }}+f_{\text {in }}\right) \cdot X\left(f-f_{\text {out }}\right) \cdot D(f) \cdot E(f) \text {. }
$$

In general the alias terms are given by;

$$
\begin{aligned}
& A_{\text {in }}(f, k)=C\left(f-k f_{\text {in }}\right) S\left(f-k f_{\text {in }}\right) X(f) D(f) E(f) ; \forall k \neq 0 \\
& A_{\text {out }}(f, l)=C\left(f-l f_{\text {out }}\right) S\left(f-l f_{\text {out }}\right) X\left(f-l f_{\text {out }}\right) D(f) E(f) \exp (j 2 \pi l \phi) ; \forall l \neq 0 \\
& A_{x}(f, k, l)=C\left(f-l f_{\text {out }}+k f_{\text {in }}\right) S\left(f-l f_{\text {out }}+k f_{\text {ln }}\right) X\left(f-l f_{\text {out }}\right) D(f) E(f) \exp (j 2 \pi l \phi) ; \forall k, l \neq 0
\end{aligned}
$$

where the phase factors in the latter two equations are due to a possible offset between the origins of the input and output sampling lattices.

An 'ideal' frequency response for a filter can be found by minimising the impairments. This is most easily achieved using a least mean square approach, in which the sum of the squares of the individual impairments are minimised. The total impairment, $I(X)$, as a function of the interpolation filter $\mathrm{X}$ is given by;

$$
I^{2}(X)=\int_{-\infty}^{+\infty}\left\{|L(f)|^{2}+\sum_{k+0}\left|A_{i n}(f, k)\right|^{2}+\sum_{l+0}\left|A_{\text {out }}(f, l)\right|^{2}+\sum_{k, l+0}\left|A_{x}(f, k, l)\right|^{2}\right\} d f
$$

This equation assumes that all the impairments add independently. This condition is usually met in practice, but would be violated if $\mathrm{kf}_{\mathrm{in}}=\mathrm{If}_{\text {out }}$ for small values of $\mathrm{k}$ and $\mathrm{l}^{4}$. The minimum impairment is found by differentiating I with respect to $\mathrm{X}$. This gives the 'ideal' interpolation filter frequency response as;

$$
X_{\text {idear }}(f)=\frac{S^{2}(f) C(f) D(f) E^{2}(f)}{\sum_{\forall k} \sum_{\forall l} S^{2}\left(f+k f_{\text {in }}\right) C^{2}\left(f+k f_{\text {in }}\right) D^{2}\left(f+l f_{\text {out }}\right) E^{2}\left(f+l f_{\text {out }}\right)}
$$

The effect of input noise can be included as a further impairment giving the following modified result;

$$
X_{\text {idear }}(f)=\frac{S^{2}(f) C(f) D(f) E^{2}(f)}{\sum_{\forall k} \sum_{\forall l} S^{2}\left(f+k f_{\text {in }}\right) C^{2}\left(f+k f_{\text {in }}\right) D^{2}\left(f+l f_{\text {out }}\right) E^{2}\left(f+l f_{\text {out }}\right)+N^{2}(f)}
$$

where $\mathrm{N}$ is the input noise as a function of frequency.

This analysis is similar to the derivation of Wiener filters for the removal of additive random noise. Hence the 'ideal' standards conversion filter derived in this way might be

${ }^{4}$ If $\mathbf{k f}_{\mathrm{in}}=\mathrm{lf}_{\text {out }}$ then the cross alias term may be coherent with the loss. This would result in an increase or decrease in the effective loss depending on the phase factor for the cross alias. 
described as a 'pseudo Wiener' filter. In this case, however, the principle picture impairments are resolution loss and aliasing. These impairments are, essentially, treated as uncorrelated noise sources. Furthermore the integration in equation 19 is more complicated than for Wiener filtering since a change of variable is needed to integrate the last term.

The 'ideal' filter's frequency response is more easily interpreted after being factorised. The 'ideal' frequency response can be rewritten as;

$$
X_{\text {idead }}(f)=\left\{\frac{1}{C(f)} \cdot \frac{S^{2}(f) C^{2}(f)}{\sum_{\forall k} S^{2}\left(f+k f_{\text {in }}\right) C^{2}\left(f+k f_{i n}\right)}\right\}\left\{\frac{1}{D(f)} \cdot \frac{D^{2}(f) E^{2}(f)}{\sum_{\forall l} D^{2}\left(f+l f_{\text {out }}\right) E^{2}\left(f+l f_{\text {out }}\right)}\right\}
$$

Assume that the display and the eye are perfect (ie that their frequency responses are flat) and that the output sampling rate is very high. Then the interpolation filter is minimising impairments due to input sampling alone. That is, it is functioning as an input post filter. Hence we can write;

$$
X_{p o s t}(f)=\frac{1}{C(f)} \cdot \frac{S^{2}(f) C^{2}(f)}{\sum_{\forall k} S^{2}\left(f+k f_{i n}\right) C^{2}\left(f+k f_{i n}\right)}
$$

The input post filter response has two terms. The first, 1 over $\mathrm{C}(\mathrm{f})$, term corrects for the losses in the camera. The second term minimises the impairments from resolution loss and aliasing. Similarly, by assuming flat scene spectrum, perfect camera and high input sampling rate, we obtain an equation for the output prefilter ie;

$$
X_{\text {pre }}(f)=\frac{1}{D(f)} \cdot \frac{D^{2}(f) E^{2}(f)}{\sum_{\forall l} D^{2}\left(f+l f_{\text {out }}\right) E^{2}\left(f+l f_{\text {out }}\right)}
$$

Again this has two terms, the first correcting for the losses in the display and the second minimising impairments due to output sampling. Therefore, in common with sub-section 3.2.1, we have;

$$
X_{\text {ideal }}(f)=X_{\text {past }}(f) \cdot X_{\text {pre }}(f)
$$

This section has presented an objective way of determining a suitable specification for a standards conversion filter. The equations presented are for a least mean square minimisation of the impairments due to loss of resolution and aliasing. Picture impairments are treated as uncorrelated noise in a fashion analogous to Wiener filtering. The analysis requires a knowledge of the spectral characteristics of the picture source, camera, display and the response of the human visual system. 


\section{4. 'Optimum' approximations to 'Ideal' Filters.}

There are two parts to the design of a practical filter. Firstly there is the 'specification problem'. That is generating a description of what we wish to achieve with the filter. This problem is addressed, for standards conversion, in the previous section. The second part of filter design is the 'approximation problem'. This second problem is addressed in this section.

The specification of the desired response of a filter is seldom realisable in practice. Usually the desired impulse response is of infinite extent and this is certainly the case for the 'ideal' standards conversion filters presented in the previous section. All that can be done, in practice, is to provide an approximation to the ideal response. The problem is to provide the 'best' approximation with a limited number and arrangement of filter taps. This section describes ways in which this can be done for standards conversion filters.

\subsubsection{Discrete LMS approximations.}

One way in which a practical standards conversion filter can be generated from an 'ideal' specification is to use the technique of overdetermined specification described in sub section 3.2.4. This provides a least mean square error approximation to the desired response at a number of discrete frequencies. Provided that the response is approximated at a sufficiently large number of frequencies a good approximation can be achieved.

To use this technique of filter design we must decide how closely to approximate the desired ('ideal') response at each frequency. That is we must determine the weighting factors to use in the filter design process. We could assume the same weighting at all frequencies. This, however, assumes that it is equally important to accurately approximate the 'ideal' response at all frequencies, which is definitely not the case. Instead the sensitivity of the impairment function (equation 19) to errors in the approximation can be considered. Considering a Taylor expansion of the interpolation filter about its 'ideal' value, and ignoring higher order terms, we have;

$$
I^{2}(X)=I^{2}\left(X_{\text {ideal }}\right)+\left.\frac{1}{2} \frac{d^{2}\left(I^{2}\right)}{d X^{2}}\right|_{X_{\text {ided }}}\left(X-X_{\text {ideal }}\right)^{2}
$$

Solution of the 'normal' equations minimises $W^{2}\left(X-X_{i d e a l}\right)^{2}$. Therefore the squared impairment caused by the approximation to the 'ideal' filter is minimised by choosing the weighting factor to be; 


$$
W=\sqrt{\left.\frac{d^{2}\left(I^{2}\right)}{d X^{2}}\right|_{X_{\text {deal }}}}
$$

Differentiating equation 19 we get a weighting factor for standards conversion given by;

$$
W(f)=\sqrt{\sum_{\forall k} S^{2}\left(f+k f_{\text {in }}\right) C^{2}\left(f+k f_{\text {in }}\right)} \sqrt{\sum_{\forall l} D^{2}\left(f+l f_{\text {out }}\right) E^{2}\left(f+l f_{\text {out }}\right)}
$$

Given an 'ideal' frequency response and the relative importance (weighting) of each frequency component, good approximations can be obtained using the technique of overdetermined frequency specifications. For standards conversion a large number of coefficients must be calculated because they are required on a supersampled input lattice. A typical 4 field, 4 (field) line interpolation aperture might include 4096 coefficients. The solution of simultaneous equations with thousands of variables is a time consuming process even on a fast computer. Although faster techniques are desirable, this technique is practical, given a little patience. Another disadvantage of discrete least mean square approximation is that the filter's frequency response is uncontrolled between specification points. These two drawbacks suggest the use of a continuous least mean square approximation technique described in the next sub-section.

\subsubsection{Continuous LMS approximations.}

An alternative, possibly better, method is available to approximate the 'ideal' frequency response. The discrete LMS approximation, described in the previous sub-section, only approximates the desired response at a finite number of points. In order to ensure the behaviour of the approximation between specification points the points must be closely spaced. Hence a large number of specification points are required and, thus, much computation is required. By using mathematical models of the necessary spectra, it is possible to approximate the 'ideal' frequency response at every frequency, rather than at discrete

points. This not only provides the 'best' approximation but also may reduce the computation required.

In determining a 'continuous' approximation to a desired frequency response specification we must minimise the approximation error over all frequencies. That is we must minimise an error function of the form; 


$$
E^{2}\left(\left\{c_{n}\right\}\right)=\int_{-\infty}^{+\infty} W^{2}(f)(D(f)-R(f))(D(f)-R(f))^{*} d f
$$

where $E$ is the approximation error produced by the set of coefficients denoted $\left\{c_{n}\right\}, W(f)$ is the approximation weighting function, $D(f)$ is the desired frequency response and $R(f)$ is the actual frequency response. The error function is minimised, with respect to any coefficient $c_{n}$, by differentiating with respect to $c_{n}$ and setting the result to zero. Substituting $R(f)$ from equation 3 and performing the differentiation gives;

$$
\int_{-\infty}^{+\infty} W^{2}(f) D(f) \exp \left(j 2 \pi f t_{n}\right) d f=\int_{-\infty}^{+\infty} W^{2} \sum_{m} c_{n} \exp \left(j 2 \pi f\left(t_{n}-t_{m}\right)\right) d f
$$

Interchanging integration and summation on the right hand side and recognising inverse Fourier transform integrals yields;

$$
\left.\mathscr{F}^{-1}\left\{W^{2}(f) D(f)\right\}\right|_{t_{n}}=\left.\sum_{m} c_{m} \mathscr{F}^{-1}\left\{W^{2}\right\}\right|_{t_{n}-t_{m}}
$$

Finally, by considering all the coefficients, we get a set of linear simultaneous equations;

$$
y=z . c
$$

where;

$$
y_{n}=\left.\mathscr{F}^{-1}\left\{W^{2}(f) D(f)\right\}\right|_{t_{n}} \quad \& \quad z_{n, m}=\left.\mathscr{F}^{-1}\left\{W^{2}(f)\right\}\right|_{t_{n}-t_{m}}
$$

Comparison of equation 33 with equations 14 leads to some interesting conclusions. Unsurprisingly, we can see that as the number of points considered in a discrete LMS approximation increases it converges to the continuous LMS approximation. If models exist for the various parts of the standards conversion chain it may be possible to calculate the coefficients of matrices $\mathbf{y}$ and $\mathbf{z}$ analytically. It is likely, however, that the matrix coefficients will have to be calculated numerically. The Fourier transforms can be calculated using FFT algorithms as for discrete LMS approximation. Unlike discrete LMS approximation, however,

${ }^{5}$ This result is achieved by first assuming real coefficients and then assuming imaginary coefficients. This can be shown to be valid if we decompose $D(f)$ into 'conjugate odd' and 'conjugate even' parts. That is $D(f)=(E(f)+O(f))$, where $E(-f)=E^{*}(+f)$ and

$$
O(-f)=-O^{*}(+f) \text {. }
$$


the number of points in the FFT can be tailored to the requirements of the Fourier transform and need not be related to the number of filter coefficients. This allows more efficient calculation of the matrix coefficients and also allow us to understand the approximations involved in calculating the matrix coefficients numerically.

Continuous least mean square approximation provides an efficient filter design technique for standards conversion filters. With this technique the filter's frequency response is controlled to give the least mean square approximation error over all frequencies. The coefficients for standards conversion are required on a supersampled input lattice. With discrete least mean squared approximation each coefficient must be computed explicitly. With continuous least mean square approximation, however, extra coefficients can be interpolated between those explicitly calculated. This approach is legitimate because the filter approximation is valid at all frequencies. Even with continuous least mean square approximation the filter coefficients are still required on a supersampled input lattice. The frequency response of the filter can then be defined beyond the input Nyquist frequency. This is necessary to deal correctly with aliasing in the input signal. The degree of supersampling can, however, be much less for continuously approximated filters. The input lattice need only be supersampled by a factor of, perhaps, 2 or 4 to give an adequate frequency range for the approximation. Supersampling by a factor of, perhaps, 8 or 16 is required for adequate coefficient quantisation (see next chapter). Typically then, continuous, as opposed to discrete, least mean square filter approximation might require the explicit calculation of only 512 , rather than 4096, coefficients. Other coefficients required can then be interpolated from those explicitly calculated. This reduction in the number of coefficients to be calculated in the initial filter design process gives a very significant reduction in the computational requirements (which are proportional to $\mathrm{N}^{2}$ or $\mathrm{N}^{3}$ depending on algorithm).

\subsubsection{Time domain constraints.}

It is possible to include time domain constraints in the filter design process in addition to frequency domain constraints. It might, for example, be desirable to generate a smooth impulse response which approximates a desired frequency response. The smoothness requirement is a time domain constraint achieved by minimising the squared gradient of the impulse response.

Any linear function (eg the gradient) of the impulse response can be constrained to approximate a desired shape. This is most easily understood by considering a discrete least mean square approximation process. For an $N$ tap filter with $L(>N)$ specification points the actual shape, $\mathbf{a}$, of the linear function of the filter coefficients is given by;

$$
a=g \cdot c
$$


where $\mathbf{c}$ is an $\mathrm{N}$ component column vector of the filter coefficients and $\mathrm{g}$ is an $\mathrm{L}$ by $\mathrm{N}$ matrix specifying a linear function of the coefficients. Consider the gradient of the impulse response as an example. The filter coefficients correspond to a continuous impulse response function given by;

$$
c(t)=\sum_{N} c_{n} \operatorname{sinc}\left(\frac{\pi\left(t-t_{n}\right)}{t_{s}}\right)
$$

where $t_{s}$ is 1 over twice the Nyquist frequency for the (sampled) system. The gradient of the impulse response is given by;

$$
c^{\prime}(t)=\frac{d c}{d t}=\left.\sum_{N} c_{n} \frac{\pi}{t_{s}} \frac{d(\operatorname{sinc}(x))}{d x}\right|_{\frac{\pi\left(t-t_{n}\right)}{t_{s}}}
$$

Hence for the gradient we have the components of $\mathbf{g}$ (equation 34) given by;

$$
g_{l, n}=\left.\frac{\pi}{t_{s}} \frac{d(\operatorname{sinc}(x))}{d x}\right|_{\frac{\pi\left(t_{l}-t_{n}\right)}{t_{s}}}
$$

A weighted least mean approximation to the desired shape, $\mathbf{s}$ ( $\mathrm{L}$ rows), of function, $\mathbf{g}$ ( $\mathrm{L}$ by $\mathrm{N}$ ), of the coefficients, $\mathbf{c}$ ( $\mathrm{N}$ rows), is given by solving the normal equation s corresponding to;

$$
q \cdot s=q \cdot g \cdot c
$$

where $\mathbf{q}$ is a diagonal $\mathrm{L}$ by $\mathrm{L}$ matrix of weighting factors. Hence solving;

$$
g^{h} W^{2} s=g^{h} W^{2} g c
$$

for $\mathbf{c}$ minimises $\sum_{L} q_{l, l}^{2}\left|s_{l}-a_{l}\right|^{2}$ (a is the actual shape). For a smoothness constraint $\mathbf{g}$ represents the gradient function and $\mathbf{s}=\mathbf{0}$ (ie ideally we want zero gradient everywhere). These give a solution to equation 39 of all coefficients equal zero. Hence a smoothness constraint alone is not useful and must be combined with additional (probably frequency domain) constraints.

Time and frequency domain constraints are easily combined for a discrete least mean square approximation. This is done by simply stacking up the rows for the frequency domain constraints (equation 10) and for the time domain constraints. This gives a combined specification of; 


$$
\Gamma \cdot \alpha=\Gamma \cdot \beta . c \quad ; \quad \alpha=\left[\begin{array}{l}
D \\
s
\end{array}\right], \quad \beta=\left[\begin{array}{l}
P \\
g
\end{array}\right] \text { \& } \Gamma=\left[\begin{array}{ll}
W & 0 \\
0 & q
\end{array}\right]
$$

Hence the corresponding normal equations $\left(\alpha^{h} \Gamma^{2} \alpha=\alpha^{h} \Gamma^{2} \beta c\right)$ can be solved for the coefficients which minimise the approximation error to both the time and frequency domain constraints.

Dual time/frequency domain constraints can also be used for filter design using a continuous least mean square approximation technique. In this case it is necessary to minimise an error function of the form;

$$
E^{2}\left(\left\{c_{n}\right\}\right)=\int_{-\infty}^{+\infty} W^{2}(f)|D(f)-R(f)|^{2} d f+\int_{-\infty}^{+\infty} q^{2}(t)\left|s(t)-g\left(\left\{c_{n}\right\}, t\right)\right|^{2} d t
$$

where $g\left(\left\{c_{n}\right\}, t\right)$ is a continuous function of time depending on the filter coefficients, for example the gradient of the filter impulse response. The approximation error can be minimised by substituting for $R(f)$ (from equation 3 ) and for $g(t)$ (from equation 34) differentiating with respect to the filter coefficients and setting the result to zero. This minimisation process yields a set of $\mathrm{N}$ simultaneous equations which can be solved to give the filter coefficients.

Using both time and frequency domain constraints for filter design may be useful if the standards conversion chain were modelled in terms of both time and frequency response. Certainly the response of the human visual system is sufficiently complex that a dual time/frequency approach might be useful. Such models are not yet available and this method of filter design remains to be investigated. The approach is also useful for designing filters with smoothness constraints eg 'maximally flat' filters. Dual time/frequency approach to filter design may prove a fruitful avenue of research in the future.

\subsubsection{Eigenfilters.}

An interesting technique for filter design is the generation of 'eigenfilters' (Vaidyanathan \& Nguyen 1987, Pei \& Shyu 1990). In this technique filter coefficients are derived as an eigenvector of a matrix. This is a general technique for the design of FIR filters. It can be computationally intensive to find eigenvectors for large matrices as required by this technique. There are, however, some shortcuts which minimise the amount of computation required. As in the previous sub-section it is also possible to include time domain constraints in the design of eigenfilters. A special case of eigenfilter design is described in the appendix (chapter 11) to derive optimum window functions for digital signal processing. 
Eigenfilters are another type of least mean square approximation to a desired filter specification. In this case an error function is derived as a Hermitian form, which is minimised by finding the eigenvector with smallest eigenvalue. As in sub-section 3.4 .2 we wish to minimise an error function of the form;

$$
E^{2}\left(\left\{c_{n}\right\}\right)=\int_{-\infty}^{+\infty} W^{2}(f)(D(f)-R(f))(D(f)-R(f))^{*} d f
$$

where $E\left(\left\{c_{n}\right\}\right)$ is the approximation error as a function of the set of filter coefficients, $\left\{c_{n}\right\}$, $R(f)$ is the actual filter response, $D(f)$ is the desired filter response and $W(f)$ is an error weighting function. As before we can compute the actual filter response in terms of the filter coefficients;

$$
R(f)=\sum_{n=1}^{N} c_{n} \exp \left(-j 2 \pi f t_{n}\right)
$$

which can be written in matrix form as;

$$
R(f)=c^{t} \varphi(f) \quad ; c=\left[\begin{array}{c}
c_{1} \\
c_{2} \\
\cdot \\
\cdot \\
c_{N}
\end{array}\right] \quad \& \quad \varphi(f)=\left[\begin{array}{c}
\exp \left(-j 2 \pi f t_{1}\right) \\
\exp \left(-j 2 \pi f t_{2}\right) \\
\cdot \\
\cdot \\
\exp \left(-j 2 \pi f t_{N}\right.
\end{array}\right]
$$

In order to formulate the problem as an eigen problem we can write;

$$
D(f)=D(f) \frac{R\left(f_{r}\right)}{D\left(f_{r}\right)}=\frac{D(f)}{D\left(f_{r}\right)} c^{\prime} \varphi\left(f_{r}\right)
$$

where $f_{r}$ is a fixed reference frequency at which the desired frequency response is defined to be equal to the actual frequency response. This is not an obvious step and is motivated purely by the need to include vector $\mathbf{c}$ in the desired frequency response specification. Substituting equations 44 and 45 into 42 gives;

$$
E\left(\left\{c_{n}\right\}\right)=\int_{-\infty}^{+\infty} W^{2}(f) c^{n}\left(\frac{D(f)}{D\left(f_{r}\right)} \varphi\left(f_{r}\right)-\varphi(f)\right)^{*}\left(\frac{D(f)}{D\left(f_{r}\right)} \varphi\left(f_{r}\right)-\varphi(f)\right)^{t} c d f
$$

where superscript $h$ represents the Hermitian transpose (complex conjugate of the transpose) and superscript $t$ represents the transpose. This can be written in matrix form as; where $\mathbf{A}$ is; 


$$
\begin{gathered}
E\left(\left\{c_{n}\right\}\right)=c^{k} A c \\
A=\int_{-\infty}^{+\infty} W^{2}(f)\left(\frac{D(f)}{D\left(f_{r}\right)} \varphi\left(f_{r}\right)-\varphi(f)\right)^{*}\left(\frac{D(f)}{D\left(f_{r}\right)} \varphi\left(f_{r}\right)-\varphi(f)\right)^{t} d f
\end{gathered}
$$

that is the coefficients of $\mathbf{A}$ are given by;

$$
\begin{aligned}
A_{m, n}=\int_{-\infty}^{+\infty} W^{2}(f) & \left(\frac{D(f)}{D\left(f_{r}\right)} \exp \left(-j 2 \pi f_{r} t_{m}\right)-\exp \left(-j 2 \pi f t_{m}\right)\right)^{*} . \\
& \left(\frac{D(f)}{D\left(f_{r}\right)} \exp \left(-j 2 \pi f_{r} t_{n}\right)-\exp \left(-j 2 \pi f t_{n}\right)\right) d f
\end{aligned}
$$

$A$ is Hermitian $\left(A^{b}=A\right)$ and therefore the error function can be minimised by choosing the coefficient vector, $\mathbf{c}$, to be the eigenvector of $\mathbf{A}$ with the smallest eigenvalue ${ }^{6}$.

Eigenfilters have some interesting properties. They are generated by finding the eigenvector with smallest eigenvalue for matrix $\mathbf{A}$ above. They give a weighted least mean square approximation to the desired frequency response at all frequencies except $\mathrm{f}_{\mathrm{r}}$. At the reference frequency, $\mathrm{f}_{\mathrm{r},}$ the actual frequency response exactly equals the desired response. This is different from the continuous least mean square approximation technique of sub-section 3.4.2. With that filter design technique the actual frequency response need not exactly equal the desired frequency response at any frequency at all. The facility to exactly specify the actual frequency response at one frequency, afforded by eigenfilter design, is convenient in practice. For standards conversion the obvious reference frequency is zero. At D.C. it is desirable, for practical reasons, to have a gain of exactly one. This can be achieved by eigenfilter design, but cannot be guaranteed by the technique of subsection 3.4.2. As in subsection 3.4.2 we can evaluate the matrix coefficients in terms of inverse Fourier transforms of functions of $W(f)$ and $D(f)$. This provides an efficient way of numerically performing the integration in equation 49 using FFT algorithms. Vaidyanathan \& Nguyen 1987, suggest that the desired eigenvector can be efficiently determined iteratively. This is possible because the ratio of second smallest to smallest eigenvalue is usually large.

Eigenfilters can also be designed to include simultaneous frequency and time domain constraints. Consider the previous example (sub section 3.4.3) in which a smooth impulse response was desired. This can be achieved by modifying the error function to be minimised so that we simultaneously minimise the frequency response approximation error and the square of the gradient. The appropriate error function is given by;

${ }^{6}$ This can be shown by expressing $\mathbf{c}$ as a sum of the eigenvectors of $\mathbf{A}$. 


$$
E^{2}\left(\left\{c_{n}\right\}\right)=\int_{-\infty}^{+\infty} W^{2}(f)|D(f)-R(f)|^{2} d f+\int_{-\infty}^{+\infty} q^{2}(t)\left|g\left(\left\{c_{n}\right\}, t\right)\right|^{2} d t
$$

where, for a smoothness constraint, $g\left(\left\{c_{n}\right\}, t\right)$ is the gradient of the impulse response given by;

$$
g\left(\left\{c_{n}\right\}, t\right)=g c \quad ; g_{l, n}=\left.\frac{\pi}{t_{s}} \frac{d(\operatorname{sinc}(x))}{d x}\right|_{\frac{\pi\left(t_{t}-t_{n}\right)}{t_{s}}}
$$

Substituting equation 51 into 50 gives a sum of two Hermitian forms. These are simultaneously minimised by simply adding the matrices together prior to finding the eigenvector with smallest eigenvalue.

\subsection{The Design of Adaptive and Motion Compensated Filters.}

The performance of 'conventional' standards converters is limited because the same interpolation aperture is used to interpolate all pixels. The characteristics of the interpolation filter are a compromise to cope with all picture characteristics which may be encountered. Conventional standards conversion filters are generated using 'typical' spectra for the image and response of the human visual system. Inevitably, when processing atypical pictures ${ }^{7}$, picture degradations will occur.

To improve performance adaptive standards converters have been proposed. A good general description of adaptive image processing can be found in Lim 1990 section 9.2.3. The general principle of adaptive processing is indicated in figure 3.10. Local characteristics of the image are determined and used to adapt the processing to the specific characteristics of that part of the image. For standards conversion a local 'motion' parameter is sometimes derived and used to control the interpolation aperture.

A local 'motion' parameter is typically provided by a 'motion detector' such as that in figure 3.11 (eg. Wade 1987 section $8.2 \&$ 8.3, Roberts 1985, Drewery, Storey \& Tanton 1984). The motion parameter is used to select an 'appropriate' interpolation aperture. Since the motion parameter is largely heuristic there is no objective way of determining what the appropriate aperture should be. Therefore the aperture may be taken as a linear combination of the apertures suitable for 'still' and for 'moving' pictures. A high output from the motion detector selects the 'moving' aperture whilst a low motion detector output selects the 'still' aperture. The non-linearity is adjusted, subjectively, to optimise the change from 'still' to

${ }^{7}$ Most images are 'atypical' in one sense or another! 
'moving' aperture. This is precisely what is done in the 'ADAC' standards converter made by AVS.

The performance of the motion detector can be understood by considering the model of 'typical' scene spectra presented in chapter 2. For simplicity let us define a 'motion' parameter $\mathrm{M}$ as;

$$
M=\frac{1}{\text { area }} \int_{\text {negion }}(g(x, y, t)-g(x, y, t+T))^{2} d x d y
$$

where $g(x, y, t)$ is the image intensity, $T$ is a picture period and integration over a region, divided by its area, is equivalent to a (lowpass) filtering operation. The motion parameter defined in this way is very similar to that generated by figure 3.11 , but is much more tractable mathematically. After a little manipulation, a relation to the normalised autocorrelation function of the image emerges, ie;

$$
M=2 \sigma^{2}(1-a(x=0, y=0, t=T))
$$

where $\sigma^{2}$ is the variance and $a(x, y, t)$ is the normalised autocorrelation function of the signal. Substituting the model of the autocorrelation function presented in chapter 2 we get;

$$
M=2 \sigma^{2}\left(1-\exp \left(-\frac{T}{\gamma}\right)\right) \sim 2 T \frac{\sigma^{2}}{\gamma} \quad \text { assuming } T<\gamma
$$

where $\gamma$ is a temporal correlation parameter (defined in chapter 2). Hence we can see that the conventional 'motion' parameter depends on two properties of the signal, the temporal correlation and the signal variance. The temporal correlation would be a useful parameter to control adaptive filtering but the motion parameter is ambiguous because it also depends on the amount of detail in the picture (signal variance). That is the motion parameter could be large either because of motion or for a detailed stationary picture.

The ambiguity in the output from a conventional 'motion' detector makes it difficult to use. Usually the 'motion' detector controls the transition between 'static' and 'moving' filter characteristics. However the 'static' characteristic can only reliably be used when the 'motion' signal is very low. Otherwise there is the risk of degrading detailed static pictures. Hence the benefits of the 'static' filter characteristic can only safely be applied to static pictures without excessive detail. The pictures for which the 'static' filter characteristic can be used therefore benefit little from its use!

It is possible to derive an appropriate adaption parameter from the television signal. If we rename $M$, in equation 52 to be $\Delta^{2} t$ then the temporal correlation parameter (from the model in chapter 2) is given by; 


$$
\gamma=2 T \frac{\sigma^{2}}{\Delta^{2} t}
$$

where gamma is temporal correlation parameter, $T$ is the picture period, $\sigma^{2}$ is signal variance and $\Delta^{2} t$ is the mean square picture difference across a picture period. Hence we can calculate the temporal correlation parameter appropriate to a small region of the image. In a similar fashion we can derive (local) spatial correlation parameters as;

$$
\begin{aligned}
& \alpha=2 X \frac{\sigma^{2}}{\Delta^{2} x} \\
& \beta=2 Y \frac{\sigma^{2}}{\Delta^{2} y}
\end{aligned}
$$

where $\alpha \& \beta$ are horizontal and vertical correlation parameters, $X \& Y$ are horizontal and vertical pixel spacings and $\Delta^{2} x \& \Delta^{2} y$ are mean square picture differences across one pixel horizontally and vertically.

Given suitable, local, picture parameters, a filter aperture appropriate to that region of the picture can be used for interpolation. Given a local value of temporal correlation, $\gamma$, derived as described above, the model from chapter 2 can be used to give a local scene spectrum. This process is, essentially, using a measurement of the autocorrelation function at a single lag, plus a priori knowledge of the shape of the spectrum, to give a local spectral model. This local spectrum can then be used to derive an appropriate filter specification and a realisable approximation to it.

The benefits of adaptive standards conversion are likely to be small, even using appropriate adaption parameters. There are two reasons for this. For all but very small movements there is very significant temporal aliasing in television pictures. Because of this aliasing the optimum interpolation aperture is not very sensitive to the temporal width of the scene spectrum. Therefore adaption would only give small changes in the interpolation used and, correspondingly, only small improvements in picture quality. Furthermore this type of adaption largely ignores the response of the human visual system. The eye is an 'active' sensor, that is its response changes with the input image. Specifically the eye 'tracks' moving objects giving an enormous change in its frequency response. Therefore a suboptimal interpolation aperture must be used, even for adaptive systems, because there is no knowledge of the movement of the eye.

To benefit from adaptive processing a knowledge of both the local scene spectrum and the response of the eye is required. This can be achieved by motion compensated interpolation. This process is described in detail in later chapters. However this is an appropriate place to discuss briefly some aspects of motion compensation relevant to filter 
design. Measuring the movement of objects in a scene gives the gross features of the (local) scene spectrum. Assuming the eye tracks the moving object it also gives the gross features of the response of the eye. Given this knowledge of the local scene spectrum and eye response an 'ideal' filter specification can be derived. The ideal filter specification is then approximated to give a practical set of filter coefficients. The approximation process is assisted by moving the coefficient positions to track the moving object. Hence motion compensation adapts the interpolation process allowing for the scene spectrum and the response of the eye. It also optimises the filter approximation process by changing the coefficient positions.

Motion compensation adapts the interpolation to allow for the gross features of the scene spectrum and eye's response. Motion compensated interpolation can be embellished by measuring the fine details of the local scene spectrum. Picture parameters, such as the correlation parameters $\alpha, \beta \& \gamma$ described above, can be measured after allowing for the measured motion. The motion compensated interpolation aperture can then be 'fine tuned' using these parameters. This type of interpolation might be referred to as 'adaptive, motion compensated processing'. This generalisation of motion compensation might appear to be 'gilding the lily'. However the temporal correlation parameter may arise naturally from appropriately designed motion estimation hardware. Therefore the information might as well be used in the interpolation process.

\subsection{Summary.}

This chapter has examined the way in which filters can be designed for interpolation in standards converters. Only transversal (finite impulse response) filters were considered because of their inherent linear phase response and ease of implementation. It is also convenient that transversal filters are easier to design than recursive (infinite impulse response) filters. A broadly historical perspective has been followed starting with the windowing technique of filter design and finishing with recent technique of 'eigenfilter' design. A number of new ideas have been presented, particularly from section 3.3 onwards.

The discussion started with the concept of the Brillouin zone as an ideal filter characteristic. This is the multidimensional analogue of a sharp cut lowpass filter in the one dimensional case. The use of the Brillouin zone was shown to be less than ideal because of its ill-defined shape for a vertical/temporal filter and its failure to allow for aliasing inherent in the signal. Then the frequency sampling technique of filter design was discussed. Although it has been successfully used for the design of standards conversion apertures, it is neither very flexible nor convenient to use. Generalisations of the frequency sampling filter design technique were also presented. These allowed arbitrarily positioned coefficients and frequency specifications. It was shown how an arbitrarily large number of frequency domain 
specifications could be used by using a least mean square approximation technique. However these frequency sampling techniques are essentially heuristic in nature, since they rely on the design engineer's idea of a 'good' filter characteristic.

Section 3.3 presented an objective method of determining a filter response specification appropriate for interpolating television pictures. This was based on the technique of Wiener filtering. In this case loss of resolution and aliasing were treated as uncorrelated noise sources. The 'ideal' response was proposed to be that which minimised the mean square impairment. Input noise can also be included as an impairment in this analysis.

Several techniques were proposed for producing 'optimum' approximations to an 'ideal' frequency specification. These techniques rely on a 'weighting' function which indicates the amount of (picture) impairment caused by approximating the ideal response. This sensitivity to approximation (the weighting function) was determined by extending the derivation of the 'ideal' filter presented in section 3.3. The characteristics and computational requirements of the various approximation techniques were discussed. For most standards conversion problems the filter produced by all the approximation techniques will be very similar. It was briefly indicated how time domain constraints (requiring, for example, a 'smooth' impulse response) could be included in the filter design process. This may be of use if more sophisticated, dual 'space-time/frequency domain', models of the response of the human visual system become available.

The results of using the filter design techniques, of section 3.3 onward, will be shown by some examples in chapter 5 . That chapter also describes the results of experiments applying these new filter apertures in comparison with existing filter designs. Postponing consideration of examples of these filter design techniques allows the implementation of standards conversion interpolation filters to be discussed first in the next chapter.

Finally the design of adaptive and motion compensated filter responses was considered. It was shown that to achieve benefits from adaptive filtering care must be taken in choosing the correct adaption parameter. Even with a well chosen adaption parameter the benefits of adaptive processing are likely to be small. To achieve significant improvements in picture quality motion compensation, the subject of later chapters, is required.

\subsection{References.}

1. Brigham, E.O. 1974. The fast Fourier transform. Prentice Hall. ISBN 0-13-307496-X.

2. Brigham, E.O. 1988. The fast Fourier transform and its applications. Prentice Hall 1988, ISBN 0-13-307547-8.

3. Clarke, C.K.P. \& Tanton, N.E. 1984. Digital standards conversion: interpolation theory and aperture synthesis. BBC Research Department Report, BBC RD 1984/20, December 1984. 
4. Clarke, C.K.P., 1990. Interpolation. Digital television, Sandbank, C.P. (Editor), John Wiley \& Sons Ltd, 1990, ISBN 0-471-92360-5, pp 287-374.

5. Crochiere, R.E. \& Rabiner, L.R., 1988. Multirate processing of digital signals. Advanced Topics in Signal Processing, Lim, J.S. \& Oppenheim, A.V. (Editors), Prentice Hall, 1988, ISBN 0-13-013129-6 025, pp 123-198.

6. Drewery, J.O., Storey, R. \& Tanton, N.E. 1984. Video noise reduction. BBC Research Department Report, BBC RD 1984/7, July 1984.

7. Drewery, J.O., 1990. Digital filtering of television signals. Digital television, Sandbank, C.P. (Editor), John Wiley \& Sons Ltd, 1990, ISBN 0-471-92360-5, pp 215286.

8. Dubois, E. 1985. The sampling and reconstruction of time-varying imagery with application in video systems. Proceedings of the IEEE, Vol. 73, No. 4, April 1985.

9. Dudgeon, D.E. \& Mersereau, R.M. 1984. Multidimensional digital signal processing. Prentice Hall 1990, ISBN 0-13-604959-1.

10. Jain, J. R. 1979. An efficient algorithm for a large Toeplitz set of linear equations. IEEE Transactions on acoustics, speech and signal processing, Vol. ASSP-27, No. 6, pp 612-615, December 1979.

11. Jennings, A. 1977. Matrix computations for engineers and scientists. John Wiley \& Sons 1977.

12. Kumar, R. 1985. A fast algorithm for solving a Toeplitz system of equations. IEEE Transactions on acoustics, speech and signal processing, Vol. ASSP-33, No. 1, pp 254267, February 1985.

13. Lim, J.S., 1990. Two-dimensional signal and image processing. Prentice Hall 1990, ISBN 0-13-934563-9.

14. Oppenheim, A.V. \& Schafer, R.W., 1989. Discrete-time signal processing. Prentice Hall Inc, 1989, ISBN 0-13-216771-9.

15. Parks, T.W. \& Burrus, C.S. 1987. Digital filter design. John Wiley \& Sons 1987, ISBN 0-471-82896-3.

16. Pearson D. 1991. Image processing theories. Image Processing, Don Pearson (editor). McGraw-Hill 1991, ISBN 0-07-707323-1, pp 15-39.

17. Pei, S. \& Shyu, J. 1990. 2-D FIR eigenfilters: a least squares approach. IEEE Transactions on circuits and systems, Vol. 37, No. 1, January 1990.

18. Preis, D.H. 1972. The Toeplitz matrix: its occurrence in antenna problems and a rapid inversion algorithm. IEEE Transactions on antennas and propagation, pp 204-206, March 1972.

19. Rabiner, L.R. \& Gold, B. 1975. Theory and application of digital signal processing. Prentice Hall 1975, ISBN 0-13-914101-4. 
20. Roberts, A. The improved display of 625-line television pictures. BBC Research Department Report, BBC RD 1983/8, July 1983.

21. Roberts, A. The improved display of 625-line television pictures: adaptive interpolation. BBC Research Department Report, BBC RD 1985/5, May 1985.

22. Storey, R. 1986. HDTV motion adaptive bandwidth reduction using DATV. BBC Research Department Report, BBC RD 1986/5, 1986.

23. Tonge, G.J. 1981. The sampling of television images. Independent Broadcasting Authority, Experimental \& Development Report 112/81, May 1981.

24. Tonge, G.J. 1982. Three-dimensional filters for television sampling. Independent Broadcasting Authority, Experimental \& Development Report 117/82, June 1982.

25. Trench, W.F. 1964. An algorithm for the inversion of finite Toeplitz matrices. J. Soc. Indust. Appl. Math., Vol 12, No. 3, pp 515-522, September 1964.

26. Vaidyanathan, P.P \& Nguyen, T.Q. 1987. Eigenfilters: a new approach to least squares FIR filter design and applications including Nyquist filters. IEEE Transactions on circuits and systems, Vol. CAS-34, No. 1, January 1987.

27. Wade, J.G., 1987. Signal coding and processing. Ellis Horwood Ltd, 1987, ISBN 0470-20778-7.

28. Zohar, S. 1969. Toeplitz matrix inversion: the algorithm of W.F. Trench. Journal of the Association for Computing Machinery, Vol. 16, No. 4, October 1969, pp. 592-601.

29. Zohar, S. 1974. The solution of a Toeplitz set of linear equations. Journal of the Association for Computing Machinery, Vol. 21, No. 2, April 1974, pp. 272-276. 


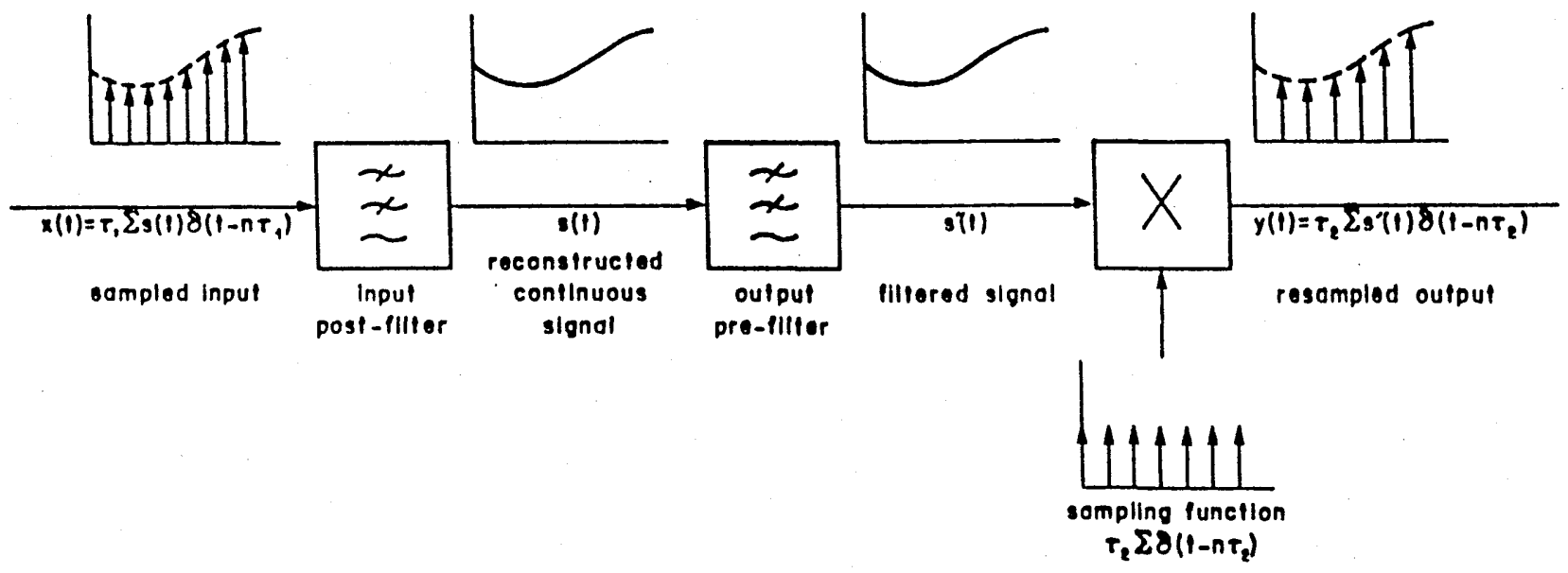

Figure 3.1 : 1 Dimensional resampling theory
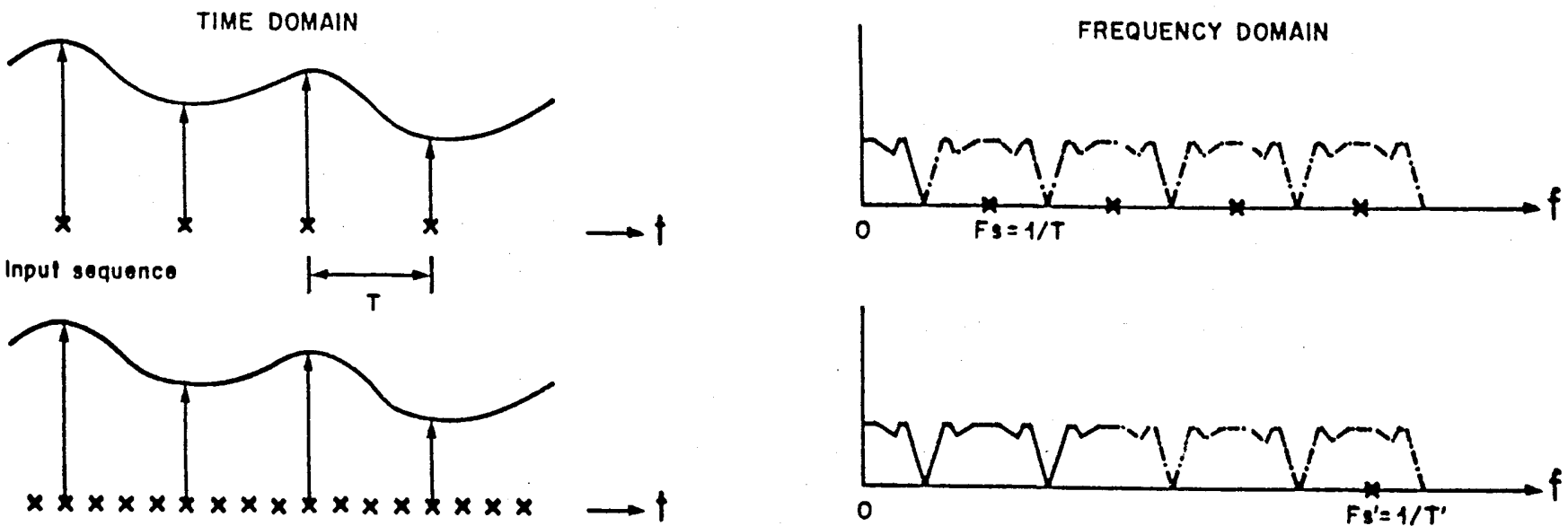

Input sequence podded with zeros $\rightarrow$
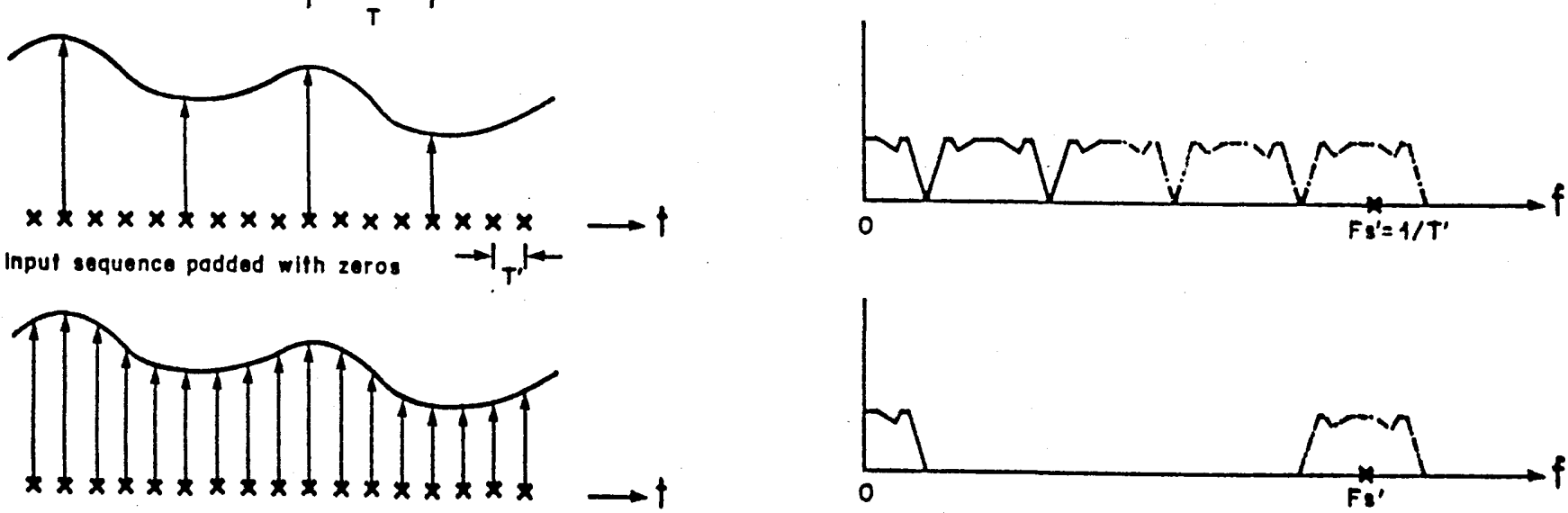

flltered podded sequence
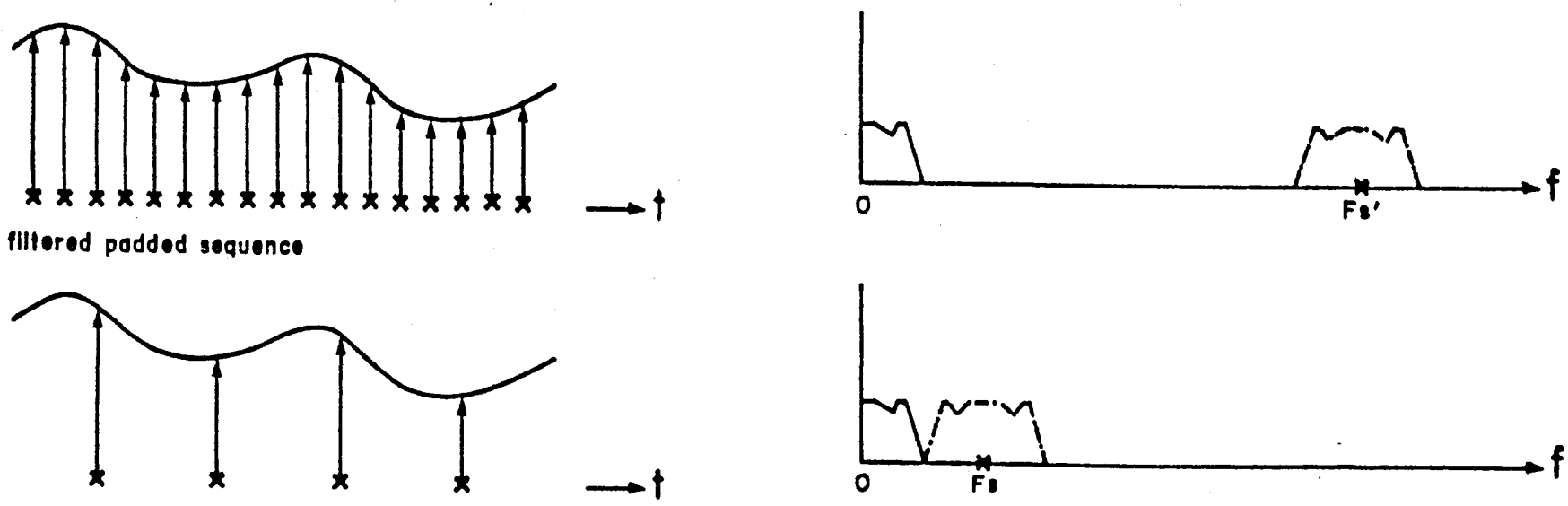

reoumpled sequence

Figure 3.2 : Sampling lattice conversion 
Figure $3.3 a$

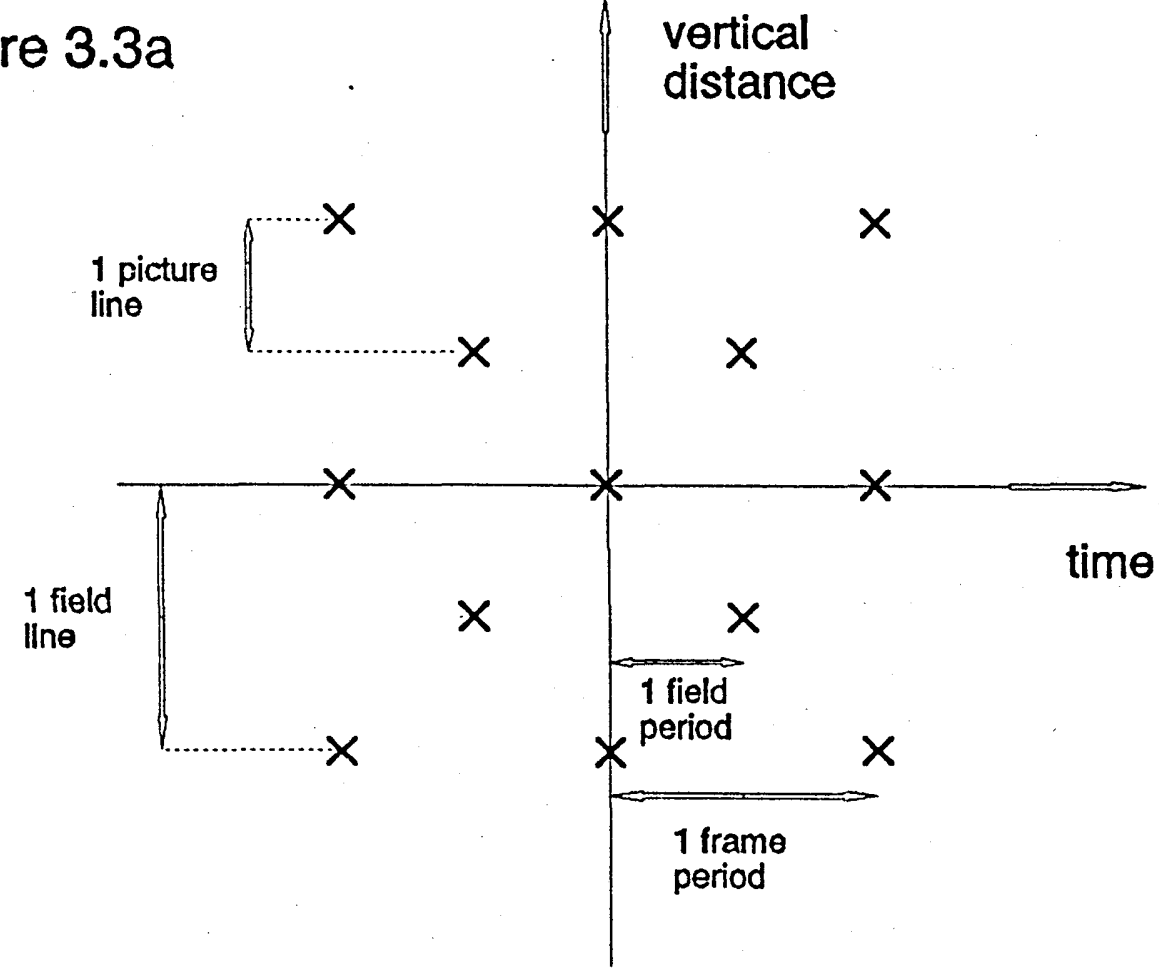

Interlaced sampling lattice for television signals

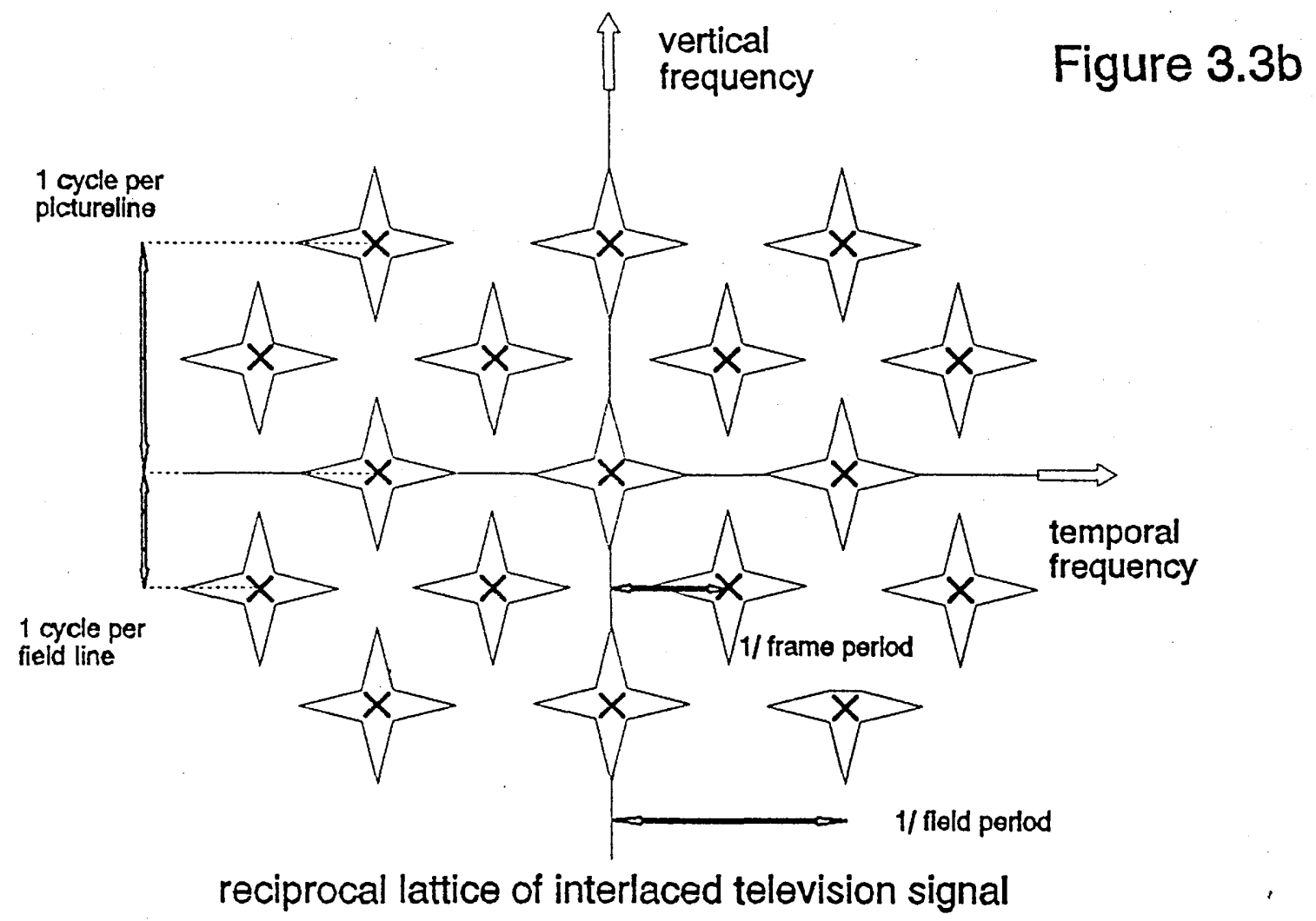




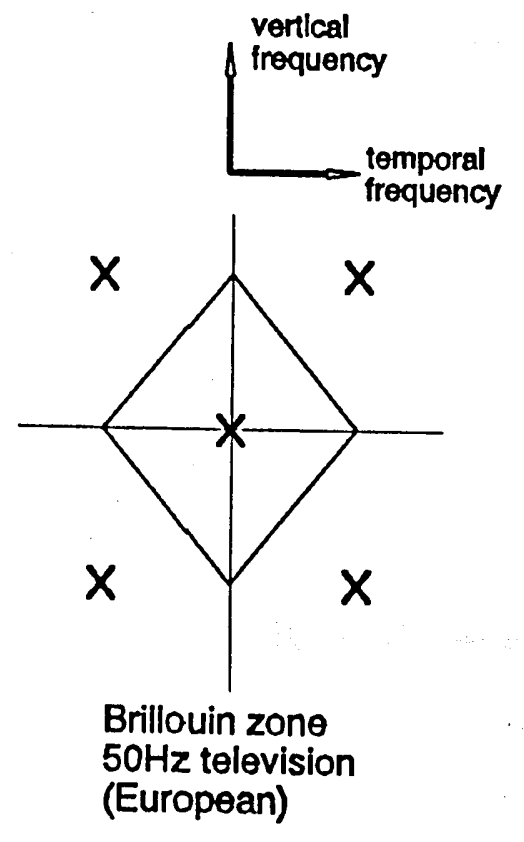

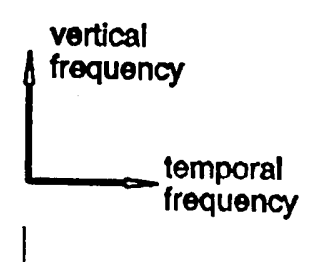

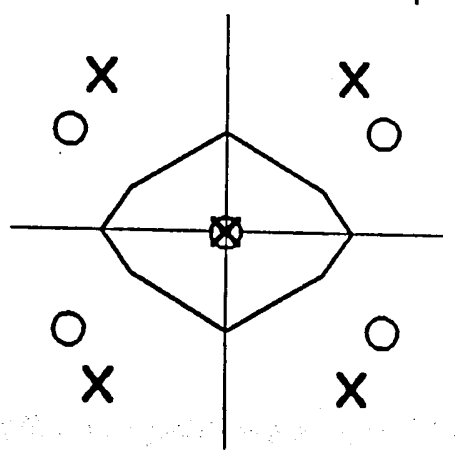

Intersection of Brillouin zones

Figure 3.4

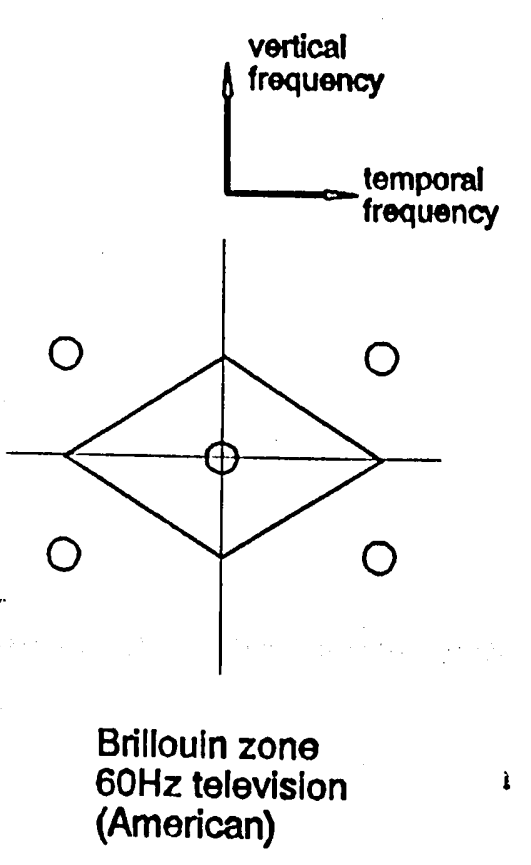

Brillouin zone (American) 


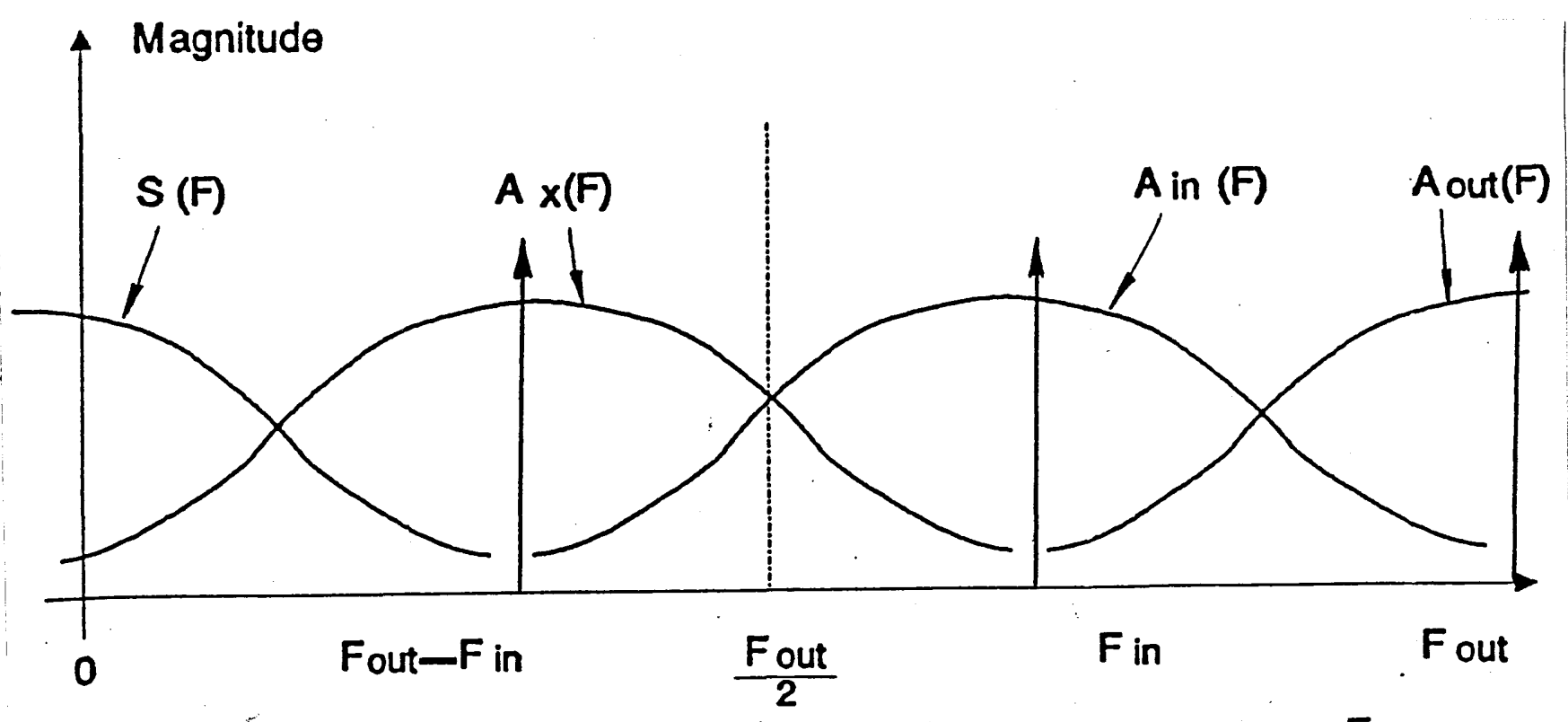

Frequency

Figure 3.9: Alias contributions in ID sample rate changing

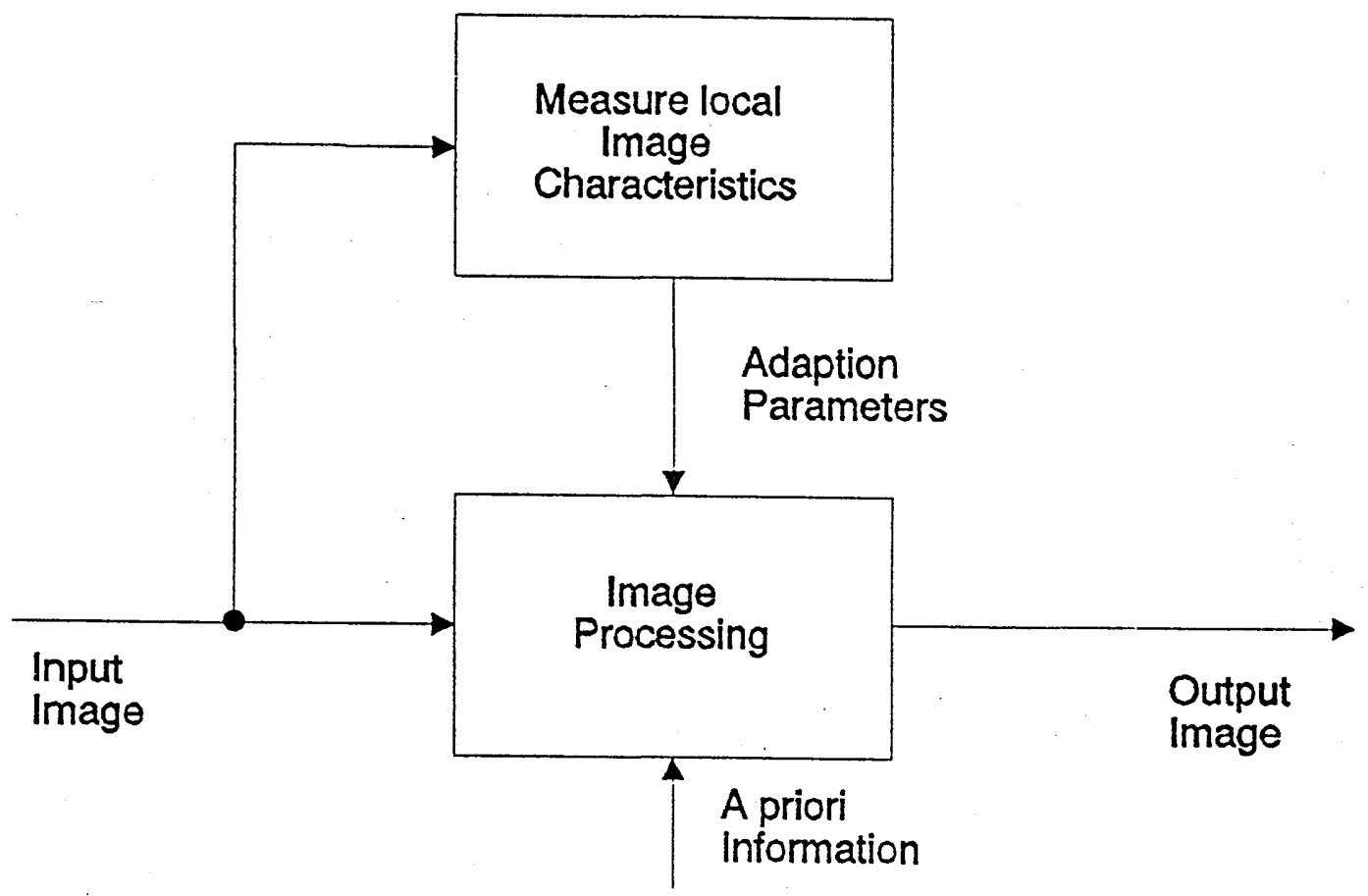

Figure 3.10: The principle of adaptive processing

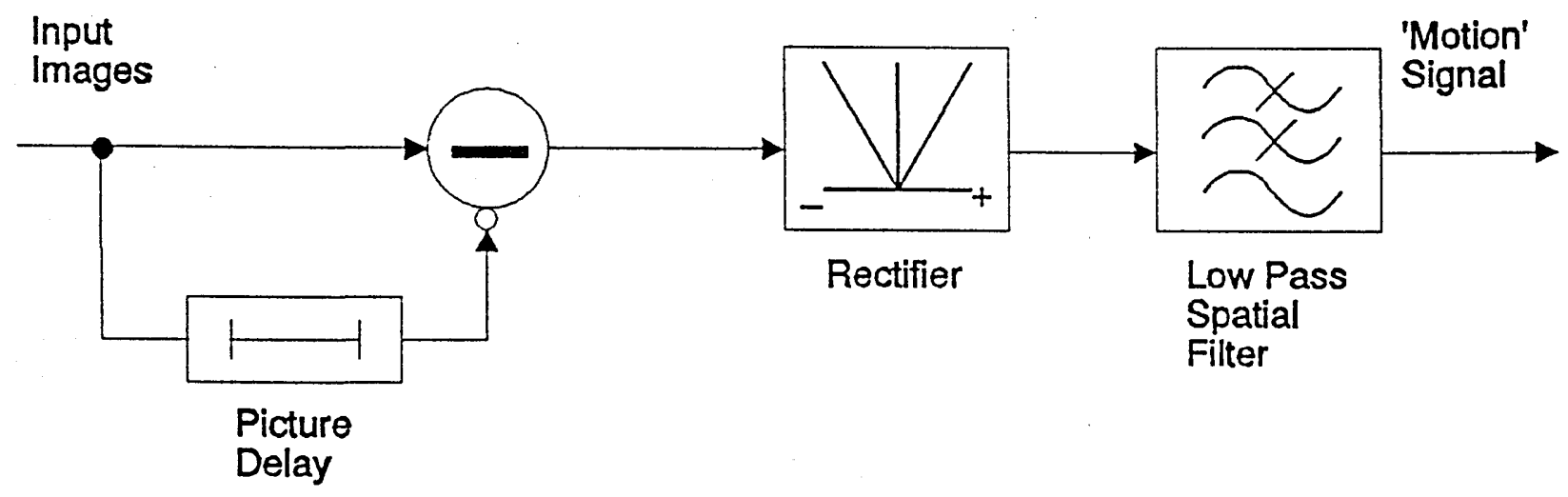

Figure 3.11 : Typical Motion Detector 


\subsection{Introduction.}

This chapter presents a detailed examination of the many ways in which standards converters can be built in practice. The possible standards converter architectures are examined in a unified and systematic way; an analysis which does not appear to have been undertaken hitherto. In particular this chapter presents two new filter architectures (figures 4.3 and 4.4) which are well suited to bidirectional standards conversion (eg interconversion of European and American television). Only a very few standards conversion architectures have ever been built. The best way to build a standards converter depends on the type of conversion required (eg whether the number of lines is increased or decreased) and the commercially available integrated circuit building blocks. The analysis in this chapter should allow the best standards conversion architecture to be selected for any particular application.

The chapter starts with a description of the practical realisation of FIR (finite impulse response) filters. The way in which digital filters can be used for interpolation is explained. In particular it is noted that the standards conversion process strictly requires two distinct filters, an input post-filter and an output pre-filter. These two filters are usually combined into a single composite (polyphase or time varying) filter operating on either the input or output sampling lattice. One dimensional configurations of input and output lattice filters (defined below) are described along with the way in which the filter coefficients are stored (aperture quantisation). Since pipelined input or output lattice filters are not simultaneously suitable for both increasing and decreasing the sampling rate (as is required in a practical bi-directional standards converter) non-pipelined input and output lattice filters are also considered.

The one dimensional theory is extended to multiple dimensions. It is shown that the principle difference between input and output lattice filters is whether the input or output samples are stored (input or output lattice filters respectively), that is whether data is stored before or after interpolation. The complication of aperture quantisation and address generation caused by scanning a multidimensional signal is also considered. Finally the principle of motion compensated interpolation is described and the changes this requires to the interpolation filter are considered.

\subsection{Fixed Filtering.}

The process of interpolation for standards conversion is, essentially, a filtering operation. Therefore it seems appropriate to start by considering the most basic implementation of 1 dimensional filtering. Video filtering is usually performed by transversal (FIR) filters, as explained in chapter 3. Figures 4.1 and 4.2 show the two basic forms of 
transversal filters. Only 3 taps are shown for brevity, but the structures can be extended to give filters with any number of taps. Filters of this type implement discrete convolution defined by;

$$
y(n)=x(n) * c(n)=\sum_{i=0}^{N-1} x(i) c(n-i)
$$

where $x(n)$ is the input sequence, $y(n)$ the output and $c(n)$ the coefficients. Note that the impulse response is the reverse sequence of the coefficients, that is; impulse_response(n)= coefficient(- $n)$. For standards conversion the impulse response is usually symmetric about its centre and this sequence reversal does not matter. The transposed implementation requires a little more hardware than the direct implementation. This is because the output of multipliers must be stored, and these signals would normally require more bits than the input signal.

The filters of figures 4.1 and 4.2 are discussed in more detail in Oppenheim \& Schafer 1989, chapter 6 and Wade 1987 chapter 7. Drewery 1990 (p240 -244) describes a number of variations on these implementations which can be used for fixed filtering (ie with non-varying coefficients). Both direct and transpose implementations are available commercially as integrated circuits.

Two variations of the basic filter structures are shown in figures 4.3 and 4.4. These structures are important because they can be developed into efficient time varying, polyphase, filters for standards conversion. However they are not efficient implementations for fixed filtering. These variations are described as non-pipelined, since data does not cascade from one filter section to the next.

In figure 4.3 each 'stage' of the filter operates on only one in three of the input samples. A new input sample is taken by each stage at a (different) point in the three phase cycle defined by the address sequence. For the remainder of the 3 phase cycle the same input sample is stored in the delay/multiplexer arrangement. The input sample is multiplied, by each of the 3 filter coefficients in turn. The coefficients are stored in ROM and addressed by the address sequence. The results from all three stages are summed to give the filter output. In the direct form filter (figure 4.1) the signal sample in each stage changes at each clock pulse, while the coefficients remain fixed. In the non-pipelined variation, by contrast, it is the coefficients which change at each clock pulse while the signal samples remain within a single stage.

The filter of figure 4.4 operates in a similar way to that of figure 4.3 . In this case, however, each filter 'stage' operates, in turn, to generate one in three output samples. Each stage generates an output by multiplying consecutive input samples by the appropriate coefficient and accumulating the result. The timing of the system is, again, orchestrated by a 3 phase address sequence. In the non-pipelined filter of figure 4.4 the output samples 'flow' through the filter while the coefficients remain fixed. In the transpose realisation of figure 4.2 , 
by contrast, the output samples remain within a single stage while the coefficients cycle through their sequence.

Figures 1 to 4 represent the four basic filter structures which will be developed in the remainder of the chapter to implement interpolation for standards conversion. Each of the four filters has different characteristics. In figures 1 and 2 the signal (input or output) 'flows' through the filter stages while the coefficients remain fixed. In figures 3 and 4 the signal samples do not move between stages but the filter coefficients cycle through their sequence. In figures 1 and 3 it is the input signal which is stored whereas in figures 2 and 4 the output signal is stored. For this reason I shall refer to filters 1 and 3 as 'input lattice' filters and filters 2 and 4 as 'output lattice' filters. The reason for this distinction will become more apparent in later sections of the chapter.

\subsection{Sample Rate Changing.}

The process of standards conversion is essentially that of interpolating a signal sampled on an input lattice, at points on an output lattice. In one dimension this process is known as sample rate changing and is discussed, for example, in Oppenheim \& Schafer 1989 section 3.6 and Crochiere \& Rabiner 1988. Sample rate changing is, conceptually, a two stage process, illustrated in figure 4.5. First the input signal is interpolated to give a continuous function. This continuous signal is resampled at the appropriate output instants. Interpolation of the input to give a continuous function requires an input post-filter to remove aliases due to input sampling. Prior to output sampling, an output pre-filter should be applied to remove signal components which would alias in the sampled output.

In practice the continuous signal is approximated by interpolation to a much higher sampling rate. This avoids the noise penalty of interconverting between analogue and digital domains. Discrete time sample rate changing only allows the sample rate to change by rational ratios, but this is sufficient for all practical purposes. The discrete time process of sample lattice conversion is illustrated in figure 4.6. Conversion to the higher sampling rate is accomplished by 'padding with zeros', that is inserting zeros value samples between the input samples. Aliases due to padding with zeros are removed by (discrete time) filtering the signal. The filter also removes frequency components which would otherwise alias when the 'supersampled' signal was sampled at the output rate. The filter characteristic depends on the input and output sampling rate and was discussed in detail in the previous chapter.

The padded input signal which is filtered contains many zero values. So although, in principle, the filter structures of figures 1 to 4 could be used, this would be extremely inefficient, since much of the time the multipliers would be multiplying by zero. Furthermore the processing would have to be performed very quickly to generate the high intermediate sample rate, even though the required output rate might be much lower. Hence in practice a 
time varying filter structure is used. This only calculates the output samples which are required and, although the zero padding values are implicit, multiplication of the padding zeros is not actually performed. Time varying filters of this type are discussed by Crochiere \& Rabiner 1988 and Clarke 1990.

One way to envisage sample rate changing using a time varying filter is to imagine a 'supersampled' coefficient sequence superimposed on the input sequence at the position of the desired output sample. Figure 4.8 illustrates this process and figure 4.7 shows a suitable time varying filter to implement it. The output sample is calculated by multiplying corresponding values in the (padded) input sequence and the ('supersampled') coefficient sequence and summing the products. In the example of figure 4.8 the sampling rate is increased by $4 / 3$. The value of output 3 is given by; out $3=$ in $1 . c 0+$ in $2 . c 4+$ in $3 . c 8$. When calculating the value of output 4 the filter coefficients used change so that out $4 ;=$ in $2 . c 1+$ in $3 . c 5+$ in $4 . c 9$ and so on for successive output samples.

When performing sample rate changing the differences between input lattice and output lattice filters become more apparent. When increasing the sampling rate it is more important to remove input aliasing with an input post filter. Subsequent resampling at the higher sampling rate will not introduce any new aliases and so the output prefilter is not required (but see chapter 3). The filter required depends on the input sampling rate, irrespective of how much the output sampling rate is increased. Therefore it is logical to define the filter characteristic with respect to the input sampling lattice, where it is invariant to any increase in sampling rate. For decreasing the sampling rate the reverse situation applies. The output prefilter is now more important since it will remove input aliases as well as frequency components which would alias in the output. The filter required depends on the output sampling rate irrespective of how much faster the input sampling rate is. Therefore, when decreasing the sampling rate, it is logical to define the filter characteristic with respect to the output sampling lattice, where it is invariant to any higher input sampling rate.

Figure 4.7 shows a time varying filter structure suitable for increasing the sampling rate. This, input lattice, filter is based on the basic structure of figure 4.1. The filter coefficients are sampled on the supersampled input lattice as shown in figure 4.8. Each filter stage includes a different ROM storing $1 / 3$ of the coefficients. The output from the filter is successive samples at the new, higher, sampling rate. The filter operates at the, higher, output sampling rate. Therefore a new input sample is not required every clock cycle. A FIFO is used to re-time the input samples.

The coefficient address, in figure 4.7, represents the current position of the interpolation aperture, in units of input sampling periods. Since, in this example, the input signal has been supersampled by a factor of 4 , a coefficient address of ' 4 ' represents one input sample period. Therefore the coefficient address is incremented by 3 for each successive output sample, that is by $3 / 4$ of an input sample. In each clock cycle a new sample is generated 
using an aperture centred $3 / 4$ of an input period from the previous output sample. Thus 4 output samples are generated for every 3 input samples.

The output samples from figure 4.7 are the weighted sum of 3 input samples. The output sampling rate is higher than the input sampling rate. Therefore, sometimes, two consecutive output samples must be generated from the same 3 input samples (using different coefficients). To do this input samples can be held fixed in the same filter stage by the delay/multiplexer arrangement at the top of the diagram. When the shift signal is asserted the input samples flow through the delays in the same way as in figure 4.1. When the shift signal is not asserted the input samples remain fixed in a particular filter stage. In this example the filter is generating 4 output samples for every 3 input samples. Hence the shift signal is only asserted for 3 clock periods in every 4 , that is two output samples in every 4 are generated using the same 3 input samples. The shift signal is asserted when a new input sample is needed. That is, when the leading edge of the interpolation aperture passes an input sample. This occurs when incrementing the coefficient address gives an overflow (ie the result is greater than 4 in this example). Generation of the coefficient address and shift signal is described in more detail below.

Figures 9 and 10 show the structure and timing of a filter suitable for decreasing the sampling rate. This, output lattice, filter is based on the basic structure of figure 4.2. In this case, in contrast to figure 4.7 , the filter coefficients are sampled on a supersampled output lattice as shown in figure 4.10. As before each filter stage includes a different ROM storing $1 / 3$ of the coefficients. The output from the filter is sampled at the new, lower, sampling rate. This filter structure operates at the, higher, input sampling rate. Therefore it does not need to generate a new output sample every clock period. The extra time available is used to increase the length of the filter aperture. Thus a longer aperture, of 3 output periods rather than 3 input periods, can be achieved. This ensures the filter's frequency response is constant, with respect to the output sampling rate, irrespective of how much the sampling rate is decreased. A FIFO is used to re-time the output samples.

The coefficient address, in figure 4.9, represents the current position of the interpolation aperture, in units of output sample periods. In this example the input has been supersampled by a factor of 3 and the output period is $4 / 3$ of the input period (ie longer). A coefficient address of ' 3 ' represents one input sample period and a coefficient address of ' 4 ' represents an output sample period. Therefore the coefficient address is incremented by 3 for each successive output sample, that is by $3 / 4$ of an output sample period. A new output sample is generated when the trailing edge of the interpolation aperture passes an output sample point.

The operation of figure 4.9, for decreasing the sampling rate is, perhaps, less obvious than the operation of figure 4.7. The output samples from figure 4.9 are the weighted sum, in this example, of 4 input samples. Sometimes, a specific input sample is required for the 
generation of three consecutive output samples. In this case the, partially computed, output samples remain fixed in one filter stage, instead of 'flowing' through the stages as in figure 4.2. The 'flow' of the output samples is through the multiplexer/delay arrangement at the bottom of figure 4.9. When the shift signal is asserted the input samples flow through the delays in the same fashion as in figure 4.1. When the shift signal is not asserted the input samples remain fixed in a particular filter stage. In this example the filter is generating 3 output samples for every 4 input samples. Hence the shift signal is only asserted for 3 clock periods in every 4 , that is only 3 output samples are generated for every 4 input samples. The shift signal is asserted when a new output sample has been generated. That is, when the trailing edge of the interpolation aperture passes an output sample. This occurs when incrementing the coefficient address gives an overflow (ie the result is greater than 4 in this example).

Figure 4.11 shows how the control signals required by the filters of figures 7 and 9 can be generated. Essentially the arrangement of adders and delays is an integrator which calculates the aperture position as a function of time. A constant increment is added to the position of the aperture every clock cycle. For an input lattice filter (eg figure 4.7) we require the current position of the interpolation aperture in terms of input periods. Therefore the increment used is 'output period divided by input period'. Hence the example of figures 7 and 8 would require an increment of $3 / 4$. For an output lattice filter (eg figure 4.9), the situation is reversed and we require the current position of the interpolation aperture in terms of output periods. Therefore the increment used is 'input period divided by output period'. Hence the example of figures 9 and 10 would also require an increment of $3 / 4$.

The fractional part of the current aperture position is used as the address for the coefficient ROMs in figures 7 and 9. For an input lattice filter (eg figure 4.7) a new input sample is required whenever the leading edge of the interpolation aperture passes an input sample. This happens when there is a carry from the fractional part of the adder, in figure 4.11 , to the integer part of the adder. Hence the carry signal is used as the shift signal in figure 4.7, indicating that a new input sample is needed. Similarly for an output lattice filter the carry, from the fractional to the integer part of the adder, signifies that the trailing edge of the interpolation aperture has passed an output sample position. That is the carry signifies that a new sample has been generated. Thus the carry signal is the shift signal for figure 4.9.

For use in a practical standards converter we may require an interpolation filter which can be used to both increase and decrease the sampling rate. The filter structure of figure 4.7 is well suited to increasing the sampling rate. Similarly the filter structure of figure 4.9 is well suited to decreasing the sampling rate. However figure 4.7 cannot be directly used for decreasing the sampling rate and figure 4.9 cannot be used for increasing the sampling rate. The problem, in these cases, can be seen by considering the aperture position generation in figure 4.11. In both cases the aperture position increment would have to be greater than 1 . 
This implies a single shift is required every clock cycle, and a double shift is required when there is carry between fractional and integer adders. The filters of figures 7 and 9 are not able to implement a double shift. There are various ways in which they could be modified to implement a decrease or increase in sample rate respectively. These modifications are, however, inelegant and inefficient. To achieve a filter structure capable of both increasing or decreasing the sampling rate it is more appropriate to develop the filter structures of figures 3 and 4.

Figures 12 and 13 are time varying filter structures derived from figures 3 and 4 . They are 'non-pipelined' input and output lattice filters respectively. That is, in the input lattice filter of figure 4.12 the coefficients are sampled on a supersampled input lattice and interpolation is performed after storage, and vice versa for figure 4.13. In these filters, as in figures 3 and 4, the (input or output) samples remain within a single filter 'stage' while the filter coefficients change at the start of every clock cycle. The 'integrator' of figure 4.11 can be used to generate the coefficient addresses in a similar way to its use for figures 7 and 9 . In this case, however, each of the coefficient ROMs contains the whole of the impulse response and hence the coefficient address must cover, in this example, 12 rather than 4 coefficients. The coefficient address must, therefore, be generated from both the fractional and the integer parts of the aperture position. The coefficient ROM address is given by;

\section{ROM address = Integer (aperture position) Modulo( number of taps) + Fraction(aperture position)}

where 'Integer' represents truncation 'Fraction' gives the fractional part of a number (ie the remainder after truncation). Hence, in this example, the coefficient ROM address is given by the integer part of the aperture position, modulo 3, plus the fractional part of the aperture position. If, as is often the case, there are $2^{\mathrm{N}}$ filter taps, then the modulo operation simply involves taking the lowest $\mathrm{N}$ bits of the integer part of the aperture position. Each stage of the filter structures perform all the required multiplications for, in this example, one in three of the input or output samples.

The advantage of the filter structures of figures 12 and 13 is that both can conveniently be used for either increasing or decreasing the sampling rate. Generation of the aperture position remains unchanged. Some modification is required to the FIFOs on the input or output to make them into more flexible stores. These modifications are, however, significantly less than the modifications which would be required to figures 7 and 9. Consider the input lattice filter of figure 4.12. This filter operates synchronously with output sampling, and is ideal for increasing the sampling rate. If it were used for decreasing the sampling rate then the aperture position increment (figure 4.11) would be greater than 1 . Hence a double shift signal (carry + LSB integer) would sometimes be generated. The double shift indicates that the trailing edge of the filter aperture has passed two, rather than one, input samples. In 
this case the input sample required by the filter is not the next one but rather the next but one. This can be accommodated either by modifying the input FIFO or by simply discarding one output sample and waiting for the correct input sample to be presented by the FIFO. The situation is similar for the output lattice filter of figure 4.13. This filter operates synchronously with input sampling and is ideal for decreasing the sampling rate. When used for increasing the sampling rate a double shift signal may be generated, indicating that the leading edge of the aperture has passed 2 , not 1 , output samples. In this case the filter will have generated two output samples in one input clock period. Hence the output FIFO must be modified so that it can store two samples in a single input clock period.

There is, of course, a price to be paid for making the filter structures more flexible. Firstly using the filters of figures 12 and 13 rather than 7 and 9 means that each coefficient ROM must store the whole, rather than part, of the filter impulse response. A fairly typical filter size might be 16 taps. Hence the additional storage can represent a significant overhead. Secondly the use of the 'wrong' filter structure (ie figure 4.12 for decreasing or figure 4.13 for increasing the sampling rate) is inefficient. If figure 4.12 is used for decreasing the sampling rate the output is generated more quickly than it is required. Consequently the filter is idle for part of the time. With a 2:1 decrease in sampling rate the filter would be idle half the time. Furthermore the filter aperture is smaller than necessary, because it is defined on the 'denser' input lattice, rather than the 'sparser' output lattice. If figure 4.13 is used for increasing the sampling rate, inefficiency arises because the filter operates at the slower input rate, rather than the faster output rate. Again the filter aperture is smaller than necessary because it is defined on the 'denser' output lattice rather than the 'sparser' input lattice. The degree of inefficiency depends on the ratio of input and output sampling rates. For standards conversion between European and American television standards the sampling rate ratio is close to unity. Therefore, in this case, there is little inefficiency in using a flexible filter structure for bidirectional conversion.

\subsection{Aperture Quantisation.}

The interpolation aperture is a continuous function and must be sampled and quantised for use in a digital system. Hence the aperture is only specified at a limited number of positions and each value will only have limited accuracy.

It is seldom convenient to sample the interpolation aperture in the way suggested by the ratio of input to output sampling rates. Let the ratio of output rate to input rate be $P / Q$. For an input lattice filter this would suggest sampling the aperture function at $\mathrm{P}$ times the input rate. Conversely, this sampling rate ratio would suggest sampling the aperture function at $\mathbf{Q}$ times the output rate for an output lattice filter. This is inconvenient since semiconductor read memories usually have $2^{n}$ locations. Furthermore if the sampling rate ratio is changed 
then the positions at which the aperture is sampled would also have to change. Finally either $\mathrm{P}$ or $\mathrm{Q}$ may be so large that it is impractical to store the coefficients.

In practice the aperture function is sampled at a fixed multiple of the input or output rate, irrespective of the sampling rate ratio. The multiple is usually $N=2^{n}$ so that it corresponds to the size of available memory chips. A continuous approximation to the actual aperture function is assumed which is constant from $1 /(2 \mathrm{~N})$ th of a sample before a sampling point to $1 /(2 \mathrm{~N})$ th of a sample after it. Examples of this, with $\mathrm{N}$ equals 4 , are shown in figure 4.14. In these figures the dotted line represents the true, continuous, aperture function. The solid line represents the continuous approximation to the aperture function generated by sampling the true aperture function at the numbered crosses.

For a practical system we need to know how frequently we should sample the aperture function. That is, if the aperture function is sampled $N=2^{n}$ times per input or output sample, what is an appropriate value for $\mathrm{N}$ ? The approximation of a continuous aperture function from its samples is precisely the same as the familiar problem of interpolating a sampled signal using a DAC followed by a zero order hold. This approximation problem is discussed, in the context of aperture quantisation, by Clarke 1990 (p310 to 304). Essentially the aperture samples are convolved with a rectangular pulse to approximate the continuous function. This convolution process attenuates aliasing introduced by sampling the aperture function but may also attenuate high frequencies in the aperture's frequency response. Increasing the value of ' $\mathrm{N}$ ' increases the attenuation of unwanted aliases and decreases the attenuation of the wanted frequency response. This is quantified in the table 4.1 taken from Clarke 1990.

Table 4.1: Filtering errors due aperture quantisation.

\begin{tabular}{|c|c|c|c|}
\hline $\mathrm{n}$ & $\mathrm{N}=2^{\mathrm{n}}$ & $\begin{array}{c}\text { Maximum attenuation } \\
\text { of wanted signal. } \\
(\mathrm{dB})\end{array}$ & $\begin{array}{c}\text { Minimum attenuation } \\
\text { of unwanted aliases. } \\
(\mathrm{dB})\end{array}$ \\
\hline 1 & 2 & -4 & -10 \\
\hline 2 & 4 & -0.2 & -17 \\
\hline 3 & 8 & -0.05 & -24 \\
\hline 4 & 16 & -0.02 & -30 \\
\hline 5 & 32 & -0.01 & -36 \\
\hline
\end{tabular}


The third column represents the maximum attenuation of the wanted signal. The loss of wanted signal energy depends on the aperture, but is likely to be much less than the maximum attenuation. Similarly the attenuation of alias energy is likely to be much higher than the minimum attenuation. Therefore, in the context of video standards conversion, it seems unlikely to be necessary to sample the aperture function at more than 32 times the input or output sampling rate.

The position at which the aperture function is sampled, relative to its centre, must also be considered in practice. Figure 4.14 a to 4.14 d show 4 ways in which aperture functions can be sampled for 2 or 3 tap filters. The dotted line represents the true, continuous, aperture function. The solid line represents a continuous, quantised, approximation to the true aperture function. In each of the examples the aperture function is sampled at 4 times the input (or output) sampling rate. The 'offset' represents the phase at which the aperture function is sampled relative to the input (or output) sampling lattice. It is the distance of the centre of the aperture function from the nearest preceding input sampling point. This has been indicated in terms of aperture sampling periods. For example an 'offset' of 1 means that the centre of the aperture function is one aperture sampling period after an input sampling point.

Figure 4.14a represents a simple example of sampling an aperture for a two tap filter. The fractional part of the aperture position, generated in figure 4.11 , is used to select which coefficient in each 'block' to apply to generate a given output sample. Coefficients 0 and 4 are selected if the fractional aperture position is between 0 and $1 / 4$. Coefficients 1 and 5 are selected if the fractional aperture position is between $1 / 4$ and $1 / 2$ etc. Hence the coefficient address can be obtained by simply truncating the fractional aperture position to 2 bits. This is very convenient in hardware. The way the aperture function has been sampled ensures that aperture sample closest to the required value is selected.

An alternative way of sampling the aperture for a two tap filter is shown in figure 4.14b. In this case we wish to select coefficients 0 and 4 if the fractional aperture position is between $-1 / 8$ and $+1 / 8$. Coefficients 1 and 5 should be selected if the fractional aperture position is between $1 / 8$ and $\% / 8$, and so on. Therefore, when the aperture is quantised in this way, we must round the fractional aperture position to 2 bits. This method of aperture quantisation can be more convenient, in practice, for test purposes.

Sampling the aperture function is slightly different when using an odd, rather than even, numbers of filter taps. Examples of sampling a 3 tap aperture are given in figures $4.14 \mathrm{c}$ and $4.14 \mathrm{~d}$. Generation of the coefficient address is again different for these two cases. In general the coefficient address is given by;

$$
\text { coefficient address = Integer }(N . \text { Fraction (aperture position }+ \text { offset }) \text { ) }
$$

where $\mathrm{N}$ is the number of aperture samples per input (or output) sample, and offset is the phase of sampling the aperture relative to input (or output) sampling positions. 'Integer' 
represents truncation and 'Fraction' gives the fractional part of a number (ie the remainder after truncation). Considering the non-pipelined filters of figures 12 and 13, the ROM address is given by;

\section{ROM address $=N .($ Integer ( aperture position+ offset $)$ Modulo (number of taps $))^{(4)}$ + coefficient address}

Notice that aperture position appears added to offset in both equations 3 and 4 above. Hence the offset can be included into the initial value loaded into the aperture position integrator of figure 4.11. The ROM address is then given by truncating the output of the aperture position integrator. In this way the need for rounding can be avoided irrespective of the way in which the aperture function was sampled. Hence the hardware requirement for all these ways of sampling is the same.

Suitable sampling of the (continuous) aperture function allows sample rate changing by any ratio. The resolution of the sampling rate ratio is determined solely by the number fractional bits used in the aperture position integrator. It does not depend on the way in which the aperture position has been sampled. Sampling the aperture introduces errors in the aperture function. These errors are reduced by increasing the number of aperture samples used per input (or output) sample. In a practical video standards converter it is unlikely that more than 32 aperture samples would be required per input sample.

\subsection{Multidimensional Filtering.}

Television standards converters require the use of multi-dimensional sample rate changing. So far this chapter has only considered one dimensional sample rate changing. The principles are, however, easily extended to multiple dimensions. For conversion between European and American television standards only a 2 dimensional (vertical-temporal) interpolator is usually required. This is because the two standards are usually sampled at the same horizontal coordinates. Other types of standards converter, for example conversion from wide screen HDTV to conventional television, may require a 3 dimensional (spatiotemporal) interpolator.

It is straight forward to generate a multidimensional filter from 1 dimensional 'prototypes'. All that is required is that the multipliers of a filter in one dimension be replaced by filters operating in a different dimension. An example of this is shown in figure 4.15. In this example a filter, of the type in figure 4.1, operating in the vertical dimension (using line delays), has been extended to a spatial filter. The multipliers in figure 4.1 have been replaced by horizontal filters (using pixel delays), also of the type in figure 4.1. All the other filter structures can be extended to multiple dimensions in this way, however the diagrams become 
rather complicated. The ordering of the dimensions does not matter and is usually determined as a matter of practical convenience.

Input or output lattice filters can be used for filtering in any of the dimensions. For example we could have an input lattice vertical filter combined with an output lattice temporal filter. Mixed input/output lattice multidimensional filters have been used commercially. However, it is usually more convenient to use either input or output lattice filters for all of the dimensions. If this is done the result is a multidimensional input or output lattice filter.

Figures 16 and 17 show generalised multidimensional input and output lattice filters. For the input lattice filter of figure 4.16 the input samples are stored prior to interpolation. The aperture function is stored on a supersampled input lattice. The input store provides a number of output samples which are multiplied by appropriate coefficients and summed to give a single output sample. The output lattice filter of figure 4.17 stores partially computed output samples and uses the aperture function sampled on a supersampled output lattice. At any time a number of different output samples are being computed. At each clock cycle the current input sample is included in all the appropriate output samples by using a Readmodify-write cycle in the output store. When the computation of an output sample is complete it can be read from the output store.

For time varying interpolation filters a small 'block' of coefficients corresponds to each filter tap. One coefficient is selected from each block, at each clock cycle, depending on the current position of the filter aperture. The division of the aperture function into blocks of coefficients is aperture quantisation, which was discussed in the previous section and illustrated, for 1 dimension, in figure 4.14. Figure 4.18 illustrates an example of aperture quantisation in two dimensions. The positions of the blocks of (non-zero) coefficients defines the region of support of the filter.

In multiple dimensions we have considerable freedom in defining the region of support for a filter. The number of filter taps defines the area (or volume) of the region of support. The positions of the blocks of coefficients defines its shape. Figure 4.19 illustrates some possible regions of support for a 9 tap, 2 dimensional filter. In some hardware implementations it is convenient to have a rectangular region of support as illustrated in the top left of figure 4.19. This shaped region of support is inefficient for implementing filters with, for example, elliptical passbands. This is because the coefficient values at the corners of a rectangular region of support will be relatively small when implementing an elliptical passband filter. In this case a different shape region of support, eg the top right of figure 4.19, may be appropriate. Some hardware configurations, particularly those for motion compensated processing, allow almost complete freedom in the placement of blocks of coefficients. The bottom of figure 4.19 gives two examples of more unusual regions of support. The most desirable shape for the region of support is determined by the shape of the aperture function. 
The design of aperture functions, for non-rectangular regions of support, is discussed in chapter 3 and the appendix (chapter 11).

\subsection{Scanning Standards.}

The purpose of this section is to explain the calculation of the, multidimensional, position of the interpolation aperture. A number of different scanning methods are used for television. Scanning is the process by which a multidimensional signal is converted into a 1 dimensional signal. The way in which a television signal is scanned determines the (spatiotemporal) coordinates of each pixel. The use of different scanning standards complicates the calculation of the correct, multidimensional, position of the interpolation aperture.

Digital processing of a multidimensional signal, such as television, requires that it both scanned and sampled. Sometimes the signal is scanned before it is sampled (eg when using a tube camera), and sometimes it is sampled before it is scanned (eg when using a CCD camera). Unfortunately the processes of scanning and sampling are not commutative. That is it makes a difference whether the signal is scanned before sampling or vice versa. The situation can be even more complicated, with the signal being scanned first in some dimensions and sampled first in others. For example, a television signal might commonly originate from film scanned by a flying spot telecine (ie using a CRT). In this case the signal is sampled first temporally but scanned first spatially! The different sources of television signals are discussed in chapter 2.

The inverse of scanning and sampling occurs when television signals are displayed. A television signal displayed on a CRT display is the inverse of scanning then sampling. Conversely a television signal displayed on an LCD screen is the inverse of sampling then scanning. More complicated situations can arise in telerecording (the conversion of television to film). The different television displays are discussed in chapter 2.

The proliferation of scanning methods only usually causes a problem when standards converting between different scanning standards. If a (moving) picture is 'filmed' with a tube camera and displayed on a CRT display then all the samples will appear in their correct (spatiotemporal) position. If a scene is 'filmed' with a CCD camera and displayed on a CRT display some distortion will occur. In this case horizontal edges will appear to slope slightly and vertical edges will acquire a, velocity dependent, slope. These are, however, very minor impairments and usually go unnoticed.

If the wrong scanning method is assumed when performing standards conversion significant picture impairments can be produced. Some commercial standards converters 
assume both input and output to have been sampled rather than scanned ${ }^{1}$. This corresponds to 'filming' a scene with a CCD camera, to be displayed on an LCD screen. If, as is much more likely, the pictures originated via a tube camera and are displayed on a CRT display, picture impairments will be introduced. These impairments arise because the output samples are displayed in their wrong, spatiotemporal, location. This is most serious in the time domain where mispositioned samples result in (extra) temporal aliasing which is perceived as judder. Vertical mispositioning of samples is, subjectively, less serious but will result in additional vertical aliasing.

To avoid impairments in standards conversion we must take account of the source and display scanning methods. There are 16 possible combinations of input and output scanning methods detailed in the table 4.2 below. Each of these cases might, realistically, be expected to occur in practice. The relationship between the input and output scanning lines are shown in figures 20 and 21.

To calculate the current position of the interpolation aperture, for a three dimensional interpolator, 3 integrators are required. The calculation of the 3 dimensional aperture position is shown in figure 4.22. The integrators are more complicated than the one dimensional one shown in figure 4.11. This is because the input and output sampling structures are not quite orthogonal. A total of 6 increments is required to calculate the current position of the interpolation aperture in all 3 dimensions. The increments, denoted $k$, are distinguished by 2 subscripts. The first subscript ( $x, y$ or $z$ ) indicates which coordinate of the interpolation aperture is to be incremented. The second subscript ( $p, 1$ or $f$ ) indicates when that increment is to be added to the aperture position. Subscript $p$ indicates that the increment is to be added every pixel, subscript 1 indicates that the increment is to be added every line and subscript $f$ indicates the increment is to be added every field. The aperture position increments can be calculated from the input and output scanning parameters as described in the appendix to this chapter (section 4.11).

${ }^{1}$ Henceforth a signal which has been sampled then scanned will be referred to as 'sampled'. If a signal has been scanned then sampled it will be referred to as 'scanned'. 
Table 4.2: Combinations of input and output scanning methods.

\begin{tabular}{|c|c|c|}
\hline Case & Input Scanning & Output Scanning \\
\hline 1 & sampled, sequential & sampled, sequential \\
\hline 2 & sampled, sequential & sampled, interlaced \\
\hline 3 & sampled, interlaced & sampled, sequential \\
\hline 4 & sampled, interlaced & sampled, interlaced \\
\hline 5 & sampled, sequential & scanned, sequential \\
\hline 6 & sampled, sequential & scanned, interlaced \\
\hline 7 & sampled, interlaced & scanned, sequential \\
\hline 8 & sampled, interlaced & scanned, interlaced \\
\hline 9 & scanned, sequential & sampled, sequential \\
\hline 10 & scanned, sequential & sampled, interlaced \\
\hline 11 & scanned, interlaced & sampled, sequential \\
\hline 12 & scanned, interlaced & sampled, interlaced \\
\hline 13 & scanned, sequential & scanned, sequential \\
\hline 14 & scanned, sequential & scanned, interlaced \\
\hline 15 & scanned, interlaced & scanned, sequential \\
\hline 16 & scanned, interlaced & scanned, interlaced \\
\hline
\end{tabular}

The integrator arrangement of figure 4.22 can be used to calculate the current aperture position required by an interpolation filter. Provided the aperture position increments are correctly calculated, no additional aliasing (other than that inherent in the signal) will be generated by the interpolation process. A large number of different input and output scanning methods may be encountered in practice but all can be accommodated by the same hardware arrangement of figure 4.22 . 


\subsection{Interlaced Signals.}

The use of interlaced signals further complicated the implementation of standards conversion. In an interlaced signal the even numbered lines $(0,2,4 \ldots)$ in a frame are scanned first, to create an 'even' field, the odd numbered lines are scanned after the even lines to create an 'odd' field. The effect of this, viewed in the vertical temporal plane is to create a quincuncial sampling structure. The reasons for interlaced scanning is the subject of some debate. A good discussion can be found in Clarke 1987. For better or worse the interlaced scanning system is almost universally used for television systems and practical standards converters must be able to deal with it.

Interlaced outputs cause few problems for input lattice filters. All that is necessary is for the aperture position to be calculated properly using the techniques described in the previous sections and detailed in the appendix to this chapter (section 4.11). Interlaced outputs do, of course, complicate the design of the interpolation aperture, but this does not affect the practical implementation of a standards converter.

Interlaced inputs to an input lattice filter require special treatment. The easiest way to deal with them is to pad the input signal with zeros to produce a sequential signal. However this approach is very inefficient since half the multipliers, at any instant, are multiplying by zero. A better solution is to use the interlaced input directly. Only about half the number of filter coefficients are then required. The filter coefficients must now, however, change according to the position of the output pixel relative to the input lattice. In an input lattice filter the aperture position is equivalent to the output sample position and is specified in terms of the input sampling lattice. By superimposing the interpolation aperture on the input samples, at different output sample positions, it can be seen that different subsets of filter coefficients are required. Consider, for example, (software) image processing systems in which video signals are usually stored as complete frames (ie pairs of fields). In this case four subsets of filter coefficients are required as illustrated in figure 4.23 . The 4 different subsets of coefficients can be equated to 4 filtering modes denoted 0 to 3 . The filtering mode used depends on the parity of the vertical and temporal coordinates of the integer part of the aperture/output sample position. If even parity is equated to zero and odd parity to unity we have, for figure 4.23 , the following equation;

$$
\text { filter mode }=\text { vertical parity + 2.temporal parity }
$$

For example if the current output pixel is at 23.3 (picture) lines and 175.1 fields, the integer parts of both vertical and temporal coordinates are odd, hence both vertical and temporal parity is 1 and filtering mode 3 should be used. In hardware (rather than software) implementations of video processing the fields are usually considered separately rather than in pairs. In this case only two subsets of filter coefficients (or filter 'modes') are required. 
Output lattice filters have similar problems dealing with interlaced signals. In this case, however, interlaced inputs present little problem, being accommodated by the correct calculation of the aperture position. For output lattice filters problems arise in generating interlaced outputs. If an output lattice filter were used directly a sequential output would be produced. This could be subsampled to give an interlaced output. This, however, would be very inefficient since either twice as many multipliers would be required or only half as large an aperture could be used. A better solution is to modify the output lattice filter so that it only generates the required output samples. This requires that the calculation of a new output sample should only commence when the trailing edge of the interpolation aperture has just passed an interlaced rather than a sequential output sample. This in turn changes the way in which the coefficients 'flow' through the filter stages. Hence it is necessary to use multimode filtering in a similar fashion to an input lattice filter.

With care both input and output lattice filters can be used to process interlaced inputs to produce interlaced outputs. Only minor changes are required to the control and address generation circuits for sequential signals.

\subsection{Motion Compensation.}

The use of motion compensation has been suggested as a means of improving the performance of television standards conversion. Indeed it is one of the purposes of this thesis to investigate the degree to which the benefits sought can be achieved in practice. The theory and some results of motion compensated processing are presented in subsequent chapters. The purpose of this section is to discuss the way in which the requirements of motion compensated processing can be achieved in practice.

The basis of motion compensation is to process moving objects, within pictures, along their motion trajectory. This idea is illustrated in figure 4.24. In conventional linear interpolation, interpolating a moving object results in multiple images in the output pictures, which is perceived as judder. This is a result of the temporal aliasing inherent in the signal. The interpolation process can be motion compensated by shifting the moving object, in each of the contributing input pictures, to its correct position in the output picture. This removes multiple imaging and hence eliminates judder.

Motion compensation is achieved by skewing the interpolation aperture to follow an object's motion. To do this we must first measure the motion of every pixel in the image. This is the subject of chapter 7. For the purpose of this chapter let us assume that a motion field is available sampled on an appropriate lattice. The sampled motion field is applied by combining it with the aperture position, as calculated in figure 4.22.

Figures $25 \& 26$ illustrate the concept of generalised motion compensated input or output lattice filters. Only 2 taps are shown for brevity. For the purposes of explanation it is 
assumed that these are two temporal taps. Nevertheless the principles are easily extended to any number of spatial and temporal taps. Essentially the interpolators are conceptually the same as for a non-motion compensated implementation. The difference with motion compensation is that the sample and coefficient addresses can change in an unpredictable manner from pixel to pixel. The unpredictable nature of these addresses preclude the use of a number of economies which can be made in non-motion compensated interpolators, where the pattern of addresses is known in advance. Therefore, in practice, motion compensated interpolators require considerably more hardware (perhaps double) than non-motion compensated ones.

Figure 4.27 illustrates the vector processing required to combine motion vectors and aperture coordinates for an input lattice filter. its operation can be understood in conjunction with figure 4.24. Assume that an output field is being generated between two input fields. Let it be generated $\alpha$ input field periods after the preceding input field, and (1- $\alpha)$ input field periods before the succeeding input field. In the case of an input lattice filter we require that the motion vectors be sampled coincident with the output field instants and the input spatial sampling. The input position, in the preceding field, corresponding to the current output pixel, is given by the current aperture position plus $\alpha$ times the motion vector. That is the point in the input field, used for interpolation, is displaced from the output pixel position by an appropriate multiple of the motion vector. The input position, in the succeeding field, corresponding to the current output pixel, is given by the current aperture position plus (1- $\alpha)$ times the motion vector. The temporal position of the second tap is one input field period after the first tap. Hence an offset of 1 input field period has been added to the temporal coordinate of the second address.

The vector processing for an output lattice filter is very similar to that for an input lattice filter. For an output lattice filter the aperture is specified in terms of the output sampling lattice. Therefore the motion vectors must be sampled, both temporally and spatially, on the output sampling lattice. In an output lattice filter a number of output samples, 2 in the case of figure 4.26 , are being calculated simultaneously from the same input sample. Each output sample corresponds to a different output position spatially and/or temporally. This implies that each sample being calculated requires a different motion vector. This, in turn, requires that the motion vectors used for the calculation of each motion compensated aperture coordinate be appropriately delayed. In hardware terms this means that the multipliers in figure 4.27 should be preceded by delays. In the example of figure 4.26/4.27 addresses ' 1 ' correspond to an earlier output instant than addresses ' 2 '. Therefore, in this case, a field delay should be introduced before the upper multiplier in figure 4.27.

Both input and output lattice filters can successfully be used for motion compensated processing. Output lattice filters are somewhat more complicated, for motion compensation, because of the need to delay the motion vectors by a different amount for each tap. Input 
lattice filters are efficient ways to implement input post filters, which remove spectral repeats in the (sampled) input signal. In non-motion compensated processing no aliasing is introduced by sampling the output signal faster than the input. In motion compensated processing, however, output aliasing can be introduced by changes in the motion vector between adjacent output samples. Hence, ideally, an output prefilter, which can be efficiently implemented by an output lattice filter, should be used as well. The use of cascaded input and output lattice filters for motion compensation is unlikely to be implemented in practice because of hardware complexity.

\subsection{Summary \& Conclusions.}

This chapter has described some ways in which interpolators for standards conversion can be implemented. The basic theory of interpolation using linear filtering was described. The changes required to basic filter structures for use as interpolators were considered.

Two types of filter, input or output lattice filters, were considered. These correspond to the direct and transposed implementations of an FIR filter in a single sampling rate filter. For interpolation, input lattice filters are efficient for removing repeat spectra (aliases) in the sampled input signal. These filters store the input samples prior to interpolation and the interpolation aperture (filter response) is specified on the input lattice. Input lattice filters are best for increasing the sampling rate. Output lattice filters are efficient for removing spectral components which would alias in the output signal. These filters store output samples while they are calculated and the interpolation aperture is specified on the output lattice. Output lattice filters are best for decreasing the sampling rate.

Two implementations of interpolation filters, pipelined or non-pipelined, were considered. Pipelined filters are efficient and convenient structures for either increasing or decreasing the sampling rate (but not both). They are inconvenient to use in circumstances where the same hardware may be required to either increase or decrease the sampling rate. This is usually the case for standards conversion between European and American television standards. Non-pipelined filters can conveniently be used to both increase and decrease the sampling rate. The price paid for this flexibility is a more complex filter structure and a loss of computational efficiency either increasing or decreasing the sampling rate (depending on whether an input or output lattice filter is used).

Theoretically the interpolation aperture is a continuous function. In practice it is stored at points on a supersampled input or output lattice. The degree of supersampling (aperture quantisation) determines how closely the sampled aperture function approximates the desired continuous one. It is unlikely to be necessary to sample the aperture function more than 32 times faster than the input or output sampling rate (in each dimension). The accuracy of the 
input/output sampling rate ratio does not depend on aperture quantisation. It is solely dependant on the precision to which aperture position, as a function of time, is calculated.

The plethora of ways in which television pictures are scanned complicates the calculation of the aperture position, as a function of time. Nevertheless the same hardware can be used to calculate the aperture position for all scanning methods. It is important to calculate the aperture position correctly. If this is not done aliasing, in addition to that inherent in the signal, is introduced to the interpolated pictures. This results in a degradation of picture quality.

The use of interlace in television picture further complicates the implementation of standards converters. It can be accommodated by selecting the filter coefficients for each sample, dependent on the relative position of the input and output sampling lattices. This complicates control of the interpolation filter but does not significantly increase the hardware required.

Motion compensation can be implemented in both (multidimensional) input and output lattice filters. Conceptually relatively modest changes are required to the calculation of sample and coefficient addresses. In practice significantly more hardware is required to allow much more flexible access to stored data samples. For non-motion compensated interpolators the hardware requirements of input and output lattice filters are very similar. When they are motion compensated, however, output lattice filters are significantly more complicated because it is necessary to store motion vectors as well as the output samples.

\subsection{References.}

1. Clarke, C.K.P, 1987. Future television systems : comparison of sequential and interlaced scanning. BBC Research Department Report No. BBC RD 1987/11. November 1987.

2. Clarke, C.K.P., 1990. Interpolation. Digital television, Sandbank, C.P. (Editor), John Wiley \& Sons Ltd, 1990, ISBN 0-471-92360-5, pp 287-374.

3. Crochiere, R.E. \& Rabiner, L.R., 1988. Multirate processing of digital signals. Advanced Topics in Signal Processing, Lim, J.S. \& Oppenheim, A.V. (Editors), Prentice Hall, 1988, ISBN 0-13-013129-6 025, pp 123-198.

4. Drewery, J.O., 1990. Digital filtering of television signals. Digital television, Sandbank, C.P. (Editor), John Wiley \& Sons Ltd, 1990, ISBN 0-471-92360-5, pp 215286.

5. Oppenheim, A.V. \& Schafer, R.W., 1989. Discrete-time signal processing. Prentice Hall Inc, 1989, ISBN 0-13-216771-9.

6. Wade, J.G., 1987. Signal coding and processing. Ellis Horwood Ltd, 1987, ISBN 0470-20778-7. 


\subsection{Appendix : Calculating the aperture position.}

The position of the interpolation aperture, as a function of time, can be calculated using the arrangement of integrators in figure 4.22. The aperture position increments $(\mathrm{k})$ can be calculated from the parameters describing the input and output scanning methods.

A brief digression is in order to consider the parameters which define a scanning standard. These are needed in the calculation of the aperture position increments. Obviously we must consider the full number of samples per line, lines per frame, fields per second and whether scanning is interlaced. If the standard is interlaced the frame rate is half the field rate, otherwise they are synonymous. Not all of the samples on each line, nor all of the lines, convey picture information. Some pixels are intentionally left blank to allow beam deflection circuits, in CRT displays, time to operate. Hence we also need to consider the number of active samples per line and lines per frame, which actually convey picture information. Finally, we need to know whether the pictures have been scanned first then sampled, or vice versa, in both the temporal and spatial dimensions. Note that the aspect ratio of the picture (the ratio of its width to its height) is not a scanning parameter, since it depends only on the shape of the display.

It is not always desirable to calculate the whole of the output picture. For example we may wish to only calculate the active part (ie picture information) of the output picture and not output blanking. The aperture position increments depend on how much of the whole output picture is calculated. 
The aperture position increments, for an input lattice filter, are given by;

$$
\begin{aligned}
& k_{x p}=\frac{\text { asamples }_{\text {in }}}{\text { asamples }_{\text {out }}}
\end{aligned}
$$

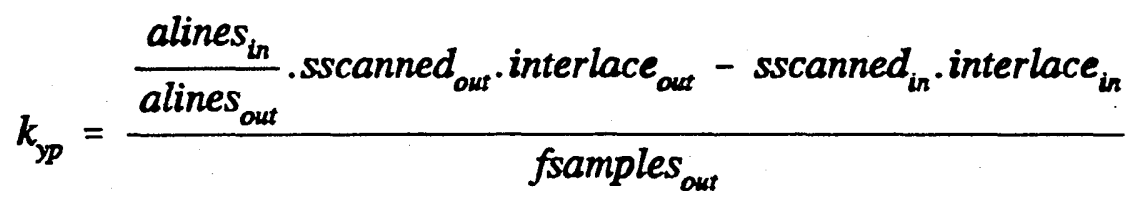

$$
\begin{aligned}
& k_{y l}=\frac{\text { alines }_{\text {in }}}{\text { alines }_{\text {out }}} \cdot \text { interlace }_{\text {out }}-k_{y p} \cdot x \text { xlength }+k_{y p}
\end{aligned}
$$

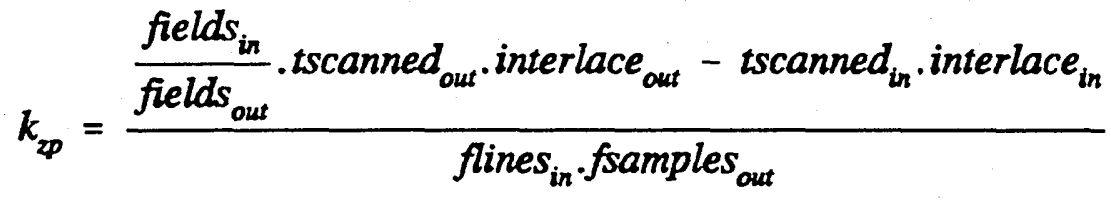

$$
\begin{aligned}
& k_{z l}=k_{z p}\left(\text { fsamples } s_{\text {out }}-x \text { length }\right)+k_{q p} \\
& k_{v f}=\frac{\text { fields }_{i_{n}}}{\text { fields }_{\text {out }}} \cdot \text { interlace }_{\text {out }}-k_{z l} \cdot \text { ylength }+k_{z l}
\end{aligned}
$$

where (with examples from digital 625 line European television);

fsamples is the full number of samples per line (eg. 864),

flines is the full number of lines per frame (eg. 625),

fields is the field rate (eg $50 \mathrm{~Hz}$ ),

interlace is the order of interlace, 1 for sequential and 2 for interlaced scanning,

asamples is the number of active samples per line (eg 720),

alines is the number of active lines per frame (eg 576),

sscanned is 1 if the signal was spatially scanned before sampling, or 0 if the signal was sampled before scanning spatially.

tscanned is 1 if the signal was temporally scanned before sampling, or 0 if the signal was sampled before scanning temporally.

xlength is the number of pixels calculated horizontally per output line, usually this will equal 'fsamples' if the whole image (including blanking) is calculated, or will equal 'asamples' if only the active part of the picture is calculated.

ylength is the number of lines calculated per output frame, usually this will equal 'flines' if the whole image (including blanking) is calculated, or will equal 'alines' if only the active part of the picture is calculated. 
The subscripts indicate whether a variable is an input or output scanning parameter. The aperture position increments, for an output lattice filter, can be determined by swapping the input and output subscripts.

Note that the vertical coordinate is calculated modulo the full number of lines per frame. For interlaced signals all will be well provided that there are an odd number of lines per frame. This is (obviously) the case for 525 and 625 lines signals. However interlaced HDTV standards have been proposed with 1050 and 1250 lines per frame. For interlaced signals with an even number of lines per frame it is necessary to add 1 to the vertical coordinate at the start of odd fields and subtract 1 at the start of even fields. 


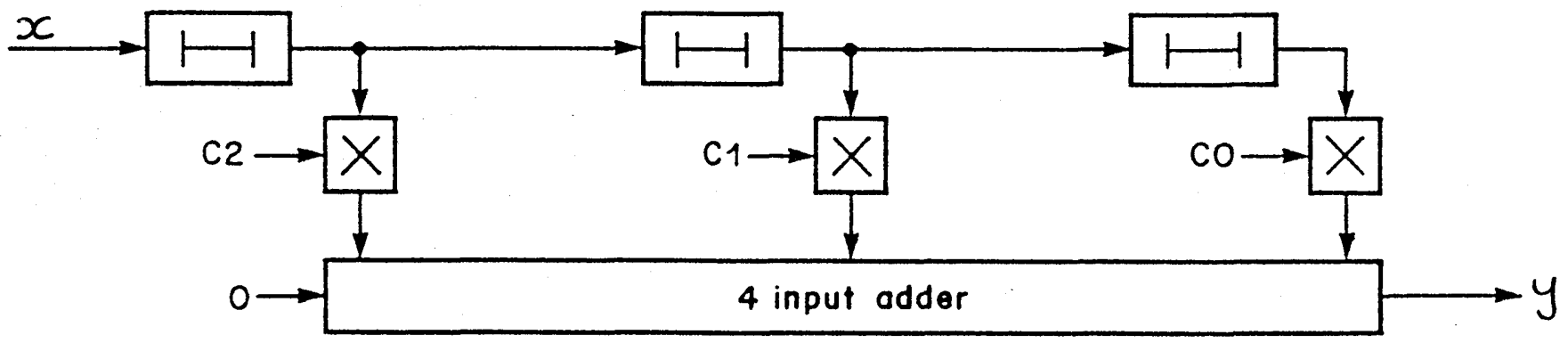

Figure 4.1 : Direct form realisation of an FIR filter

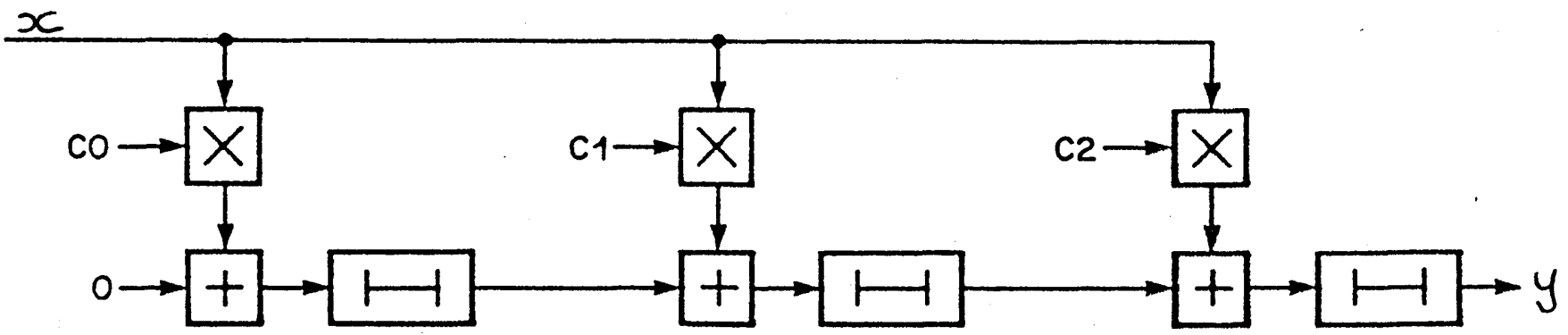

Figure 4.2 : Transposed direct form realisation of an FIR filter 


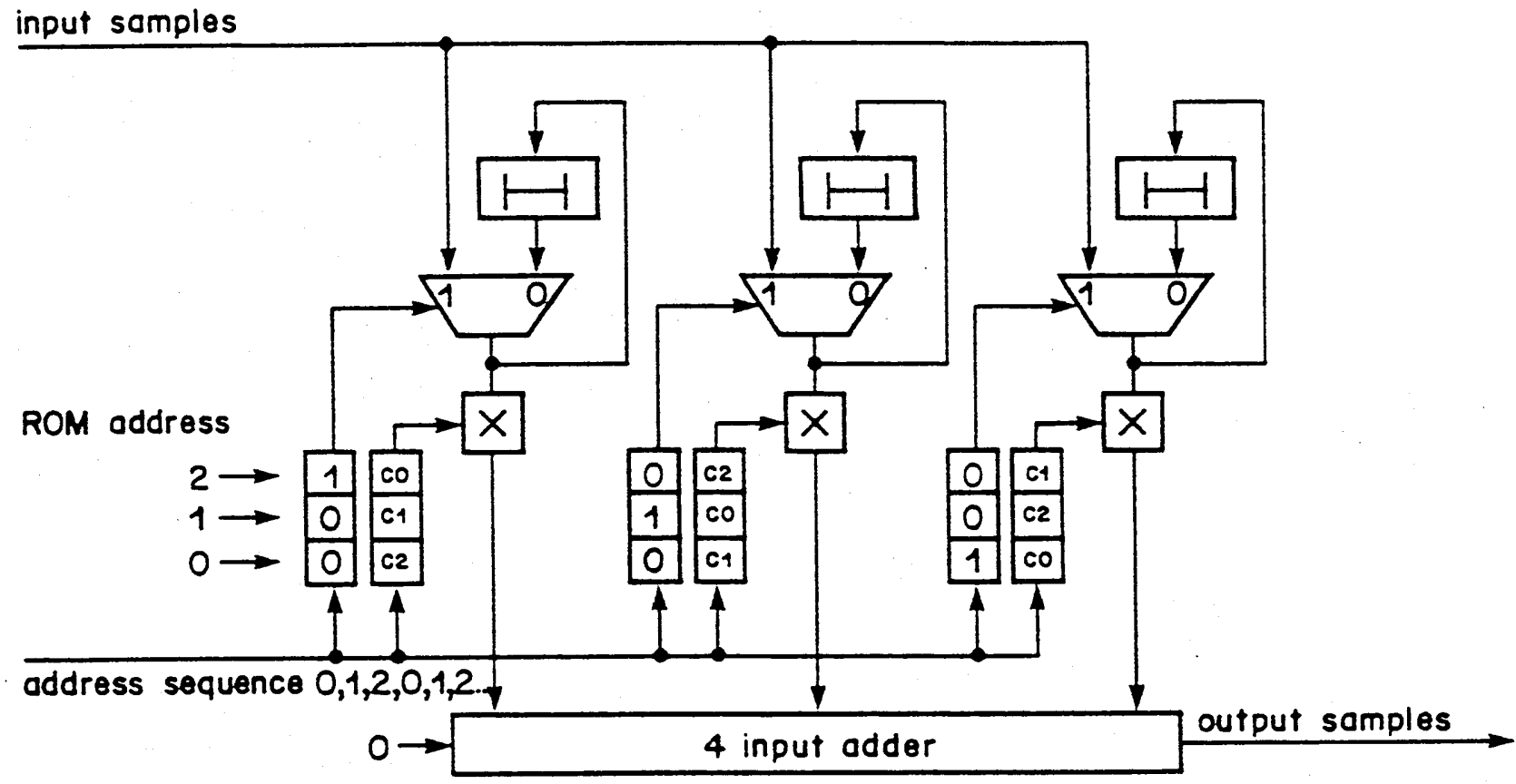

Figure 4.3 : Non-pipelined FIR filter

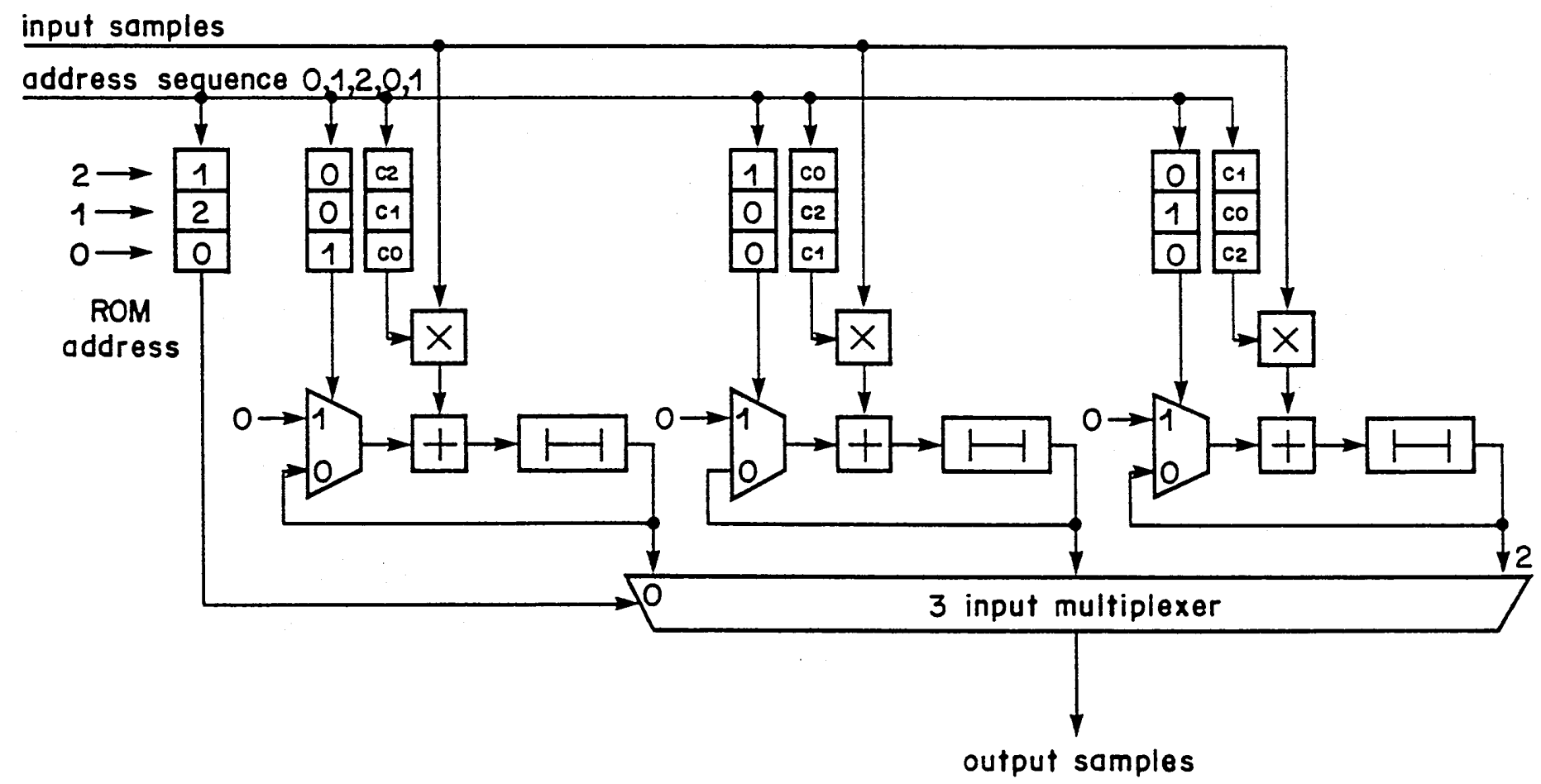

Figure 4.4 : Non-pipelined transposed FIR filter 


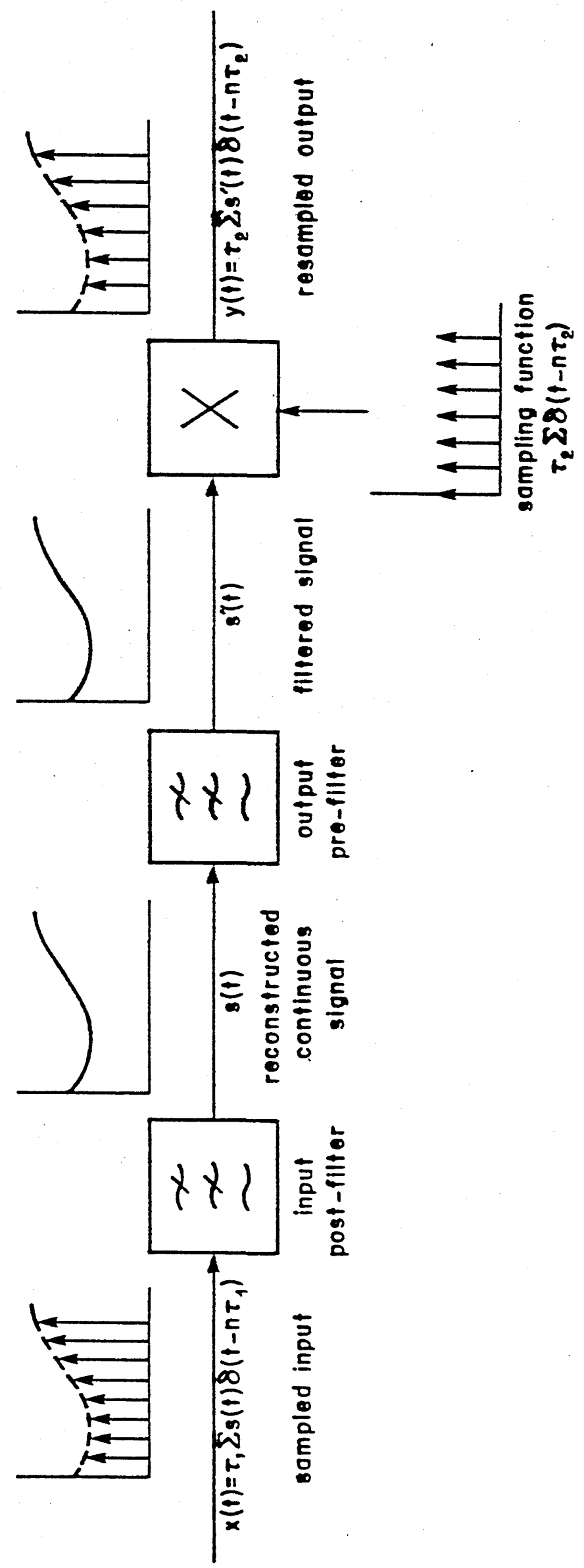

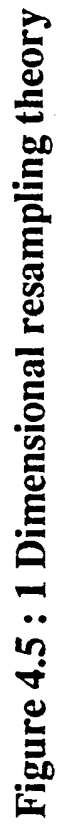




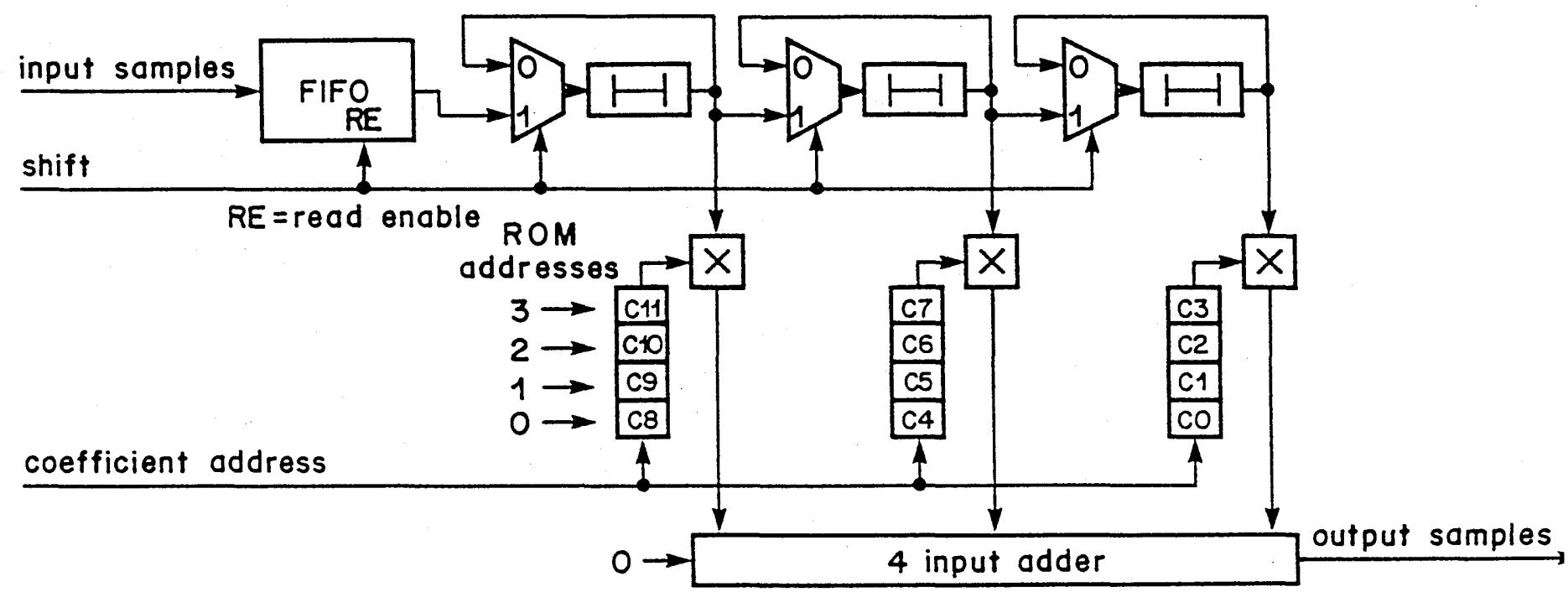

Figure 4.7 : Time varying input lattice filter

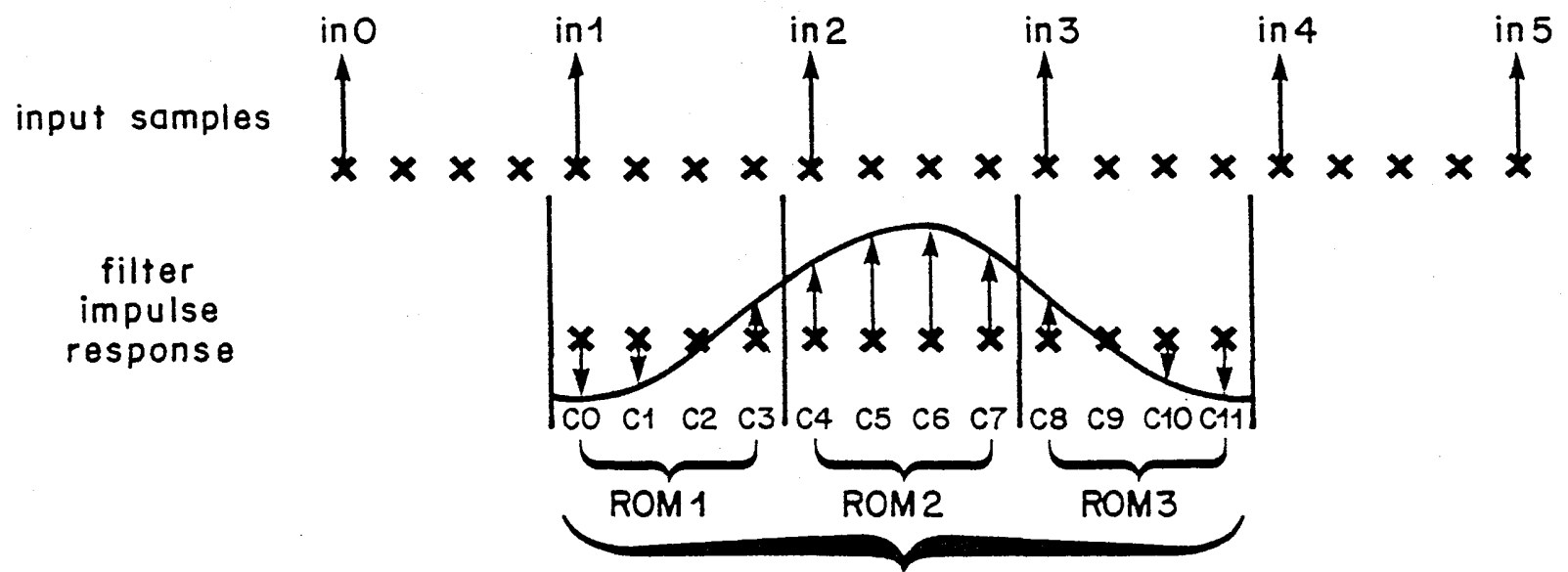

filter aperture

output samples

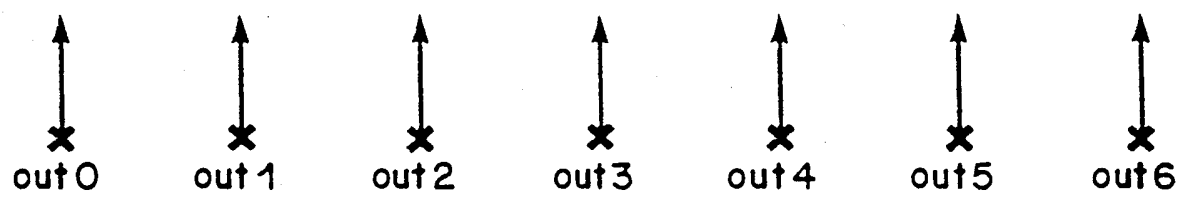

Figure 4.8 : Timing relationship between input and output samples for input lattice filter 


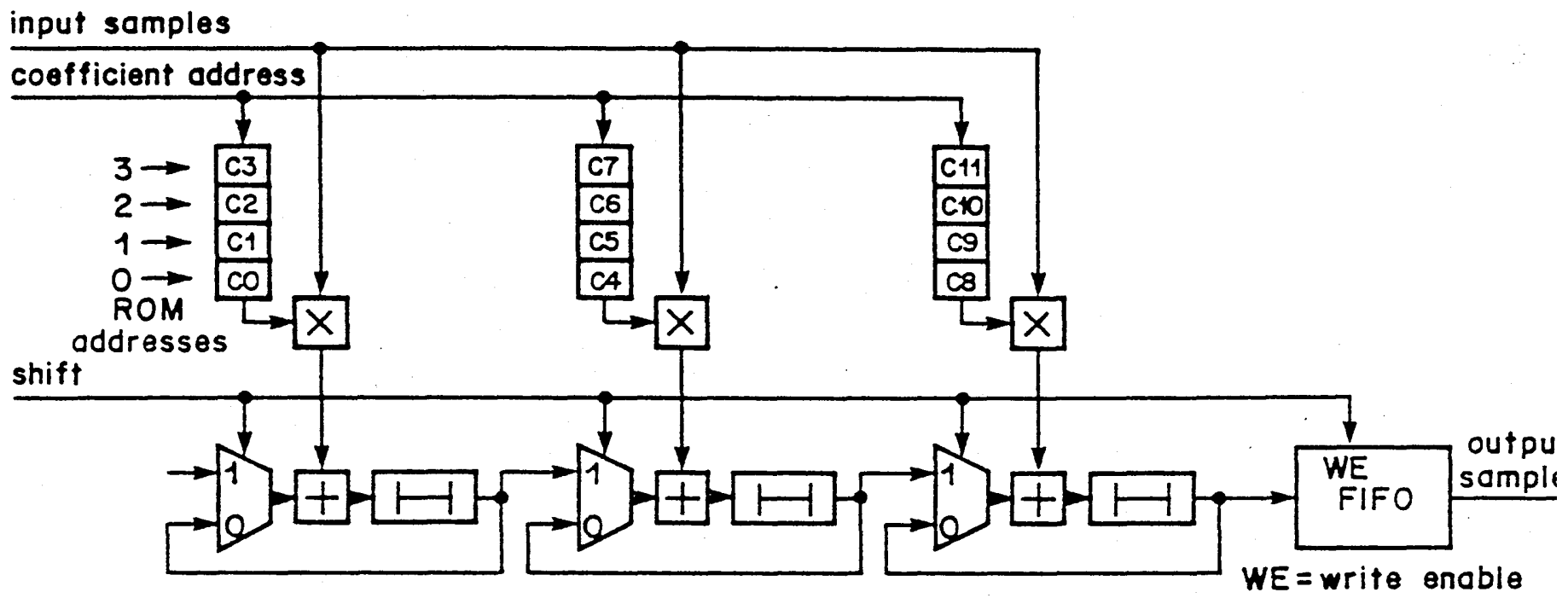

Figure 4.9 : Time varying output lattice filter

input samples

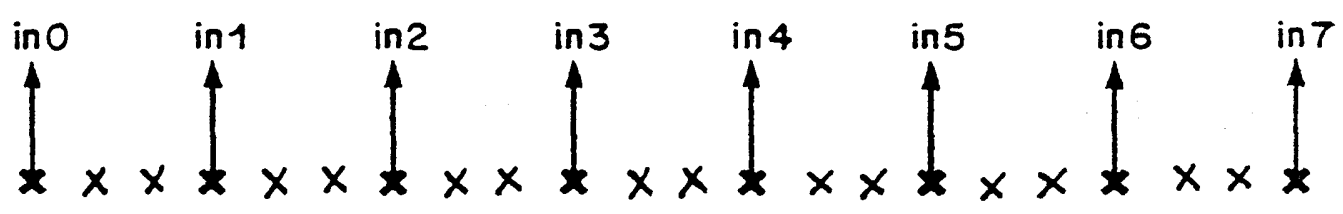

filter

impulse

response

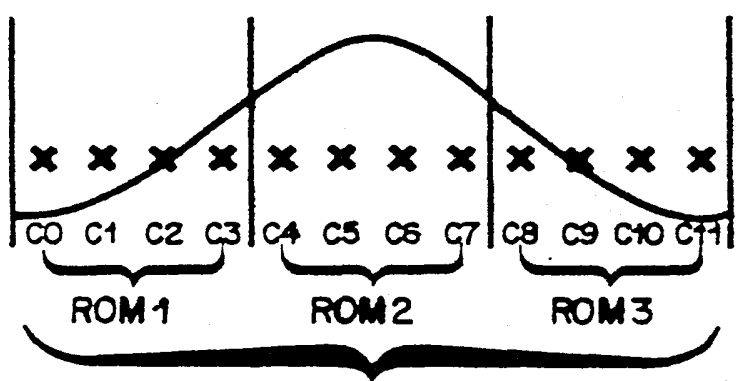

filter aperture
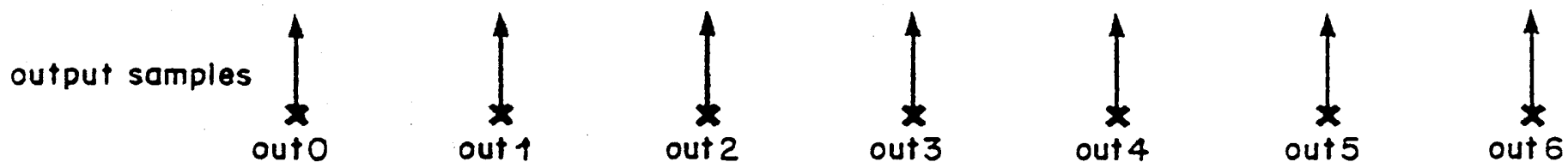

Figure 4.10 : Timing relationship between input and output samples for output lattice filter 


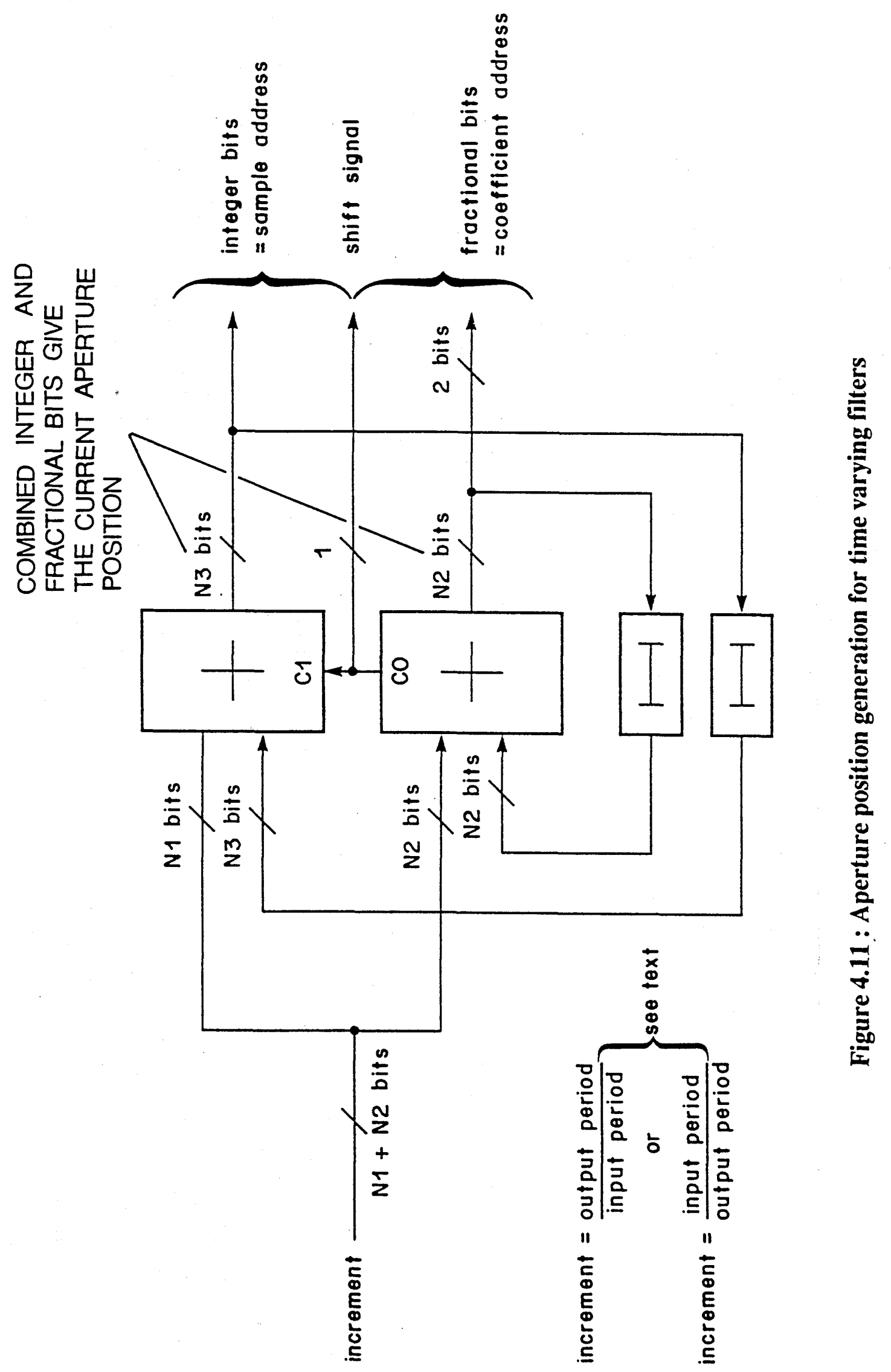




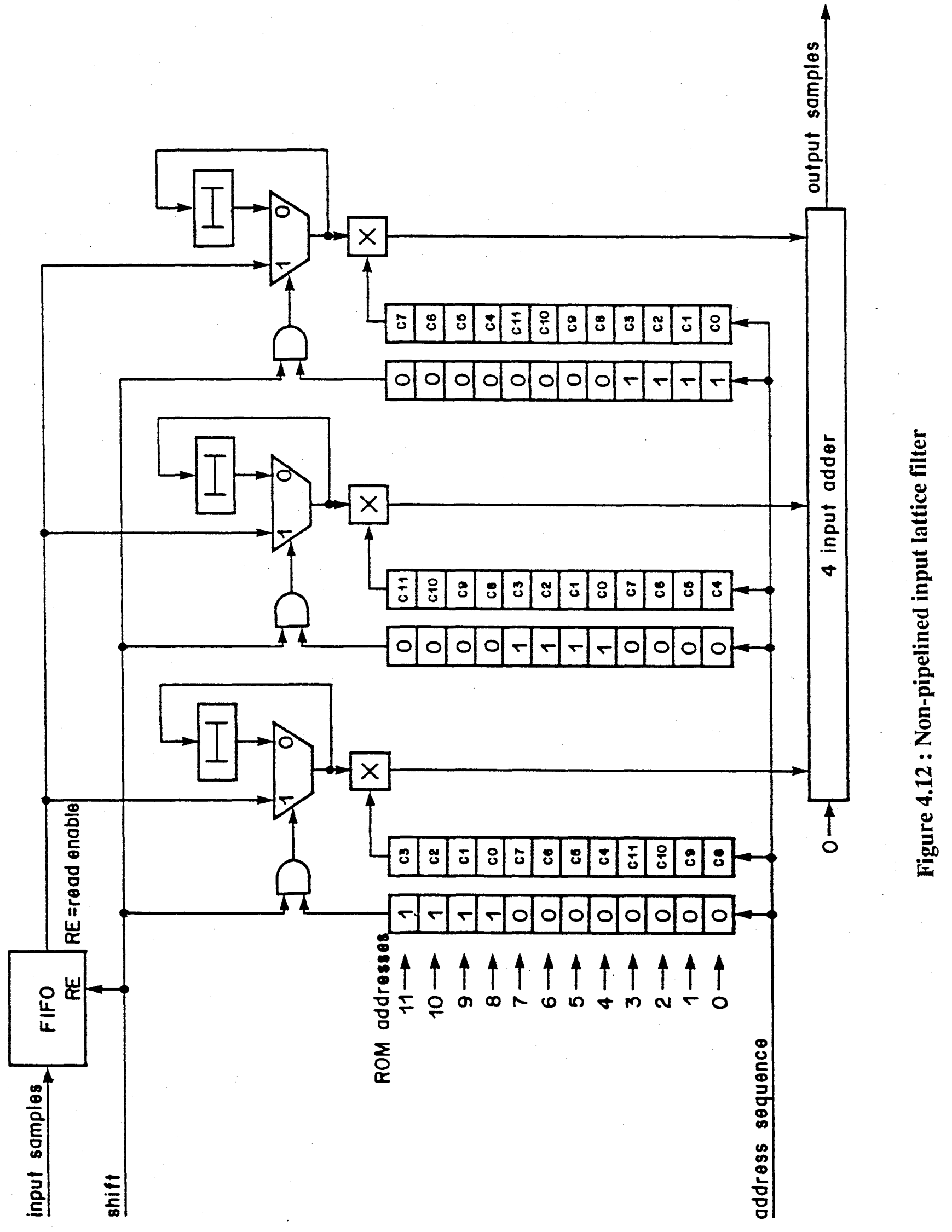


input samples

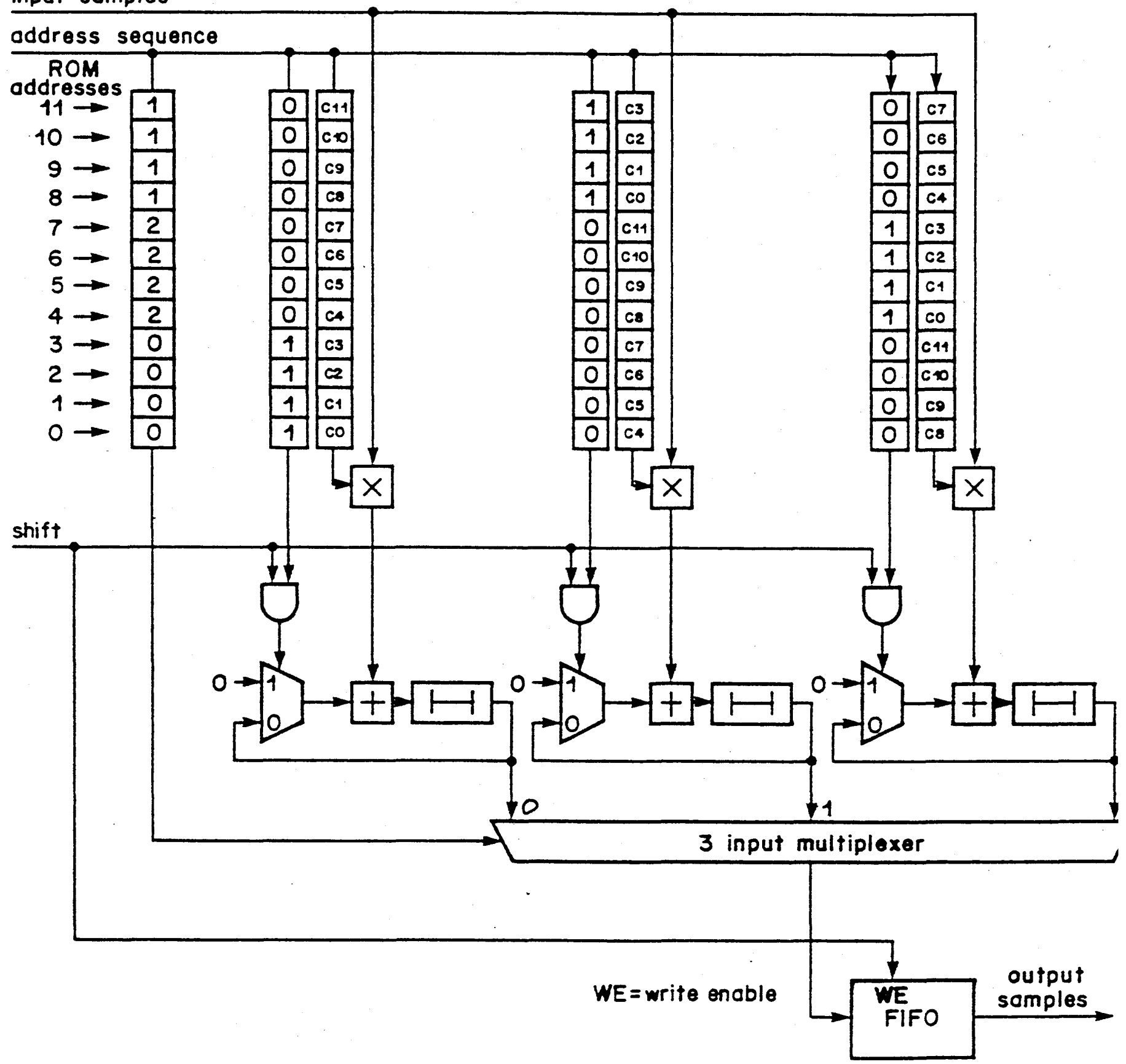

Figure 4.13 : Non-pipelined output lattice filter 

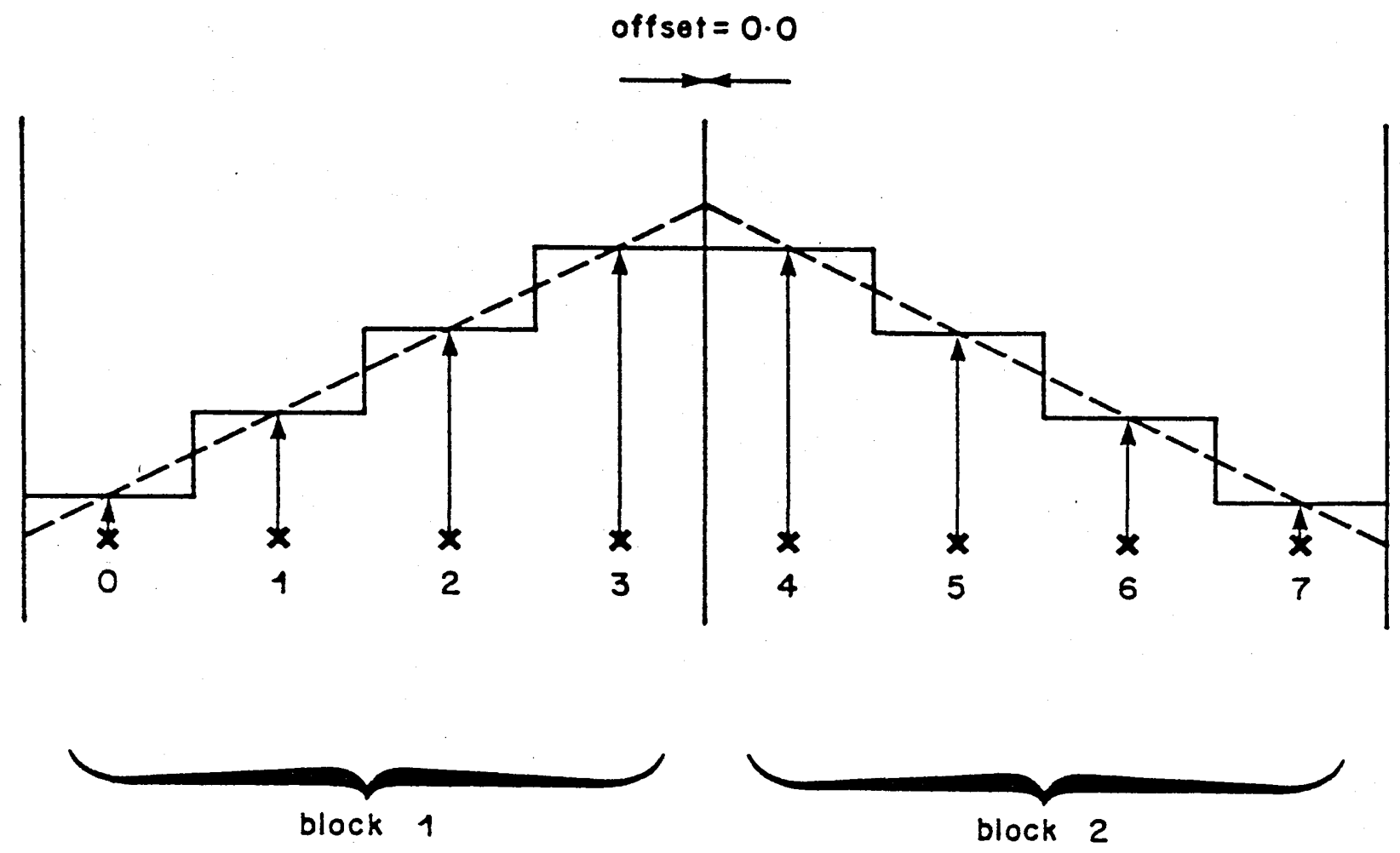

Figure 4.14a

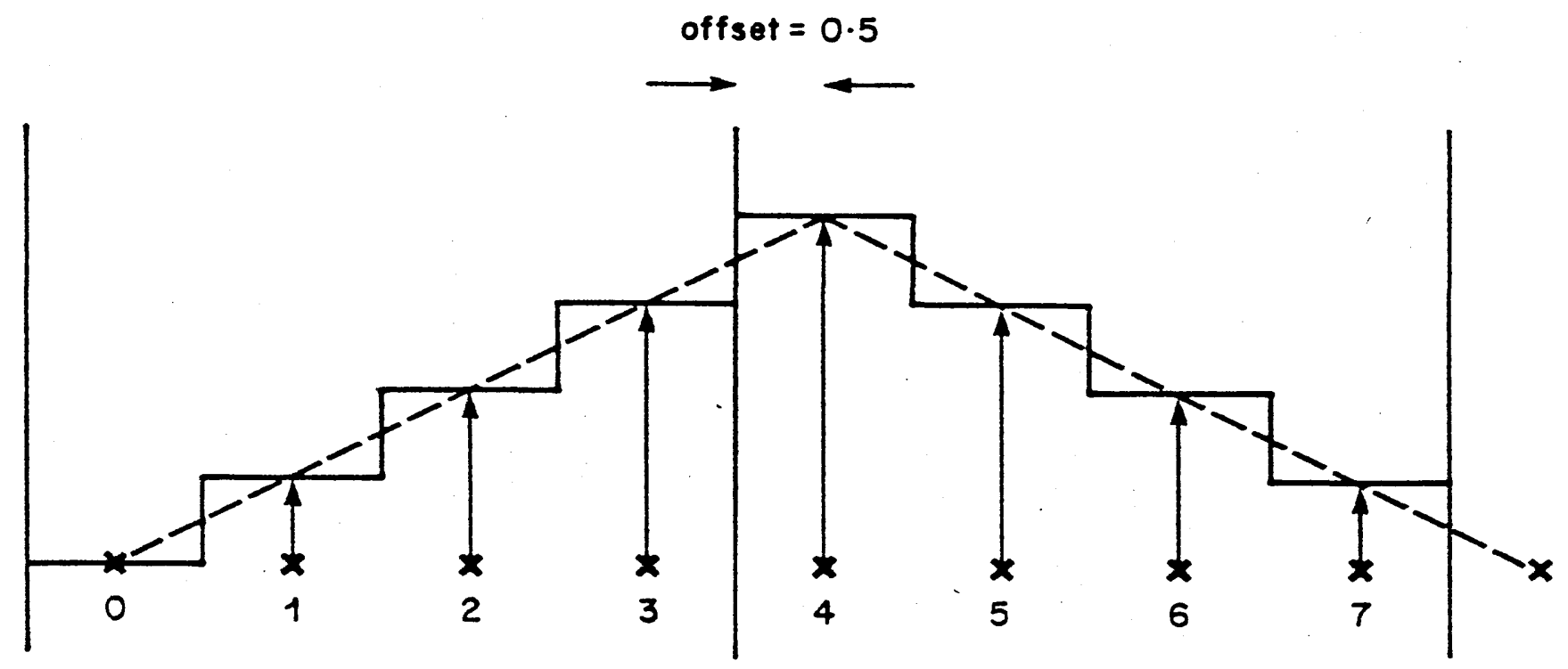

block 1

block 2

Figure 4.14b

Figure 4.14 : Examples of aperture quantisation 
Examples of aperture quantisation
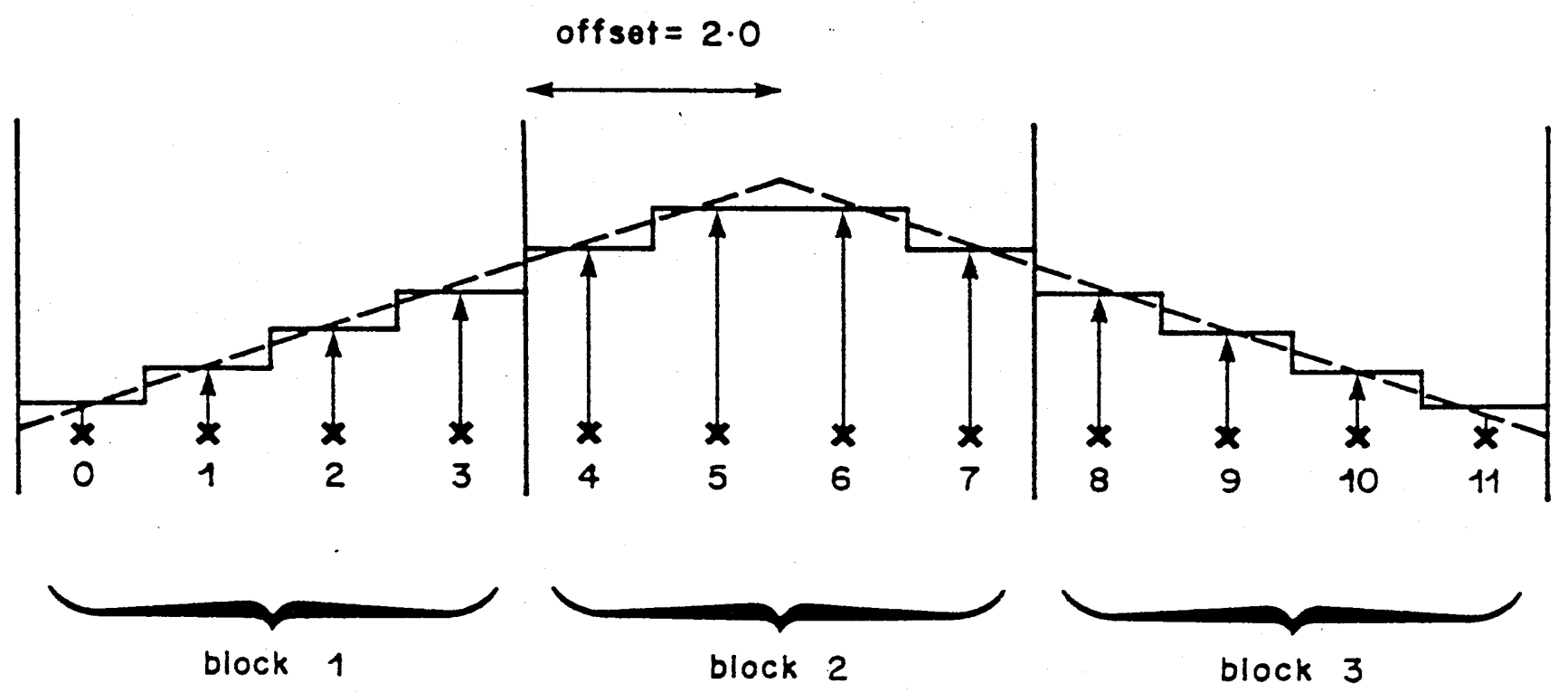

Figure 4.14c
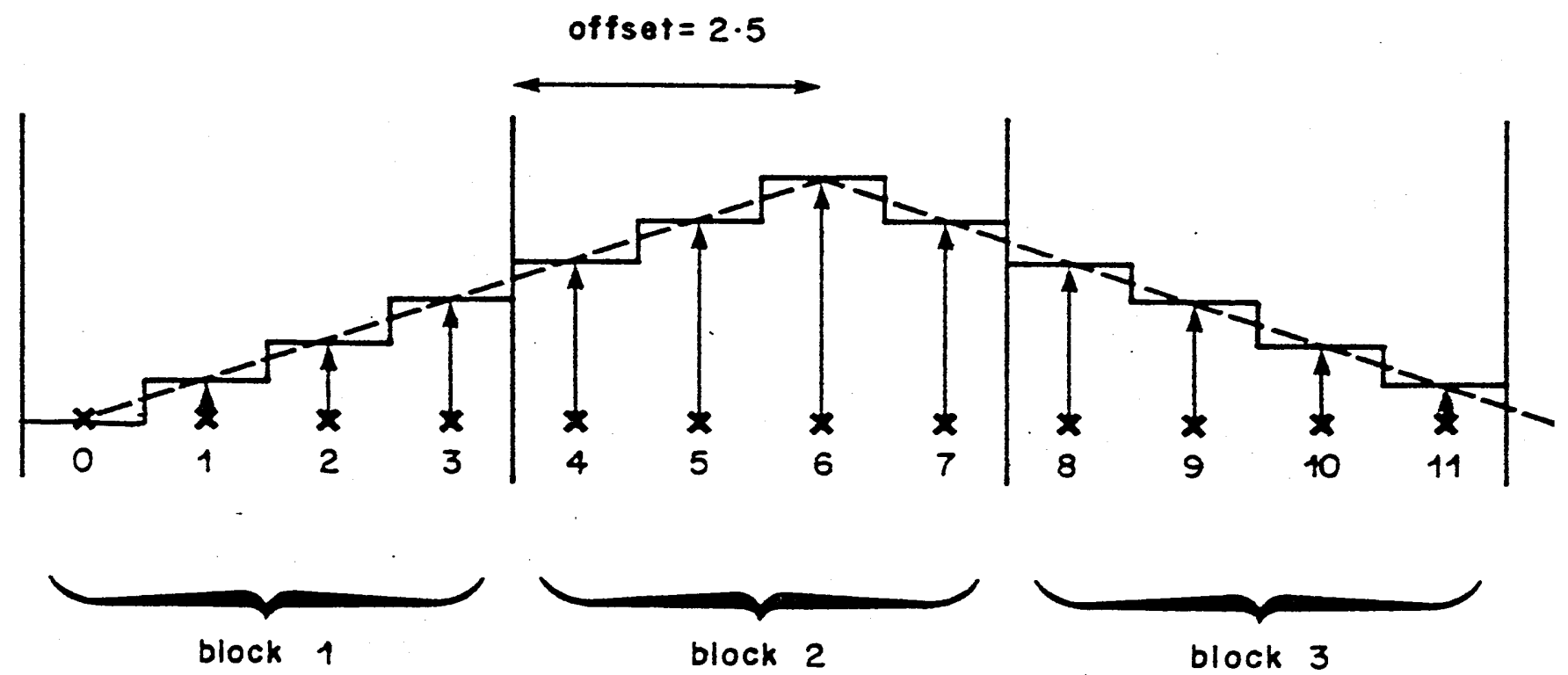

Figure 4.14d

Figure 4.14 : Examples of aperture quantisation 


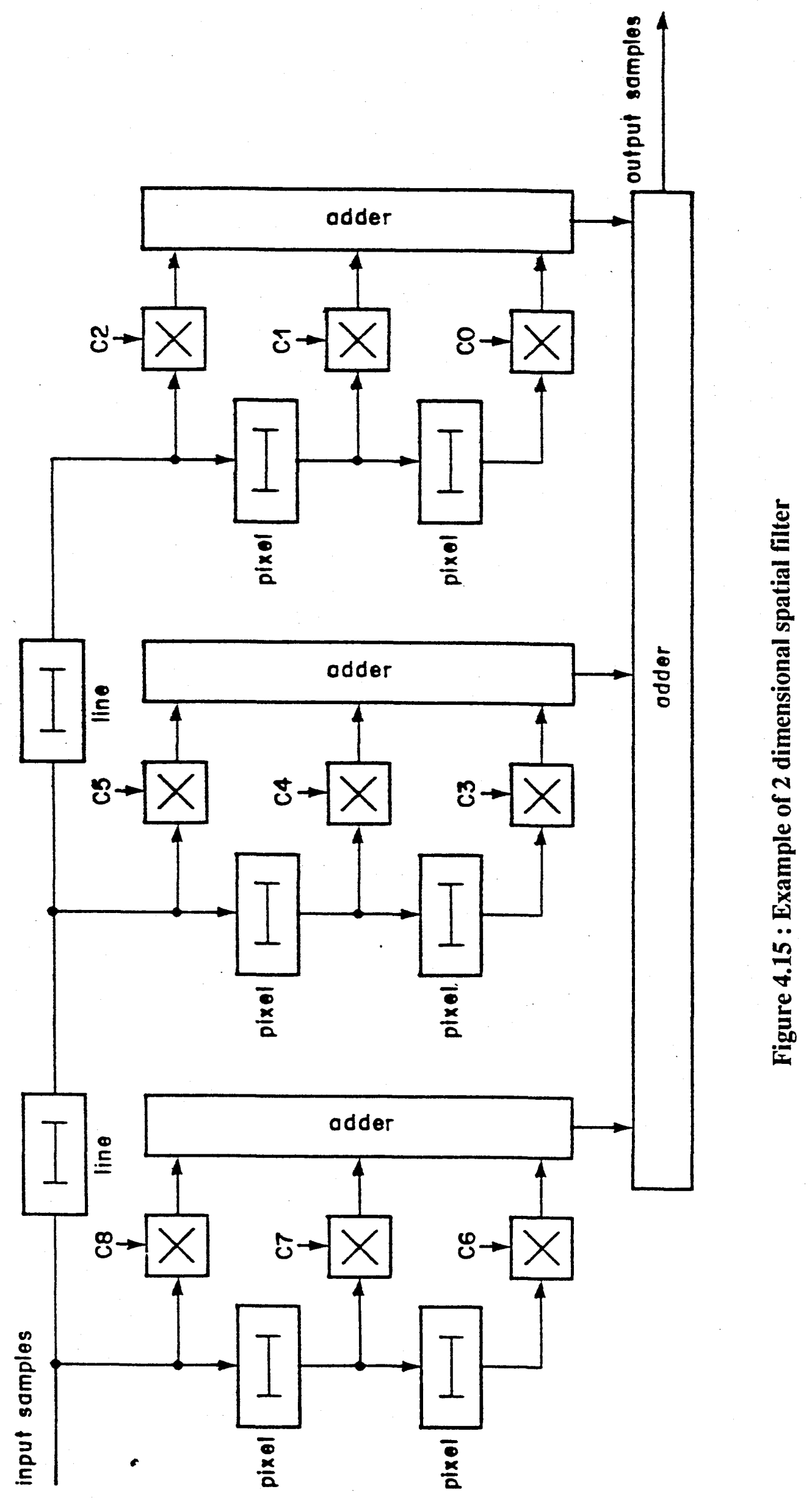




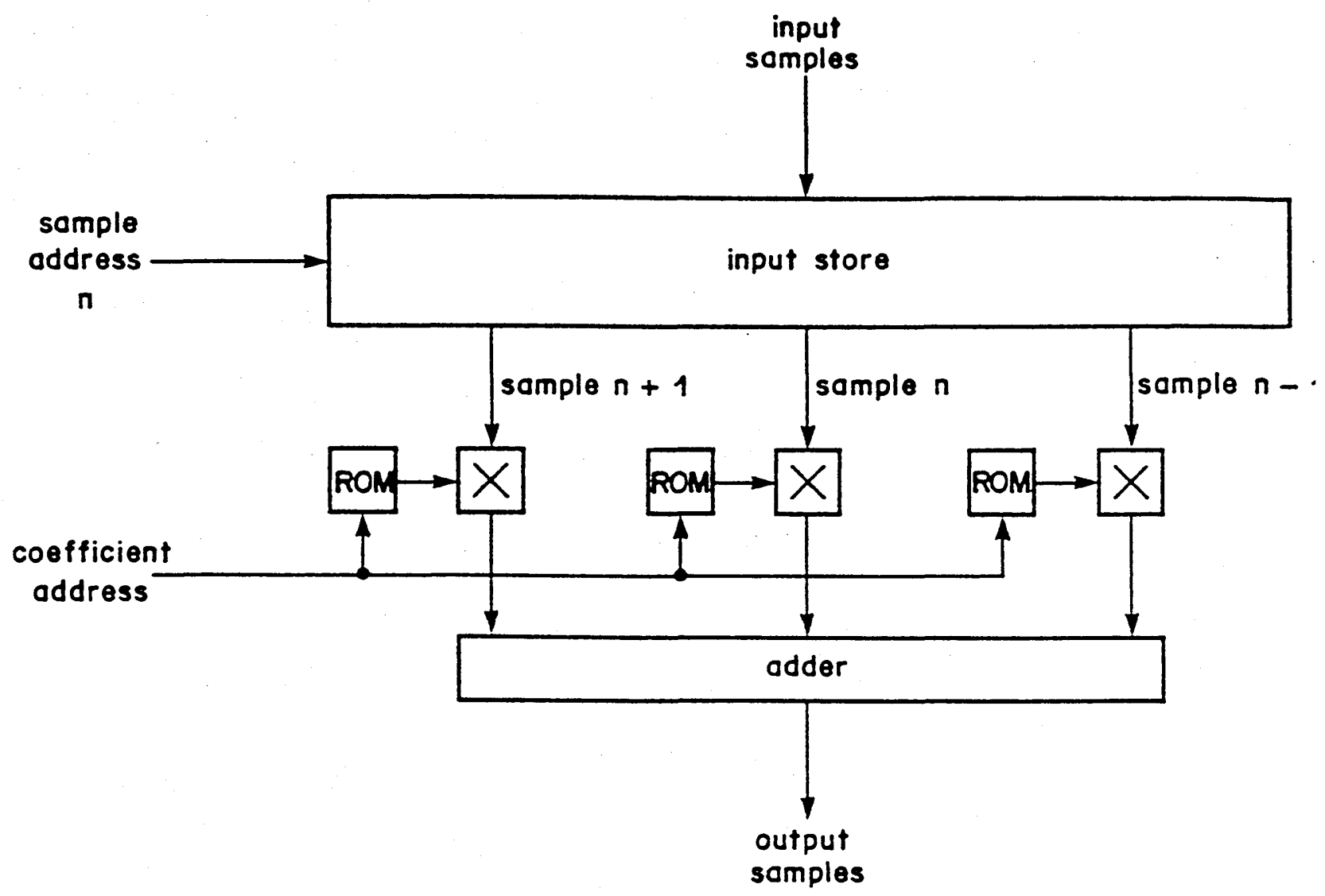

Figure 4.16 : Generalised input lattice filter

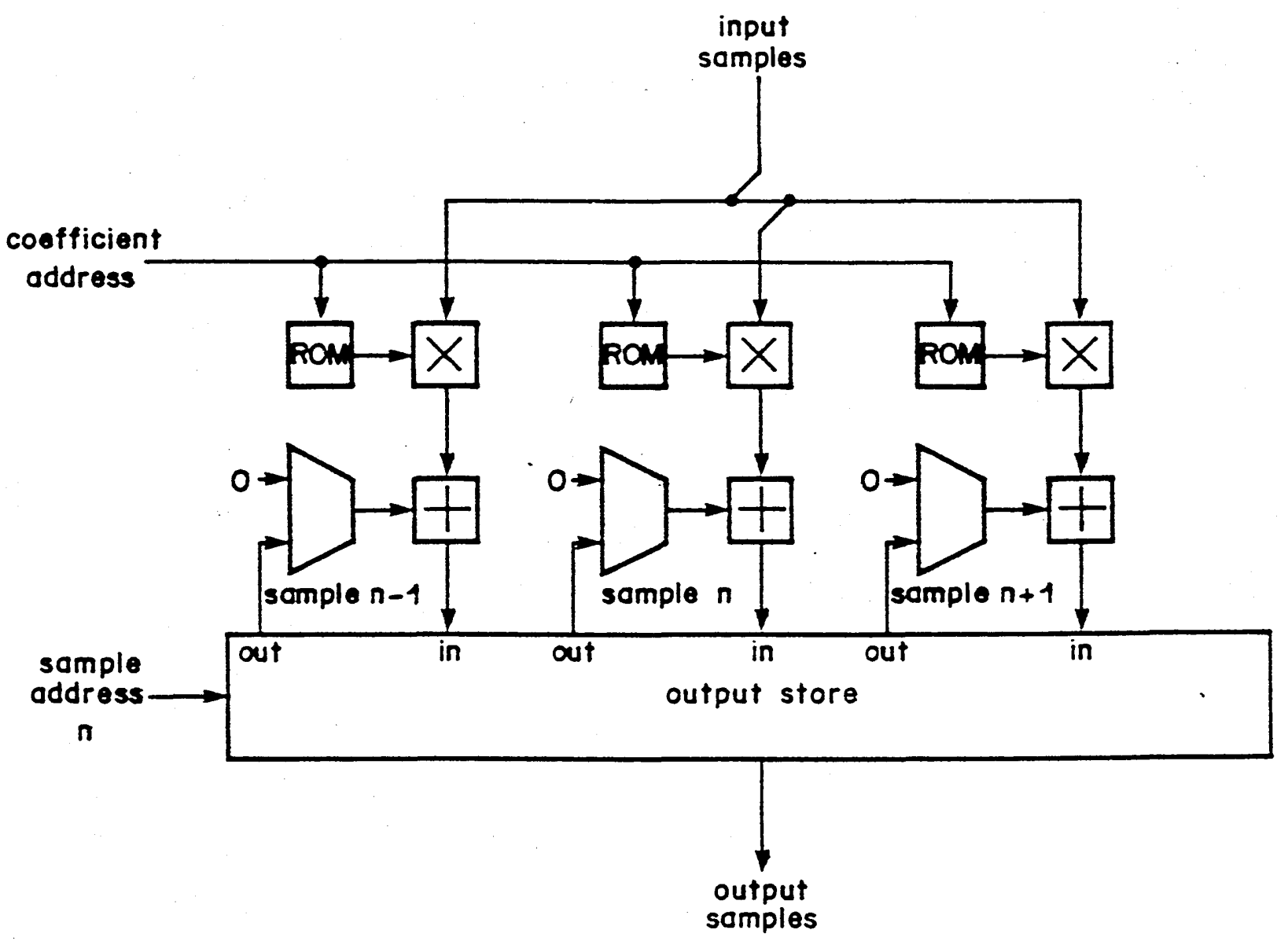

Figure 4.17 : Generalised ouput lattice filter 


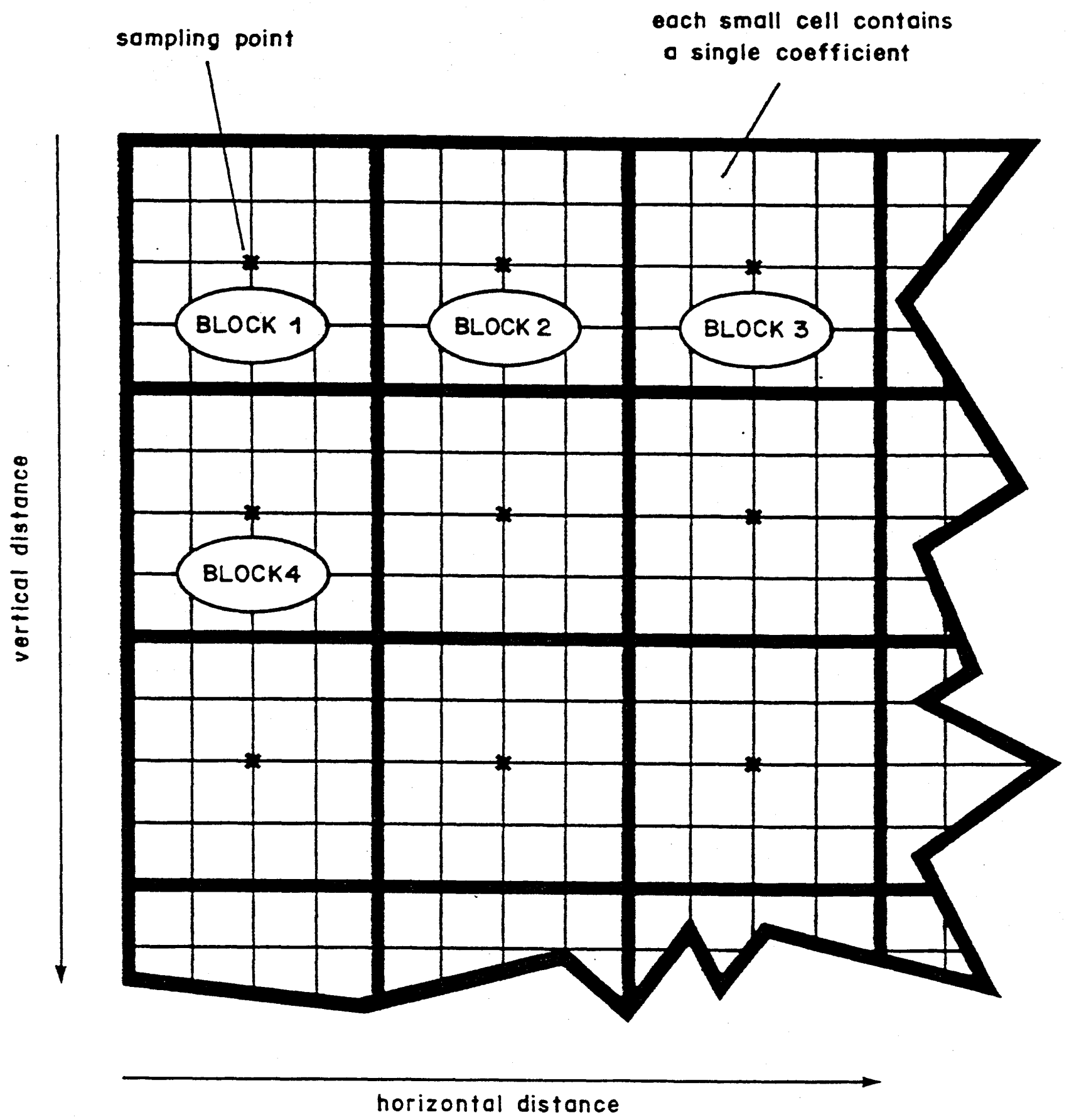

Figure 4.18 : Example segmentation of part of a 2D impulse response into coefficient blocks 

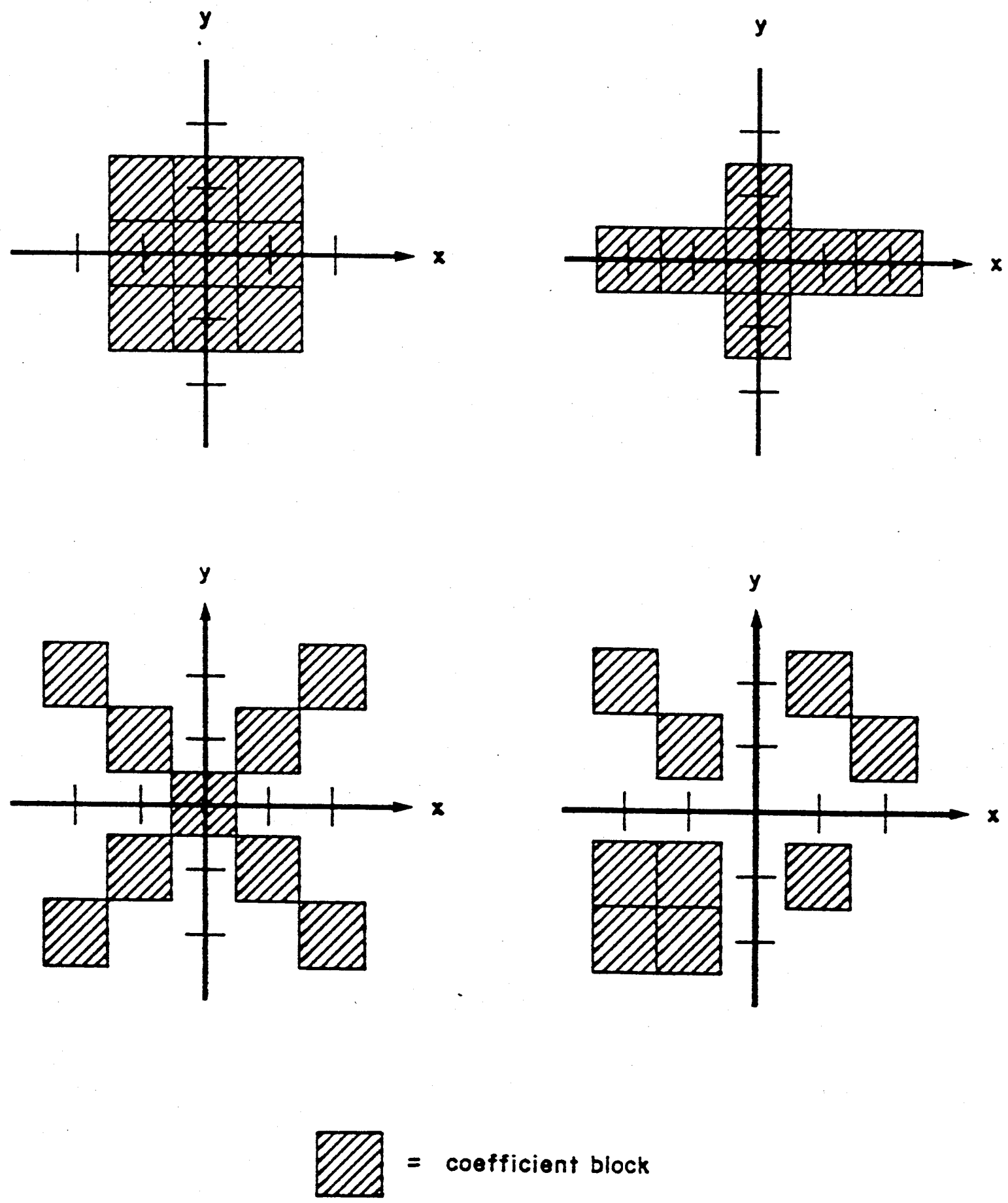

Figure 4.19 : Some possible coefficient block layouts 


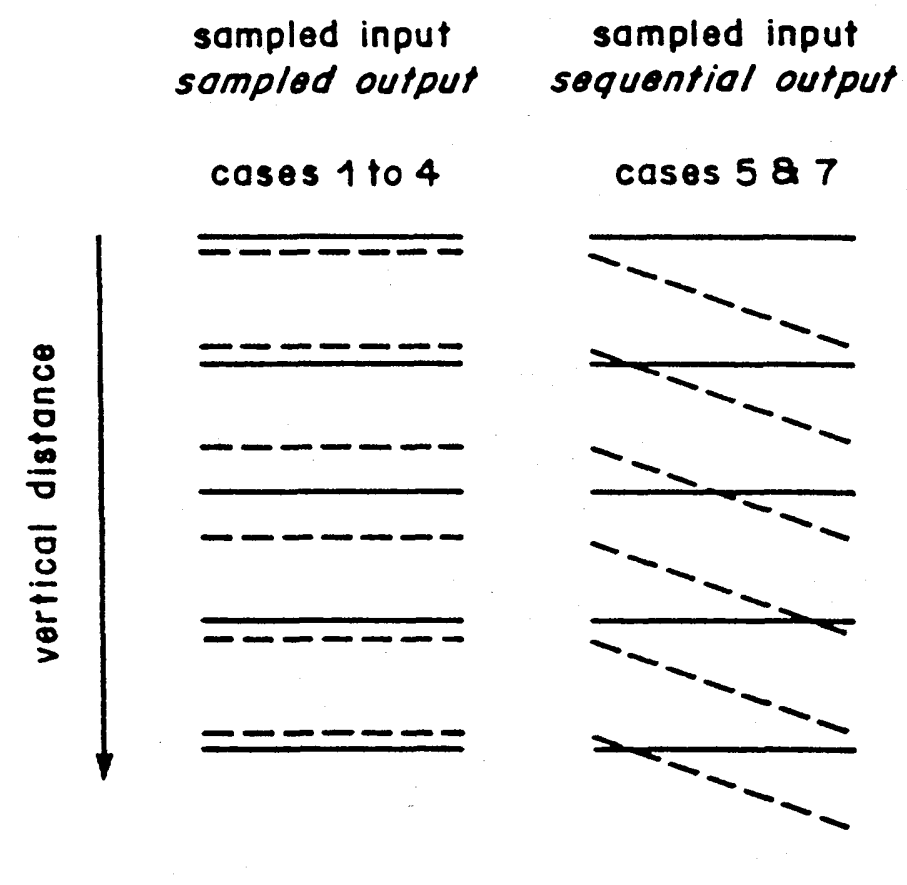

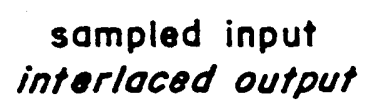

sequential input sompled output

horizontal distánce

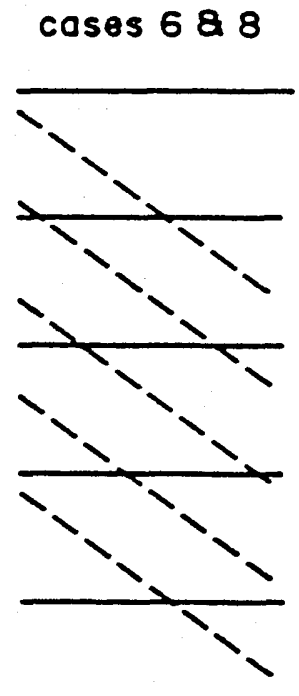

$\operatorname{coses} 9 \& 10$

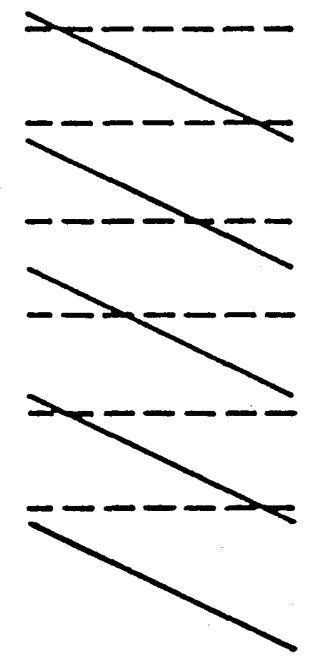

sequential input sequential input interlaced input sequential output interlaced output sompled output case 14

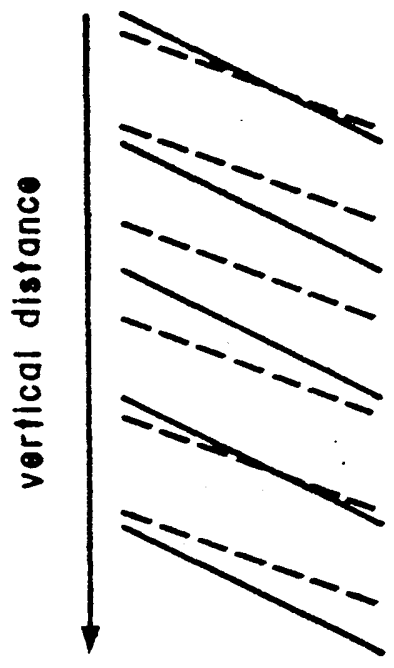

$\operatorname{coses} 11 \& 12$

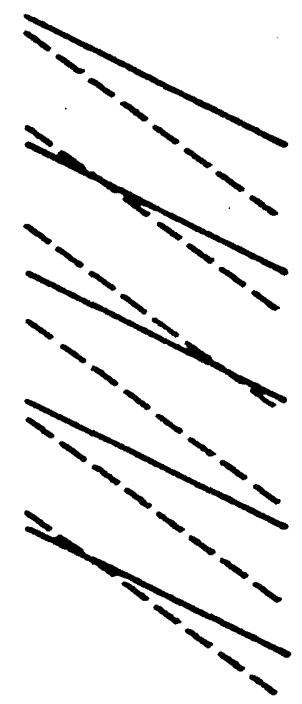

horizontal distance interlaced input interlaced input sequential output interlaced outpul 

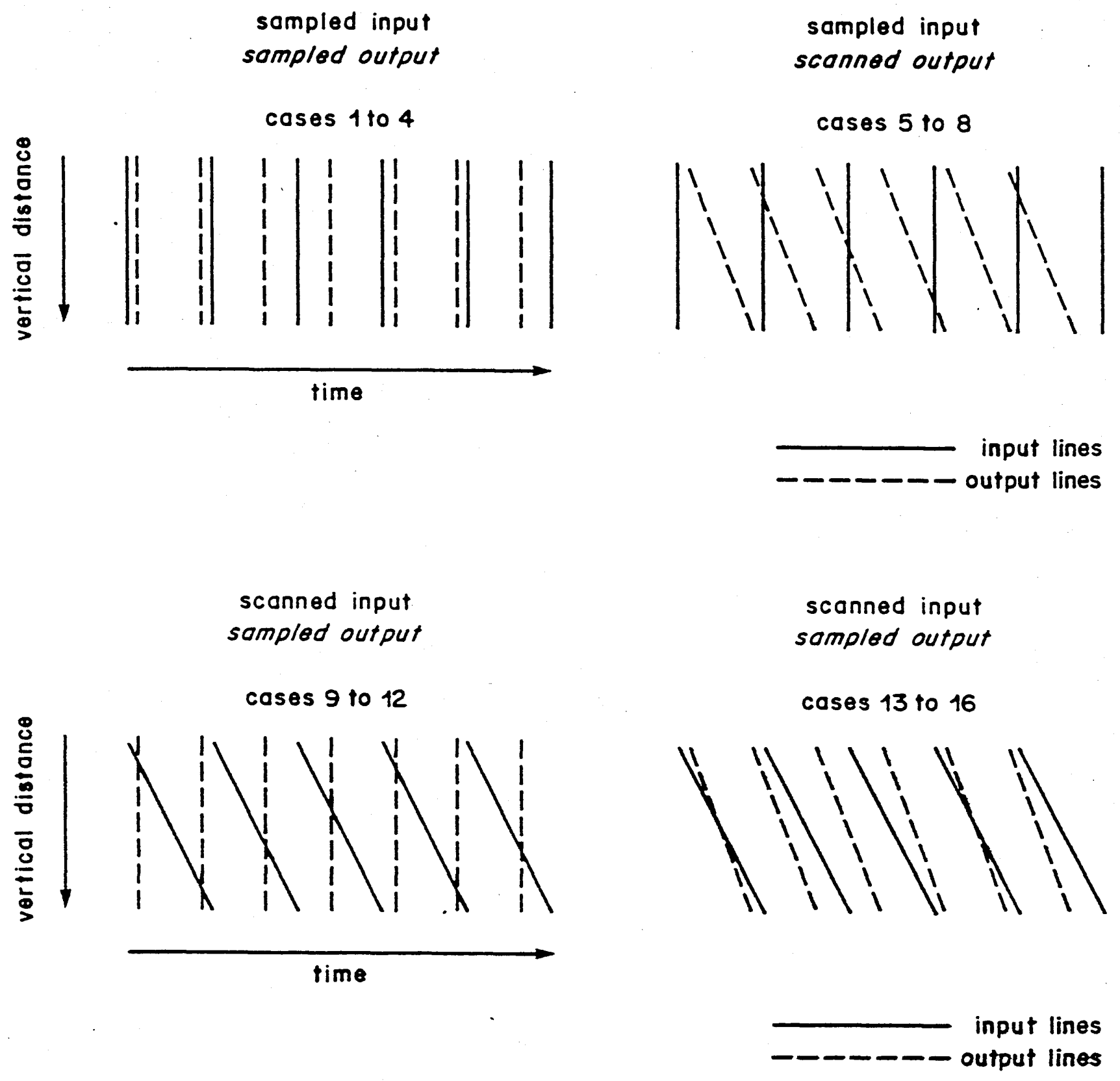

Figure 4.21 : Vertical/Temporal relationship between input and output scanning formats 

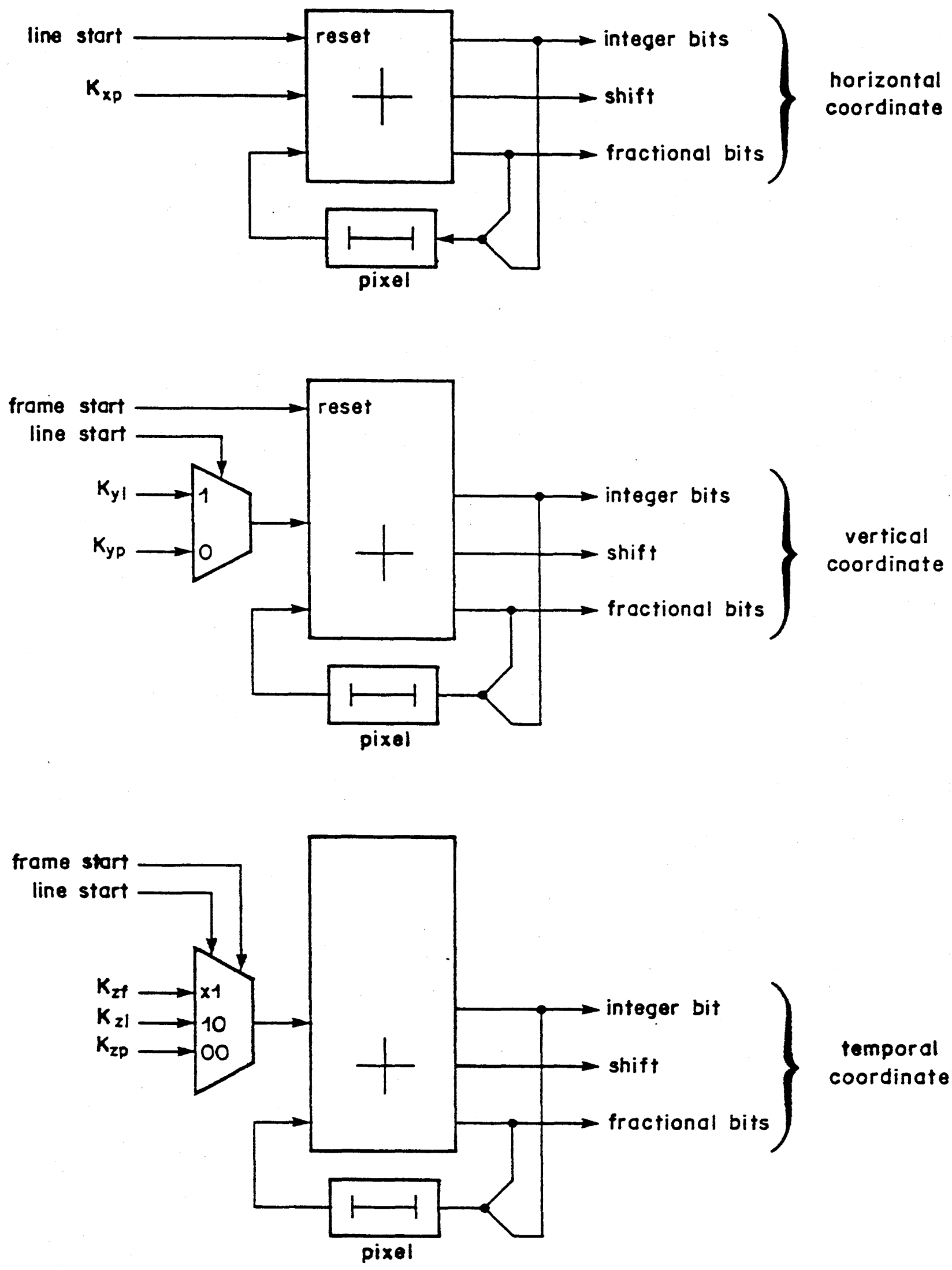

Figure 4.22 : Multidimensional aperture position generation 


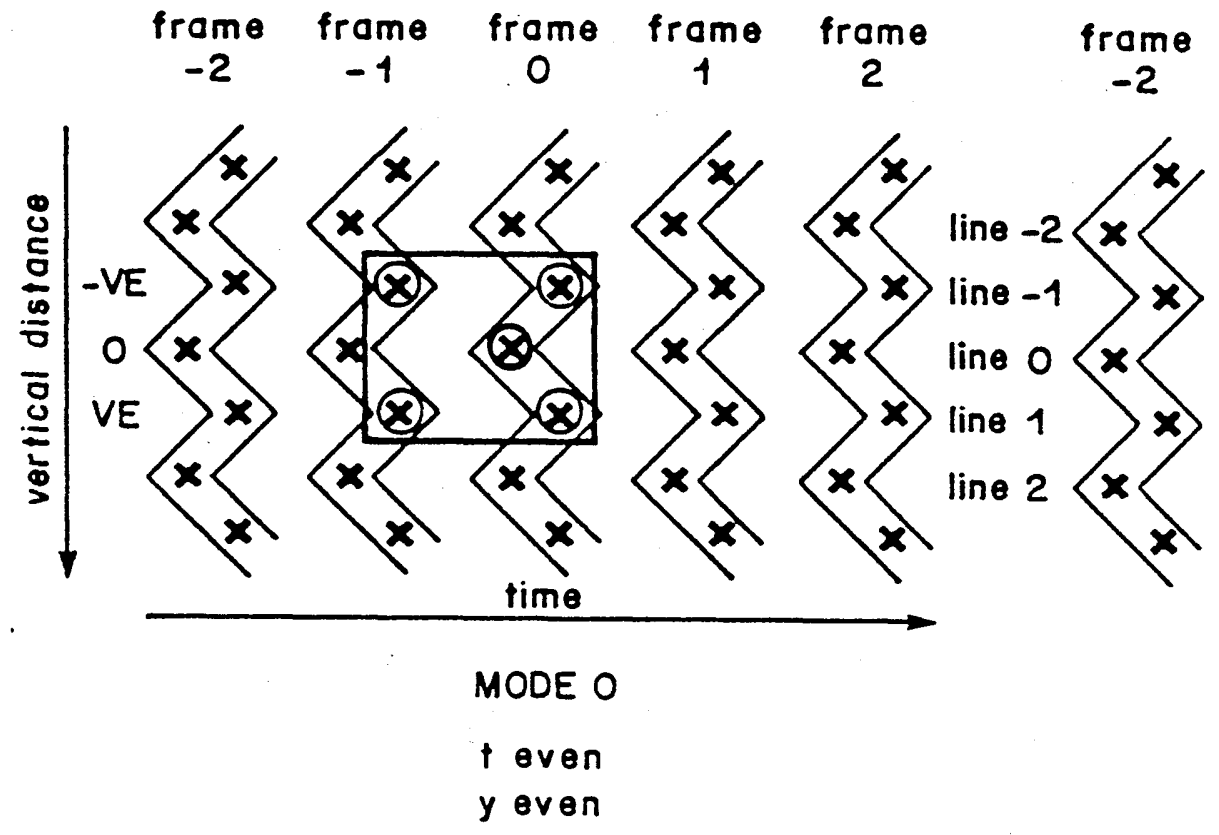

$\begin{array}{cccc}\text { frame } & \text { frame } & \text { frame } & \text { frame } \\ -1 & 0 & 1 & 2\end{array}$

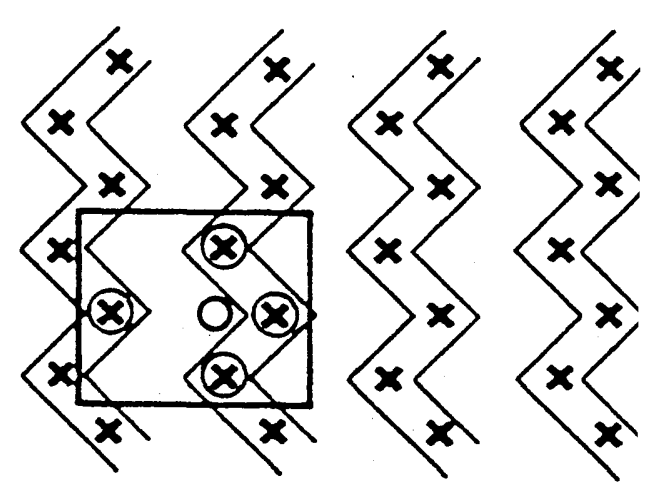

MODE 1

t even

$y$ odd

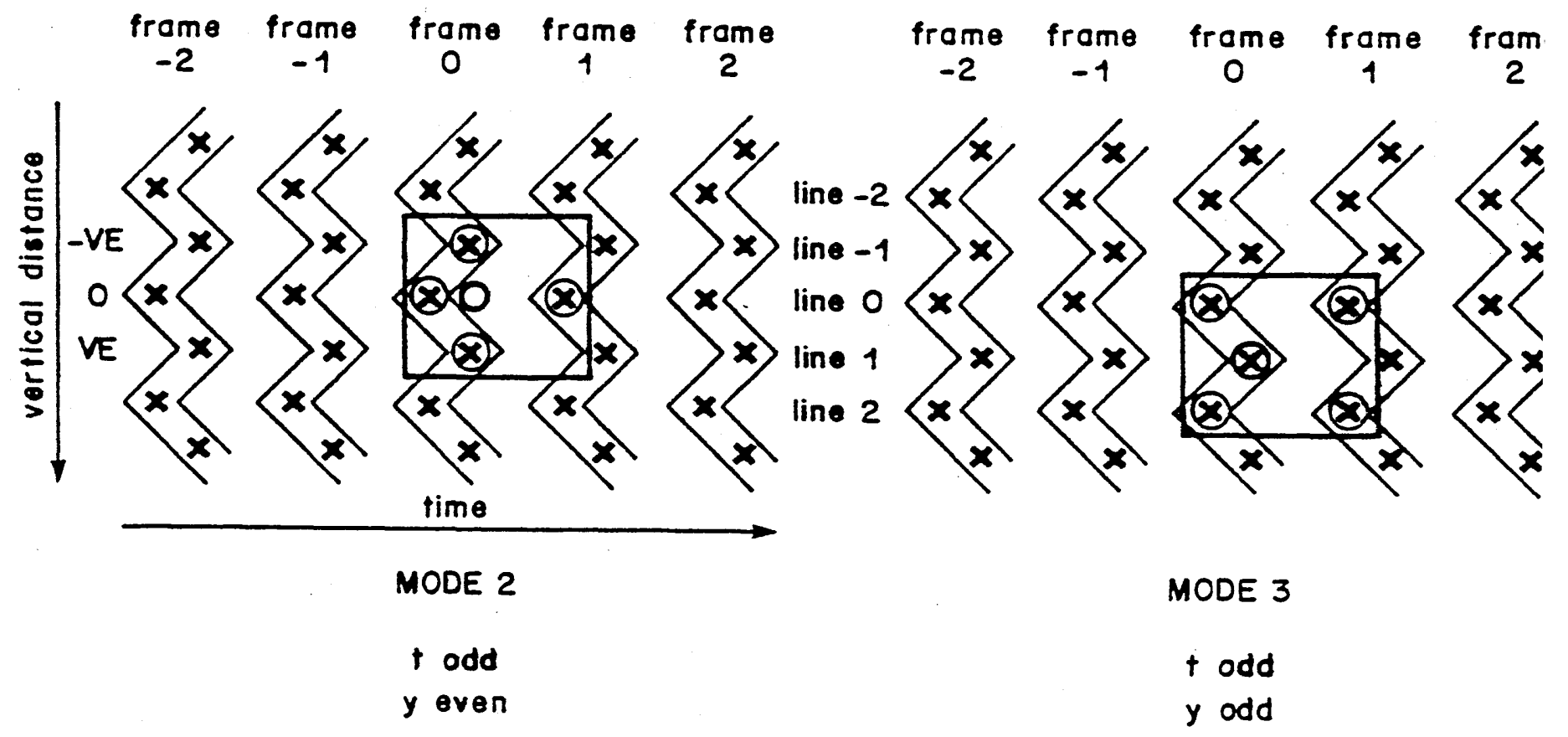

Figure 4.23 : The four subsets of filter coefficients required for filtering interlaced pictures 


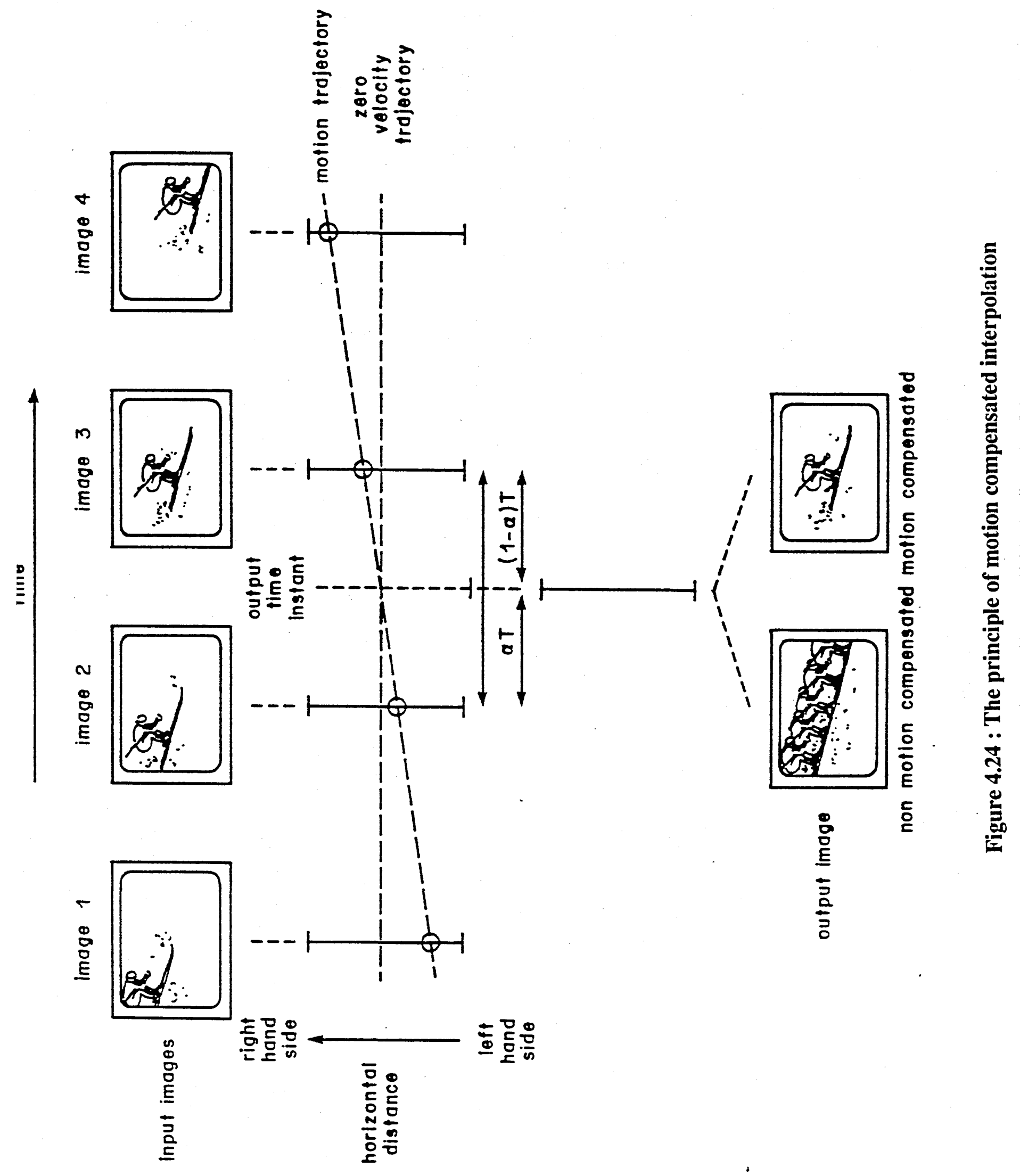




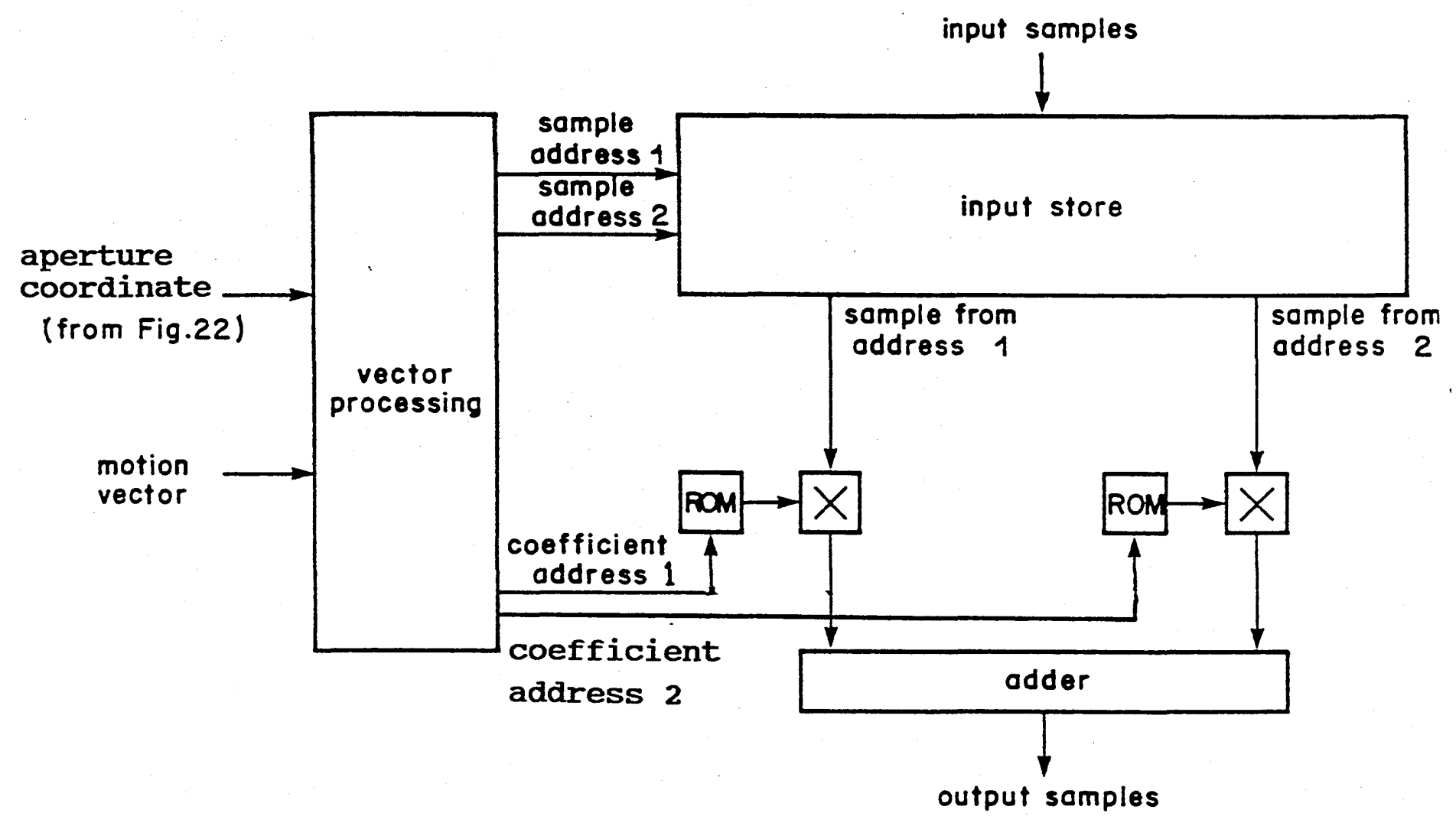

Figure 4.25 : Motion compensated input lattice filter

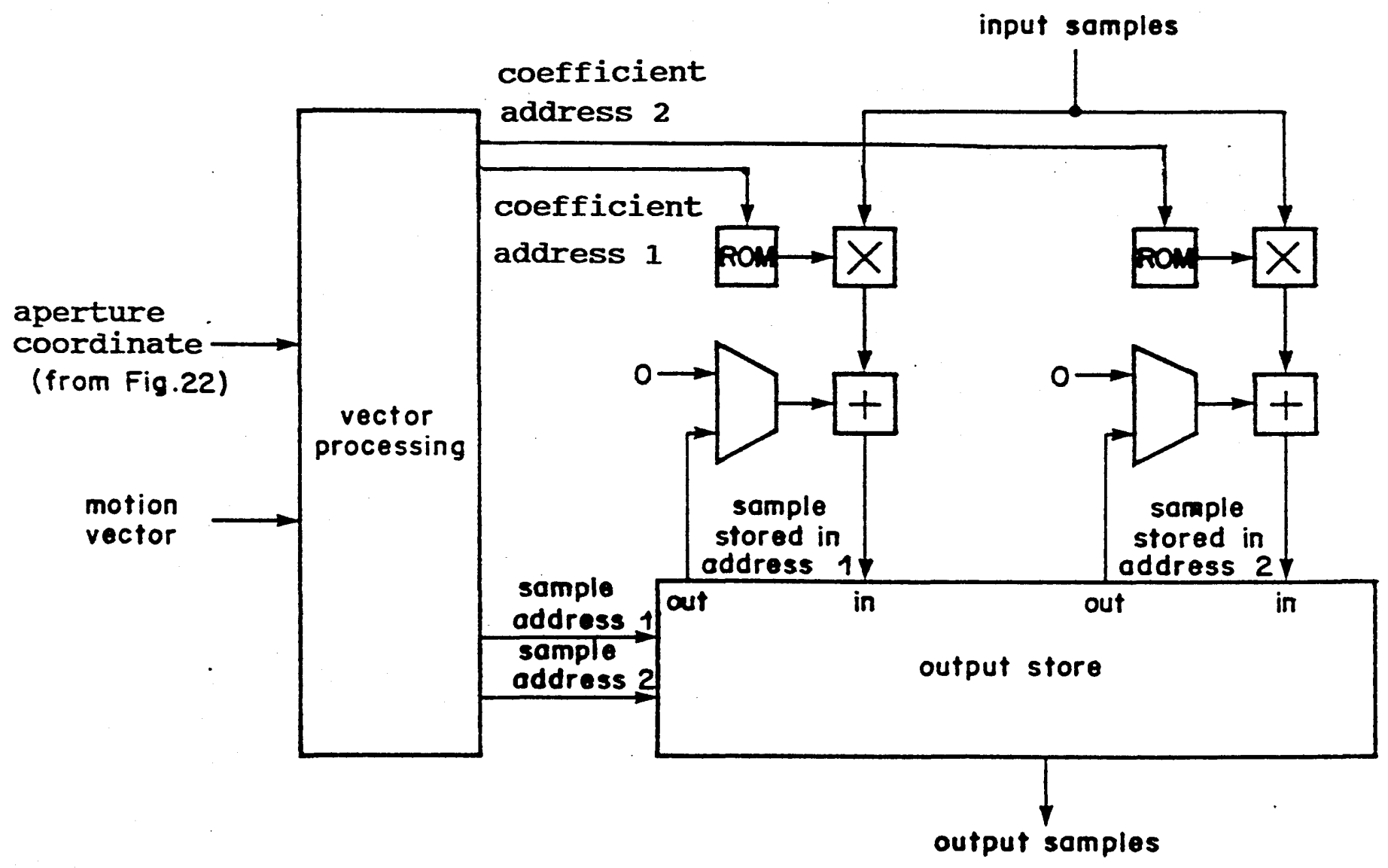

Figure 4.26 : Motion compensated output lattice filter 


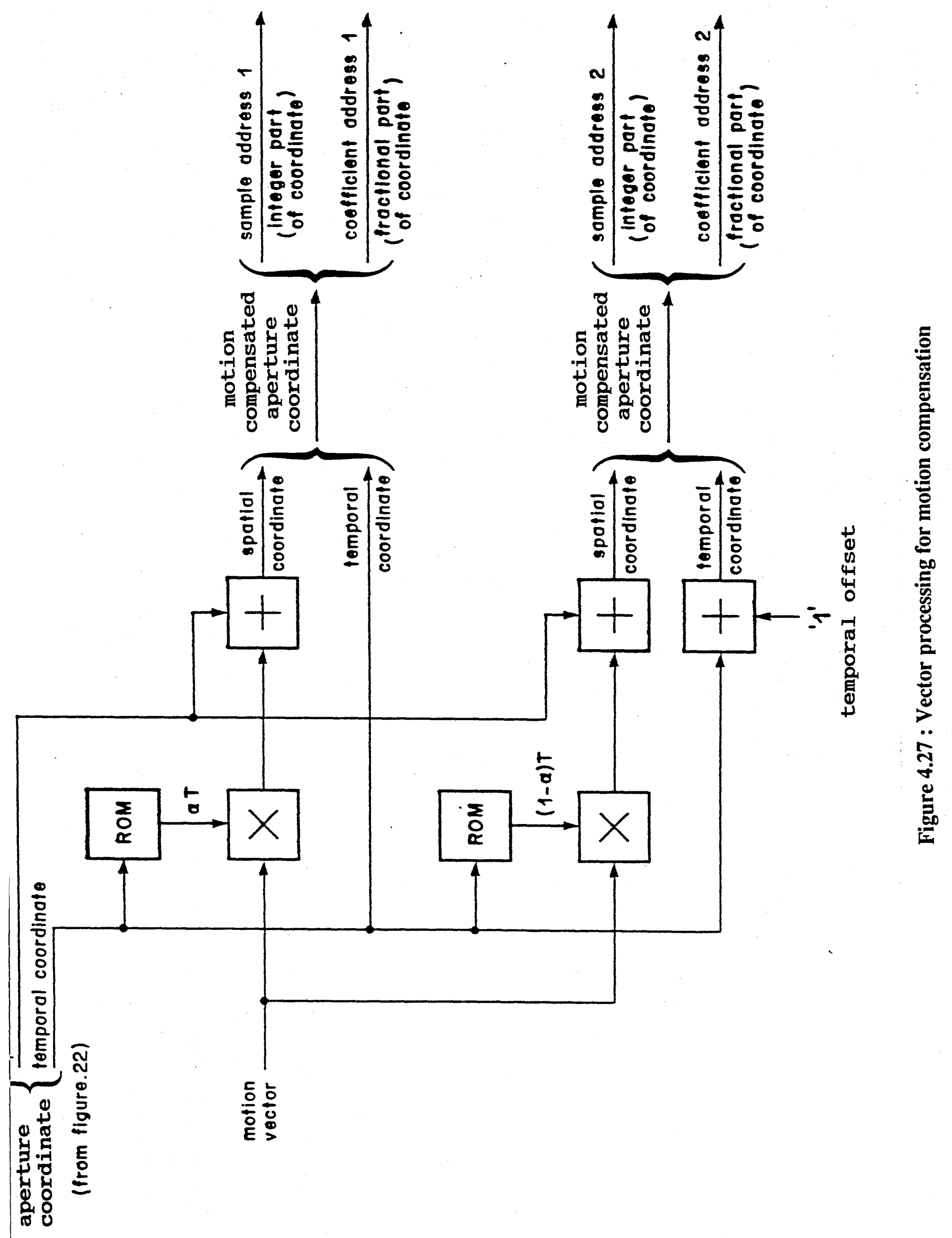




\section{The Performance of Conventional Standards Converters.}

\subsection{Introduction.}

This chapter describes the experimental implementation of two standards conversion processes. The first process is known as field rate upconversion. That is, increasing the field rate to improve the appearance of the displayed pictures. This particular investigation concerns doubling the field rate from $50 \mathrm{~Hz}$ to $100 \mathrm{~Hz}$. The second process is the interconversion between the television formats used in Europe and America.

The conversion processes described below use the same interpolation aperture for each pixel. That is, these are 'conventional' standards conversions and involve neither adaption nor motion compensation. The adjective 'conventional' is, perhaps, a little misleading. Although both processes have been investigated previously (see below) the approach to filter design used here is new.

The filters used in these experiments were designed using the techniques presented in chapter 3. The filters are approximations to the 'pseudo Wiener' filters described in section 3. The practical approximations used were obtained by the discrete least mean square approximation technique of section 3.4.1. For these examples the continuous least mean square and eigenfilter approaches would have given very similar results. Part of the purpose of this chapter is to compare the efficacy of these new filter design techniques to those which were used previously.

These experiments were performed using 'input lattice' filters as described in the previous chapter. That is, the picture samples were stored prior to, rather than after, interpolation and the interpolation aperture was defined in terms of the input, rather than output, sampling structure. Upconversion was performed by computer simulation whereas the European/American interconversion was performed using real time hardware.

\subsection{Field rate upconversion.}

\subsubsection{Introduction.}

This section describes an investigation into improving the display of television pictures by increasing the temporal sampling rate. In this investigation the field rate was doubled from an input field rate of $50 \mathrm{~Hz}$ (interlaced) to an output field rate of $100 \mathrm{~Hz}$ (interlaced). The processing was performed by computer simulation. That is, a sequence of images were captured in a computer store, processed by computer, and then displayed from the computer's memory. 
The primary objective of field rate upconversion is to reduce large area flicker by increasing the field rate. Large area flicker is the result of perceiving the field rate at which the pictures are displayed. It is apparent as a rapid flickering of the whole picture. In some viewing conditions large area flicker can be quite disturbing. Ideally flicker would be removed by the combined filtering action of the display and the human visual system (henceforth the 'eye'). Unfortunately both the display and the 'eye' have a significant response at the $50 \mathrm{~Hz}$ or $60 \mathrm{~Hz}$ field rates used for television display. The response of the display and the 'eye' decrease with frequency and so flicker is reduced by increasing the field rate. The field rate could be increased by broadcasters but this would require a profligate, uneconomic use of transmission bandwidth. Hence the only realistic option is to interpolate additional fields at the receiver.

When television broadcasts began receiver displays were small, quite dim and display defects were accepted as inevitable. Thirty years later displays are much larger and brighter. The effects of large area flicker are much more apparent on large, bright displays. This is firstly because flicker is perceived more in peripheral vision and is thus more obvious in larger displays. Secondly the temporal frequency response of the 'eye' increases with brightness at higher frequencies (eg Wentworth 1955). The trend towards large, bright displays is continuing with the development of HDTV and computer displays. Hence field rate upconversion will become increasingly important in the future.

Various techniques have been tried previously for performing field rate upconversion (eg Roberts 1983, 1985 and Parker \& van der Polder 1984). Typically such techniques involve repeating whole frames or fields. In either case vertical (intra field/frame) interpolation may be used to allow for interlace by correcting the vertical position of the pictures. Such conventional linear and adaptive techniques for upconversion do not preserve both spatial resolution and motion fidelity. For example, picture repeat preserves spatial detail but can only be used for stationary images because of its poor motion portrayal. Field repeat, by contrast, has good motion fidelity but worse spatial resolution. Adaptive methods, switching between these techniques, have been used to try to achieve the best of both worlds. However this has met with only limited success for the reasons discussed in section 3.5.

\subsubsection{Filter Design.}

Field rate upconversion is a more complicated standards conversion than might at first appear. Although in this case we are simply doubling the field rate to $100 \mathrm{~Hz}$, the presence of interlace complicates the alias structure making interpolation more difficult.

The alias structure for $50 \mathrm{~Hz}$ interlace to $100 \mathrm{~Hz}$ interlace conversion is shown in figure 5.1. The crosses represent the centres of spectral repeats caused by the input sampling lattice. The circles represent repeats of the response of the eye due to the output sampling lattice. 
Aliases of the response of the eye are significant because they cause high frequencies in the signal to be perceived as low frequencies.

The sharp cut filters customarily used for sample rate changing are inappropriate for television signals because of aliasing in the input signal. The presence of aliasing means that the filters used must take a more complicated shape. The interpolation filter used for upconversion introduces two impairments to an aliased signal. Firstly filtering reduces the resolution of the interpolated signal. Secondly insufficient filtering will pass unwanted alias components. The objective of the filter design is to reach a good compromise between loss of resolution and aliasing.

The improved filter design techniques, described in chapter 3, were used to try to enhance the performance of upconverters. The procedure is first to determine an 'ideal' filter response and then to generate a realisable approximation to it. The ideal filter response was taken to be the 'pseudo Weiner' filter derived in section 3.3. This simultaneously minimises the mean square loss of resolution and the mean square aliasing impairment. For simplicity it was assumed that the response of both the camera and the display were perfect. This is a reasonable approximation, particularly since both devices may incorporate compensation for losses ('aperture correction'). The scene spectrum used was 'model 2' from chapter 2. The response of the human visual system was interpolated from the data published by Kelly 1972.

Two filters are required in a standards conversion system. Firstly an input post filter is required to attenuate repeat spectra in the (sampled) input signal. Secondly an output pre filter is needed to remove components which would otherwise alias when resampled on the output lattice. With the simplifications described in the previous paragraph the equations for 'ideal' post and pre filters are given by;

$$
\begin{aligned}
& \text { Ideal post filter }=\frac{S^{2}(f)}{\sum_{\text {all } n} S_{n}^{2}(f)} \\
& \text { Ideal pre filter }=\frac{E^{2}(f)}{\sum_{\text {all } n} E_{n}^{2}(f)}
\end{aligned}
$$

where $f=(m, n, f)$ is the 3 dimensional frequency,

$S(f)$ is the magnitude of the average scene spectrum,

$S_{n}(f)$ is the $n^{\text {th }}$ alias of the scene spectrum,

$\mathrm{E}(\mathrm{f})$ is the magnitude of the 'eye's' response,

$E_{n}(f)$ is the nth alias of the 'eye's' response.

An 'ideal' overall response for the interpolation filter is given as the product of the 'ideal' post and pre filter responses, that is; 
The 'ideal' upconversion filter must be implemented as' an approximate practical realisation. This approximation was performed using the 'discrete least mean square' approximation technique from chapter 3. This is an optimisation in which the total, weighted, squared, frequency response errors are minimised for a set of frequency points. The weighting function determines how accurately the ideal response is approximated at each frequency point. The weighting function is given by;

$$
\text { (Weighting) }^{2}=\sum_{\text {all } n} S_{n}^{2}(f) \times \sum_{\text {all } n} E_{n}^{2}(f)
$$

assuming, as above, a perfect camera and display.

Upconversion was performed as a three stage process. First the $50 \mathrm{~Hz}$ interlaced input was padded with zero values to produce a $100 \mathrm{~Hz}$ sequential (non-interlaced) signal. Then the $100 \mathrm{~Hz}$ sequential signal was filtered to remove aliasing. Finally the filtered $100 \mathrm{~Hz}$ sequential signal was subsampled to give the $100 \mathrm{~Hz}$ interlaced output.

'Optimum' filters for two aperture sizes were calculated. One had a small 3 by 3 filter kernel, while the other had a larger 7 by 7 kernel. Note that, because the processing was performed as a three stage process (described above) the aperture size is in terms of a $100 \mathrm{~Hz}$ sequential signal

The derivation of the ideal and 'optimum' interpolation filters is shown graphically in figures 5.2 to 5.9. The weighting function (figure 5.9), used to derive these 'optimum' filters, has high values around the signal aliases. This ensures that the aliases are well suppressed. The values of the weighting function away from the signal aliases are much smaller but are still significant. If the ideal filter is approximated using a weighting function which is zero except at the centre of the signal spectrum and its aliases, a bilinear ${ }^{1}$ interpolator is produced! The frequency response of the bilinear interpolator (figure 5.14) is very different from that of the equivalent size ( 3 by 3 ) 'optimum' filter. Hence we can see that the weighting function used in the approximation process has a significant effect on the practical realisation of the ideal filter.

Two other filters were used, for comparative purposes, in this investigation. These filters are best described in terms of the outputs that they generate. The input is a sequence of odd and even fields denoted $A B A B$. Let $A^{\prime}$ represent the opposite type of field to $A$ derived by averaging adjacent lines of field A (similarly for $B^{\prime}$ ). The first filter generated an output of AA'B'B. The second filter was a bilinear interpolator generating an output sequence

\footnotetext{
${ }^{1} \mathrm{~A}$ bilinear interpolator is a linear interpolation between nearest neighbours in two dimensions, in this case vertical and temporal.
} 
$A\left(A^{\prime}+B\right) B^{\prime}\left(B+A^{\prime}\right)$. The first of these is the motion algorithm from Roberts 1985 . It is known to give quite smooth motion portrayal with some loss of vertical resolution. It suffers from detail flicker at $25 \mathrm{~Hz}$ and combing on moving edges. This was included as a reference for the other algorithms. The bilinear interpolator is a simple filter which appears to have been overlooked in previous work and so was included for assessment here. The frequency responses of field repeat $(\mathrm{ABAB})$, the 'motion' algorithm $\left(\mathrm{AA}^{\prime} \mathrm{B}\right.$ ' $\left.\mathrm{B}\right)$ and bilinear interpolation are illustrated in figures 5.12 to 5.14 .

\subsubsection{Experimental Procedure.}

The upconversion in this investigation was performed using computer simulation (described above). Each of the 4 filter apertures described above was tested on each of 4 sequences. The 4 sequences used were called 'Renata', 'Blonde', 'Balkans' and 'Panzoom'. Renata features a presenter walking in front of a slowly moving background. Blonde is a shot of head and shoulders. Balkans shows costumed musicians playing stringed instruments and Panzoom is a fast pan and zoom of a motor racing scene. All four sequences include movement but Renata and Blonde contain little fast motion whereas Balkans and Panzoom do. The first two sequences were chosen to be typical of uncritical material and the latter two were intended to contain difficult material. Only monochrome sequences were processed. The capability of the simulation system restricted the simulations to a 100 frames of 360 pixels by 288 picture lines.

Some difficulty was experienced in trying to assessthe results. The simulation system was unable to display results at $100 \mathrm{~Hz}$. Therefore the processed sequences were displayed at $50 \mathrm{~Hz}$ resulting in slow motion. To allow for this the 'optimum' filters were derived for display at $50 \mathrm{~Hz}$ rather than $100 \mathrm{~Hz}$. Nevertheless display at $50 \mathrm{~Hz}$ does allow a valid comparison between filter apertures. Impairments at $100 \mathrm{~Hz}$ should be less visible than at $50 \mathrm{~Hz}$ because of the increased temporal bandwidth of the display. No direct comparison of input and processed pictures is possible because they have different field rates. Comparison between input and output spatial resolution is possible but may be misleading because of confusion by spatio/temporal aliasing. Therefore the best method of assessment is probably to compare the output using the different filters.

The four sequences were processed using each of the 4 filter apertures. The 4 results for each sequence were displayed simultaneously for comparison and assessment. The input sequences could also be displayed for a comparison of static resolution. 


\subsubsection{Results, Discussion \& Conclusions.}

The picture quality of the processed sequences was better than expected. A small amount of resolution loss could just be discerned on some details (all filters). Judder could be seen on Balkans and Panzoom using the AA'B'B interpolation but was at a low level. It was difficult to see any motion defects with the other 3 filters. In general it was difficult to distinguish between the processed output using the bilinear, $3 \times 3$ or $7 \times 7$ filters. For this reason a larger filter aperture was not tried.

The assessment conditions may have obscured some less obvious impairments. Only short quarter picture sequences were used and longer full size sequences would have been better. This problem is inherent to computer simulations, which are thus more suitable for dismissing bad algorithms than differentiating between good ones. Since the results were displayed at 50 rather than $100 \mathrm{~Hz}$ a direct comparison between input and output was not possible. Motion defects would have been visible at $50 \mathrm{~Hz}$ but some blurring may have gone unnoticed for lack of a reference picture.

The two 'optimum' filters work well relative to the known performance of AA'B'B interpolation. This is a vindication of the filter design strategy. The performance on moving pictures, of both bilinear and the two 'optimum' filters, was better than that of the AA'B'B algorithm. The AA'B'B algorithm was used as the 'motion' algorithm by Roberts 1985 because of its good motion performance. Since the other algorithms in this investigation perform better on moving images we can conclude that good motion portrayal is possible for upconversion using modest hardware. The results of this investigation, and the spatial frequency response of the 'optimum' filters, suggests that good spatial resolution is also achievable in the same filter, of modest complexity.

It is interesting to note that when, by mistake, just an (optimum) input post filter was used serious motion artifacts were easily visible. This shows that a non optimum filter response will give poor performance in this application. Hence the good results achieved in this investigation are because good filter apertures were used and not simply because upconversion is an inherently easy process.

\subsection{Intercontinental standards conversion.}

\subsubsection{Introduction.}

This section describes the investigation of new filter apertures for intercontinental standards conversion. The purpose of the investigation was to compare the performance of the filter design techniques of chapter 3 with filters already used for this type of standards conversion. If the comparison were favourable then simple filter design would probably be 
possible, for many other standards conversion problems, using the new filter design techniques. It would be an additional bonus if the new filter designs performed better than those already in use. This investigation was performed using real time hardware which allowed a large amount of standards converted picture material to be assessed.

The process of intercontinental standards conversion is vital for the international exchange of television pictures. The historical development of intercontinental standards conversion is discussed in the introductory chapter of this thesis. The aliasing inherent in television picture prevents artifact free intercontinental standards conversion using conventional (non-adaptive, linear filtering) standards converters. Nevertheless because of the vital nature of the process it is important to achieve the best results which are possible in practice.

Intercontinental standards conversion interconverts American 525 line $60 \mathrm{~Hz}$ interlaced television pictures to European 625 line $50 \mathrm{~Hz}$ interlaced pictures. The sampling structure used for digital processing of television pictures has been standardised by international agreement (CCIR Rec 601 1986). For both European and American television standards the overall data rate is the same (13.5 Msamples/s) and the number of pixels per line is also the same (720 pixels/line). Hence the conversion between these two standards is, conventionally, achieved by vertical/temporal filtering, since pixels are in the same positions horizontally in both standards.

To convert from European to American television the field rate must be increased and the number of lines decreased. The reverse situation applies when converting in the opposite direction. In practice the same hardware must be used to convert in both directions. This means that both increasing and decreasing the field rate and number of lines is required in the same hardware. This leads to considerable complication in practice (see chapter 4). The temporal and vertical sampling rates are similar for both European and American television. Hence the degree of impairment introduced by the standards conversion process is similar in both directions. The nature of the impairments are, of course, slightly different for conversion in the two directions.

\subsubsection{Filter Design.}

The process of filter design for intercontinental standards conversion is very similar to that for field rate upconversion (see section 5.2). As for upconversion the interpolation filter response is designed to jointly minimise the effects of aliasing and loss of resolution (both spatially and temporally). The same simplifications and spectral models were used to design filters for intercontinental standards conversion as were used for upconversion. Hence equations 1 to 4 from section 5.2 also apply in this case. The positions of aliasing due to both the input and output sampling structure are, of course, different from upconversion and are 
shown in figures 5.15 and 5.27. The crosses represent the centres of spectral repeats caused by the input sampling lattice. The circles represent repeats of the response of the eye due to the output sampling lattice. Aliases of the response of the eye are significant because high frequencies in the signal are perceived as low frequencies.

A significant difference between upconversion and intercontinental standards conversion is the number of filter coefficients that have to be calculated. For upconversion the filter coefficients were calculated on a supersampled input lattice corresponding to a 625 line, $100 \mathrm{~Hz}$ non-interlaced signal. For standards conversion the output pixel positions can have any relation to the input lattice. This is because the input and output sampling lattice are generated by different clocks which can drift in frequency relative to each other. Ideally a continuous interpolation aperture would be used. In practice however the aperture is quantised to a finite number of points as described in the previous chapter. In this example the filter coefficients were calculated on a lattice which was supersampled 16 times vertically and 8 times temporally with respect to the input lattice. This corresponds (with a 625 line input) to sampling the coefficients on a 10000 line, $400 \mathrm{~Hz}$ non-interlaced lattice. The experimental hardware used could implement an interpolation aperture up to 4 fields long and 12 picture lines high. Hence, with the supersampling ratio used, up to 6144 coefficients had to be calculated. This number contrasts strongly with the 49 ( $7 \times 7$ aperture) coefficients which were needed for upconversion. Of course only a subset of (up to 24) coefficients were used for interpolating any given output pixel. However different output pixels require different subsets of coefficients (depending on the pixel's position) and hence all 6144 are needed for the complete standards conversion process.

The large number of filter coefficients to be calculated requires that the computational aspects of the filter design be considered. In this example the discrete LMS approximation technique (section 3.4.1) was used. The computation of each interpolation aperture took a considerable time (hours) on a modern computer. The results of using discrete LMS approximation were probably indistinguishable from using the continuous LMS approximation technique (section 3.4.2), since a large degree of oversampling was used (relative to the input lattice). The use of continuous LMS approximation or eigenfilter design (section 3.4.4) would probably have been significantly quicker. In this example computational speed was not a problem. If, however, a large number of apertures were required (perhaps for motion compensated interpolation) the computational requirements of the discrete LMS approximation technique might be prohibitive and one of the other approximation techniques would be more appropriate.

Although vertical/temporal filters were used for standards conversion the 'ideal' interpolation aperture is actually 3 dimensional (horizontal, vertical \& temporal). The 'ideal' three dimensional aperture was calculated using equations 1 to 3 . Realisable two dimensional approximations to it were then made. For these examples the 'ideal' frequency response was 
specified at $\pm 50 \mathrm{~Hz}, \pm 37.5 \mathrm{~Hz}, \pm 25 \mathrm{~Hz}, \pm 12.5 \mathrm{~Hz}$ as well as at $0 \mathrm{~Hz}$ prior to producing an 'optimum' vertical/temporal approximation to the ideal filter response.

The derivation of the 'ideal' interpolation filters and the 'optimum' practical approximations to them are shown graphically in figures 5.15 to 5.24 and 5.27 to 5.36 . The weighting function governs how closely the 'ideal' response is approximated at each frequency. As for upconversion, weighting is high where there is a lot of signal energy or where the 'eye' has a strong response. This ensures that aliases are well suppressed and resolution is preserved where it is most needed. 'Optimum' filters were designed for European to American conversion and for conversion in the opposite direction. Two 'optimum' interpolation apertures were calculated in each case for aperture sizes of 4 fields by 8 picture lines (input lattice) and 4 fields by 6 picture lines. The frequency responses of both 'optimum' apertures were very similar and so only one is shown.

\subsubsection{Experimental Procedure.}

Standards conversion for this investigation was performed using real time hardware. Hence the output of the converter could be studied for long periods using each of the interpolation apertures. This makes assessment rather easier than for computer simulation. All apertures used were stored in semiconductor memory and the active aperture could be changed at the push of a switch. This enabled detailed comparison between apertures. A wide variety of material was available from a digital video tape recorder.

Objective measurement of standards converter performance is extremely difficult. Ideally standards converted pictures would be compared to non standards converted pictures. However this would require that identical scenes were 'filmed' using both European and American standard cameras. This is extremely difficult to arrange in practice and was not possible for this investigation. The alternative, used here, is to compare the output using different interpolation apertures. For this purpose the apertures published by Clarke \& Tanton 1984 were used as a reference. These apertures are generally accepted to give 'good' performance on a wide range of picture material.

\subsubsection{Results, Discussion \& Conclusions.}

The overall quality of the standards converted pictures was broadly similar using either the 'optimum' filters or the reference apertures from Clarke \& Tanton 1984. A slight preference was expressed by some observers for the slightly 'sharper' pictures from the 'optimum' filters. The filter design algorithm was therefore judged successful.

The impairments in standards converted pictures are blurring and the effects of aliasing. The interpolation filter design is a compromise to minimise the joint effects of all 
the impairments. Blurring of either stationary or moving detail is due to wanted parts of the spectrum being filtered out. Aliasing is due to unwanted spectral energy being left in the output signal. The effect of aliasing depends on where the alias energy occurs in the output spectrum. The spectral regions giving rise to different type of alias impairment are illustrated in figure 5.39. The modulation of vertically moving detail is sometimes known as the 'crankshaft effect' (Clarke 1990, p 336).

All the interpolation apertures (both reference and 'optimum') are beneficial in reducing the effects of aliasing. A simple standards conversion, which simply took the nearest available pixel, was implemented for comparison. As expected this showed disturbing levels of all artifacts on various scenes. All the apertures tested gave greatly improved performance over this simple standards conversion algorithm. The filter considered best depended both on the type of scene and varied between observers. This would suggest, as expected, that the compromises inherent in each of the filter apertures were better suited to some types of pictures than others. It was very difficult to distinguish between the two 'optimum' filters on any picture material. On many pictures it was difficult to distinguish between any of the filters. The overall quality of converted pictures using each of the apertures was broadly comparable.

Clarke and Tanton 1984 distinguish between two types of motion. Firstly there are rapid, short duration movements such as arm of leg movements of a dancer. This type of movement predominates in studio pictures. The duration of each movement is short so there is no opportunity for the observer to track the motion. Hence any judder which is present is not noticeable. Blurring of these rapid movements is both noticeable and objectionable. Therefore a wider temporal frequency response is desirable to preserve detail at the expense of judder. The second type of movement is relatively slow, steady and sustained. This typically occurs when a camera pans to follow a moving foreground object. Such camera induced movement often occur in outside broadcasts, particularly of sporting events. Since this type of motion is sustained it can be tracked by an observer and hence judder becomes a very significant impairment. Although blurring is still noticeable it is less objectionable than judder. Therefore, for this type of motion, a narrower temporal frequency response is desirable to minimise judder at the expense of detail. A third movement category became apparent in this investigation. That is the movement of highly detailed computer graphics and special effects. Such pictures have not even been subject to the mild prefiltering action of the camera. Their high detail and rapid motion is a severe test for conventional standards converters. Similar high resolution rapid motion is produced by CCD cameras using a short shutter time.

The performance of the interpolation apertures varied with the type of movement in the scene. Clarke and Tanton give two apertures for each direction of conversion, one has a wider temporal frequency response and is intended for studio pictures (Tables $9 \& 11$ from Clarke \& Tanton '84), the other has a narrower temporal response and is intended for outside 
broadcasts (Tables $8 \& 10$ from Clarke \& Tanton '84). The frequency response of these reference apertures is shown in figures 5.25, 5.26, 5.37 and 5.38. As expected the reference apertures performed best on the picture material for which they were intended. The 'optimum' apertures gave improved resolution on all scenes. This was expected because of their much wider frequency response (see figures $5.24 \& 5.36$ ). The 'optimum' apertures also performed well for both studio and panning movement. Judder was, perhaps, slightly worse using the 'optimum' filters for panning motion. However the overall quality of the pictures was comparable to using the reference filters because the resolution was better. Computer graphics and short shutter time $\mathrm{CCD}$ pictures had significant artifacts using all the filters. Motion artifacts for these pictures were worse with the

'optimum' filters as would be expected because of their wider temporal frequency response.

It is interesting to compare the frequency responses of the reference and 'optimum' apertures. Comparing figures $5.25 \& 5.26$ (the reference apertures) to figure 5.24 (the 'optimum' aperture) we can see that the frequency response of the 'optimum' aperture is much wider than the reference aperture ${ }^{2}$. It is surprising, in view of the big difference in frequency response, that there is relatively so little difference in their performance when interpolating real pictures. The improved response of the 'optimum' aperture along the vertical frequency axis is because the 'optimum' filter has a sharper cutoff than the reference filter. The same is true, to some extent, along the temporal frequency axis. The pictures interpolated with the 'optimum' filter do appear a little sharper as a consequence. Stopband ripples in the reference filters are concentrated along the vertical and temporal frequency axes, whereas they are more evenly spread in the 'optimum' filter. This allows the 'optimum' filter to have a wider passband and sharper cutoff while maintaining the same degree of stopband suppression. Some of the differences between reference and 'optimum' filters are probably due to the filter design methods used. A basic frequency sampling technique was used by Clarke and Tanton whereas an optimising techniques was used for the 'optimum' filters. Optimised filter design can achieve significant improvements in filter response, compared to heuristic design procedures, for the same size aperture. This is demonstrated, in another context, in the appendix (chapter 11). The small difference in performance between the filters is, perhaps, explained by the low level of higher frequency components in typical pictures.

The most significant picture impairment with either reference or 'optimum' filter is judder. Judder is usually caused by sustained horizontal panning to follow a foreground object. Horizontal motion, at typical speeds, tends to spread energy, due to horizontal detail,

${ }^{2}$ This comparison is for conversion from European to American television. We could also compare the frequency response of the reference (figures 37 \& 38) and optimum (figure 36) apertures for conversion from American to European television. The conclusions in both cases are broadly the same. 
evenly along the whole temporal frequency axis. This is a result of temporal aliasing caused by the motion. Temporal filtering using a pure vertical/temporal filter cannot remove judder, merely reduce its effect. For example if frequencies above half the (temporal) Nyquist frequency are removed half the aliasing which causes judder will be removed but so will half the signal. Judder cannot be eliminated, with a vertical/temporal filter without removing all the detail! It might be possible to reduce judder without removing too much detail by using a true three dimensional filter aperture. This, however, would require a very significant increase in hardware complexity. Another approach to eliminating judder (and other artifacts) is the use of motion compensation, which is the subject of later chapters in this thesis.

\subsection{Summary.}

This chapter describes the implementation of two standards conversion processes using the filter design techniques described in chapter 3. The purpose of the investigation was to validate the filter design technique and to determine the performance of 'conventional' (ie non-adaptive, linear filtering) standards conversion. Two standards conversion processes were investigated; field rate upconversion to improve picture display and intercontinental standards conversion to allow the international exchange of television programs.

Field rate upconversion was investigated using computer simulation. Upconversion reduces display flicker by increasing the number of fields displayed per second. Comparisons were made of interpolation using 'optimum' filters versus interpolation using filters previously reported in the literature. It seems that a carefully optimised filter can give better performance than those suggested previously. The performance seems to be sufficiently good to obviate the need for adaptive processing that has been used previously. A relatively small aperture (1.5 input fields by 3 picture lines) is sufficient to give good performance. Large apertures do not give significantly better performance in spite of their extra complexity.

The more complex process of intercontinental standards conversion was investigated using real time hardware. Comparison of the performance of interpolation using 'optimised' filters were made with successful filters previously reported in the literature. The characteristics of the 'optimised' and previously reported 'reference' filters were compared on a variety of picture material. The results from the 'optimised' filters were broadly comparable to those from the 'reference' filters.

Several general conclusions can be drawn from the results. The filter design algorithm used produced acceptable results for both the standards conversion processes and will therefore probably produce good results for other applications. Good results for field rate upconversion can be achieved using a relatively simple non-adaptive linear filter. It may be possible to gain a small improvement in the quality of intercontinental standards conversion by using very carefully optimised filters. Even when this has been done, however, there 
remain very noticeable impairments (particularly judder) in the quality of the converted pictures. To achieve 'transparent' intercontinental standards conversion (ie without noticeable artifacts) it will be necessary to use more sophisticated interpolation techniques. A suitable technique may be the use of motion compensated processing discussed in subsequent chapters.

\subsection{References.}

1. CCIR. 1986. Encoding parameters of digital television for studios. CCIR Recommendation 601-1, XVIth Plenary Assembly, Geneva 1986, XI-1, pp. 319-328.

2. Clarke, C.K.P. \& Tanton, N.E. 1984. Digital standards conversion: interpolation theory and aperture synthesis. BBC Research Department Report, BBC RD 1984/20, December 1984.

3. Clarke, C.K.P., 1990. Interpolation. Digital television, Sandbank, C.P. (Editor), John Wiley \& Sons Ltd, 1990, ISBN 0-471-92360-5, pp 287-374.

4. Kelly, D. H., 1972. Adaption effects on spatio-temporal sine wave thresholds. Vision Research, 12, 1972, pp. 89-101.

5. Parker, D.W. \& L.J. van der Polder 1984. Display standards for extended definition component television systems. International Broadcasting Convention 1984. IEE Publication No. 240.

6. Roberts, A., 1983. The improved display of 625-line television pictures. BBC Research Department Report, BBC RD 1983/8, July 1983.

7. Roberts, A., 1985. The improved display of 625-line television pictures: adaptive interpolation. BBC Research Department Report, BBC RD 1985/5, May 1985.

8. Wade, J.G., 1987. Signal coding and processing. Ellis Horwood Ltd, 1987, ISBN 0470-20778-7.

9. Wentworth, J.W. 1955. Colour television engineering. McGraw-Hill pp 51-53. 


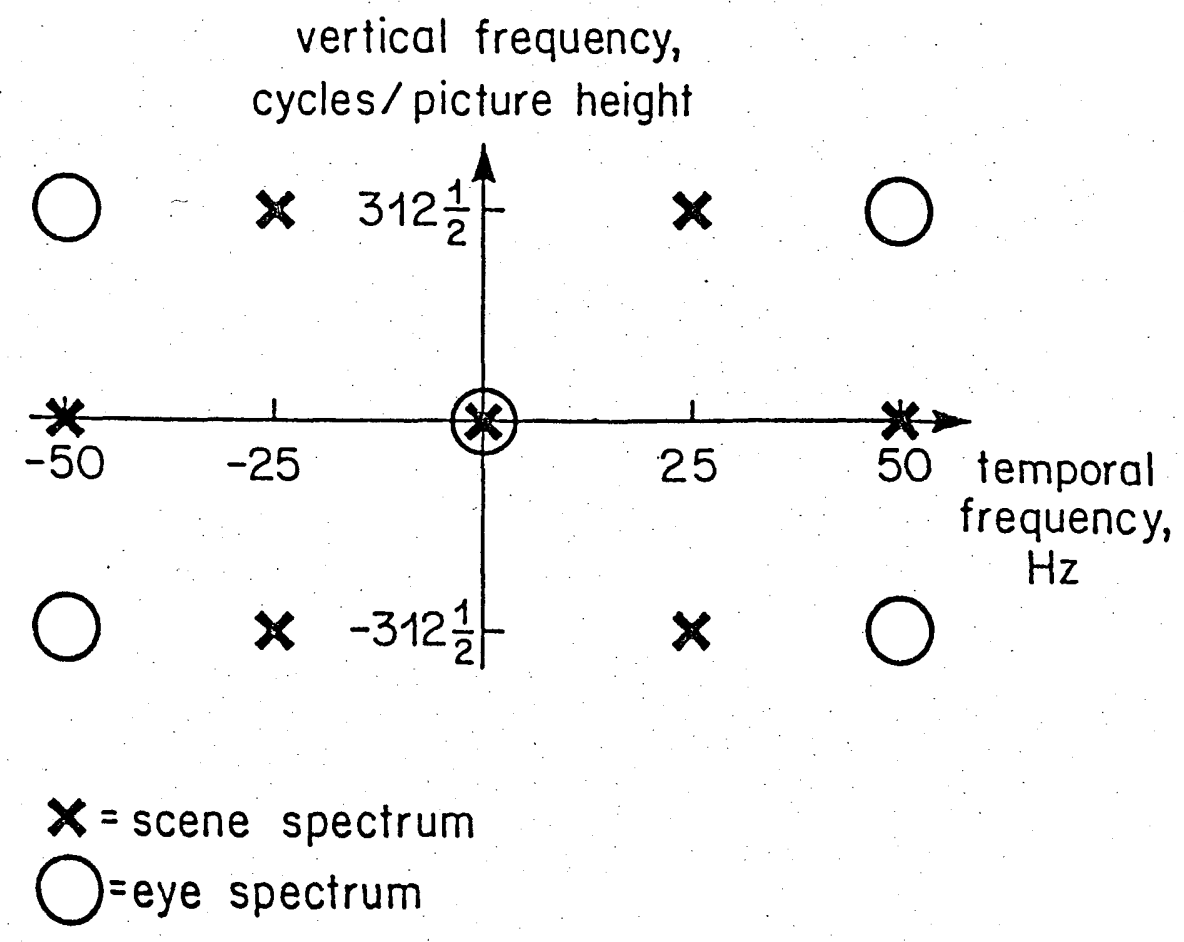

Figure 5.1 : Alias diagram for $50 \mathrm{~Hz}$ to $100 \mathrm{~Hz}$ upconversion 


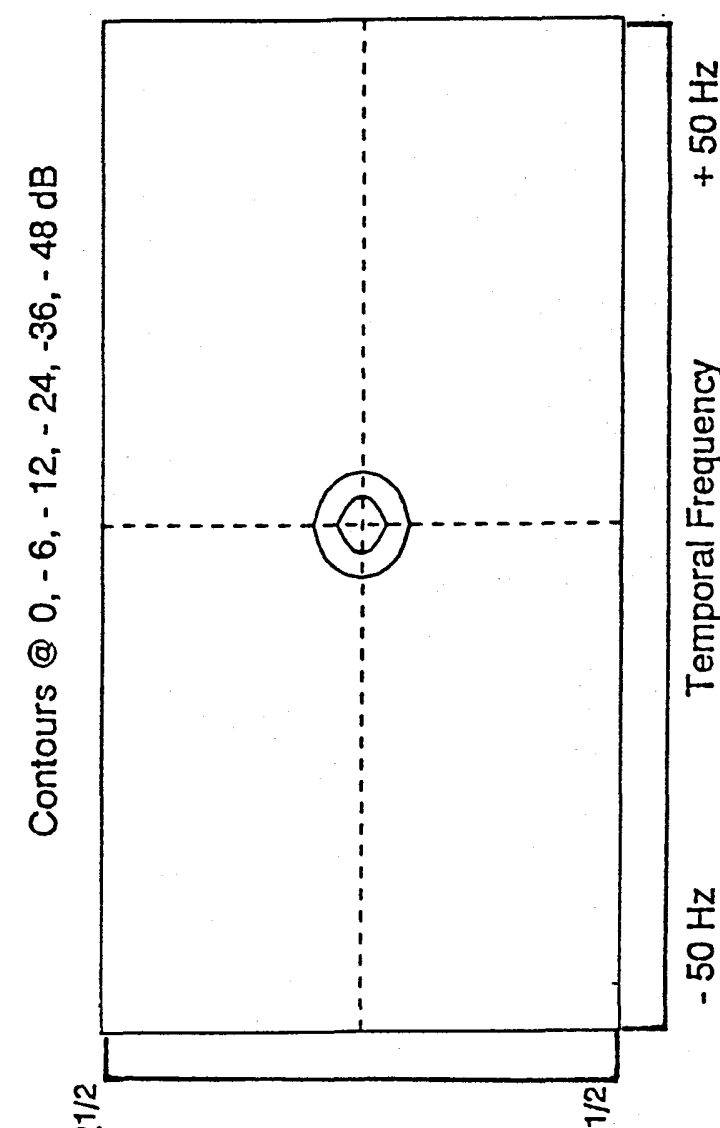

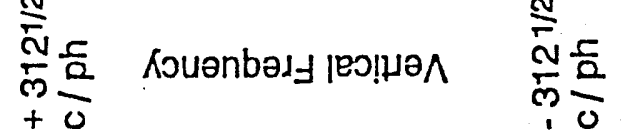

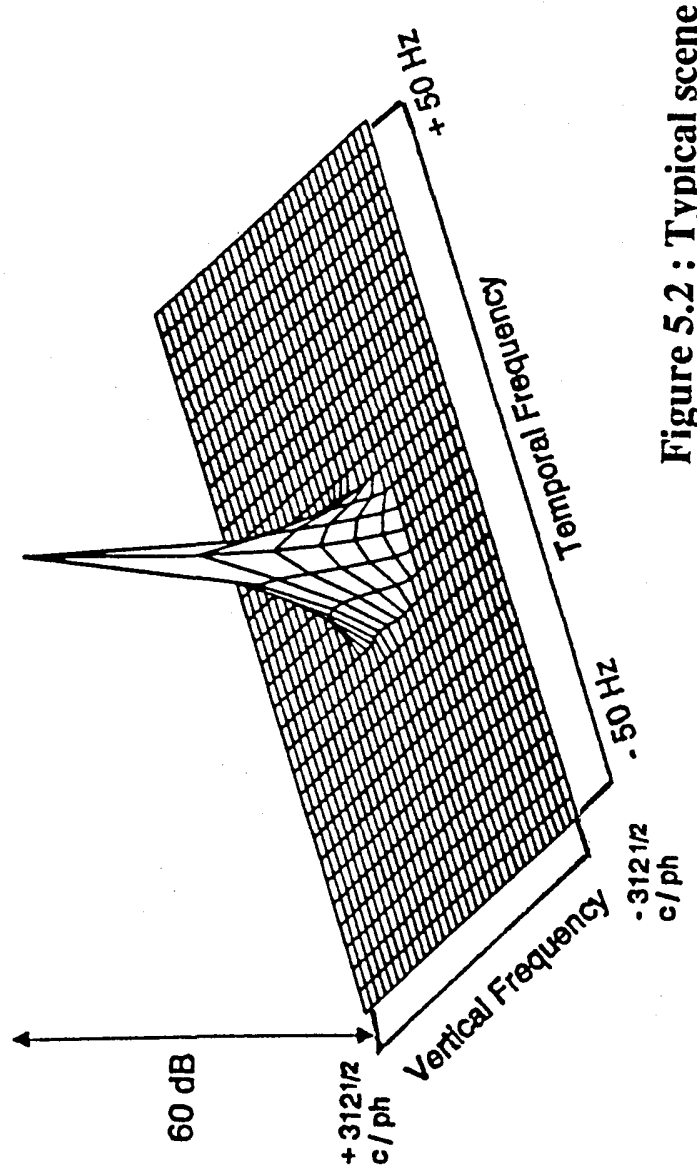

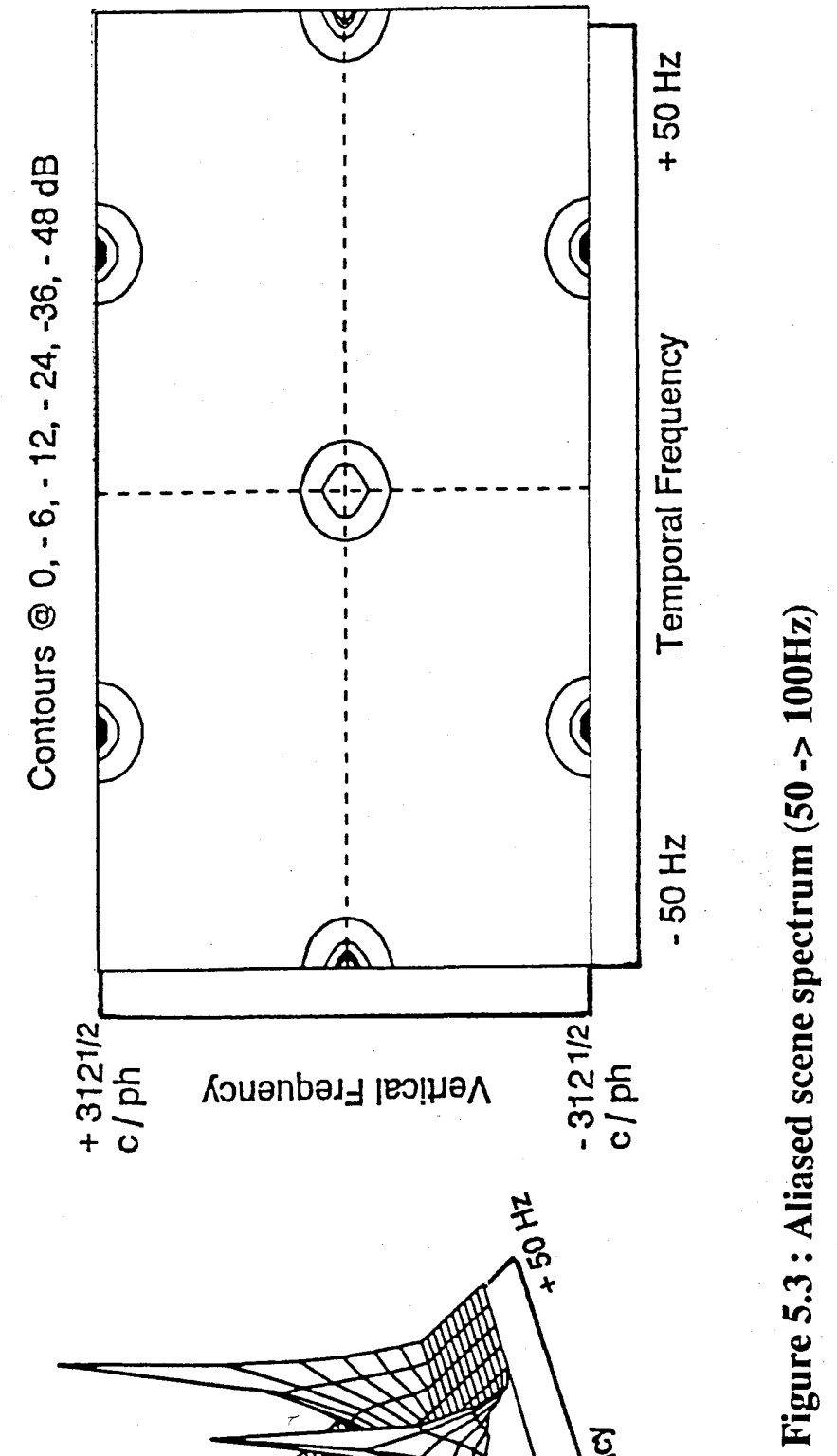

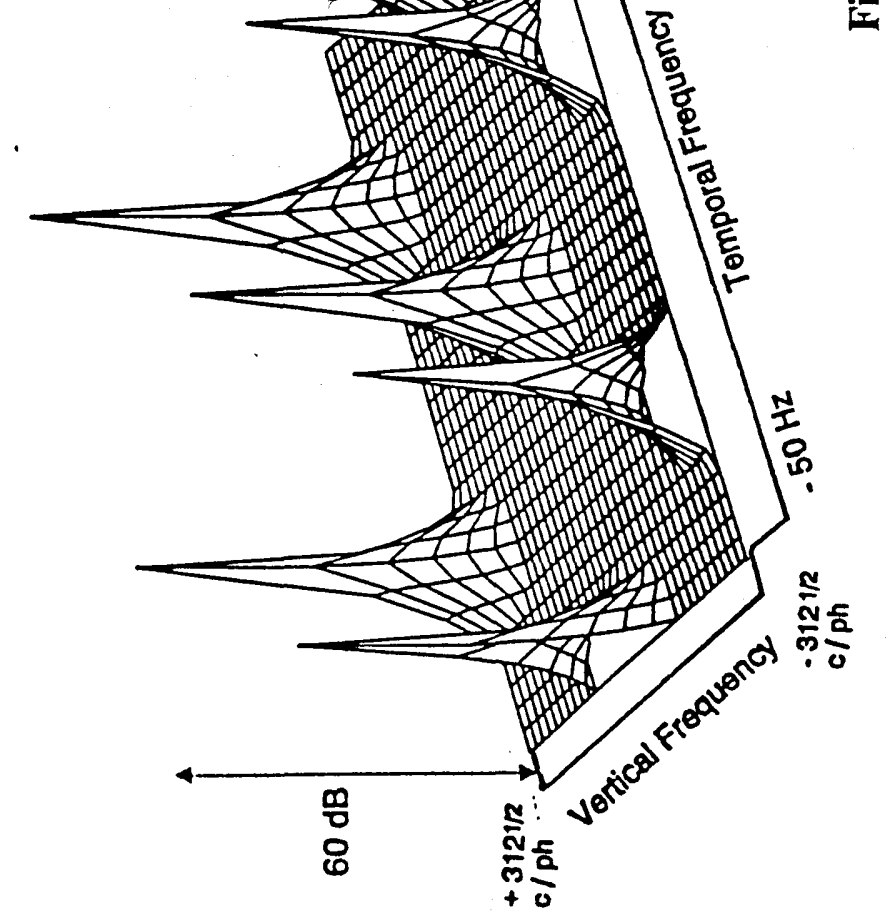




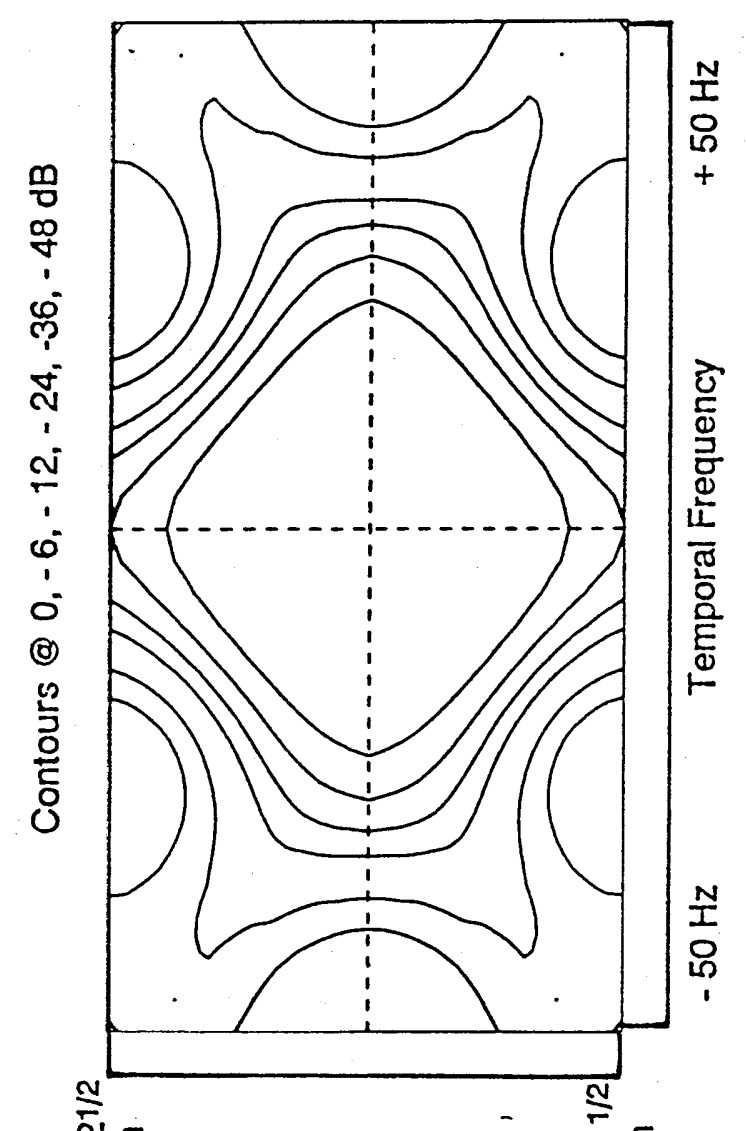

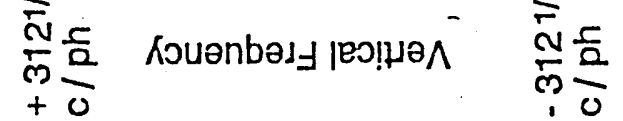

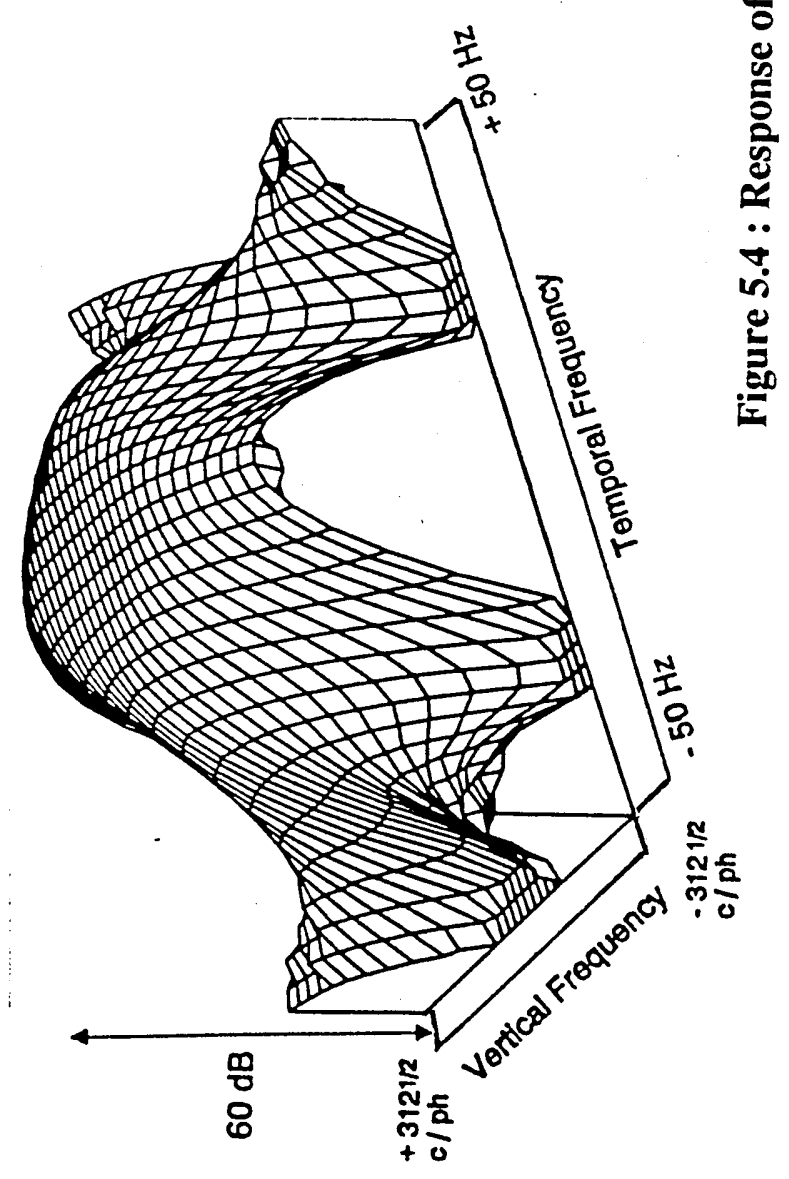

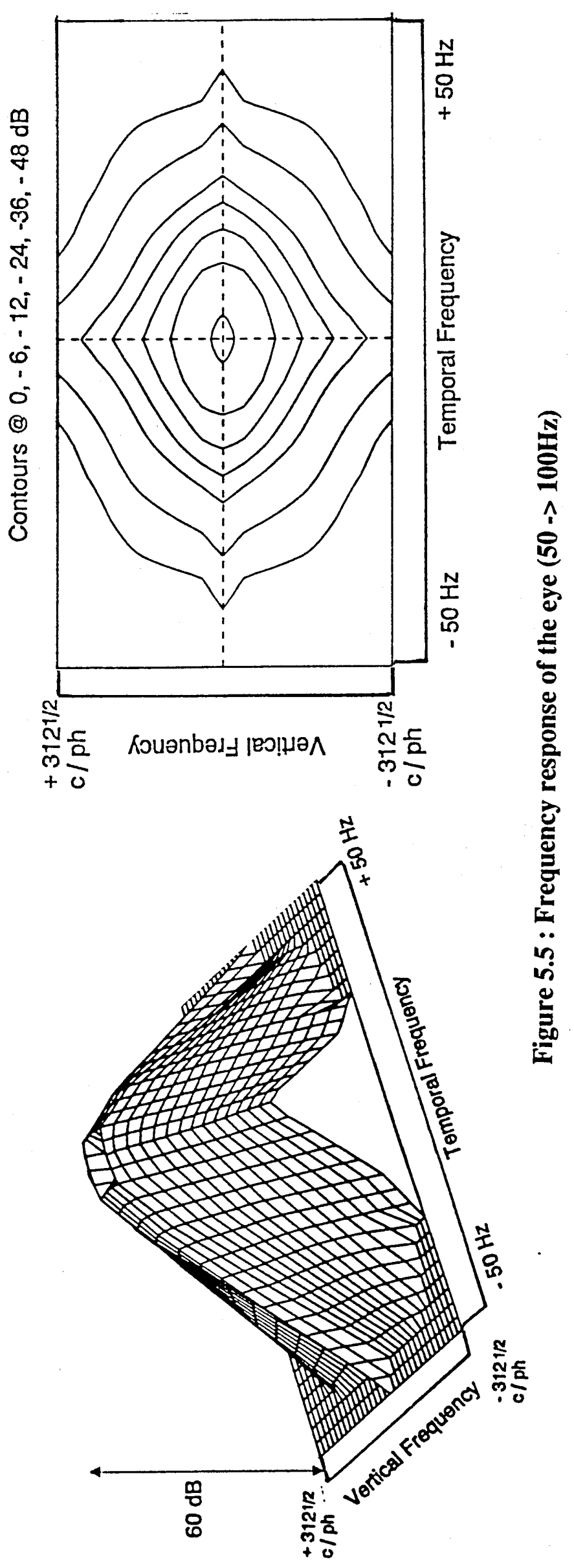



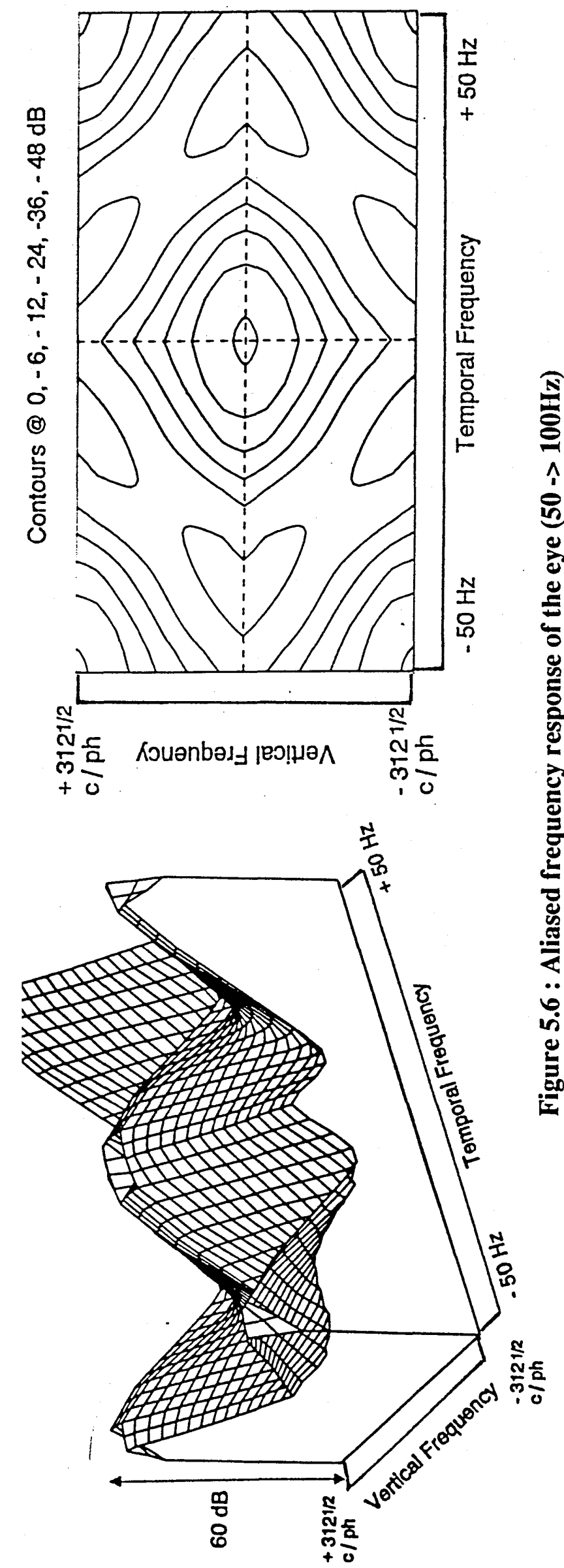

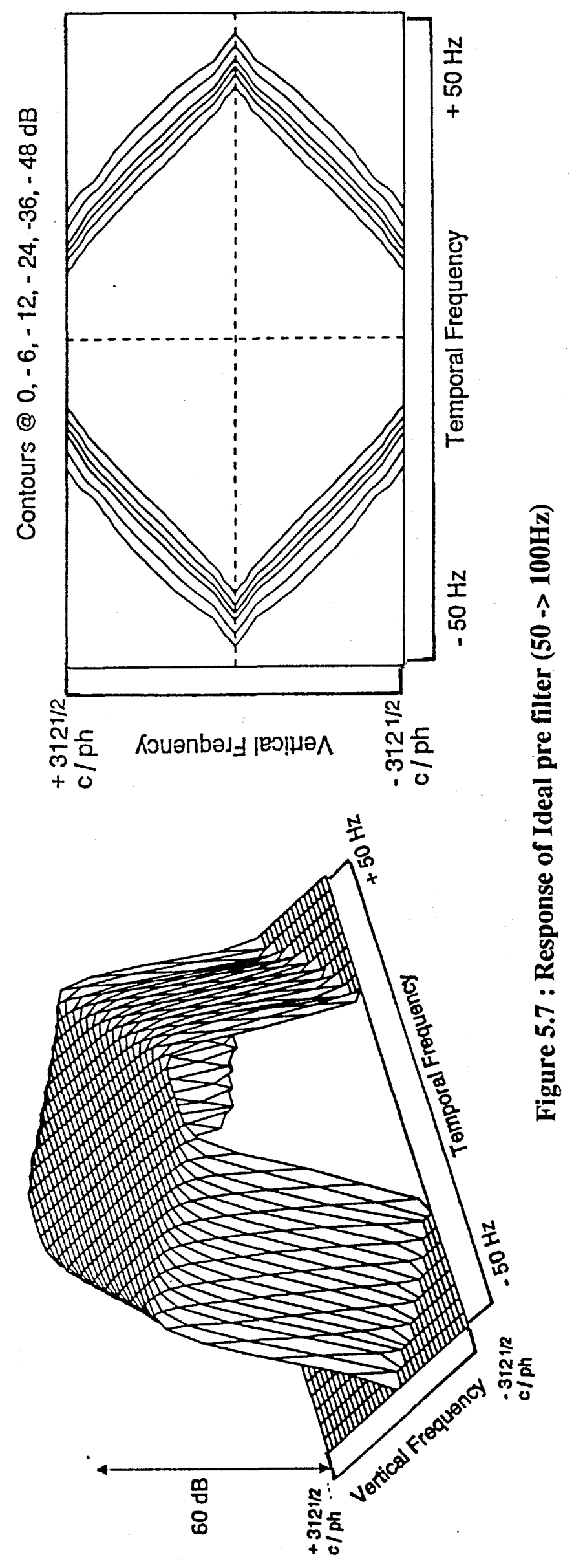



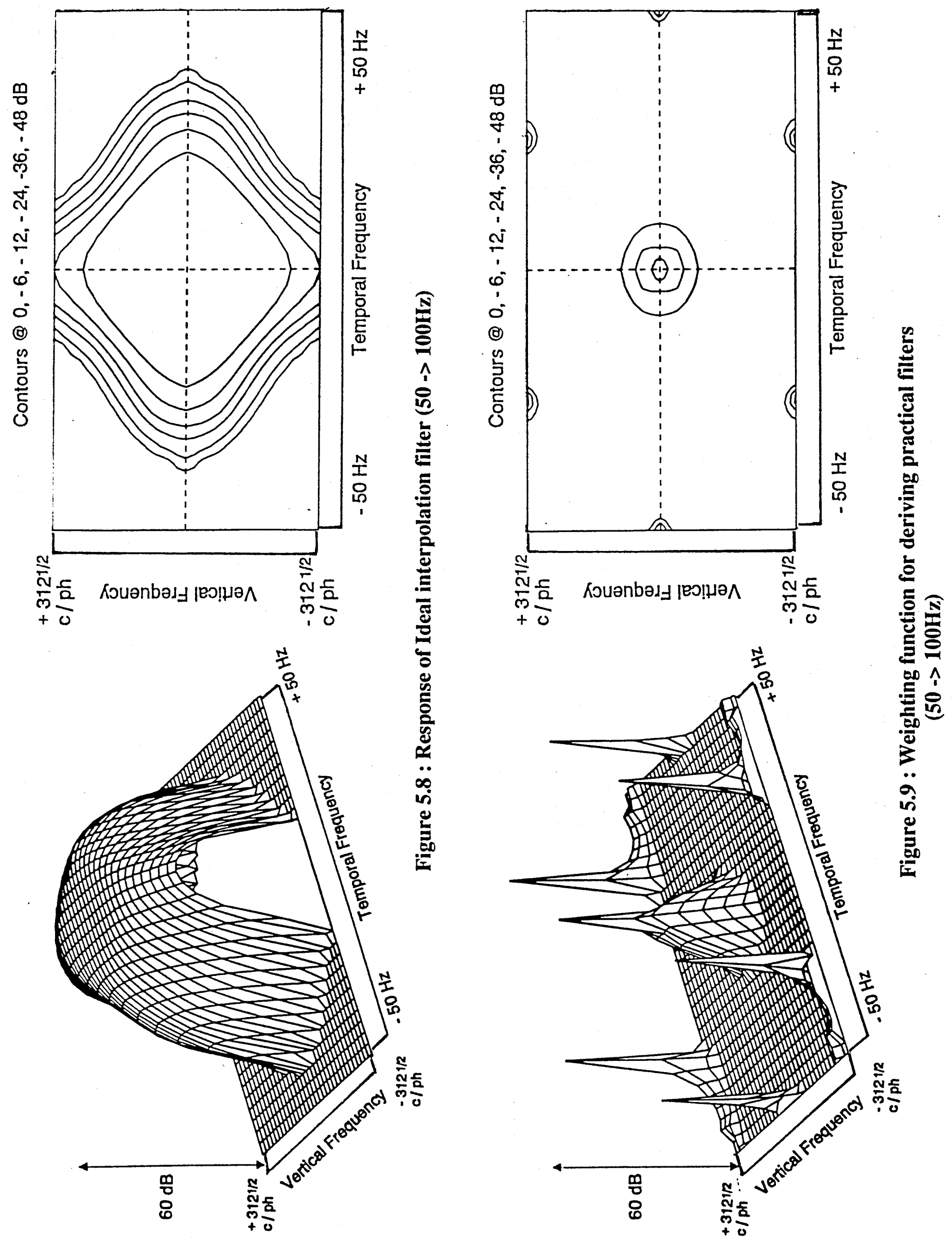

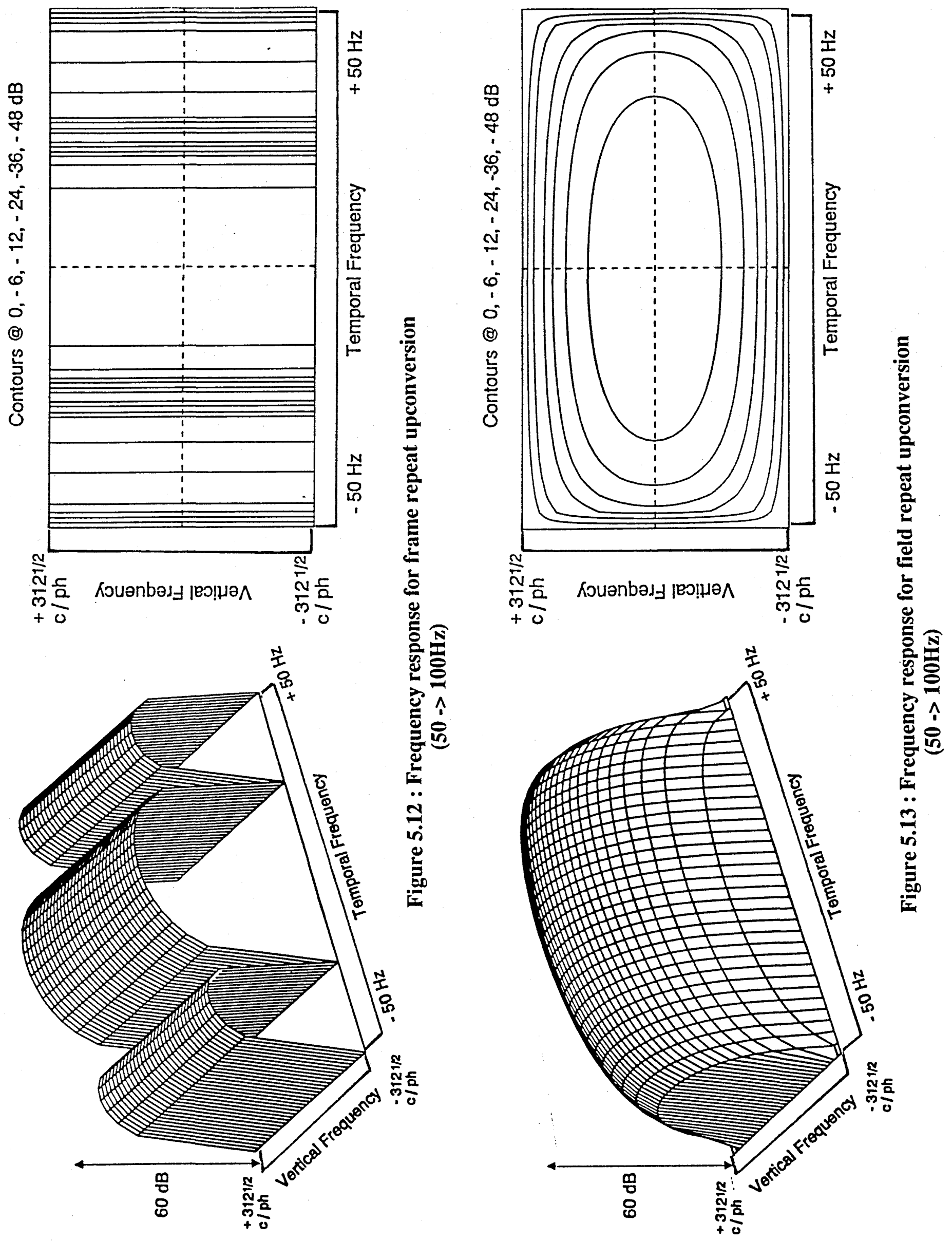


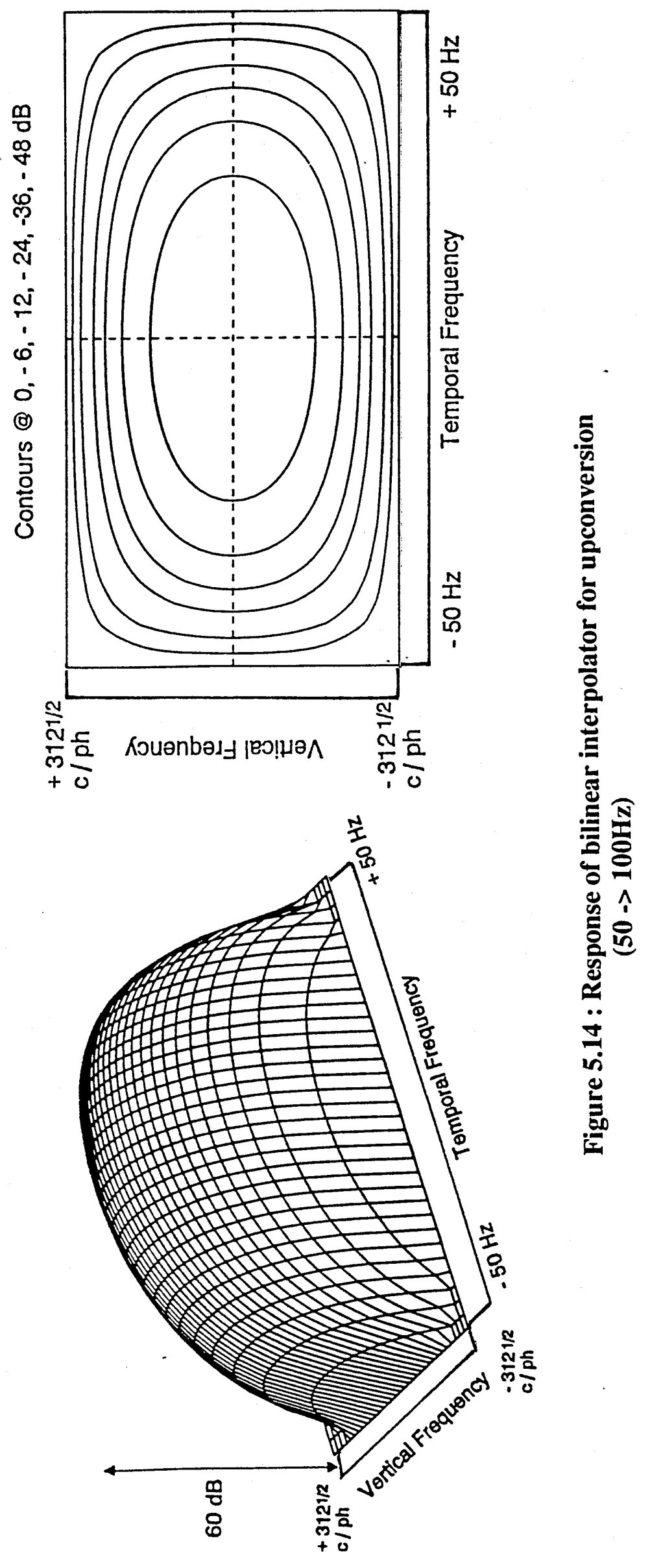


Centres of Eye's Response

(\& Allases)

X Centres of Scene Spectra

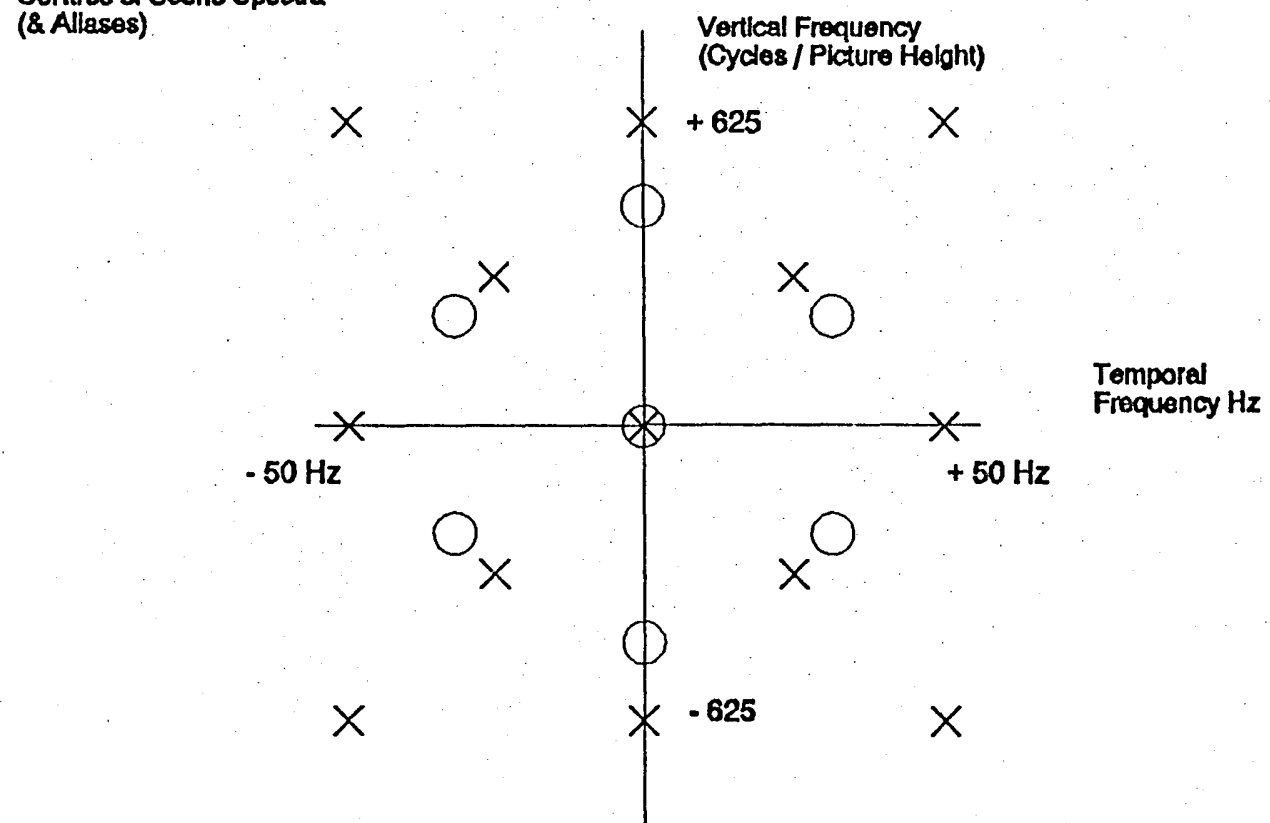

Figure 5.15 : Alias Diagram for $50 \mathrm{~Hz}$ to $60 \mathrm{~Hz}$

Standards Conversion 
$0,-6,-12,-24,-36,-48 d B$

Figure 5.16 : Typical scene spectrum $(50$-> 60Hz)
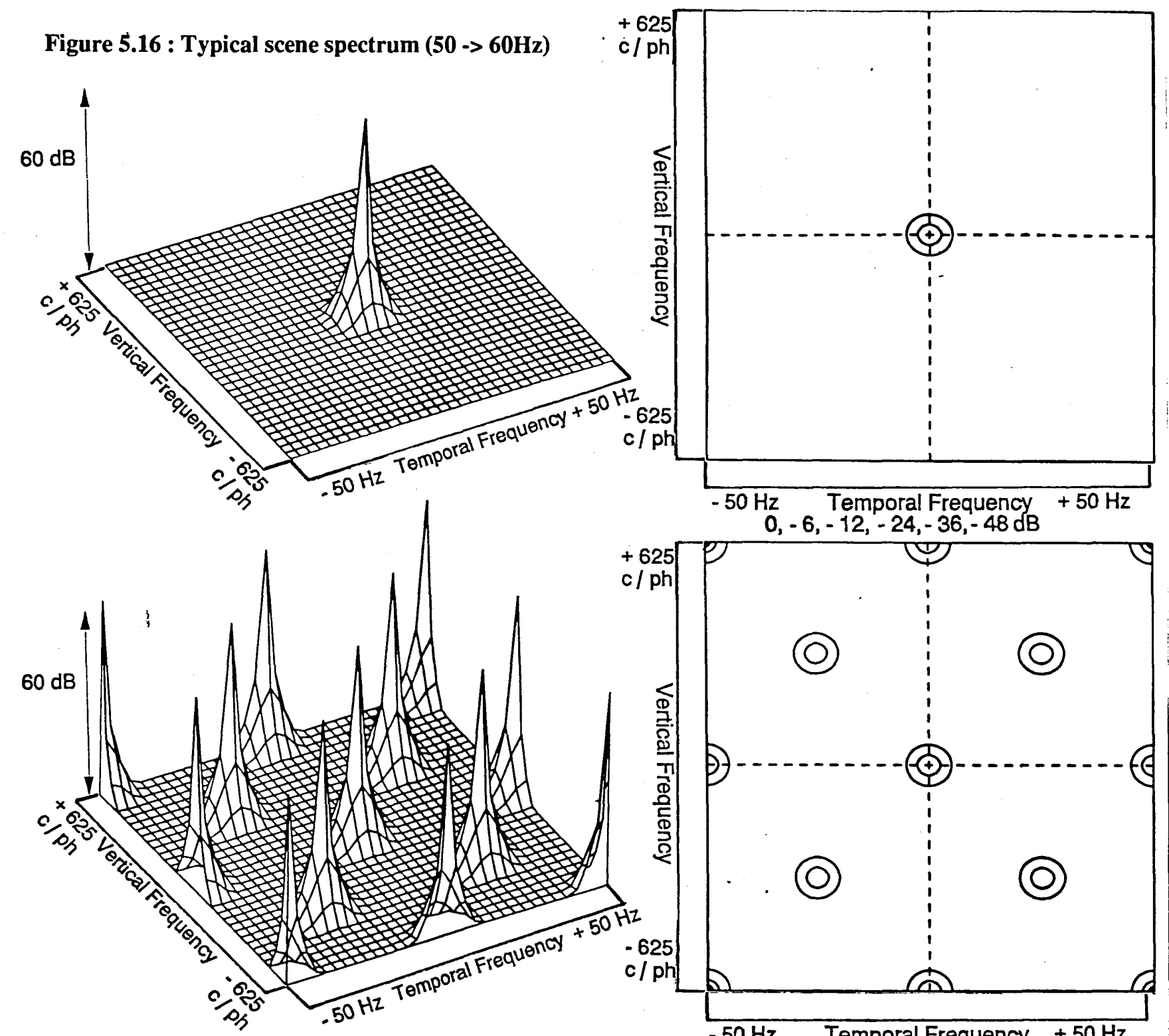

Figure 5.17 : Aliased scene spectrum $(50$-> 60Hz)
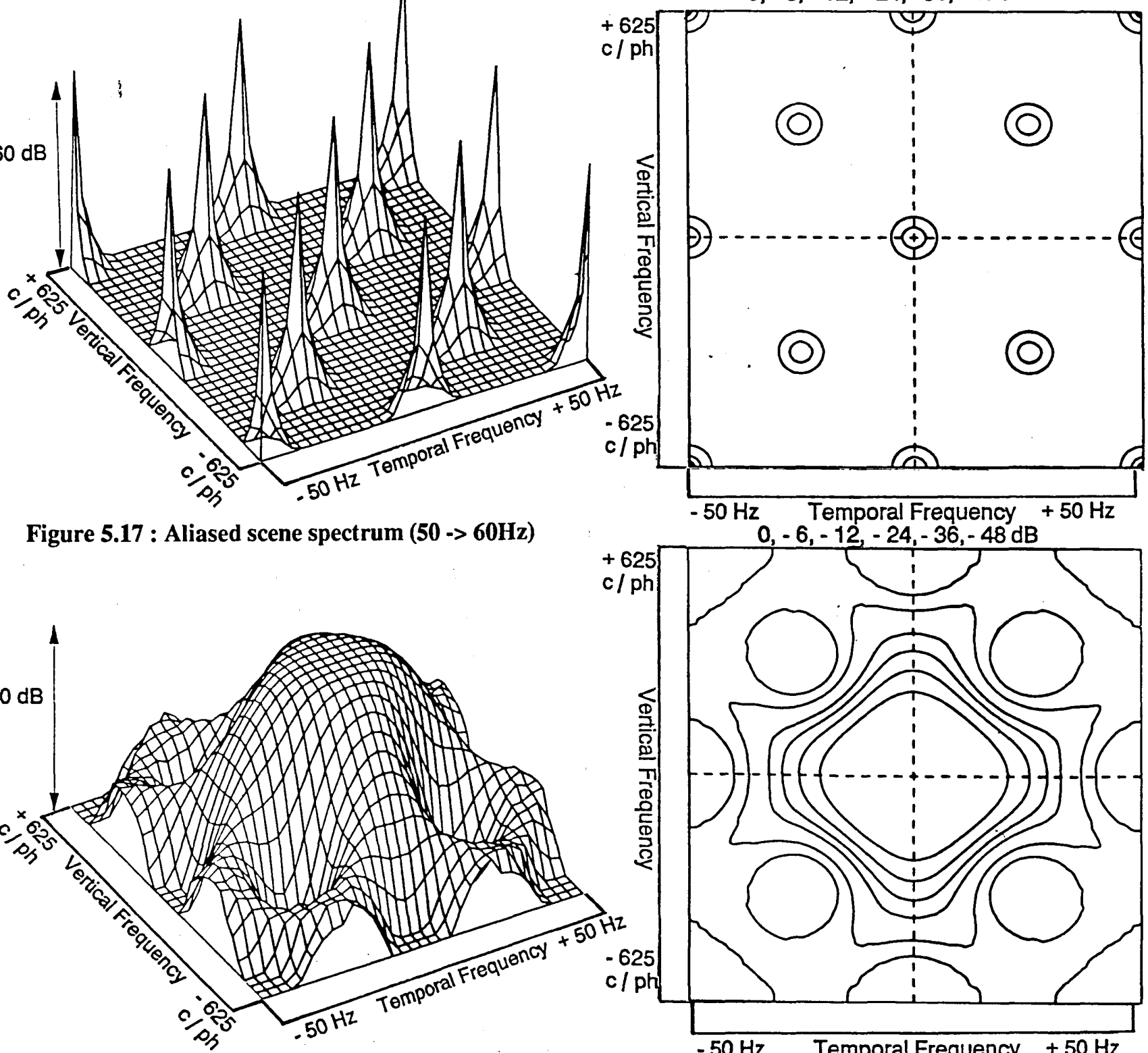

Figure 5.18 : Response of Ideal post filter $(50->60 \mathrm{~Hz})$

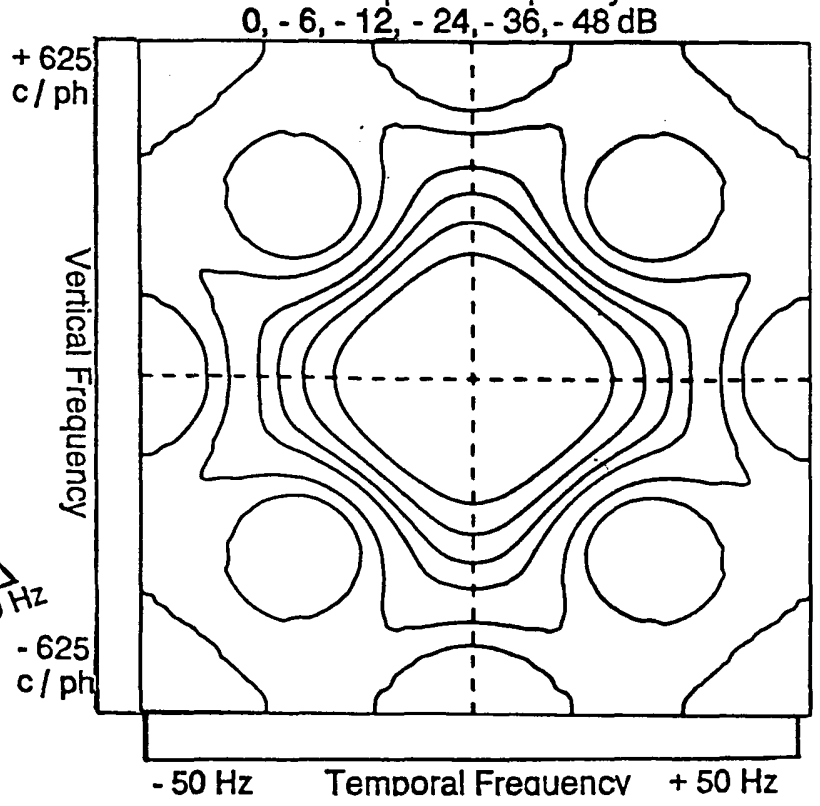


$0,-6,-12,-24,-36,-48 d B$

Figure 5.19 : Frequency response of the eye $(50->60 \mathrm{~Hz})$
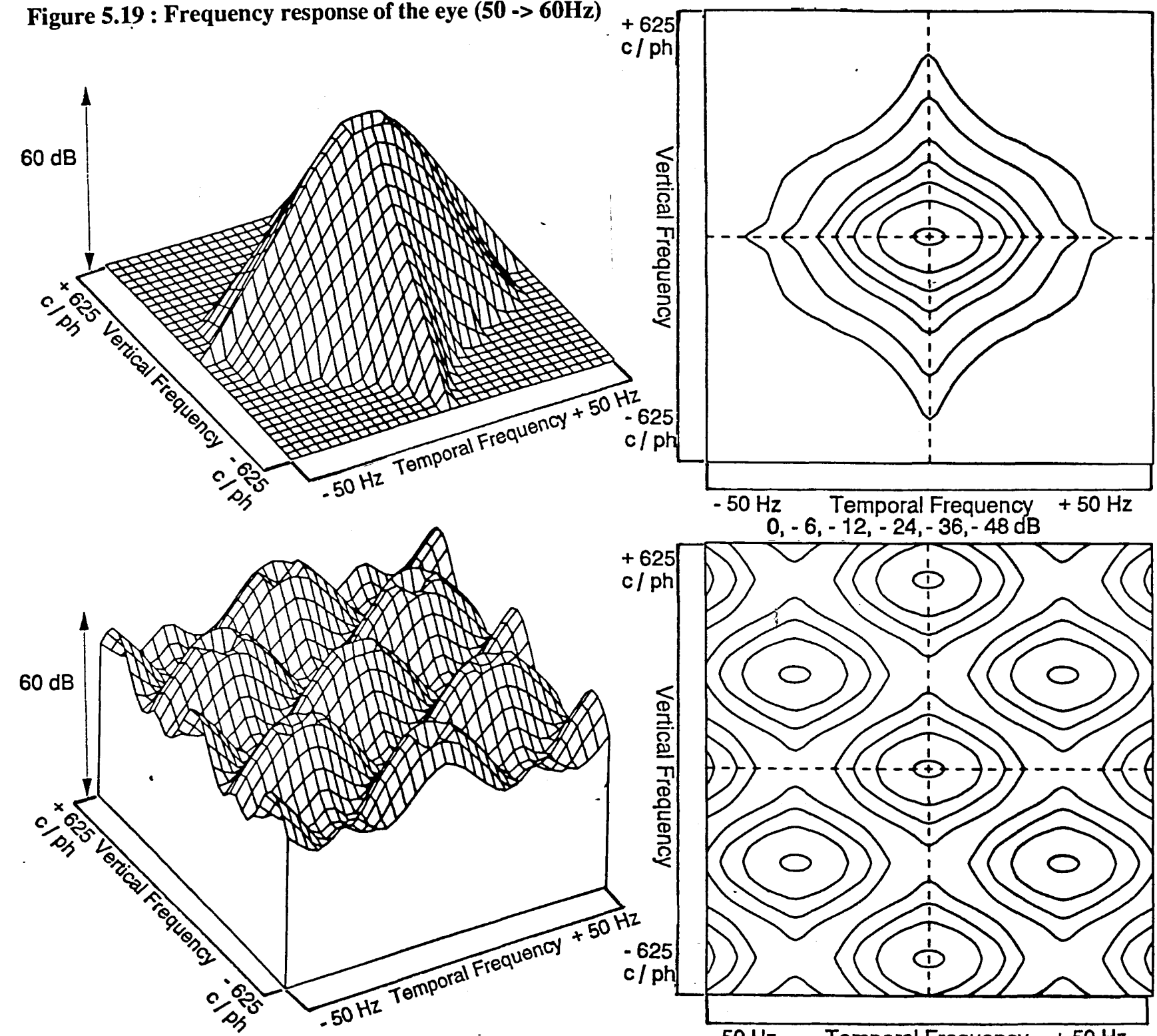

Figure 5.20 : Aliased frequency response of the eye $(50 \rightarrow 60 \mathrm{~Hz})$
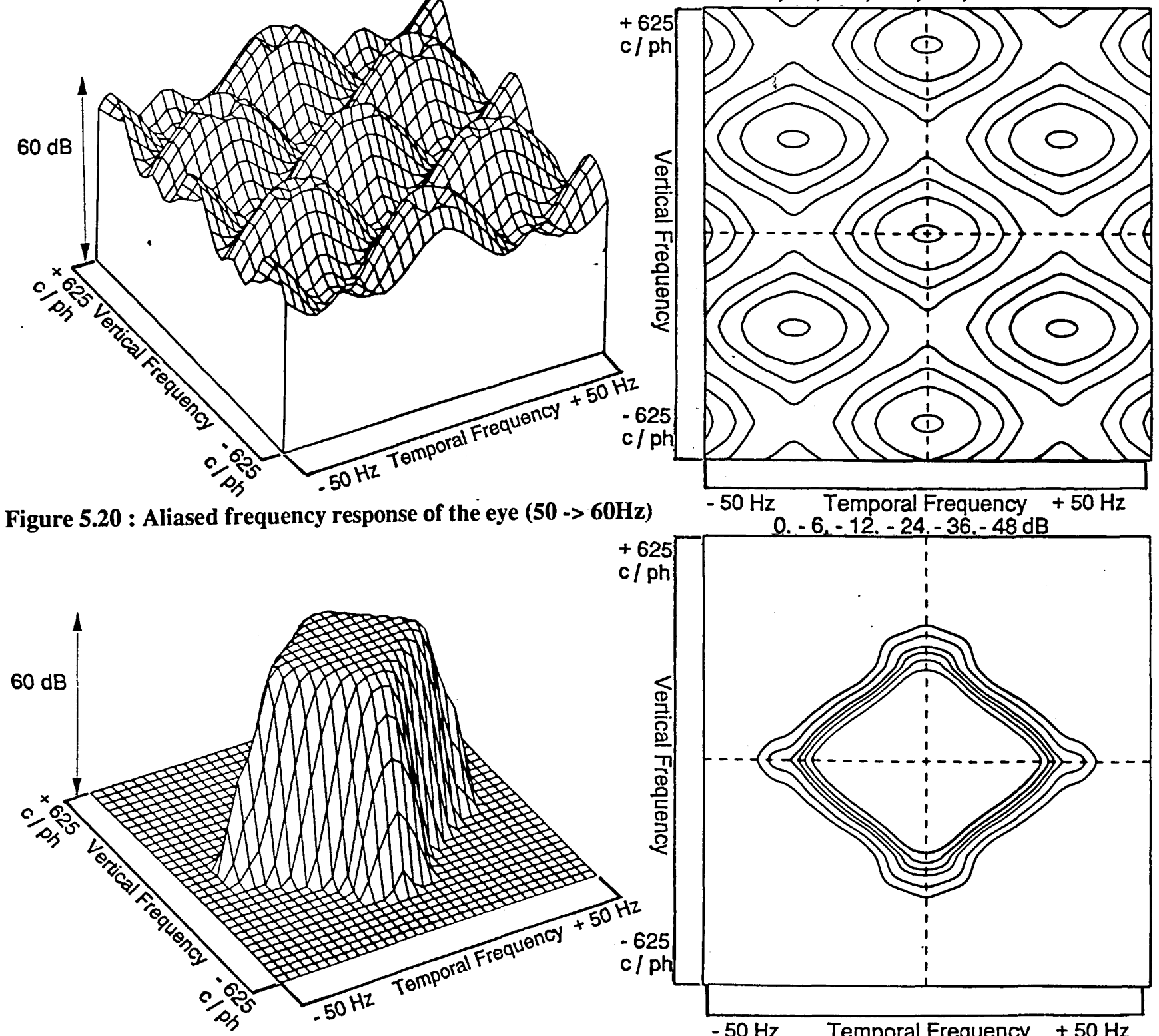

Figure 5.21 : Response of Ideal pre filter $(50->60 \mathrm{~Hz})$

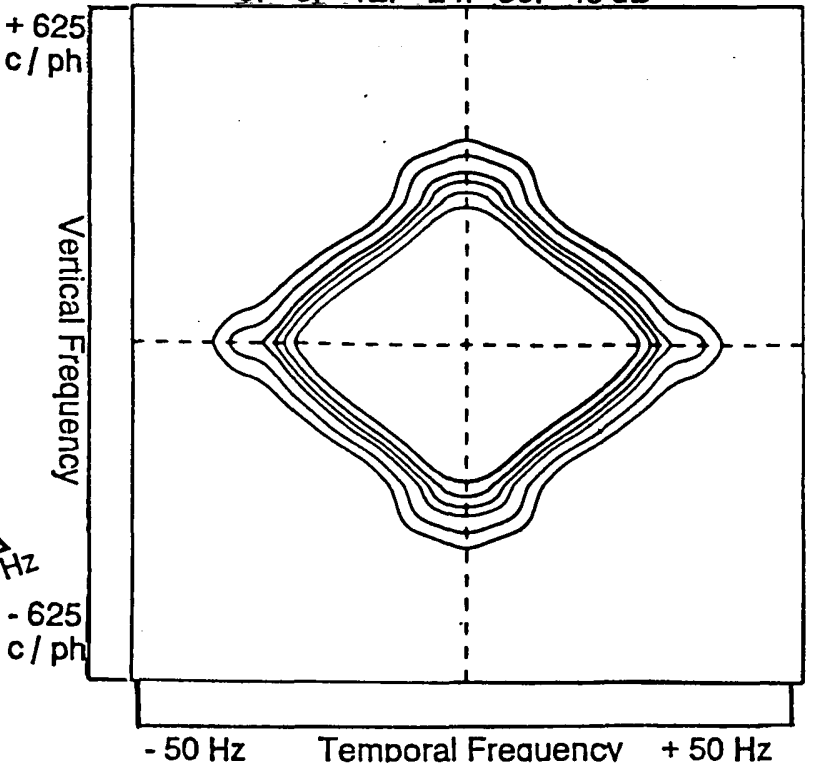


Figure 5.22 : Response of Ideal interpolation filter $(50->60 \mathrm{~Hz})$

$0,-6,-12,-24,-36,-48 d B$
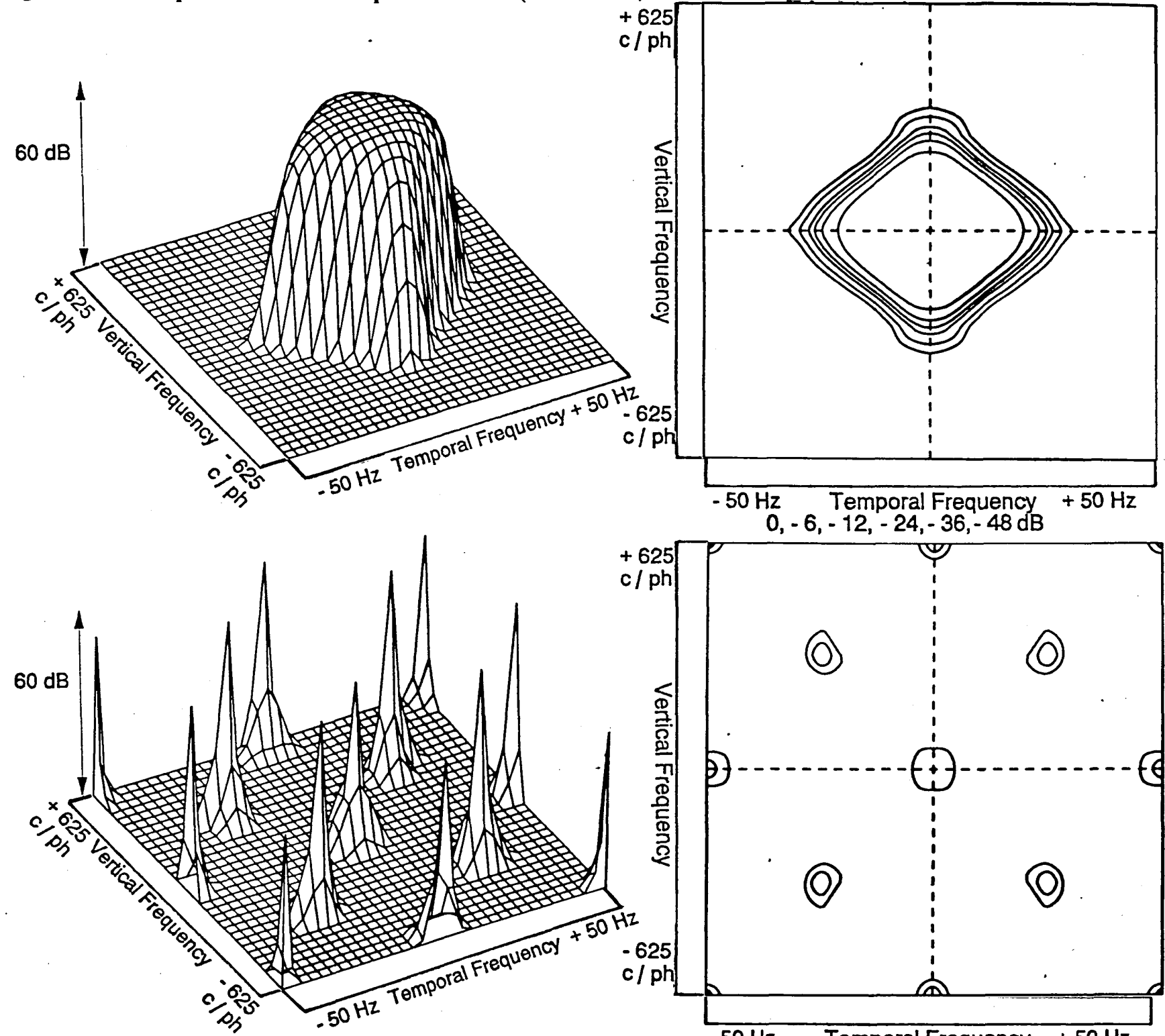

Figure 5.23 : Weighting function for deriving practical filters

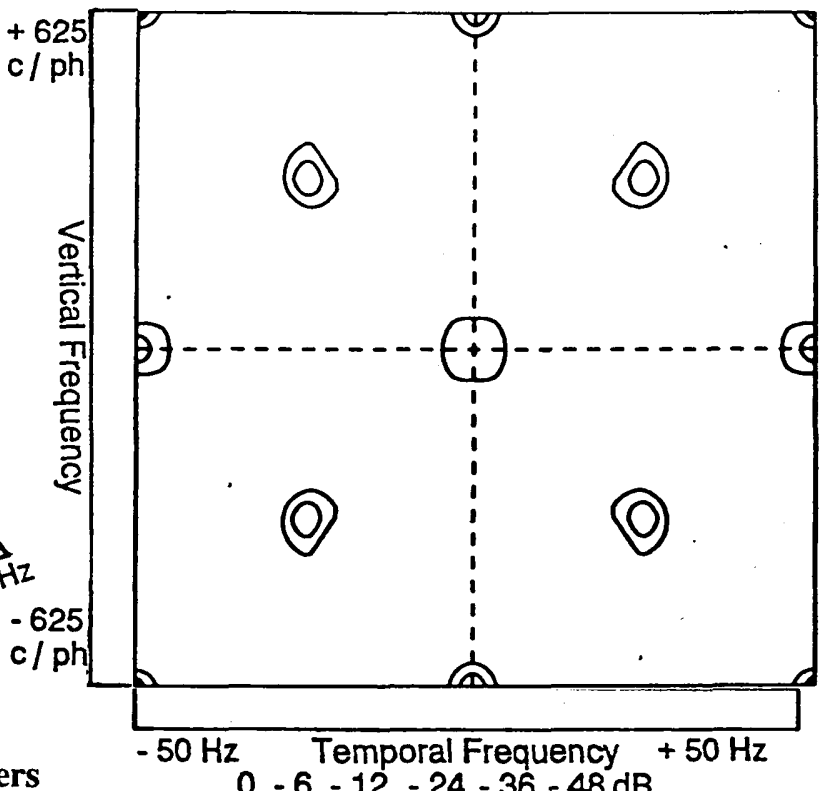
$(50->60 \mathrm{~Hz})$

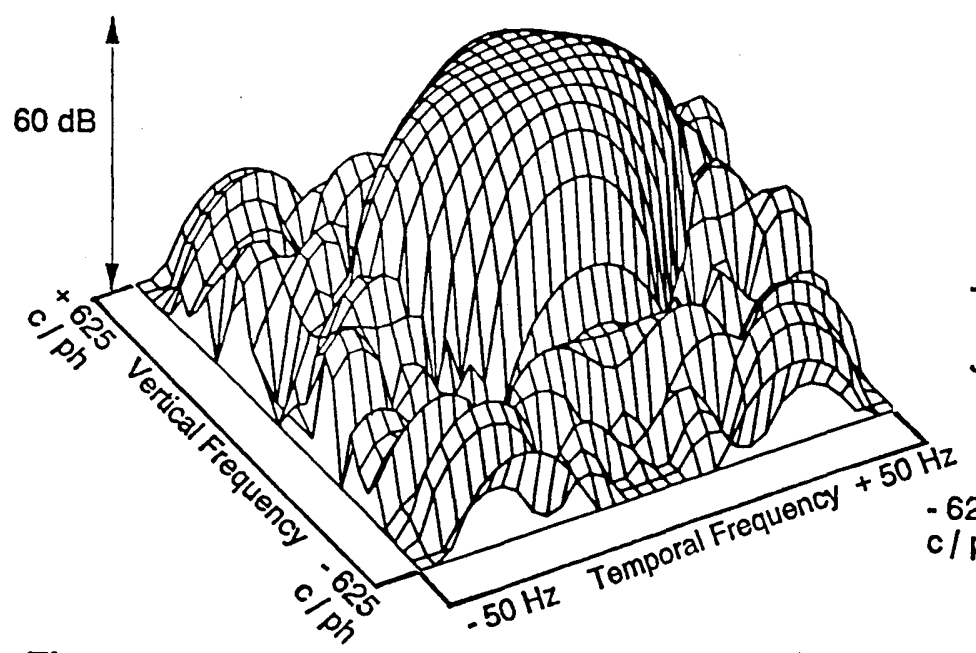

Figure 5.24 : Practical interpolation filter $(50 \mathrm{~Hz}->60)$

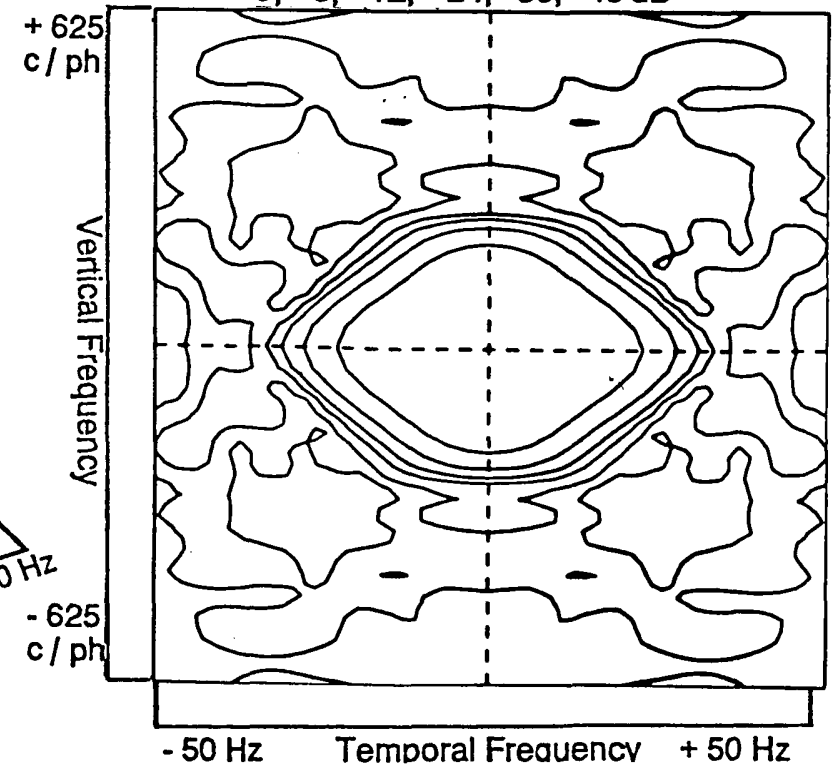



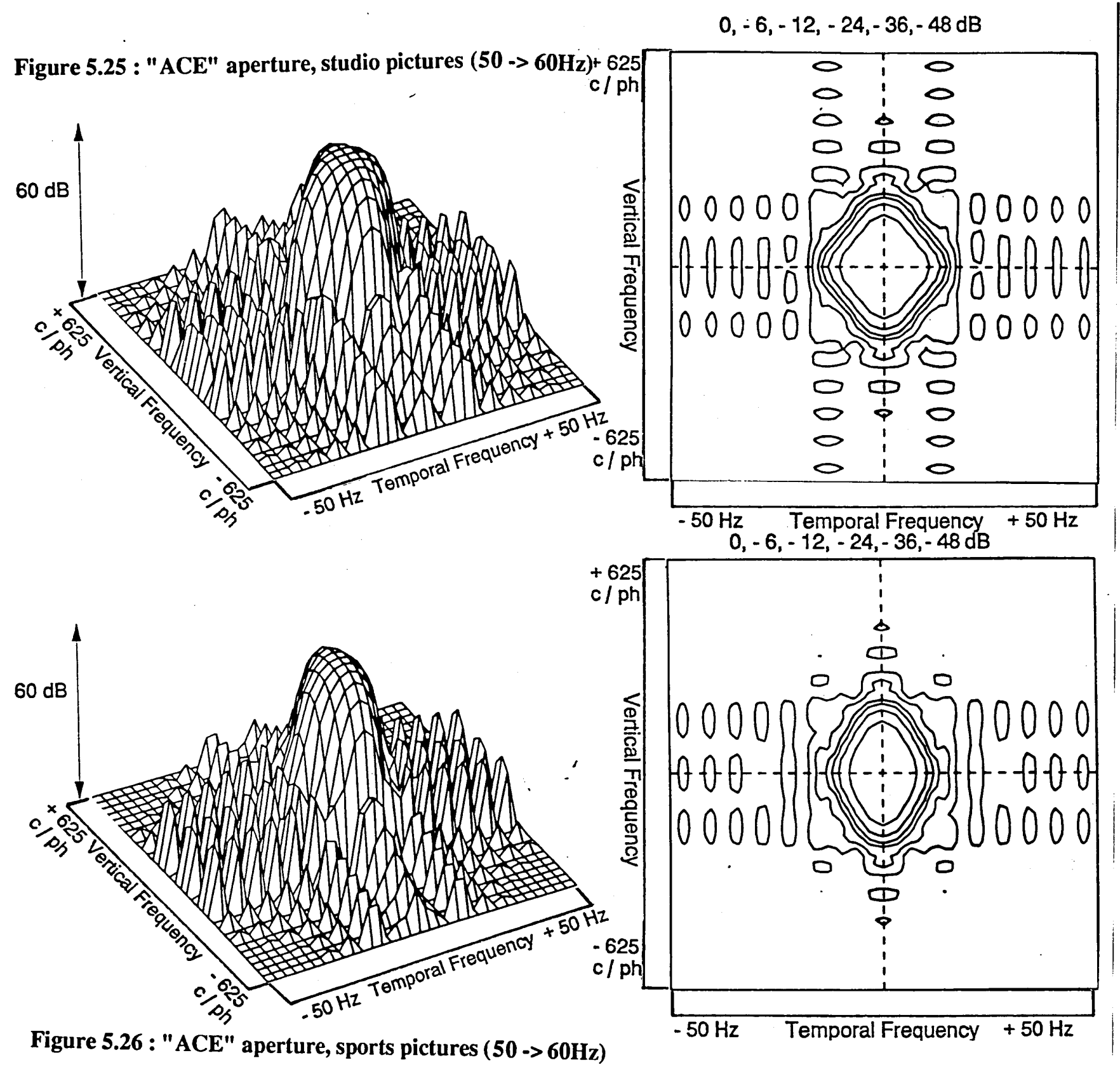


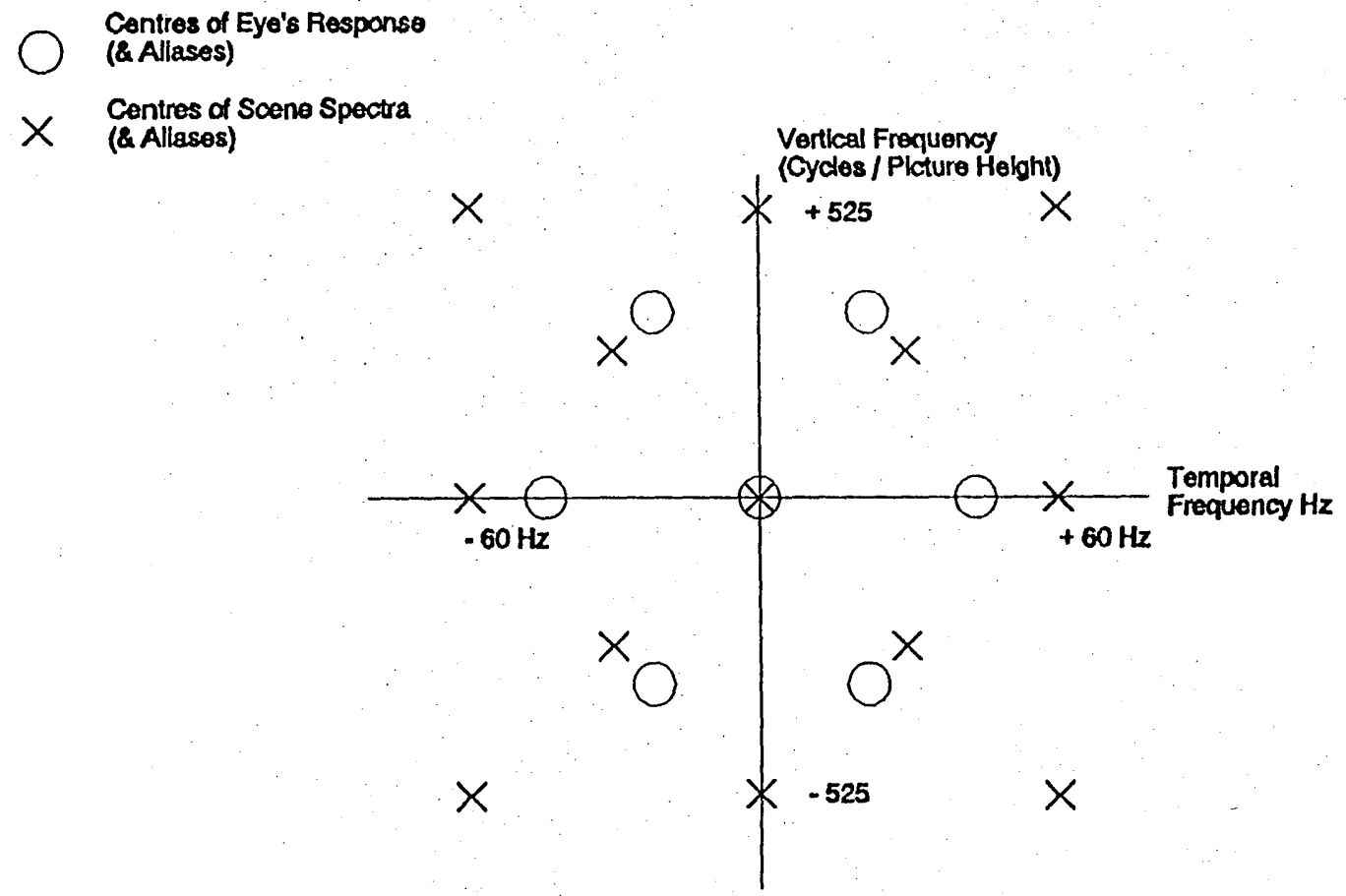

Figure 5.27: Alias Diagram for $60 \mathrm{~Hz}$ to $50 \mathrm{~Hz}$

Standards Conversion 

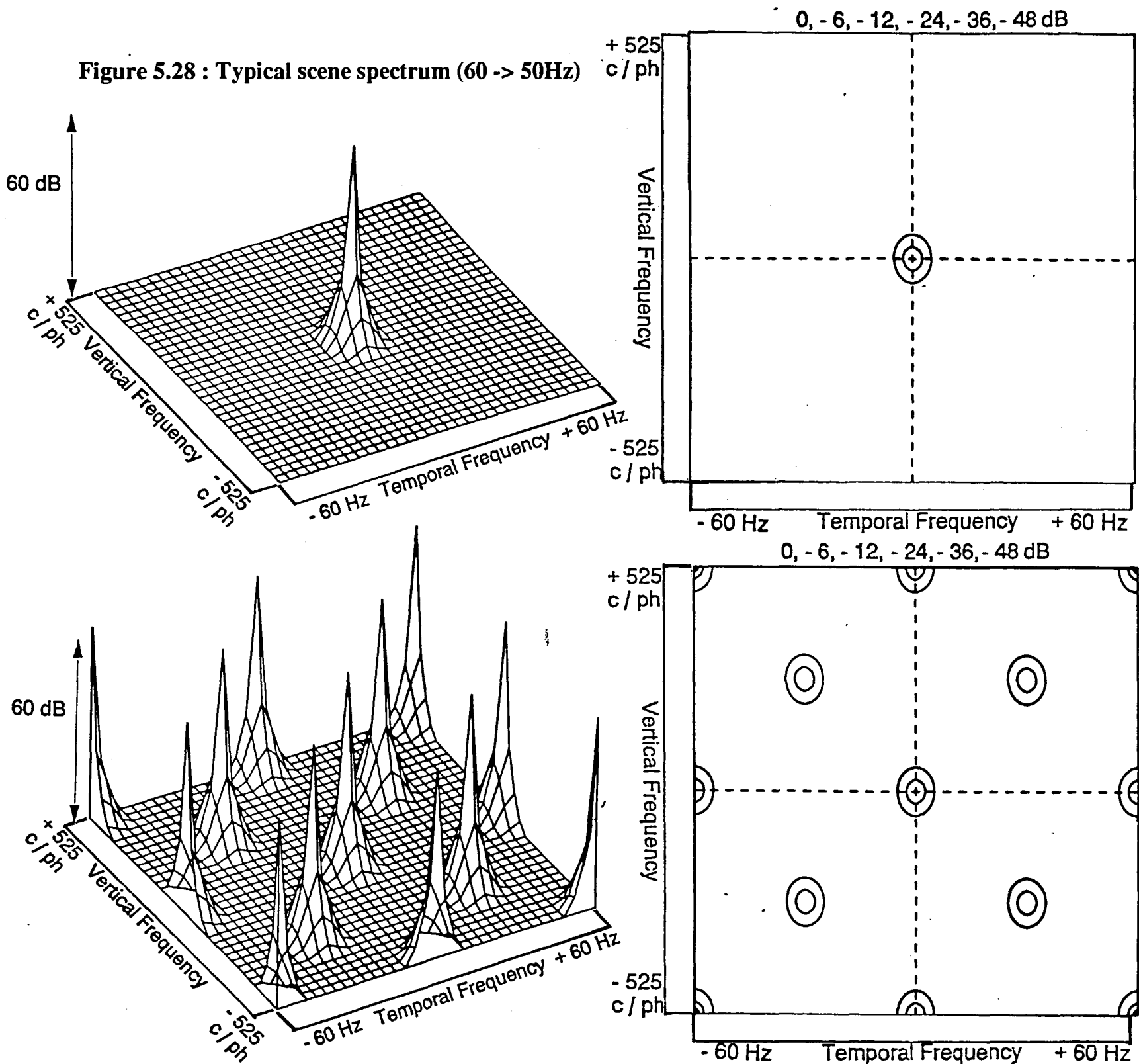

Figure 5.29 : Aliased scene spectrum $(60->50 \mathrm{~Hz})$
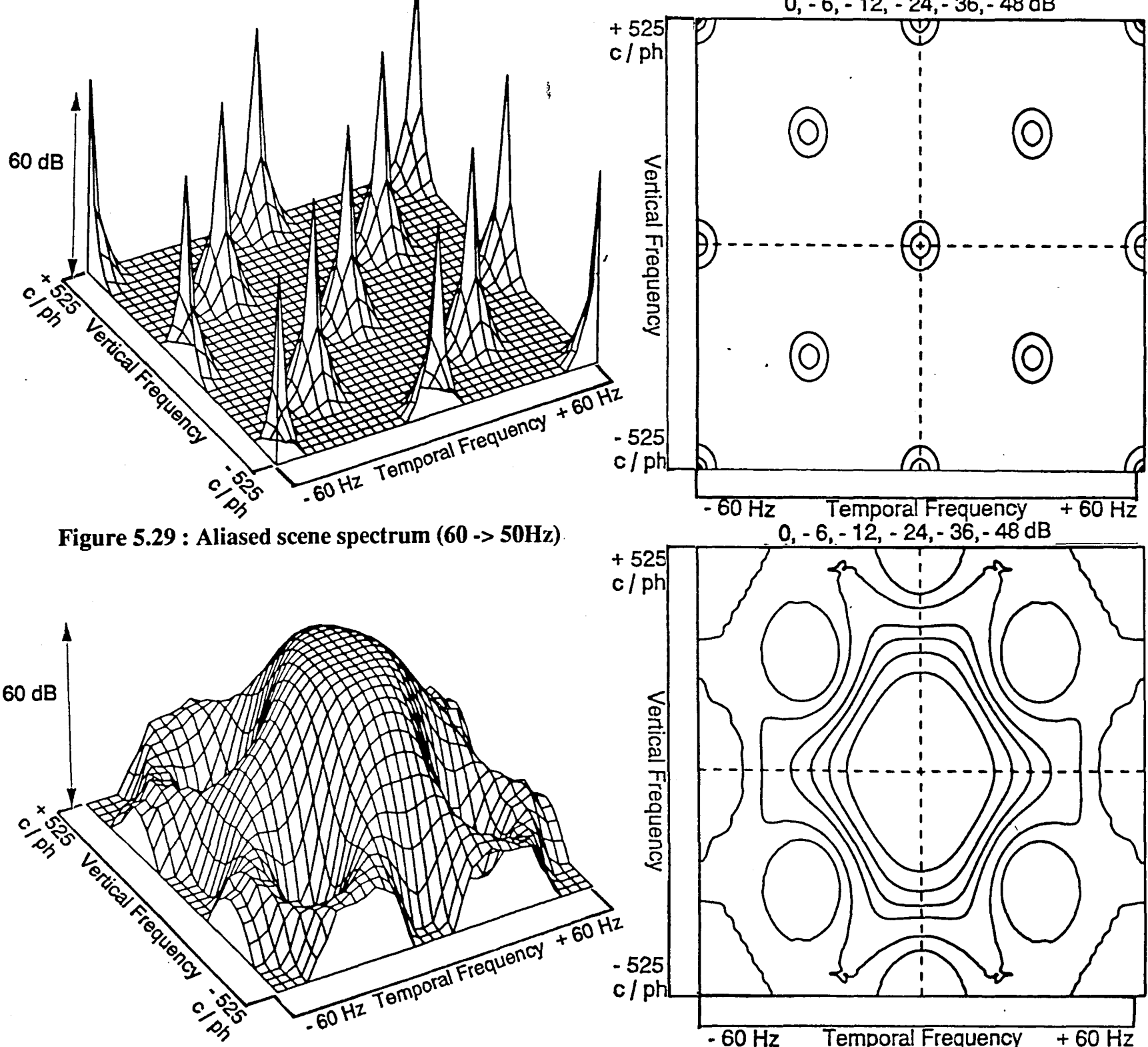

Figure 5.30 : Response of Ideal post filter $(60->50 \mathrm{~Hz})$

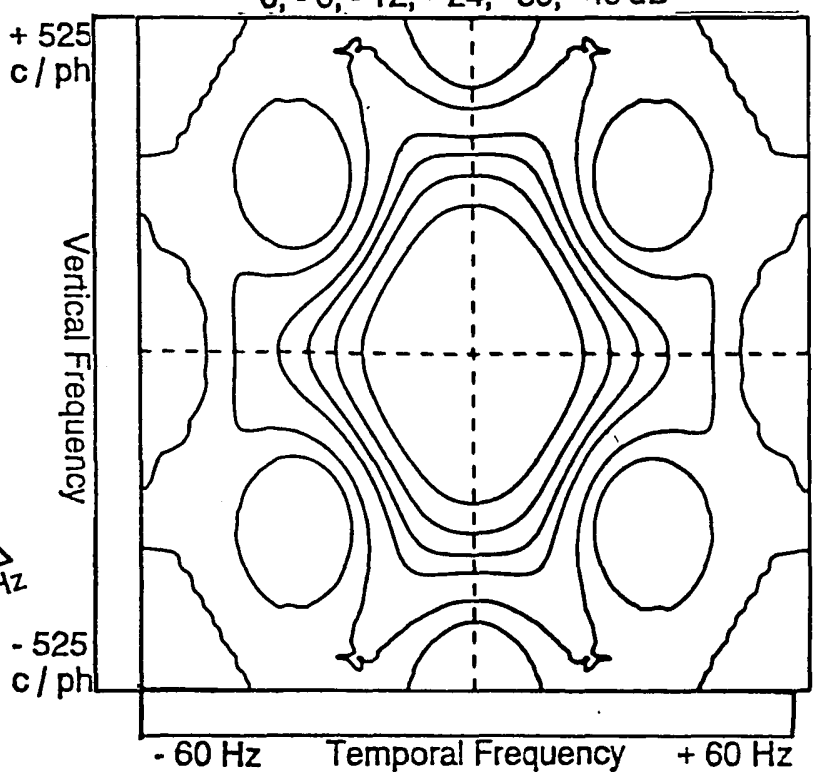



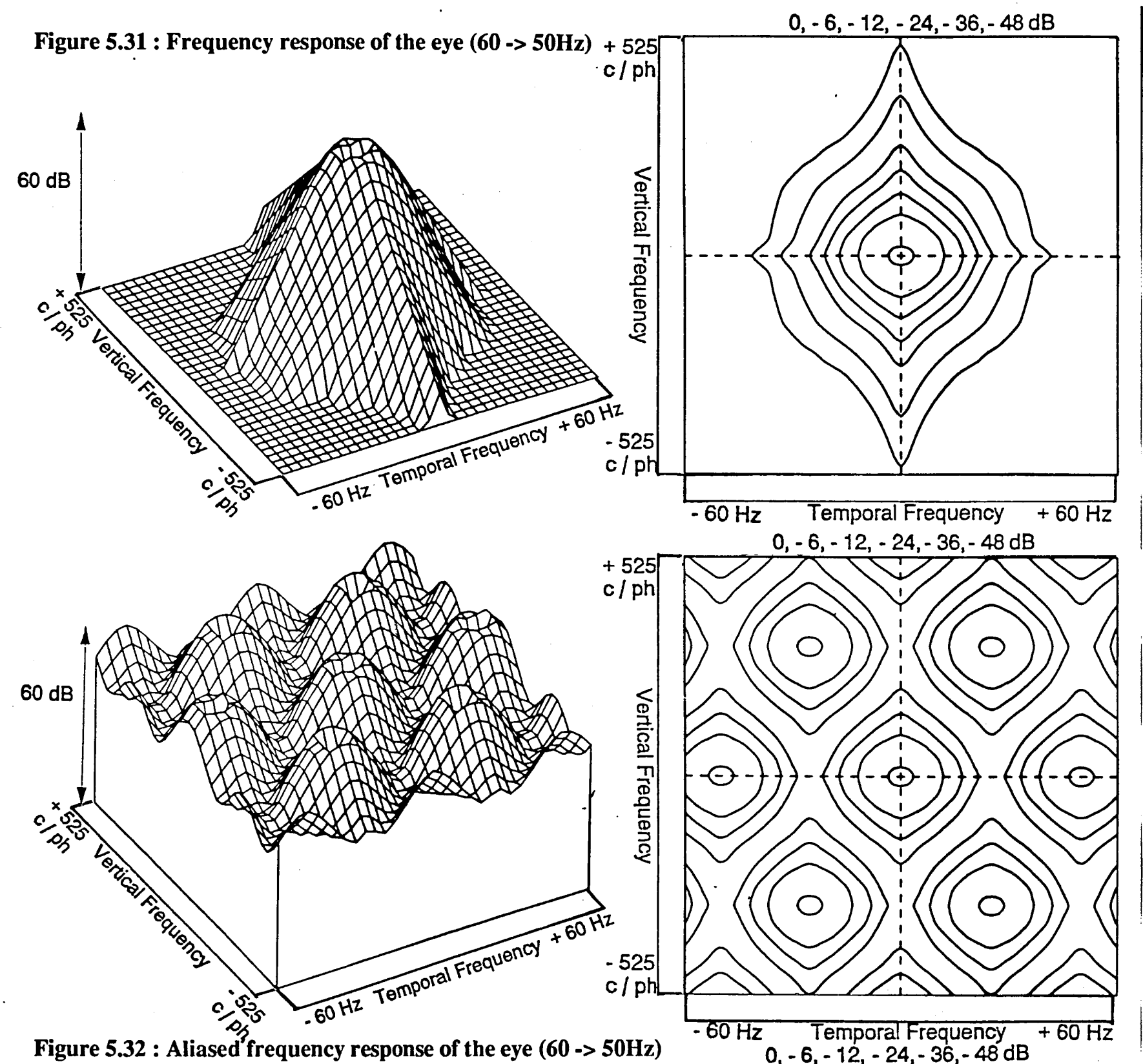

Figure 5.32 : Aliased frequency response of the eye $(60->50 \mathrm{~Hz})$
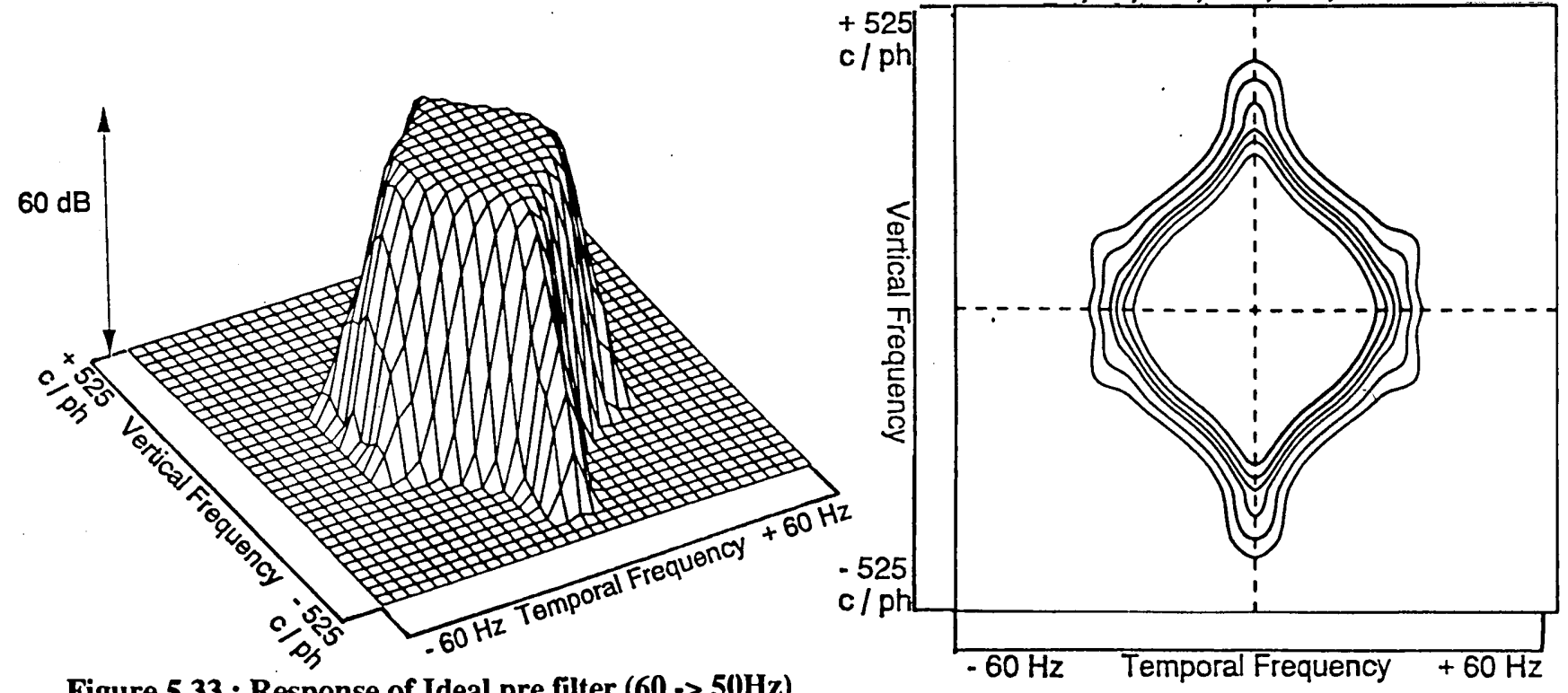

Figure 5.33 : Response of Ideal pre filter $(60->50 \mathrm{~Hz})$ 
Figure 5.34 : Response of Ideal interpolation filter

$(60$-> 50Hz)
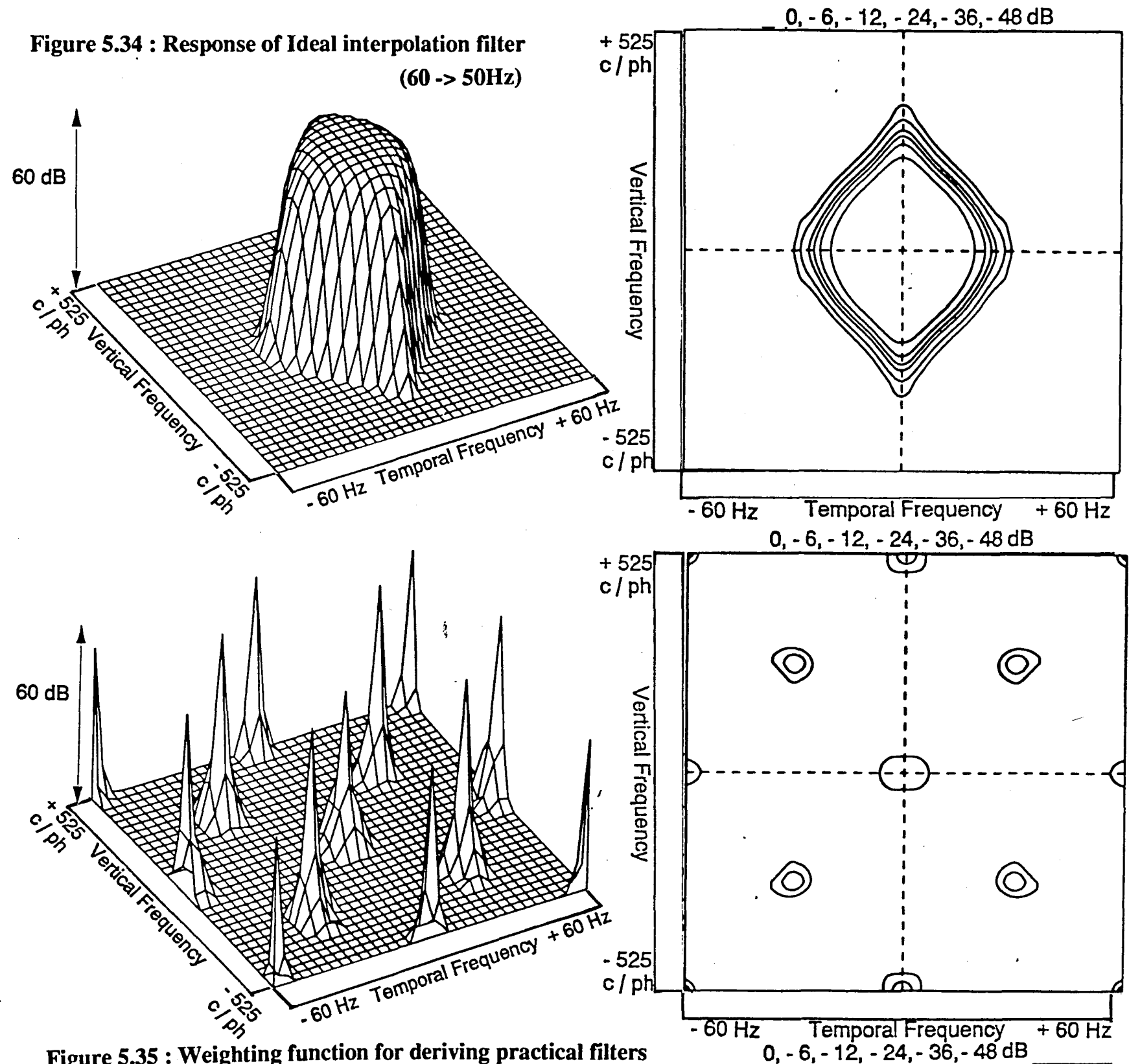

Figure 5.35 : Weighting function for deriving practical filters

$$
(60->50 \mathrm{~Hz})
$$

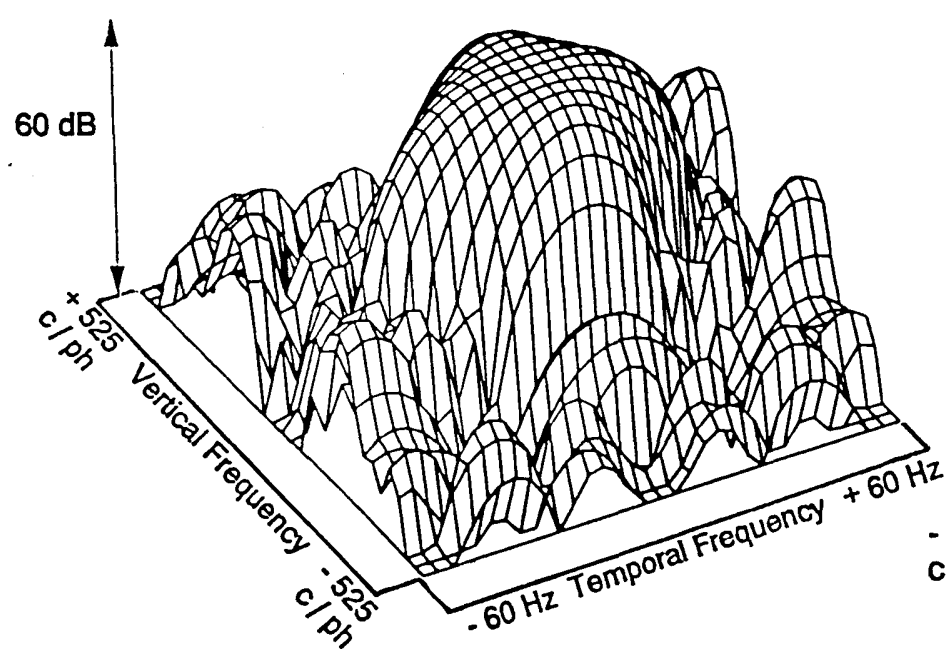

Figure 5.36 : Practical interpolation filter (60 -> 50Hz)

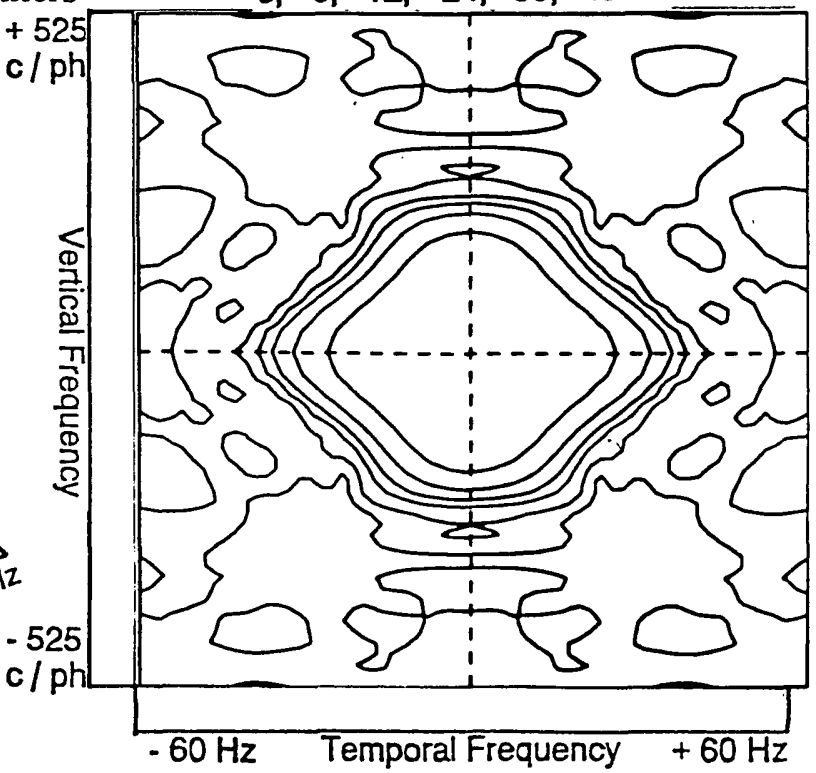




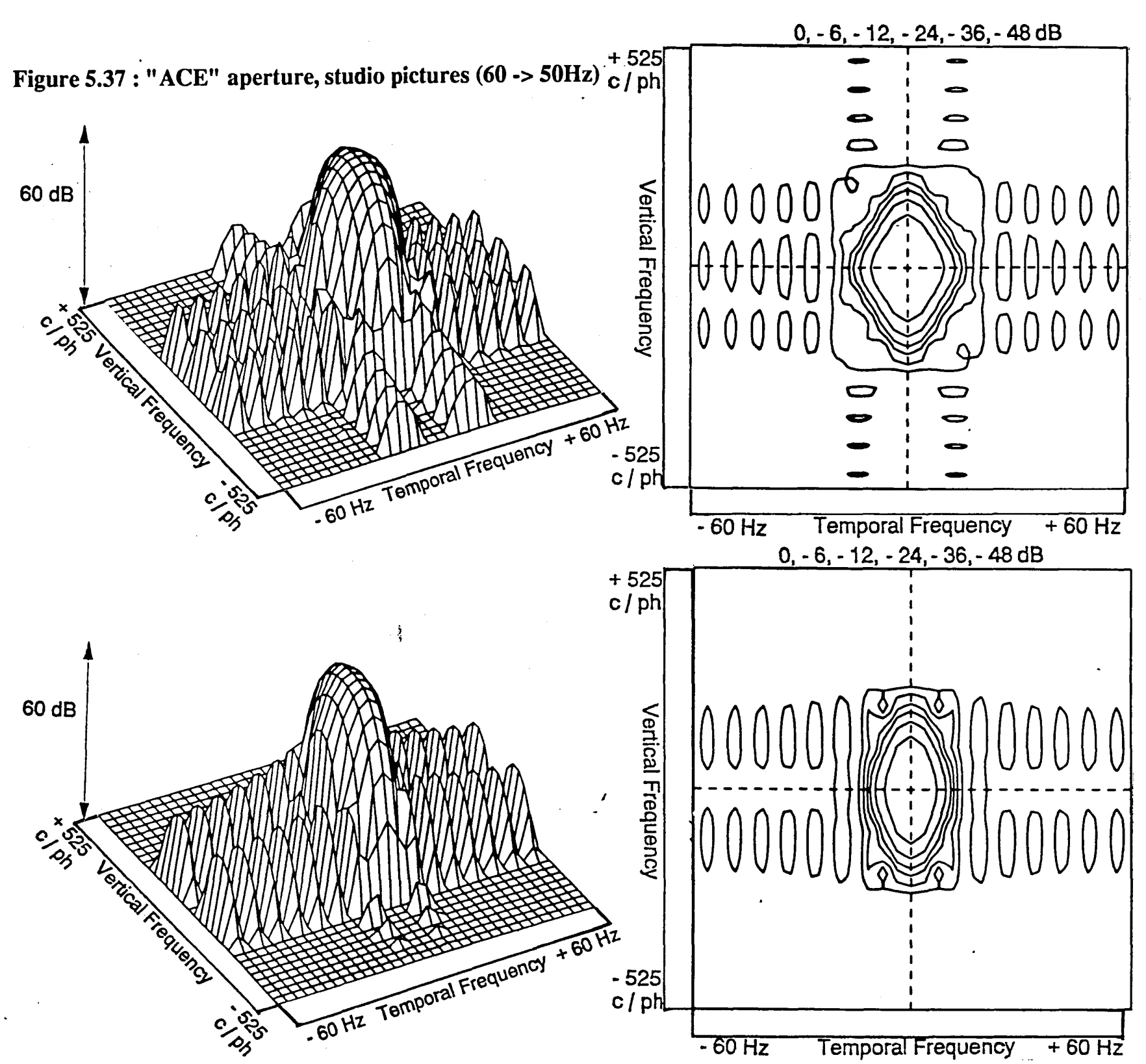

Figure 5.38 : "ACE" aperture, sports pictures $(60->50 \mathrm{~Hz})$ 


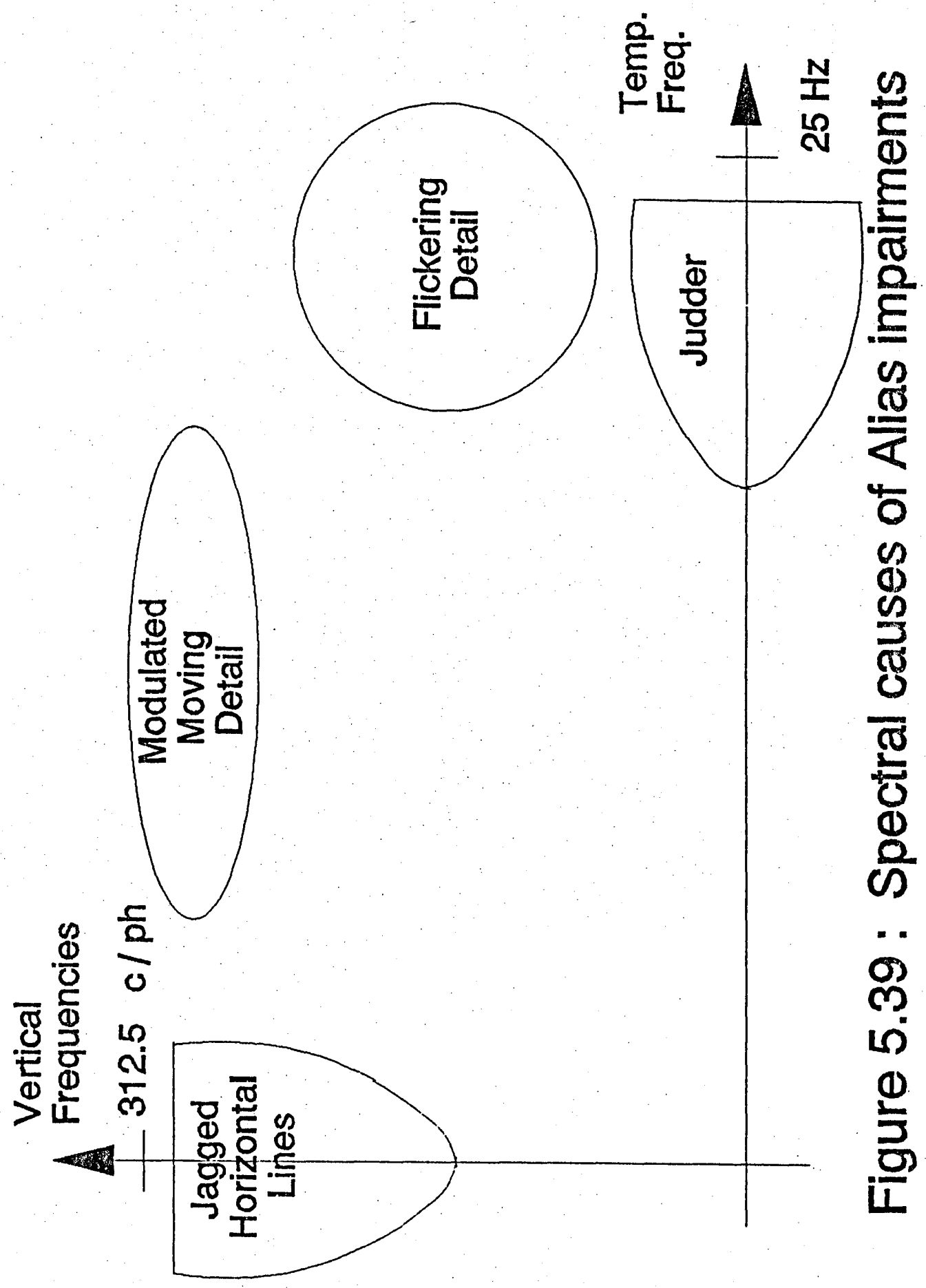




\subsection{Introduction.}

Until the late 1980's the performance of standards converters was constrained by the available semiconductor technology. This limited standards converters to interpolation using non-adaptive, linear filtering techniques. On fast moving pictures, such as sport, the impairments generated by this technique were only too obvious as judder and blurring of the pictures. As semiconductor technology improved it was possible to contemplate more complex algorithms. One of the most promising of these is the use of motion compensation.

This chapter discusses the theory of motion compensated processing. The occurrence of movement in television pictures presents great difficulties for picture processing. These arise because, considering conventional sampling theory, the input images are grossly undersampled temporally. Temporal undersampling is due to the very high spatio-temporal frequencies which can be generated by even relatively slow movement in images. Motion compensated processing provides a way of circumventing some of these difficulties.

The chapter starts by discussing the way that moving images are perceived by the human visual system (henceforth 'the eye'). It then considers how processing may be adapted to allow for the operation of the eye. This leads to the idea of motion compensated processing which is discussed in section 6.3. Using motion compensation allows the temporal interpolation of moving images without generating artifacts due to temporal aliasing. Since television images are inherently aliased, it seems a contradiction of Nyquist's sampling theorem that we should be able to interpolate them without alias artifacts. This apparent paradox is discussed in section 6.4. The chapter is intended as a general overview of the motivation for and principles of motion compensated processing. Details of the implementation are discussed in other chapters.

\subsection{The perception of motion in television pictures.}

It is curious that, although television pictures are significantly undersampled temporally, nevertheless temporal aliasing is seldom perceived by the viewer. Temporal aliasing only becomes apparent when the image has been processed in some way, such as for standards conversion. The absence of perceived temporal aliasing is due to interaction between the moving image and the eye. This interaction is because the eye is an 'active' sensor. In particular the eye moves to follow ('track') the movement of objects thereby keeping their image stationary on the retina.

The perception of television motion is, most easily, explained in the frequency domain. Fundamental to this discussion is the spectrum of a linearly moving object. Consider the 
image of an object, defined by its spatial brightness function $h(x, y)$. This object has a corresponding (spatial) spectrum $\mathrm{H}(\mathrm{m}, \mathrm{n})$. If the object moves with a constant velocity it is defined by a (spatio/temporal) brightness function, $g(x, y, t)$, given by;

$$
g(x, y, t)=h(x-u t, y-v t)
$$

where $(u, v)$ is the object's velocity. The moving object has a corresponding 3 dimensional spectrum (derived in appendix, section 6.7) given by;

$$
G(m, n, f)=H(m, n) 8(f+m u+n v)
$$

That is the moving object's spectrum lies on the plane $f+m u+n v=0$. In practice, of course, the moving object's spectrum spreads away from the plane due to slight changes in the appearance of the object (eg due to changing illumination) and non-linearities in its motion (see appendix, section 6.7). Nevertheless the spectrum of real moving objects remains concentrated about a plane.

Consider first the perception of a stationary television image. For simplicity let's assume that the camera and display introduce no loss in the signal chain. The perceived image is then due only to the object, sampling of the television signal and the eye's response. Figure 6.1 shows the horizontal/temporal spectrum for this case. The circular area is a diagrammatic representation of the frequency response of the eye. The elongated shapes represent the spectrum of the (stationary) object. Note that there are repeat spectra (aliases) caused by sampling the image (the crosses represent the centres of the repeat spectra). Parts of the object spectrum within the response of the eye are perceived by the viewer, while parts outside go unnoticed. In the case of a stationary object the eye perceives the correct spectrum. In addition, spatial and temporal aliasing may also be detected, depending upon viewing conditions. Spatial aliasing (in this example horizontal aliasing) may be perceived as the image being composed of discrete pixels rather than a continuous image. Temporal aliasing may, sometimes, be detected as flicker of the whole picture. Neither of these aliasing artifacts are particularly severe.

If an object in a television picture moves, and the eye remains stationary, then picture impairments will be perceived by the viewer. This situation is illustrated in figure 6.2 for relatively slow movement. From this figure it can be seen that the baseband part of the object's spectrum is no longer completely within the response of the eye. The part of the baseband spectrum outside the eye's response results in a loss of resolution. This is perceived as blur because the image on the retina is blurred by the object's motion. It can also be seen that parts of repeat spectra start to encroach within the eye's response. This results in aliasing, which is manifest as multiple images on the retina, caused by the combined action of 
movement and temporal sampling. Severe aliasing, perhaps from a shuttered CCD camera with a $1 \mathrm{~ms}$ integration time, may be perceived as multiple images. The severity of these picture impairments increases with the speed of motion.

Impairments of moving objects are not usually seen, provided there is no temporal processing in the signal chain. This happy state of affairs is because the eye is an active sensor and tracks the motion of the object. By doing this the object's image is kept stationary on the retina. The effect of the eye's motion is to skew its frequency response, to match the spectrum of the object, as illustrated in figure 6.3. It can be seen that when the eye tracks a moving object the loss of resolution and aliasing, which would result from a stationary eye, are avoided.

It can be helpful to view the response of eye in the frame of reference of the retina rather than the stationary television sampling structure. From this point of view, when a moving object is tracked by the eye, the image on the retina is a stationary but sampled on a moving lattice. This situation is illustrated in figure 6.4. The effect of motion, in the eye's frame of reference, is to shift the origins of the repeat spectra in proportion to the temporal sampling frequency and their spatial frequency. The arrows in figure 6.4 indicate the corresponding positions of the repeat spectra for a stationary object.

Alias artifacts due to the sampling of moving objects are seldom perceived because the eye tracks the motion of moving objects. This maintains a stationary image on the retina and minimises alias effects. The effects of temporal aliasing are usually only seen when the picture has undergone processing such as standards conversion. The interaction of the eye with a moving image and the requirements this poses for temporal sampling rates is discussed further in Tonge 1986.

\subsection{Motion compensated processing.}

By tracking the motion of objects the eye manages to avoid most of the effects of temporal aliasing in television pictures. This is despite the temporal sampling rate being far too low, using the conventional Nyquist criterion. Motion compensated processing is an attempt to emulate this feature of the eye to obtain similar freedom from aliasing when processing moving pictures.

The principle of motion compensated image processing is illustrated in figures 6.5 \& 6.6. In figure 6.5 consecutive fields are combined in a temporal filtering operation (eg for interpolation for standards conversion) using a non-adaptive filter. If there is significant movement in the scene then effect of temporal aliasing in the undersampled scene is to produce multiple images in the processed picture. If we know the motion of the objects in the scene then we can allow for it when we perform temporal processing. This is done by shifting the position of moving objects to the appropriate position for the output image before 
performing filtering (or other operations). By this means we can avoid multiple imaging (the result of aliasing) and produce an alias free output image. This is precisely analogous to tracking action of the human eye. One of the earlier suggestions for the use of this technique was by Netravali \& Robbins 1979. Since then there has been increasing interest in the technique for example Girod \& Thoma 1985, Chiariglione et al 1986, Bierling \& Thoma 1986, Marozzi \& Tubaro 1987, Borer 1987, Martinez 1987.

The fundamental basis of motion compensated processing is to process moving objects, within an image, in their own (moving) frame of reference rather than in a stationary frame of reference. This is based on the assumption that the eye tracks the motion of moving objects. Therefore, if this assumption is correct, motion compensated processing is performed in a frame of reference which is stationary with respect to the eye. Evidently since there may be multiple objects in a single image it is necessary to process different part of the image in different frames of reference. This requires that the image is segmented into regions with different motion. This is the job of motion estimation and is discussed in detail in later chapters.

It is interesting to consider motion compensated image processing in the frequency domain. Consider the process of interpolation which is central to this thesis. The filter required for this operation is the product of an input prefilter (to remove input repeat spectra) and an output post filter (to prevent the output aliasing). If the output sampling rate is very high then the interpolation filter's sole job is to remove repeat spectra in the (sampled) input signal. Figure 6.7 shows an idealised horizontal/temporal post filter passband for non-motion compensated standards conversion. This effectively separates the baseband and repeat spectra provided the object's motion is less than about 1 pixel per field period. Above this velocity the interpolated image starts to loose resolution as the signal spectrum extends outside the passband. At the same velocity temporal aliasing artifacts, such as judder, start to become apparent, as repeat spectra encroach into the passband. In a motion compensated standards converter the passband is skewed to follow the signal spectrum as shown in figure 6.8. Skewing the passband occurs as a result of allowing for the object's motion and thereby processing the image along the motion trajectory. Provided the motion of the object is correctly estimated the, motion compensated, interpolation filter aperture successfully separates baseband and repeat spectra at all velocities, thereby avoiding processing artifacts.

One of the difficulties in motion compensated processing is the problem of revealed and occluded background. As the name implies revealed background occurs when a foreground object moves and reveals the background image behind it. At the same time as revealing one part of the background image a different part of it is occluded by the moving object. In the frequency domain this is equivalent to a dramatic change in the shape of the spectrum's baseband region at the spatio/temporal boundaries of an object. The spectrum at a point in an image is a measure of the characteristics of local region surrounding that point. 
In motion compensation each object is treated differently according to its motion. Therefore the spectrum of an object should only be calculated from points within that object. Revealed/occluded background occurs at the temporal boundary of an object. Hence the spectrum of revealed/occluded background should be calculated looking only forward or only backwards in time (ie using only future or past samples). This is somewhat analogous to the use of one-sided Laplace transforms in control theory. In practice this means that the (motion compensated) filter used to process revealed background should only use future samples. Similarly the processing for occluded background should use only past samples. This implies processing which can switch between using both past and future samples and past or future samples.

\subsection{The Nyquist paradox.}

The preceding section suggests that alias free interpolation of moving scenes is possible even though the temporal Nyquist criterion for sampled systems has not been met! This paradox is a little disquieting because it suggests there may be a fallacy in the above reasoning. Fortunately there is an explanation for this apparent contradiction.

For a one dimensional signal the Nyquist criterion, to allow alias free reconstruction of a sampled signal, is that the sampling frequency must be at least twice the frequency of the signal's highest frequency component. For three dimensional image processing there is rather more flexibility in interpreting the Nyquist criterion. In 3 dimensions the product of the 3 sampling rates must be 8 (ie $2^{3}$ ) times the spectral volume of the signal. The only restriction on the shape of the baseband region is that it must tessellate to fill the entire frequency domain. Motion compensation changes the shape of the baseband region, according to the object's velocity, to give the most advantageous baseband shape for that velocity. Note that the baseband region in figure 6.8 will always tessellate to fill the frequency plane irrespective of velocity.

Changing the shape of the baseband region for motion compensation does not, itself, entirely avoid the Nyquist paradox. After all knowledge of the object's velocity has, presumably be determined from the same, aliased, input signal. At this point we have to rely on a priori knowledge of the signal. We have implicitly assumed that the scene consists of a number of linearly translating, rigid objects. By making this assumption we are able to estimate the object's velocity (see chapter 7). Given the measured velocity we can then decide what the most appropriate shape is for the baseband region of the sampled signal. Therefore, by making this assumption, we have used more knowledge of the signal than is inherent in the sampled signal itself. The use of this additional, a priori, knowledge allows us to perform better interpolation than the Nyquist criterion would imply. 
Some deviation for the strict assumption of a scene consisting of linearly translating rigid objects is permissable. If the motion is not quite linear (see appendix, section 6.7), or the object changes shape, then the spectrum will spread away from the plane corresponding to its mean motion. Providing the spread is not too large it can be accommodated within the passband of the motion compensated filter. For some types of picture, for example noise like pictures, the model of linearly moving objects does not apply and motion compensation cannot be used. For these cases we have to 'fall back' to processing which does not use a priori knowledge of the signal. For standards conversion this would probably be interpolation using a non-adaptive linear filter. Motion compensation can only be used for that subset of images which conforms to the assumption of linearly moving objects. Fortunately most television images fall into this category.

\subsection{Summary.}

This chapter has considered the motivation and principles of motion compensated image processing. By considering the reason that the human eye does not usually perceive temporal aliasing in television picture we are led to the principle of motion compensation. This principle is that images of moving objects are processed in their own moving, rather than a stationary, frame of reference. That is processing is performed along an object's motion trajectory.

The use of motion compensation rests on several underlying assumptions. It is assumed that the eye tracks the motion of moving objects. This is usually true, but when this assumption fails we cannot obtain the subjective benefits of motion compensation. It is also assumed that the image consists approximately of linearly translating rigid objects. Motion compensation might, therefore, be considered as a form of model based image processing. The model is, however, highly general and non-specific. When the model of moving objects fails we cannot use motion compensation and must fall back to another processing algorithm. Arguably, however, motion that does not correspond to the model is less accurately perceived by the eye, and hence deficiencies in the signal processing are less serious. Fortunately motion compensation seems to be applicable to most television pictures.

The discussion in this chapter has highlighted the principles and possible benefits of motion compensation. The principal benefit is freedom from temporal aliasing artifacts in spite of television's relatively low temporal sampling rate. this promises significant improvements in the quality of standards conversion for fast moving pictures. These benefits are contingent on the ability to accurately determine the motion of objects in a moving image. This is a difficult and complicated subject and much of the research in motion compensation has concentrated on the problem of motion estimation. The problem of motion estimation is discussed in detail in the next chapter. 


\subsection{References.}

1. Bierling, M., Thoma, R. 1986. Motion compensating field interpolation using a hierarchically structured displacement estimator. Signal Processing, Volume 11, No. 4, December 1986, pp. 387-404. Elsevier Science Publishers.

2. Balanza, M. \& Cortelazzo, G.M. 1990. On the effects of acceleration in the frequency domain. Signal Processing of HDTV, II. Proceedings of the third international workshop on HDTV, Turin, Italy, 30th August - 1st September 1989, Chiariglione, L. (editor), Elsevier science publishers, ISBN 044488833 0, pp 879-885.

3. Borer, T.J. 1987. The use of Motion Vectors for Broadcast Applications in Television. I.E.E. Colloquium on 'Motion Compensated Image Processing'. Friday October 23 1987.

4. Chiariglione, L., Corgnier, L. and Guglielmo, M. 1986.Pre- and Post-Processing in a Video Terminal using Motion Vectors. 1986. I.B.C. Brighton 1986.

5. Girod, B. \& Thoma, R. 1985. Motion-compensating field interpolation from interlaced and non-interlaced grids. 2nd International Technical Symposium on Optical and Electo-Optical Science and Engineering: Image Processing Symposium, Cannes, December 1985.

6. Marozzi, E., Tubaro, S. 1987. Motion compensated interpolation. SPIE Vol. 804, Advances in image processing, p61-68.

7. Martinez, D.M. 1987. Model-based motion estimation and its application to restoration and interpolation of motion pictures. RLE Technical Report No. 530. June 1987. Research Laboratory of Electronics, Massachusetts Institute of Technology, Cambridge, MA 02139 USA.

8. Netravali, A.N., Robbins, J.D. 1979. Motion compensated television coding, Part 1. Bell Syst. Tech. J., vol. 58, pp. 631-670, March 1979.

9. Tonge, G.J. 1986. Time sampled motion portrayal. Proceeding of the second international conference on 'Image processing and its applications', Imperial College of Science and Technology, London, 24-26 June 1986, organised by the I.E.E., pp 216-219.

6.7. Appendix: The spectrum of a moving object.

Fundamental to the analysis of the perception of the eye and to the principles of motion compensation, is the frequency domain analysis of moving images. Therefore this appendix derives the form of the spectrum of images of moving objects. 
Assume that we have an object whose image is defined by the brightness function $h(x, y)$. If the object moves, a 3 dimensional brightness function, $g(x, y, t)$ is generated. Then $\mathrm{g}(\mathrm{x}, \mathrm{y}, \mathrm{t})$ is given by;

$$
g(x, y, t)=h(\alpha(x, y, t), \beta(x, y, t))
$$

where $\alpha$ and $\beta$ are the coordinates of a point on the object. Let us assume motion depending on time with a polynomial law so that;

$$
\begin{aligned}
& \alpha(x, y, t)=x-\sum_{k=1}^{K} u_{k} t^{k} \\
& \beta(x, y, t)=y-\sum_{k=1}^{K} v_{k} t^{k}
\end{aligned}
$$

(this assumes $\alpha \& \beta$ are zero at $\mathrm{t}=0$ ). Then the three dimensional brightness function of the moving object is given by;

$$
g(x, y, t)=h\left(\left(x-\sum_{k=1}^{R} u_{k} t^{k}\right),\left(y-\sum_{k=1}^{R} v_{k} t^{k}\right)\right)
$$

If $\mathrm{K}=1$ we have linear motion, for $\mathrm{K}=2$ we have accelerating motion and for higher values of $\mathrm{K}$ we have more complex motion.

Given the two dimensional spectrum of the object, defined by;

$$
H(m, n)=\mathscr{F}(h(x, y))=\int h(x, y) \exp (-j 2 \pi(m x+n y)) d x d y
$$

we can determine the 3 dimensional, spatio-temporal, spectrum of the moving object. This is defined by;

$$
G(m, n, f)=\mathscr{F}(g(x, y, t))=\int g(x, y, t) \exp (-j 2 \pi(m x+n y+f t)) d x d y d t
$$

Substituting equations $3 \& 4$ in 7 gives;

$$
G(m, n, f)=\int h\left(\left(x-\sum_{k=1}^{K} u_{k} t^{k}\right),\left(y-\sum_{k=1}^{K} v_{k} t^{k}\right)\right) \exp (-j 2 \pi(m x+n y+f t)) d x d y d t
$$

Substituting $\alpha \& \beta$ from equation 4 above allows $G(m, n, f)$ to be rewritten as;

$$
G(m, n, f)=\int h(\alpha, \beta) \exp (-j 2 \pi(m x+n y)) d x d y \int \exp \left(-j 2 \pi\left(\sum_{k=1}^{K}\left(m u_{k}+n v_{k}\right) t^{k}+f t\right)\right) d t
$$


That is;

$$
G(m, n, f)=H(m, n) \delta\left(f+m u_{1}+n v_{1}\right) \star C_{2}(m, n, f) \star C_{3}(m, n, f) \ldots \star C_{K}(m, n, f)^{(10)}
$$

where the star represents the operation of convolution.

If we have linear translatory motion then $K=1$ and;

$$
G(m, n, f)=H(m, n) 8(f+m u+n v)
$$

that is the three dimensional spectrum of the object lies in a plane. For more complex type of motion $\mathrm{K}>1$ and the convolving factors $\mathrm{C}_{2}$ to $\mathrm{C}_{\mathrm{K}}$ spread the spectrum away from a single plane. Balanza \& Cortelazzo 1990 have derived expressions for convolving factors $C_{2}$ and $C_{3}$. The expression for $\mathrm{C}_{2}$;

$$
C_{2}(m, n, f)=\left\{\begin{array}{cc}
\delta(f) & ; m u_{2}+n v_{2}=0 \\
\frac{\exp \left(-j \frac{\pi}{4} \operatorname{sign}\left(m u_{2}+n v_{2}\right)\right) \exp \left(\frac{j \pi f^{2}}{m u_{2}+n v_{2}}\right)}{\sqrt{m u_{2}+n v_{2}}} & ; m u_{2}+n v_{2} \neq 0
\end{array}\right.
$$

is interesting because it shows that the effect of accelerated motion is to spread the energy, in the moving object's spectrum, away from a single plane along the temporal frequency axis. 


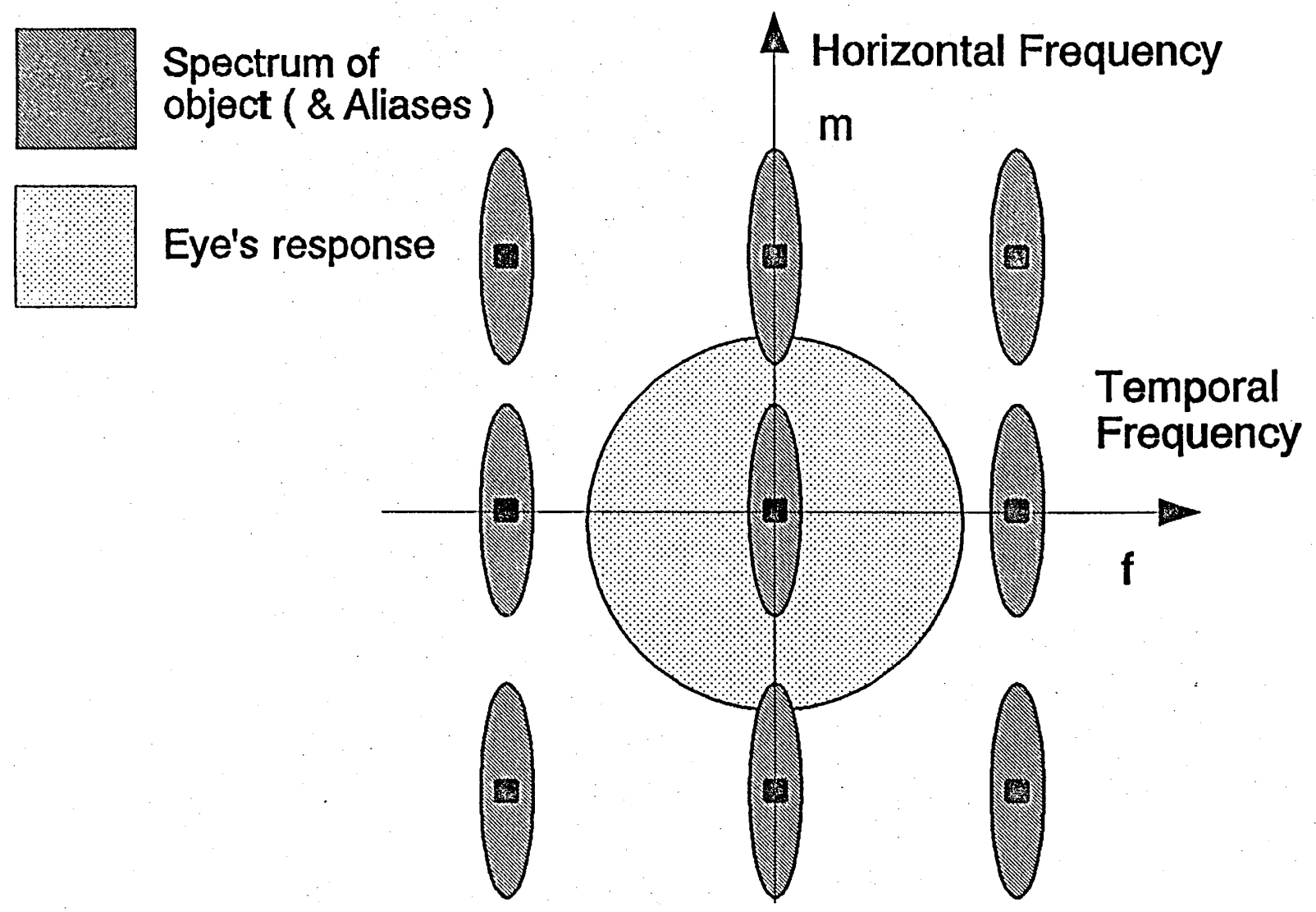

Figure 6.1 : Response of a stationary eye to a stationary object

Spectrum of object ( \& Aliases )
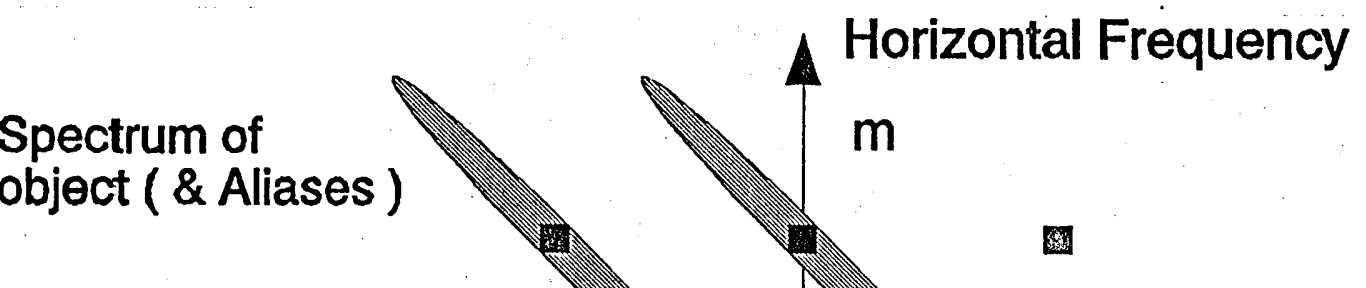

Eye's response

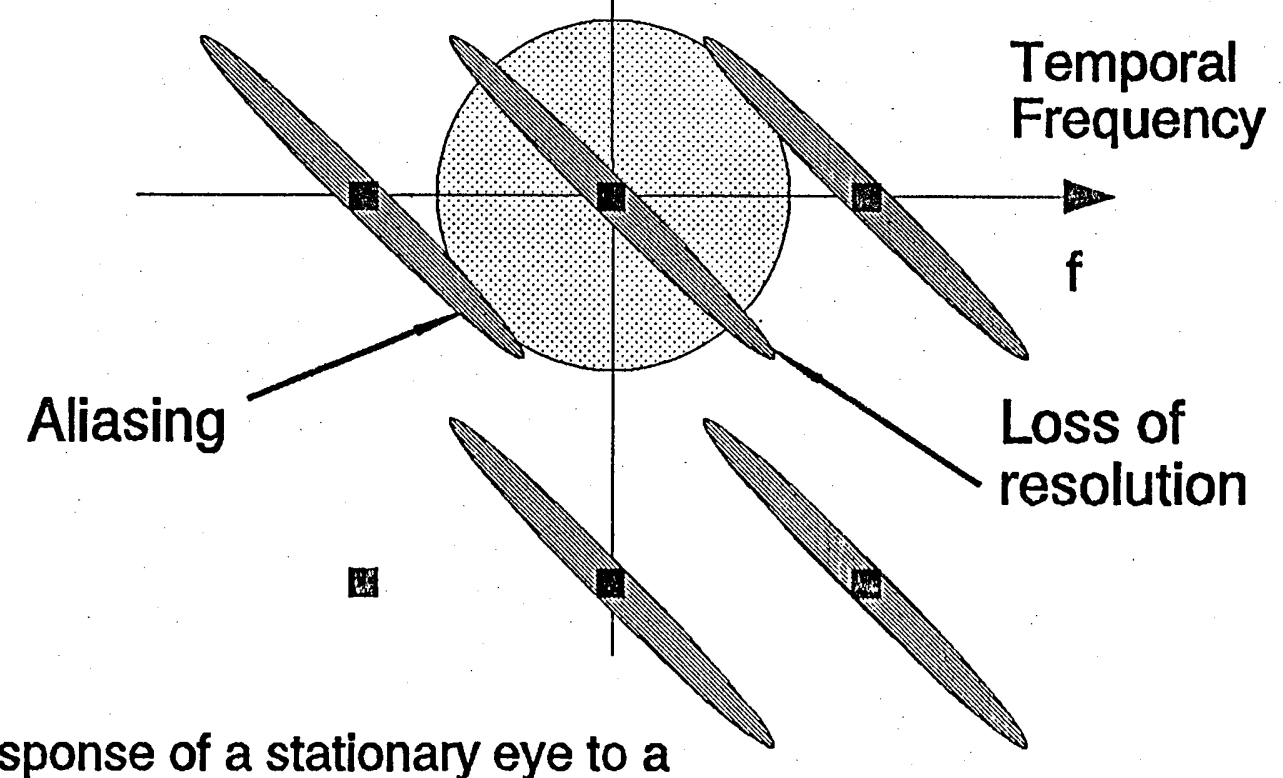

Figure 6.2: Response of a stationary eye to a moving object 
Spectrum of object ( \& Aliases )

\section{$\triangle_{\text {Horizontal Frequency }}$}

\section{)}

Eye's response

Figure 6.3 : Response of a tracking eye to a

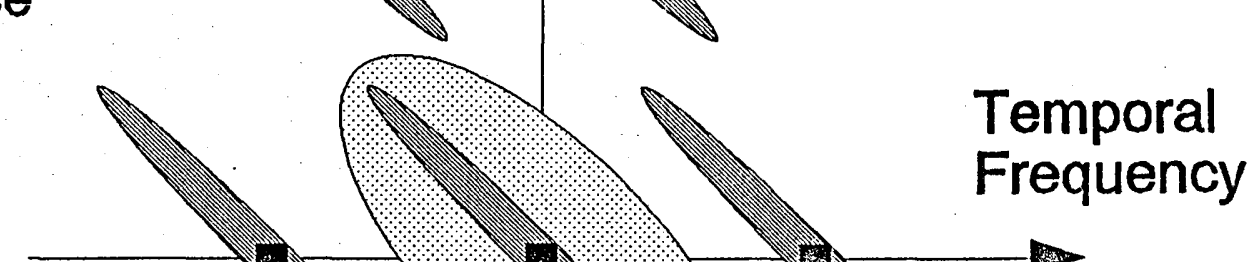
moving object

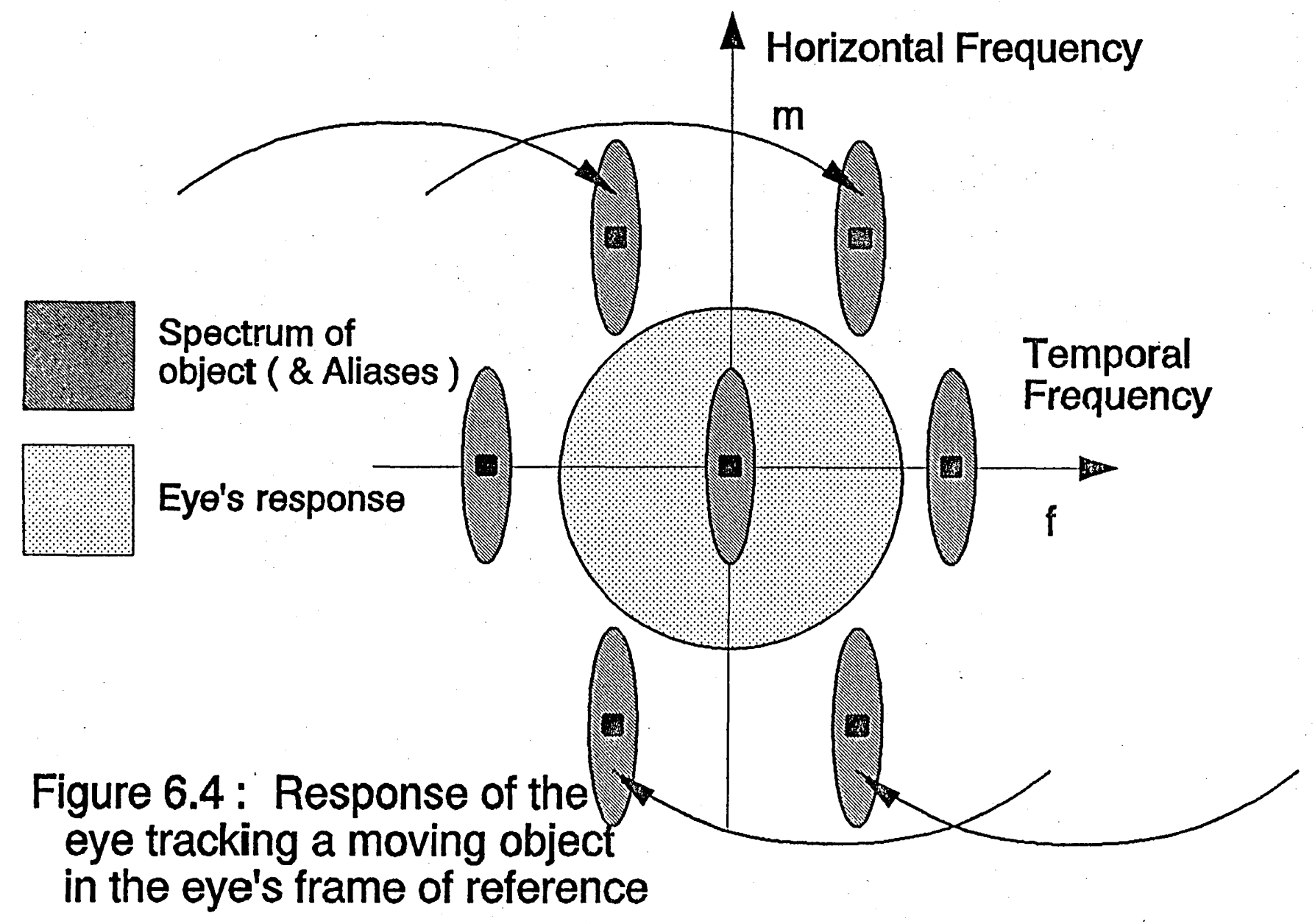


Field 1

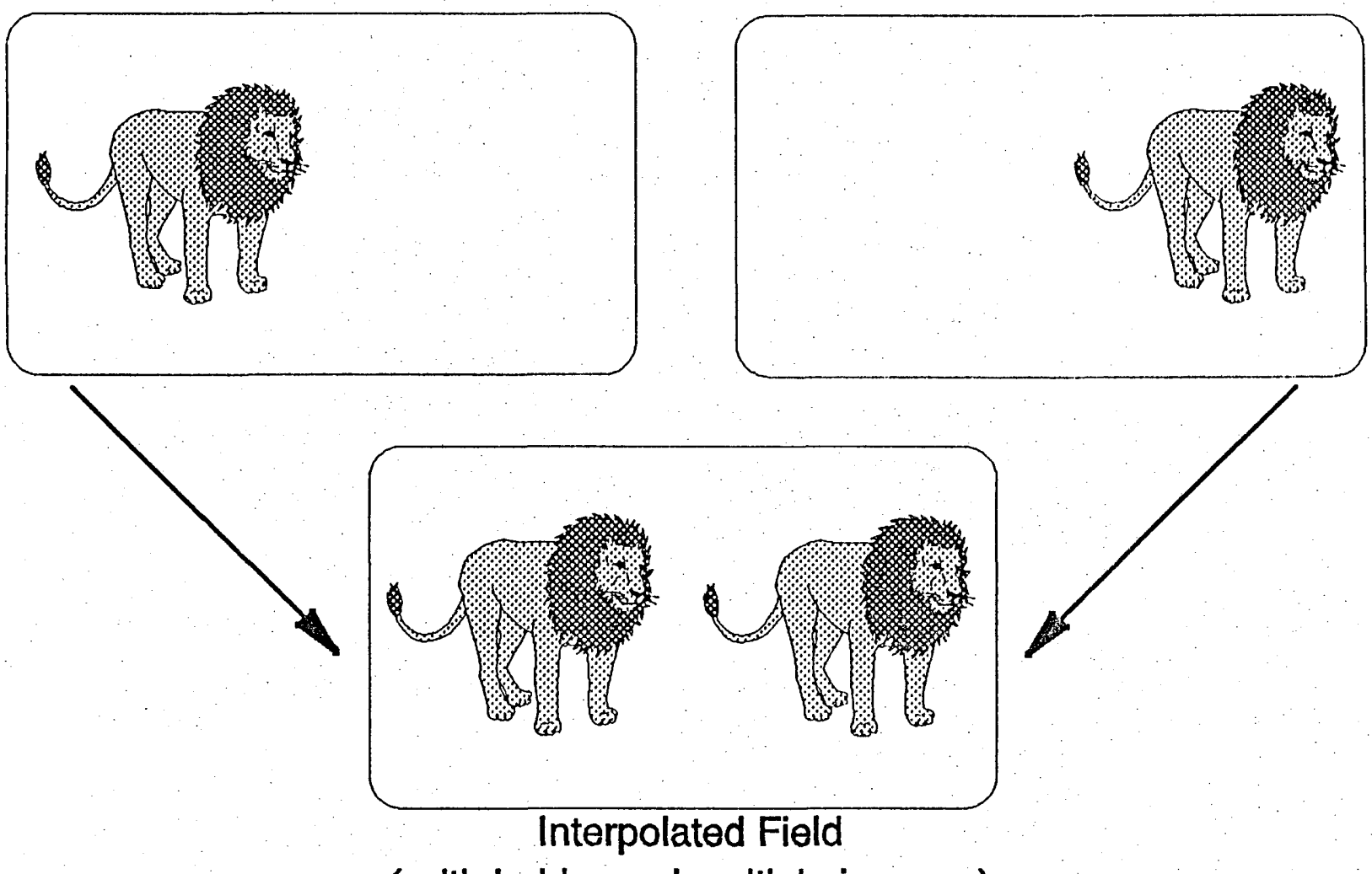

( with judder and multiple images )
Field 1

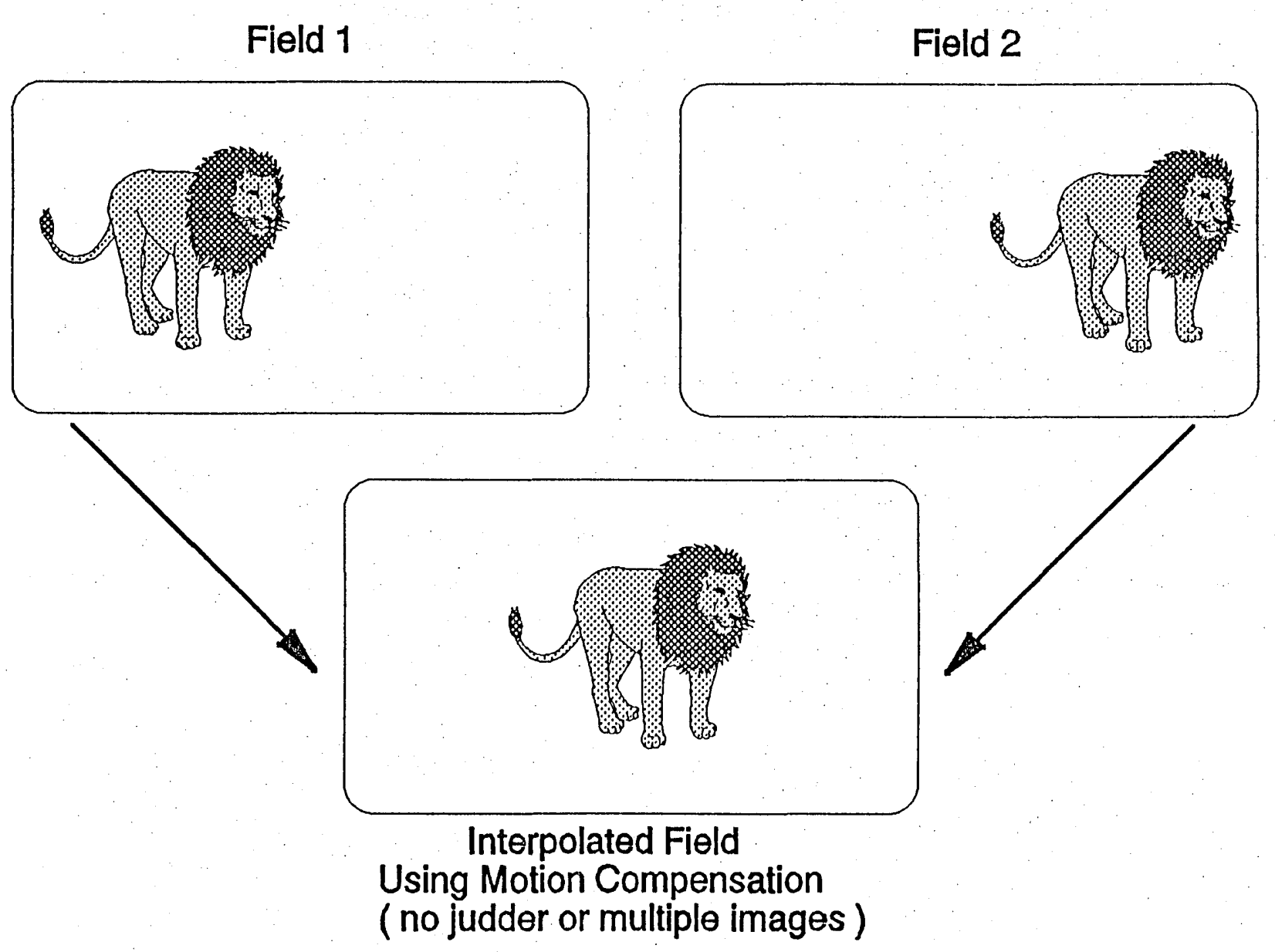

Field 2

Figure 6.5: Conventional Standards Conversion

Figure 6.6: Motion compensated Standards Conversion 


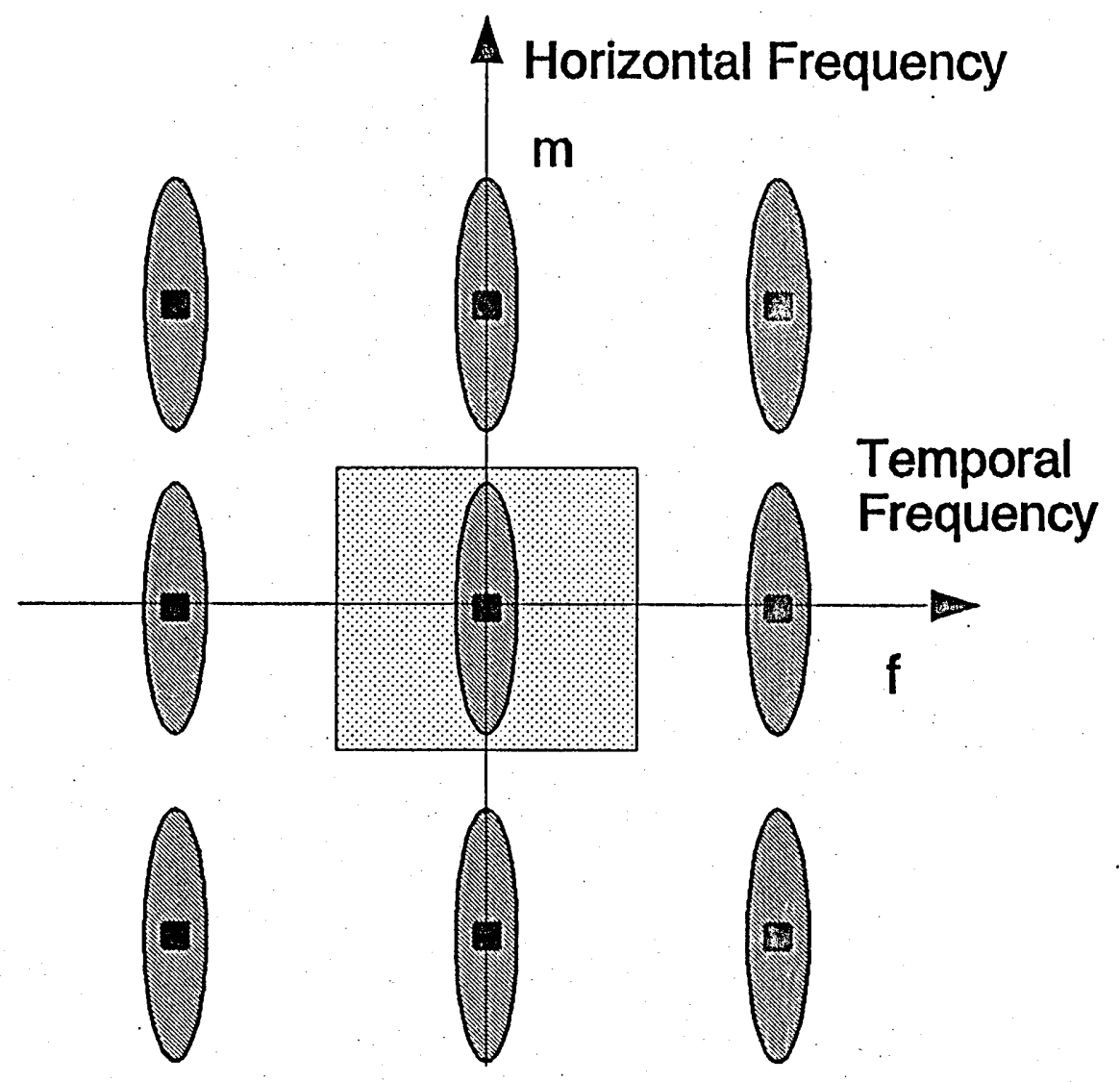

Figure 6.7: Idealised passband of non-motion compensated standards converter

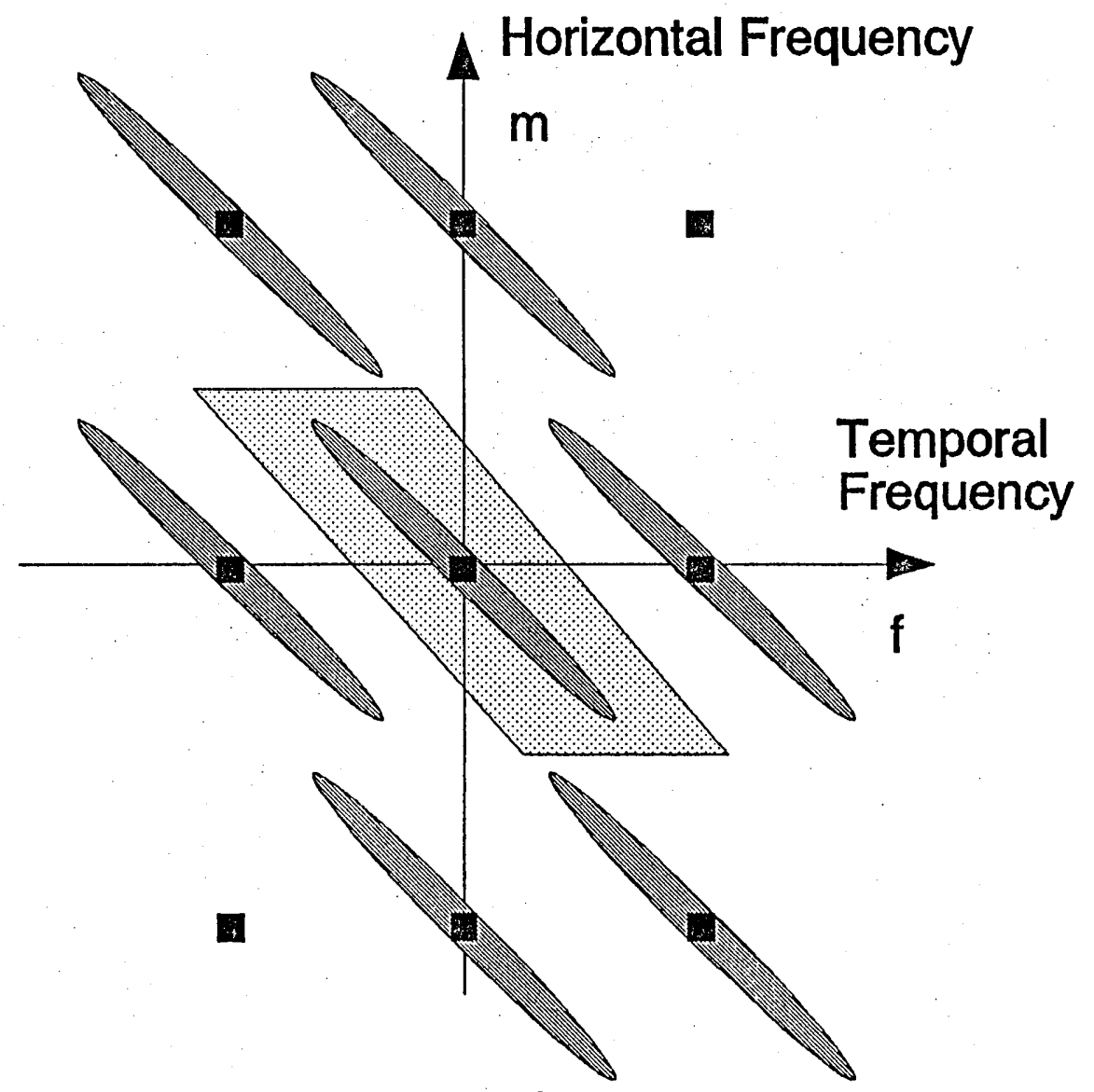

Figure 6.8 : Passband of a motion compensated standards converter for a slowly moving objest. 


\subsection{Introduction.}

This chapter presents a review of the subject of motion estimation, followed by the details of an experimental investigation. The chapter starts with a discussion of the requirements and the problems of motion estimation in general, and for standards conversion in particular. This is followed by a review of motion estimation algorithms previously published in the literature. The published algorithms are reviewed and assessed for their suitability for motion compensated standards conversion. Two algorithms, phase correlation and motion correlation, were selected for further investigation by computer simulation. The results from the simulation of phase correlation confirms previous results and extends these to indicate the performance that might be achieved in a real time system with typical pictures. Motion correlation appears to be a new technique of motion estimation. Comparison of simulation results suggests that motion correlation may give better performance that phase correlation, albeit at the cost of additional complexity.

Motion estimation is the process of constructing a description of an environment, in terms of objects and their movement through space, from an image sequence. It is worth stressing that motion information cannot simply be computed from the image data since, even under ideal conditions, there may be many motion fields which are compatible with the data. The problem of motion estimation, therefore, is to find an appropriate motion field, suitable to the application at hand. It might be assumed that the 'best' motion field would be that closest to the true motion field. This may not be the case for standards conversion. The human visual system 'expects' to see motion that is compatible with real world motion. Thus a motion field which is somewhat in error, but realistic, may be preferable to one which is closer to the true motion, but inconsistent with the real world. Furthermore care must be taken in choosing a definition of 'closest' to the true motion. Some motion estimation algorithms can be shown to converge to the true motion on average. However if the variance of the motion estimate is high, the motion field will be disjointed and unrealistic. This type of motion estimator would be unsuitable for standards conversion.

Many motion estimation algorithms have been derived for a variety of applications. It seems to be a common misconception that any working motion estimator can produce a motion field which would be equally suitable for many different applications. In fact motion estimators are usually tailored to a specific application and require modification for different uses. There are three common applications for motion estimators, image processing (including 
standards conversion), image analysis (machine vision etc) and bitrate/bandwidth reduction ${ }^{1}$. These applications have different requirements. Hence, an algorithm which is less suitable for standards conversion is not necessarily a 'bad' algorithm, merely inappropriate for that specific application.

To discuss motion estimation some terminology must be defined and considered. The velocity field may be defined as the instantaneous velocity of a point, in the image plane, as a function of position and time (ie. $\mathbf{v}(x, y, t)$ ). These velocities are related, through a perspective transformation, to the velocities of objects in the original ( 3 dimensional) scene. For temporally sampled images (as in television) a function related to the velocity field is the displacement field. This establishes a correspondence between points in the current frame and points in either the previous or subsequent frame. These two displacement fields may be referred to as the backwards and forward displacement fields respectively (eg Cafforio 1990). The displacement field is only defined for a specific pixel if the corresponding pixel exists in the previous or subsequent frame. The forward and backward displacement field are thus different, since backward displacement is not defined for regions which have been newly revealed, and forward displacement is not defined for regions about to be obscured. It is usually the displacement, rather than the velocity, field which is used for motion compensated image processing. The difference between the forward and backward displacement fields is quite significant since it is directly related to regions of revealed or obscured background. The more general term motion field can refer to either a velocity or a displacement field.

Consider the nature of the velocity field. The projection of each point in a (3 dimensional) scene traces a trajectory in the image plane, as long as it remains visible in the image. The collection of all motion trajectories defines the motion of the image. This collection of the motion trajectories is sometimes known as the 'optic flow' of the image. A trajectory can be considered a curve in 3 dimensional (spatio-temporal) space starting when and where the image point first appears and ending where it disappears. The reason for wanting to estimate the motion field (for standards conversion) is to overcome some of the problems caused by the slow sampling rate (and hence temporal aliasing) of the image. Considering the velocity field as above, shows that it has at least as much spatio-temporal detail as the original image. Therefore great care must be taken when estimating, and using, sampled motion fields, to avoid aliasing of the motion field itself. Furthermore, care must be taken, in motion estimation, not to assume an unaliased image or velocity field. Since the velocity field is so (spatio-temporally) detailed it may be difficult to use estimates of the displacement field intended for one application, for a different one.

1 The difference between bitrate and bandwidth reduction is that bitrate reduction is a purely digital process, whereas bandwidth reduction involves the transmission of analogue information. 
In order to perform motion estimation all algorithms make certain, a priori, assumptions about the image. An image is formed by the projection of a 3 dimensional scene on to 2 dimensions. For motion estimation it is assumed that the scene comprises of a number of moving objects, and that each point in the image corresponds to a unique point on the surface of an object. This model is violated in a number of cases, for example with transparent, diffuse (eg smoke) and reflecting scene components. A relationship must also be assumed between the observed image and the unknown motion field. The assumption usually made is of the constancy of image features along the motion trajectory. This assumption is known as the structural model (Dubois 1990). A number of different image features could be used, most commonly the image luminance. Another commonly used feature is the filtered luminance, for example the gradient of the image might be used. Using the image gradient, rather than intensity, improves the validity of the structural model in the cases where there are changes of illumination or shadow, because it removes the zero frequency component of the image. More generally a 3 component colour vector can be taken as constant along the motion trajectory (Dubois ICASSP '90). The structural model is not, in itself, sufficient to uniquely define the motion field. Even in ideal circumstances the structural model may be satisfied by many different motion fields. In practice the motion field is even less well defined due to noise and inadequacies in the structural model. Therefore an additional assumption must be made about the motion field. This is that the motion field is generally smooth, with discontinuities at (spatio-temporal) object boundaries. This assumption is occasionally explicit (eg Horn 1981). More often it is implicit in the algorithm (eg in the use of post processing of the motion field (Weiss 1990) or in the use of trial vectors in phase correlation (Thomas 1987)).

Standards conversion imposes particular requirements on motion estimation. First the relevant parameters will be considered, and then discussed in more detail in subsequent paragraphs. One such parameter is the resolution required of the motion field. That is, how many pixels each measured motion vector corresponds to. The necessary range and resolution of the motion vectors must also be considered. These are the largest velocity likely to be encountered and the precision to which motion vectors are measured. The characteristics of the input images, from which the motion must be estimated, are also important. Finally, since all motion estimation algorithms sometimes fail, the conditions under which an algorithm fails, and its behaviour in these circumstances should be considered.

Ideally for motion compensated standards conversion a distinct motion vector is required for each image pixel. That is the resolution of the motion field should be the same as the original image. This is in contrast to bitrate reduction applications, where such a high resolution motion field is not required and, indeed, would be impractical because of the large 
bit rate required for its transmission ${ }^{2}$. A high resolution vector field for standards conversion allows the precise segmentation of the input image into regions with different velocities (ie different objects). Precise segmentation is essential to produce high quality interpolations. In practice it may be impossible to obtain the motion field to the same resolution as the input image. This may be due to imperfections in the input images (eg from vertical/temporal aliasing caused by interlace). Therefore, in practice, it may be necessary to use a lower resolution motion field, which must then be interpolated to the resolution required for motion compensated processing.

The motion field must be estimated in a way that will allow the range of velocities likely to be encountered, to be measured. Again standards conversion is significantly different from bit rate reduction. Bit rate reduction algorithms typically only require relatively small velocities (eg \pm 8 pixels/field period). For larger velocities bit rate reduction algorithms generally switch to an alternative mode of operation (eg spatial subsampling with intra field interpolation). For standards conversion there is no good 'fallback' option for interpolation and large velocities are precisely those for which motion compensation is most needed. Therefore standards conversion applications require the measurement of relatively large velocities (at least \pm 32 pixels/field period). This difference in the range of velocities required can have a significant effect on the motion estimation algorithm used. For example, the 'block matching' algorithm, often used for bit rate reduction, does not scale well to the larger velocities required for standards conversion.

It is difficult to assess the precision to which motion vectors should be estimated. That is how many fractional accuracy bits are need to describe the vectors. Girod and Thoma (December 1985) have shown that a signal containing translatory motion can be perfectly interpolated using integer pixel displacement vectors. This sets a lower limit on the required resolution (ie the velocity need only be resolved to the nearest pixel/frame period). However this result is only applicable for pure translatory motion and assuming an infinite number of input images can be filtered. In practice neither of these conditions are met and accuracy to better than integer pixel displacements is required to maintain the full resolution of the input in the interpolated images. The size of the (motion compensated) interpolation aperture used and the nature of source images (eg resolution, interlace etc) both influence the accuracy to which the vectors should be measured. For typical television images the resolution of the image decreases with object velocity because of the camera integration time (this effect should not be over estimated since integration time can be very short with modern CCD cameras). Therefore the measurement accuracy of the motion vectors can decrease with speed. A constant fractional error would probably be appropriate. For 'typical' (interlaced) images,

${ }^{2}$ Usually bit rate reduction algorithms use a subsampled motion field, where each vector is taken to apply to a small block of pixels (typically $8 \times 8$ ). 
and an interpolation aperture including only a few fields, a vector accuracy of, perhaps, $1 / 4$ or $1 / 8$ pixel/field period might be appropriate at low velocities.

Finally, from a practical point of view, it should be remembered that motion estimation can be computationally expensive. Therefore, in order to produce real time hardware, it may be necessary to use a sub-optimal algorithm to achieve a practical hardware complexity. The practical limit to hardware complexity depends on the application. Fortunately, high quality standards conversion in the television studio can probably afford to be more complex than in other applications (eg bit rate reduction for teleconferencing).

\subsection{A Review of motion estimation algorithms.}

This part of the chapter is devoted to a review of motion estimation algorithms described in the literature. Where appropriate, comments are included on their suitability for motion compensated standards conversion. A number of reviews of motion estimation are available (eg Musmann 1985, Dubois 1990, Konrad 1990, Vega-Riveros 1989, Lim 1990). The review here is from the perspective of the algorithm's applicability to motion compensated standards conversion.

Motion estimation algorithms may be broadly classified into 3 groups, spatio-temporal constraint techniques, region matching methods and frequency domain techniques. Each of these groups of algorithms is discussed, in turn, below. In order to minimise the computational complexity, the first two methods are often implemented recursively, as described below. Motion estimation techniques can also be applied in a hierarchical manner to deal with different object sizes and large velocities. In hierarchical processing, motion is estimated at a number of different image scales. Typically a pyramid of different image resolutions is used. The lowest level of the pyramid is the original image and successively higher levels in the pyramid are obtained by low pass filtering the lower level image (perhaps with subsampling to reduce computation). Large regions of the image are examined, in low resolution, at the top of the pyramid, while small regions are examined, in high resolution, at the bottom. This process is illustrated in figure 7.1.

The use of iterative (or recursive) techniques is common to both region matching and constraint techniques and also, potentially, to frequency domain methods. The basis of the technique is to use a motion estimation technique to improve the accuracy of an initial velocity estimate. The new estimate can then be used as the basis for another iteration to improve accuracy further. The process of recursive motion estimation is illustrated in figure 7.2. For example, given an image $g(x, y, t)$ and an initial estimate of the velocity $\left(u_{0}, v_{0}\right)$ an 'improved' estimate can be made by applying the motion estimation algorithm to $g\left(\left(x+u_{0} t\right),\left(y+v_{0} t\right)\right)$. That is, a motion estimate can be improved by compensating for the initial velocity estimate before performing motion estimation. This gives a new velocity estimate 
$\left(\mathrm{p}_{1}, \mathrm{q}_{1}\right)$. The 'improved' velocity estimate is then given by $\left(\mathrm{u}_{0}+\mathrm{p}_{1}, \mathrm{v}_{0}+\mathrm{q}_{1}\right)$. The process can be summarised as follows. Let ME be a motion estimation operator which acts on a region of a moving image to give an estimate of the motion ie:

$$
M E(g(x, y, t)) \quad-(u, v)
$$

Then the iterative process can be described as;

$$
\begin{aligned}
& \operatorname{ME}\left(g\left(\left(x+u_{n} t\right),\left(y+v_{n} t\right), t\right)\right) \Rightarrow\left(p_{n+1}, q_{n+1}\right) \\
& \left(u_{n+1}, v_{n+1}\right)=\left(\left(u_{n}+p_{n+1}\right),\left(v_{n}+q_{n+1}\right)\right)
\end{aligned}
$$

A variety of 'recursive' techniques arise from different choices of the initial estimate and the number of iterations to be performed. If initial velocity estimate is zero and iteration is performed as described above, the process is known as motion compensated iteration (Bierling 1986). This is often used with spatio-temporal constraint techniques. Alternatively either spatial or temporal recursion may be performed (Netravali 1979, Paquin 1983). In these techniques the initial estimate is taken from an adjacent pixel, either spatially or temporally. Both spatial and temporal recursion risk error propagation at (spatial or temporal) object boundaries. For spatial recursion the accuracy of the motion estimate also depends on the relative directions of object motion and the recursion (Robert 1985). This problem can be avoided by performing spatial recursion in 2 (or more) directions simultaneously and selecting the best result (Cafforio 1990, Hann 1990). Unfortunately it is difficult to guarantee the convergence of motion compensated, spatial and temporal recursive schemes.

One way of implementing hierarchical motion estimation is as a form of recursion. Hierarchical motion estimation, generally, uses information from the image at a variety of different scales. This is achieved by applying the motion estimation algorithm to different sized regions of the image to extract the maximum information. Usually the larger regions are filtered and subsampled to minimise computation. One form of hierarchical motion estimation uses the recursive technique described above (equations $1 \& 2$ ). The initial velocity estimate comes from a larger, lower resolution, region of the image (Bierling 1986, Martinez 1987). The largest, lowest resolution, image region used would typically take zero as the initial velocity estimate. This hierarchical technique allows the measurement of large velocities with high accuracy and produces a high resolution (dense) velocity field. With this form of motion estimation the maximum measurable velocity depends on the resolution of the lowest resolution image used. However, with low resolution images it may not be possible to detect the movement of small objects. There is, thus, a relationship between the highest velocity which can be measured and the smallest object which can be detected.

Some more notation is required before proceeding further. Let the image feature (eg gamma corrected intensity, perhaps after filtering) be represented by the function $g(x, y, t)$. In 
this section the image feature will be referred to as intensity; however it should be borne in mind that a different image feature might be used in practice. In the context of time sampled images, let $g\left(x, y, t_{0}\right)$ represent the current image and $g\left(x, y, t_{-1}\right)$ and $g\left(x, y, t_{1}\right)$ represent the preceding and succeeding images respectively. Similarly spatial arguments can be subscripted for spatially sampled data. For example $\mathrm{g}\left(\mathrm{x}_{-2}, \mathrm{y}_{+1}, \mathrm{t}_{0}\right)$ represents a pixel two pixels left and one line down relative to the origin or current pixel (depending on context) in the current image. For motion estimation the image is assumed to represent a moving object. Hence the image 'intensity', $\mathrm{g}$, may be expressed as,

$$
g(x, y, t)=h(\alpha(x, y, t), \beta(x, y, t), t)
$$

where $h$ is the 'intensity' of the object (at $t=0$ ) and $\alpha$ and $\beta$ are the (horizontal and vertical) coordinates of a point on the object. The functions $\alpha$ and $\beta$ are the spatially varying transformation of the object as a function of time. The dependence of the object intensity, $h$, on time, allows for changes of illumination etc with time and will, in general, be quite small. Note that this representation of the scene allows for quite general distortions of the object. In the particular case of an object translating with a uniform velocity, (u,v);

$$
\begin{aligned}
h\left(\alpha, \beta, t_{1}\right) & =h\left(\alpha, \beta, t_{2}\right) \quad \forall t_{1}, t_{2} \\
\alpha(x, y, t) & =x-u t \\
\beta(x, y, t) & =y-v t
\end{aligned}
$$

The first of these equations indicates that the appearance of the object does not change with time, while the latter two equations show that the transformation is a simple translation. For more complex types of motion (eg zooms, rotations, sheers and accelerations) it is possible to formulate more general expressions for $\alpha$ and $\beta$, see, for example, Schalkoff 1984, Martinez 1987 or Wu 1990.

\subsubsection{Spatio-temporal constraint methods.}

Spatio-temporal constraint methods are based on the relationship between the partial derivatives of the image intensity. The principle of this technique is illustrated in figure 7.3, in which an image, of linearly increasing brightness, moves between frames 1 and 2 . It can be seen from this diagram that the displacement is the ratio of the temporal difference to the spatial difference in brightness between the two frames. More generally the relationship, between the partial derivatives of the image intensity, can be derived by differentiating equation 3 and using equation 4, ie;

$$
\frac{\partial g(x, y, t)}{\partial x}=\frac{\partial h}{\partial \alpha} \frac{\partial \alpha}{\partial x}+\frac{\partial h}{\partial \beta} \frac{\partial \beta}{\partial x}=\frac{\partial h}{\partial \alpha}
$$




$$
\begin{gathered}
\frac{\partial g(x, y, t)}{\partial y}=\frac{\partial h}{\partial \alpha} \frac{\partial \alpha}{\partial y}+\frac{\partial h}{\partial \beta} \frac{\partial \beta}{\partial y}=\frac{\partial h}{\partial \beta} \\
\frac{\partial g(x, y, t)}{\partial t}=\frac{\partial h}{\partial \alpha} \frac{\partial \alpha}{\partial t}+\frac{\partial h}{\partial \beta} \frac{\partial \beta}{\partial t}+\frac{\partial h}{\partial t}=-u \frac{\partial h}{\partial \alpha}-v \frac{\partial h}{\partial \beta}+\frac{\partial h}{\partial t}
\end{gathered}
$$

from which;

$$
\frac{\partial h(x, y, t)}{\partial t}=u \frac{\partial g(x, y, t)}{\partial x}+v \frac{\partial g(x, y, t)}{\partial y}+\frac{\partial g(x, y, t)}{\partial t}=0
$$

This equation is known as the spatio-temporal constraint equation. It relates the partial derivatives of the moving image, when the appearance of the moving object does not change (ie when $\partial \mathrm{h} / \partial \mathrm{t}=0$ ). It is exact for a scene comprising a uniformly translating object. By using more general equations for $\alpha$ and $\beta$ it can be generalised to include other motions such as zooms, rotations and sheers (see Martinez 1987).

The spatio-temporal constraint equation is sometimes derived as a first order Taylor expansion of $\mathrm{g}(\mathrm{x}, \mathrm{y}, \mathrm{t})$ (eg Vega-Riveros 1989 and Thomas 1991) ie;

$$
\text { (2) } g(x+\delta x, y+\delta y, t+\delta t)=g(x, y, t)+\delta x \frac{\partial g}{\partial x}+\delta y \frac{\partial g}{\partial y}+\delta t \frac{\partial g}{\partial t}+\text { error terms }
$$

The constraint equation arises from cancelling common terms, dividing by $\delta$ t and ignoring the error terms. This approach fails to show that the spatio-temporal constraint equation is exact for uniformly translating objects. A comparison with the approach in the previous paragraph leads to the conclusion that the error terms in the Taylor expansion are due to some form of non-uniform motion and/or changes in illumination.

The velocity at an image point can be estimated by minimising the following error function;

$$
\text { Error }=\iint_{(x, y, t) \in R}\left(\frac{\partial h}{\partial t}\right)^{2} d x d y d t=\iiint_{(x, y, t) \in R}\left(u \frac{\partial g(x, y, t)}{\partial x}+v \frac{\partial g(x, y, t)}{\partial y}+\frac{\partial g(x, y, t)}{\partial t}\right)^{2} d x d y d t
$$

The error function is minimised over a region, $R$, for which the velocity $(\mathrm{u}, \mathrm{v})$ is assumed constant. The size of the region must be carefully selected. If it is too small there will be insufficient information to form a reliable motion estimate, if it is too large the assumption of a single velocity may be false. Different region sizes can be used in a hierarchical approach.

One method of minimising the error function above, for sampled data, is to form a constraint equation for each sample point. That is, 


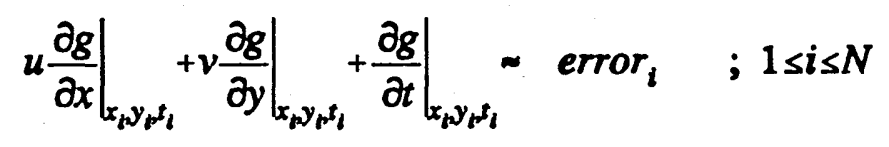

Where the error term would ideally be zero if all the appropriate assumptions are met. A group of these equations form an overdetermined set of equations for $u$ and $v$,

$$
S v=t
$$

Where;

$$
S=\left[\begin{array}{cc}
\left.\frac{\partial g}{\partial x}\right|_{x_{1} y_{1} s_{1}} & \left.\frac{\partial g}{\partial y}\right|_{x_{1} y_{1} s_{1}} \\
\cdot & \cdot \\
\cdot & \cdot \\
\left.\frac{\partial g}{\partial x}\right|_{x_{N} y_{N} t_{N}} & \left.\frac{\partial g}{\partial y}\right|_{x_{N} y_{N} s_{N}}
\end{array}\right], \quad v=(u, v)^{T}, \quad t=\left[\begin{array}{c}
-\left.\frac{\partial g}{\partial t}\right|_{x_{1} y_{1} s_{1}} \\
\cdot \\
\cdot \\
-\left.\frac{\partial g}{\partial t}\right|_{x_{N} y_{N} t_{N}}
\end{array}\right]
$$

The error function;

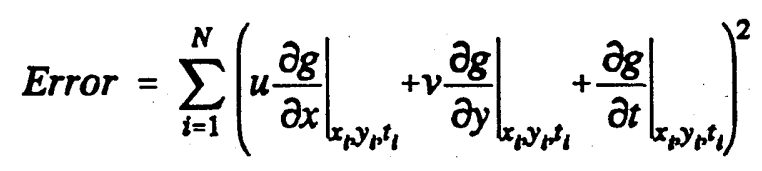

can be minimised by solving the corresponding 'normal equations' ie;

$$
v=\left(S^{T} S\right)^{-1} S^{T} t
$$

More generally the error function integral in equation 10 can be minimised by differentiating with respect to $\mathrm{u}$ and $\mathrm{v}$ and setting the results to zero. This leads to a pair of linear equations;

$$
W v=\gamma
$$

where; 


$$
\begin{gathered}
W=\left[\begin{array}{cc}
\iint_{(x, y, t) \in R}\left(\frac{\partial g}{\partial x}\right)^{2} d x d y d z & \iint_{(x, y, t) \in R} \frac{\partial g}{\partial x} \frac{\partial g}{\partial y} d x d y d z \\
\iiint_{(x, y, t) \in R} \frac{\partial g}{\partial x} \frac{\partial g}{\partial y} d x d y d z & \iiint_{(x, y, t) \in R}\left(\frac{\partial g}{\partial y}\right)^{2} d x d y d z
\end{array}\right] \\
\gamma=(u, v)^{T} \\
\gamma=\left[\begin{array}{c}
-\iint_{(x, y, t) \in R} \frac{\partial g}{\partial x} \frac{\partial g}{\partial t} d x d y d z \\
-\iiint_{(x, y, t) \in R} \frac{\partial g}{\partial y} \frac{\partial g}{\partial t} d x d y d z
\end{array}\right]
\end{gathered}
$$

Neither of the above approaches guarantees that a solution for the motion vector $(u, v)$ can be found. For example the equations are not solvable for an image point in a plain region of the image. Furthermore, if the image is the edge of an object only the velocity normal to that edge can be determined. These problems have been investigated by Martinez (1987), who has drawn the following conclusions. The nature of the solution to these equations is determined by the eigenvalues of matrix $\mathbf{W}$ (or $\mathbf{S} . \mathbf{S}^{\mathrm{T}}$ ). If both eigenvalues are small the determinant (which equals the product of the eigenvalues) will be small and the equations ill conditioned. This happens in plain areas and hence, in this case, it is appropriate to set the velocity to zero. If one eigenvalue is small this indicates a moving edge. In this case the velocity can be set normal to the edge. This is done by taking the solution to the equations (the 'raw' velocity) and projecting it along the eigenvector with largest eigenvalue. In other cases, where both eigenvalues are reasonably large, the solution of the above linear equations is valid.

The solution of the spatio-temporal constraint equation, outlined above, must be performed assuming a constant velocity in the neighbourhood of the current pixel. It is not possible to estimate a complete, spatially varying, velocity field simply by global minimisation of $(\partial \mathrm{h} / \partial \mathrm{t})^{2}$ in equation 10 . The minimisation of

$$
\iint_{\text {image }}\left(\frac{\partial h(x, y, t)}{\partial t}\right)^{2} d x d y=\iint_{\text {image }}\left(u(x, y) \frac{\partial g(x, y, t)}{\partial x}+v(x, y) \frac{\partial g(x, y, t)}{\partial y}+\frac{\partial g(x, y, t)}{\partial t}\right)^{2} d x d y
$$

is evidently underdetermined since there is only one constraint per pixel, whereas an estimate of two velocity components is required. Obviously another constraint is needed. Horn \& Schunck (1981) proposed the constraint that the velocity field should be smooth. This is achieved by minimising the squared gradients of the two velocity components. If 


$$
e_{u}^{2}=\left(\frac{\partial u(x, y)}{\partial x)}\right)^{2}+\left(\frac{\partial u(x, y)}{\partial y}\right)^{2}, \quad e_{v}^{2}=\left(\frac{\partial v(x, y)}{\partial x}\right)^{2}+\left(\frac{\partial v(x, y)}{\partial y}\right)^{2}
$$

then the velocity field $(\mathrm{u}(\mathrm{x}, \mathrm{y}), \mathrm{v}(\mathrm{x}, \mathrm{y}))$ is determined by minimising

$$
\iint_{\text {image }}\left(\frac{\partial h}{\partial t}\right)^{2}+\lambda\left(e_{u}^{2}+e_{v}^{2}\right) d x d y
$$

The (heuristic) parameter $\lambda$ is a compromise between generating a smooth velocity field and allowing changes at the boundary of objects. Nagel \& Enkelmann (1986) suggested improving Horn \& Schunck's original algorithm by using a spatially varying smoothness constraint, dependent on the image structure.

An alternative technique for determining a spatially varying motion field (rather than the average velocity of a region of the image) is to apply the constraint equation to each of the image's spatial derivatives (Tretiak 1984, Bertero 1988). If the image intensity is constant along the motion trajectory then the spatial derivatives of the intensity will also be zero. If the constraint equation is differentiated with respect to both $\mathrm{x}$ and $\mathrm{y}$ the following matrix equation is obtained;

$$
\nabla\left(\nabla^{r} g\right) \cdot v+\frac{\partial}{\partial t}\left(\nabla^{T} g\right)=\frac{\partial}{\partial t}\left(\nabla^{T} h\right)
$$

where,

$$
\nabla\left(\nabla^{\top} g\right)=\left[\begin{array}{ll}
\frac{\partial^{2} g}{\partial x^{2}} & \frac{\partial^{2} g}{\partial y \partial x} \\
\frac{\partial^{2} g}{\partial x \partial y} & \frac{\partial^{2} g}{\partial y^{2}}
\end{array}\right], \quad v=(u, v)^{T}, \quad \frac{\partial}{\partial t}\left(\nabla^{T} g\right)=\left[\begin{array}{l}
\frac{\partial^{2} g}{\partial t \partial x} \\
\frac{\partial^{2} g}{\partial t \partial y}
\end{array}\right], \quad \frac{\partial}{\partial t}\left(\nabla^{T} h\right)=\left[\begin{array}{l}
\frac{\partial^{2} h}{\partial t \partial x} \\
\frac{\partial^{2} h}{\partial t \partial y}
\end{array}\right]
$$

These simultaneous equations, in $u$ and $v$, can then be solved for each pixel in the image. However, the robust computation of second order image derivatives (especially temporal ones) is extremely difficult.

How may equations 15 and 16 be used to find the motion field in practice? The first (and major problem) is to determine the partial differentials of the image sequence with respect to space and time. Two approaches have been used to evaluate these differentials. One is to estimate the differentials from finite differences (Bierling \& Thoma 1986). The other is to fit a (multi-dimensional) polynomial, to a small region of the image sequence, from which 
the differentials can be calculated analytically (Martinez) ${ }^{3}$. The problem with both these techniques is that they fail at the boundaries between objects. This is not, perhaps, too severe spatially. Temporally, however, even with moderate motion, the same pixel in successive frames may correspond to different objects, thus rendering any estimate of the temporal derivative meaningless. Essentially the above techniques are difficult to apply because of the problem of estimating the partial derivatives. To quote Bierling \& Thoma: 'A displacement estimate, obtained by evaluating a differential displacement algorithm, is often far away from the true displacement, even if the present motion is restricted to pure translatory motion. This is due to the fact that the actual image signal differs drastically from the mathematical image model the algorithm is based on.'

The problem of estimating (particularly) the temporal partial derivative of the image stems principally from temporal undersampling of the image. In the context of motion compensated standards conversion it is precisely because of temporal undersampling that motion estimation is required. The maximum displacements that can be extracted depend on the local frequency content of the image. For example in random dot patterns (white spatial noise) the maximum displacement that can be extracted is one pixel (Fennema 1979). Essentially the accuracy of the temporal derivative can only be guaranteed if the velocity is less than 1 pixel per frame (or field). For higher velocities it must be assumed that the source image does not contain the highest frequencies which can be supported by the (spatial) sampling lattice. This assumption may or may not be true, and becomes increasingly unlikely as the motion speed increases.

The problems indicated above lead to the use of iterative (or recursive) techniques to improve the accuracy of constraint techniques. Recursive techniques are described at the beginning of this section. Using zero as an initial velocity estimate and applying equations

${ }^{3}$ Let $f(x, y, t)$ be an approximation to the function $g(x, y, t)$ such that;

$$
f(x, y, t)=\sum_{i=1}^{N} c_{i} \phi_{i}(x, y, t)
$$

For example we might have the following functions for $\mathrm{N}=9$.

$$
\phi_{i}(x, y, t)=1, x, y, t, x^{2}, y^{2}, x y, x t, y t
$$

The coefficients can be determined by minimising

$$
\text { Error }=\left.\sum \sum_{(l, m, n) \in \psi} \sum_{f}\left(g(x, y, t)-\sum_{i=1}^{N} c_{i} \phi_{i}(x, y, t)\right)^{2}\right|_{x=L x, y=m Y, t=n T}
$$

Where typically the region $\psi$ might contain 50 pixels; 5 for 1,5 for $\mathrm{m}$ and 2 for $\mathrm{n}$. Minimising the error expression with respect to the coefficient $c_{i}$ results in a set of $\mathrm{N}$ linear equations. 
1 \& 2 directly results in motion compensated iteration (Bierling 1986, Wu 1990). Alternatively the motion of an adjacent pixel can be used as an initial estimate, to give spatially or temporally recursive schemes. Unfortunately these schemes cannot be guaranteed to converge and may not give the 'true' velocity even if they do.

A specific iterative scheme, based on the constraint equation, has been suggested by Robert, Cafforio \& Rocca (1985). If the velocity estimate is performed on single pixel regions the matrix $\mathrm{W}$ (equation 17) is singular. The eigenvector with non-zero eigenvalue points in the direction of the luminance gradient. The velocity estimate can, therefore, only be updated in that direction. The recursive formula they derived, for updating the velocity estimate in the direction of the image gradient, is given by;

$$
v_{i+1}=v_{i}-\frac{\frac{\partial g}{\partial t} \cdot \nabla g}{|\nabla g|^{2}+\varepsilon^{2}}
$$

where $\mathrm{g}$ is the image luminance, $\nabla$ represents the spatial gradient and $\varepsilon$ is a damping factor. In their implementation Robert, Cafforio \& Rocca estimated the differentials by finite differences.

A potentially better solution to the problem of estimating the temporal derivative, in constraint techniques, is the use of hierarchical motion estimation. Problems in estimating the temporal derivative can be removed by eliminating, potentially harmful, high spatial frequencies by low pass filtering. A reliable motion estimate can thus be made on the low resolution image. The velocity cannot be measured very accurately because of the low resolution of the filtered image. However the motion estimate can be improved upon by taking it as the basis of second iteration using a higher resolution image as described above (Bierling 1986). Unfortunately, since the process starts with a low resolution image, it may not be possible to detect the motion of small objects.

Constraint techniques of motion estimation are typically used for bit rate reduction for teleconferencing. They are quite suitable for this application since expected motion is small. Furthermore, absolute fidelity of the motion field is not required because errors in motion estimation can be corrected by the rest of the bit rate reduction system. The use of a recursive technique yields an effective, computationally efficient algorithm. For standards conversion, by contrast, large velocities may be expected and good motion fidelity is required. To achieve this a hierarchical approach is necessary and good estimates of the differentials from polynomial fitting may be required. Hence this type of motion estimator, for standards conversion applications, would be much more complex than for, say, teleconferencing. Furthermore there is the risk that the motion of small objects may not be detected. Hence this type of algorithm is not, perhaps, ideal for standards conversion. 


\subsubsection{Region matching methods.}

Region matching methods are based on the assumption that the correct motion transformation will maximise the correspondence between regions in successive images. If this is true then, in the terminology of equations $3 \& 4$ above, the correct motion transformation, $\alpha(x, y, t) \& \beta(x, y, t)$, will minimise;

$$
\left|g\left(x, y, t_{0}\right)-g\left(\alpha(x, y, \Delta t), \beta(x, y, \Delta t), t_{-1}\right)\right| \quad ; \Delta t=t_{0}-t_{-1}
$$

Which says that, having allowed for the motion in the image, the difference between the two images is entirely due to changes in the appearance (shape, illumination) of the object. This equation becomes clearer when restricted to objects translating with uniform motion. Such a restriction is usually imposed to limit computational complexity. In this case expression 26 becomes;

$$
B D F D\left(x, y, t_{0}\right)=\left|g\left(x, y, t_{0}\right)-g\left((x-u \Delta t),(y-v \Delta t), t_{-1}\right)\right| \quad ; \Delta t=t_{0}-t_{-1}
$$

where BDFD is called the backward displaced frame difference. There is also a corresponding forward displaced frame difference. In general either the forward or the backward displaced frame difference will be denoted 'DFD'. Motion is estimated by minimising a suitable norm of the displaced frame difference. The $L_{n}$ norm of the displaced frame difference is defined by;

$$
\|D F D(x, y, t)\|_{n}=\sqrt[n]{\iint_{(x, y) \in R}(|D F D(x, y, t)|)^{n} d x d y}
$$

where $\boldsymbol{R}$ is a region of the image for which the velocity is assumed constant. Suitable norms might be $\mathrm{L}_{1}, \mathrm{~L}_{2}, \mathrm{~L}_{\infty}$ which are the mean modulus, root mean square and maximum value respectively. Mean modulus and root mean square are commonly used norms in this application.

Image motion is determined, in region matching algorithms, by minimising a norm of the displaced frame difference. Let the error function be defined as;

$$
\operatorname{error}(u, v)=\|D F D(x, y, t)\|_{n}
$$

then the velocity, of a point, is defined to be that velocity which gives the minimum error at that point. This is a classic, non-linear, optimisation problem, examples of which can be found in other branches of image processing eg filter design and image restoration (eg. maximum entropy techniques). The ( 2 dimensional) space of possible velocities must be searched to find a global minimum. This process is complicated by the possible presence of multiple local 
minima. It is simplified because the search space is only 2 dimensional. The various region matching, motion estimation methods are different methods for solving this minimisation problem, in real time and with practical hardware. The algorithms used can be grouped into 'recursive or gradient' and 'block matching' techniques. Region matching can also be extended to deal with more general motion such as rotation and zooms; however this increases the dimension of the search space and the problem becomes very much more complicated (Keesman 1988).

Before considering the minimisation of the error function in equation 29 , there follows a brief digression to consider the subject of displaced frame differences. The backward displaced frame difference is defined in equation 27 . The forward displaced frame difference is, correspondingly, defined by;

$$
F D F D\left(x, y, t_{0}\right)=\left|g\left(x, y, t_{0}\right)-g\left((x+u \Delta t),(y+v \Delta t), t_{1}\right)\right| \quad ; \Delta t=t_{0}-t_{-1}
$$

The forward and backward displaced frame differences are related, in most areas of the image by,

$$
F D F D\left(x, y, t_{0}\right)=B D F D\left((x+u \Delta t),(y+v \Delta t), t_{1}\right)
$$

ie. the forward displaced frame difference can be obtained by projecting the subsequent backward displaced frame difference, backwards along the motion trajectory. The difference between forward and backward displaced frame differences is important because it indicates regions of revealed or obscured detail. If the FDFD is minimised to find the motion, the vector found is valid for projecting the image at $t_{0}$ forward to a time between $t_{0}$ and $t_{1}$. Similarly a motion vector obtained from the BDFD is valid for projecting the image at $t_{0}$ backward to a time between $t_{-1}$ and $t_{0}$. Normally these two vectors would be the same. If, however, a region in the image at $t_{0}$ has been newly revealed between $t_{-1}$ and $t_{0}$, then the vector for that region can be found by minimising the FDFD, but the BDFD will always be large. Similarly the velocity for regions about to be obscured can be found from the BDFD but not the FDFD. Hence the image can be segmented into regions of uniform motion, and revealed and obscured areas by comparing the minima of the forward and backward DFDs. The velocity can be determined by minimising one, or both, of the DFDs.

The $\mathrm{L}_{2}$ norm (root mean square) of the DFD is particularly convenient for mathematical analysis. This is because it is related to the autocorrelation function of the signal. Assuming linear translation of a rigid body;

$$
\begin{aligned}
& \|D F D(u, v)\|_{2}=\iint_{(\alpha, \beta) \in R}(h(\alpha, \beta)-h(\alpha-u \Delta t, \beta-v \Delta t))^{2} d \alpha d \beta \\
& =\iint_{(\alpha, \beta) \in R} h^{2}(\alpha, \beta)-2 h(\alpha, \beta) h(\alpha-u \Delta t, \beta-v \Delta t)+h^{2}(\alpha-u \Delta t, \beta-v \Delta t) d \alpha d \beta \\
& \quad \sim 2 A \sigma^{2}-h \circ h(u \Delta t, v \Delta t)
\end{aligned}
$$


where $\mathrm{A}$ is the area of region $\mathrm{R}, \sigma^{2}$ is the image variance and hoh is the autocorrelation function of the moving object. The approximate equality in the third line is because of the finite size of the region R. Hence minimising the $\mathrm{L}_{2}$ norm of the DFD is equivalent to finding the maximum of the autocorrelation function. The Wiener-Khintchine theorem tells us that the autocorrelation function is the Fourier transform of the object's power spectrum. Since the spectrum of a typical image decays quite rapidly with frequency, the peak in the autocorrelation function would be expected to be quite broad. Hence there may be some difficulty in locating its exact position.

The minimisation of the norm of the DFD is a classic optimisation problem which can be approached by a number of well known techniques (eg. Stearns 1988, Lim 1990). For example the steepest descent (Netravali 1979) or Newton-Raphson (Lucas 1981) algorithms may be used to minimise the DFD. In general these types of algorithm are iterative with each iteration giving an improved estimate. They may be described by the general iterative scheme;

$$
v_{i+1}=v_{i}-\varepsilon R \cdot \nabla\left(\left\|D F D\left(v_{i}\right)\right\|_{2}^{2}\right)
$$

where $\mathbf{v}_{\mathrm{i}+1}$ is an improvement to the estimate $\mathbf{v}_{\mathrm{i}}$ and $\mathbf{R}$ is a recursion matrix (the mean square of the DFD is used as an example). As discussed previously the use of a recursive scheme permits a plethora of algorithms using spatial, temporal, motion compensated or hierarchical iteration. For the Newton-Raphson method;

$$
\boldsymbol{R}=\left[\begin{array}{ll}
\frac{\partial^{2}}{\partial x^{2}} & \frac{\partial^{2}}{\partial y \partial x} \\
\frac{\partial^{2}}{\partial x \partial y} & \frac{\partial^{2}}{\partial y^{2}}
\end{array}\right] \cdot\left\|D F D\left(v_{i}\right)\right\|_{n}
$$

The Newton-Raphson algorithm gives the correct solution in a single iteration if the DFD is a quadratic function of $\mathbf{v}$. However, it cannot be guaranteed to converge if the DFD is not a quadratic function of $\mathbf{v}$ and the estimate is not near the true value. Hence the, somewhat safer, approach of steepest descent is often used. In this case the recursion matrix is the identity matrix. Considering steepest descent with an image region of only one pixel;

$$
v_{t+1}=v_{i}-\varepsilon \nabla\left(D F D\left(v_{i}\right)^{2}\right)
$$

and after a little manipulation; 


$$
v_{t+1}=v_{t}-2 \varepsilon D F D\left(v_{t}\right) \cdot \nabla\left(g\left(x-v_{t} \Delta t\right)\right)
$$

which is the iterative equation derived by Netravali \& Robbins (1979). Note that the gradient in equation 35 is with respect to $\mathbf{v}$, whilst in equation 36 it is with respect to $\mathbf{x}(=(\mathrm{x}, \mathrm{y}))$.

Care must be exercised when using this type of recursive motion estimation technique. Moorhead et al (1987) have analysed the convergence of equation 36 and shown that the expected value of the velocity converges to the true value. However the variance of the measured velocity is proportional to the step size $\varepsilon$, which also determines the rate of convergence. Hence there is a tradeoff between the rate of convergence and the variance of the measured velocity at convergence. This type of iterative scheme is often used because of its low computational complexity. For computational simplicity there is usually only one iteration per pixel. Hence a rather noisy velocity field would be expected. In addition to these problems there is the same difficulty in determining the image gradient as for constraint techniques of motion estimation. Thus, used directly, this iterative scheme would only be expected to work for quite small velocities. In order to achieve the velocity range and accuracy required for standards conversion a hierarchical scheme, possibly with multiple iterations per stage, would probably have to be used. These embellishments greatly increase the computational complexity.

An alternative, straight forward, way to minimise the norm of the DFD is to quantise the search space $(\mathrm{u}, \mathrm{v})$ and transform a continuous minimisation problem into a discrete search. This technique is known as block matching and is illustrated in figure 7.4. The norm of the DFD is calculated using;

$$
\|D F D(v)\|_{n}=\sqrt[n]{\sum_{(L, m) \in R}\left(g\left(l X, m Y, t_{0}\right)-g\left((l X-u \Delta t),(m Y-v \Delta t), t_{-1}\right)\right)^{n}}
$$

for a discrete number of values of velocity. These values are then searched to find the minimum. The velocity must be quantised in integer pixels per frame unless image interpolation is used. The maximum velocity which can be measured is determined by the size of image region used. If the velocity range is doubled, the number of possible quantised velocities is quadrupled (2 dimensions) and the computation per pixel also quadruples. Hence the computational complexity increases as the forth power of maximum velocity. While block matching is a reasonable option for regions of $8 \times 8$ pixels, typically used for bit rate reduction, it rapidly becomes impractical for the velocities required for standards conversion. Furthermore a discrete search does not necessarily give an accurate estimate of the velocity. If the image region contains more detail in one orientation than at right angles, then the minimum discrete value of the DFD can be much more than one pixel from the true minimum in the continuous DFD surface. This situation is illustrated in figure 7.5. In this example the 
shape of the minimum in the DFD surface is very elongated, rather than being circularly symmetric. This may occur at the edge of an object, or if there is considerable camera integration due to a high velocity. The minima of the DFD is actually at $(0,1 / 2)$ but, because of the minima's shape, a discrete search would find the minimum at either $(-2,0)$ or $(2,1)$. Thus a discrete search of the DFD, as in block matching, can give errors greater than $\pm 1 / 2$ a pixel. This problem is really a manifestation of the aperture effect caused by considering too small a region of the image. It causes difficulties for block matching algorithms because computational complexity limits the size of the image region used. These problems can be ameliorated by using a hierarchical strategy as in Weiss 1990.

The computational complexity of block matching can be reduced by using the fast Fourier transform. As noted above, block matching, using the $\mathrm{L}_{2}$ norm of the $\mathrm{DFD}$, is equivalent to locating the maxima in the cross correlation function of two adjacent frames. This cross correlation is equivalent to the autocorrelation function of the object, assuming linear translation. The cross correlation, for two consecutive images, can be calculated from;

$$
g\left(t_{0}\right) \circ g\left(t_{1}\right)=\mathscr{F}_{x}^{-1}\left(G\left(t_{0}\right) \cdot G\left(t_{1}\right)^{*}\right) \quad ; G(t)=\mathscr{F}_{x}(g(t))
$$

where $F_{x}$ represents a spatial Fourier transform. The computational complexity of cross correlation (using the FFT algorithm) is of order $\mathrm{v}_{\max }{ }^{2} \cdot \ln \left(\mathrm{v}_{\max }\right)$ rather than $\mathrm{v}_{\max }{ }^{4}$ for the direct computation of mean square of the DFD. Thus, if large image regions are used for region matching (as is required to measure large velocities for standards conversion), it is much more efficient to use the cross correlation function calculated via the frequency domain.

A fundamental difficulty with block matching techniques is that the velocity resolution depends on the shape of the cross correlation peak (or the shape of the null in the norm of the DFD). If, as is typical for television images, the image spectrum decreases quite rapidly with frequency, then the peak in the cross correlation function will be broad. This makes the precise location of the peak (ie the velocity) subject to error. Hass \& Brubaker (1980) used a spectral whitening filter on the image to sharpen the cross correlation peak. If the cross correlation is performed via the frequency domain it is easy to apply an adaptive filter to achieve a white power spectral density. This process results in what is known as a "phase correlation' function (Thomas 1991).

The phase correlation function of two consecutive images is defined by;

$$
\Phi\left(g\left(t_{0}\right), g\left(t_{1}\right)\right)=\mathscr{F}_{x}^{-1}\left\{\frac{G\left(t_{0}\right) \cdot G\left(t_{1}\right)^{*}}{\left|G\left(t_{0}\right) \cdot G\left(t_{1}\right)^{*}\right|}\right\}
$$

It is essentially the crosscorrelation function of, the spectrally whitened, images. It was first used by Pearson et al (1977) for image registration and later by (Thomas 1987) for television motion measurement. An interesting alternative interpretation of the phase correlation function 
is as the probability distribution of velocities (Girod 1989). In practice the definition may be modified to;

$$
\Phi\left(g\left(t_{0}\right), g\left(t_{1}\right)\right)=\mathscr{F}_{x}^{-1}\left\{\frac{G\left(t_{0}\right) \cdot G\left(t_{1}\right)^{*}}{\sqrt{\left|G\left(t_{0}\right) \cdot G\left(t_{1}\right)^{*}\right|^{2}+N^{2}}}\right\}
$$

(where $\mathrm{N}^{2}$ is the expected noise power spectral density) to avoid excessive noise amplification. Effectively this is equivalent to applying a Wiener filter to spectrally whiten the image's cross power spectrum.

Calculation of a phase correlation function for every pixel in an image would be too computationally intensive if large image regions are used. Furthermore, the use of large image regions would risk missing the motion of small objects. Hence, a hierarchical approach is used. If there is more than one moving object in the image region used, then multiple peaks can be detected in the phase correlation function. Each peak in the phase correlation surface corresponds to the velocity of a different object. It is assumed that all objects in the image region are moving with one of these velocities. A local norm of the DFD is calculated for each pixel, for each of the possible motion vectors. The motion assigned to each pixel is that which gives the smallest norm DFD. In this way the image is segmented into regions corresponding to each velocity. Hence a dense motion field can be calculated. Computational complexity for the second stage of the hierarchy is low since the region used in calculating the norm of the DFD is relatively small.

\subsubsection{Frequency domain methods.}

The frequency domain properties of a moving object, both spatially and spatiotemporally, can be used for motion analysis of moving sequences. Consider a linearly translating object defined by;

$$
g(x, y, t)=h((x-u t),(y-v t))
$$

then the spatial Fourier transform is given by;

$$
G(m, n)=\mathscr{F}(g(x, y, t))=\exp (-j 2 \pi(m u+n v) t) \cdot H(m, n) \quad ; H(m, n)=\mathscr{F}(h(x, y))
$$

Note that equation 41 is more restrictive than equation 3 applying only to uniform linear translation. From these equations it can be seen that the (unwrapped) phase difference, at a given frequency $\left(m_{i}, n_{i}\right)$, from time $t_{a}$ to time $t_{b}$, is given by; 


$$
\Delta \phi\left(m_{i}, n_{i}\right)=2 \pi\left(m_{l} u+n_{i} v\right) \Delta t \quad ; \Delta t=t_{a}-t_{b}
$$

This equation can be used to estimate the velocity by forming, from the measured phase differences, an overdetermined set of equations for $u$ and $v$ at various frequencies. The velocity can be determined from a least mean square solution of these equations (Haskell 1974, Huang 1981). That is, given

$$
\boldsymbol{W} . \boldsymbol{d}=\Delta \phi \quad ; \boldsymbol{W}=\left[\begin{array}{cc}
2 \pi m_{1} & 2 \pi n_{1} \\
2 \pi m_{2} & 2 \pi n_{2} \\
\cdot & \cdot \\
\cdot & \cdot \\
2 \pi m_{N} & 2 \pi n_{N}
\end{array}\right], d=\left[\begin{array}{l}
u \Delta t \\
v \Delta t
\end{array}\right], \Delta \phi=\left[\begin{array}{c}
\Delta \phi\left(m_{1}, n_{1}\right) \\
\Delta \phi\left(m_{2}, n_{2}\right) \\
\cdot \\
\cdot \\
\Delta \phi\left(m_{N}, n_{N}\right)
\end{array}\right]
$$

find the LMS solution given by;

$$
d=\left(W^{T} W\right)^{-1} W^{T} \Delta \phi
$$

This is, however, a somewhat awkward process, since it involves the calculation of unwrapped phase.

Alternatively the spatio-temporal spectrum of a moving image may be used for motion analysis. Consider a linearly translating object defined by;

$$
g(x, y, t)=h((x-u t),(y-v t), t)
$$

then its spatio-temporal Fourier transform is given by;

$$
G(m, n, f)=\mathscr{F}(g(x, y, t))=\delta(f-m u-n v) * H(m, n, f) \quad ; H(m, n, f)=\mathscr{F}(h(x, y, t))
$$

Note that equation is more restrictive than 3 but less restrictive than 41 . It assumes uniform linear translation of an object but does allow some change of shape/lighting with time. Equation 47 says than the moving objects spectrum lies in a plane in spatio-temporal frequency space. Heegar (1987) uses a set of, quadrature pairs of, Gabor filters to estimate the 3D spectrum. The output of the filters is normalised, with respect to spatial frequency, and a least mean square approach is used to find the best fitting plane and hence the velocity. This approach has been generalised by Jacobson and Wechsler (1987) via the use of the Wigner distribution.

The use of frequency domain techniques for motion analysis suffers from the fundamental uncertainty relations of Fourier transforms. Hence the more accurately an object's velocity is determined the less accurately its position is defined. Therefore an additional technique (eg local block matching) must be used to assign a specific velocity to each pixel, 
thereby segmenting the image into regions of different motion. This is a hierarchical motion estimation technique very similar to that used for phase correlation (described in the previous section). As with other techniques large regions must be used (for spectral estimation) in order to determine large velocities. As usual, when large regions are used, there is the risk of not detecting the motion of small objects. Again this points to the use of hierarchical techniques.

As part of this research a frequency domain technique has been investigated which appears to be novel. Essentially this technique is based on the fact that the energy of a moving objects 3 dimensional spectrum is concentrated in a single plane. The spatial phase and magnitude information in the spectrum, which describes the object, is removed by a normalisation process. This leaves a modified spectrum containing only the motion information. The spatio-temporal spectrum of a moving image is estimated over a region of the image. The energy at each spectral point is normalised by dividing by the total energy at that spatial frequency. Finally the modified spectrum is retransformed to the spatio-temporal domain. The result of this processing, for a uniformly moving object, is to generate a spatial delta function moving along the motion trajectory. Sampling the retransformed spectrum at a specific time, gives a two dimensional function with a peak at a position corresponding to the object's motion. The process can be summarised as follows:

i) Estimate the image spectrum;

$$
G(m, n, f)=\mathscr{F}(g(x, y, t))
$$

ii) Normalise the spectrum;

$$
G^{\prime}(m, n, f)=\sqrt[2]{\frac{G(m, n, f) G^{*}(m, n, f)}{D^{2}(m, n)}} \quad ; D^{2}(m, n)=\int G(m, n, f) G^{*}(m, n, f) d f
$$

iii) Retransform the modified spectrum to the spatio-temporal domain;

$$
g^{\prime}(x, y, t)=\mathscr{F}^{-1}\left(G^{\prime}(m, n, f)\right)
$$

iv) Sample the resulting time domain function at a specific time;

$$
M(x, y)=g^{\prime}\left(x, y, t_{s}\right)
$$

v) Locate maximum in function $M$ to determine the velocity;

$$
\operatorname{maximum}\{M(x, y)\}=M\left(u t_{s}, v t_{s}\right)
$$


For a moving object in the image;

$$
g(x, y, t)=h((x-u t),(y-v t))
$$

then the functions $g^{\prime}(x, y, t)$ and $M(x, y)$ are given by;

$$
g^{\prime}(x, y, t)=\delta((x-u t) \cdot(y-v t)) \quad ; M(x, y)=\delta\left(\left(x-u t_{s}\right) \cdot\left(y-v t_{s}\right)\right)
$$

The function $M(x, y)$, in equation 51 above, is, in some ways, analogous to the cross correlation or phase correlation function described in section 7.2.2 above. $\mathrm{M}(\mathrm{x}, \mathrm{y})$ will be referred to as a 'motion correlation' function because of the analogy. The motion correlation function can be used for motion estimation in much the same way as the phase correlation function. A region of the image is chosen to calculate the motion correlation function, the region's size being determined by the maximum velocity required. The motion correlation function is searched for maxima, corresponding to possible movements within this region. The image is then segmented into areas (objects) corresponding to each possible motion. The segmentation is performed, as for phase correlation, on the basis of the minimum local norm of the displaced frame difference. Essentially, the whole process is a two stage, hierarchical motion estimation system. In contrast to previous methods, however, this is a mixed hierarchy, since the first stage is based on frequency domain analysis while the second stage is based on region matching. Like phase correlation, the technique can produce a dense motion field which contains a limited number of movements detected as maxima in the motion correlation function.

What are the differences that would be expected between, frequency domain, motion correlation and, region matching, cross or phase correlation? A principle difference is that motion correlation measures velocity, whereas region matching measures displacement. Motion correlation would also be expected to have greater noise immunity and, perhaps, greater accuracy because more information (from multiple rather than just 2 fields) is used.

The difference between measuring velocity and displacement is clarified by the following example. Studio processing such as colour decoding or noise reduction can apply temporal filtering to television signals. Analysis of temporally filtered signals by phase correlation or motion correlation will give significantly different results. Assume an image, panning at velocity $\mathrm{v}$, has been temporally filtered using an aperture of 3 fields. The filtered image at $t_{0}$ contains contributions from times $t_{1}, t_{0}$ and $t_{1}$, and the image at $t_{1}$ contributions from $t_{0}, t_{1}$ and $t_{2}$. A cross or phase correlation, between filtered images at $t_{0}$ and $t_{1}$, will include peaks corresponding to correlations between all permutations of these contributions. Thus there will be a peak corresponding to velocity $v$ from contributions at $t_{0}$ and $t_{1}$, however there will also be a peak corresponding to velocity $3 \mathrm{v}$ (for example) from the contributions at $t_{-1}$ and $t_{2}$. Altogether there will be 5 peaks corresponding to velocities at $-v, 0, v, 2 v$ and 
3v. A motion correlation function, for the same, temporally filtered, image, would, by contrast, produce a single peak corresponding to the true velocity $\mathrm{v}$. This is because motion correlation is based on the idea that the spectral energy of a moving object lies in a plane in spatio-temporal frequency space. This feature of the spectrum is little affected by the type of (usually low pass) temporal filtering applied to television signals.

An experimental investigation was undertaken to assess the performance of both phase and motion correlation motion estimation algorithms. This investigation is described in the next section.

\subsubsection{Summary.}

This section has given an overview of the different techniques which can be used for motion estimation. It has attempted to highlight the pros and cons of each technique, in particular with respect to their application to motion compensated standards conversion. Both constraint techniques and region matching methods have been used for motion compensated interpolation. Region matching is frequently used for bit rate reduction in the form of block matching algorithms. Block matching is also used for video processing, as are recursive implementations of region matching and constraint techniques. Frequency domain techniques appear, so far, to have received little attention for motion compensated interpolation, nevertheless they seem well suited to this application.

Algorithms for motion estimation, used to date, have largely concentrated on reducing the computational complexity required. This has resulted in compromises in the quality of the estimated motion field. This section has tried to show that motion estimation, of the quality required for standards conversion, is likely to be a complex process. Having accepted that computational complexity is probably inevitable for this application, more complex, high quality, methods of motion estimation can be considered. Furthermore computational complexity will become progressively more acceptable in the light of technological developments in signal processing.

\subsection{An experimental investigation of two motion estimation algorithms.}

This section gives a description of an experimental investigation of two motion estimation algorithms. The two algorithms chosen are phase correlation and motion correlation, both described in the previous section. Although very different in their theoretical basis, the two techniques can be implemented in a similar fashion in practice. This allows a fair comparison of a region matching and a frequency domain motion estimation technique. Phase correlation has been investigated by Thomas (1987) and found to be suitable for television processing. It is therefore used as the basis of the experimental, real time, 
implementation of motion estimation described in chapter 8. Thomas' investigation only used a limited number of small test images. More general test patterns were used in this investigation, in order to generalise the results. Extra processing has been used in this investigation (in addition to that used by Thomas) in order to achieve the best possible performance. Motion correlation requires rather more computation than phase correlation and, at the start of this research, had not been validated by computer simulation. It was not, therefore, chosen for real time hardware implementation. The results of this investigation, however, indicate that its performance may be superior to that of phase correlation.

Both phase and motion correlation algorithms are hierarchical in nature. A relatively large area of the image is first analysed to detect movements which may be present in that region. The image is then segmented into regions corresponding to these different velocities by selecting, for each pixel, the velocity giving the minimum norm DFD. The method of analysis used is the calculation of either the phase or motion correlation function, followed by a search to find maxima within it. The norm used for segmentation is either the local mean absolute DFD or the local root mean square DFD. This investigation concentrates on the motion analysis stage. Segmentation of the image into regions of constant velocity (ie. objects) depends on how the motion field is to be used. That process has been implemented, in different ways, in hardware, and details and results are discussed in subsequent chapters.

This investigation was conducted by computer simulation using specially devised test sequences. The algorithms may be described as 3 stages, image preprocessing (signal conditioning), correlation processing, and searching for maxima of the correlation functions. The difference between phase and motion correlation is purely in the type of correlation processing used. Results are given for the two algorithms, which show the accuracy and spread of motion measurements and the effect of input noise on the measurements.

\subsubsection{Image Preprocessing.}

The processing applied to the image sequence, prior to correlation, are described in this section. Two types of processing are used; filtering to improve performance of the motion analysis, and windowing to divide the image area into smaller regions for correlation analysis. The filtering used has 3 functions. Firstly it greatly reduces aliasing in the input images. Secondly it is used to reduce the effect of changes in illumination across the scene. Finally it reduces the effect of spectral leakage, caused by performing spectral analysis using the discrete Fourier transform.

Spatial filtering reduces the deleterious effects of vertical aliasing in the input images. Standards conversion requires the motion estimation of interlaced images, which are universally used for television. Consider a European ' 625 line' image. Each television field samples about 288 lines of the image. A typical camera has considerable response above the 
vertical Nyquist frequency of 144 cycles/active picture height (see chapter 2 and C.K.P. Clarke 1987). This allows a little more vertical detail to be extracted from a pair of interlaced fields (a frame) for near stationary pictures. From the point of view of motion estimation, however, camera response above $144 \mathrm{c}$ /aph is a nuisance which merely confuses the analysis. In very severe cases vertical aliasing can extend down to zero vertical frequencies. This is unusual and typically the lower vertical frequency components are fairly free from alias.

If vertical aliasing is ignored errors result in the vertical velocity which is measured. The aliased parts of an image 'move' in a different way to the true motion of the object. Typically this might result in errors of about \pm 1 picture line (ie \pm 0.5 field lines) per field period. Such errors are unacceptably large for standards conversion, as discussed in section 7.1. Thomas (1987) alleviates the problem, by correlating across 2 fields (ie 1 frame). This technique avoids problems with vertical aliasing, for stationary images, but has two disadvantages. Firstly, motion estimation errors due to vertical aliasing reappear with even small vertical movements. Such small vertical motion might be caused by a vibration of the camera. The second disadvantage to correlating across a frame, rather than a field, is to halve the motion range for the same size analysis region. In order to achieve the same velocity range the area of the analysis region would have to be quadrupled. This, in turn, would lead to increased computational complexity and would risk failing to detect the movement of small objects.

In this investigation the effects of vertical aliasing were reduced by low pass vertical filtering. Vertical frequency components above $72 \mathrm{c} / \mathrm{aph}$ were removed (ie frequencies above $1 / 2$ Nyquist frequency for a single field). Frequency components below this cutoff point are little affected by alaising. Since the vertical bandwidth of the signal was halved 2:1 vertical subsampling was used to reduce the computational complexity. To avoid a large disparity between the horizontal and vertical measurement accuracy the image was also horizontally filtered to half its original bandwidth and subsampled 2:1. This also gives a useful reduction in computational complexity. Thus image analysis was performed on filtered sequential (ie. non-interlaced) images of 360 pixels by 144 lines at $50 \mathrm{~Hz}$.

The filtering used also removed the very low horizontal and vertical frequencies. These frequencies add little to motion estimation (since motion cannot be detected in a plain image!). Illumination usually changes slowly over the image giving rise to low spatial frequency components. By removing these frequencies some resistance is gained to the effects of changing illumination. Furthermore since images typically contain considerable energy at zero frequency (see chapter 2) it is a major cause of spectral leakage when performing Fourier transforms using the discrete Fourier transform. Therefore, eliminating low frequencies reduces spectral leakage. The characteristics of the filtering used is given in figures 7.6 and 7.7. 
Windowing is used to divide the image into smaller regions for correlation analysis. An analysis region used for motion estimation is defined by;

$$
r\left(l X_{i}, m Y_{i}, n T_{i}, p X_{w}, q Y_{w}, s T_{w}\right)=g\left(l X_{i}, m Y_{i}, n T_{i}\right) \cdot w\left(\left(l X_{i}-p X_{w}\right),\left(m Y_{i}-q Y_{w}\right),\left(n T_{i}-s T_{w}\right)\right)
$$

where $g(x, y, t)$ is the image, sampled at intervals of $X_{i}, Y_{i}, T_{i}$ horizontally, vertically and temporally respectively, and $w(x, y, t)$ is the window function, centred at intervals of $X_{w}, Y_{w}, T_{w}$ horizontally, vertically and temporally respectively. Thus, in this definition the analysis regions are centred on a regular orthogonal lattice. A windowed region of the image may be regarded as a 6 dimensional function of the pixel position and the centre of the region in both space and time. Phase and motion correlation differ because motion correlation uses temporal windowing, whereas phase correlation inherently does not. Thus, for phase correlation only, $w(x, y, t)$ is zero at times other than zero.

The size of the analysis region, ie. the size of the window, determines the maximum velocity which can be measured. The size chosen for this investigation was 128 pixels by 128 picture lines in the original image (only 64 horizontal samples by 32 vertical samples were actually used in calculating the DFTs, the reduction being due to subsampling discussed in previous paragraphs). This size region allows velocities approaching 64 pixels/field period to be measured and is the smallest region able to measure the high velocities needed for standards conversion. A window of 5 fields duration was used for motion correlation.

What properties should be chosen for the window function? One desirable property is that the sum of all the windowed regions is equal to the original image. If this condition is not satisfied the motion estimator will be biased to finding the motion in some areas of the image rather than others. This condition can be expressed mathematically as;

$$
g\left(l X_{i}, m Y_{i}, n T_{i}\right)=\sum_{p} \sum_{q} \sum_{s} r\left(l X_{i}, m Y_{i}, n T_{i}, p X_{w}, q Y_{w}, s T_{w}\right)
$$

and substituting equation 55 and rearranging gives;

$$
\sum_{p} \sum_{q} \sum_{s} w\left(\left(l X_{i}-p X_{w}\right),\left(m Y_{i}-q Y_{w}\right),\left(n T_{i}-s T_{w}\right)\right)=1 \quad ; \forall l, m, n
$$

In general the window functions will overlap so that each pixel is present in number of different regions. Another condition on the window function is given by considering the Fourier transform of the windowed image. The Fourier transform is denoted by;

$$
R\left(a M, b N, c F, p X_{w}, q Y_{w}, s T_{w}\right)=\mathscr{F}_{x_{N}, x}\left\{r\left(l X_{i}, m Y_{i}, n T_{i}, p X_{w}, q Y_{w}, s T_{w}\right)\right\}
$$

where $\mathscr{F}_{x, y, t}$ is a spatio-temporal discrete Fourier transform operating on a sampled signal $\mathrm{r}(\mathrm{lX}, \mathrm{mY}, \mathrm{nT})$ to give a sampled spectrum $\mathrm{R}(\mathrm{aM}, \mathrm{bN}, \mathrm{cF})$. The function $\mathrm{R}$ is essentially a 
representation of the local spectrum of the image as a function of the window's position. The subject of local spectra is dealt with in considerable detail in Allen 1977, Nawab 1988 and the appendix (chapter 11). For now it is sufficient to note that the spectral component at a given frequency, $\mathrm{R}\left(\mathrm{pX}, \mathrm{w} \mathrm{X}_{\mathrm{w}}, \mathrm{sT}_{\mathrm{w}}\right)$, is a three dimensional sampled function. As with any sampled function, it must be sampled sufficiently often to avoid aliasing. This can be done by a suitable choice of window function. It turns out that the sampling rate required depends on the Fourier transform of the window function. This is best clarified by an example. Consider horizontal regions of 128 pixels separated by 32 pixels. In this case each pixel appears in four, adjacent, horizontal regions. This may be referred to as an overlap factor of 4. In order that the local spectrum is properly sampled the following condition is required of the window function;

$$
W(a M)=\mathscr{F}_{x}\left\{w\left(l X_{i}\right)\right\}=0 \quad ; a \geq 4
$$

That is taking the discrete Fourier transform of a 128 point window and overlapping it by a factor of 4 (ie successive windows separated by 32 pixels) the DFT of the window must be essentially zero at frequency samples other than $0, \pm 1, \pm 2$ and \pm 3 . If this condition is satisfied then the condition in equation 57 is automatically satisfied as well!

The preceding paragraph says that the spectrum of the window function used should depend on the degree by which adjacent regions overlap. As the degree of overlap increases the bandwidth of the window function can also be allowed to increase. In practice it is impractical to use a large degree of overlap, as this greatly increases the computational complexity. In this investigation an overlap factor of 2 has been used, both vertically and horizontally. That is, a given pixel appears in 2 adjacent regions both horizontally and vertically, so that it appears in a total of 4 regions altogether. Unfortunately no conventional window function (eg Hamming, Hanning or Kaiser) satisfies the requirement (equation 59) for an overlap factor of 2 . This is because an overlap factor of 2 requires that the spectrum of the window has a very narrow bandwidth. The nearest conventional approach is to use a rectangular window, but its sinc function frequency response is far from ideal. The window function used in this investigation is the product of discrete prolate spheroidal functions. These have an optimum energy compaction property as discussed in the appendix (chapter 11). The ones used have in excess of $98 \%$ of their energy within the required bandwidth. They therefore constitute a good approximation to the ideal window functions required by this application. The window used is a variables separable product of horizontal, vertical and temporal prolate spheroidal functions. Note the temporal window function was also calculated for an overlap factor of 2 . Nevertheless the motion correlation function was calculated every field.

This section has shown that considerable care was taken in signal conditioning the image sequence, prior to correlation analysis. Careful filtering was applied to reduce the 
effects of vertical aliasing, changes in scene illumination and spectral leakage when calculating Fourier transforms for correlation. Careful windowing was performed to ensure that motion estimation was not biased towards motion in particular parts of the image. Care was taken to ensure that the local spectra used for correlation analysis were correctly sampled.

\subsubsection{Correlation processing.}

The correlation processing, described in this subsection, constitutes the principle difference between phase and motion correlation analysis.

\subsubsection{Phase Correlation.}

The phase correlation processing implemented equation 40 to produce a separate phase correlation function for each spatial region, for every field. Forward spatial Fourier transforms were implemented by direct application of the discrete Fourier transform (Brigham 1974) to the filtered and windowed signal, produced by the preprocessing described above. A fast Fourier transform algorithm (Brigham 1974) was used to minimise the amount of computation.

The noise term in the denominator of equation 40 was independent of frequency (ie white noise was assumed) and equivalent to an input signal to noise ratio of about 8 bits 4 . In principle the noise term could be a function of frequency. In this investigation, however, the signal was truncated to 8 bit accuracy after filtering, and so noise was mainly due to truncation and therefore independent of frequency.

Non linear processing of a signal's spectrum generally requires that the processed Fourier transform is sampled more often than the input transform. For example, if a signal's autocorrelation function is calculated via the frequency domain the sampling rate required for the output Fourier transform is twice that required to properly sample the input transform. This is because the autocorrelation function is twice the duration of the input signal. Essentially squaring a signal doubles the duration of its Fourier transform and doubles the sampling rate required for the processed spectrum. If this double sampling rate is not used in calculating the autocorrelation function the result is a circular, rather than true, autocorrelation. In the case of phase correlation processing, however, the processing is designed to produce a smooth output spectrum (ideally giving a uniform spectral amplitude). This tends to decrease the detail in the processed spectrum and give a highly localised signal in the time domain. The whole purpose of phase correlation is, fundamentally, to generate a

${ }^{4}$ The input used was truncated to 8 bits after filtering and would therefore be expected to have a RMS noise level of $1 / \sqrt{3}$ lsbs. A slightly higher noise level of $1 / 2$ lsb RMS was used. 
function containing sharp peaks. Hence it was not felt necessary to increase the sampling rate used for the processed spectrum, above that required by the input signal. This gave a considerable saving in the amount of computation required.

A window function was applied to the processed spectrum prior to performing the inverse (discrete) Fourier transform. If no window is used the shape of a 'perfect' phase correlation peak is a sinc function (ie the transform of a uniform amplitude spectrum). This causes problems when the peak of phase correlation function does not lie on a sampling point. In that case spurious peaks are generated by the side lobes of the sinc function. Essentially 'noise' (in the form of these spurious peaks) is generated in the phase correlation function from 'spectral leakage' generated by performing the discrete Fourier transform. The processed spectrum was, therefore, multiplied by a window function to avoid this problem. Initially a (discrete) prolate spheroidal function was used as the window. The transform of this window was a peak about 2 samples wide (ie the same width as the sinc function) with low amplitude side lobes. This window functioned correctly, producing narrow peaks (and low noise) in the phase correlation function. These peaks, however, were so narrow that it proved difficult to accurately determine the location of a maximum, if it did not lie close to a sampling point. Hence for the results below, a 'softer' window, which produced wider correlation peaks, was used. The window function chosen was a Gaussian, whose transform was about 4 samples wide (ie. twice the width of the sinc function). Although this gave wider peaks in the correlation function, location of the maxima was easier and better overall results were obtained.

\subsubsection{Motion Correlation.}

Motion correlation processing implemented equation 51 to produce a separate motion correlation function for each spatial region for every field. In general the processing used for motion correlation was very similar to that used for phase correlation. Forward spatial Fourier transforms were implemented by direct application of the discrete Fourier transform to the filtered and windowed signals produced by the preprocessing described above. In this case a 3 dimensional Fourier transform was taken of a 3 dimensional windowed image sequence. Phase correlation, by contrast, only used 2 dimensional processing.

The denominator of equation 49 was modified to avoid excessive amplification of noise. The modified equation used here was; 


$$
G^{\prime}(m, n, f)=\sqrt[2]{\frac{G(m, n, f) G^{*}(m, n, f)}{D^{2}(m, n)+N^{2}}} \quad ; D^{2}(m, n)=\int G(m, n, f) G^{*}(m, n, f) d f
$$

where $\mathrm{N}^{2}$ is the expected noise energy in the signal. The noise term used was independent of frequency (ie white noise) and equivalent to an input signal to noise ratio of about 8 bits.

The normalising factor given in equation 49 was approximated by a finite sum of the discrete Fourier transform coefficients. That is;

$$
D^{2}(a M, b N)=\sum_{c=-2}^{2} G(a M, b N, c F) \cdot G^{*}(a M, b N, c F)
$$

The remaining processing was much as for phase correlation. The Fourier transforms were, as above, only calculated to the resolution required by the input signal. A window function was used prior to inverse transforming the processed spectrum (ie $G^{\prime}(m, n)$ in equation 50). For this part of the processing only a spatial window function was used. The same window function was used as for phase correlation.

After the inverse ( 3 dimension) Fourier transform had been calculated the function was sampled at $\mathrm{t}=1$ (ie the 4 th of 5 images, which start at $\mathrm{t}=-2$, in the retransformed sequence). This gave a motion correlation function as described in equation 51 . The retransformed sequence was sampled at $t=1$ so that the motion correlation function corresponded as closely as possible to the phase correlation function. Using this sampling instant, motion in the input sequence should give maxima at the same position in both phase and motion correlation functions. A sampling instant of $t=2$ could have been chosen. This would have given half the velocity range but twice the resolution.

\subsubsection{Searching for correlation maxima.}

Having calculated the correlation functions the maxima of these functions were located. In order to achieve the velocity accuracy necessary for standards conversion, the location of maxima had to be determined to an accuracy of better than one sample. This was achieved by fitting a continuous function to the (sampled) correlation function in the vicinity of a maximum. The position of the maxima could then be determined, to the required accuracy, from this continuous function.

A two dimensional quadratic function was fitted to the sampled correlation functions. This function was chosen because it is the most general multidimensional polynomial having a single unique maximum. The quadratic surface corresponding to a particular pixel was determined by a least mean square fit to that pixel and its 8 immediate neighbours, achieved as follows. The correlation function, $s(x, y)$, is approximated by $z(x, y)$ defined as; 


$$
z(x, y)=a x^{2}+b y^{2}+c x y+d x+e y+f
$$

The error between this approximation and the true correlation function is given by;

$$
\text { error }=\sum_{x=-1}^{1} \sum_{y=-1}^{1}(s(x, y)-z(x, y))^{2}
$$

This can be written in matrix form ie.

$$
\text { error }=e . e^{T} ; e=s-z \& z=M . c
$$

where;

$$
s=\left[\begin{array}{l}
s(-1,-1) \\
s(0,-1) \\
s(1,-1) \\
s(-1,0) \\
s(0,0) \\
s(1,0) \\
s(-1,1) \\
s(0,1) \\
s(1,1)
\end{array}\right], \quad M=\left[\begin{array}{rrrrrr}
1 & 1 & 1 & -1 & -1 & 1 \\
0 & 1 & 0 & 0 & -1 & 1 \\
1 & 1 & -1 & 1 & -1 & 1 \\
1 & 0 & 0 & -1 & 0 & 1 \\
0 & 0 & 0 & 0 & 0 & 1 \\
1 & 0 & 0 & 1 & 0 & 1 \\
1 & 1 & -1 & -1 & 1 & 1 \\
0 & 0 & 0 & 0 & 1 & 1 \\
1 & 1 & 1 & 1 & 1 & 1
\end{array}\right], \quad c=\left[\begin{array}{l}
a \\
b \\
c \\
d \\
e \\
f
\end{array}\right]
$$

The error is minimised, with respect to the coefficient vector $\mathbf{c}$, by solving the corresponding normal equations, ie.

$$
c=\left(M^{T} M\right)^{-1} M^{T} S
$$

Note that 9 points of the correlation function are used to fit only 6 coefficients describing the surface. By using more values of the correlation function than there are coefficients, and performing the LMS fit, the sensitivity of the fitting process to noise is reduced.

The coefficients defining the 2 dimensional quadratic function were used to determine position of maxima in the (sampled) correlation function. Given the polynomial coefficients, a to $f$ defined above, the existence and location of a (local) maximum can be determined by some straight forward multidimensional differential calculus (Kaplan 1973, pp 176-184). The result of this analysis is that there is a maxima if, and only if; 


$$
4 a b-c^{2}>0 \text { \& } a+b<0
$$

located at;

$$
x_{\max }=\frac{c e-2 b d}{4 a b-c^{2}} \& y_{\max }=\frac{e d-2 a e}{4 a b-c^{2}}
$$

Several other methods have been suggested for locating the maxima of (sampled) correlation functions. Thomas (1987) suggests fitting quadratic functions separately in the horizontal and vertical directions. One coordinate of the correlation function maximum is found from the location of the maximum in each of the two (1 dimensional) quadratic functions which are fitted. Later (Thomas 1990) he suggests fitting two other functions, an inverted ' $\mathrm{V}$ ' and a sinc function, to the measured correlation function. Fadzil (1991) suggests determining the peak location by calculating the 'centre of gravity' of a region of the (sampled) correlation surface near the peak. All of these techniques appear to work well in the reported computer simulations. Nevertheless, in certain, realistic, situations, they can give highly erroneous results. The problem arises, fundamentally, because these methods determine the correlation peak's two coordinates separately. That is, they treat the problem of locating the maximum as variables separable. Fitting a two dimensional polynomial, as has been done in this investigation, treats the problem as non-variables separable.

Erroneous velocity measurements can result from treating peak location in a 'variables separable' manner. All is well provided the peak in the correlation surface is both horizontally and vertically symmetric, or the maximum is located at a sampling site. These conditions often apply in computer simulations but may not apply when processing real pictures. Consider diagonal motion of an object. Camera integration may blur the object in the direction of motion while leaving resolution, normal to the motion vector, unaffected. This, in turn, can give rise to a correlation peak which is stretched in the direction of motion. Edge like features in images can also give rise to elongated peaks. In these situations the conditions necessary for variable separable peak location are not met and errors can result. The problem is indicated in table 7.1. Peak 1 is a circularly symmetric peak, about 4 samples wide, defined by;

$$
\operatorname{Peak} 1(x, y)=\exp \left\{-0.6\left(x^{2}+y^{2}\right)\right\}
$$

note the coordinates used are relative to the centre of the peak and the units are 'samples'. Peak 2, defined as, 


$$
\operatorname{Peak} 2(x, y)=\exp \left\{-0.6\left(0.53 x^{2}-0.94 x y+0.53 y^{2}\right)\right\}
$$

is 4 times wider in one direction than at right angles and is orientated at $45^{\circ}$. Both peaks were centred equidistant between 4 sampling sites. Note that peak 2 has been chosen to represent a realistic situation which often occurs in practice. The first 3 peak location techniques are fitting various functions, separately in the horizontal and vertical directions as described by (Thomas 1987 and Thomas 1990). The forth technique is the 'centre of gravity' technique described by (Fadzil 1991). The final technique is that used in this investigation and described above.

Table 7.1: Comparison of peak location techniques.

\begin{tabular}{|l|c|c||}
\hline Peak Location Technique & $\begin{array}{c}\text { Peak 1 } \\
\text { Measurement Error } \\
\text { (samples) }\end{array}$ & $\begin{array}{c}\text { Peak 2 } \\
\text { Measurement Error } \\
\text { (samples) }\end{array}$ \\
\hline \hline Separable Quadratic & 0.0000 & 0.4500 \\
\hline Separable Inverted ' $\mathrm{V}$ ' & 0.0000 & 0.4091 \\
\hline Separable sin(x)/x & 0.0000 & 0.3914 \\
\hline Centre of Gravity & 0.1957 & 0.4696 \\
\hline Non-separable polynomial & 0.0942 & 0.0998 \\
\hline
\end{tabular}

The errors given are for one coordinate only (the magnitude error is $\sqrt{2}$ times greater). From the table it can be seen that all the techniques work fairly well with a circularly symmetric peak. When, however, the peak is elongated in shape, very significant errors result with all techniques, except fitting a non-variables separable, 2 dimensional polynomial.

After completing the computer simulations it was realised that the accuracy of peak location, by fitting a 2 dimensional polynomial, could be improved. By taking the logarithm of the correlation surface, peaks with a gaussian profile are converted into peaks with a quadratic profile. The gaussian profile of peaks in the correlation function is the result, in favourable cases, of using a Gaussian window in the correlation processing. In less favourable cases a (broader) gaussian peak would be expected as a result of the central limits theorem. The error in locating the correlation maximum, indicated in table 7.1 , is due to the difference 
in shape between quadratic and Gaussian functions. By converting the peak profile from Gaussian to quadratic, this error should be largely eliminated.

\subsubsection{Results and Discussion.}

This section gives results obtained using phase and motion correlation motion estimation techniques and discusses their implications. Results, given for the two algorithms, show the accuracy and spread of motion measurements and the effect of input noise on these measurements. The intention was to attempt to determine the performance that might be obtained in a real time system working on real images.

The two motion estimation techniques were used on carefully chosen image sequences. It might be thought desirable to test motion estimation techniques on a wide range of real scenes with known motion. This would require an astronomical amount of computation. Furthermore it is extremely difficult to accurately determine the true motion in real scenes (hence the need for motion estimation techniques!). Therefore, for this investigation, carefully generated synthetic image sequences were used.

The test image sequences used were of 'pink' noise with a mean spectral amplitude 'typical' of real images (see chapter 2). One frame of the test image sequence is shown in figure 7.8. The mean spatial autocorrelation function assumed for the image spectrum was;

$$
a(x, y)=\exp \left\{-\sqrt{\frac{x^{2}}{\alpha^{2}}+\frac{y^{2}}{\beta^{2}}}\right\}
$$

where parameters $\alpha \& \beta$ are both 25 pixels. The corresponding power spectral density is given by;

$$
|S(m, n)|^{2}=\frac{2 \pi \alpha \beta}{\left(1+k^{2}\right)^{3 / 2}} \quad ; k=2 \pi \sqrt{m^{2} \alpha^{2}+n^{2} \beta^{2}}
$$

The test sequences were generated by calculating the discrete inverse Fourier transform of the corresponding spatio-temporal spectrum. That is;

$$
t(x, y, t)=\mathscr{F}_{x, y, z}^{-1}\left(S(m, n) \frac{\sin (f-m u-n v)}{L \cdot \sin ((f-m u-n v) / L))}\right)
$$

where $F_{x, y, t}^{-1}$ represents the inverse discrete Fourier transform and $x, y$ and $t$ range from 0 to 719 pixels, 0 to 576 picture lines and 0 to 15 fields respectively. Variables $u$ and $v$ are the horizontal and vertical velocity components respectively. Note that the discrete Fourier 
transform kernel $^{5}(\mathrm{~L}=16)$ has been used rather than the continuous one. Values of the phase component of the spectrum were generated randomly between $-\pi$ and $\pi$. Test sequences generated in this way are periodic in all three dimensions. Hence no discontinuous 'edge effects' are generated at the edge of the image due to motion. The test sequences thus generated are sequentially (or progressively) scanned. Therefore they were vertically subsampled to give an interlaced, 8 frame sequence. These interlaced test sequences were then processed as described in sections 7.3.1, 7.3.2 and 7.3.3 above.

The true and measured motion for a variety of test sequences, with different horizontal and vertical motion, are compared in tables 7.2 to 7.5 below. Using the 8 frame test sequences described above, image motion was measured in 1620,128 pixel square, regions for phase correlation and 1296 regions for motion correlation. The tables show the true and measured velocity of the test image, the standard deviation of the measured velocity and the mean value of the correlation function at largest and second largest maxima. The 'measured horizontal velocity' is the mean of the velocity measurements in all the regions measures. The 'RMS error' is the standard deviation of the velocity measurements for all regions. When interpreting these results it should be remembered that the signal has been subsampled 2:1 horizontally and 4:1 vertically. Hence a velocity of 4 picture lines/20ms actually only corresponds to a single (vertical) sampling period of the correlation function. Therefore care must be used when comparing these results with other published results (eg Thomas 1987).

A number of conclusions and inferences can be drawn from these results. Consider first the random error of the velocity measurements. This is about 0.1 pixels or 0.2 picture lines per field period for phase correlation. For motion correlation it is about 0.02 pixels or picture lines per field period. Motion correlation appears to have between 5 and 10 time less random error than phase correlation. The measurement error, for motion correlation, is approximately equal in the horizontal and vertical directions. This is a little unexpected because the test image, although originally isotropic, was subsampled twice as much vertically than horizontally. This result suggests that the measurement accuracy of the motion correlation technique is being limited by the original image sequence. Phase correlation, by contrast, has about 2:1 difference in random error between vertical and horizontal directions. This, in conjunction with the contrasting result from motion correlation, suggests that phase correlation is being limited by the processing used.

${ }^{5}$ The discrete Fourier transform kernel is given by $\sin (\pi \mathrm{k}) / \mathrm{L} \cdot \sin (\pi \mathrm{k} / \mathrm{L})$ where $\mathrm{L}$ is the transform length (equals 16 in this case). This is in contrast to the continuous Fourier transform kernel, $\sin (\pi x) / \pi x$. In the limit, as $L$ becomes very large, the two kernels become the same. 
Table 7.2: Phase Correlation, horizontal pans.

\begin{tabular}{||l|l|l|l|l||}
\hline $\begin{array}{l}\text { Actual } \\
\text { Horizontal } \\
\text { Velocity } \\
\text { pixels/20ms }\end{array}$ & $\begin{array}{l}\text { Measured } \\
\text { Velocity } \\
\text { pixels/20ms }\end{array}$ & $\begin{array}{l}\text { RMS error } \\
\text { (horizontal) }\end{array}$ & Peak Height & Peak Height \\
\hline 0.0 & 0.0004 & $8.758 \times 10^{-2}$ & 0.7250 & $3.899 \times 10^{-2}$ \\
\hline 0.5 & 0.3930 & $3.936 \times 10^{-2}$ & 0.6850 & $4.307 \times 10^{-2}$ \\
\hline 1.0 & 0.9924 & $8.362 \times 10^{-2}$ & 0.6537 & $4.356 \times 10^{-2}$ \\
\hline 1.5 & 1.5978 & $6.330 \times 10^{-2}$ & 0.6794 & $4.404 \times 10^{-2}$ \\
\hline 2.0 & 1.9988 & $9.772 \times 10^{-2}$ & 0.6822 & $4.387 \times 10^{-2}$ \\
\hline 4.0 & 3.9996 & $9.686 \times 10^{-2}$ & 0.6740 & $4.488 \times 10^{-2}$ \\
\hline 8.0 & 8.0002 & $9.568 \times 10^{-2}$ & 0.6973 & $4.142 \times 10^{-2}$ \\
\hline 16.0 & 16.0000 & $9.382 \times 10^{-2}$ & 0.6548 & $4.496 \times 10^{-2}$ \\
\hline 32.0 & 31.9942 & $1.099 \times 10^{-1}$ & 0.5293 & $5.388 \times 10^{-2}$ \\
\hline
\end{tabular}

Table 7.3: Phase Correlation, vertical tilts.

\begin{tabular}{||l|l|l|l|l||}
\hline $\begin{array}{l}\text { Actual } \\
\text { Vertical } \\
\text { Velocity } \\
\text { pixels/20ms }\end{array}$ & $\begin{array}{l}\text { Measured } \\
\text { Vertical } \\
\text { Velocity } \\
\text { picture } \\
\text { lines/20ms }\end{array}$ & $\begin{array}{l}\text { RMS error } \\
\text { (vertical) } \\
\text { picture } \\
\text { lines/20ms }\end{array}$ & $\begin{array}{l}\text { Peak Height } \\
\text { (highest }\end{array}$ & $\begin{array}{l}\text { Peak Height } \\
\text { (second } \\
\text { highest peak) }\end{array}$ \\
\hline \hline 0.0 & -0.0001 & $1.720 \times 10^{-1}$ & 0.7250 & $3.899 \times 10^{-2}$ \\
\hline 1.0 & 0.7768 & $1.915 \times 10^{-1}$ & 0.7015 & $3.771 \times 10^{-2}$ \\
\hline 2.0 & 2.0312 & $2.789 \times 10^{-1}$ & 0.6278 & $4.456 \times 10^{-2}$ \\
\hline 3.0 & 3.2752 & $1.935 \times 10^{-1}$ & 0.6740 & $4.203 \times 10^{-2}$ \\
\hline 4.0 & 4.0281 & $1.742 \times 10^{-1}$ & 0.7059 & $4.012 \times 10^{-2}$ \\
\hline 8.0 & 8.0485 & $1.783 \times 10^{-1}$ & 0.6798 & $4.197 \times 10^{-2}$ \\
\hline 16.0 & 16.0763 & $9.076 \times 10^{-2}$ & 0.6324 & $4.607 \times 10^{-2}$ \\
\hline 32.0 & 32.1964 & $2.072 \times 10^{-1}$ & 0.4482 & $8.268 \times 10^{-2}$ \\
\hline
\end{tabular}


Table 7.4: Motion Correlation, horizontal pans.

\begin{tabular}{||l|l|l|l|l||}
\hline $\begin{array}{l}\text { Actual } \\
\text { Horizontal } \\
\text { Velocity } \\
\text { pixels/20ms }\end{array}$ & $\begin{array}{l}\text { Measured } \\
\text { Velocizontal } \\
\text { Velocity }\end{array}$ & $\begin{array}{l}\text { RMS error } \\
\text { (horizontal) }\end{array}$ & Peak Height & Peak Height \\
\hline \hline 0.0 & -0.0007 & $4.810 \times 10^{-3}$ & 0.7240 & $2.642 \times 10^{-2}$ \\
\hline 0.5 & 0.5192 & $9.656 \times 10^{-3}$ & 0.6512 & $2.940 \times 10^{-2}$ \\
\hline 1.0 & 1.0008 & $2.142 \times 10^{-2}$ & 0.6452 & $2.602 \times 10^{-2}$ \\
\hline 1.5 & 1.6046 & $9.544 \times 10^{-3}$ & 0.6564 & $3.144 \times 10^{-2}$ \\
\hline 2.0 & 1.9882 & $1.440 \times 10^{-2}$ & 0.6535 & $2.665 \times 10^{-2}$ \\
\hline 4.0 & 3.9950 & $1.666 \times 10^{-2}$ & 0.6438 & $2.681 \times 10^{-2}$ \\
\hline 8.0 & 7.9980 & $1.982 \times 10^{-2}$ & 0.6551 & $2.814 \times 10^{-2}$ \\
\hline 16.0 & 15.996 & $2.470 \times 10^{-2}$ & 0.6412 & $2.969 \times 10^{-2}$ \\
\hline 32.0 & 31.994 & $3.026 \times 10^{-2}$ & 0.5624 & $3.408 \times 10^{-2}$ \\
\hline
\end{tabular}

Table 7.5: Motion Correlation, vertical tilts.

\begin{tabular}{||l|l|l|l|l||}
\hline $\begin{array}{l}\text { Actual } \\
\text { Vertical } \\
\text { Velocity } \\
\text { pixels/20ms }\end{array}$ & $\begin{array}{l}\text { Measured } \\
\text { Vertical } \\
\text { Velocity } \\
\text { picture } \\
\text { lines/20ms }\end{array}$ & $\begin{array}{l}\text { RMS error } \\
\text { (vertical) } \\
\text { picture } \\
\text { lines/20ms }\end{array}$ & $\begin{array}{l}\text { Peak Height } \\
\text { peak) }\end{array}$ & $\begin{array}{l}\text { Peak Height } \\
\text { (second } \\
\text { highest peak) }\end{array}$ \\
\hline \hline 0.0 & 0.0001 & $2.490 \times 10^{-3}$ & 0.7240 & $2.642 \times 10^{-2}$ \\
\hline 1.0 & 0.9140 & $9.640 \times 10^{-3}$ & 0.7114 & $2.655 \times 10^{-2}$ \\
\hline 2.0 & 1.9860 & $1.893 \times 10^{-2}$ & 0.6085 & $3.008 \times 10^{-2}$ \\
\hline 3.0 & 3.2516 & $1.422 \times 10^{-2}$ & 0.6944 & $2.688 \times 10^{-2}$ \\
\hline 4.0 & 4.004 & $1.719 \times 10^{-2}$ & 0.7182 & $2.707 \times 10^{-2}$ \\
\hline 8.0 & 8.040 & $2.486 \times 10^{-2}$ & 0.6945 & $2.826 \times 10^{-2}$ \\
\hline 16.0 & 16.056 & $2.567 \times 10^{-2}$ & 0.6177 & $3.589 \times 10^{-2}$ \\
\hline 32.0 & 32.172 & $2.923 \times 10^{-2}$ & 0.4886 & $4.668 \times 10^{-2}$ \\
\hline
\end{tabular}


Both phase and motion correlation exhibit a small systematic error for some velocities. These errors occur when the maximum of the correlation function lies between sampling sites. It seems likely that these errors are due to polynomial fitting technique used to locate the position of the maxima. This error results from the mismatch between the actual shape of the maximum and the shape of the polynomial fitted. These systematic errors should be greatly reduced by non-linear processing of the correlation surfaces prior to locating the maxima, as described at the end of section 7.3.3.

The signal/noise ratio of the correlation surfaces is approximately the same, about 25 , for both phase and motion correlation. This signal to noise ratio is roughly the ratio between the largest (wanted) maximum relative to the second largest maximum (due to 'noise'). The signal to noise ratio suggests that 5 or 6 bits would be sufficient to represent the correlation surface. However, it must be remembered that, for unfavourable images, the correlation maxima may be broad and therefore the maximum value lower. A dynamic range of greater than 5 or 6 bits is required to cope with this situation.

The value of the correlation maximum reduces only slowly with velocity, for both phase and motion correlation. Hence it is possible to accurately measure large velocities with either technique, as required for standards conversion.

The effect of input noise on the motion estimation process is shown in tables 7.6 and 7.7 below. These tests used static test sequences, to which a known amount of noise had been added. The noise used was approximately Gaussian white noise. The test images were stored in 8 bit integer format, with signal values ranging from 16 to 240 . The RMS noise level added was;

$$
N=\frac{2^{b}}{\sqrt{12}}
$$

where $b$ is the signal to noise ratio in bits. This definition corresponds to the signal to noise ratio in a signal rounded to $b$ bits. 
Table 7.6: Phase Correlation, effect of input noise.

\begin{tabular}{||l|l|l|l|l||}
\hline $\begin{array}{l}\text { Signal to } \\
\text { Noise Ratio }\end{array}$ & $\begin{array}{l}\text { RMS error } \\
\text { (horizontal) } \\
\text { pixels/20ms }\end{array}$ & $\begin{array}{l}\text { RMS error } \\
\text { (vertical) } \\
\text { picture } \\
\text { lines/20ms }\end{array}$ & $\begin{array}{l}\text { Peak Height } \\
\text { (highest }\end{array}$ & $\begin{array}{l}\text { Peak Height } \\
\text { (second } \\
\text { highest peak) }\end{array}$ \\
\hline \hline 8 & 0.0868 & 0.1700 & 0.7365 & $3.82 \times 10^{-2}$ \\
\hline 7 & 0.0848 & 0.1696 & 0.7212 & $3.87 \times 10^{-2}$ \\
\hline 6 & 0.0884 & 0.1756 & 0.7102 & $3.99 \times 10^{-2}$ \\
\hline 5 & 0.0868 & 0.1792 & 0.6914 & $4.06 \times 10^{-2}$ \\
\hline 4 & 0.1066 & 0.1720 & 0.6670 & $4.28 \times 10^{-2}$ \\
\hline 3 & 0.1560 & 0.2008 & 0.5884 & $5.25 \times 10^{-2}$ \\
\hline 2 & 0.2052 & 0.2580 & 0.4246 & $6.67 \times 10^{-2}$ \\
\hline 1 & 1.2264 & 0.5416 & 0.2799 & $7.55 \times 10^{-2}$ \\
\hline
\end{tabular}

Table 7.7: Motion Correlation, effect of input noise.

\begin{tabular}{||l|l|l|l|l||}
\hline $\begin{array}{l}\text { Signal to } \\
\text { Noise Ratio } \\
\text { (Bits) }\end{array}$ & $\begin{array}{l}\text { RMS error } \\
\text { (horizontal) } \\
\text { pixels/20ms }\end{array}$ & $\begin{array}{l}\text { RMS error } \\
\text { (vertical) } \\
\text { picture } \\
\text { lines/20ms }\end{array}$ & $\begin{array}{l}\text { Peak Height } \\
\text { (highest } \\
\text { peak) }\end{array}$ & $\begin{array}{l}\text { Peak Height } \\
\text { (second } \\
\text { highest peak) }\end{array}$ \\
\hline \hline 8 & 0.0040 & 0.0024 & 0.7270 & $2.62 \times 10^{-2}$ \\
\hline 7 & 0.0054 & 0.0036 & 0.7205 & $2.66 \times 10^{-2}$ \\
\hline 6 & 0.0090 & 0.0060 & 0.7185 & $2.67 \times 10^{-2}$ \\
\hline 5 & 0.0162 & 0.0112 & 0.7091 & $2.74 \times 10^{-2}$ \\
\hline 4 & 0.0348 & 0.0224 & 0.6787 & $2.81 \times 10^{-2}$ \\
\hline 3 & 0.0792 & 0.0444 & 0.5985 & $3.00 \times 10^{-2}$ \\
\hline 2 & 0.1850 & 0.0860 & 0.4335 & $3.31 \times 10^{-2}$ \\
\hline 1 & 0.4632 & 0.1872 & 0.2062 & $4.21 \times 10^{-2}$ \\
\hline
\end{tabular}


Various conclusions and implications can be drawn from the results in tables 7.7 and 7.7. Both phase and motion correlation algorithms appear to be very robust with respect to the effects of input noise. The effect of input noise seems to be to reduce the value of the correlation maximum, rather than increase the noise in the correlation function. Hence accurate motion estimation remains possible so long as the principle correlation maximum is sufficiently greater (say 3 times greater) than the next highest (noise) maximum. Certainly good motion estimation should be possible at, or below, a signal to noise ratio of 3 to 4 bits. It is interesting to note the difference in performance between phase and motion correlation. Motion correlation starts, at high signal to noise ratios, with very low velocity measurement errors which increase monotonically as the signal to noise ratio degrades. Phase correlation, by contrast, starts with a higher velocity measurement error which does not, however, decrease until the signal to noise ratio degrades to about 4 bits. As with the results in tables 7.2 to 7.5 , this suggests that motion correlation is being limited by the input image, whereas phase correlation is limited (at high $\mathrm{S} / \mathrm{N}$ ratios) by the processing used.

This section has discussed an experimental investigation of two motion estimation techniques. One of these (phase correlation) has been investigated previously, while motion correlation appears to be new. The intention of this investigation was to try and gain an indication of the performance of these algorithms if used in a real time system for standards conversion. Care was taken with the processing used, to try to achieve the best results from both algorithms. Since very similar processing was used for both techniques it is possible to compare fairly a region matching algorithm (phase correlation) with a frequency domain algorithm (motion correlation). In general, motion correlation appears to perform somewhat better than phase correlation, although it is more computationally complex.

\subsection{Summary.}

This chapter has discussed the requirements and techniques of motion estimation for standards conversion. The 3 main categories of motion estimation techniques, namely spatiotemporal constraint, region matching and frequency domain were reviewed with reference to the published literature. The importance of hierarchical motion estimation, to measure the large velocities required by standards conversion, was discussed; as was the use of recursive motion estimation techniques.

A wide variety of spatio-temporal constraint and region matching motion estimation techniques have been described in the literature. However both phase and motion correlation motion estimation techniques appear more suitable for the particular application of standards conversion. The reason why phase correlation has, perhaps, received less attention than other techniques may be because it appears to be computationally complex. This chapter has tried 
to show that, for the large range and high accuracy of motion vectors needed for standards conversion, the complexity of other techniques is very much greater than in other applications. Therefore, for standards conversion, phase correlation may be less complex than some of the alternatives, and can be demonstrated to give good performance.

The experimental investigation, using computer simulation, of 2 motion estimation techniques was described. The two techniques, phase correlation and motion correlation, were selected as being particularly suitable for standards conversion. Results of the simulations allowed the performance of the two algorithms to be assessed and for them to be compared. The phase correlation technique has been reported previously. The results presented here confirm the published results and extend them to indicate the performance of a real time system operating on typical pictures. The motion correlation technique appears to be new. Results from simulating this algorithm suggest it may be capable of better performance than the phase correlation technique, albeit at the expense of additional complexity. Both algorithms have the performance required by standards conversion applications. Motion correlation may have an advantage over phase correlation because of greater accuracy, better noise performance and resilience to the effects of prior temporal filtering of the pictures.

\subsection{References.}

1. Allen, J.B., 1977. Short term spectral analysis, synthesis and modification by discrete Fourier transform. IEEE Transactions on Acoustics, Speech and Signal Processing, Vol. ASSP-25, No. 3, June 1977, pp 235-238.

2. Allen, J.B., Rabiner, L.R. 1977. A Unified Approach to Short-Time Fourier Analysis and Synthesis. Proceedings of the IEEE, Vol. 65, No 11, November 1977.

3. Bertero, M., Poggio, T., Torre, V., 1988. Ill-posed problems in early vision. Proc. IEEE, Vol. 76, pp 869-889, August 1988.

4. Bierling, M., Thoma, R. 1986. Motion compensating field interpolation using a hierarchically structured displacement estimator. Signal Processing, Volume 11, No. 4, December 1986, pp. 387-404. Elsevier Science Publishers.

5. Brigham, E.O. 1974. The Fast Fourier Transform. Prentice Hall. ISBN 0-13-307496-X.

6. Cafforio, C., Rocca, F. 1983. The differential method for image motion estimation. Image sequence processing and dynamic scene analysis (ed. T.S. Huang). Springer-Verlag, pp 104-124, 1983.

7. Cafforio, C., Rocca, F., Tubaro, S., 1990. Motion Compensated Image Interpolation. IEEE Trans. on Comm. Vol. 38, No. 2, February 1990, pp 215-222.

8. Clarke, C.K.P, 1987. Future television systems : comparison of sequential and interlaced scanning. BBC Research Department Report No. BBC RD 1987/11. November 1987. 
9. Dubois, E., Konrad, J., 1990. Review of techniques for motion estimation and motion compensation. Fourth International Colloquium on Advanced Television Systems, Ottawa, Canada, June 1990. Organised by CBC Engineering, Montreal, Quebec, Canada.

10. Fadzil, A., 1991. Video image sequence coding at low bit rates. Ph.D. Thesis, Department of Electronic Systems Engineering, University of Essex, England.

11. Fennema, C.L., Thompson, W.B., 1979. Velocity determination in scenes containing several moving objects. Computer Vision, Graphics and Image Processing, Vol. 9, pp 301-315, 1979.

12. Girod, B. \& Thoma, R. 1985. Motion-compensating field interpolation from interlaced and non-interlaced grids. 2nd International Technical Symposium on Optical and Electo-Optical Science and Engineering: Image Processing Symposium, Cannes, December 1985.

13. Girod, B., Kuo, D. 1989. Direct estimation of displacement histograms. Image understanding and machine vision 1989, Technical digest series, Vol. 14, Conference edition, pp 73-76.

14. Haass, U.L., Brubaker, T.A., 1980. Estimation of cloud motion from satellite pictures. Proc. IEEE, International Conference on Acoustics, Speech and Signal Processing, (ICASSP ‘80) Denver, Colorado, 1980, pp 422-425.

15. Hann, G., Huijgen, H., 1990. new algorithm for motion estimation. Signal processing of HDTV, II, L. Chiariglione (editor), Proceedings of the Third International Workshop on HDTV, Turin, Italy, 1989, pp 109-116.

16. Haskell, B.G, 1974. Frame to frame coding of television pictures using twodimensional Fourier transforms. IEEE transactions on Information Theory, Vol. IT-20, pp 119-120, 1974.

17. Heegar, D.J., 1987. Optical flow from spatiotemporal filters. Proceedings of the First International Conference on Computer Vision, 1987, pp 181-190.

18. Horn, B.K., Schunck, B.G. 1981. Determining optical flow. Artif. Intell., 1981, 17, pp. 185-203.

19. Huang, T.S., Tsai, R.Y., 1981. Image sequences analysis : Motion estimation. Image Sequence Analysis, T.S. Huang (editor), Springer-Verlag, Berlin, Germany, 1981, pp 1-18.

20. Jacobson, L., Wechsler, H., 1987. Derivation of optical flow using a spatiotemporalfrequency approach. Computer Vision, Graphics and Image Processing, Vol. 38, No. 1, April 1987, pp 29-65.

21. Kaplan, W., 1973. Advanced Calculus, second edition. Addison Wesley. 
22. Keesman, G., 1988. Motion estimation based on a model incorporating translation, rotation and zoom. Signal Processing IV, Lacoume, J.L., Chehikian, A., Martin, N., Malbos, J. (editors), Elsevier Science Publishers, pp 31-33.

23. Konrad, J., 1990. Issues of accuracy and complexity in motion compensation for ATV systems. Contribution to 'Les Assises Des Jeunes Chercheurs', CBC, Montreal, June 1990.

24. Konrad, J., Dubois, E. 1990. Use of colour information in Bayesian estimation of 2-D motion. Proc. IEEE international Conference on Acoustics, Speech and Signal Processing (ICASSP '90), 1990, pp 2205-2208.

25. Lim, J.S., 1990. Two-dimensional signal and image processing. Prentice Hall 1990, ISBN 0-13-934563-9, pp 497-511.

26. Lucas, B.D., Kanade, T., 1981. An iterative image registration technique with an application to stereo vision. IJCAI, pp. 674-679, 1981.

27. Martinez, D.M. 1987. Model-based motion estimation and its application to restoration and interpolation of motion pictures. RLE Technical Report No. 530. June 1987. Research Laboratory of Electronics, Massachusetts Institute of Technology, Cambridge, MA 02139 USA.

28. Moorhead, R.J., Rajala, S.A., Cook, L.W. 1987. Image sequence compression using a pel-recursive motion-compensated technique. IEEE Journal on selected areas in communications, vol. sac-5., No. 7, August 1987.

29. Musmann, H.G., Pirsch, P., Grallert, H.J. 1985. Advances in picture coding. Proceeding of the IEEE, Vol. 73, No. 4, 1985, pp 523-548.

30. Nagel, H.H., Enkelmann, W., 1986. An investigation of smoothness constraints for the estimation of displacement vector fields from image sequences. IEEE Transactions on Pattern Analysis and Machine Intelligence, Vol. PAMI-8, pp 565-593, September 1986.

31. Nawab, S.H., 1988. Short-time Fourier transform. Advanced Topics in Signal Processing, Lim, J.S. \& Oppenheim, A.V. (Editors), Prentice Hall, 1988, ISBN 0-13-013129-6 025, pp 289-337.

32. Netravali, A.N., Robbins, J.D. 1979. Motion compensated television coding, Part 1. Bell Syst. Tech. J., vol. 58, pp. 631-670, March 1979.

33. Paquin, R., Dubois, E., 1983. A spatio-temporal gradient method for estimating the displacement vector field in time-varying imagery. Computer Vision, Graphics and Image Processing, Vol. 21, 1983, pp 205-221.

34. Pearson, J.J., Hines, D.C., Golosman, S., Kuglin, C.D. 1977. Video rate image correlation processor. SPIE, Vol. 119, Application of digital image processing (IOCC 1977). 
35. Robert, P., Cafforio, C., Rocca, F., 1985. Time/Space recursion for differential motion estimation. Spie Symp., Cannes, France, November 1985.

36. Schalkoff, R.J., Labuz, J., 1984. An infrared spatio-temporal model and recursive algorithm for image motion estimation. Proceedings of the 7th International Conference on Pattern Recognition, Montreal, Canada, 1984, pp 530-533.

37. Stearns, S.D., 1988. Fundamentals of adaptive signal processing. Advanced Topics in Signal Processing, Lim, J.S. \& Oppenheim, A.V. (Editors), Prentice Hall, 1988, ISBN 0-13-013129-6 025, pp 246-288.

38. Thomas, G.A., 1987. Television Motion Measurement for DATV and other Applications. BBC Research Department Report No BBC RD 1987/11. September 1987.

39. Thomas, G.A., 1990. Motion estimation and its application to broadcast television. Ph.D. Thesis, Department of Electronic Systems Engineering, University of Essex, England.

40. Thomas, G.A. 1991. Motion and motion estimation. Image Processing, Don Pearson (editor). McGraw-Hill 1991, ISBN 0-07-707323-1, pp 40-57.

41. Tretiak, O., Pastor, L., 1984. Velocity estimation from image sequences with second order differential operators. Proceedings of IEEE International Conference on Pattern Recognition (ICPR ‘84), 1984, pp 16-19.

42. Vega-Riveros, J.F., Jabbour, K. 1986. Review of motion analysis techniques. IEE Proceedings, Vol 136, Pt I, No. 6, December 1989.

43. Weiss, P., Christensson, B., 1990. Real time implementation of subpixel motion estimation for Broadcast applications. IEE Colloquium on 'Applications of motion compensation', October 1990, Digest No. 1990/128.

44. Wu, S.F., Kittler, J., 1990. A differential method for the simultaneous estimation of rotation, change of scale and translation. Image Communication, Vol. 2, No. 1, May 1990, pp 69-80.

45. Yamaguchi, H., 1989. Iterative method of movement estimation for television signals. IEEE Trans. on Comm., Vol. 37, No. 12, December 1989, pp 1350-1358. 


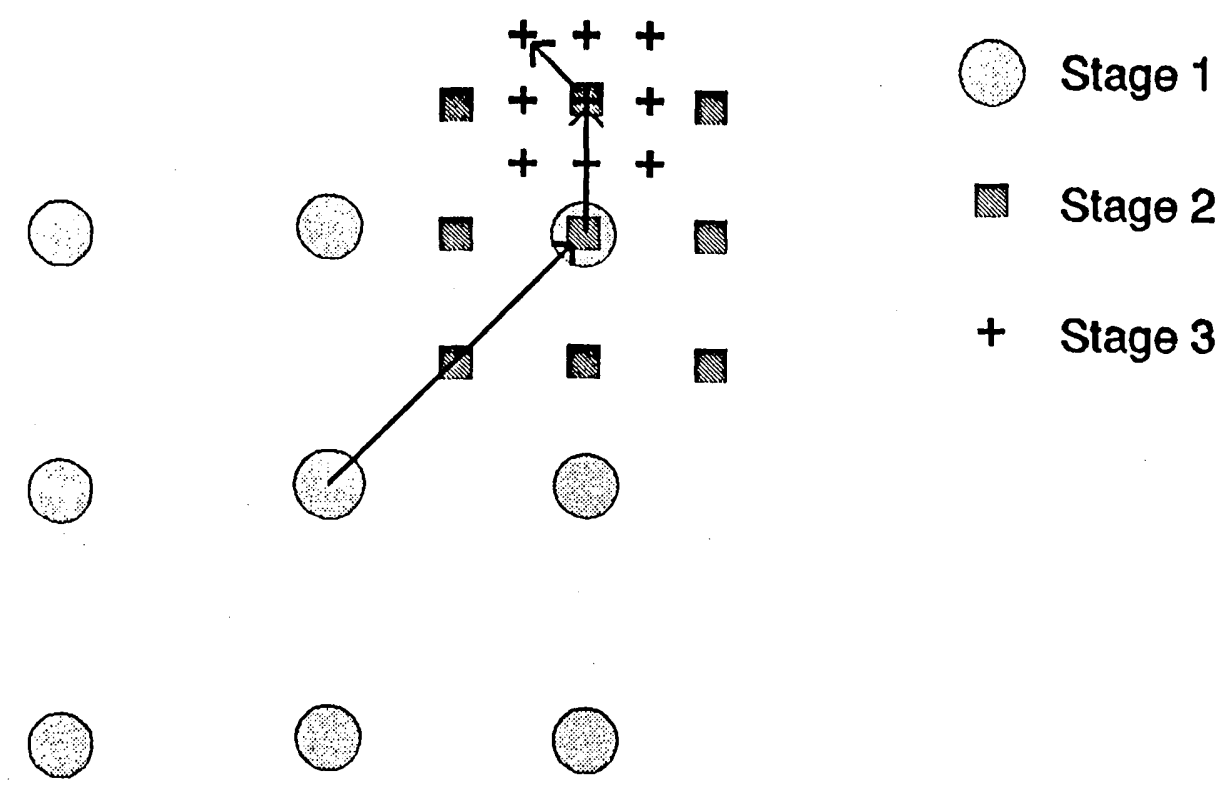

Figure 7.1 : Hierarchical Motion Estimator ( 3 stages )

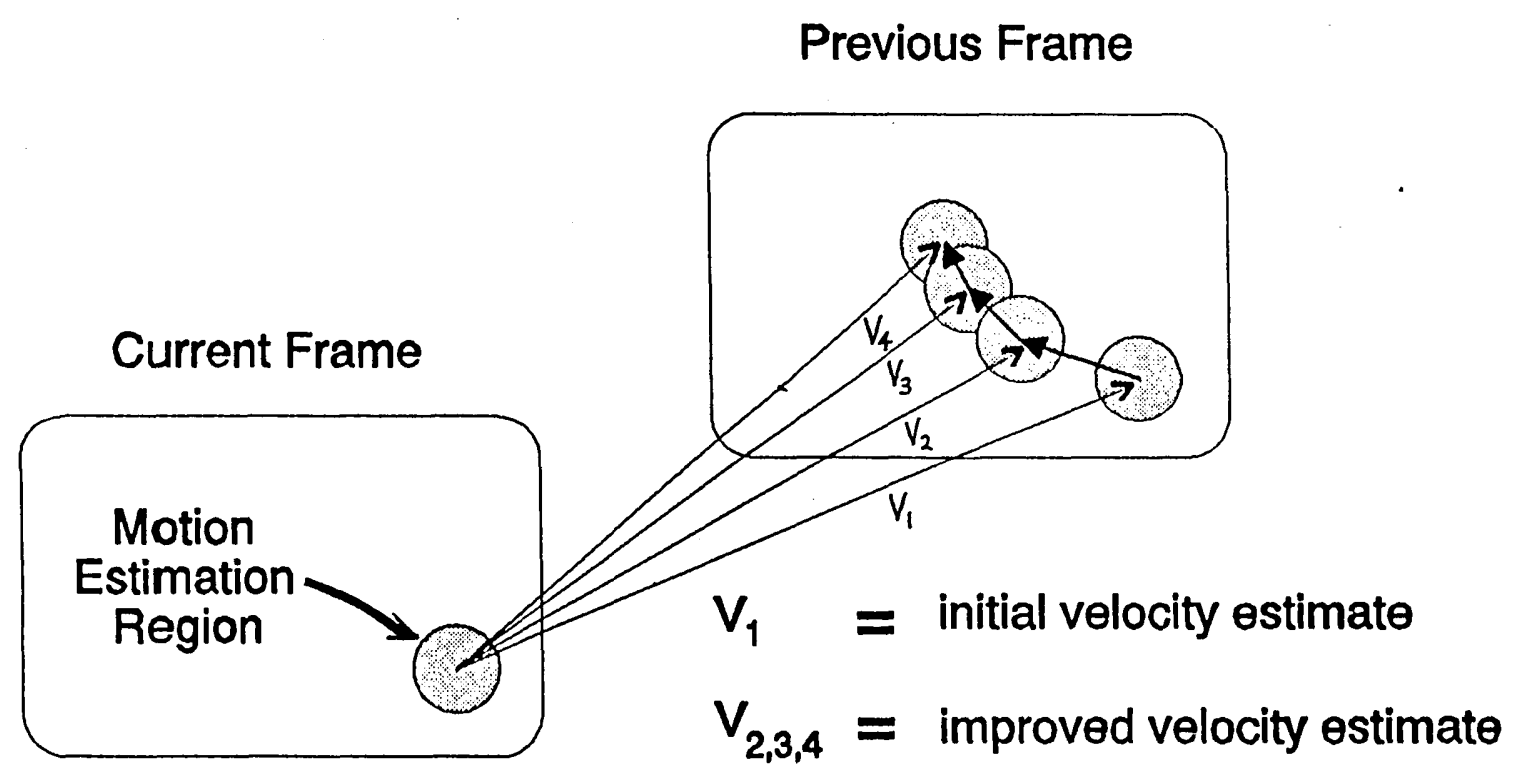

Figure 7.2 : Recursive Motion Estimation 


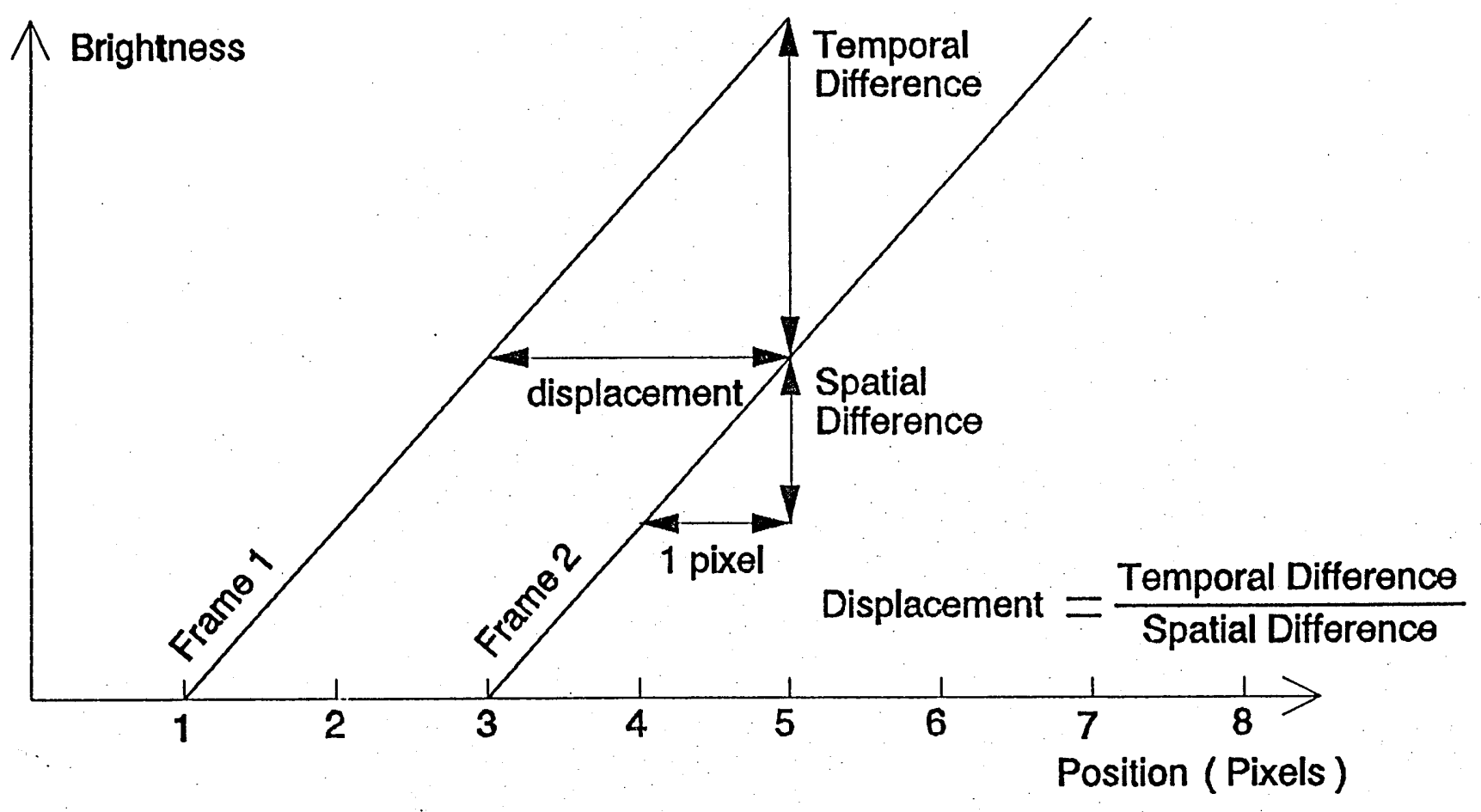

Figure 7.3 : Estimating displacement from Brightness differences

Previous Frame

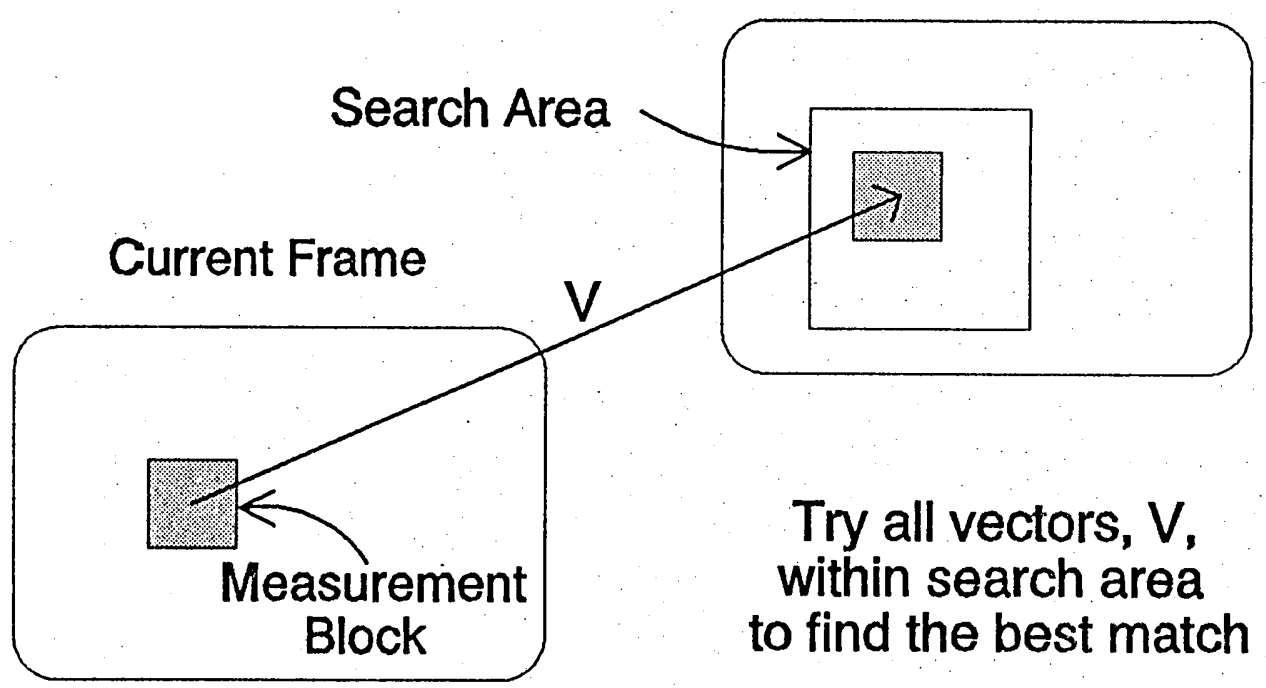

Figure 7.4 : Motion estimation using Block Matching 


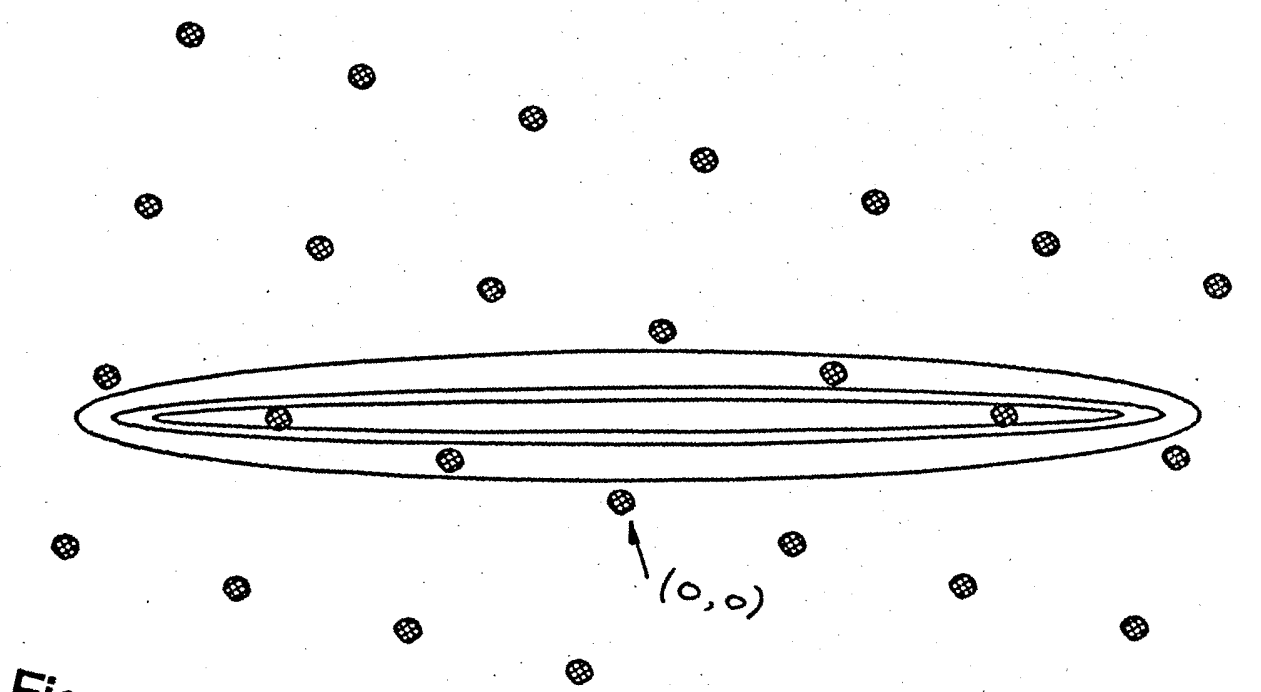

Figure 7.5 :

(2) Contour lines of a displaced frame
difference function 


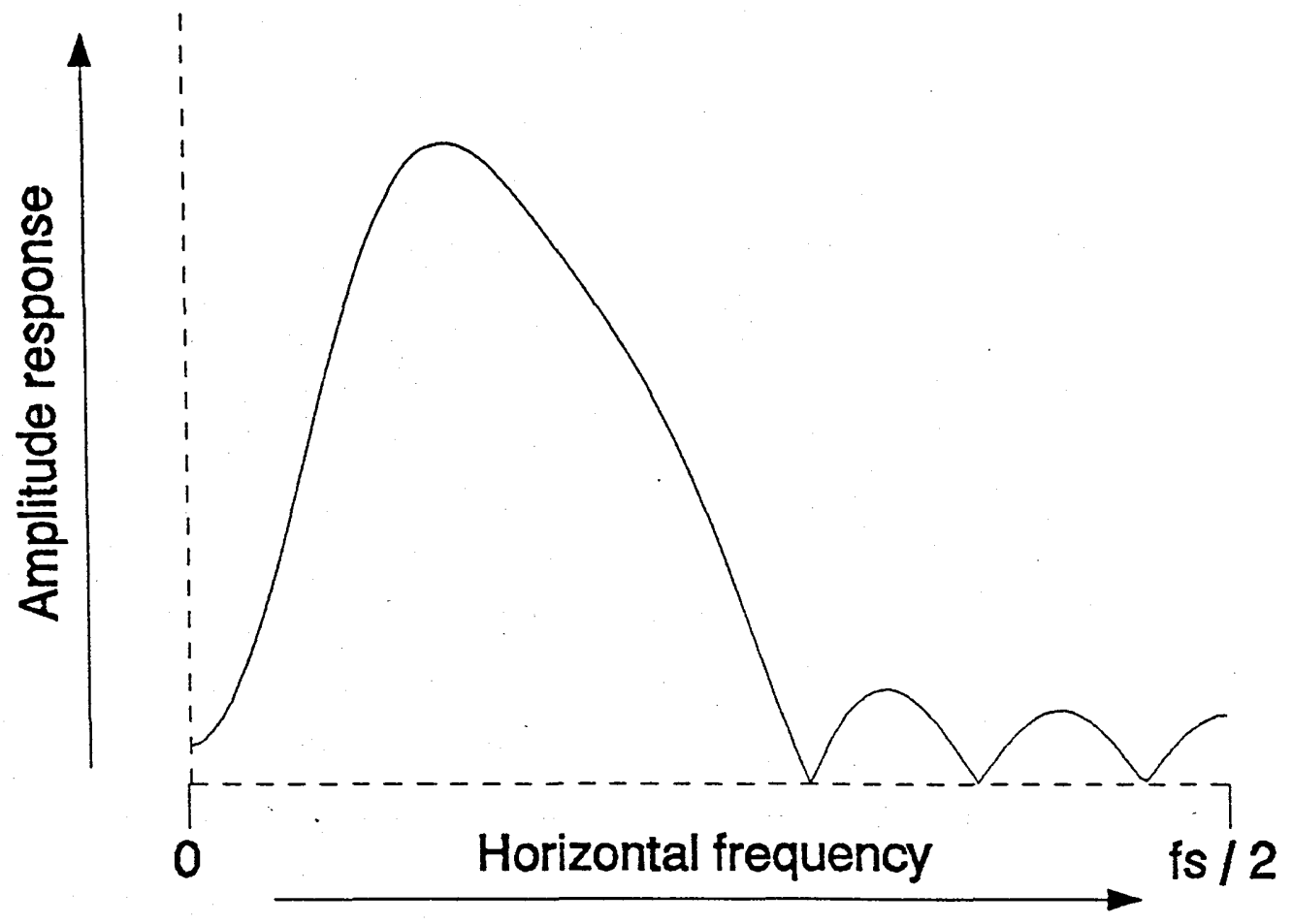

Figure 7.6 : Frequency response of horizontal prefilter

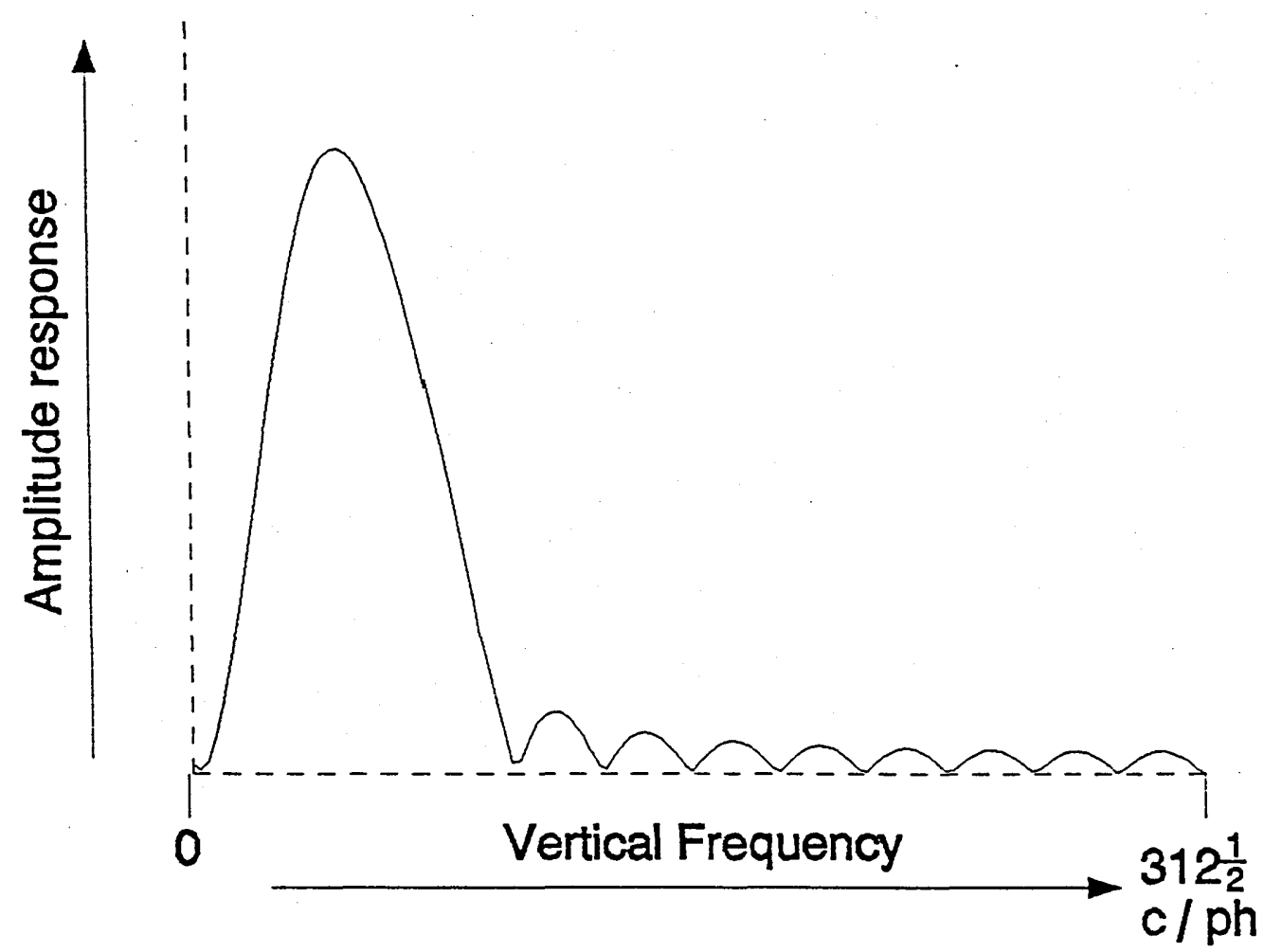

Figure 7.7 : Frequency response of vertical prefilter 


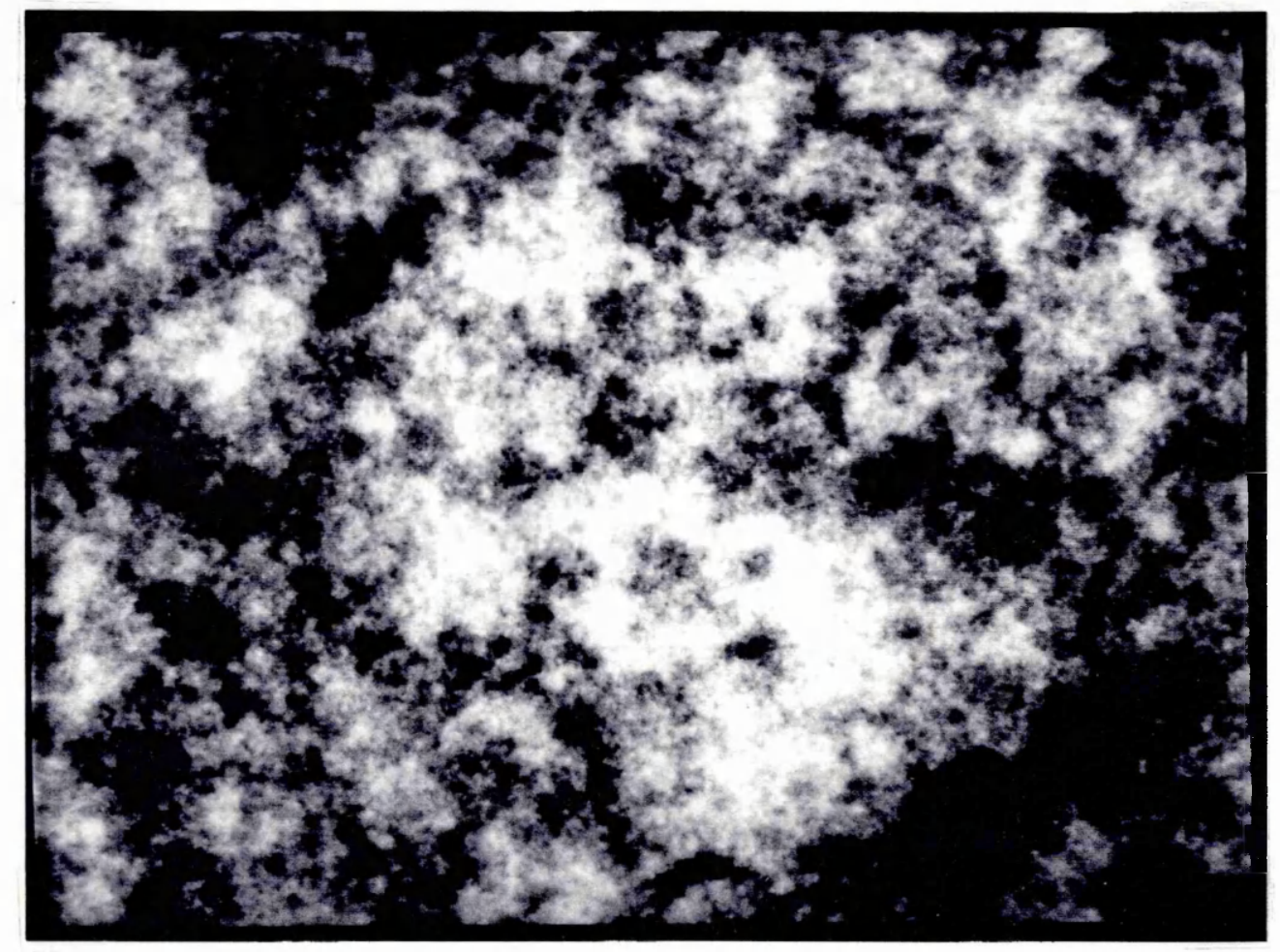

Figure 7.8 : Test image used for motion analysis 


\subsection{Introduction.}

This chapter presents a description of an experimental real time motion estimation system, and the results obtained from it. The motivation for building this system was to investigate improvements in the processing of moving images using motion compensation (described in chapter 6). An essential prerequisite for motion compensated processing is a high quality motion estimator. Indeed, the results in this thesis suggest that, the quality of motion compensated systems may depend more on the quality of the motion estimator than the implementation of motion compensated processing. Therefore this system was built with the intention of producing the highest quality motion vector field, given the state of knowledge at the beginning of the project. A number of different applications were envisaged for the equipment including motion compensated standards conversion, bandwidth reduction and noise reduction. Of particular interest, in this thesis, is the application of motion compensation to standards conversion, including both intercontinental $(50$ to $60 \mathrm{~Hz}$ ) standards conversion and upconversion (50 to $100 \mathrm{~Hz}$ ).

The motion estimator described below, is based on the technique of phase correlation detailed in chapter 7. It was designed to generate accurate vectors describing the motion of all objects in a conventional television picture. At the inception of the project no real time motion estimation equipment, based on phase correlation, had been built. Therefore this experimental system was built in a modular fashion so that it was as flexible as possible. This allowed the motion estimation algorithm to be modified, both in the light of experimental results and for slightly different algorithms to be used for different applications.

This chapter concentrates on the implementation of, and results from, the motion estimator. Section 8.2 gives a detailed explanation of each of the parts of the motion estimator in turn. Section 8.3 presents results from the system both in terms of qualitative descriptions and with photographs. Detailed numerical results, for the computer simulation of a comparable system, were given in the previous chapter. The ultimate test of a motion estimator is, of course, how well it works in real image processing application. Such results, for experimental motion compensated standards conversion systems, will be presented in the next chapter.

\subsection{Hardware description.}

The motion estimation equipment is divided into a number of distinct parts as indicated in figure 8.1. The objective of the system is to generate a distinct motion vector for each pixel in the input television signal. The luminance part of the input signal is divided into 
a number of small, rectangular, sub-images ('measurement blocks') which, taken together, cover the whole image. Each part (measurement block) of the input image is analyzed to determine possible motions which may be occurring within that block. The motion analysis, for each measurement block, is performed using the technique of phase correlation described in the previous chapter. Motion analysis, in this context, generates a list of motion vectors which may be present in the picture. These possible, or 'candidate', vectors are then tested to determine which, if any, of them correspond to the motion of each pixel. The correct vector, for each pixel, is selected on the basis of having the smallest, modulus, displaced field difference in a small region around that pixel. The displaced field difference, defined in the previous chapter, is the difference between two consecutive fields allowing for the (assumed) motion between them. By testing each candidate vector for each pixel, the image is effectively segmented into regions with different motion, corresponding to the different objects in the picture.

The development of the motion estimator took place over several years. The results from the earliest system suggested modifications which were included in later developments of the system. These developments, and the reasons for them, will be discussed in the relevant parts of the description below.

\subsubsection{Signal Conditioning.}

Figure 8.2 shows a block diagram of the signal conditioning used in the final version of the motion estimator. The processes performed in this part of the system, while simple, have a significant effect on its overall performance.

The first signal conditioning process is low pass spatial filtering. This was implemented as a spatial running average over a small region of the image (the size of the region was programmable). For the results presented here a size of 3 pixels horizontally by 2 field lines was found to give satisfactory performance. Towards the end of the project a more sophisticated filter was designed, but was not available in time to be included in these results. The purpose of the spatial filter is two fold. Firstly, for the standards conversion applications presented in the next chapter, image segmentation was performed on the basis of the displaced field difference signal. In order to avoid aliasing, caused by the interlaced nature of the input television signal, a vertical lowpass filter is required. This filter removed higher vertical frequencies which were most contaminated by aliasing due to interlace. For other applications, where image segmentation was performed on the basis of displaced frame difference, this vertical filter would not be required. The second function of the filter is as a prefilter prior to subsampling described below.

Spatial subsampling by a factor of 2 , both horizontally and vertically, is performed for several reasons. Firstly it enables the size of the measurement blocks, used for motion 
analysis, to be increased. This, in turn, doubles the range of motion vectors which can be measured. Secondly spatial subsampling allows the measurement blocks, which cover the input image, to overlap rather than being merely juxtaposed. This makes the analysis of motion, for objects near the edge of a measurement block, more reliable, since any object will be present in more than one block. Overlapping the measurement blocks also allows them to be 'windowed' prior to performing Fourier transforms for motion analysis. Windowing the measurement blocks reduces 'spectral leakage' when performing (numerical) Fourier analysis (FFTs). Reduced leakage increases the signal to noise ratio of the phase correlation surface and, thereby, improves motion analysis.

Following subsampling the remaining pixels in the picture are rearranged into a form suitable for motion analysis. The output from image restructuring is in the same format as a conventional video signal. This allows the output from the picture conditioning section to be observed on a conventional monitor to verify correct operation. The picture information comprises an array of 12 (horizontal) by 9 (vertical) juxtaposed, measurement blocks. Each measurement block is 64 pixels by 32 field lines in size. Since 2:1 spatial subsampling was used the measurement blocks correspond to an area of 128 pixels by 64 field lines in the original image. This size of measurement block allows motion vectors up to \pm 62 pixels or \pm 30 field lines per frame to be measured. The output from picture restructuring is derived from only $1 / 4$ of the pixels in a normal television image (because of subsampling). Therefore each (subsampled) pixel appears in 4 (spatially adjacent) measurement blocks. This improves the motion analysis of objects near the edge of a block (as discussed above).

When first built the motion estimator omitted the signal conditioning part of the system! The measurement blocks were, at first, 64 pixels by 32 field lines in the original image. The measurement blocks were, originally, juxtaposed and not overlapped. That is motion analysis was performed directly on the input image. This allowed velocities up to \pm 31 pixels and \pm 15 field lines per frame to be measured. Computer simulations had indicated that this velocity range was sufficient for the vast majority of television pictures. When the motion estimator was first interfaced to a standards converter, it was quickly apparent that this velocity range was quite inadequate. Subsequently velocities up to 160 pixels per frame have been measured! This, perhaps, illustrates the dangers of relying too heavily on computer simulation. By introducing spatial subsampling, and the other signal conditioning described above, the maximum measurable velocity was increased to a more realistic range.

\subsubsection{Motion analysis.}

Motion analysis, in this system, is performed using phase correlation (see chapter 7). Motion analysis is a two stage process as illustrated in figure 8.3. First a phase correlation 
surface is generated for each measurement block. Then each surface is searched to find the position of maxima within it.

Generation of the phase correlation surfaces is illustrated in figure 8.4. The mathematical operation performed by the phase correlator is given by;

$$
\Phi\left(g\left(t_{0}\right), g\left(t_{1}\right)\right)=\mathscr{F}_{x}^{-1}\left\{\frac{G\left(t_{0}\right) \cdot G\left(t_{1}\right)^{*}}{\left|G\left(t_{0}\right) \cdot G\left(t_{1}\right)^{*}\right|} \frac{\left|G\left(t_{1}\right)\right|^{2}}{\sqrt{\left|G\left(t_{1}\right)\right|^{2}+N^{2}}}\right\}
$$

where $\Phi$ is the phase correlation function of images $g\left(t_{0}\right) \& g\left(t_{1}\right), G\left(t_{0}\right) \& G\left(t_{1}\right)$ are the spatial Fourier transforms of the two images, $\mathrm{N}$ is a constant (the noise level) and $\mathscr{F}_{\mathbf{x}}$ represents a spatial Fourier transform. This is very similar to equation 40 in chapter 7 and becomes identical if the spectral magnitudes of the two fields are the same. The difference is that the magnitude normalising factor, which reduces the effect of noise, only depends on the most recent image. The two dimensional Fourier transforms in figure 8.4 are performed using custom FFT hardware, utilising VLSI complex multiplier chips. The Fourier transform hardware also implements windowing in both the forward and inverse directions. Fourier transforms are performed using the real/imaginary representation of the complex data. The computations, in the frequency domain, to calculate the correlation surface are most conveniently performed on the phase and magnitude parts of the spectrum separately. Therefore components of the spectrum are converted between real/imaginary and magnitude/phase representations by coordinate conversion. Note that the phase correlation processing is performed across a frame rather than field period. This minimises the effects of aliasing caused by the interlaced nature of the input signal. Dabner 1990 provides further details on the implementation of the phase correlation processing. Conveniently the output of the phase correlation unit is formatted as a conventional video signal. This allows the correlation surfaces to be observed, in real time, on a CRT monitor. An example of the output from the phase correlation unit is shown in figure 8.8.

The second part of the motion analysis process is to locate the positions of maxima ('peaks') in the phase correlation surface. Peaks in the correlation surface indicate the presence of motion in the corresponding measurement block. The position of the peak gives the value of the motion vector. For example a peak at $(2,4)$ represents a velocity of $(4,8)$ pixels per frame period (remember 2:1 subsampling has been used). The process of locating peaks in the correlation surface is known as 'peak hunting'. It is performed by an array of fast, DSP, microprocessors. This allows easy modification of the details of the peak hunting algorithm. Up to 3 peaks may be found in each measurement block. The list of 'candidate' vectors for each measurement block is derived, heuristically, from the location of peaks found in that, and the immediately adjacent blocks. The output from the peak hunter is an ordered list of up to 8 candidate vectors for each measurement block. These candidate vectors are 
ordered according to the probability of the vector. The list of candidate vectors then forms the basis of image segmentation described in the next section.

\subsubsection{Image segmentation.}

The third part of the motion estimation system is segmentation of the image into regions with different velocities. These regions correspond to the different objects within the scene. It is this part of the system which can be tailored to the specific requirements of a particular, motion compensated, processing application. Several, slightly different, methods of segmentation were used for different applications. The implementation of these different methods is described below. However, the reasons for the precise method of image segmentation are intimately related to the way in which the motion vectors are used by each application. Therefore the rationale for the different methods of segmentation will be discussed in the next chapter, which considers the experimental implementation of motion compensated standards conversion. An additional, important, function of this part of the motion estimator is to indicate when no reliable motion vector has been determined. This allows the motion compensated processing application to use an alternative algorithm for that part of the picture.

Image segmentation in this system is based, essentially, on the comparison of local, mean, absolute displaced field differences. For brevity these local, mean, absolute displaced field differences will be referred to as 'error' functions. The error function, for a particular candidate vector, $v_{n}=\left(u_{n}, v_{n}\right)$, is defined by;

$$
\operatorname{error}\left(v_{n}, t_{0}, t_{1}\right)=\frac{1}{\text { area }} \int_{\text {region }}\left|g\left(x, y, t_{0}\right)-g\left(x+u \Delta t, y+v \Delta t, t_{1}\right)\right| d x d y ; \Delta t=t_{1}-t_{0}
$$

where $g\left(x, y, t_{0}\right)$ is the earlier field, $g\left(x, y, t_{1}\right)$ is the later field and 'area' is the area of a local region over which integration is performed. The arguments $t_{0} \& t_{1}$, of the error function, refer to the temporal location of the fields which are being compared. Note that this error function in equation 2 corresponds to the forward displaced field difference discussed in the previous chapter.

The hardware implementation used for calculating the error functions is illustrated in figure 8.5. The displaced image is calculated by spatially shifting the image. This process may be regarded as changing the length of a variable delay according to the motion vector. If the variable delay simply introduces an integer number of clock cycles delay then it is only possible to apply integer pixel displacements to the image. One of the advantages of the phase correlation technique of motion estimation is that sub-pixel accuracy can be achieved. Therefore it was considered important to be able to displace the image to sub-pixel accuracy. This was achieved, in this implementation, by the use of a bilinear interpolator. Integration 
of the modulus displaced picture difference is indicated by a low pass filtering operation. In this implementation all the filter coefficients had the same value to achieve an estimate of the integral of the (sampled) signal.

The simplest implementation of image segmentation is illustrated in figure 8.6. Error functions are calculated for each of the candidate vectors generated by the, preceding, motion analysis stage. The candidate vector giving the lowest error function is assumed to be the correct motion vector. Comparison of the error functions, for all the candidate vectors, yields the number of the candidate vector which is most likely to correspond to the actual motion. The motion vector output from image segmentation is simply the candidate motion vector, which gives the lowest error function, selected on a pixel by pixel basis. The motion estimator was built to allow the error function, corresponding to the selected candidate vector, to be displayed on a CRT monitor. This allows fault free operation of the image segmentation process to be verified. It also gives an indication of the performance of the image segmentation process. Examples of the error functions for various moving scenes are given in figures 8.9 to 8.16 and are described in section 8.3. It is also possible to display the motion vectors on a colour CRT monitor. This is done by substituting the horizontal and vertical motion vectors for the colour components $(U \& \mathrm{~V})$ of the original signal. When this is done we obtain colours, superimposed on the original (monochrome) image, which correspond to the motion of the image. Examples of motion vectors presented as a coloured overlay are given in figures 8.17 to 8.24 and are described in section 8.3.

In some circumstances the error function for all of the candidate vectors may be quite high. This indicates that either the motion analysis has failed to detect the correct motion vector, or that more complex, non-translatory, motion is occurring in that part of the picture. Indication of the failure of the motion estimator, for a part of the image, is an important feature of the image segmentation process. It allows an alternative, non-motion compensated, algorithm to be applied for processing parts of the image for which no motion vector can be found. It can also be used, with additional processing, to indicate regions of revealed and obscured background (see next chapter). A signal, indicating failure of the motion estimator, is generated for any pixel for which all the error functions are above a threshold value.

Image segmentation, performed by comparing the error functions defined above, generates a 'forward pointing' motion vector field. Such a forward pointing vector field is appropriate for projecting an image forward (but not backwards) in time along the motion trajectory. The error function could, alternatively, have been based on backward (rather than forward) displaced field difference (equation 27 chapter 7). Had this been done the vector field generated would have been appropriate for projecting the image backwards (but not forwards) in time along the motion trajectory.

For some applications better image segmentation can be achieved by comparing combinations of more than one basic error functions. Three such 'image segmentation modes' 
are illustrated in figure 8.7. Each of these image segmentation modes has been used for one of the motion compensated standards conversion applications described in the next chapter. Other image segmentation modes, appropriate to specific applications, are presented in Borer, Hulyer \& Parker 1990 and Thomas \& Lau 1990. The first mode, in figure 8.7, illustrates the simplest image segmentation mode described above. For mode one the combined error function', $\varepsilon$, upon which segmentation is based, is simply the basic error function, ie;

$$
\varepsilon_{1}\left(v_{n}, t_{0}\right)=\operatorname{error}\left(V_{n}, t_{0}, t_{1}\right)
$$

where the subscript on $\varepsilon$ indicates the image segmentation mode, $v_{n}$ is the candidate vector and the temporal argument, $t_{0}$, is the instant to which the combined error function applies. Mode one generates different motion vectors for each field, even though new candidate vectors are only generated at frame rate (ie for every pair of fields). The pixels at either end of the arrows, in figure 8.7, are compared to generate the error function. The value of the error function, thus generated, is located at the head of the arrow.

Combinations of basic error functions were compared in the segmentation process used for motion compensated field rate upconversion. This is illustrated as 'Mode 2' in figure 8.7. This second image segmentation mode is a little more complicated than the basic 'mode 1'. Image segmentation is achieved by comparing combined error functions defined as;

$$
\varepsilon_{2}\left(v_{n}, t_{0}\right)=\operatorname{error}\left(v_{n}, t_{0}, t_{1}\right)+\operatorname{error}\left(2 v_{n}, t_{0}, t_{2}\right)
$$

The first component of the combined error function is the error function between fields 1 and 2 and the second component is the error function between fields 1 and 3. By using the combination of two error functions in this way the hardware requirements have been doubled. Hence new motion vectors are only generated once per frame rather than once per field. The advantage of this mode of segmentation is that 'double check' is provided on the candidate vector using displacements with two different magnitude. This reduces the number of incorrect vector assignments, particularly those due to periodic structure in the input image. The vectors generated by this segmentation mode are appropriate for projecting an image forwards (but not backwards) in time along the motion trajectory.

Combinations of basic error functions were also used in the segmentation process for motion compensated, intercontinental, standards conversion. This process is illustrated as 'Mode 3' in figure 8.7. In this mode image segmentation uses both forward and backward displaced field difference signals. This has the advantage that, image regions which are obscured or revealed between fields are flagged as having no valid vector. For more details see the next chapter and Borer 1991. The combined error function for this mode is defined as; 


$$
\varepsilon_{3}\left(v_{n}, t_{0}\right)=\operatorname{maximum}\left(\operatorname{error}\left(v_{n}, t_{0}, t_{1}\right), \operatorname{error}\left(-v_{n}, t_{1}, t_{0}\right)\right)
$$

That is the combined error function is the maximum of a 'forward' error function and a 'backward' error function. The vectors generated in this manner are valid at any instant between fields 0 and 1 . They can be used for projecting the image both backwards and forwards in time.

This subsection has described how the image can be segmented into regions with different motion vectors. These regions correspond to different objects in the original image. Image segmentation is performed, essentially, by comparing 'error functions' for the different candidate vectors. More complex methods are used for the real applications described in the next chapter.

\subsection{Results \& Discussion.}

This section describes, with the aid of photographs, the results obtained from the experimental motion estimation system. First the parameters used in the motion estimator to obtain these results will be described. Then two sets of photographs will be described and discussed. The first set is examples of the error function corresponding to the chosen motion vector. These give an indication of the performance of the image segmentation process. The second set of photographs is of motion vectors superimposed, as a coloured 'overlay', on the original (monochrome) image. These can be used to assess the quality of the motion vectors.

The motion estimation system was built so that many parameters of the algorithm could be easily changed in software. The parameters actually used for these experiments are described here. The signal conditioning, described in sub-section 8.2.1, was used for these experiments. The lowpass filtering, used prior to both motion analysis and image segmentation, was a running average of an area of 3 pixels by 2 field lines. 2:1 subsampling was used prior to motion analysis. Sub pixel location of correlation peaks was achieved by fitting an inverted ' $V$ ' function as described in the previous chapter and Thomas 1987. Five candidate vectors were selected from the current and surrounding measurement blocks. These were selected as the highest correlation peaks in the measurement blocks, provided candidate vectors differed by at least 1 pixel (or field line) per frame. In addition a candidate vector of zero was always used. A mixture of image segmentation modes 1 and 3 (figure 8.7) were used. The error function for the 2 most probable candidate vectors (ie those corresponding to the biggest correlation peaks) was calculated using mode 3. The error function for the remaining 3 (plus stationary) candidate vectors was calculated using mode 1 . Ideally all error functions would have been calculated using mode 3 , but this was impossible because of hardware limitations. The filter used in calculating the error functions was 19 pixels by 11 field lines. This corresponds to the integration region in equation 2 If the (minimum) error 
function exceeded 32 ( 8 bit) grey levels failure of motion estimation was assumed for that pixel.

Figure 8.8 provides an example of the output from the phase correlator. The magnitude of the correlation function was displayed, as brightness, on a CRT monitor which was then photographed. One (or more) peaks can be seen in each of the measurement blocks which make up the picture. The phase correlation signal has a larger dynamic range than can conveniently be displayed on a CRT monitor and so there is more information in the phase correlation functions than may be apparent from this photograph.

Figures 8.9 to 8.16 show examples of error functions corresponding to the selected motion vector. These images indicate the performance of the image segmentation process. The original images are shown in figures 8.17 to 8.24 (with superimposed colours) and in the next chapter (figures 9.11 to 9.18). A zero value of the error function is represented, in these photographs, by black and large values by white. Ideally these images would be completely black. Typically the error function does correspond to only a few grey levels in the displaced field difference signal. In order to show anything the error functions have been greatly amplified prior to display. This results in considerable contouring of the image, and transitions of 1 bit in the error function (from an original 8 bit signal) can easily be seen. These pictures generally show little detail from the original image, indicating that the image segmentation process usually works quite well. When a constant offset, of 0.1 pixel per field period, was added to all the candidate vectors, there was a marked increase in the error function across the whole image. This suggests that the motion analysis process is typically accurate to a fraction of a pixel per field period for real scenes.

Figures 8.9 to 8.16 illustrate some interesting features of image segmentation error functions. Figures 8.9 to 8.11 are examples from a sequence of ice dancing. Generally the error function for the background is very low indicating that image segmentation is working well for those parts of the image. The error function increases slightly at horizontal and vertical edges in the original image because of non-ideal filtering of the (modulus) displaced field difference signal. The error function increases around the dancers because their motion cannot be described as simple translation. Nevertheless the error function is still sufficiently low that the motion vectors can usefully be used. The camera is panning to the right, in figures 8.9 to 8.11 , so higher error functions can be seen at the left of the picture, corresponding to appearance of new background. Figure 8.14 is an example from an ice hockey sequence. Again the error function is generally low for the background with slightly higher values around the players. Figure 8.12 is from a down hill skiing sequence involving a very fast camera pan. Motion analysis has failed to detect some of the very high velocities in this scene. The error function assumes a relatively high value for the background. For some regions the error function exceeds a threshold level of 32 grey levels, so that motion estimation is deemed to have failed. Nevertheless the error function, for the near stationary 
skier in the foreground, is relatively low and motion estimation has succeeded for this part of the picture. Figure 8.13 is from down hill skiing with a slightly slower pan. In this case the motion analysis has succeeded in detecting the correct motion vectors and the error signal is relatively low for the whole scene. Higher error functions can be seen at the edges of the picture because of revealed and obscured background. Note that there is a region of high error both in front and behind the skiers head. This is because the head has moved by more than its own width between frames. This effect is a feature of 'Mode 3' image segmentation. Finally figure 8.15 is a pan across a town scene and figure 8.16 is a car driving in front of a building. These show relatively low error functions throughout, showing the image segmentation is working well.

Figures 8.17 to 8.24 show motion vectors, represented as colours, superimposed on the original (monochrome) scene. In these pictures the horizontal vector has replaced the $U$ colour difference signal and the vertical vector has replaced $V$. This results in the magnitude of the vector being represented by the saturation ('depth') of the colour and the direction by its hue. With this representation cyan represents upwards motion, blue, motion to the right, red, downward motion and (yellowy) green, leftward motion. These pictures were photographed from a CRT monitor. Unfortunately the vagaries of the photographic process have not resolved some of the subtler hues. Nevertheless the general features of the motion vector fields can still be seen. The examples selected correspond to the examples of the error functions (above) and also to the results of motion compensated standards conversion presented in the next chapter.

Some features of the motion vectors from this system are illustrated in figures $\mathbf{8 . 1 7}$ to 8.24. Measurement of the predominant motion (the 'pan vector') is remarkably consistent. There appears to be a single colour representing motion of the background. This is despite the fact that the pan vector is independently measured in each of the measurement blocks. Significant areas of most of the examples are not coloured. This indicates that a zero vector was chosen for that area. As stated above, a candidate vector of zero was tried over the whole picture. Hence in areas containing little detail the zero vector is as likely to be selected as the true velocity. It can be seen that uncoloured areas generally correspond to undetailed areas or stationary objects ${ }^{1}$. The bright green regions in figure 8.20 indicate that the error function was greater than the preset threshold ( 32 grey levels) and that motion estimation is deemed to have failed. This bright green colour is used solely to represent failure of the motion estimator and does not represent a vector. The motion estimator does not fail in the other

${ }^{1}$ Greater absolute fidelity of the vector field might have been achieved by omitting the stationary candidate vector (if it was not found in a relevant measurement block). If, however, a stationary vector fits the data as well as a moving one then it is often better to select the stationary vector. This is because motion compensated processing applications generally fail less severely if an erroneous stationary, rather than an erroneous moving vector is chosen. 
examples. In general the motion estimator fails only rarely, in areas of complicated or very rapid motion. Although the motion estimator does not always select the correct vector it is usually very consistent. Real time, block matching motion estimators, by contrast, (eg Weiss \& Christensson 1990) can generate a large number of different vectors within the same moving object. In some examples (eg the bottom right corner of figure 8.19) the size and shape of the measurement blocks can be seen. This is because some of the measurement blocks have failed to detect movement because of limited detail in the image.

\subsection{Summary \& Conclusions.}

This chapter has described the implementation of, and results from, an experimental, real time, motion estimation system. The chapter started by describing the hardware implementation of the motion estimator. The system is based on the process of phase correlation and may be divided into 3 parts, signal conditioning, motion analysis and image segmentation. Signal conditioning performs initial processing such as filtering and subsampling on the original image. Motion analysis calculates phase correlation functions and locates the maxima of these functions. This generates a list of motions which may be present in the image. Finally image segmentation selects which particular vector applies to each pixel and, thereby, segments the image into regions of different velocity.

Many hours of video signals have been processed with this motion estimator. This allows the qualitative assessment of very much more material than would ever be possible using computer simulation. Assessment of the motion estimator output allowed significant improvements to be made to the system. Undoubtedly considerable further improvements could be made to similar systems built in the future, some of which are suggested in the previous chapter. Nevertheless this system shows that high fidelity motion estimation can be performed by real time motion estimation systems of this type. Of course the ultimate test of any motion estimator is its performance in a real motion compensated processing application. Such, standards conversion, applications are discussed in the next chapter.

\subsection{References.}

1. Borer, T.J., Hulyer, M.G. \& Parker, D.W. 1990. Motion compensated display field rate upconversion. Proceedings of the International Broadcasting Convention, September 1990, Brighton, England, I.E.E. Publication No. 327, ISBN 0 85296000, pp 321-325.

2. Borer, 1991. British Patent Application No. PCT/GB91/00982.

3. Dabner, S.C. 1990. Real time motion measurement hardware: Phase correlation unit. BBC Research Department Report No. BBC RD 1990/11. 
4. Thomas, G.A., 1987. Television Motion Measurement for DATV and other Applications. BBC Research Department Report No BBC RD 1987/11. September 1987.

5. Thomas, G.A. \& Lau, H.Y.K. 1990. Generation of high quality slow-motion replay using motion compensation. Proceedings of the International Broadcasting Convention, September 1990, Brighton, England, I.E.E. Publication No. 327, ISBN 085296000 , pp 121-125.

6. Weiss, P., Christensson, B., 1990. Real time implementation of subpixel motion estimation for Broadcast applications. IEE Colloquium on 'Applications of motion compensation', October 1990, Digest No. 1990/128. 


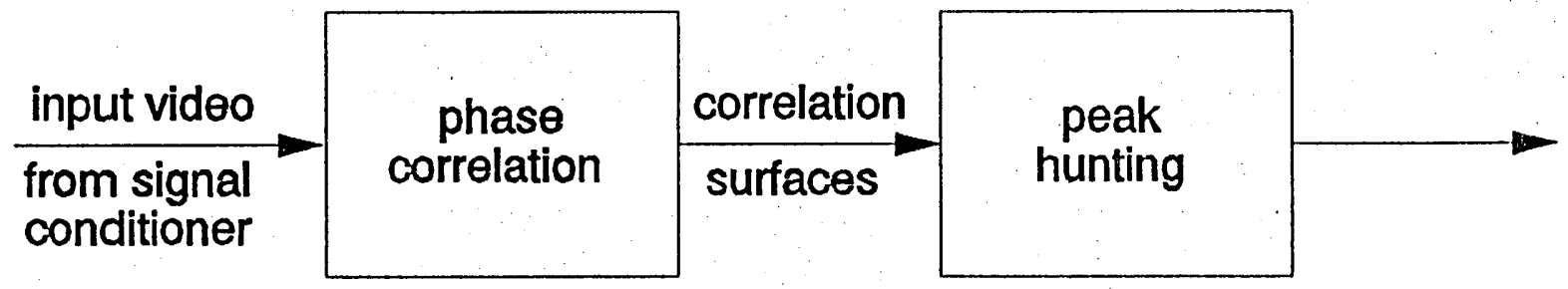

Figure 8.3 : Motion analysis processing

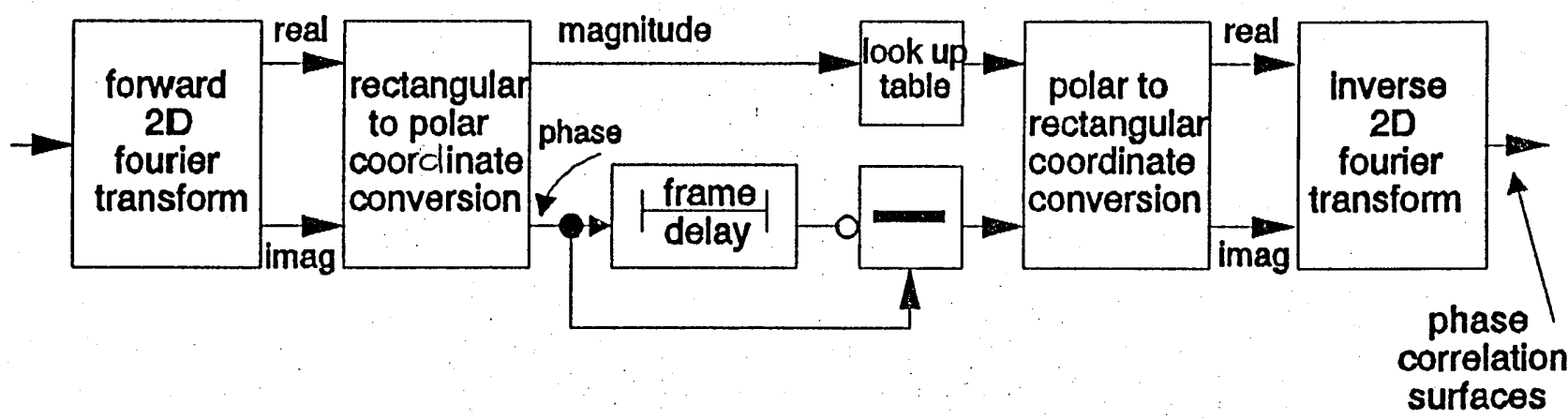

Figure 8.4: Phase correlation processing 
Video Input

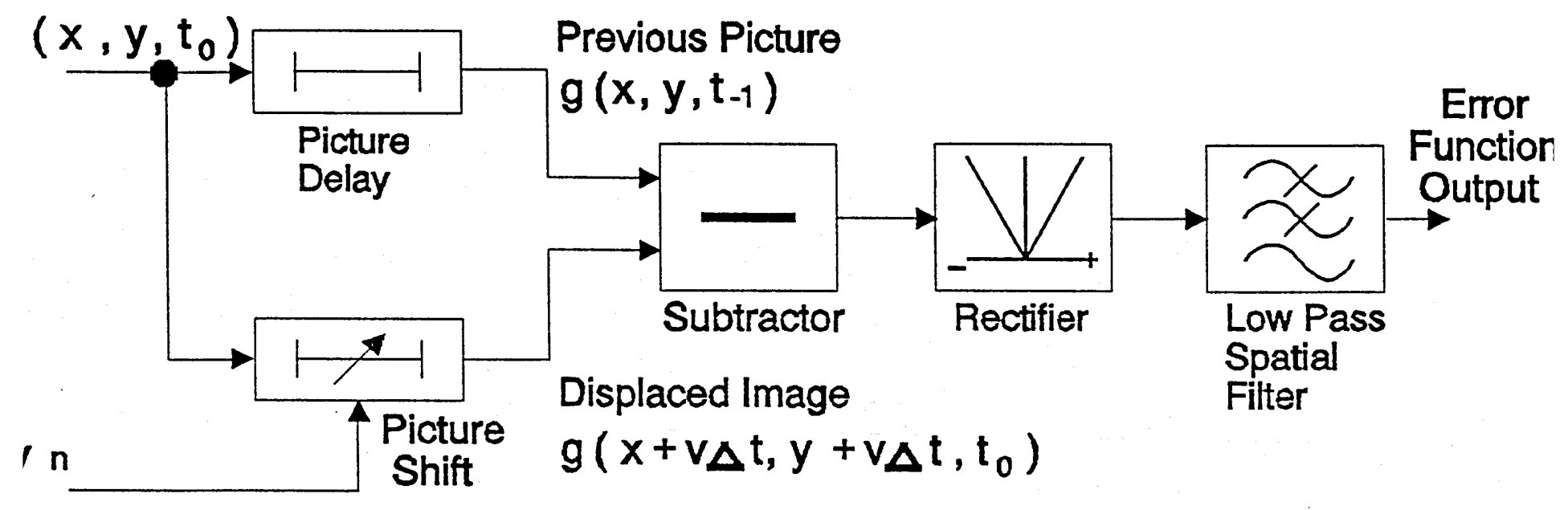

Figure 8.5: Calculation of the Error Function

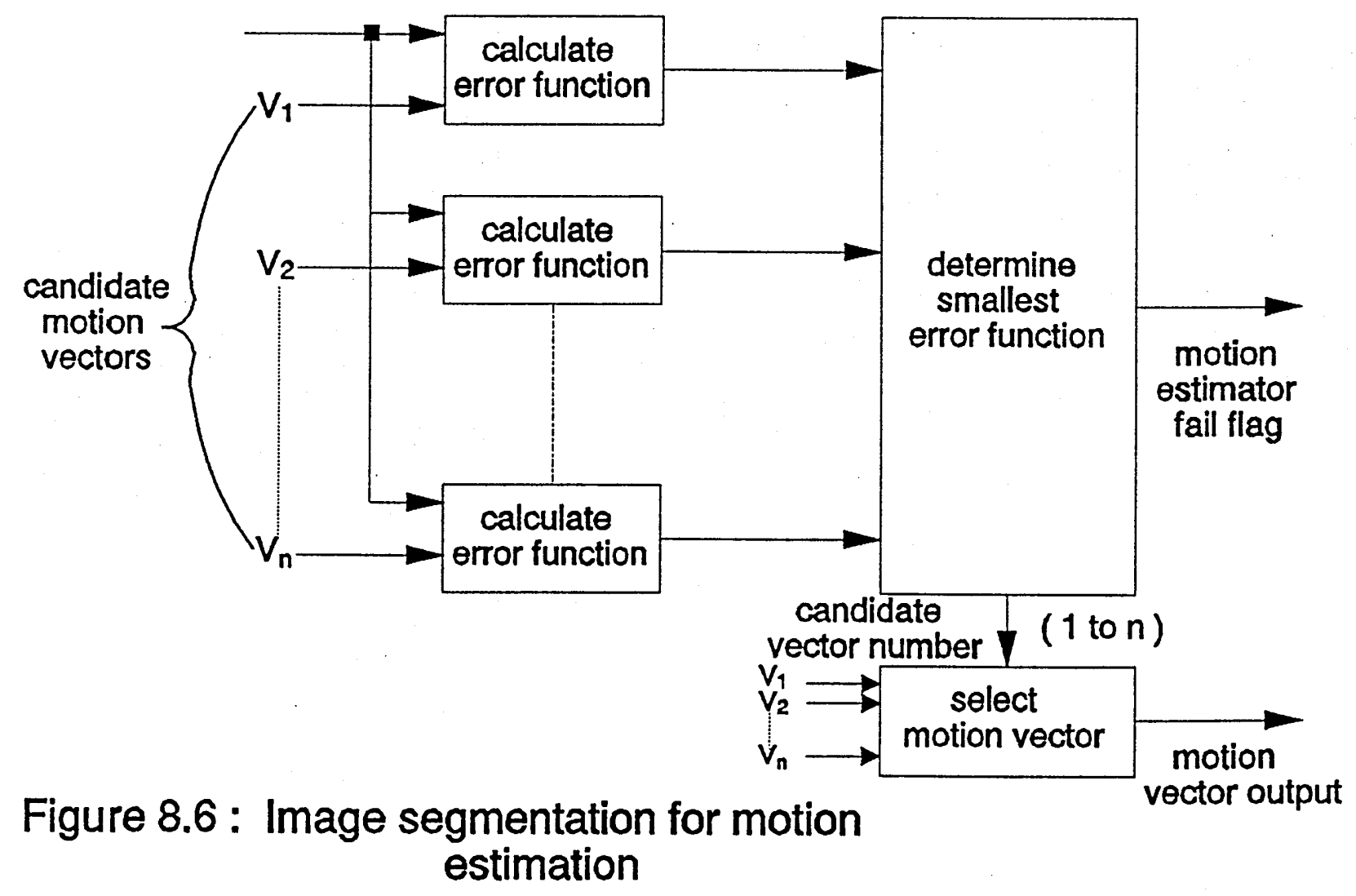



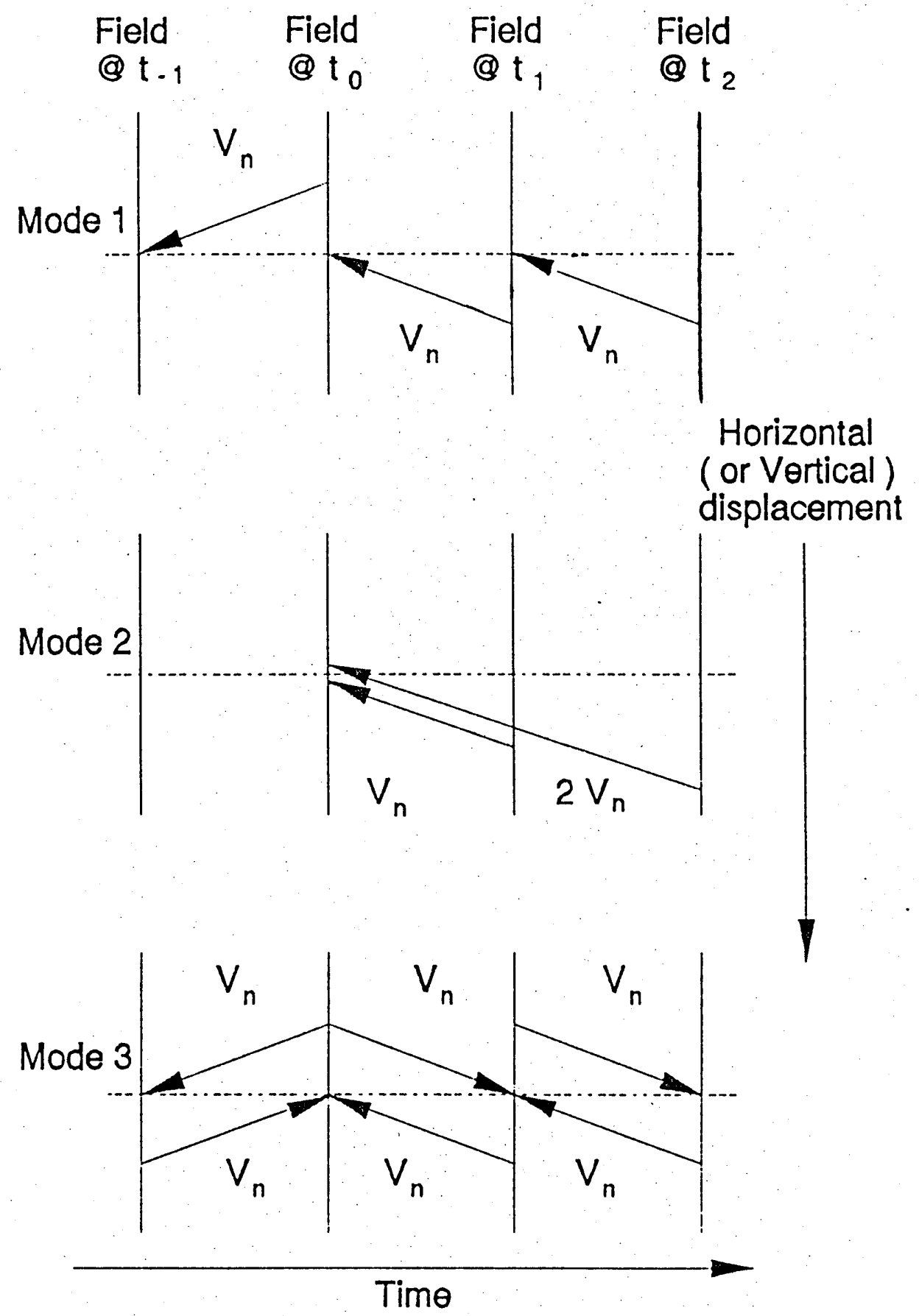

Figure 8.7: Image segmentation modes 


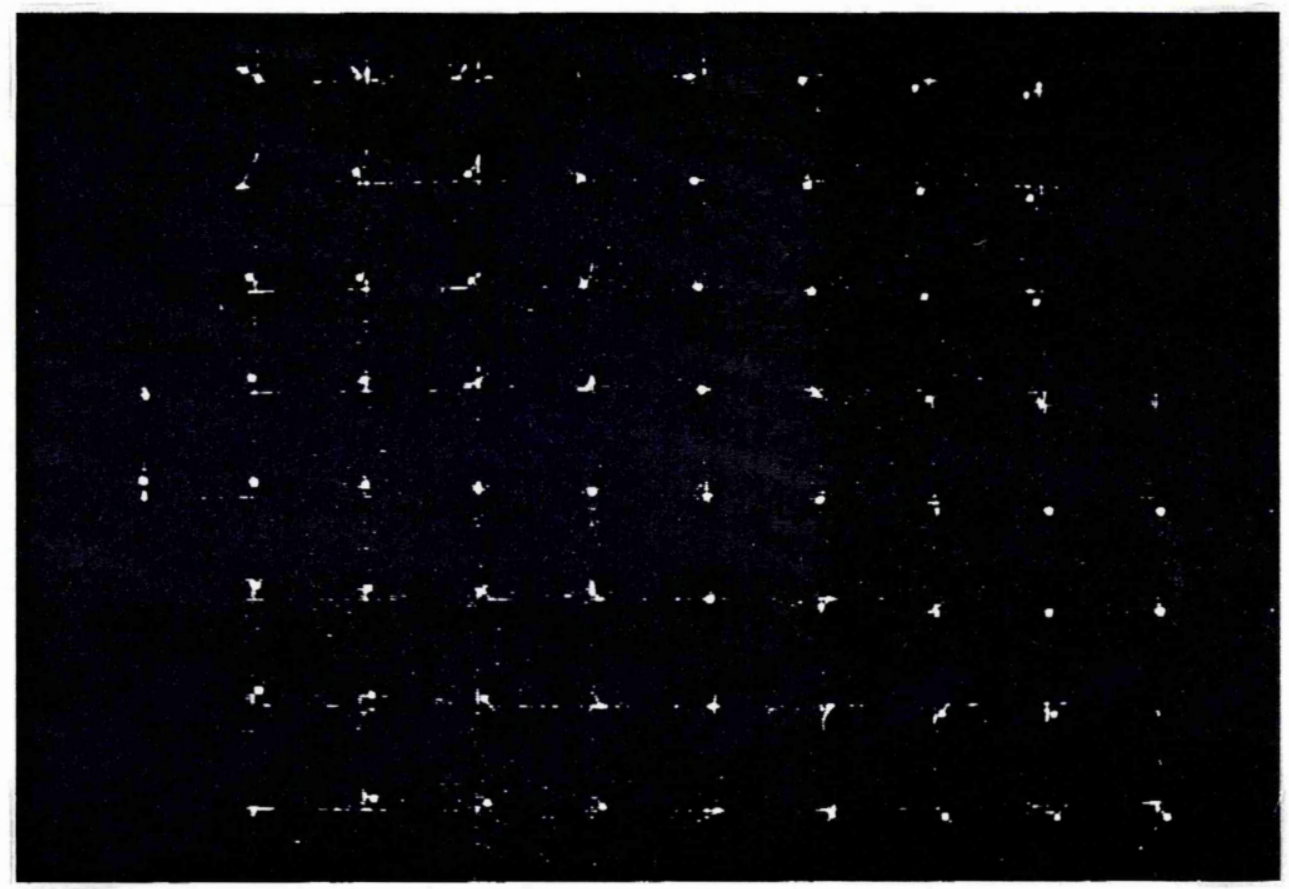

Figure 8.8 : Example output from phase correlator 


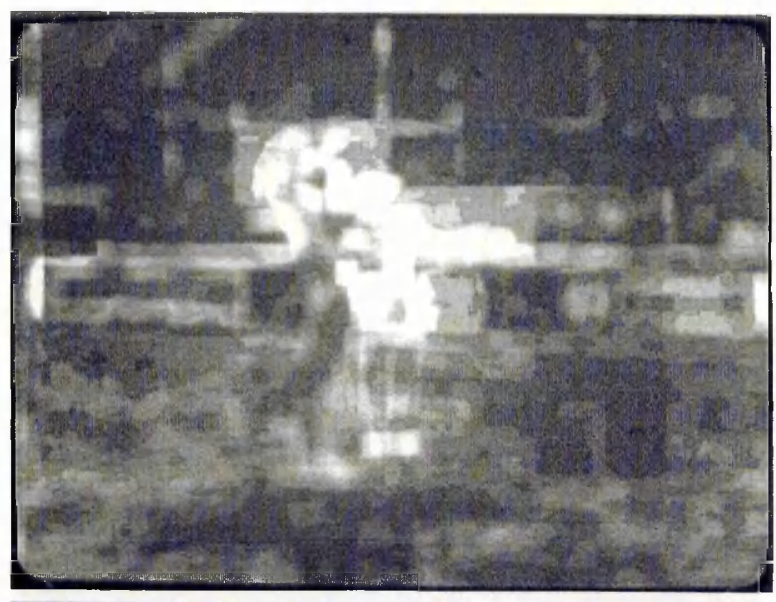

Fig 8.9

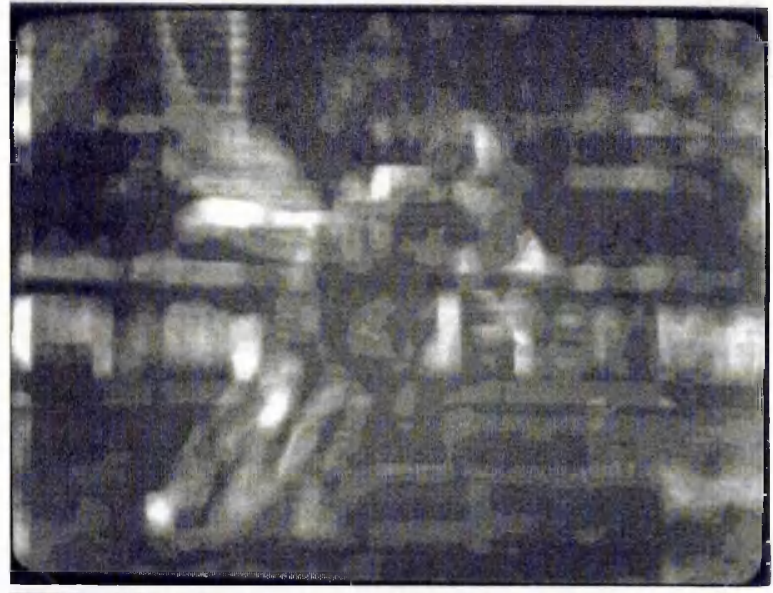

Fig 8.10
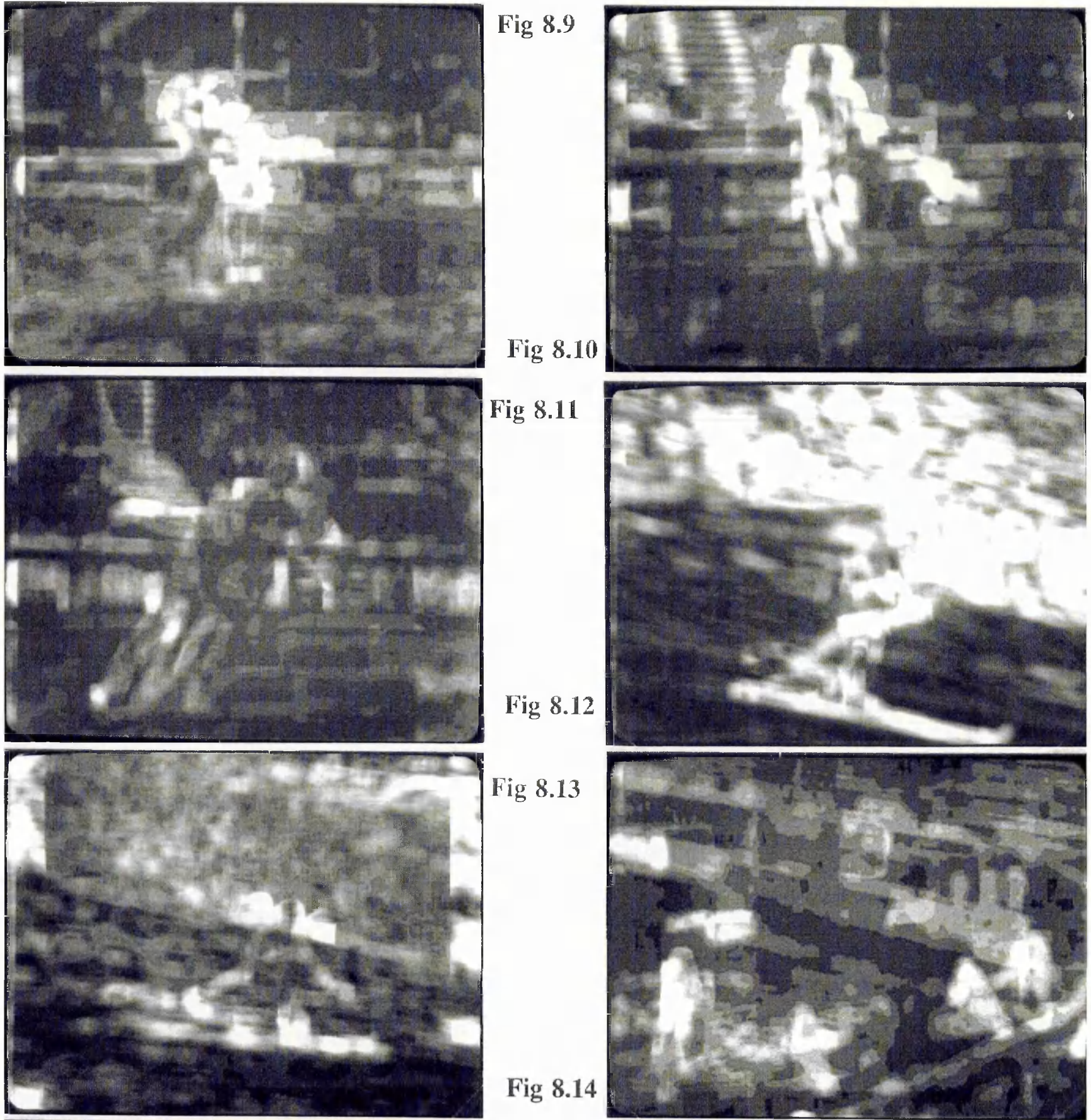

Fig 8.12

Fig 8.11

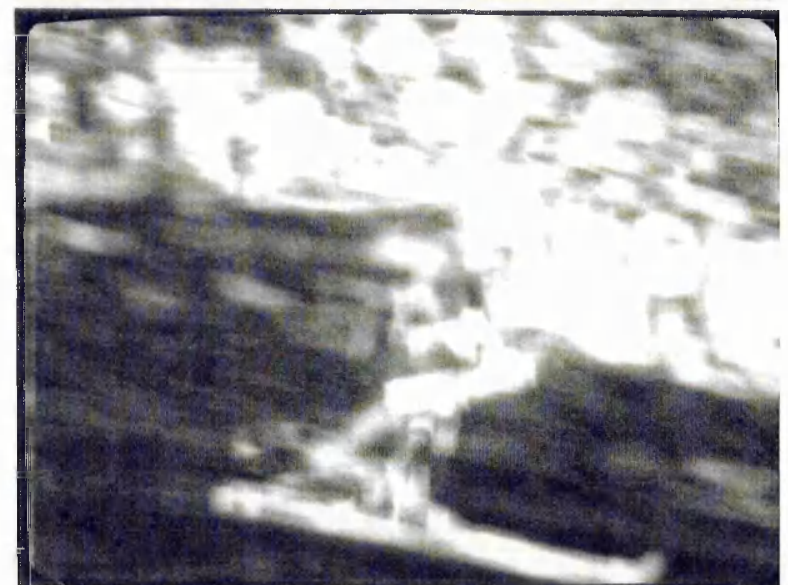

Fig 8.13

Fig 8.14
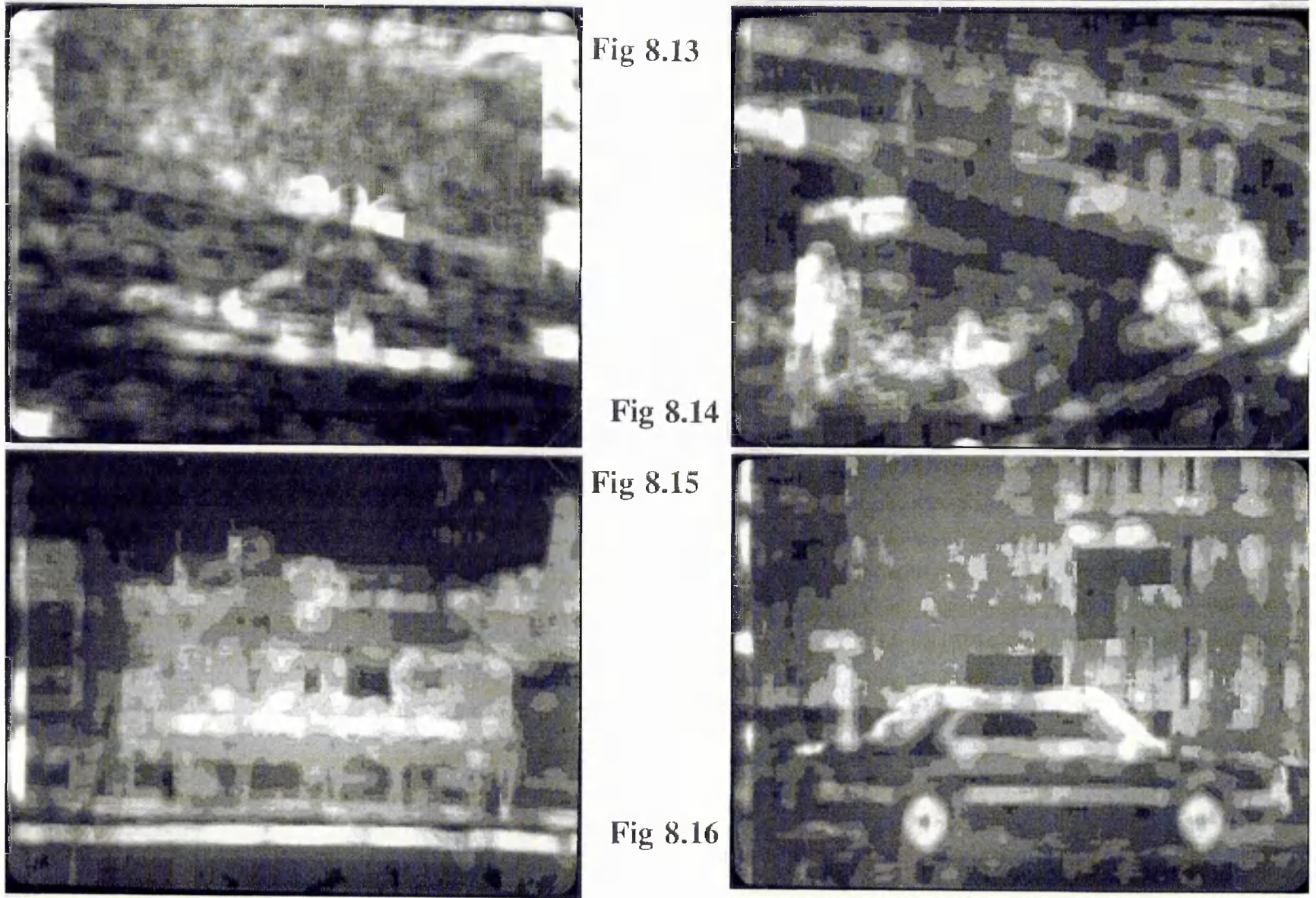

Figs 8.9 - 8.16: Examples of error functions 


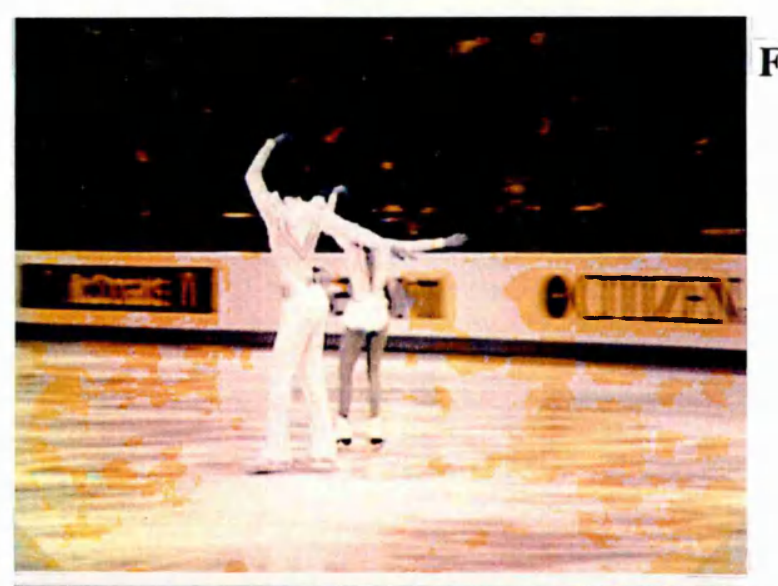

Fig 8.17

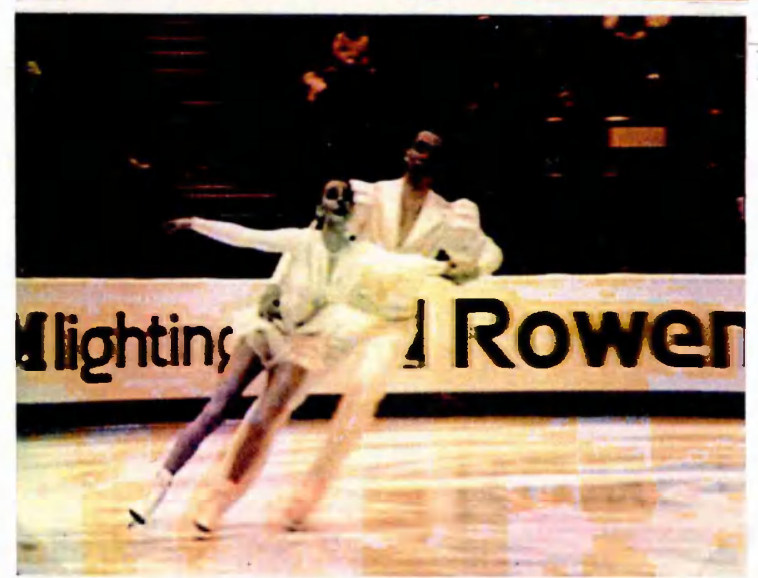

Fig 8.19

Fig 8.18
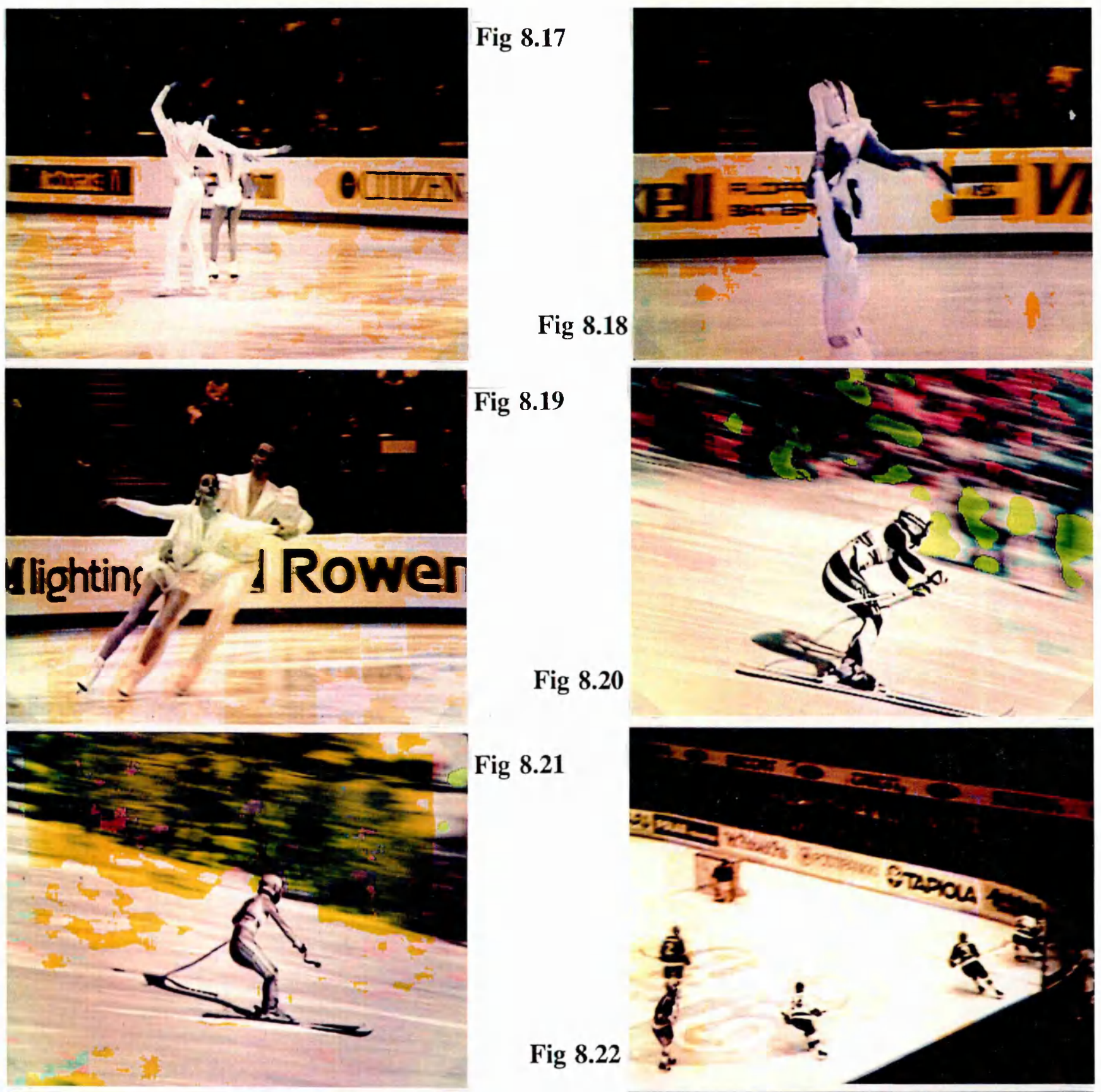

Fig 8.21

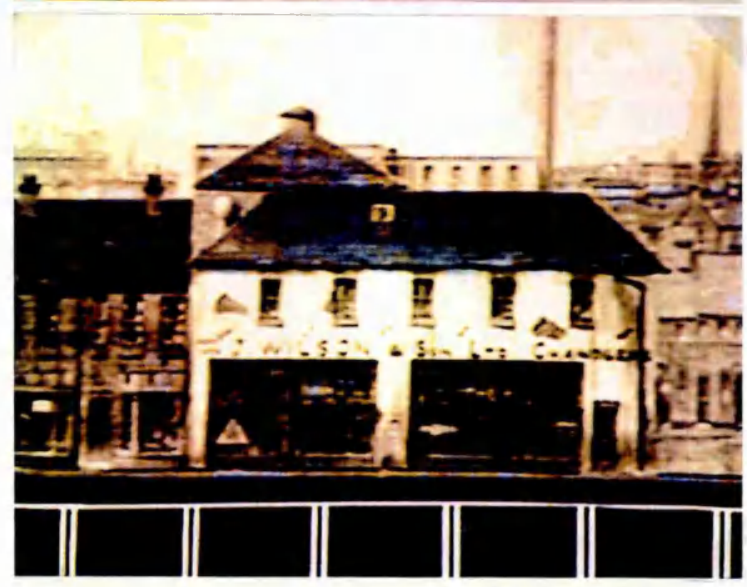

Fig 8.22

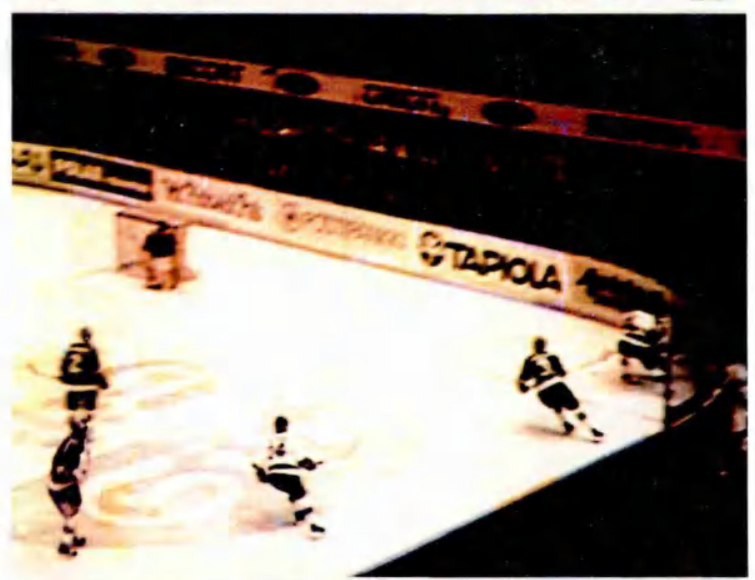

Fig 8.23

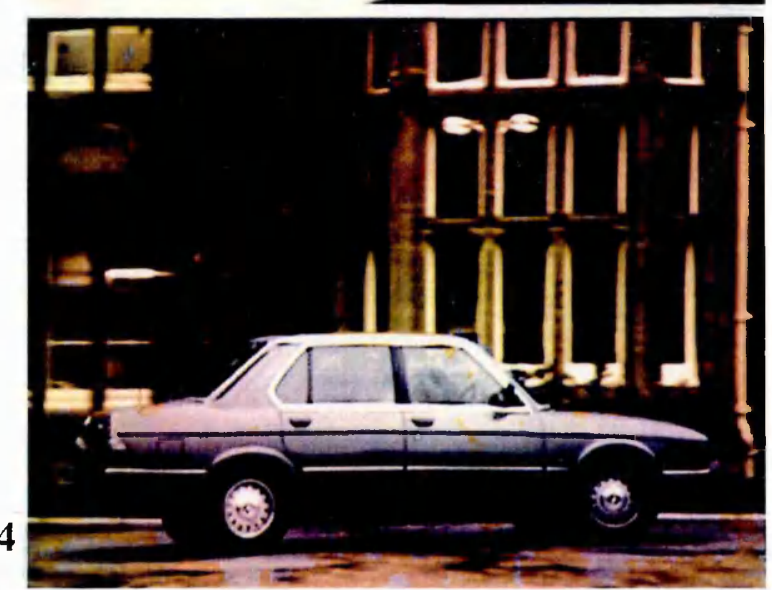

Figs 8.17 - 8.24: Motion vectors presented as colours 


\section{The Implementation of Motion Compensated Standards Conversion}

\subsection{Introduction.}

Some details of the practical implementation of motion compensation are considered in this chapter. Following this the results of two examples of experimental, real time, motion compensated standards conversion are presented. As previously in this thesis the examples chosen are those of motion compensated display field rate upconversion and intercontinental standards conversion.

Since the late 1980's there has been considerable interest in the use of motion compensation for standards conversion and related applications (eg. Marcozzi '87, Nishizawa '87, Fernando '87 \& '88, Thoma '89, Reuter '89, Robert '89). The emphasis in most of these papers is, however, on the motion estimation algorithms. This, perhaps, reflects the difficulty of performing reliable motion estimation. It also indicates the intimate relationship between motion estimation and motion compensation. The subject of motion estimation was discussed in detail in chapter 7 and will not be revisited here. Generally speaking the motion compensated interpolation, discussed in these papers, may be described as 'linear interpolation along the motion trajectory'. Reuter ('89) discusses rather more complicated filtering but gives little detail.

Most of the results of motion compensation, reported in the literature, are based on computer simulations. This is an extremely valuable technique for such investigations. Computer simulation allows new algorithms to be tested (relatively) quickly and easily, and avoids the need to build complex and expensive hardware. If an algorithm is found to work poorly it can be improved, or discarded in favour of a better one. Computer simulation does, however, have the inevitable disadvantage that, because of limited computing power, only short sequences can be processed. The short, processed sequences (at best, usually a few hundred frames) can give valuable insights into the algorithms used. Short sequences are not, however, well suited to investigating and comparing potentially good algorithms. Hence, there is no substitute for processing much longer sequences using real time hardware. This allows motion compensated standards conversion algorithms to be assessed with a wide variety of testing picture material.

The results presented in the second half of this chapter are from experimental, real time, motion compensated standards converters. This inevitably restricts the complexity of the processing which can be performed. On the other hand, the ability to view very long sequences (hours) has proved invaluable, as may be seen from the results presented in sections $9.4 \&$ 9 9.5. Several production and prototype motion compensated standards converters have recently been presented at conferences or on the broadcast equipment market (Nishizawa '87, Rutter '90, Weiss '91 \& Richards '90). Motion estimation for the Oki Lt 
2000 machine is based on a recursive, constraint based technique (Yamaguchi 1987), while that for the Vistec 'Vector' is based on hierarchical, block matching technique (Nowak '90). Motion estimation for the other two, prototype, machines is described in the papers in which they are presented. Much of the information on these machines is proprietary and relatively little can be gleaned from the published papers.

Information on the details of motion compensated interpolation is rather limited. This is disappointing since there are different ways in which this process can be implemented. Chapter 4 has already presented techniques which can be used to implement both conventional and motion compensated standards converters. Subsequent sections, in this chapter, consider further the implementation of motion compensated interpolation and, in particular, the crucial way in which motion estimators and standards converters can be combined to form a single system.

\subsection{Implementing Motion Compensation.}

The process of motion compensated standards conversion is, essentially, that of interpolation along the motion trajectory. This process is illustrated in figure 9.1. The assumptions behind this type of motion compensated interpolation are that the scene comprises a number of linearly translating rigid objects. This simple model is adequate for most areas of the picture. It breaks down, however, in regions of revealed or obscured background. Care must be taken, in dealing with these areas of the image, as they can have a disproportionate effect on the overall quality of the interpolated pictures.

In order to perform motion compensated interpolation we must displace the image of objects in the input pictures to the appropriate position in the output picture. This is achieved with the aid of, what may be called, a 'picture shifter', illustrated in figure 9.2. The picture shifter restructures the input image so that all the objects within it are moved to their correct position in the output image. These, motion compensated input fields, can then be combined to produce an output image as illustrated in figures $9.3,9.4 \& 9.5$. By combining the position of the interpolation aperture with the motion vector (as described in section 4.8 ) the frequency response of the interpolation filter automatically skews to follow the motion of the object. This process, of skewing the interpolation aperture to follow motion, and its effects are discussed in chapter 6.

The picture shifting function, in motion compensated systems, is often performed by using a variable delay. In a raster scanned television system the introduction of a delay in the signal path produces a spatial shift in the image. Small delays give horizontal shifts while delay, by a multiple of the number of pixels in a line, gives a vertical shift. Any spatial displacement can be produced by introducing the correct delay. By introducing a variable delay we have a mechanism for dynamically shifting the position of objects within an image. 
A variable delay can, conveniently, be implemented by a circular buffer, made from random access memory. Such a circular buffer is illustrated in figure 9.6. If the memory contains $\mathrm{N}$ locations, then a circular buffer is created by considering that the first location immediately follows the Nth location. This is equivalent to calculating the memory addresses modulo N. A fixed delay is produced by writing to one side of the circular buffer and reading from the opposite side. That is, the write address increments every clock period and the read address is the write address plus $N / 2$. The delay can be varied by changing the difference between the read and the write pointers. Sub pixel shifts, of part of the image, can be achieved by taking a weighted sum of the outputs from several variable delays, thereby performing a filtering operation. This process is described in more detail in chapter 4.

There is another way in which picture shifts can be achieved in motion compensated systems. This can also, conveniently, be described in terms of a circular buffer consisting of random access memory. In this case it is the read pointer which is incremented regularly, every clock cycle, and the write pointer is varied according to the motion vector. For a constant delay the write pointer is the read pointer minus $\mathrm{n} / 2$. If the motion vector is added to the write pointer the effect on the image is to 'project' it forward (in time) along the motion trajectory. Similarly, if the motion vector is subtracted from the write pointer the image is projected backwards along the motion vector. Using this method of motion compensation sub pixel shifts can be achieved by writing (scaled) pixel values to multiple locations simultaneously.

Some problems can be associated with 'projecting' images along the motion trajectory as described in the previous paragraph. These problems occur at the boundaries between regions with different motion vectors. That is, in regions of revealed and obscured background. If the vector field is diverging (eg. revealed background) then the output image will contain gaps where no information has been projected. Similarly if the vector field is converging (eg. obscured background) then parts of the output image will have been written to twice. This problem of overwriting can be dealt with by carefully combining the multiple contributions written to a single location. The write cycle then becomes a read-modify-write cycle. Gaps in the interpolated image can be avoided by combining successive images projected forwards and backwards in time. Nevertheless the technique of 'projecting' images is rather more awkward to implement than the 'variable delay' technique presented above. Projection is mentioned here because it is used (for the vector field) in the example in section 9.4.

The difference between motion compensation using 'variable delays' and 'image projection' is the same as between input and output lattice filters described in chapter 4 . The use of variable delays implements a motion compensated, input lattice filter. In this case it is the uninterpolated input samples which are stored. Image projection implements a motion compensated output lattice filter. Using this technique the interpolated pixels are built up in 
memory locations which correspond to output pixels. Generalised, motion compensated, input and output lattice filters are shown in figures $4.25,4.26 \& 4.27$ (chapter 4). Both types of motion compensated interpolator will produce identical results in regions of the picture which have a constant motion vector. It is in the way in which revealed and obscure background are treated that the results will vary.

\subsection{The Interface between Motion Estimator and Standards Converter.}

In order to make a motion compensated standards converter the motion estimator and interpolator must be integrated into a single system. The interface between the motion estimator and the interpolator is crucial to the performance of the system as a whole. This fact was highlighted, in this investigation, because motion estimator and the motion compensated interpolators were all distinct, separately designed, electronic systems. In a computer simulation the division between the motion estimator and interpolator is much less distinct.

Motion compensated input lattice ('variable delay') or output lattice ('picture projection') interpolators require motion vectors in different formats. Input lattice interpolators store the input samples and take a weighted sum of these input samples to generate each output pixel. The interpolator needs to know the position of the input samples required to generate the output pixel. Therefore input lattice ('variable delay') interpolators require that the motion vectors it uses have been sampled on the output sampling lattice. Output lattice ('picture projection') interpolators, by contrast, project each input field to the correct positions required in the output pictures. Therefore output lattice interpolators requires that the motion vectors it uses are sampled on the input lattice.

Motion estimators, for standards conversion, can generate motion vectors on either the input or output sampling lattice. These, sampled, vector fields can be significantly different, because the temporal undersampling of television signals also applies to the motion vector field. Most motion estimators, including the one described in chapter 8 , generate motion vectors on the input sampling lattice. Motion estimators can, however, be designed to generate motion vector on the output sampling lattice. For example Thomas \& Lau (1990) have proposed a modification to the image segmentation part of a phase correlation motion estimator. They propose to use this for the slow motion replay of television signals using motion compensation. Essentially, in the terminology of chapter 8, they propose performing image segmentation on the basis of error functions sampled on the output lattice.

The interface between motion estimator and interpolator depends on how both of these sub-systems have been implemented. If the motion estimator generates motion vectors on the same sampling lattice as is required by the interpolator all is well. This would happen if the motion vectors were generated on the output lattice and an input lattice ('variable delay') interpolator were used. Alternatively, the motion vector could be generated on the input lattice 
and an output lattice ('picture projection') interpolator could be used. It, is however, most convenient to generate motion vectors on the input lattice and to use them (with an input lattice interpolator) on the output lattice. This is what has been done in the examples presented in this chapter.

If the sampling lattice of the motion vectors is different from that required by the interpolator, then sampling lattice conversion (ie. standards conversion) of the motion vectors is required. This is the case for the examples in this chapter. Motion vectors are generated on the input sampling lattice and pictures are interpolated using an input lattice ('variable delay') interpolator. There are two options for standards converting the motion vectors themselves. Either a motion compensated, or a non-motion compensated standards conversion can be used. Each of these possibilities is used for one of the examples in this chapter.

For display field rate upconversion (section 9.4) the motion vectors are converted from the input to output sampling lattice using motion compensation. The way this was done (Borer, Hulyer \& Parker '90) was to project the vectors forward along their motion trajectory. That is, an output lattice interpolator was used for standards converting the motion vectors. This type of interpolation has problems at discontinuities in the vector field (see above). It is possible, either for gaps to be left in the output vector field, or for multiple vectors to be written to the same output pixel. For simplicity the hardware used assumed zero velocity for gaps and the most recently written vector was used in the case of multiple assignment.

For intercontinental standards conversion (section 9.5) a simple non-motion compensated standards conversion was performed on the motion vectors. The vector field is undersampled, and therefore temporally aliased, at least as much as the original video. Hence it was inappropriate to perform (temporal) linear interpolation (ie. conventional standards conversion) on the motion vectors. Therefore, the simple strategy was used, of taking the nearest sample in the input vector field which immediately preceded the output time. This produced unsatisfactory artifacts in the interpolated pictures and required a modification to the motion estimator. This is discussed in more detail in section 9.5.

\subsection{Motion Compensated Display Field Rate Upconversion.}

This section describes the experimental implementation of motion compensated display field rate upconversion. That is, in this example, doubling the field rate of the display from $50 \mathrm{~Hz}$ to $100 \mathrm{~Hz}$. Upconversion was performed, in monochrome only, using real time hardware. The motion estimator used was that described in chapter 8.

The primary objective of field rate upconversion is to reduce large area flicker by increasing the field rate. Large area flicker is the result of perceiving the field rate at which the pictures are displayed. It is apparent as a rapid flickering of the whole picture. In some viewing conditions large area flicker can be quite disturbing. Ideally flicker would be removed 
by the combined filtering action of the display and the human visual system (the 'eye'). Unfortunately both the display and the 'eye' have a significant response at the $50 \mathrm{~Hz}$ or $60 \mathrm{~Hz}$ field rates used for television display. The response of the display and the 'eye' decrease with frequency and so flicker is reduced by increasing the field rate. The field rate could be increased by broadcasters but this would require a profligate, uneconomic use of transmission bandwidth. Hence the only realistic option is to interpolate additional fields at the receiver.

The motivation for this work was to improve the display of large, bright, high definition television pictures. Under these conditions the effects of large area flicker will be much more obvious, and irritating, than they are for conventional domestic television. To ease the hardware requirements this investigation was undertaken for upconverting conventional 625 line pictures. This also allowed the use of mature camera and display technology. Hence it was hoped to be able to study problems which might not be apparent with early generations of HDTV equipment. The pictures were displayed on a conventional, monochrome, CRT display which had been modified to display 100 fields/second. Under the viewing conditions used large area flicker was not a problem with $50 \mathrm{~Hz}$ displays used (which were insufficiently bright at $70 \mathrm{~cd} / \mathrm{m}^{2}$ to demonstrate this problem). Thus this experiment allowed us to assess imperfections in the picture processing but would not actually improve the pictures!

\subsubsection{Implementation.}

The experimental, motion compensated upconverter comprised two distinct parts, the motion estimator and adaptive filter hardware for interpolation. The motion estimator has been described in some detail in chapter 8 . The interpolation hardware was designed so that a variable number of filter taps, taken from two consecutive fields, could be used in the interpolation. This structure allowed comparison with conventional algorithms, such as field or frame repeat, discussed in chapter 5 . The physical interface between motion estimator and interpolation hardware was via a single (CCIR Rec. 656) digital video interface. The interpolation hardware operated in monochrome only. Hence the colour part of the video channel could be used to convey motion vector information. The horizontal motion vector replaced the ' $U$ ' colour component and the vertical vector replaced the ' $V$ ' component (as described in chapter 8). This allowed the two parts of the hardware to be developed separately, and results from the motion estimator to be conveyed to the interpolator via digital video tape recordings.

Some features of the motion estimation hardware are specific to the upconversion process. Image segmentation 'Mode 2' (chapter 8) was employed to assign a particular 'candidate vector' to each output pixel. This image segmentation mode generates motion vectors at $25 \mathrm{~Hz}$ frame rate. The vectors produced are valid for projecting the image forward in time for 1 frame period. This is important since projection was the method used to convert 
motion vectors on the input sampling lattice to the output sampling lattice. The vectors used were spatially subsampled by a factor of 2 , both horizontally and vertically, with respect to the input image. Thus each motion vector applied to a group of 4 (2x2) pixels. This subsampling allowed all the vector information to be conveyed using only one field of the 'colour channel'. The data capacity of the other field was not used. The motion vectors used spanned a range of \pm 32 pixels/frame period and were accurate to $1 / 6$ pixel/frame period.

The interpolation hardware comprised a two stage process. The first part was conversion of the motion vectors from the input to the output sampling lattice. This was followed by interpolating the output fields. To convert the motion vectors they were first written in to a location in a circular RAM buffer, the location being determined by the integer part of the motion vector. The motion vectors used for interpolation were then read out, sequentially, from the buffer. The motion compensated interpolation used was linear interpolation along the motion trajectory between two consecutive fields. Sub-pixel picture shifts were performed using 4 point (bilinear) interpolators on both input fields. The structure of the video interpolator is illustrated in figures $9.7 \& 9.8$.

\subsubsection{Results, discussion and conclusions.}

After a few initial difficulties the two parts of the upconverter were successfully coupled together. Upconversion of long picture sequences could then be studied. As expected no reduction in flicker could be perceived because of the viewing conditions (see above). Nevertheless, observers were favourably surprised by the absence of processing errors on the interpolated pictures. After watching hours of typical program material only a very few picture artifacts were seen (and these could possibly have come from occasional hardware faults). The picture material came from a variety of sources including both tube and CCD cameras. When very difficult test sequences were used (eg slow zooms on high contrast periodic patterns!) significant artifacts could be seen due to failure of the motion estimator. No problems were noted with regard to revealed or obscured background. A slight loss of resolution, compared to uninterpolated pictures, could be discerned on careful inspection. This was, presumably, due to frequency response losses in the bilinear interpolator.

To facilitate comparison with other (non motion compensated) interpolation algorithms a 'split screen' facility was included in the interpolation hardware. This allowed the top half of the screen to be interpolated with one algorithm, while the bottom half was interpolated with a different one. Using this facility it was possible to compare motion compensated upconversion with the more conventional field repeat (AA'B'B) algorithm. This is the 'motion' algorithm from Roberts 1985 and was selected (by Roberts) for its relatively good performance on moving images (though at the expense of some spatial resolution). Using this facility the difference between the motion compensated and non-motion compensated 
algorithms was readily apparent. The loss of resolution apparent on moving objects without motion compensation was virtually eliminated using motion compensation. Furthermore the motion artifacts of the non-motion compensated algorithm (variously perceived as 'combing' or 'judder' by various observers) were absent. It was apparent that motion compensation was a significant improvement on the field repeat algorithm.

The lack of processing artifacts on upconverted pictures was somewhat surprising. This could be explained either because the motion compensated upconverter was working particularly well, or because field rate upconversion is not a particularly testing application. The explanation is probably somewhere between these two extremes. Since the fields are displayed for a relatively short period, perhaps processing artifacts are less visible than for other applications. This cannot, however, be the complete explanation since artifacts using field repeat were clearly visible. In chapter 5 it was shown that other, relatively simple, nonmotion compensated upconversion algorithms give better performance than field repeat. Perhaps motion compensation might not have been such a significant improvement compared to these algorithms. This comparison was not made because of the hardware limitation of the motion compensated interpolator. It is regrettable that, due to the dynamic nature of the images there appears to be no practicable way to show these results as still pictures.

\subsection{Motion Compensated, Intercontinental, Standards Conversion.}

This section describes the experimental implementation of real time, motion compensated, intercontinental standards conversion. This investigation of standards conversion was performed in one direction only, from 50 to $60 \mathrm{~Hz}$, for instrumental simplicity. Nevertheless the results would be expected to be broadly similar for either direction of conversion because of the similarity of frame rates. Standards conversion was performed in colour, which improved the overall picture quality and helped in assessing the results.

Standards conversion, as discussed previously, is an essential prerequisite for the international exchange of television programs. The essence of an intercontinental standards converter is the ability to interpolate output images at time instants different from the input sampling instants. It is also necessary to resample the image spatially, but this is a less critical process. A considerable number of ('conventional') standards converters are manufactured, which use linear filtering techniques to interpolate output images. The theory of this type of standards conversion has been discussed in previous chapters. This type of interpolation can provide good pictures for some types of picture material, for example for (studio based) drama. These types of programs contain few fast pans and little rapid motion. Temporal undersampling is, therefore, less severe and so interpolation is less difficult. The most prestigious events are often outside broadcasts particularly sport, for example the Olympic Games. Pictures from these type of events, in contrast to studio drama, often contain very 
rapid pans and fast motion. It is for these prestigious events that standards conversion is most critical and for which 'conventional' standards converters perform least well. The motivation for this investigation was to try to improve standards conversion, for these prestigious events, by using motion compensated interpolation.

The results of this investigation are presented as a series of photographs in figures 9.11 to 9.42. These will be described and discussed, in detail, in later subsections. For comparison the original images, for 8 pictures, are shown in figures 9.11 to 9.18 . Figures 9.19 to 9.26 illustrate the results of 'conventional' standards conversion. Early motion compensated standards conversion results are presented in figures 9.27 to 9.34 . Later, improved, motion compensated standards conversion is shown in figures 9.34 to 9.42 . These photographs were taken from a CRT monitor screen. The pictures had first been recorded on a digital video tape recorder. Single fields were then 'frozen' by the video recorder and photographed.

\subsubsection{Implementation.}

The motion compensated standards converter was, as for upconversion, composed of two distinct parts: the motion estimator and the interpolation filter. The motion estimator used was, again, that described in detail in chapter 8 . The interpolation filter was an experimental version of a commercial, 'conventional' standards converter. With a little modification this standards converter was able to perform motion compensated interpolation. The interpolation hardware for standards conversion was significantly smaller than for upconversion. Both motion estimator and interpolator were sited in the same laboratory and linked via a short length of ribbon cable. The signals processed were sampled according to CCIR Rec. 601 (ie the luminance signal was sampled at $13.5 \mathrm{MHz}$ ).

Some parts of the motion estimation algorithm, described in chapter 8 , were specific to standards conversion. The motion estimator initially used 'image segmentation mode 1' to generate the motion vectors. Later mode 3 was used, as will be described below. These methods, for segmenting the motion field into regions with different motion vectors, both generate vectors at the $(50 \mathrm{~Hz})$ input field rate. The motion vectors were conveyed to the interpolator with the same (spatial and temporal) resolution as the input images. The vector range used was, initially \pm 32 pixels per frame period, but this was subsequently increased to \pm 64 pixels per frame period.

The motion vectors were processed to obtain the correct format for their use by the interpolator. Since the interpolator, essentially, used a variable delay to achieve motion compensation (ie. it was an 'input lattice' filter) motion vectors are required to be sampled on the output sampling lattice. The motion estimator generated vectors on the input sampling lattice. Thus sampling lattice conversion of the motion vectors (from the input to the output lattice) was required. This was achieved by the use of a 'synchroniser'. A synchroniser is a 
simple standards converter which produces an output pixel which is the input pixel closest (in space and time) to the desired position of the output pixel. This may be regarded as linear interpolation using an aperture, with uniform amplitude, extending $\pm 1 / 2$ pixel by $\pm 1 / 2$ field line by $\pm 1 / 2$ field period.

The interpolator was a transversal vertical/temporal filter with 4 filter taps in each of 4 consecutive input fields. This is a common configuration for 'conventional' standards converters. The difference, in these experiments, was that the whole aperture could be skewed to follow motion. The architecture of the standards converter was, essentially, a circular RAM buffer. Pixels were written in to one side of buffer, using a write pointer which was incremented every clock cycle. Sixteen pixels were read from the opposite side of the buffer, corresponding to the 16 points in the filter aperture. The relative position of the 16 read pointers were fixed and the position of the centre of the read aperture was, on average, offset from the write pointer by half the length of the buffer. Motion compensation was achieved by moving the position of all 16 read pointers, in unison, according to the motion of the output pixel.

A conventional standards impulse response was used for these experiments. The coefficients were taken from Clarke \& Tanton 1984. These coefficients produce relatively good conventional interpolation even for slowly moving parts of the picture. By using this interpolator it was considered that small errors in the vector field would be less noticeable. That is the system would be robust to some errors in motion estimation. It was also convenient, in practice, to use these coefficients. In future work it may be desirable to vary the interpolation coefficients with velocity and, possibly, with signals from the motion estimator indicating the probability of the current vector. This was not possible in this investigation.

For some parts of the picture the motion estimator is unable to determine a valid motion vector. An important feature of the motion estimator is its ability to reliably identify such areas. In areas for which the interpolator has no vector it 'falls back' to using a conventional ( 4 field) interpolation algorithm. In practice this is achieved by simply setting the vector to zero for these parts of the picture.

\subsubsection{Initial results.}

When the motion estimator and interpolation hardware were first connected together significant impairments were obvious in the interpolated pictures. The existence of artifacts was not unexpected since this was a new process, for which there was little previous experience. Where motion compensation succeeded, which it did for the majority of the picture area, high spatial resolution was maintained and judder virtually eliminated. The severity of the artifacts, however, greatly outweighed the improvements. 
The major artifact was apparent as objects suddenly 'breaking up' as they moved. This was emphasised by the high resolution which they maintained until the instant when they suddenly disintegrated. This artifact was caused by the sudden failure of the motion estimator to measure the velocity of moving objects. It was quickly realised that this happened when an object's velocity exceeded the measurement range of the motion estimator. At this initial stage of the investigation the velocity measurement range was only \pm 32 pixels/frame period. This had been considered adequate on the basis of previous computer simulations (Thomas 1990). A relatively few minutes of pictures processed using the real time standards converter was sufficient to demonstrate the error of this assumption. The motion estimator was then modified to double the range of vectors which could be measured. This was done by spatially subsampling the input video as described in chapter 8 . Fortuitously this had other benefits as well. With the velocity range increased to \pm 64 pixels/frame period the problem of objects breaking up was greatly reduced and the picture quality correspondingly improved.

With the increased velocity range another, less severe but still unacceptable, picture impairment became apparent. This took the form of an objectionable 'ghost' following the trailing edge of moving objects. The effect is illustrated in figures 9.27 to 9.34 . It is clearly visible to the left of the ice dancers in figures 9.27 and 9.28 and is even more dramatically illustrated behind the skiers in figures 9.30 and 9.31. These trailing 'ghosts' are not only objectionable as a static impairment but they come and go depending on the relative phase of the output picture relative to the input lattice. This dynamic aspect of the impairment gives it further emphasis on moving pictures. After considerable thought, it was realised that the cause of this impairment was the way in which the motion vectors had been converted from the input to the output sampling lattice. The origin of the problem, and the solution that was adopted are described in the next section.

\subsubsection{Improved motion estimation.}

In the initial results an objectionable 'ghost' could sometimes be seen flickering behind the trailing edge of a moving object. This effect is caused by the implementation of the motion estimator and the way it is interfaced to the interpolation hardware. The combination of the image segmentation mode used in the motion estimator ('Mode 1' chapter 8) and the simple way the vectors were converted to the output sampling lattice (using a synchroniser) can generate motion vectors which are inappropriate for interpolation.

Initially the segmentation of the image, into regions with different motion vectors, generated vectors valid for projecting the image forward in time ('Mode 1'). Vectors were assigned to each pixel on the basis of the match between the current and subsequent picture. In regions which were obscured between the two pictures no match could be found between them. This process is illustrated on the left hand side of figures $9.9 \& 9.10$. In these diagrams 
the hatched area represents an object moving in front of a (moving) background. $V_{0}$ represents the velocity of the object and $V_{b}$ that of the background. For areas of obscured background, at the leading edge of the object, there is no correspondence between the two pictures and no vector is assigned to these regions. Such regions are indicated 'NM' (no match). When a synchroniser is used to convert the vector field, from input to output lattice, the vector field (generated at picture 1) is used for the whole of the period between the two pictures. At the trailing edge of objects, apparently valid vectors are presented to the interpolator for regions of newly revealed background. It is these incorrect vectors in regions of revealed background which cause the objectionable 'ghost' artifact. The validity of this explanation is demonstrated since the artifact only appeared at the trailing edge, and never the leading edge, of moving objects.

It would have been possible to have used the complementary method of image segmentation, denoted 'backward assignment' on the right hand side of figures $9.9 \& 9.10$. In this method of segmentation, vectors are assigned to pixels on the basis of the correspondence between the current and previous pictures. The vectors, thus produced, are thus valid for projecting images backwards along their motion trajectory. If this method of image segmentation had been used the 'ghost' artifact at the trailing edge of objects would have been removed, only to be replaced by a similar artifact at the objects's leading edge!

To eliminate the 'ghost' artifact both forward and backward assignment techniques were combined to generate the vector field. Regions for which no match could be found using either forward or backward assignment techniques were flagged as having no reliable vector. This technique is image segmentation 'Mode 3' described in chapter 8. This technique ensures that regions of the picture which are revealed or obscured are flagged as having no reliable vector. In these regions the pictures are interpolated using a conventional 4 field linear interpolation algorithm, that is the motion vector is set to zero. Further details of this technique can be found in Borer 1991.

\subsubsection{Results, conclusions and discussion.}

The results of this investigation are presented in the photographs of figures 9.11 to 9.42. These comprise 4 groups of 8 photographs. The groups represent the unprocessed input images, interpolation using non-motion compensated ('conventional') interpolation, initial results (illustrating the 'ghost' artifact) and the final results using the modified image segmentation algorithm ('Mode 3'). The photographs were taken, as described above, using 'frozen' field from a digital video tape recorder.

Figures 9.11 to 9.18 are original, uninterpolated, images. It is important to be aware of the quality of these unprocessed images when assessing the results. In general they have much lower resolution than single pictures originated with a conventional photographic 
camera. This is because of motion blur caused by the $(20 \mathrm{~ms})$ integration time of the television camera. Generally it is the background which is blurred while the object in the foreground is relatively sharp. This results from the cameraman panning the camera to follow the object of interest. It should be realised that when these images are shown dynamically they do not look blurred. This illustrates the difficulty of trying to demonstrate dynamic image processing using static images. Therefore these results should be interpreted with care. Ice dancing (figures 9.11 to 9.13) is, conventionally, a difficult standards conversion problem. Fast pans, across the advertising hoardings in the background, can generate very objectionable judder. Skiing (figures $9.14 \& 9.15$ ) produces very high velocities. Figure 9.16 shows ice hockey (a typical team sport) while figure 9.17 is a slow pan across a (simulated) town scene. Figure 9.18 is a car driving in front of building.

Interpolation using conventional standards conversion (ie using linear filtering) is shown in figures 9.19 to 9.26 . This was performed using the motion compensated standards converter with the vectors set to zero. Caution is needed in interpreting the dynamic behaviour of a standards converter from still pictures. Nevertheless some of the impairments of conventional standards conversion can be seen in these photographs. In general moving parts of the picture are subject to blurring and double imaging (perceived as judder in moving pictures). Blurring and double imaging are particularly severe in the ice dancing of figure 9.21. In this case both foreground object (the dancers) and the background (eg the advertisements) are moving. Hence both parts of the picture are impaired. Blurring is also particularly noticeable in the ice hockey of figure 9.24 and the town scene of figure 9.25 . The car, in figure 9.26, is interpolated rather well because it is stationary. The moving parts of that picture (eg wheels and the buildings windows), however, are quite blurred. In the skiing the fast moving background is relatively unimpaired. This is because most of the detail has had already been lost by camera integration in the original image. Much greater impairment of the background would have been apparent if the skiing had been filmed with a CCD camera using a shorter aperture.

The initial results of motion compensated standards conversion are shown in figures 9.27 to 9.34. These used image segmentation 'Mode 1' (chapter 8) and contain the 'ghost' artifact in some of the pictures. In general the resolution of the panning background is significantly improved, eg. figures $9.33 \& 9.34$, compared to 'conventional' interpolation. In the car scene (figure 9.34) the quality of most of the scene is comparable to the original. However, the quality of those parts, for which motion could not be measured (eg the rotating wheels), is closer to conventional standards conversion than the original. The ice dancing and skiing scenes illustrate the 'ghost' artifact, explained above, at the trailing edge of moving objects. These scenes have been chosen to emphasise this effect. The artifact is not nearly so visible in most interpolated images. The severity of the artifact depends both on the relative phasing of input and output sampling lattices and on the motion fields estimated for the 
images. The effect is emphasised on moving scenes because of its dynamic nature (ie. it flashes at $10 \mathrm{~Hz}$ ). Clearly this artifact is unacceptable in the interpolated pictures. Hence the image segmentation mode was modified to eliminate it, as described above.

The final results of motion compensated standards conversion, after modifying the motion estimation algorithm, are shown in figures 9.35 to 9.42 . In these figures the advantages of motion compensated interpolation observed initially (figures 9.27 to 9.34 ) are maintained whilst the objectionable 'ghost' artifact has been eliminated. Generally improved resolution and freedom from double imaging are attained in most parts of most pictures. Overall the quality of the interpolated images is closer to the originals than to images interpolated using 'conventional' standards conversion. The (few) problems that remain appear to result from errors in the motion estimation process rather than from interpolation or the interface between the two. For example, some artifacts, from imperfect motion estimation, can be seen in the background of figure 9.38. Nevertheless this image is quite acceptable when viewed as part of a dynamic sequence.

The results from the experimental motion compensated standards converter illustrate the potential and feasibility of this method of interpolation. Although the picture quality is generally closer to the original images than to 'conventional' standards conversion a few artifacts remain in the pictures. These arise from errors in motion estimation, which seems to be the most critical part of the whole process. For conventional standards conversion interpolation errors appear in the moving background. Unfortunately, for motion compensated interpolation it is motion estimation errors for stationary, foreground, objects that produce the most noticeable artifacts. Therefore the quality of motion estimation is paramount and it is improvements to motion estimation which are likely to have the greatest beneficial effect. For these experiments motion estimation might have been improved by using more candidate vectors in the image segmentation process (5 were used). Improved filtering in signal conditioning for motion estimation and the use of the original colour information might also have improved the motion vector fields.

In these experiments no specific action was taken to deal with areas of revealed and obscured background. Such regions were interpolated using a 'fallback' algorithm (ie. the motion vector was set to zero). This might be expected to produce impaired interpolation at the leading and trailing edge of moving objects. However, this effect was not observed subjectively. If other artifacts, due to errors in motion estimation, were reduced it might become more important to use greater sophistication in interpolating revealed and obscured background. 


\subsection{Summary \& Conclusions.}

This chapter has considered the practical implementation of motion compensated standards conversion. The results of two examples of motion compensated standards conversion, upconversion and intercontinental standards conversion, were presented. It is worth emphasising that these examples used real time processing and not computer simulation. This allows very much longer sequences to be processed but restricts the complexity of the algorithms used.

First of all two distinct ways in which motion compensated standards conversion can be implemented were considered. The first of the techniques uses a variable delay to implement motion compensation. The second technique is to project images along their motion trajectory. The rather different properties of these two techniques were discussed in section 9.2. The two different implementations of motion compensation correspond to input and output lattice filters as described in chapter 4.

The two techniques for motion compensation require vectors in different formats. Motion compensation using variable delays requires motion vectors on the output sampling lattice, whereas projecting the image requires vectors on the input sampling lattice. It is most convenient to make a motion estimator which generates motion vectors on the input sampling lattice. For interpolation it is most convenient to use a variable delay to implement motion compensation. Therefore the motion vectors must be converted from the input to the output sampling lattice. The way in which this is done is critical to the quality of the interpolated images, as was shown from the results in section 9.5.

The results of real time motion compensated, field rate, upconversion were presented in section 9.4. This is the process of doubling the display field rate to reduce flicker. The interpolated images were, for the most part, were of very similar quality to the input pictures. A slight loss of resolution could be seen on some parts of the pictures due to the limited number of filter taps used in the interpolation. The good performance of this system was due, in part, to the efficacy of the processing used. However, it also seems likely that upconversion is more tolerant of interpolation imperfections than some other standards conversion processes.

The results of motion compensated, intercontinental, standards conversion, using real time hardware, were presented in section 9.5. This process is essential for the exchange of programs between countries using $50 \mathrm{~Hz}$ and $60 \mathrm{~Hz}$ field rates. Imperfections in the initial results highlighted inadequacies in the motion estimator and the interface between motion estimator and interpolation hardware. When these problems were corrected the quality of the interpolated images was closer to that of the original images rather than the quality achieved using 'conventional' standards conversion. Loss of resolution and double images, which are common using 'conventional' standards conversion, were largely absent when motion 
compensation was used. The remaining artifacts in the interpolated pictures seem mainly due to difficulties with motion estimation.

The two examples of real time motion compensated interpolation, presented here, demonstrate the potential, and increasing practicality of this technique. The results, while not perfect, indicated that high quality standards conversion can be achieved using motion compensation. While the technique requires a complex implementation, advances in semiconductor technology make it more and more practical.

\subsection{References.}

1. Borer, T.J., Hulyer, M.G. \& Parker, D.W. 1990. Motion compensated display field rate upconversion. Proceedings of the International Broadcasting Convention, September 1990, Brighton, England, I.E.E. Publication No. 327, ISBN 0 85296000, pp 321-325.

2. Borer, 1991. British Patent Application No. PCT/GB91/00982.

3. Clarke, C.K.P. \& Tanton, N.E. 1984. Digital standards conversion: interpolation theory and aperture synthesis. BBC Research Department Report, BBC RD 1984/20, December 1984.

4. Fernando, G.M.X. \& Parker, D.W. 1988. Motion compensated display conversion. Proceedings of the 2nd International Workshop on Signal Processing of HDTV. 29 February - 2 March 1988, L'Aquila, Italy.

5. Fernando, G.M.X. \& Parker, D.W. 1987. Motion compensated field rate conversion for HDTV display. Proceedings of the Third International Colloquium on Advanced Television Systems, Ottawa, Canada, October 1987.

6. Marcozzi, E. \& Tubaro, S. 1987. Motion compensated interpolation. SPIE, Vol. 804, Advances in image processing, pp 61-68.

7. Nishizawa, T. \& Tanaka, Y. 1987. Standards Conversion with HDTV. Proceedings of the Third International Colloquium on Advanced Television Systems, Ottawa, Canada, October 1987.

8. Nowak, T. \& Weiss, P., 1990. Vector. International Broadcast Engineer, Edition 249, January 1992, pp 20-24.

9. Reuter, T. 1989. Standards conversion using motion compensation. Signal processing 16, 1989, pp 73-82.

10. Richards, J.W., Keating, S.M. \& Gillard, C.H. 1991. A motion compensated standards converter for down conversion of 1125/60/2:1 SMPTE-240M high definition to 625/50/2:1 video. Proceedings of 17th International Television Symposium, Montreaux 1991.

11. Robert, P., Lamnabhi, M. \& Lhuillier, J.J. 1989. Advanced high-definition 50 to $60-\mathrm{Hz}$ standards conversion. SMPTE Journal, June 1989, pp 420-424. 
12. Roberts, A., 1985. The improved display of 625-line television pictures: adaptive interpolation. BBC Research Department Report, BBC RD 1985/5, May 1985.

13. Rutter, P., 1990. The Oki Lt 2000 standards converter. International Broadcast Engineer, Vol. 21, Edition 235, January 1990, pp 26-27.

14. Thoma, R. \& Bierling, M. 1989. Motion compensated interpolation considering covered and uncovered background.

15. Thomas, G.A., 1990. Motion estimation and its application to broadcast television. Ph.D. Thesis, Department of Electronic Systems Engineering, University of Essex, England.

16. Thomas, G.A. \& Lau, H.Y.K. 1990. Generation of high quality slow-motion replay using motion compensation. Proceedings of the International Broadcasting Convention, September 1990, Brighton, England, I.E.E. Publication No. 327, ISBN 0 85296000, pp 121-125.

17. Weiss, P., Christensson, B., 1990. Real time implementation of subpixel motion estimation for Broadcast applications. IEE Colloquium on 'Applications of motion compensation', October 1990, Digest No. 1990/128.

18. Yamaguchi, H., Sugi, T. \& Kinuhata, K. 1987. Movement-compensated framefrequency conversion of television signals. IEEE Trans. Comm., Vol 35, No. 10, October 1987, pp 1069-1082. 


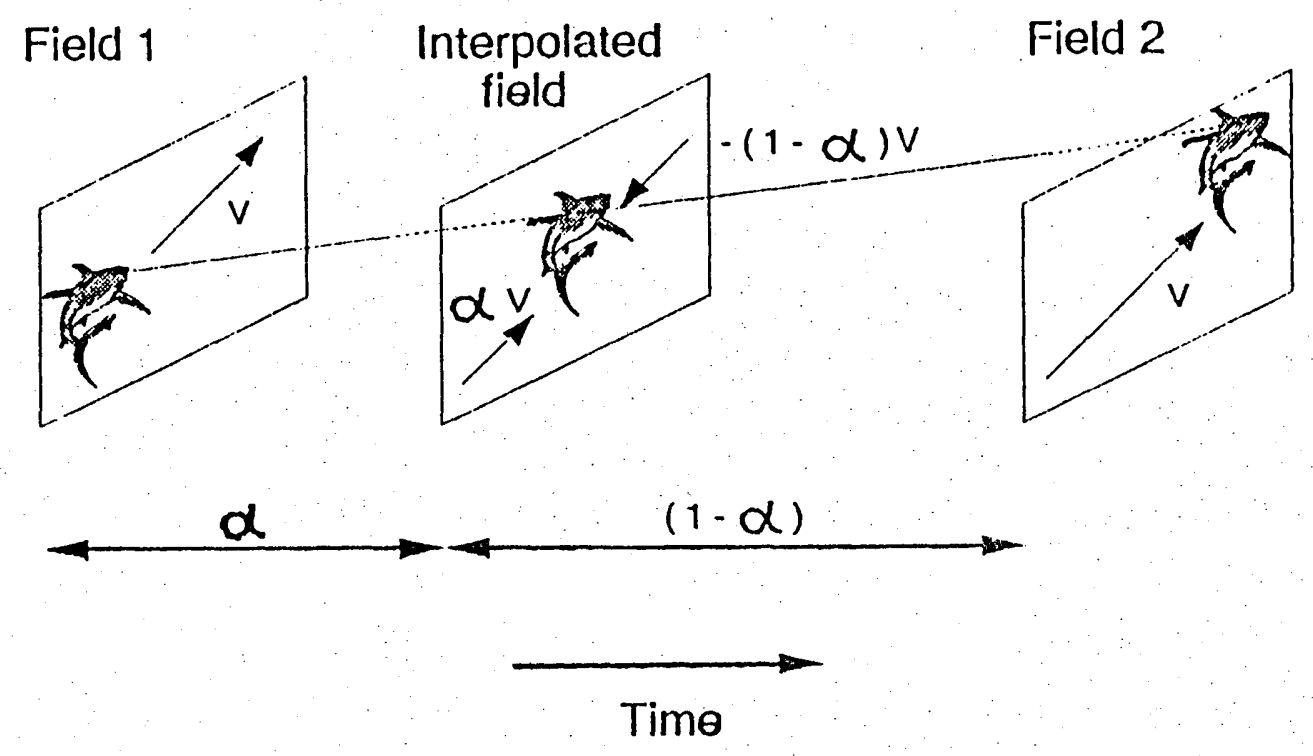

Figure 9.1: Geometry of motion compensated standards conversion

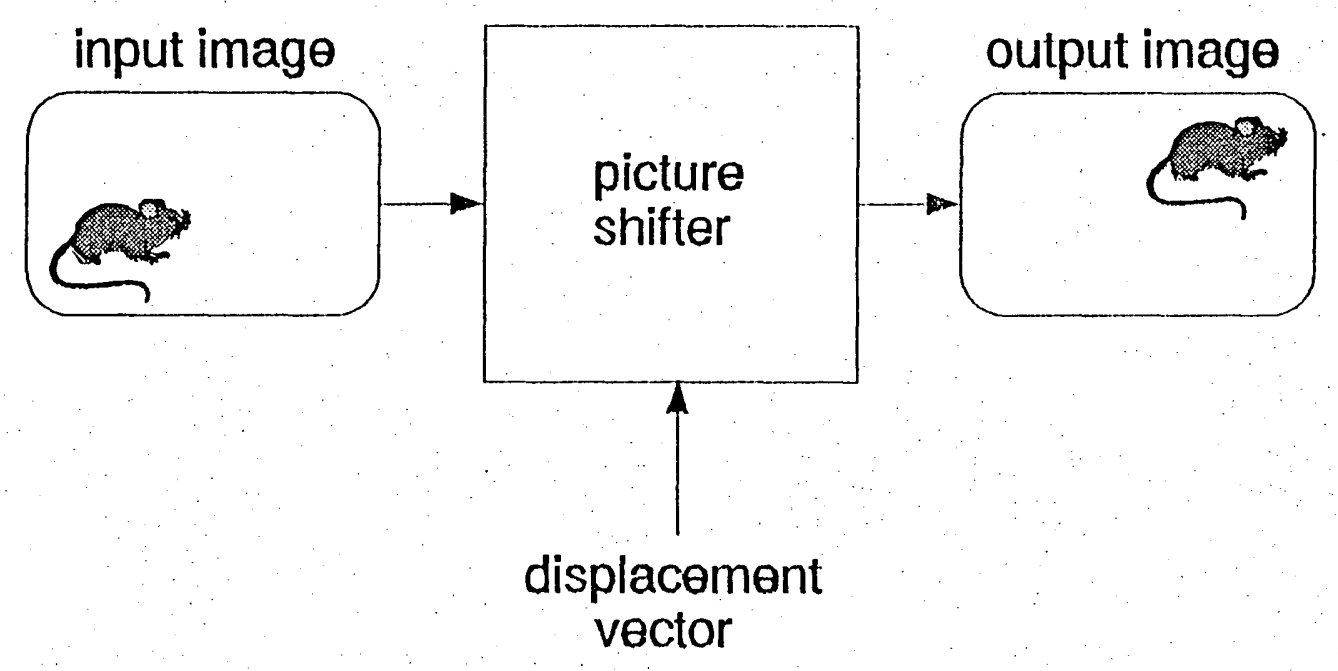

Figure 9.2: Implementing picture shifts 


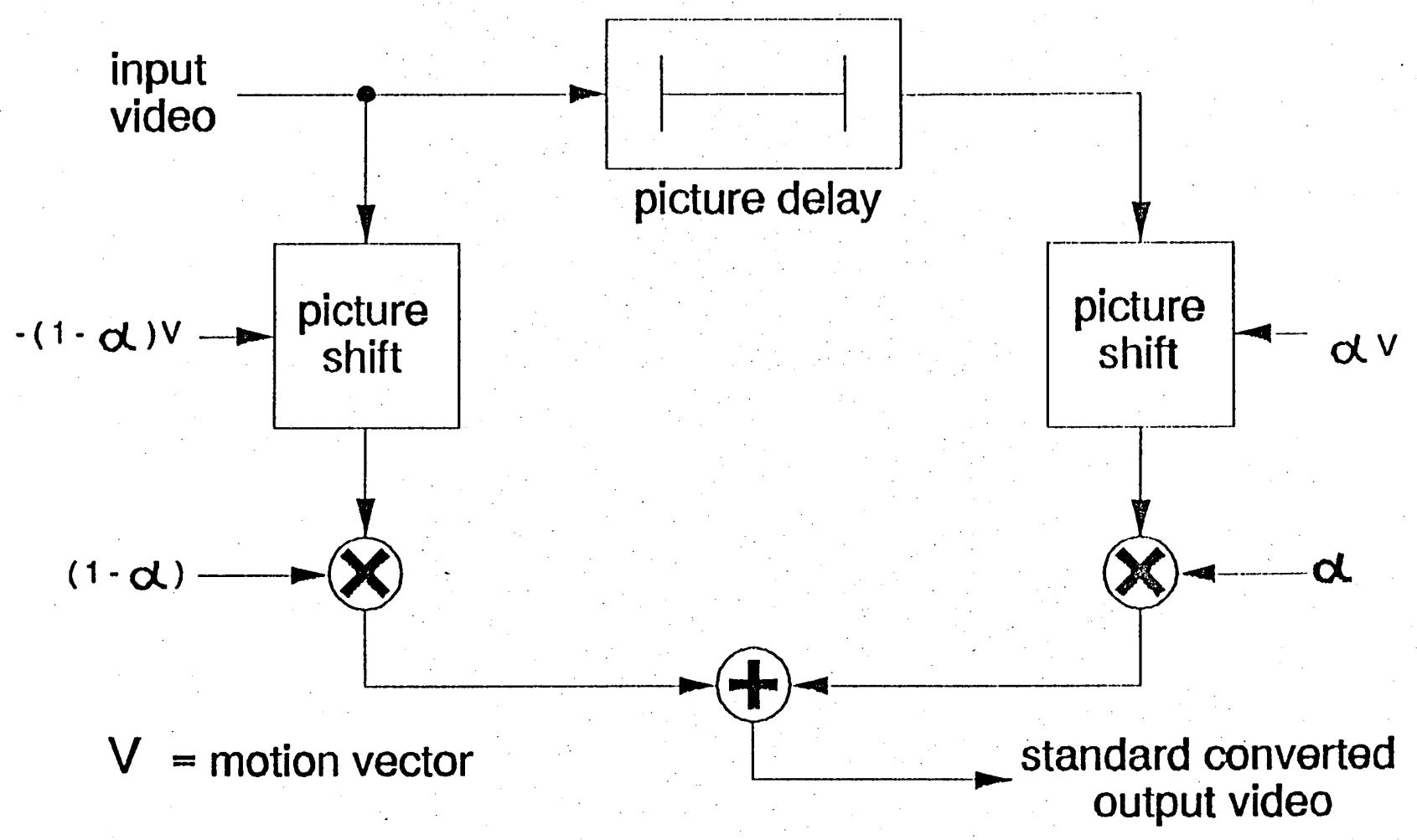

Figure 9.3: Motion compensated standards converter

Field 1

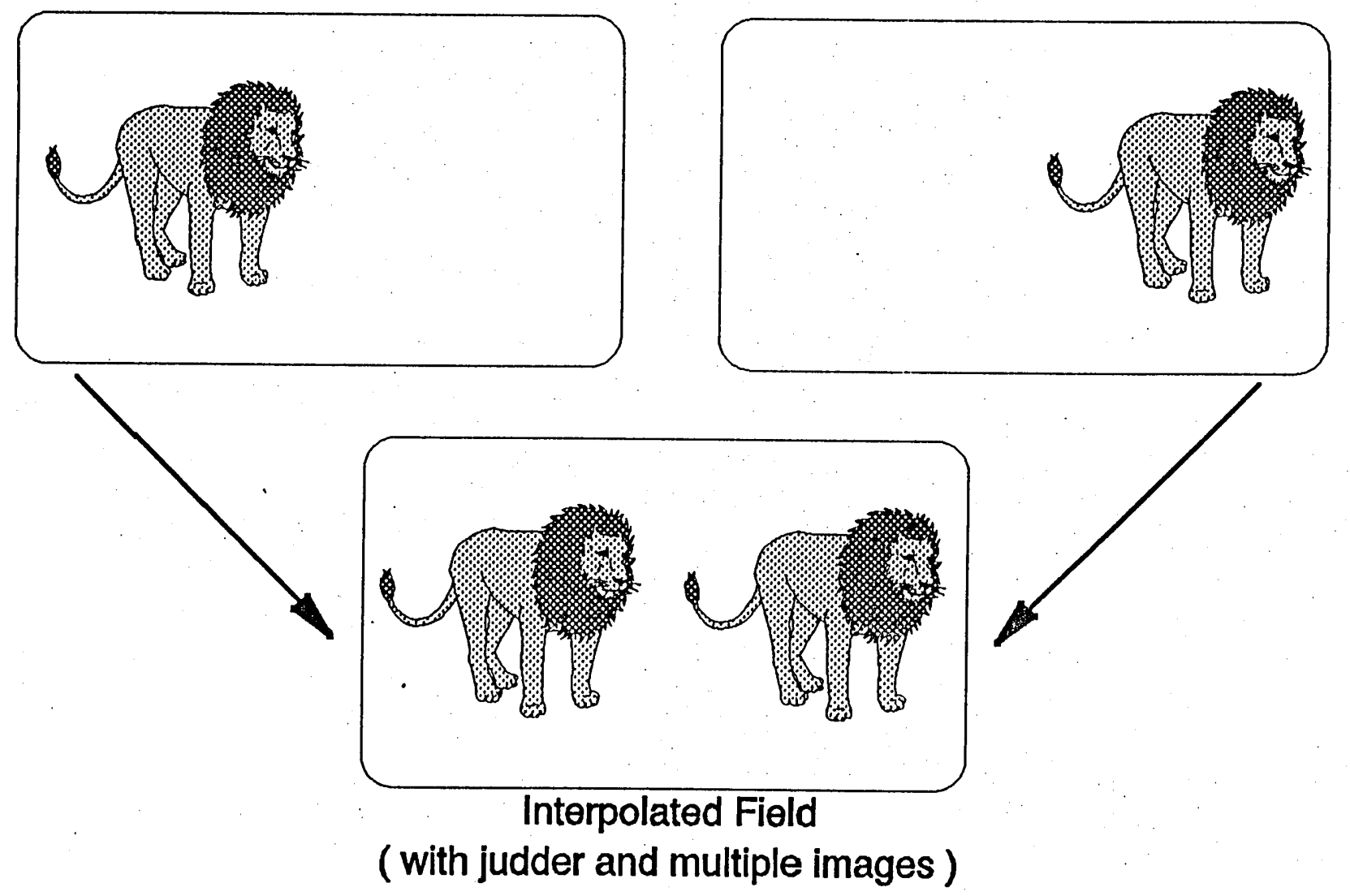

Figure 9.4: Conventional Standards Conversion 
Field 1

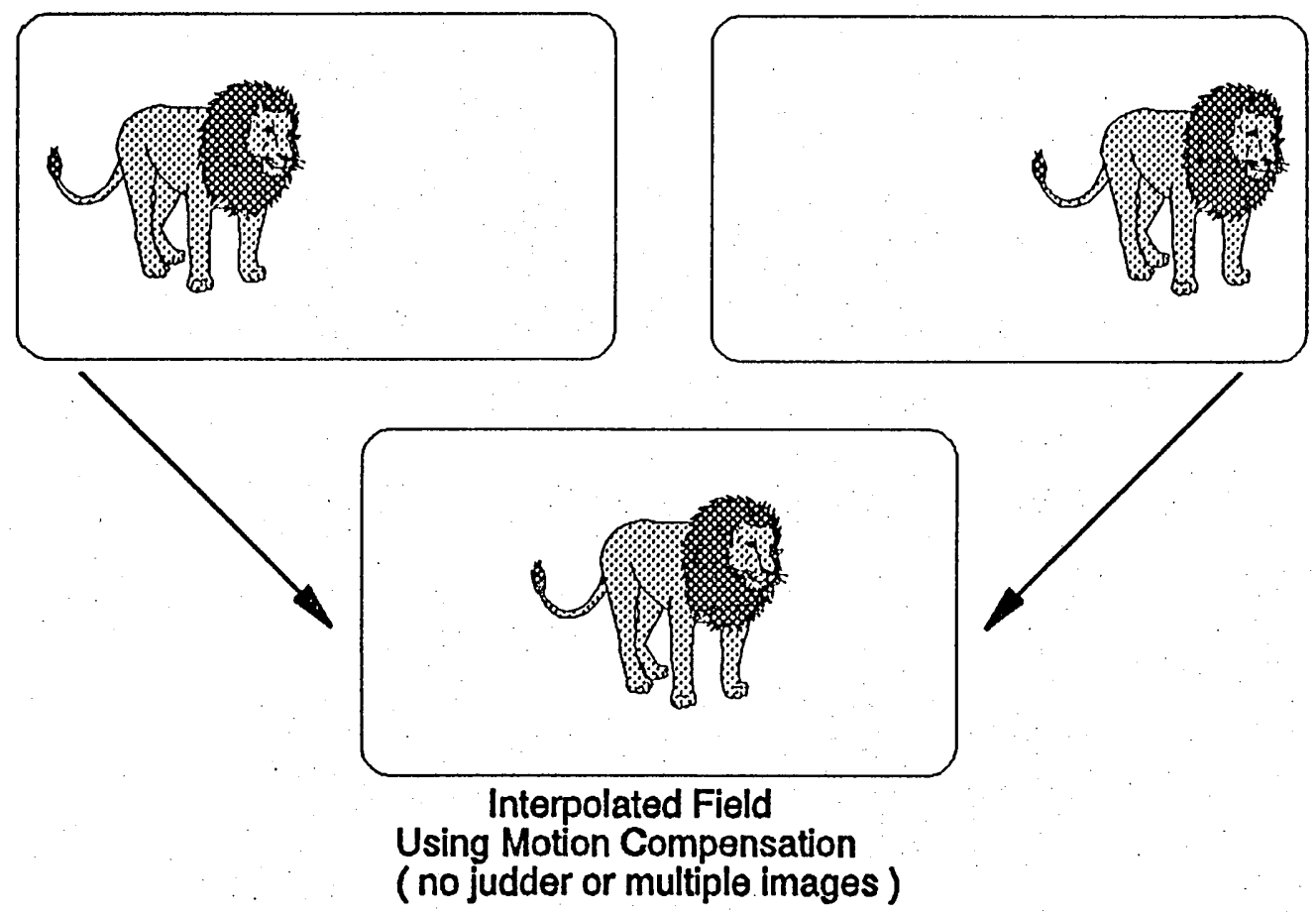

Figure 9.5 : Motion compensated Standards Conversion

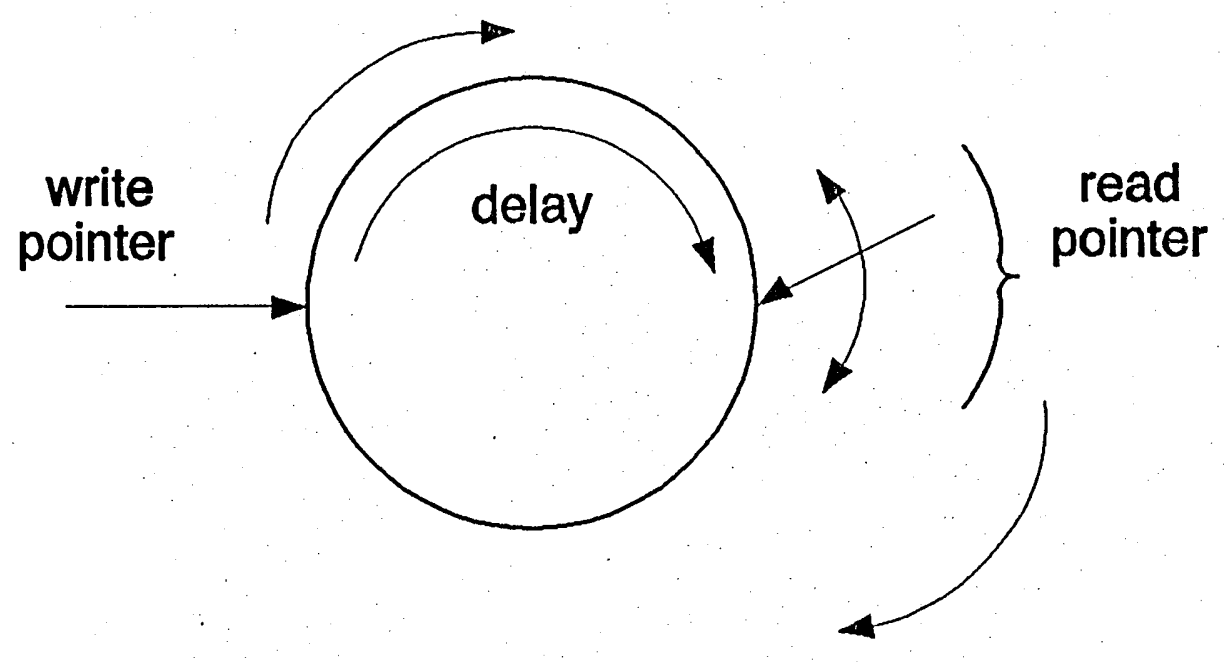

Figure 9.6 : A circular buffer for implementing motion compensation 


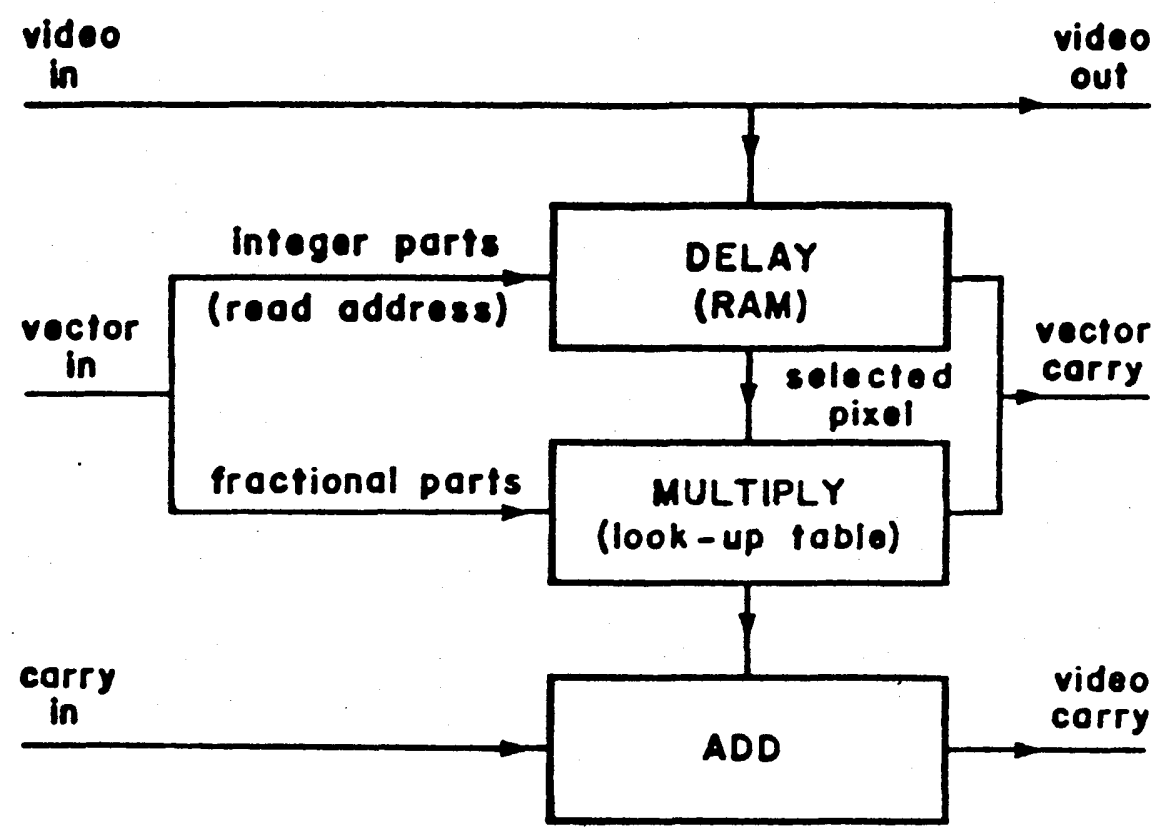

Figure 9.7 : bosic interpolation cell
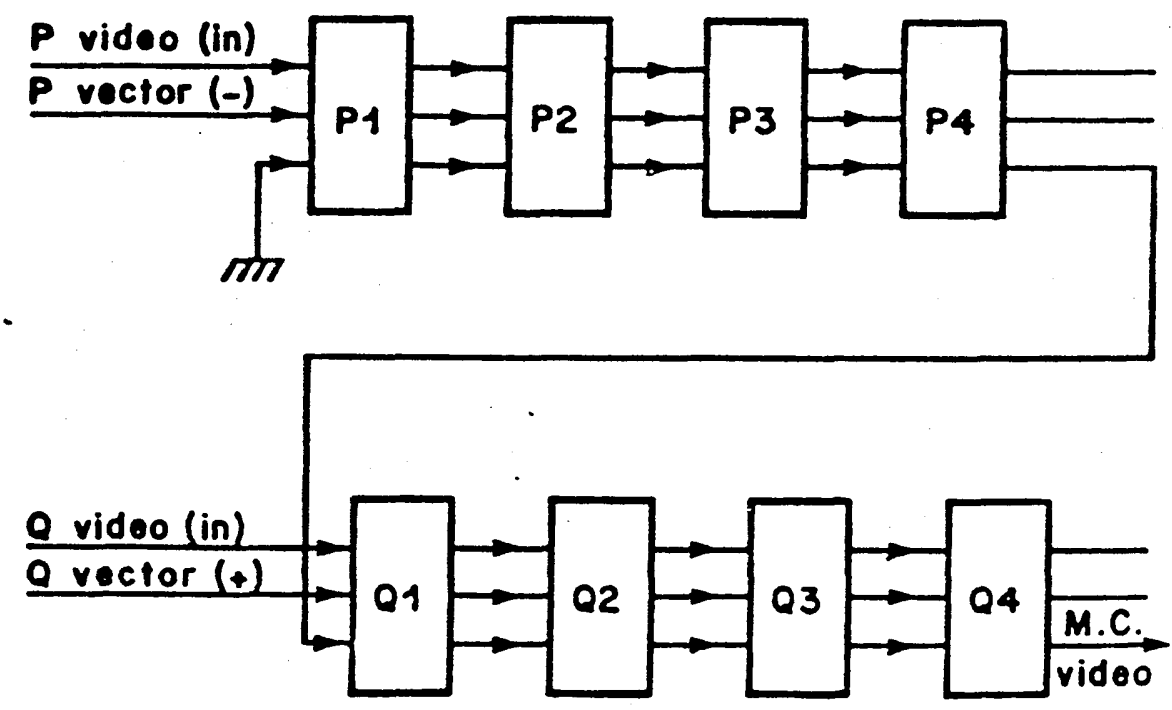

(b) Two-field configuration ( $P$ and $Q$ ) of basic interpolation cells

Figure 9.8 : Interpolator structure 

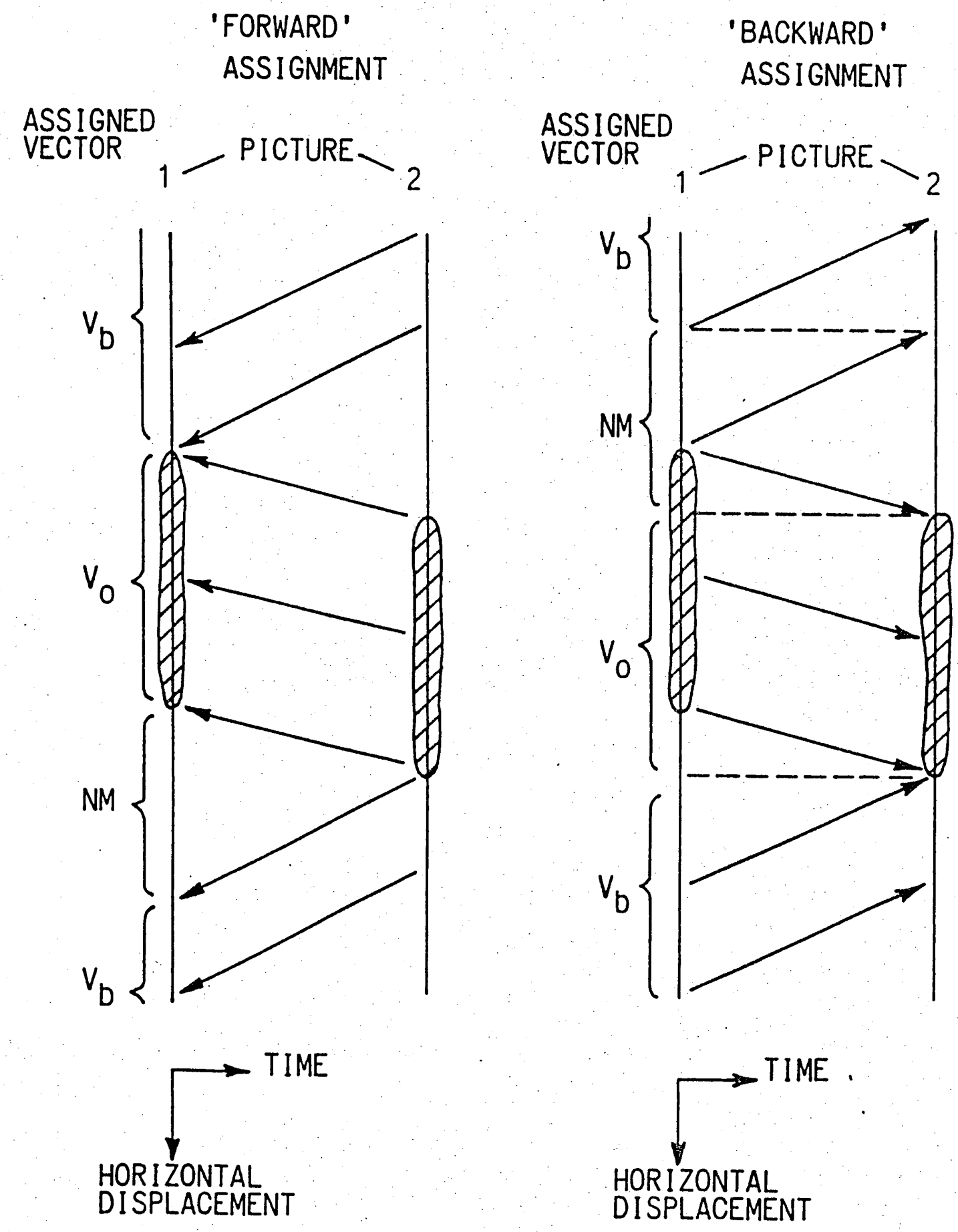

Figure 9.9 : Forward and backward assignment 


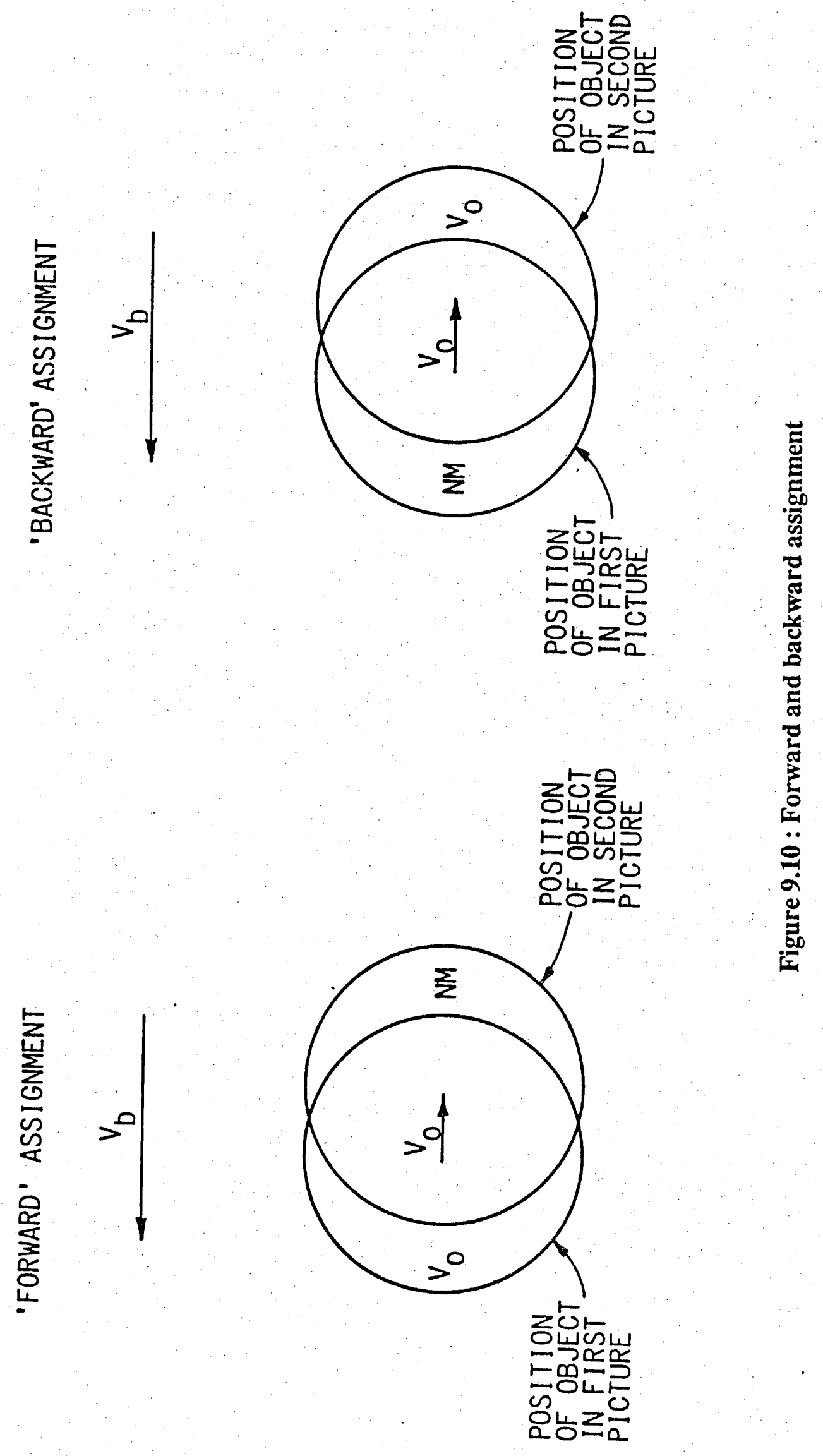




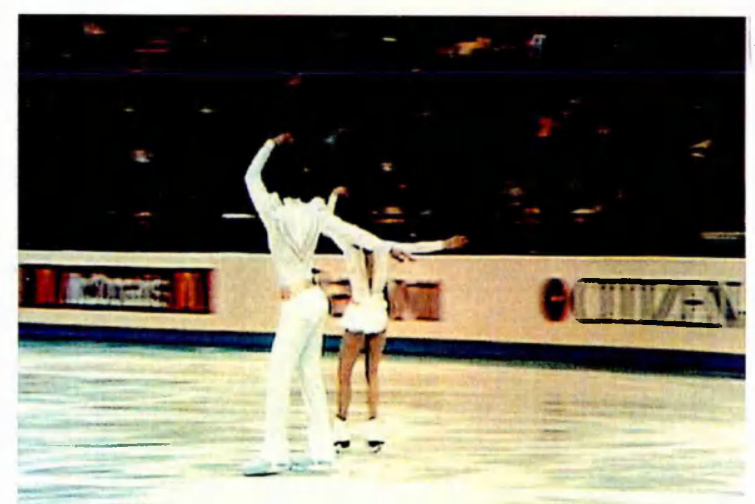

Fig 9.11

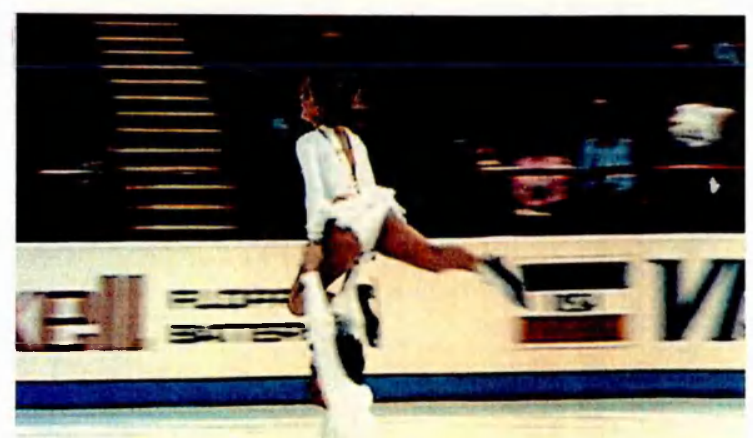

Fig 9.12

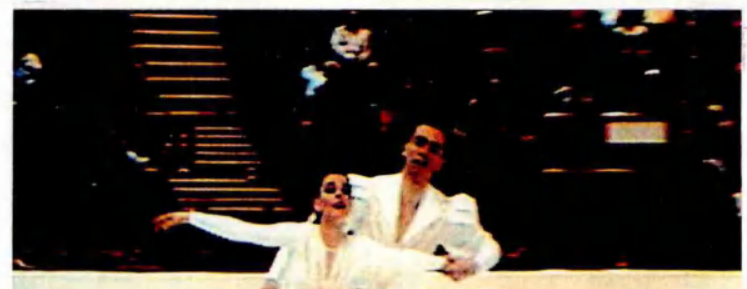

Fig 9.13

llightins 1 Rower

Fig 9.14
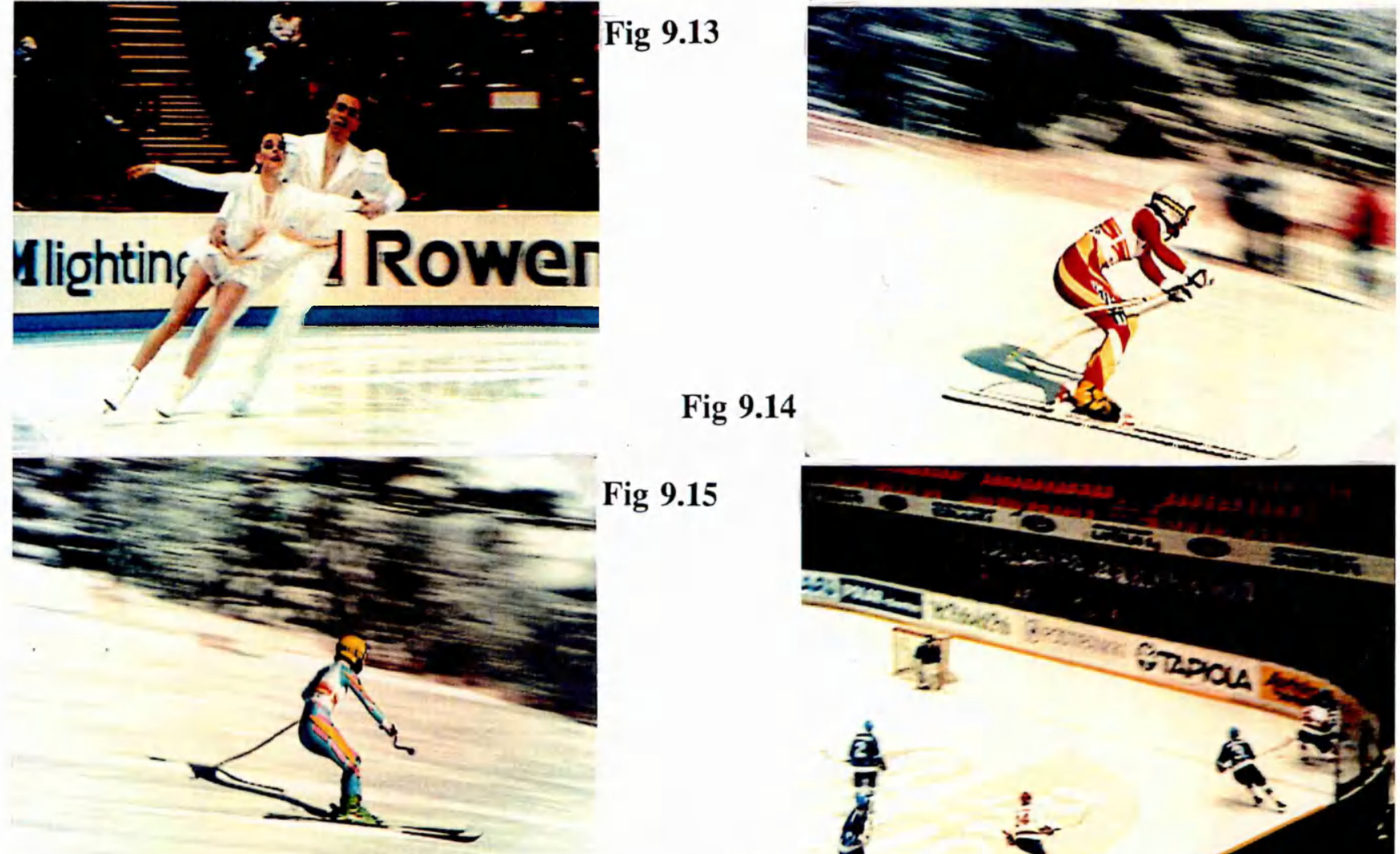

Fig 9.15

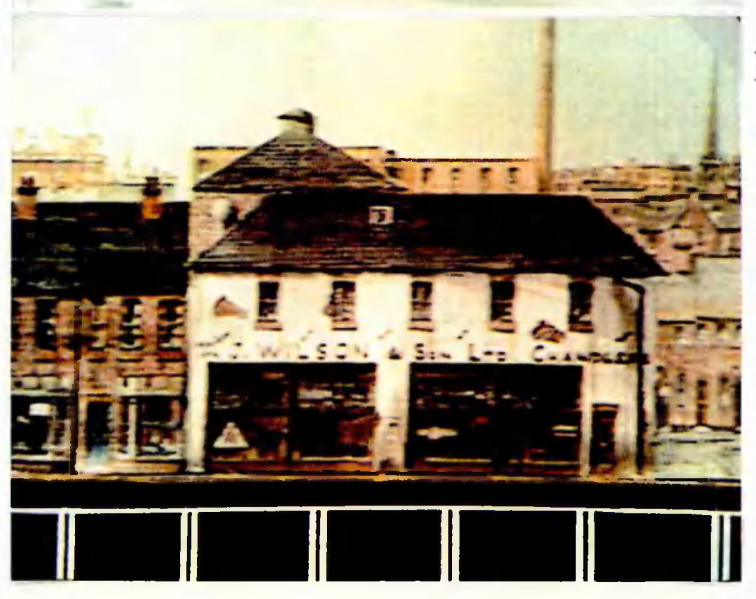

Fig 9.17

Fig 9.16
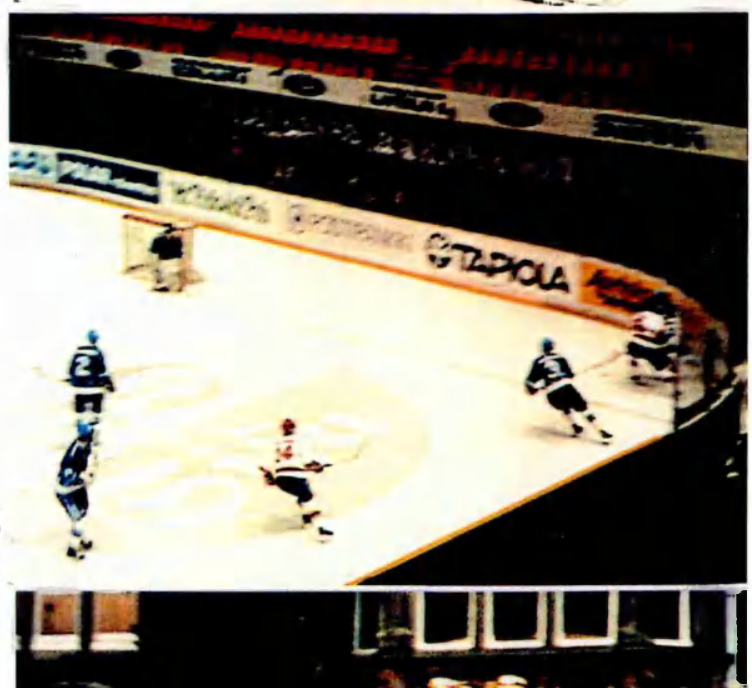

Fig 9.18

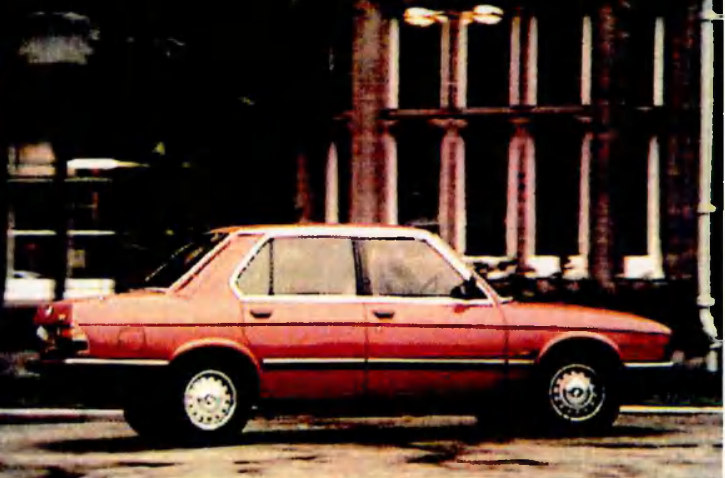

Figs 9.11 - 9.18: Original, uninterpolated test images 


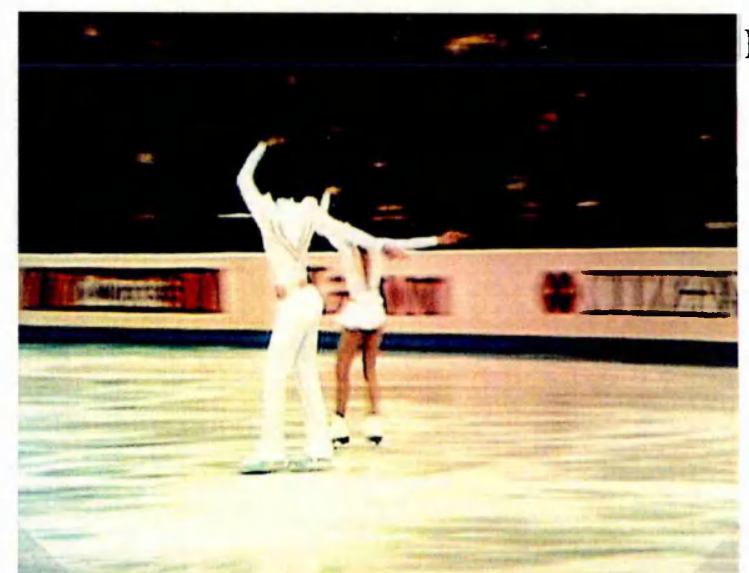

Fig 9.19

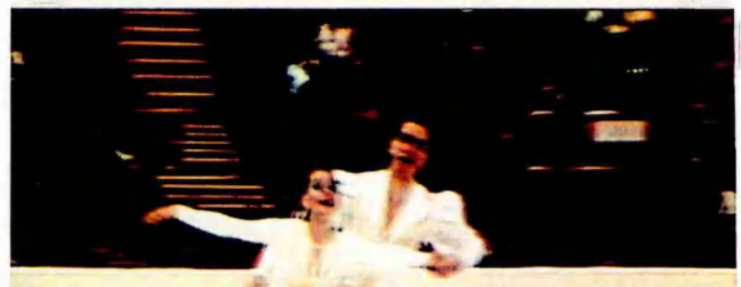

Fig 9.21
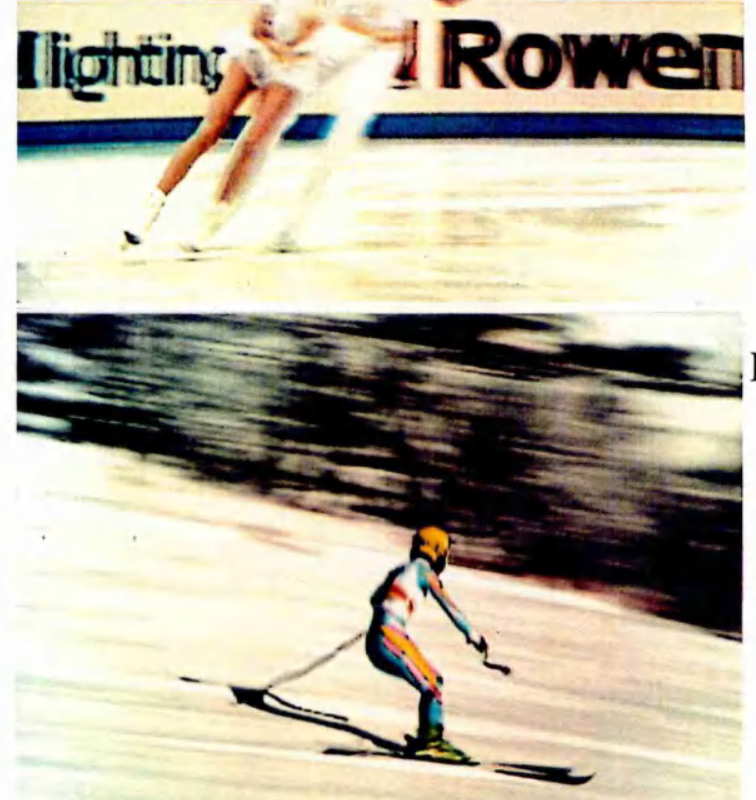

Fig 9.22

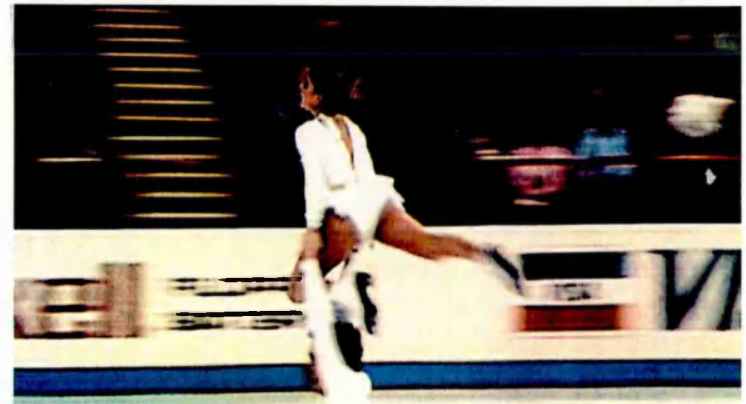

Fig 9.20
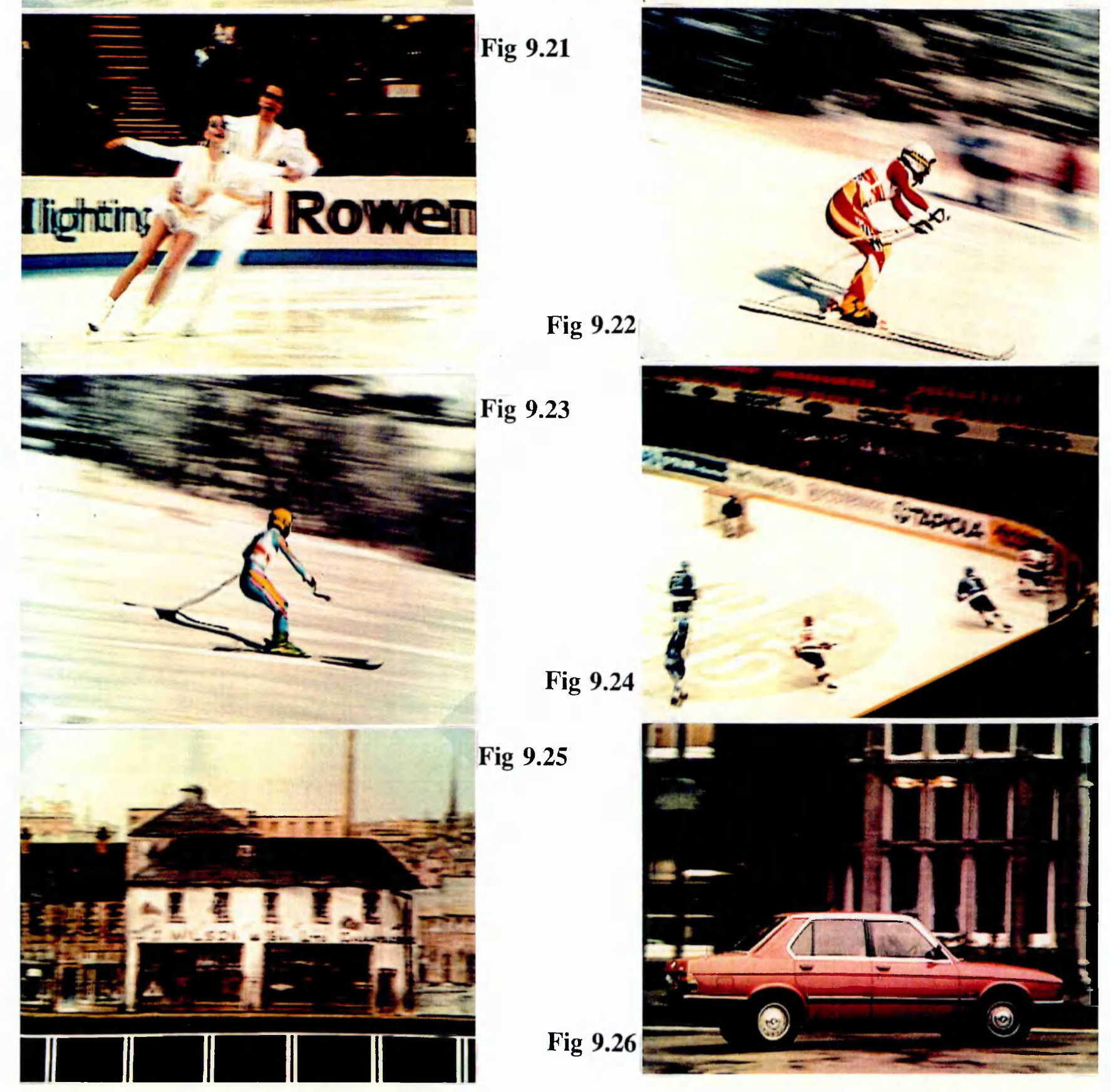

Fig 9.25

Fig 9.24

Fig $9.26=-1=$

Figs 9.19 - 9.26: Conventional standards conversion (linear filtering) 


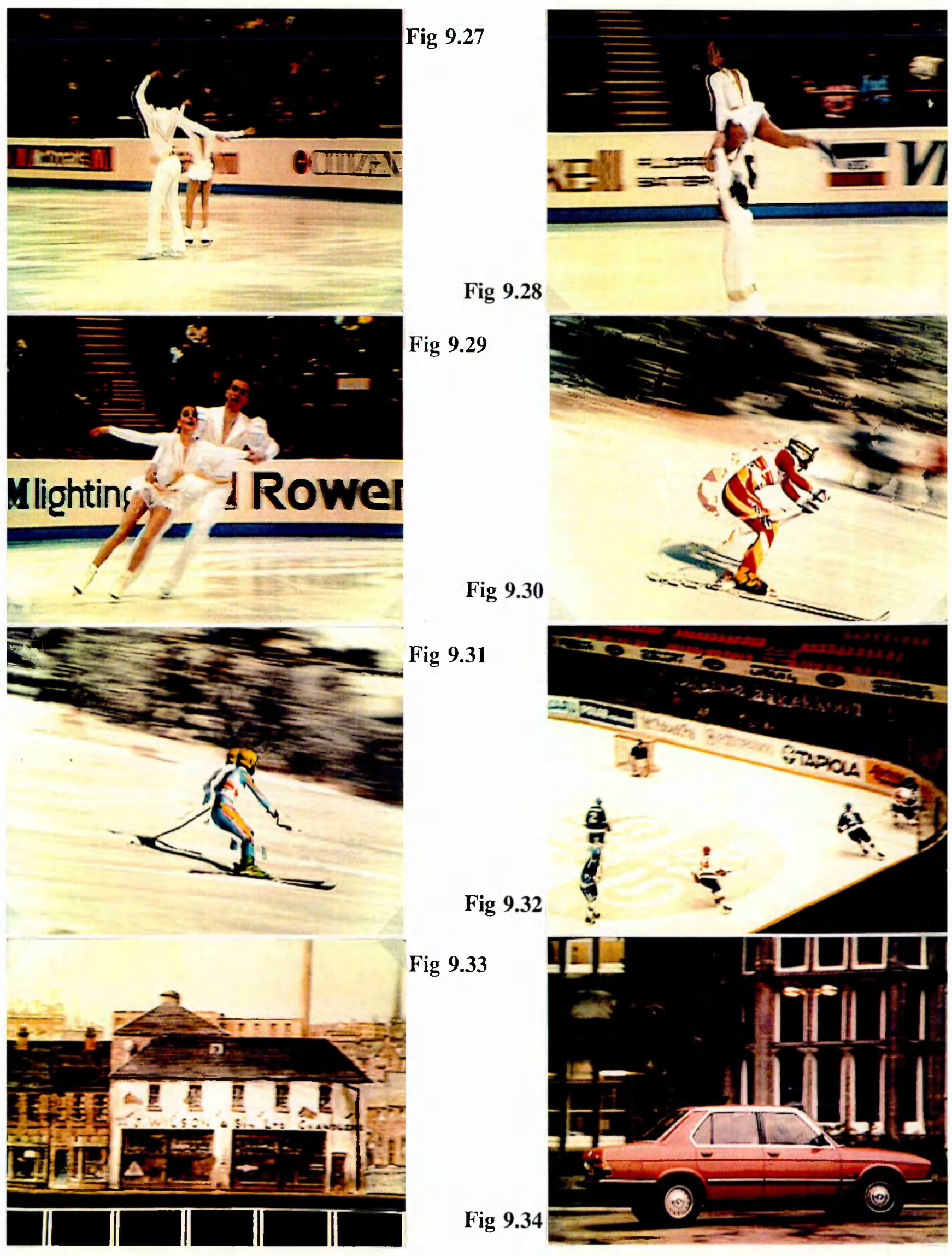

Figs 9.27 - 9.34: Initial results of motion compensated standards conversion 


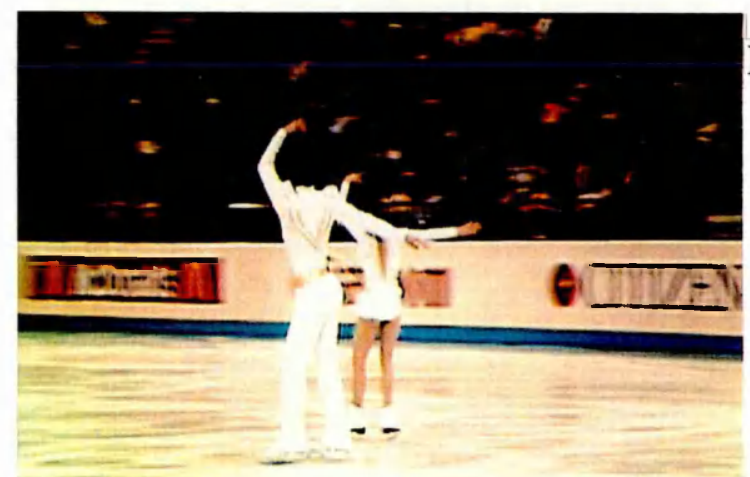

Fig 9.35

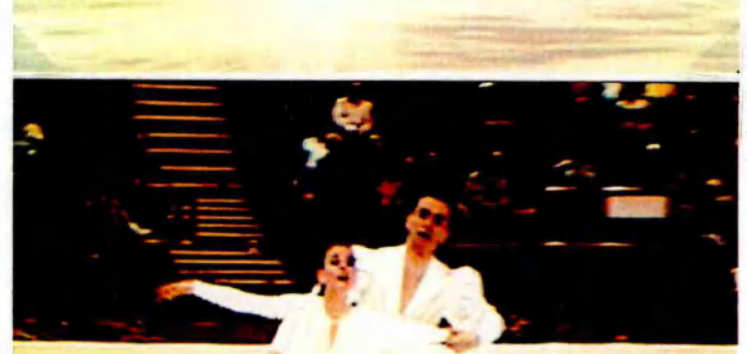

Fig 9.36
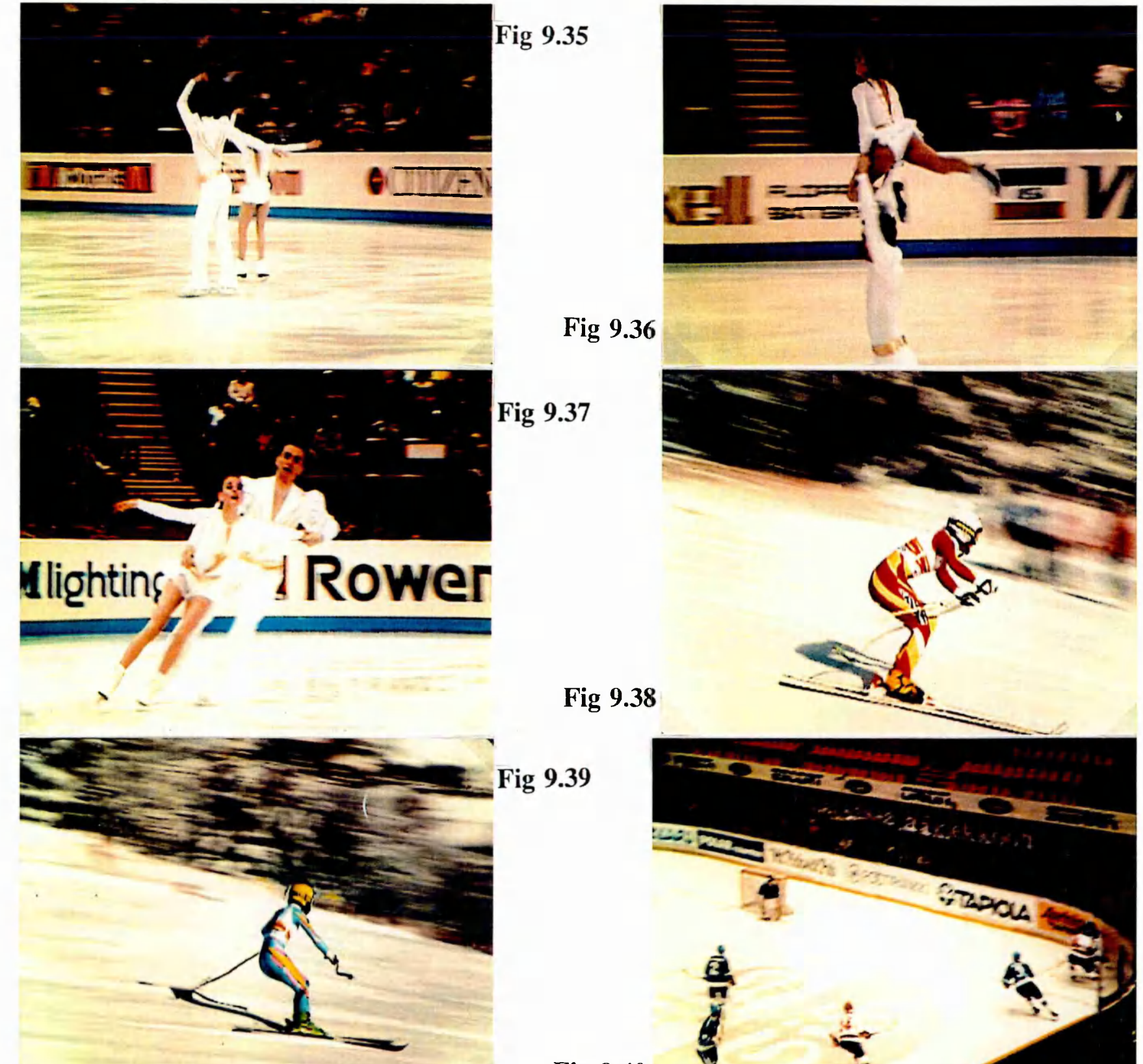

Fig 9.38

Fig 9.37

Fig 9.39

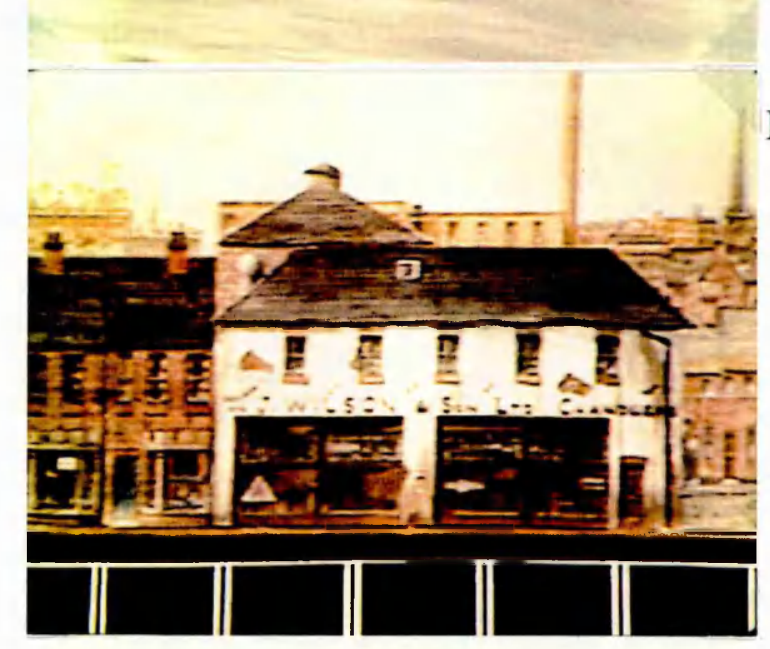

Fig 9.40

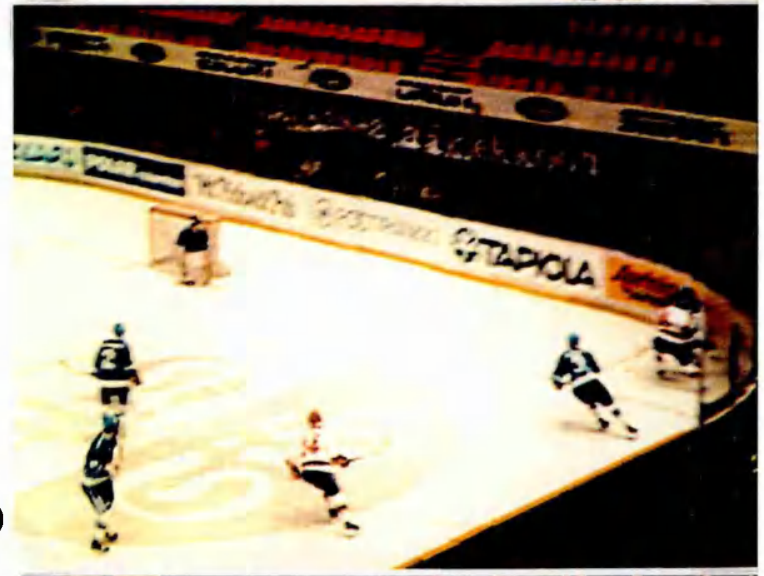

Fig 9.41

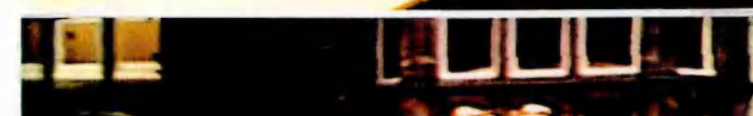

Fig 9.42

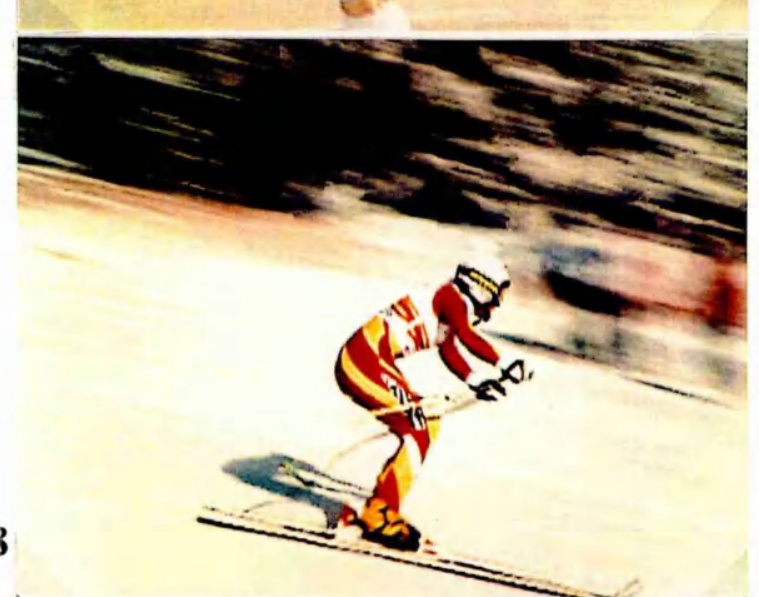


Since early in the development of television it has been necessary to convert television programmes between different television standards. One reason for this requirement is the different pictures rates originally chosen for European and American television standards, a legacy which is still with us today. Initially such standards conversion was performed by rather crude techniques, for example 'filming' a CRT display operating on one standard with a camera operating on the other standard. Such techniques gave little control over the characteristic of the interpolation process and so gave correspondingly crude results. Towards the end of the 1970's semiconductor technology had been developed to the stage where standards conversion could be performed using digital signal processing techniques. This allowed a significant improvement in the control of the interpolation characteristics. The improved interpolation yielded significantly improved standards converted pictures. Nevertheless the technology available limited standards conversion interpolation techniques using linear filtering. These techniques still produced some artifacts in the converted pictures, particularly judder and blurring. During the 1980's there was considerable interest in the technique of estimating movement in television pictures. This was initially aimed at reducing the bandwidth or bit rate for transmitting moving pictures. It was realised that if television movement could be measured and used for standards conversion, improved quality, free from judder and blurring, might be achieved. At the present time several organisations are investigating the use of motion compensation for standards conversion. Meanwhile the process of standards conversion is becoming more important as the number of standards for moving pictures increases to encompass high definition television and computer graphics.

It was the purpose of the investigation described in this thesis to study the process of converting television pictures between different television standards. Although considerable research had been conducted into standards conversion it seemed appropriate to take a fresh look at the digital signal processing techniques which could be used for standards conversion. The techniques investigated were the (conventional) linear filtering approach and the use of motion compensated interpolation. An objective of the research was to develop improved algorithms for standards converting television pictures. The use of motion compensated interpolation offered the prospect of improved standards conversion. Hence the investigation was focused on this objective and much of the study was devoted to developing real time motion compensated standards converters. Two examples of standards conversion processes were chosen for special consideration. These were 'field rate upconversion' (increasing the picture rate for improved display) and 'intercontinental standards conversion' (interconversion between $50 \mathrm{~Hz}$ and $60 \mathrm{~Hz}$ field rates). These two examples were investigated using both conventional and motion compensated interpolation techniques. 
A starting point for the study was the investigation of digital filters for interpolation. Previously the design of filter characteristics had been performed in a largely heuristic manner. This produced satisfactory results for the specific application of intercontinental standards conversion. It seemed, however, desirable to develop a more systematic method of filter design for the increasing number of standards converter applications (eg high definition television, computer graphics and motion compensation). The systematic design of standards conversion filters required a knowledge of the (frequency domain) characteristics of the parts of the television signal chain. These were determined by experimental investigation and by searching the published literature. Using these systematic design techniques interpolation filters for 'conventional' standards conversion were designed. This conventional approach to standards conversion was tested using the examples of field rate upconversion and intercontinental standards conversion. The results from this phase of the research provided a benchmark by which the performance of more advanced, motion compensated, algorithms could be judged.

A second starting point for the project was the estimation of movement in television pictures. An investigation of the published literature revealed a plethora of different motion estimation algorithms. Of these algorithms the 'phase correlation' technique of motion estimation seemed the most promising for standards conversion applications. The design of real time hardware to perform motion estimation, using phase correlation, was initiated near the start of the project. Design and implementation of the motion estimator proceeded in parallel with other investigations in order to produce results by the end of the project. When it had been built the motion estimator was coupled to experimental motion compensated standards converters to investigate motion compensated field rate upconversion and intercontinental standards conversion.

The thesis starts, in chapter 2 , by analysing the parts of the television signal chain excluding processing. This is necessary to set the process of standards conversion in its correct context. The parts of the signal chain are the scene from which the pictures originate, the camera used to detect them, the displays for showing then and the way they are perceived by the human visual system. Each of these parts of the signal chain is discussed in turn, with particular emphasis placed on the spectrum of the original scene and the response of the human visual system, since these have a significant effect on standards conversion.

The frequency domain characteristics of the television chain, discussed in chapter 2 , are useful for the design of filters and for indicating how adaptive and motion compensated processing may be applied to standards conversion. To be used for these purposes mathematical models of these characteristics are required. Chapter 2 presents a number of models, for different parts of the chain, suitable for these purposes. The models both simplify 
and generalise the true characteristics to give an approximate mathematical description of reality.

The distribution of energy in the frequency domain, for a typical scene, is investigated, in chapter 2, using computer analysis of a number of images. Experimentally it seems that the autocorrelation function (and hence power spectral density) of a typical scene can be represented by a simple model. This confirms previously reported results for the spatial autocorrelation function and extends them to 3, spatio temporal, dimensions. It is interesting to note that a suitable model for a typical, 3 dimensional scene, corresponds to a fractal function of dimension 3.5.

Chapter 2 also presents a number of models, taken from the literature, for the three dimensional frequency response of the human visual system. These provide an estimate of both the monochrome and colour response of the eye over the range of frequencies of interest in television systems. These models should be considered indicative of the frequency response rather than being particularly precise. This is true, not least, because there is considerable variation between the responses of particular individuals. Nevertheless these models are sufficiently accurate to allow some useful optimisation of interpolation filters for standards conversion.

Chapter 3 examines the design of linear, symmetric, transversal interpolation filters for standards conversion. It starts by considering the historical development of filter design techniques for television. This is followed by a more precise description of desirable filter characteristics and ways in which they can be approximated. The chapter is also intended to provide an overview of filter design for standards conversion.

The discussion of filters, in chapter 3, starts with the concept of the Brillouin zone as an ideal filter characteristic. This is the multidimensional analogue of a sharp cut lowpass filter in the one dimensional case. The use of the Brillouin zone is shown to be less than ideal because of its ill-defined shape for a vertical/temporal filter and its failure to allow for aliasing inherent in the signal. Next the frequency sampling technique of filter design is discussed. Although it has been successfully used for the design of standards conversion apertures, it is neither very flexible nor convenient to use. Generalisations of the frequency sampling filter design technique are also presented. These allow arbitrarily positioned coefficients and frequency specifications. It is shown how an arbitrarily large number of frequency domain specifications can be used in a least mean square approximation technique. Frequency sampling technique are, however, essentially heuristic in nature, since they rely on the design engineer's idea of a 'good' filter characteristic. Hence a more objective method of filter design is desirable.

An objective method of determining a filter response specification appropriate for interpolating television pictures is presented in section 3.3. It is based on the technique of Wiener filtering. For standards conversion it is assumed that loss of resolution and aliasing 
can be treated as uncorrelated noise sources. The 'ideal' response is then that which minimises the mean square impairment. Input noise can also be included as an impairment in this analysis.

Several techniques are proposed for producing 'optimum' approximations to an 'ideal' frequency specification, some of which appear to be new. These techniques rely on a 'weighting' function which indicates the amount of (picture) impairment caused by approximating the ideal response at a particular frequency. The appropriate weighting function for approximating the ideal response can be determined by extending the analysis of the 'ideal' filter presented in section 3.3. The characteristics and computational requirements of the various approximation techniques are discussed. It is briefly indicated how time domain constraints (requiring, for example, a 'smooth' impulse response) can be included in the filter design process. This may be of use if more sophisticated, dual 'space-time/frequency domain', models of the response of the human visual system become available. At the end of the chapter the design of adaptive and motion compensated filter responses is considered.

Having discussed how digital interpolation filters, for standards conversion, could be designed, chapter 4 examines the practical implementation of such filters. First the basic theory of interpolation using linear filtering is described. Then the changes required to basic filter structures, for use as interpolators, are considered. Two types of filter, input and output lattice filters, are considered. These correspond to the direct and transposed implementations of an FIR filter in a single sampling rate filter. Input lattice filters store the input samples prior to interpolation and the interpolation aperture (filter response) is specified on the input sampling lattice. They are good for increasing the sampling rate because they are efficient at removing repeat spectra (aliases) in the sampled input signal. Conversely output lattice filters store output samples and the interpolation aperture is specified on the output sampling lattice. They are good for decreasing the sampling rate because they efficiently remove spectral components which would otherwise alias in the output signal.

Two implementations of interpolation filters, pipelined or non-pipelined, are considered. Pipelined filters are efficient and convenient structures for either increasing or decreasing the sampling rate (but not both). However, they are inconvenient to use in circumstances where the same hardware may be required to either increase or decrease the sampling rate. This is usually the case for standards conversion between European and American television standards. Non-pipelined filters can conveniently be used to both increase and decrease the sampling rate. The price paid for this flexibility is a more complex filter structure and a loss of computational efficiency.

The problem of quantising a continuous interpolation aperture for use in practical hardware is considered. Theoretically the interpolation aperture is a continuous function. In practice it is stored at points on a supersampled input or output lattice. The degree of 
supersampling (aperture quantisation) determines how closely the sampled aperture function approximates the desired continuous one.

Finally chapter 4 considers the implementation of motion compensated interpolation filters. Motion compensation can be implemented in both (multidimensional) input and output lattice filters. Conceptually relatively minor changes are required to the calculation of sample and coefficient addresses. In practice significantly more hardware is required to allow much more flexible access to stored data samples. For non-motion compensated interpolators the hardware requirements of input and output lattice filters are very similar. When they are motion compensated, however, output lattice filters are significantly more complicated because it is necessary to store motion vectors as well as the output samples.

The experimental investigation of two 'conventional' standards conversion processes are considered in chapter 5 . The first process is field rate upconversion, that is, increasing the field rate to improve the appearance of the displayed pictures. This particular investigation concerned doubling the field rate from $50 \mathrm{~Hz}$ to $100 \mathrm{~Hz}$. The second process is the interconversion between the television formats used in Europe and America. The description 'convention standards conversion' means that a non-adaptive (hence non-motion compensated) linear filter was used to perform interpolation. The adjective 'conventional' is, perhaps, a little misleading. Although both processes have been investigated previously the new filter design techniques, described in chapter 3 were used for this investigation.

The purpose of the investigation of conventional standards conversion was both to validate the filter design technique and to determine the performance of 'conventional' standards conversion.

Field rate upconversion was investigated using computer simulation. Upconversion reduces display flicker by increasing the number of fields displayed per second. Comparisons were made of interpolation using 'optimum' filters versus interpolation using filters previously reported in the literature. It seems that a carefully optimised filter can give better performance than those suggested previously. The performance seems to be sufficiently good to obviate the need for adaptive processing that has been used previously. A relatively small aperture (1.5 input fields by 3 picture lines) is sufficient to give good performance. Large apertures do not give significantly better performance in spite of their extra complexity.

The more complex process of intercontinental standards conversion was investigated using real time hardware. Comparison of the performance of interpolation using 'optimised' filters were made with successful filters previously reported in the literature. The characteristics of the 'optimised' and previously reported 'reference' filters were compared on a variety of picture material. The results from the 'optimised' filters were broadly comparable to those from the 'reference' filters.

Several general conclusions can be drawn from these results. The filter design algorithm, described in chapter 3 and used in these investigations, produced acceptable results 
for both the standards conversion processes. Therefore it will probably produce good results for other applications. Good results for field rate upconversion can be achieved using a relatively simple non-adaptive linear filter. It may be possible to gain a small improvement in the quality of intercontinental standards conversion by using very carefully optimised filters. Even when this has been done, however, there remain very noticeable impairments (particularly judder) in the quality of the converted pictures.

Chapter 6 discusses the theory of motion compensated processing. The occurrence of movement in television pictures presents great difficulties for picture processing. These arise because, considering conventional sampling theory, the input images are grossly undersampled temporally. Temporal undersampling is due to the very high spatio-temporal frequencies which can be generated even by relatively slow movement in images. Motion compensated processing provides a way of circumventing some of these difficulties.

By considering why the human eye does not usually perceive temporal aliasing in television pictures we are led to the principle of motion compensation. This principle is that images of moving objects are processed in their own moving, rather than a stationary, frame of reference. That is processing is performed along an objects motion trajectory.

The use of motion compensation rests on several underlying assumptions. It is assumed that the eye tracks the motion of moving objects. This is usually true, but when this assumption fails we cannot obtain the subjective benefits of motion compensation. It is also assumed that the image consists approximately of linearly translating rigid objects. Motion compensation might, therefore, be considered as a form of model based image processing. The model is, however, highly general and non-specific. When the model of moving objects fails we cannot use motion compensation and must fall back to another processing algorithm. Fortunately motion compensation seems to be applicable to most television pictures.

The first half of chapter 7 contains a detailed literature review and discussion of motion estimation. The 3 main categories of motion estimation techniques, namely spatiotemporal constraint, region matching and frequency domain are reviewed with reference to the published literature. The importance of hierarchical motion estimation, to measure the large velocities required by standards conversion, was discussed as was the use of recursive motion estimation techniques.

Although a large variety of spatio-temporal constraint and region matching techniques have been described in the literature, either the phase or motion correlation techniques appear more suitable for the particular application of standards conversion. The reason why phase correlation has, perhaps, received less attention than other techniques may be because it appears to be computationally complex. Chapter 7 aimed to show that, for the large range and high accuracy of motion vectors, for standards conversion the complexity of other techniques is very much greater than for other applications. Therefore, for standards conversion, phase 
correlation may be less complex than some of the alternatives, and has been demonstrated to give good performance.

The second half of chapter 7 details two motion estimation algorithms which have been investigated by computer simulation. These two motion estimation algorithms, phase correlation and motion correlation, were chosen for their suitability for standards conversion applications. Phase correlation has, previously, been described in the literature and is based on analysis of the phase difference of (spatial) Fourier transforms of successive pictures. Motion correlation appears to be a new technique, and is based on analysis of the magnitude of the 3 dimensional spectrum of an image sequence. The intention of this experimental investigation was to determine the performance of these algorithms if used in a real time system for standards conversion. Care was taken with the processing used, to try to achieve the best results from both algorithms. Since very similar processing was used for both techniques it is possible to compare fairly a region matching algorithm (phase correlation) with a frequency domain algorithm (motion correlation).

Phase correlation and motion correlation algorithms both seem to give the performance required for standards conversion. They can both measure large velocities to sub pixel accuracy. Both phase and motion correlation are able to accurately measure movement in noisy pictures until the input signal to noise ratio falls below 3 or 4 bits. Motion correlation may have some advantage over phase correlation because of greater accuracy, better noise performance and resilience to the effects of prior temporal filtering of the pictures. These advantages are gained at the cost of greater computational complexity. The encouraging results from motion correlation should be treated with caution since it has not be investigated elsewhere.

Chapter 8 presents a description of the experimental real time motion estimation system, and the results obtained from it. The motion estimator was based on the technique of phase correlation detailed in chapter 7. It was designed to generate accurate vectors describing the motion of all objects in a conventional television picture. At the inception of the project no real time motion estimation equipment, based on phase correlation, had been built. Therefore this experimental system was built in a modular fashion so that it was as flexible as possible. This allowed the motion estimation algorithm to be modified, both in the light of experimental results and for slightly different algorithms to be used for different applications.

Many hours of video signals were processed with the motion estimator. This allowed the qualitative assessment of very much more material than would ever be possible using computer simulation. Assessment of the motion estimator output allowed significant improvements to be made to the system. Undoubtedly considerable further improvements could be made to similar systems built in the future. Nevertheless this system shows that high 
fidelity motion estimation can be performed by real time motion estimation systems of this type.

The motion estimator system was interfaced to 2 experimental, real time, standards converters. One performed motion compensated field rate upconversion while the other performed motion compensated intercontinental standards conversion. The results of these experiments are given in chapter 9 , following consideration of the practical implementation of motion compensation. It is worth emphasising that, in contrast to many results reported in the literature, these results used real time processing not computer simulation. This allows very much longer sequences to be processed but restricts the complexity of the algorithms used.

The output from motion compensated field rate upconversion appeared to be of very similar quality to its input (but with reduced flicker). A slight loss of resolution could be seen on some parts of the pictures due to the limited number of filter taps used in the interpolation. The good performance of this system was due, in part, to the efficacy of the processing used. However, it also seems likely that upconversion is more tolerant of interpolation imperfections than some other standards conversion processes.

The results of motion compensated, intercontinental, standards conversion, using real time hardware, were presented in section 9.5. Imperfections in the initial results highlighted inadequacies in the motion estimator and the interface between motion estimator and interpolation hardware. When these problems were corrected the quality of the interpolated images was more similar to the original images rather than those interpolated using 'conventional' standards conversion. Loss of resolution and double images, which are common using 'conventional' standards conversion, were largely absent when motion compensation was used. The remaining artifacts in the interpolated pictures seem mainly due to difficulties with motion estimation.

The two examples of real time motion compensated interpolation, presented here, demonstrate the potential, and increasing practicality of this technique. The results, while not perfect, indicated that high quality standards conversion can be achieved using motion compensation. While the technique requires a complex implementation, advances in semiconductor technology make it more and more practical.

It is hoped that this thesis provides a clear and consistent framework for understanding the process of standards converting television pictures. Nevertheless the field of standards conversion is too large to have been comprehensively covered in this project and considerable research remains to be done.

Chapters 2 and 3 considered the process of filter design using models of parts of the signal chain. Only monochrome processing was investigated experimentally. Colour has a profound effect on the perception of television images. The models presented in chapter 2 
could be used to design 'optimum' colour interpolation filters. The ideas of 'pseudo Wiener filtering' could also be extended to allow optimum encoding and decoding of television signals in a composite luminance/chrominance signal (eg. PAL). In chapter 3 it was assumed that resolution loss and aliasing are subjectively equally undesirable. This is a reasonable first approximation, as borne out by the results presented here. Nevertheless improved results might be achieved by giving different weighting to different types of impairments. Some brief informal experiments suggest that alaising is considerably more objectionable than loss of resolution. There is, thus, considerable scope for interesting future research in filter design for television systems.

Chapter 7 presented a new technique ('motion correlation') for motion estimation. Computer simulations suggested that this might give good performance and have some benefits over existing algorithms. It was not possible to pursue this line of research further in this project but the use of this technique in computer simulations or, better still, real time applications would be interesting. Further improvements in motion compensation may result from using more advanced motion estimators. In particular the image segmentation stage of phase correlation or motion correlation motion estimation could be developed to give information on areas of revealed or obscured background. Such information might result in significantly improved motion compensated interpolation. It should be clear that there is considerable scope for future research in this field.

This thesis has investigated the process of television standards conversion. That is the conversion of television pictures on one standard to a different standard. The interpolation method used determines the quality of the standards converted pictures. For some application, eg. field rate upconversion, conventional, non-adaptive linear filtering is sufficient to give good results. For other applications, eg. intercontinental standards conversion, more sophisticated techniques are required to give artifact free standards conversion.

One technique which promises to provide improved intercontinental standards conversion is the use of motion compensated processing. The success of this technique appears to depend much more critically on the performance of the motion estimator and how it is interfaced to the interpolator than on details of the interpolator itself. Considerable development work is still required in this field. Nevertheless the results presented in this thesis show that very high quality standards conversion is possible using motion compensation. 


\subsection{Introduction.}

While investigating the design of television standards converter systems it became apparent that there is a need for an improved method of designing window functions (Papoulis '77). Such window functions (often referred to simply as 'windows') have (at least) two functions in standards converters. Firstly they can be used for filter design (chapter 3 ). Second they are useful in segmenting the video signal prior to performing discrete Fourier transforms for motion estimation (chapters 7 and 8). This appendix describes the uses of window functions and an improved method of designing them.

The appendix starts with the history and use of window functions. This is followed by a suggestion for the definition of 'optimal' window functions. Next the short comings of current methods for the design of multidimensional window functions are discussed. Using the definition of 'optimal' window functions it is shown how to design multidimensional window functions which are particularly suitable for digital video processing. After this theoretical discussion some 1 and 2 dimensional filter design examples are given using optimum window functions. In order to make this document as comprehensible as possible most mathematics has been relegated to (sub) appendices.

\subsection{A Brief History of Window Functions.}

Window functions were first used in spectrum analysis and for filter design. For spectrum analysis windows are required to trade off resolution and spectral leakage (Prabhu '87). In filter design windows are used to ameliorate the effect of Gibbs' phenomenon. The problems in both these applications are caused by trying to process a finite section of an infinite signal. This problem often arises, when simultaneously considering both the time and the frequency domains, because it is impossible for a signal to be simultaneously of limited extent in both domains (Slepian '76).

The computing power available for digital signal processing was very limited when windows were first used. This had two consequences; window functions had to be easily calculated and they were only used on 1 dimensional signals (multidimensional signals required too much processing power to be considered). These limitations are no longer applicable but appear to have left a lasting legacy on the type of window functions which are commonly used.

There are a large number of window functions which have been used for signal processing. These include Bartlett (or triangular), Hanning, Hamming, Blackman, DolphChebyshev and Kaiser windows. This (non-exhaustive) list is given to indicate the bewildering 
plethora of windows available to chose from. Each of these has its own particular characteristics which may make it particularly suitable for some applications. The design of these window functions appears to have been largely heuristic, determined by the needs and resources of the moment.

Kaiser windows (Kuo \& Kaiser '66, Kaiser '74) are a particularly good and flexible family of window functions. They are an approximation to optimum (continuous) 1 dimensional window functions. Their definition includes a variable parameter which allows them to be optimised for a given application. These windows have been extensively used because they are easy to use and to calculate. True optimum window functions, however, are equally easy to use and require very modest computational power by today's standards.

\subsection{Applications of Window Functions.}

This section describes two applications of window functions. The following discussion will suggest a definition for optimum window functions which is described in the next section.

\subsubsection{Filter design.}

Window functions can be used in the design of finite impulse response (FIR or transversal) filters. The technique provides a quick and easy design strategy albeit with limitations on the response of the resulting filter.

The problem of designing an FIR filter is to take an ideal filter response specification and to generate a practically realisable filter whose response approximates the ideal response. The ideal filter specification is given in the frequency domain. The impulse response of the ideal filter is the Fourier transform of the frequency specification. Unfortunately the ideal impulse response invariably turns out to be of infinite duration. Hence we cannot simply take the Fourier transform of our frequency specification to obtain a practical filter impulse response.

The most obvious way to generate a practical filter response is to simply truncate the Fourier series corresponding to the ideal response. This certainly gives a practically realisable filter. Furthermore, this technique gives a least mean square approximation to the desired filter response. One might think that this solves the problem. There is however a serious drawback to this technique.

Although truncating the Fourier series gives a least mean squares approximation to the ideal filter, it gives large errors at the edges of discontinuities in the filter specification. This happens, for example, in low pass filters where the frequency response specification changes from +1 (passband) to 0 (stopband). This is Gibbs' phenomenon and these errors are not 
reduced by using more filter taps ${ }^{1}$. It might be considered that this problem is due to using a least mean square error criterion rather than minimising the maximum error (a minimax approach). It is, indeed, possible to use a minimax approach to filter design (eg the ParksMcCellan algorithm (Parks \& Burrus '87)) but this is quite complicated. The complexity of the minimax approach makes it impractical for some applications (for example calculating filter coefficients 'on the fly' in a standards converter). An alternative to the minimax approach is the use of window functions (Prabhu '88).

Windowed filter designs are easy to calculate and reduce the effect of Gibbs' phenomenon. The ideal filter impulse response is calculated by Fourier transforming the ideal frequency response. The ideal impulse response is then multiplied by a finite length window function to produce a practical filter. In the frequency domain windowing convolves the frequency response of the window with the ideal frequency response. The effect of this is to smooth the ideal frequency response. Hence a sharp transition in, for example, a low pass filter, becomes a transition band of finite width.

Generally, the frequency response of a window function has a large amplitude main lobe, centred at D.C., surrounded by a number of low amplitude side lobes. As the width of the main lobe increases the amplitude of the side lobes decreases. This is a manifestation of the uncertainty principle of Fourier transforms which says that we cannot, simultaneously, restrict the duration and bandwidth of a signal. The different window functions available each involve a different compromise between main lobe width and side lobe amplitude.

In a windowed low pass filter design the window function used determines the width of the transition band, pass band ripple and stop band attenuation. The width of the main lobe of the window frequency response determines the width of the transition band. The narrower the main lobe the narrower the transition band. The amplitude of the side lobes determine passband ripple and limits the achievable stop band attenuation. One can thus have a narrow transition band or a high stop band attenuation, but not both. Typical transition bandwidths are between $2 / \mathrm{Nt}_{s}$ and $6 / \mathrm{Nt}_{s}$; where $\mathrm{N}$ is the number of taps in the filter and $\mathrm{t}_{s}$ is the sampling period. These correspond to stop band attenuations between $25 \mathrm{~dB}$ and $100 \mathrm{~dB}$.

The characteristics of windowed filter designs, described above, suggest desirable properties for window functions. For a specific filter design we might start with the number of filter taps and the width of the transition band as initial parameters. The transition band width directly determines the width of the main lobe in the window's frequency response. We now wish to minimise pass band ripple and maximise stop band attenuation. These are determined by the magnitude of the side lobes. An optimum window would therefore minimise the effect of side lobes for a given main lobe width. None of the windows named

1. Mathematically this is due to a failure of uniform convergence in the Fourier series. 
above does this although the Kaiser window provides an approximation in some circumstances.

In addition to their use in windowed filter designs, windows can also be used as a filter impulse response in their own right. Used in this way windows provide a low pass filter response. The transition band effectively stretches from DC to the cutoff frequency. Low pass filters produced in this way do not have an, even approximately, flat pass band. Their frequency response falls monotonically from DC to the cutoff frequency. Although this may seem an unusual characteristic, this type of filter can be useful in some applications. For example when filtering prior to subsampling a signal it may be more important to eliminate aliasing than to preserve a flat passband. An optimum window function used as a filter in this way gives the maximum rejection of alias components. This application of window functions would be useful in parts of the signal processing used for motion estimation.

\subsubsection{Signal segmentation.}

Window functions provide a convenient way of segmenting a signal prior to processing. This is useful in circumstances where it is inconvenient or undesirable to process the entire signal. For example, segmentation is necessary when performing motion estimation using either phase or motion correlation techniques (chapter 7). If the whole image were processed only 2 or 3 different movements could be distinguished. If, however, the image is segmented into blocks, many more different movements can be found. Segmentation provides a way of combining traditional time domain and frequency domain concepts into a single framework. This is described elsewhere (Allen '77, Allen \& Rabiner '77, Nawab \& Quatieri '88), this section will give only a very brief description relevant to the appreciation of the function of windows for this type of application.

A signal can be segmented by multiplying it by a set of (possibly overlapping) windows. This gives a set of short duration signals rather than one long one. An immediate question is what shape window function should we use and how much (if at all) should the windows overlap. In addition it would be nice if the (overlapping) windows added to give a constant value. In this case the original signal would be equal to the sum of its segments. It would then seem reasonable to use this alternative description if it happened to be convenient.

The desired shape and overlap of the window function, used to segment a signal, can be determined by considering Fourier transforms. The Fourier transforms of the windowed segmented signal can be regarded as the 'time varying spectrum' of the signal. Consider a set of windows $\mathrm{N}$ samples long, overlapping by a factor of $\mathrm{O}$ (ie each sample is included in $\mathrm{O}$ consecutive windows). It turns out that if the spectrum of the window has a bandwidth no greater than $f_{N} O / N\left(f_{N}\right.$ is the Nyquist frequency ie. 1/(twice sampling period)) then the segmentation works out very neatly. In this case the sum of the overlapping windows is 
guaranteed to take a constant value. Furthermore, the samples of the 'time varying spectrum' are properly sampled in time (in the Nyquist sense). Unfortunately, it is impossible to jointly limit both the duration and bandwidth of a window function (uncertainty principle) as would ideally be required by signal segmentation. However, good (finite duration) window functions can have an approximately limited bandwidth.

For segmenting images a 2 dimensional window function is required. This can conveniently be obtained as a (variables separable) product of 2 one dimensional windows. If the horizontal and vertical overlap factors are both $O$ this implies an increase in processing power by a factor of $\mathrm{O}^{2}$ (compared to using no overlap). Therefore the required processing power increases rapidly with overlap factor. Hence it is important to use the best possible window function to minimise the required overlap and therefore processing power.

\subsection{Optimum Window Functions.}

This section describes a criterion for the design of optimum window functions. In (simple) 1 dimensional cases this criterion leads to functions known as 'prolate spheroidal wave functions' (Mathews et al '85, Slepian '61, Landau \& Pollak '61, Landau \& Pollak '62, Slepian '64, Slepian '78). In multidimensional cases (see section 11.5) we obtain similar, unnamed, functions. To the best of the author's knowledge these functions do not appear to have been used previously.

Window functions attempt to approximate a band limited frequency response from a finite duration impulse response. From a strict, mathematical, point of view this approximation is impossible. As discussed above, the frequency responses of practical windows generally have a high amplitude main lobe and low amplitude side lobes. If the amplitude of the side lobes is sufficiently low (eg. below the signal noise floor) then the window's frequency response may be regarded as essentially band limited. As the width of the main lobe is reduced the transition bandwidth for filter design, or the degree of overlap in signal segmentation, are reduced. At the same time the amplitude of the side lobes rises, increasing errors in the processed signals.

An optimum window will minimise the effect of side lobes for a given width of main lobe in the frequency response. A suitable criterion for this optimisation is to minimise the out of band energy in the window's frequency response while maintaining a constant overall energy. This can be described mathematically (for a 1 dimensional window) as maximising a parameter $\lambda$, defined in equation 1 , by varying the window function. 


$$
\lambda=\frac{\int_{-f_{c}}^{+f_{c}} S(f) d f}{\int_{-f_{n}}^{+f_{n}} S(f) d f}
$$

where $S(f)$ is the power spectrum of the window function, $f_{c}$ is the desired cutoff frequency for $S(f)$ and $f_{N}$ is the Nyquist frequency (assuming a sampled system).

In the continuous case the solution to equation 1 yields an integral eigenfunction equation defining the prolate spheroidal wave functions. In the discrete case we obtain the matrix eigenvector equation 2 (see appendix 2).

$$
E . a=\lambda a
$$

Where $a$ is a vector containing the values of the (discrete time) window function, and $\mathrm{E}$ is a matrix of coefficients. Fortunately, it is relatively easy to solve eigenvector problems using library programs. The optimum window function can thus be found as the eigenvector corresponding to the maximum eigenvalue in equation 2 . The maximum eigenvalue gives the proportion of energy (of the window) which lies in the specified bandwidth. The solutions to equation 2 are generally known as the discrete prolate spheroidal sequences.

The solution to equation 2 (derived from equation 1) provides a way of calculating optimum (1 dimensional) window functions. Although these optimum window functions have been known since the early 'seventies they have not been extensively used. The reason for this is probably the existence of the convenient, and easily calculated, family of (sub optimal) Kaiser windows. In one dimension Kaiser windows perform only marginally worse than optimum windows in most situations. Furthermore, Kaiser windows are easier to calculate than optimum windows. While an optimum window might typically take less than a second to calculate on a modern computer, it might have taken many minutes in 1970.

This section has discussed known theory with respect to one dimensional signals. From this perspective the theory and design of optimum windows is interesting, but not of vital importance. When the theory is extended to multidimensional processing it becomes much more necessary and useful.

\subsection{Multidimensional Window functions.}

This section describes the design of multidimensional window functions. It is shown that previously window designs were rather heuristic and of restricted flexibility. This section discusses how the optimum criterion described above can be extended and used for multidimensional window design. The technique is described with reference to 2 dimensional 
windows, which suffices to illustrate all the salient points of window design. The technique can, however, easily be extended to as many dimensions as required.

In the past, 2 dimensional window functions have been derived from 1 dimensional ones (Dudgeon \& Meresreau '84). This appears to have been largely a matter of expediency. Three methods used to generate a 2 dimensional window from a (continuous) 1 dimensional one are given

in equations 3,4 \& 5 below.

$$
\begin{gathered}
w_{P}(x, y)=w(x) \cdot w(y) \\
w_{C}(x, y)=w\left(\sqrt{x^{2}+y^{2}}\right) \\
w_{S}(x, y)=w(\max (x, y))=\lim _{p \rightarrow \infty} \sqrt[p]{x^{p}+y^{p}}
\end{gathered}
$$

Equation 3 defines a (separable) product window. This would be the window of choice for segmenting a signal in 2 dimensions, because 2 dimensional segmentation can be considered as a cascade of two 1 dimensional segmentations. Equations 4 and 5 define circular and square windows. Slightly modified definitions would define elliptical and rectangular windows. Although equations 4 and 5 yield passable window designs the definitions appear to be completely ad hoc and are in no sense optimal. None of these windows would be ideal for filter design.

The definition of a 1 dimensional optimum window (equation 1 ) can be modified, to equation 6, for multiple dimensions. This modification introduces an additional complication.

$$
\lambda=\frac{\iint_{R} S(m, n) d m d n}{\int_{-m_{N}}^{+m_{N}} \int_{-n_{N}}^{+n_{N}} S(m, n) d m d n}
$$

The difference between equations 1 and 6 is that the passband of the numerator in equation 6 (denoted $R$ ) now has a definite shape as opposed to merely a length. For example, the baseband (ie. the shape of the central spectral lobe) of the window could be either circular or square. Different shape passbands will result in different window functions.

A further complication with multidimensional window functions is that they can have different shaped regions of support. The region of support is the area over which the window function is non zero. For example the windows defined in equations 4 and 5 have circular and square regions of support respectively. These fixed shape regions of support can constitute a serious disadvantage of 'conventional' two dimensional windows. 
In filter design, the optimum shape region of support for a window function depends on the filter being designed. The wrong shape region of support can result in an FIR filter in which there are many small coefficients which make little contribution to the filter output. This can lead to an inefficient use of hardware which is particularly significant in video systems where the cost of each filter tap can be significant (particularly in motion compensated filters). It is, therefore, important to be able to define the region of support of a window function. This can be done by restricting window functions to those which are nonzero only on a defined region of support. From this restricted set of possible window functions we chose the one which gives the maximum energy concentration $(\lambda)$ in a defined passband in equation 6 . This is achieved by forming an eigenvector equation analogous to equation 2; details are given in appendix 2.

Optimum multidimensional window functions can be designed which have arbitrarily shaped passbands and regions of support. This flexibility, impossible with previous techniques, allows the digital signal processing system designer the ability to squeeze maximum performance out of limited hardware.

\subsection{Filter Design Examples.}

This section describes a selection of 1 and 2 dimensional filter designs using window functions. These designs are intended as a comparison of the use of different window functions and the trade offs involved. They do not show all the possible benefits and applications of window functions.

Figures 11.1, $11.2 \& 11.3$ show the frequency responses for a selection of (1 dimensional) half band low pass filters and of the window functions used to produce them. They correspond to symmetric FIR filters with a total of 33 filter taps. Figures 11.1, 11.2 \& 11.3 have window function bandwidths of $\pm 1, \pm 2 \& \pm 3$ (frequency) samples respectively (ie. $2,4 \& 61 / 33^{\text {rds }}$ of the Nyquist limit). Hence these figures correspond to progressively greater filter transition bandwidths and correspondingly less passband ripple and greater stop band attenuation. Particular care was taken to ensure that the transition bandwidths were the same within each figure so that the window functions could be meaningfully compared. Details of the window functions used are given in appendix 1. Table 11.1 gives the relative performance for each of the filter designs. The shape parameters used for the Kaiser windows are given in the table. In each case the optimum window gives the best performance, as we would expect. Perhaps of particular significance is the very large improvement of minimum stop band attenuation, from $20 \mathrm{~dB}$ to $35 \mathrm{~dB}$ with the smallest transition bandwidth. This corresponds to the sort of performance improvement which might be achieved when the number of filter taps is very limited. 
Table 11.1: Performance of 1D Windowed Filter Designs.

\begin{tabular}{|l|c|c|c|}
\hline \multicolumn{1}{|c|}{ Window Function } & $\begin{array}{c}\text { Transition } \\
\text { Bandwidth } \\
\left.\text { (samples ie. } \mathrm{f}_{\mathrm{N}} / 33\right)\end{array}$ & $\begin{array}{c}\text { Minimum Stop } \\
\text { Band Attenuation } \\
(\mathrm{dBs})\end{array}$ & $\begin{array}{c}\text { Maximum Pass } \\
\text { Band Ripple } \\
(\mathrm{dBs})\end{array}$ \\
\hline \hline Rectangular & \pm 1.0 & 20.9 & 0.912 \\
\hline Kaiser $(\alpha=0.00)$ & \pm 1.0 & 20.9 & 0.912 \\
\hline Optimum & \pm 1.0 & 35.6 & 0.203 \\
\hline Hamming & \pm 2.0 & 56.9 & 0.028 \\
\hline Kaiser $(\alpha=5.44)$ & \pm 2.0 & 61.5 & 0.014 \\
\hline Optimum & \pm 2.0 & 64.5 & 0.008 \\
\hline Blackman & \pm 3.0 & 75.2 & 0.001 \\
\hline Kaiser $(\alpha=8.89)$ & \pm 3.0 & 88.6 & 0.001 \\
\hline Optimum & \pm 3.0 & 95.2 & 0.0005 \\
\hline
\end{tabular}

Figure 11.4 shows a comparison of three, 2 dimensional, circularly symmetric, windowed filter designs. The filter designs are for a 81 point FIR filter, which is a typical size for a video filter. In order to achieve a useful separation between pass and stop bands it is rather impractical to have a transition bandwidth much greater than 1 (frequency) sample (ie. $f_{N} / 9$ ). This restriction precludes the use of circularly symmetric version (equation 4 ) of conventional windows, as they all have greater transition bandwidths. Three windows were used; rectangular, optimum $9 \times 9$ and optimum 81 point. The rectangular window has a square region of support and a roughly square passband. The optimum $9 \times 9$ window also has a square region of support but has a circular passband. The third window, optimum 81 point, has a roughly circular region of support (shown in figure 11.5) and a circular passband. Care was taken to ensure that the transition bandwidths of each of the filter designs were the same so that they could meaningfully be compared. Table 11.2 shows the relative performance of these filter designs. This shows the improvement to be gained using an optimum $(9 \times 9)$ window function (with an appropriate shape passband). The further improvement which can be 
achieved using an optimum (81 point) window with an appropriate region of support is also shown.

Table 11.2: Performance of 2D Windowed Filter Designs.

\begin{tabular}{||l|c|c|c|}
\hline \multicolumn{1}{|c|}{ Window Function } & $\begin{array}{c}\text { Transition } \\
\text { Bandwidth } \\
\left.\text { (samples ie. } \mathrm{f}_{\mathrm{N}} / 9\right)\end{array}$ & $\begin{array}{c}\text { Minimum Stop } \\
\text { Band Attenuation } \\
(\mathrm{dBs})\end{array}$ & $\begin{array}{c}\text { Maximum Pass } \\
\text { Band Ripple } \\
(\mathrm{dBs})\end{array}$ \\
\hline \hline Rectangular & \pm 1.0 & 17.2 & 3.21 \\
\hline Optimum 9x9 & \pm 1.0 & 30.1 & 0.91 \\
\hline Optimum 81 point & \pm 1.0 & 34.9 & 0.81 \\
\hline
\end{tabular}

The improvements in the 2 dimensional filter designs above, through using optimum windows, are not possible using conventional window functions. This emphasises the utility of using optimum windows for small aperture FIR filters, which are typical in video processing. There are, of course, other methods for the design of FIR filters. However these are unlikely to give significantly better results than the use of optimum windows when designing small FIR filters. Given the simplicity of filter design using windows it seems sensible to consider this design technique for these applications.

\subsection{Conclusions.}

This chapter has described the background, theory and application of window functions. Window functions, in general, provide a quick and simple method of filter design and are useful for segmenting signals into small regions.

A criterion is suggested above for designing 'optimum' window functions. These 'windows' can be used in a variety of applications with advantages over conventional windows. A number of applications and their advantages are detailed below.

1.) Optimum window functions give much improved performance with small (and especially multidimensional) FIR filter designs (eg improved ripple and stopband attenuation). This is of particular significance as these are the sort of filters which are typically used for digital video processing. 
2.) Optimum window functions can be designed with arbitrary regions of support. Conventional window functions typically have a circular or square region of support. All filter taps in the region of support must be implemented in hardware even if the coefficients are very small. Taps with small coefficients can be removed from the region of support and a new filter calculated using a smaller optimum window. The hardware required to implement a filter can thus be reduced by using an optimum rather than a conventional window, with negligible reduction in performance.

3.) Optimum window functions can perform efficient antialias filtering.

4.) Signal segmentation is more efficient using optimum rather than conventional windows. The computing power required for processing segmented signals can be reduced by at least a factor of 2 . Alternatively the computational noise floor can be reduced by tens of $\mathrm{dBs}$.

\subsection{References.}

1. Papoulis, A. 1977. Signal Analysis. McGraw-Hill. ISBN 0-07-Y66468-4.

2. Rabnier, L.R. \& Gold, B. 1975. Theory and application of digital signal processing. Prentice-Hall. ISBN 0-13-914101-4.

3. Oppenheim, A.V. \& Schafer, R.W. 1989. Discrete time signal processing. PrenticeHall. ISBN 0-13-216771-9.

4. Tretter, S.A. 1976. Introduction to discrete time signal processing. John Wiley \& Sons. ISBN 0-471-88760-9.

5. Prabhu, K.M.M. 1987. Generalized families of windows for use in signal processing. Journal of the Institution of Electronic and Radio Engineers, Vol. 57, No. 4, pages 174-177, July/August 1987.

6. Slepian, D. 1976. On bandwidth. Proceedings of the IEE, Volume 64, Number 3, March 1976.

7. Kuo, F.F. \& Kaiser, J.F. (Editors) 1966. System analysis by digital computer. John Wiley 1966.

8. Kaiser, J.F. 1974. Nonrecursive digital filter design using the $\mathrm{I}_{0}$-Sinh window function. Proceedings 1974 IEEE International Symposium on Circuits and Systems, April 2225, 1974, pages 20-23.

9. Parks, T.W. \& Burrus, C.S. 1987. Digital Filter Design. John Wiley \& Sons, Inc. ISBN 0-471-82896-3 
10. Prabhu, K.M.M. \& Bhoopathy Bagan, K. 1988. FIR digital filter design with variable parameter window families. Journal of the Institution of Electronic and Radio Engineers, Vol. 58, No. 6, pages 295-298, September-December 1988.

11. Allen, J.B. 1977. Short term spectral analysis, synthesis, and modification by discrete Fourier transform. IEEE Transactions on Acoustics, Speech, and Signal Processing. Volume ASSP-25, Number 3, June 1977.

12. Allen, J.B. \& Rabiner, L.R. 1977. A unified approach to short-time Fourier analysis and synthesis. Proceedings of the IEEE, Volume 65, Number 11, November 1977.

13. Nawab, S.H. \& Quatieri, T.F. 1988. Short-time Fourier Transform. Chapter 6, Advanced topics in signal processing, Lim, J.S. \& Oppenheim, A.V. (Editors), Prentice Hall, 1988, ISBN 0-13-013129-6 025, pp 123-198.

14. Mathews, J.D., Breakall, J.K. \& Karawas, G.K. 1985. The discrete prolate spheroidal filter as a digital signal processing tool. IEEE Transactions on Acoustics, Speech and Signal Processing. Vol. ASSP-33. No. 6. Pages 1471 to 1478. December 1985.

15. Slepian, D. \& Pollak, H.O. 1961. Prolate spheroidal wave functions, Fourier analysis and uncertainty - I. The Bell system technical journal. Pages 43 to 63. January 1961.

16. Landau, H.L. \& Pollak, H.O. 1961. Prolate spheroidal wave functions, Fourier analysis and uncertainty - II. The Bell system technical journal. Pages 65 to 84. January 1961.

17. Landau, H.L. \& Pollak, H.O. 1962. Prolate spheroidal wave functions, Fourier analysis and uncertainty - III: The dimension of the space of essentially time- and band-limited signals. The Bell system technical journal. Pages 1295 to 1336. July 1962.

18. Slepian, D. 1964. Prolate spheroidal wave functions, Fourier analysis and uncertainty IV: Extensions to Many Dimensions; Generalised prolate spheroidal functions. The Bell system technical journal. Pages 3009 to 3057. November 1964.

19. Slepian, D. 1978. Prolate spheroidal wave functions, Fourier analysis and uncertainty V: The discrete case. The Bell system technical journal. Pages 1371 to 1430 . MayJune 1978.

20. Dudgeon, D.E. \& Mersereau, R.M. 1984. Multidimensional Digital Signal Processing. Prentice Hall, Inc. ISBN 0-13-604959-1.

11.9. Appendix 1: Some sub-optimal window functions.

Four conventional window functions are used in the filter design examples of section 11.6 (in addition to the optimum window functions). These were carefully chosen to provide spectral main lobe widths for comparison with optimum windows. This appendix is intended to describe the characteristics of these windows. These window functions are described for lengths of an odd number of samples because this is how they are commonly used. They can, 
however, also be defined for an even number of samples. For more details see Rabiner \& Gold '75 p91, Oppenheim \& Schafer '89 p447 or Tretter '76 p224.

A quick explanation of notation etcetera is probably in order before presenting too many equations. The window functions described in this appendix are one dimensional and are sampled in time at multiples of $t_{s,}$ the sampling period. Time domain functions are denoted by small letters and generally have arguments of $\mathrm{nt}_{\mathbf{s}}$. Fourier transforms of window functions are continuous frequency domain functions and are denoted by capital letters. The frequency responses are given with arguments of $\mathrm{kF}_{\mathrm{s}}$. Since these are continuous functions $\mathrm{k}$ may be regarded as a continuous variable. Taking the DFT of the (sampled) window will give the frequency response sampled with integer $k$. The sampling period, sampling frequency $\left(F_{s}\right)$ and Nyquist frequency $\left(f_{N}\right)$ are related by the following two equations. $N$ is the length of the window function in sampling periods.

$$
\begin{gathered}
f_{N}=\frac{1}{2 t_{s}} \\
f_{s}=\frac{2 f_{N}}{N}=\frac{1}{N t_{s}}
\end{gathered}
$$

\subsubsection{Rectangular Window.}

The time and frequency domain characteristics are given by the following pair of equations ${ }^{2}$.

$$
w_{R}\left(n t_{s}\right)=\left\{\begin{array}{cc}
1.0 & -\left(\frac{N-1}{2}\right) \leq n \leq\left(\frac{N-1}{2}\right) \\
0.0 & \text { elsewhere }
\end{array}\right.
$$

Note that the frequency response is only equal to the familiar sinc function in the limit as $\mathrm{N}$ tends to infinity. This is because of the sampled nature of the window. The result below

${ }^{2}$ It would be more correct to define the sampled window functions as a weighted sum of $\delta$ functions. For example equation 9 would more properly be written as;

$$
w_{R}(t)=\sum_{k=-(N-1) / 2}^{k=+(N-1) / 2} \delta\left(t-k t_{s}\right)
$$

This has not been done to maintain consistency with the references and also to emphasise the relationship to the continuous time domain. 


$$
W_{R}\left(k f_{s}\right)=\frac{\sin (\pi k)}{\sin \left(\frac{\pi k}{N}\right)}
$$

includes aliasing caused by overlapping periodically spaced sinc functions.

The spectrum of a rectangular window has its first zero at a frequency of $F_{s}$. This seems to be a natural definition of the width of the main spectral lobe and is the one used in the rest of this appendix. The rectangular window has the narrowest main lobe of conventional windows. This gives rapid cut filters, an overlap factor of 2 when segmenting signals (see section 11.3.2), and high resolution for spectral analysis. The price that is paid for these good features is high pass band ripple and low stop band attenuation in filters, a very high noise floor when processing segmented signals or high leakage in spectral analysis. The very high peak side lobe amplitude $(-13 \mathrm{~dB}$ relative to $\mathrm{DC})$ generally makes the rectangular window a poor choice for most signal processing purposes.

11.9.2. Hamming Window.

$$
\begin{gathered}
w_{H}\left(n t_{s}\right)= \begin{cases}0.54-0.46 \cos \left(\frac{2 \pi n}{N}\right) & -\left(\frac{N-1}{2}\right) \leq n \leq\left(\frac{N-1}{2}\right) \\
0.0 & \text { elsewhere }\end{cases} \\
W_{H}\left(k f_{s}\right)=0.54\left[\frac{\sin (\pi k)}{\sin \left(\frac{\pi k}{N}\right)}\right]+0.23\left[\frac{\sin (\pi(k-1))}{\sin \left(\frac{\pi(k-1)}{N}\right)}\right]+0.23\left[\frac{\sin (\pi(k+1))}{\sin \left(\frac{\pi(k+1)}{N}\right)}\right]
\end{gathered}
$$

The Hamming window, defined by the above two equations, has a main lobe twice the width of the rectangular window and a corresponding reduction in the amplitude of the side lobes. The coefficients in its definition (ie $0.54 \& 0.46$ ) are chosen so that its frequency response tends to zero at $\mathrm{k}=2.5$ for large values of $\mathrm{N}$. This is an ad hoc way of reducing the amplitude of the side lobes.

Despite its heuristic design the Hamming window is easy to use and has reasonable properties. It has frequently been used for DSP applications.

\subsubsection{Blackman Window.}

The Blackman window defined above is similar in concept to the Hamming window. It has a main spectral lobe three times as wide as the rectangular window and lower side lobe amplitudes than the Hamming window. It is used where better performance than the Hamming 
window is required. This improved performance comes at the price of increased hardware requirements.

$$
\begin{gathered}
w_{B}\left(n t_{s}\right)=\left\{\begin{array}{lc}
0.42-0.50 \cos \left(\frac{2 \pi n}{N}\right)+0.08 \cos \left(\frac{4 \pi n}{N}\right) & -\left(\frac{N-1}{2}\right) \leq n \leq\left(\frac{N-1}{2}\right) \\
0.0 & \text { elsewhere }
\end{array}\right. \\
W_{B}\left(k f_{s}\right)=0.42\left[\frac{\sin (\pi k)}{\left.\sin \left(\frac{\pi k}{N}\right)\right]+0.25\left[\frac{\sin (\pi(k-1))}{\sin \left(\frac{\pi(k-1)}{N}\right)}+\frac{\sin (\pi(k+1))}{\sin \left(\frac{\pi(k+1)}{N}\right)}\right]}\right. \\
+0.04\left[\frac{\sin (\pi(k-2))}{\sin \left(\frac{\pi(k-2)}{N}\right)}+\frac{\sin (\pi(k+2))}{\sin \left(\frac{\pi(k+2)}{N}\right)}\right]
\end{gathered}
$$

\subsubsection{Kaiser Window.}

The definition of the Kaiser window includes a variable parameter $\alpha$. The Kaiser window is thus actually a family of window functions. The parameter $\alpha$ allows the width of the main spectral lobe to be traded off against the amplitude of the side lobe. This gives considerable flexibility in the design of filters etc using this type of window. Generally a Kaiser window can be found which gives better performance than the windows above. For the narrowest width main lobe the Kaiser window degenerates to a rectangular window.

The Kaiser window is derived from an approximation to (continuous) prolate spheroidal wave functions. Since the prolate spheroidal wavefunctions may be regarded as optimum continuous one dimensional window functions the good performance of the Kaiser window is unsurprising. Limitations in the performance of the Kaiser windows arise in two ways. Firstly it is the discrete and not continuous prolate spheroidal wave functions which are ideal for sampled systems. The differences become significant for small sampled windows and in these cases the performance of the Kaiser window is sub-optimal. Secondly the Kaiser window is only an approximation to the prolate spheroidal wave functions and this approximation also makes the window sub-optimal. Using this approximation, however, makes the Kaiser window quick to calculate and easy to use.

$$
w_{K}\left(n t_{s}\right)=\left\{\begin{array}{lc}
\frac{I_{0}\left(\alpha \sqrt{1-(2 n / N)^{2}}\right)}{I_{0}(\alpha)} & -\left(\frac{N-1}{2}\right) \leq n \leq\left(\frac{N-1}{2}\right) \\
0.0 & \text { elsewhere }
\end{array}\right.
$$

Where $\mathrm{I}_{0}$ is the modified Bessel function of the first kind and zeroth order. This function can be easily calculated from its power series expansion (below) which converges very quickly. 


$$
\begin{gathered}
W_{K}\left(k f_{s}\right)=\frac{N \sin \sqrt{(\pi k)^{2}-\alpha^{2}}}{I_{0}(\alpha) \sqrt{(\pi k)^{2}-\alpha^{2}}} \\
I_{0}(x)=\sum_{l=0}^{\infty}\left[\frac{x^{l}}{2^{l} n}\right]^{2}
\end{gathered}
$$

From equation 16 we can see that spectrum has its first zero at $f=k_{0} F_{s}$ such that;

$$
\sqrt{\left(\pi k_{0}\right)^{2}-\alpha^{2}}=\pi
$$

With a little rearrangement we get;

$$
\alpha=\pi \sqrt{k_{0}^{2}-1}
$$

The rectangular, Hamming and Blackman windows above have values of $\mathrm{k}_{0}$ of 1,2 \& 3 respectively. These values of $k_{0}$, when substituted into equation 19 , give values of $\alpha$ for Kaiser windows corresponding to the 3 windows above. The values of $\alpha(0.00,5.44,8.89)$, obtained in this way were used in the filter design examples of section 11.6.

\subsection{Appendix 2: Calculating optimum window functions.}

This appendix covers the details of the theory of optimum window functions. The first part of the appendix derives the theory of one dimensional window functions (the discrete prolate spheroidal wavefunctions). This theory is also presented in Mathews et al ' 85 and elsewhere. The theory is then extended to multiple dimensions in the second half of the appendix. The extended theory is not available elsewhere to the best of the author's knowledge. The notation etcetera used in this appendix is generally the same as that used in appendix 1 .

The calculation of optimum window functions starts with equation 1 (section 11.4). The parameter $\lambda$ is a measure of the efficiency of the window which we wish to maximise. Physically it represents the proportion of the total energy which lies within the main central lobe of the windows frequency response.

$$
\lambda=\frac{\int_{-f_{c}}^{+f_{c}} S(f) d f}{\int_{-f_{N}}^{+f_{N}} S(f) d f}
$$

Where $S(f)$ is the power spectral density of the window function, $f_{c}=k_{0} F_{s}$ is the upper limit of the windows main spectral lobe and $f_{N}$ is the Nyquist limit. The windows power spectral density function is defined by; 


$$
S(f)=W(f) \cdot W^{*}(f)=|W(f)|^{2}
$$

and the Fourier transform of the window function is defined by;

$$
\begin{gathered}
W\left(f=k f_{s}\right)=\sum_{n=-(N-1) / 2}^{n=+(N-1) / 2} w\left(n t_{s}\right) \exp (-j 2 \pi k n / N) \\
S\left(f=k f_{s}\right)=\sum_{n=-(N-1) / 2} \sum_{m=-(N-1) / 2}^{n=+(N-1) / 2} w\left(n t_{s}\right) w\left(m t_{s}\right) \exp [-j 2 \pi k(n-m) / N]
\end{gathered}
$$

To determine the optimum window function substitute equations $21 \& 23$ into equation 20 and integrate. This gives the following equation;

$$
\lambda=\frac{\sum_{n, m=-N}^{n, m=+N} w_{n} w_{m} \frac{\sin (n-m) \pi \epsilon}{(n-m) \pi}}{\sum_{n=-N}^{n=+N} w_{n}^{2}}
$$

where $w\left(n t_{s}\right) \& w\left(m t_{s}\right)$ have been denoted $w_{n} \& w_{m}$ for brevity and;

$$
\varepsilon=\frac{f_{c}}{f_{N}}=\frac{k_{0} f_{s}}{f_{N}}=\frac{k_{0}}{N}
$$

Thus $\varepsilon$ is width of the window's main spectral lobe as a fraction of the Nyquist frequency. Equation 24 can be rewritten more clearly in matrix form as;

$$
w^{T} E w-\lambda w^{T} w=0
$$

where vector $\mathbf{w}$ is defined as;

$$
w^{T}=\left[w_{1}, w_{2}, \ldots w_{N}\right]
$$

and the element $\mathrm{m}, \mathrm{n}$ of matrix $\mathbf{E}$ is given by;

$$
E_{m n}=\frac{\epsilon \sin [(n-m) \pi \epsilon]}{(n-m) \pi \varepsilon}=\epsilon \operatorname{sinc}[(n-m) \pi \varepsilon]
$$

From equation 26 we can see that the samples of the optimum window function satisfy the following eigenvector equation. 


$$
E w=\lambda w
$$

Thus to find the optimum window function we must solve equation 29 to find the eigenvector with the largest eigenvalue. Fortunately this can be done using readily available mathematical software libraries. Solution of equation 29 usually gives us not only the eigenvector (which is the optimum window function) but also the magnitude of the eigenvalue. This is useful since the eigenvalue is the fraction of the window's energy within its main spectral lobe. A good window will have a value of $\lambda$ greater than 0.99 ; the corresponding figure for a Hamming window, for comparison, is 0.99963 .

The section below extends the theory above to multiple dimensions. As might be expected this introduces some additional complications. As noted in the main text equation 20 becomes multidimensional to give equation 30 . This section considers the example of a three dimensional window function. A slight change of notation is necessary since $m$ and $n$ now represent spatial frequencies; variables $p$ and $q$ are used as integer indexes and bold characters represent vector quantities.

$$
\lambda=\frac{\int_{R} S(f) d f}{\int_{N} S(f) d f}
$$

where $f=(m, n, f)$ and $\mathbf{R}$ and $\mathbf{N}$ are regions of the spectrum within the passband of the window and the Nyquist limits of the spectrum respectively. In this multidimensional case the (complex) spectrum of the window is given by;

$$
W(m, n, f)=\sum_{p} w_{p} \exp \left[-j 2 \pi\left(m x_{p}+n y_{p}+f t_{p}\right)\right]
$$

where $\left(\mathrm{x}_{\mathrm{p}}, \mathrm{y}_{\mathrm{p}}, \mathrm{t}_{\mathrm{p}}\right)$ is the location of the $\mathrm{p}^{\mathrm{th}}$ coefficient, $\mathrm{w}_{\mathrm{p}}$, in the window. The power spectral density is given by;

$$
\begin{aligned}
S(m, n, f) & =W(m, n, f) W^{*}(m, n, f) \\
& =\sum_{p} \sum_{q} w_{p} w_{q} \exp \left\{-j 2 \pi\left[m\left(x_{p}-x_{q}\right)+n\left(y_{p}-y_{q}\right)+f\left(t_{p}-t_{q}\right)\right]\right\}
\end{aligned}
$$

As in the one dimensional case an eigenvector equation is derived by substituting equation 32 into equation 30 and integrating to give;

where again; 


$$
\begin{gathered}
E w=\lambda w \\
w^{T}=\left[w_{1}, w_{2}, \ldots w_{N}\right]
\end{gathered}
$$

The difference in this, multidimensional, case is that the matrix coefficients depend on the shape of the window's passband region. The matrix coefficients are given by ${ }^{3}$;

$$
E_{q p}=\frac{\int_{R} S(m, n, f) d m d n d f}{8 m_{N} n_{N} f_{N}}
$$

where subscript $\mathrm{N}$ indicates a Nyquist frequency.

Matrix coefficients have been evaluated for three different (3D) shapes of the window functions passbands. The three different passband shapes that have been evaluated are cuboid, cylindroid and ellipsoid. These correspond to evaluating the integral in equation 35 using cartesian, cylindrical polar and spherical polar coordinates respectively. Three parameters are required to define the shape of the passband in each of these three cases. These parameters, $\varepsilon, \zeta$ and $\eta$, correspond to the point where the windows passband intersects the $n, m$ and $f$ axes respectively. The parameters are fractions of the corresponding Nyquist limits. Evaluating the matrix coefficients for these three passband shapes gives the following results.

Cuboid:

$$
\begin{gathered}
E_{Q P}=\epsilon \operatorname{sinc}\left(k_{x}\right) \zeta \operatorname{sinc}\left(k_{y}\right) \eta \operatorname{sinc}\left(k_{t}\right) \\
k_{x}=\epsilon \pi\left(x_{p}-x_{q}\right) / x_{s} \\
k_{y}=\zeta \pi\left(y_{p}-y_{q}\right) / y_{s} \\
k_{t}=\eta \pi\left(t_{p}-t_{q}\right) / t_{s}
\end{gathered}
$$

where $x_{s}, y_{s}$ and $t_{s}$ are the horizontal, vertical and temporal sampling intervals.

${ }^{3}$ The denominator of equation 35 comes from evaluating the denominator of equation 30 . Two assumptions have been made in evaluating that integral. Firstly an orthogonal sampling structure has been assumed. This is not unduly restrictive although different sampling structures might give a different constant. The second assumption is that the window function is sampled on a periodic lattice. If this were not the case an optimum window function could still be calculated by solving a generalised eigenvector equation of the form;

$$
\boldsymbol{A} \boldsymbol{w}=\lambda \boldsymbol{B} w
$$

where both $\mathbf{A}$ and $\mathbf{B}$ are matrices. 
Cylindroid:

$$
\begin{gathered}
E_{q p}=\frac{\epsilon \zeta \pi}{4}\left[\frac{2 \mathrm{~J}_{1}\left(k_{x y}\right)}{k_{x y}}\right] \eta \operatorname{sinc}\left(k_{t}\right) \\
k_{x y}=\sqrt{k_{x}^{2}+k_{y}^{2}}
\end{gathered}
$$

Ellipsoid:

$$
\begin{gathered}
E_{q p}=\frac{e \zeta \eta}{8} \frac{4 \pi}{3}\left[\frac{3\left(\sin \left(k_{x y t}\right)-k_{x y t} \cos \left(k_{x y t}\right)\right)}{k_{x y t}^{3}}\right] \\
k_{x y t}=\sqrt{k_{x}^{2}+k_{y}^{2}+k_{t}^{2}}
\end{gathered}
$$



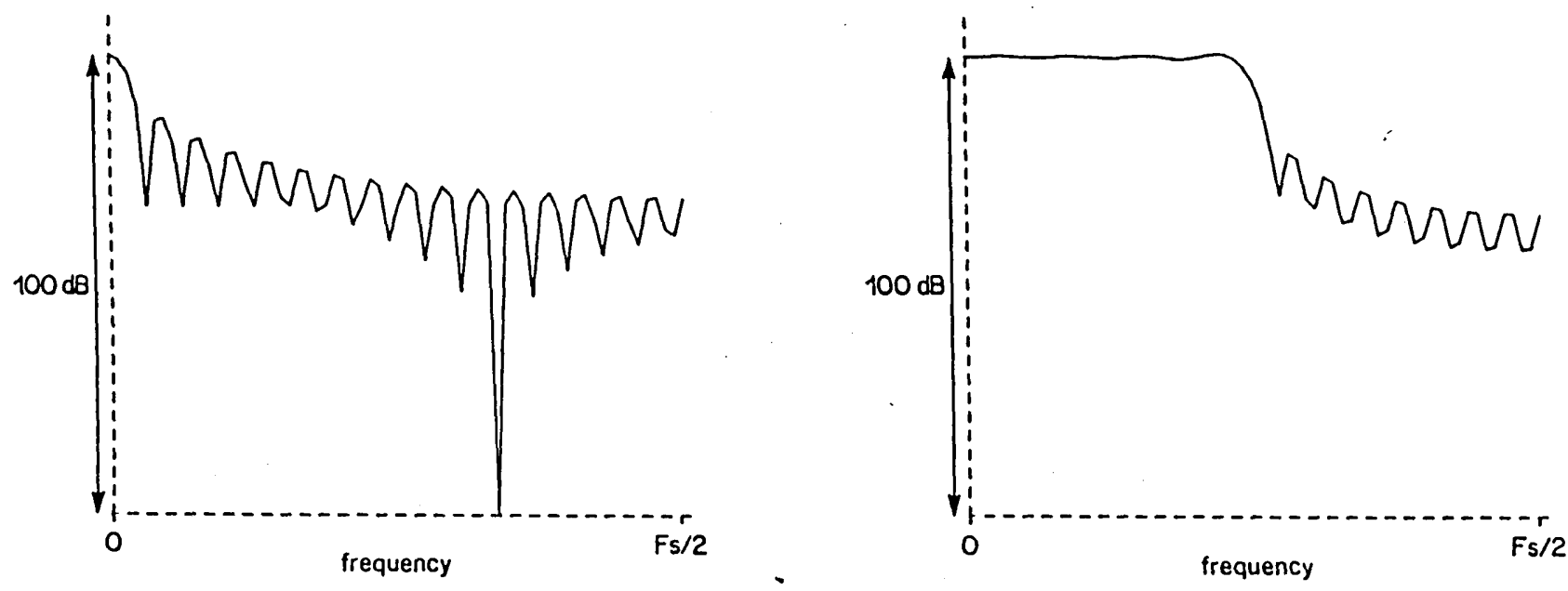

rectangular window
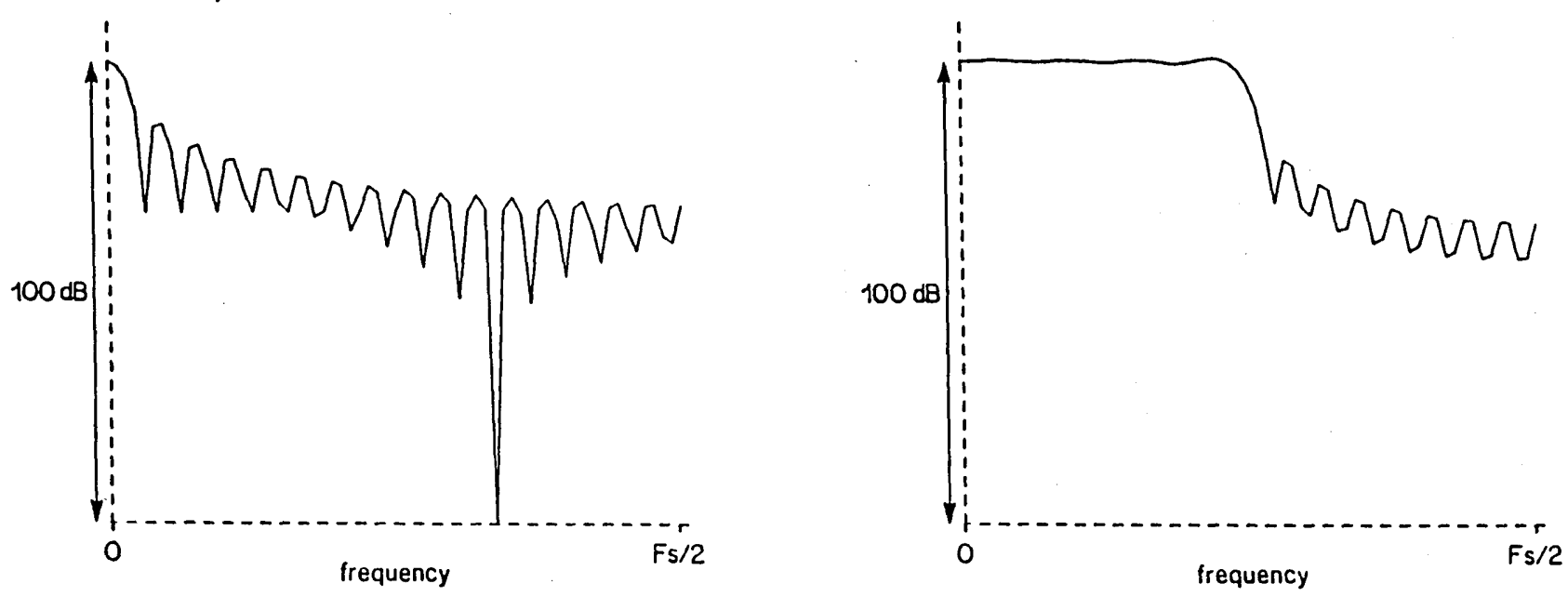

Kaiser window
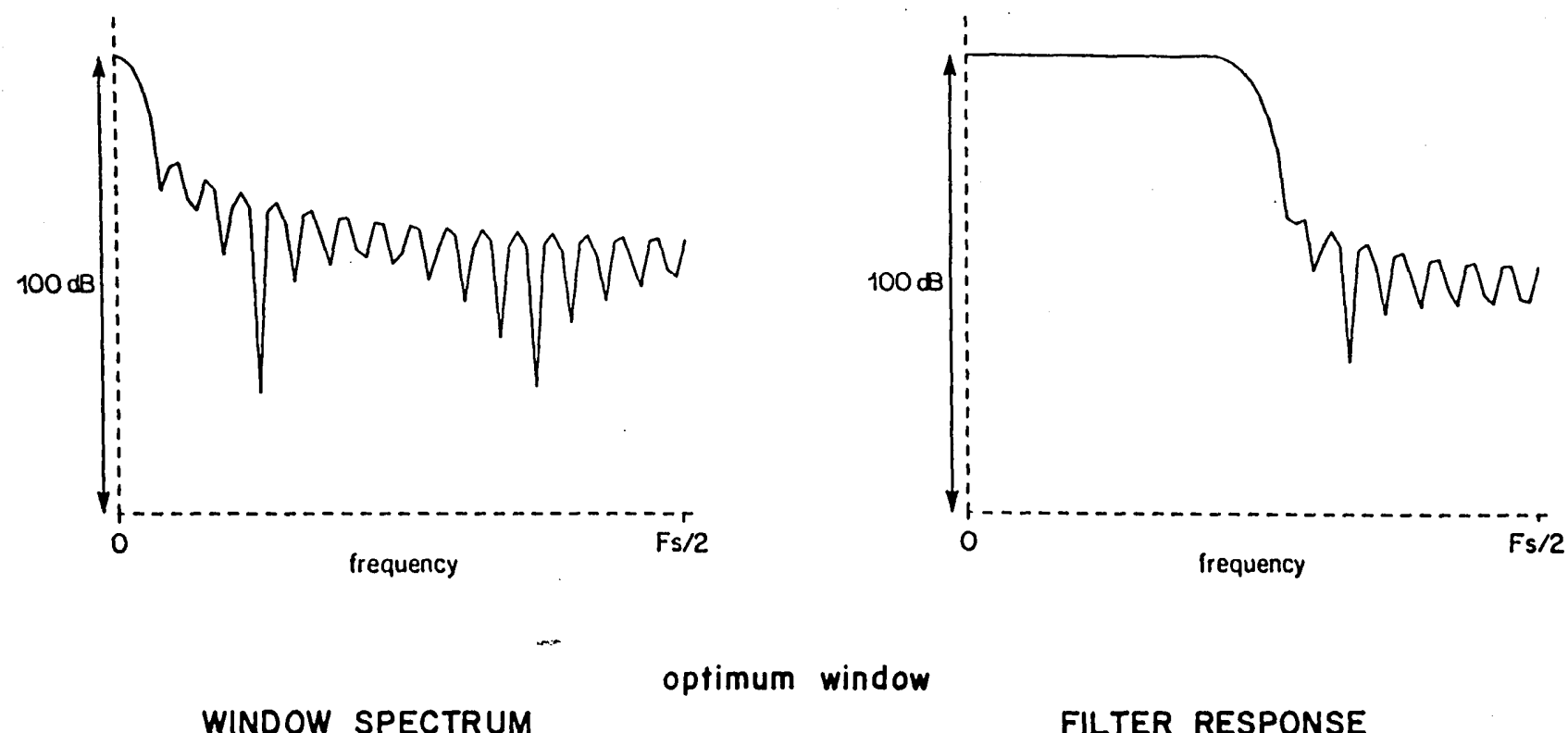

FILTER RESPONSE

Fig 11.1: Frequency responses with a transition band of \pm 1 sample 

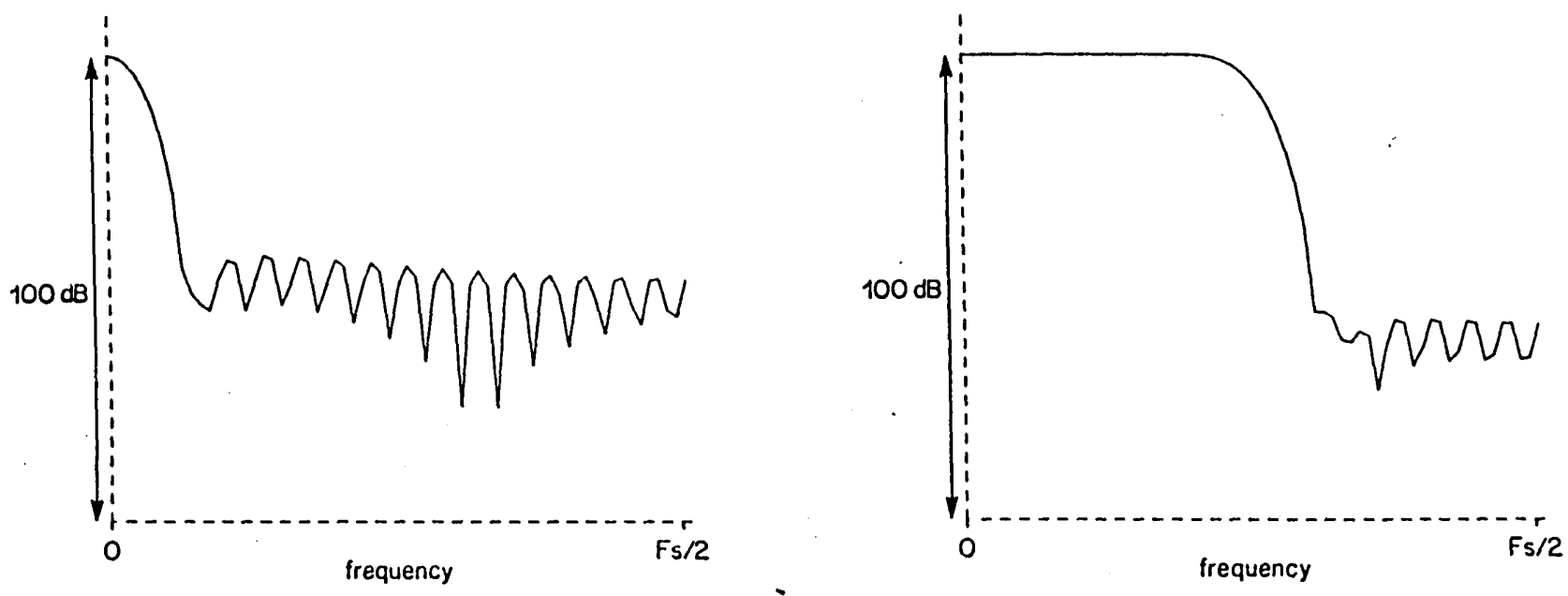

Hamming window
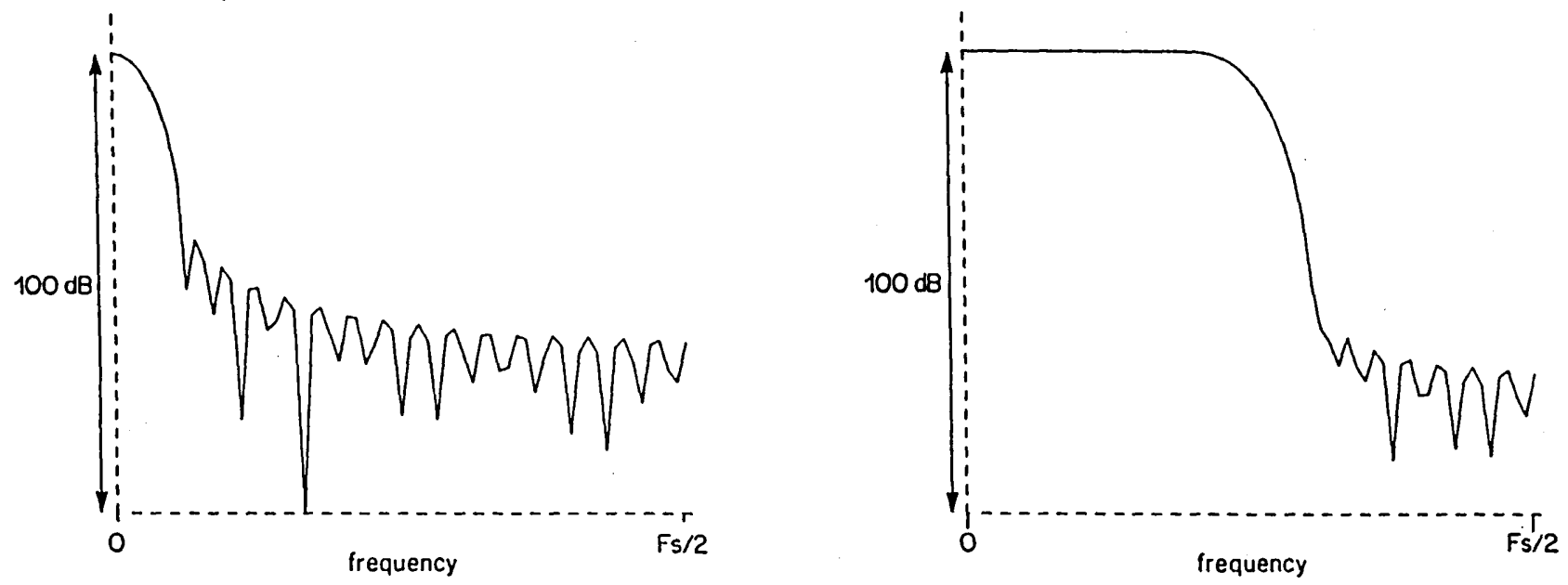

Kaiser window
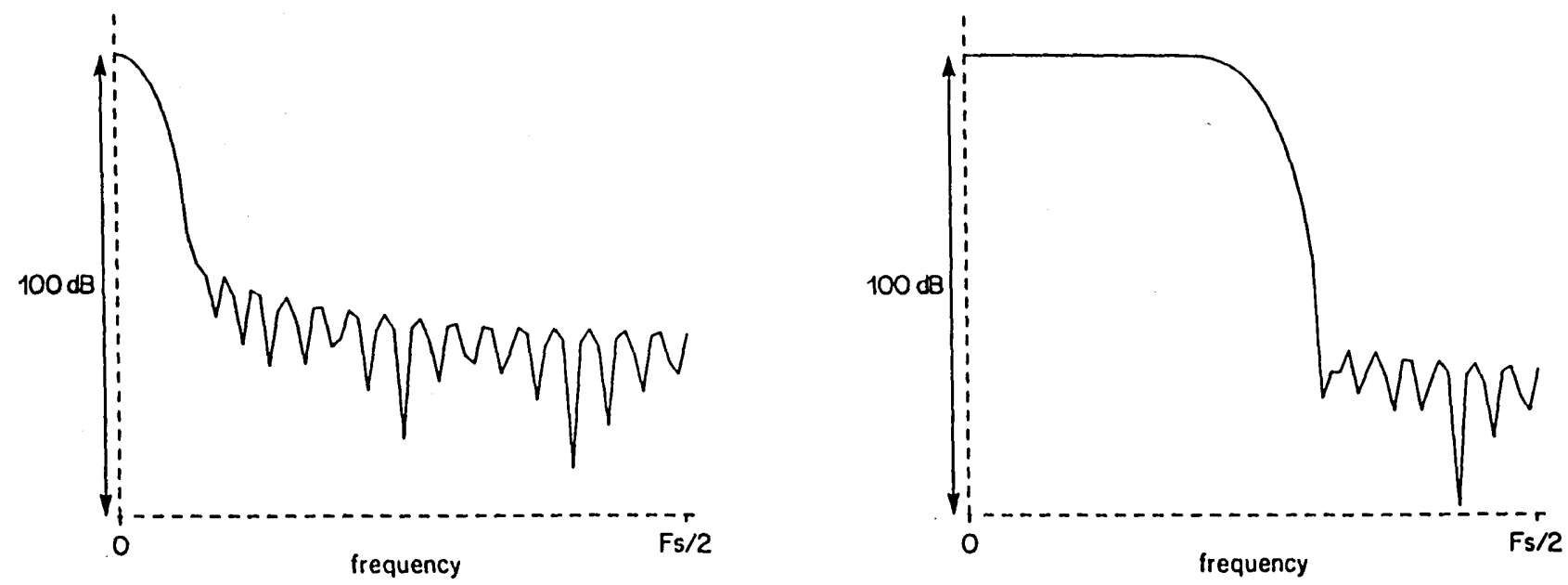

WINDOW SPECTRUM

optimum window

FILTER RESPONSE

Fig 11.2: Frequency responses with a transition band of \pm 2 samples 

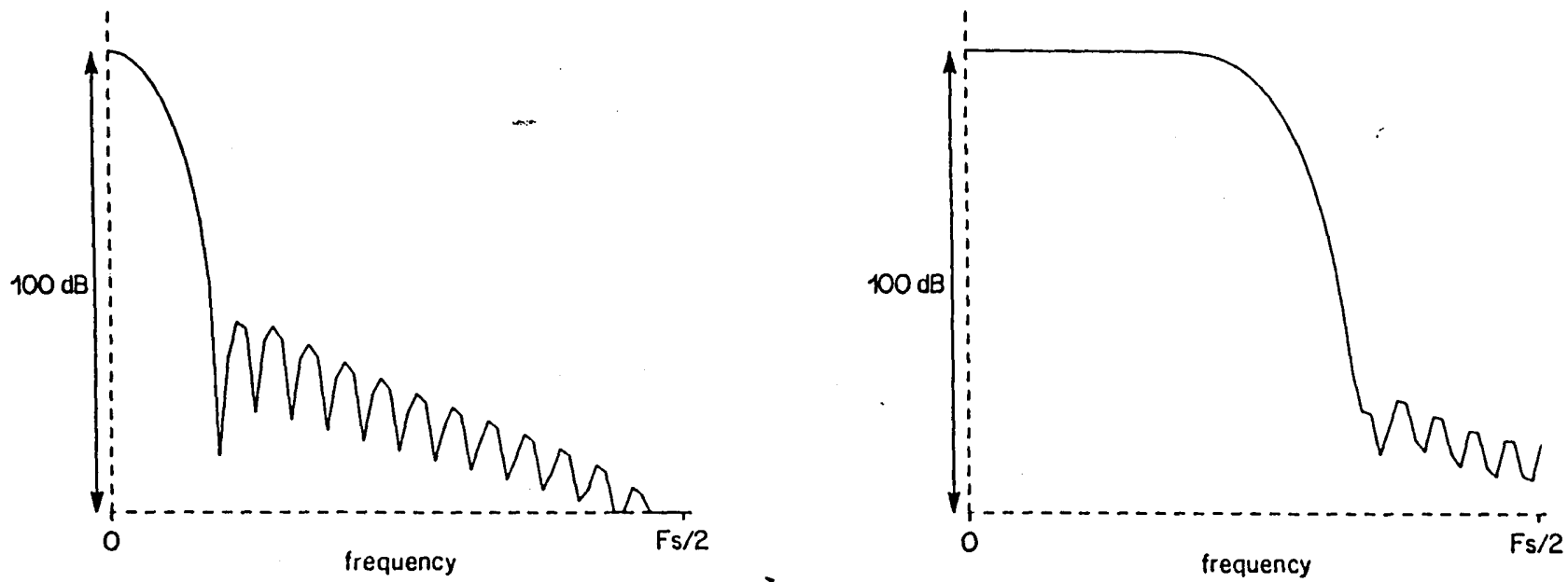

Blackman window
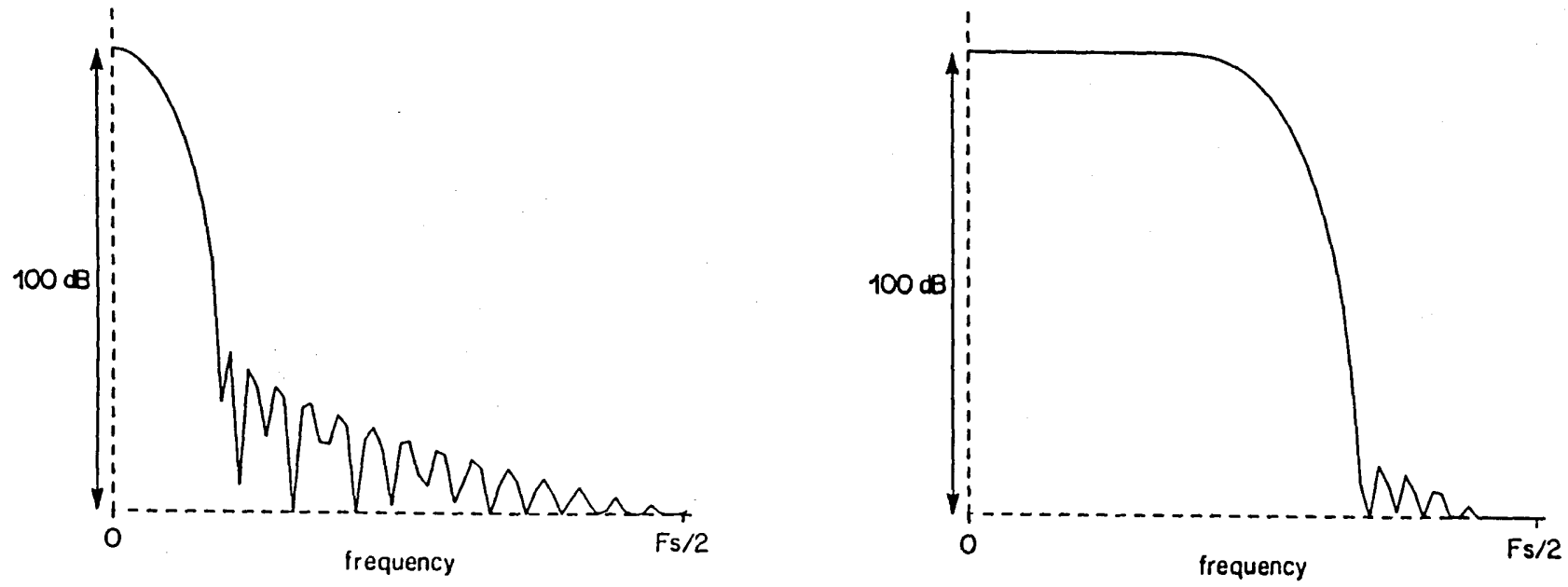

Kaiser window
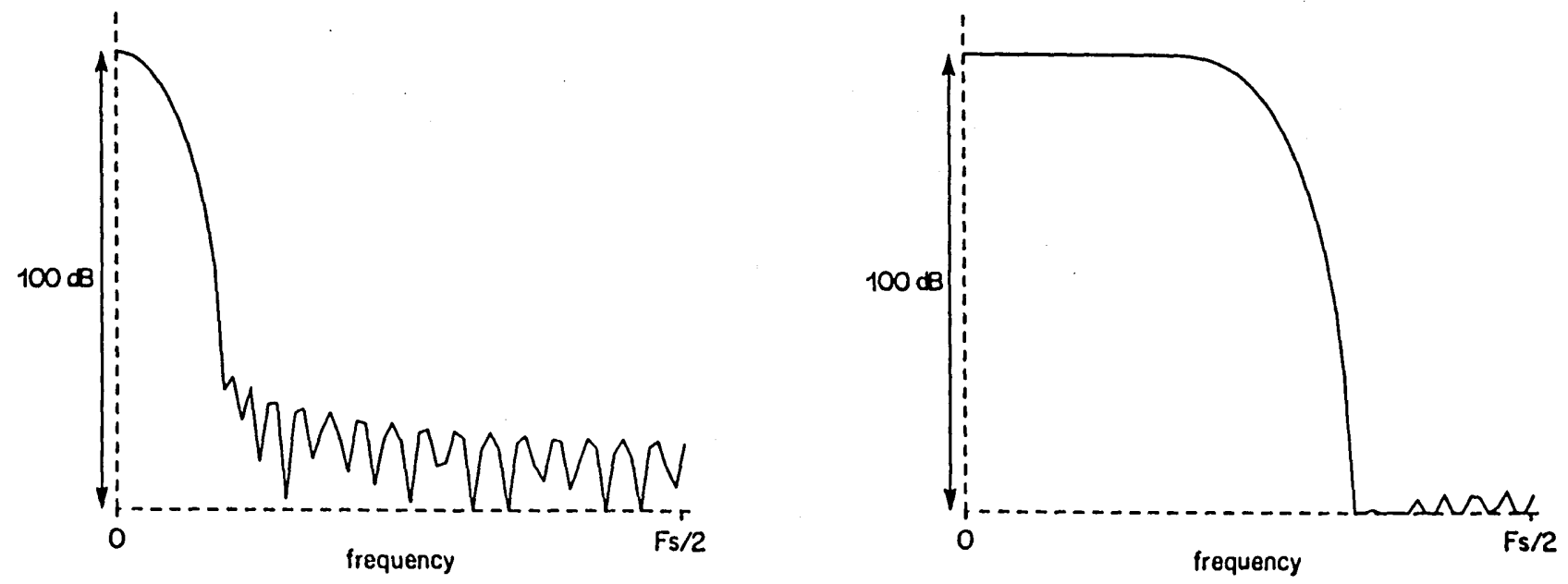

optimum window

WINDOW SPECTRUM

FILTER RESPONSE

Fig 11.3: Frequency responses with a transition band of \pm 3 samples 

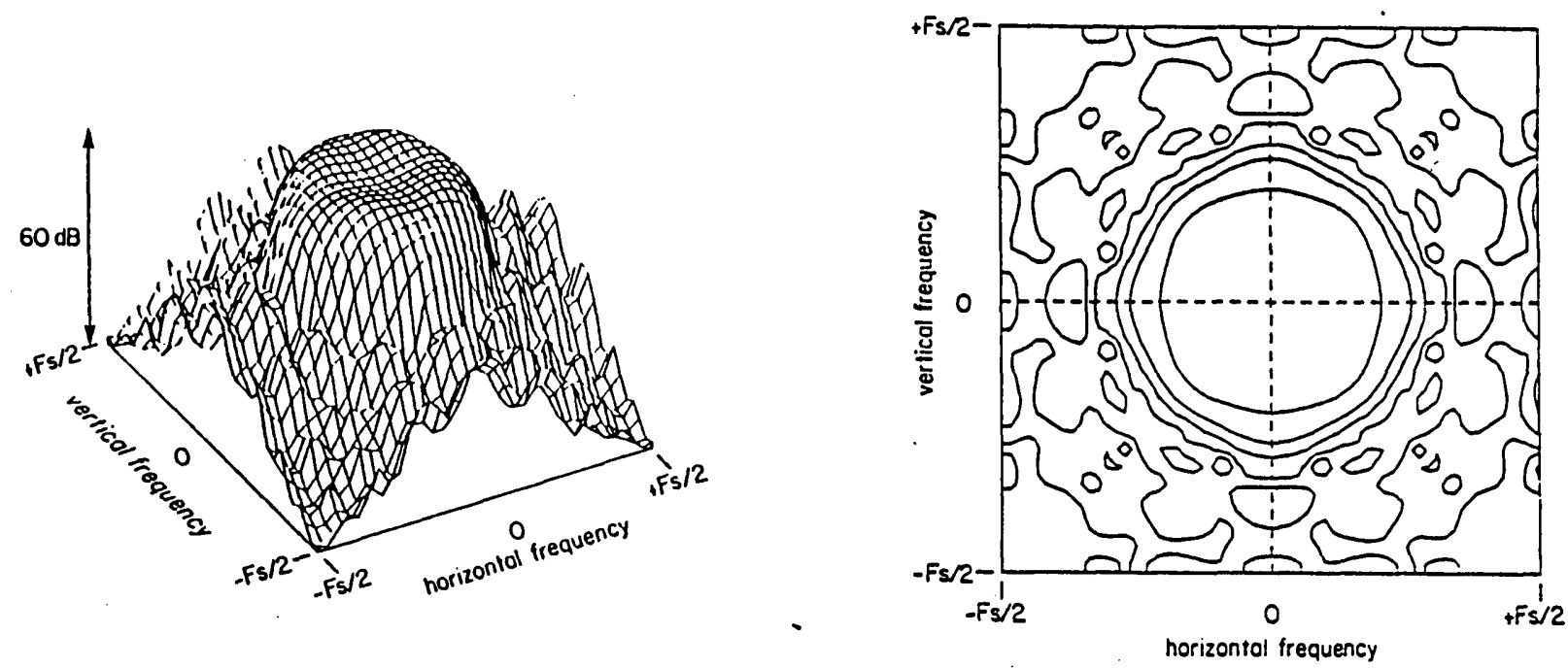

rectangular window
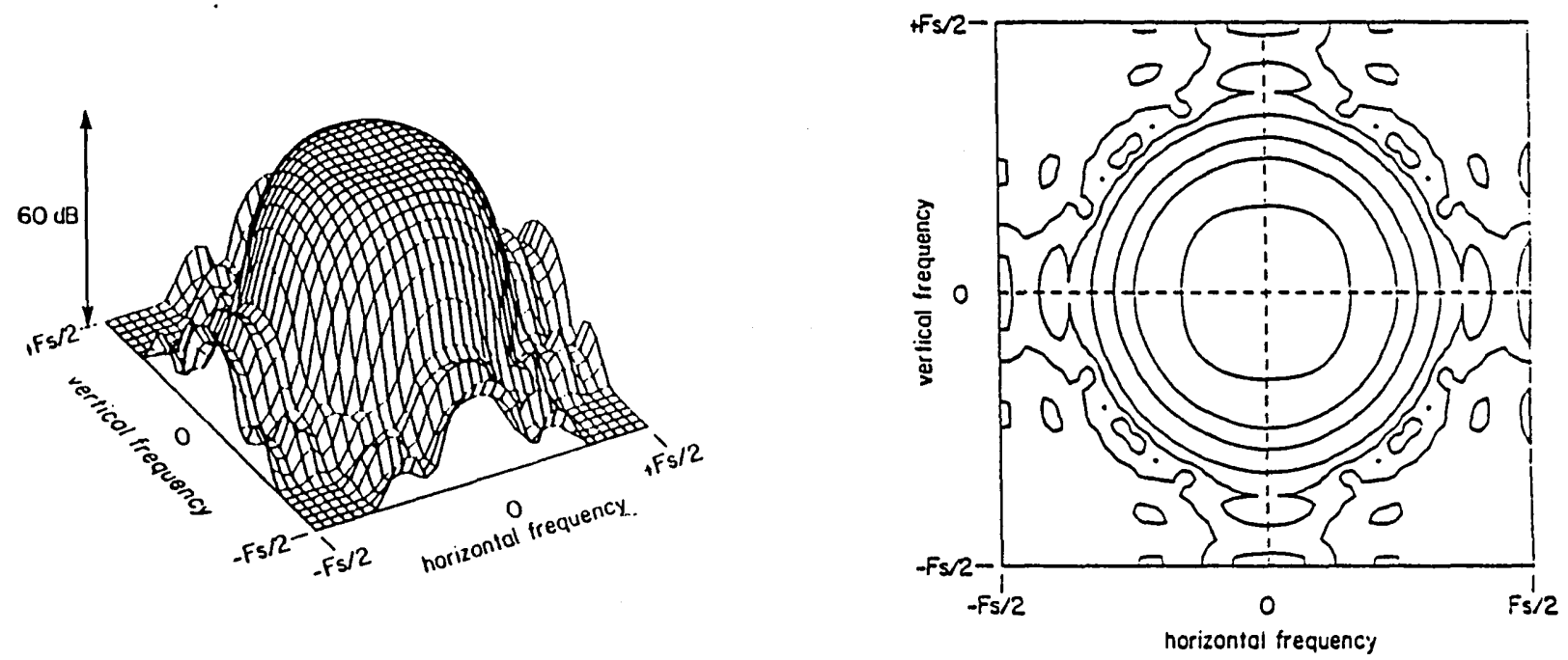

optimum $9 \times 9$ window

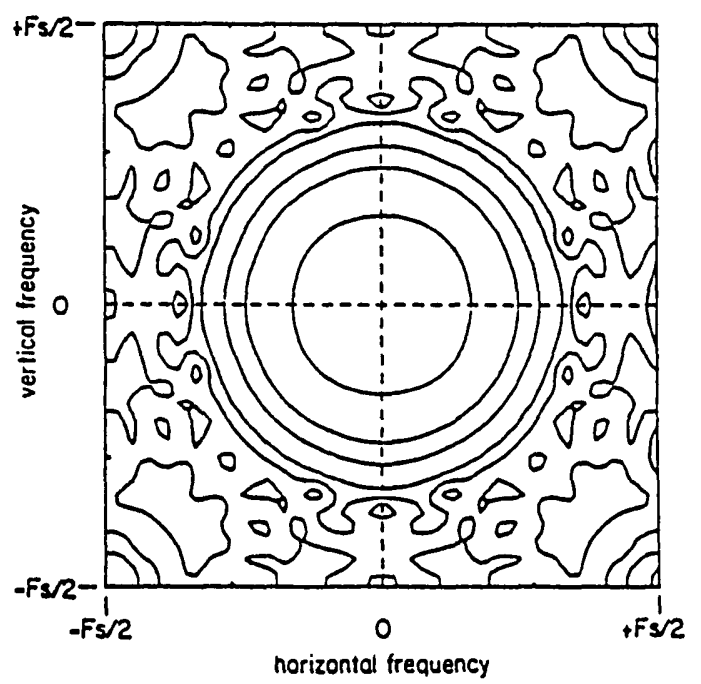




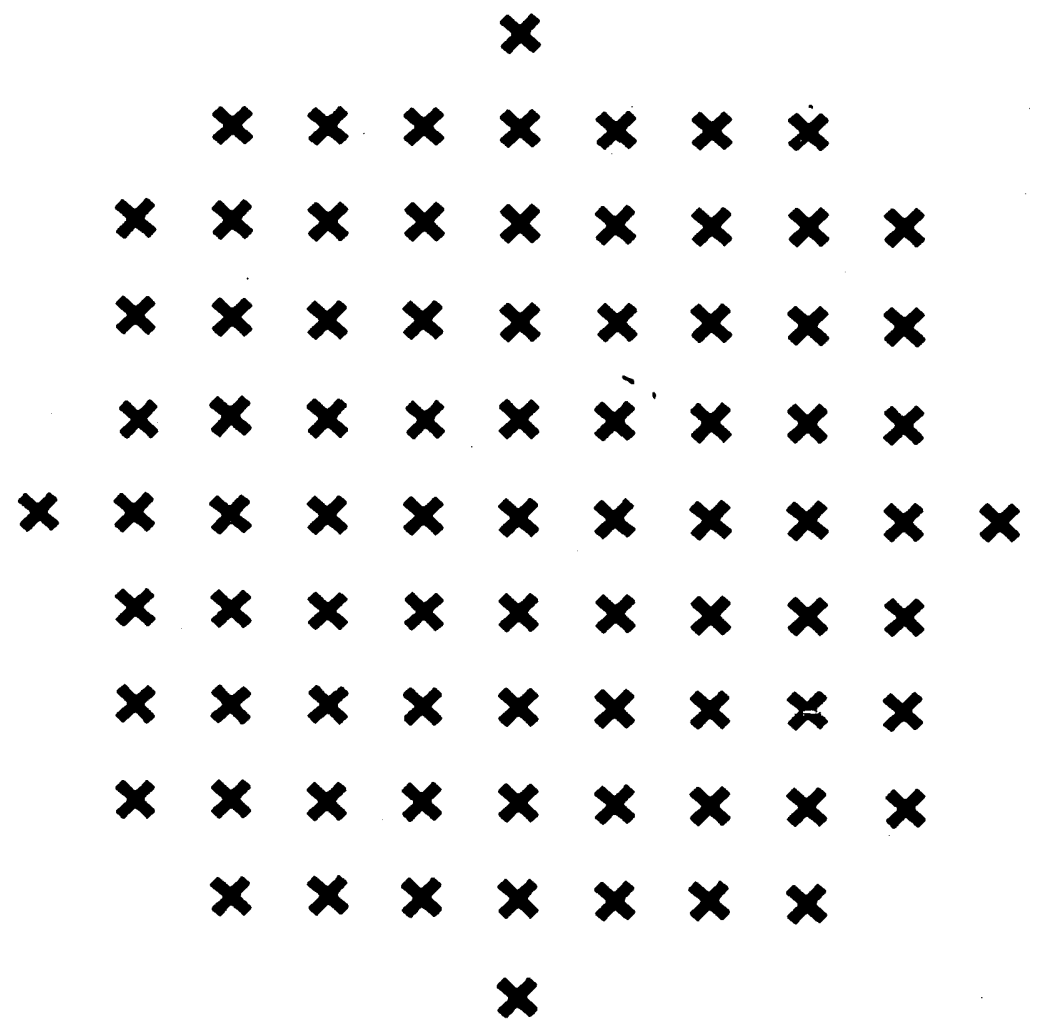

Fig 11.5: Aperture of 81 point window

UNIUERSTY OF SURTEY LIRRARY 